\title{
Abstraction in Ontology-based Data Management
}

Department of Computer, Control and Management Engineering "Antonio Ruberti"

Philosophiæ Doctor in Engineering in Computer Science - XXXII Cycle

Candidate

Gianluca Cima

ID number 1492955

Thesis Advisor

Prof. Maurizio Lenzerini
Co-Advisor

Prof. Giuseppe De Giacomo

A thesis submitted in partial fulfilment of the requirements for the degree of Philosphiæ Doctor in Engineering in Computer Science

A.Y. $2019 / 2020$ 
Thesis defended on December 07, 2020

in front of a Board of Examiners composed of:

Prof. Maria Costabile (chairperson)

Prof. Matteo Matteucci

Prof. Magdalena Ortiz (Examiner of a different EU State from where the thesis is discussed as required for the additional certificate/mention of Doctor Europaeus)

List of Reviewers:

Prof. Meghyn Bienvenu

Prof. Axel Polleres

\section{Abstraction in Ontology-based Data Management}

Philosophiæ Doctor Thesis. Sapienza - University of Rome

(C) 2020 Gianluca Cima. All rights reserved

This thesis has been typeset by $\mathrm{LT}_{\mathrm{E}} \mathrm{X}$ and the Sapthesis class.

Version: December 9, 2020

Author's Website: https://sites.google.com/view/gianlucacima

Author's Email: gianlucacima@gmail.com 


\section{Acknowledgments}

First of all, I would like to express my sincere gratitude to my advisor, Maurizio Lenzerini, for his constant support and enthusiastic encouragement during all my PhD journey. Without his help this work would never have been accomplished.

I feel very luck to have been part of the "Data Management and Service-Oriented Computing" research group at the "Department of Computer, Control and Management Engineering" of "Sapienza University of Rome". The working environment is very stimulating, and I thank all the members of the group I have worked with. In particular, I am very grateful to Antonella Poggi for her invaluable advices and the several fruitful and stimulating discussions we have had together.

I am also thankful to the members of the "Knowledge Representation and Reasoning" research group at the "Department of Computer Science" of "University of Oxford", which I have joined for six months as a visiting PhD student. I have really appreciated my staying, and I am pleased with the work we have done together.

Finally, I would like to thank all the people whose company and friendship I have enjoyed over these years, and all my family members for their abiding affection. A special thank goes to my loving girlfriend, Giulia Maria, for her continuous motivation and the way in which she pushes me to give my best in all that I do.

Gianluca Cima

Italy, July 2020 



\section{Abstract}

In many aspects of our society there is growing awareness and consent on the need for data-driven approaches that are resilient, transparent, and fully accountable. But in order to fulfil the promises and benefits of a data-driven society, it is necessary that the data services exposed by the organisations' information systems are welldocumented, and their semantics is clearly specified. Effectively documenting data services is indeed a crucial issue for organisations, not only for governing their own data, but also for interoperation purposes.

In this thesis, we propose a new approach to automatically associate formal semantic descriptions to data services, thus bringing them into compliance with the FAIR guiding principles, i.e., make data services automatically Findable, Accessible, Interoperable, and Reusable (FAIR). We base our proposal on the Ontology-based Data Management (OBDM) paradigm, where a domain ontology is used to provide a semantic layer mapped to the data sources of an organisation, thus abstracting from the technical details of the data layer implementation.

The basic idea is to characterise or explain the semantics of a given data service expressed as query over the source schema in terms of a query over the ontology. Thus, the query over the ontology represents an abstraction of the given data service in terms of the domain ontology through the mapping, and, together with the elements in the vocabulary of the ontology, such abstraction forms a basis for annotating the given data service with suitable metadata expressing its semantics.

We illustrate a formal framework for the task of automatically produce a semantic characterisation of a given data service expressed as a query over the source schema. The framework is based on three semantically well-founded notions, namely perfect, sound, and complete source-to-ontology rewriting, and on two associated basic computational problems, namely verification and computation. The former verifies whether a given query over the ontology is a perfect (respectively, sound, complete) source-to-ontology rewriting of a given data service expressed as a query over the source schema, whereas the latter computes one such rewriting, provided it exists. We provide an in-depth complexity analysis of these two computational problems in a very general scenario which uses languages amongst the most popular considered in the literature of managing data through an ontology. Furthermore, since we study also cases where the target query language for expressing source-to-ontology rewritings allows inequality atoms, we also investigate the problem of answering queries with inequalities over lightweight ontologies, a problem that has been rarely addressed. In another direction, we study and advocate the use of a non-monotonic target query language for expressing source-to-ontology rewritings. Last but not least, we outline a detailed related work, which illustrates how the results achieved in this thesis notably contributes to new results in the Semantic Web context, in the relational database theory, and in view-based query processing. 



\section{List of Publications}

The results presented in this thesis are part of the findings for my doctoral research work, and most of them are already published in the following scientific publications:

- Gianluca Cima, Maurizio Lenzerini, and Antonella Poggi. Answering Conjunctive Queries with Inequalities in DL-Lite $\mathscr{R}$. In Proceedings of the Thirty-Fourth AAAI Conference on Artificial Intelligence (AAAI 2020), pages 2782-2789, 2020 .

- Gianluca Cima, Domenico Lembo, Riccardo Rosati, and Domenico Fabio Savo. Controlled Query Evaluation in Description Logics through Instance Indistinguishability. In Proceedings of the Twenty-Ninth International Joint Conference on Artificial Intelligence (IJCAI 2020), pages 1791-1797, 2020.

- Gianluca Cima, Maurizio Lenzerini, and Antonella Poggi. Non-Monotonic Ontology-based Abstractions of Data Services. In Proceedings of the Seventeenth International Conference on Principles of Knowledge Representation and Reasoning (KR 2020), pages 243-252, 2020.

- Gianluca Cima, Domenico Lembo, Lorenzo Marconi, Riccardo Rosati, and Domenico Fabio Savo. Controlled Query Evaluation in Ontology-based Data Access. In Proceedings of the Nineteenth International Semantic Web Conference (ISWC 2020), volume 12506 of Lecture Notes in Computer Science, pages $128-146,2020$.

- Federico Croce, Gianluca Cima, Maurizio Lenzerini, and Tiziana Catarci. Ontology-based Explanation of Classifiers. In Proceedings of the Workshops of the EDBT/ICDT 2020 Joint Conference, volume 2578 of CEUR Electronic Workshop Proceedings, 2020.

- Gianluca Cima, Domenico Lembo, Riccardo Rosati, and Domenico Fabio Savo. Controlled Query Evaluation in Description Logics through Instance Indistinguishability (Extended Abstract). In Proceedings of the Thirty-Third International Workshop on Description Logics (DL 2020), volume 2663 of CEUR Electronic Workshop Proceedings, 2020.

- Gianluca Cima, Maurizio Lenzerini, and Antonella Poggi. Semantic Characterization of Data Services through Ontologies. In Proceedings of the TwentyEighth International Joint Conference on Artificial Intelligence (IJCAI 2019), pages 1647-1653, 2019. 
- Gianluca Cima, Charalampos Nikolaou, Egor V. Kostylev, Mark Kaminski, Bernardo Cuenca Grau, and Ian Horrocks. Bag Semantics of DL-Lite with Functionality Axioms. In Proceedings of the Eighteenth International Semantic Web Conference (ISWC 2019), volume 11778 of Lecture Notes in Computer Science, pages 128-144, 2019.

- Gianluca Cima, Charalampos Nikolaou, Egor V. Kostylev, Mark Kaminski, Bernardo Cuenca Grau, and Ian Horrocks. Bagging the DL-Lite Family Further. In Proceedings of the Thirty-Second International Workshop on Description Logics (DL 2019), volume 2373 of CEUR Electronic Workshop Proceedings, 2019 .

- Gianluca Cima, Federico Croce, Maurizio Lenzerini, Antonella Poggi, and Elian Toccacieli. On Queries with Inequalities in DL-Lite $e_{\mathcal{R}}^{\neq}$. In Proceedings of the Thirty-Second International Workshop on Description Logics (DL 2019), volume 2373 of CEUR Electronic Workshop Proceedings, 2019.

- Gianluca Cima, Maurizio Lenzerini, and Antonella Poggi. Reverse Engineering of Data Services. In Proceedings of the Twenty-Seventh Italian Symposium on Advanced Database Systems (SEBD 2019), volume 2400 of CEUR Electronic Workshop Proceedings, 2019.

- Gianluca Cima, Maurizio Lenzerini, and Antonella Poggi. Exploiting Ontologies for Explaining Data Sources Semantics. In Proceedings of Discussion and Doctoral Consortium Papers of the Eighteenth International Conference of the Italian Association for Artificial Intelligence (DDC@AI*IA 2019), volume 2495 of CEUR Electronic Workshop Proceedings, pages 33-35, 2019.

- Gianluca Cima, Giuseppe De Giacomo, Maurizio Lenzerini, and Antonella Poggi. On the SPARQL Metamodeling Semantics Entailment Regime for OWL 2 QL ontologies. In Proceedings of the Seventh International Conference on Web Intelligence, Mining and Semantics (WIMS 2017), pages 10:1-10:6, 2017.

- Gianluca Cima, Maurizio Lenzerini, and Antonella Poggi. Semantic Technology for Open Data Publishing. In Proceedings of the Seventh International Conference on Web Intelligence, Mining and Semantics (WIMS 2017), pages $1: 1,2017$.

- Gianluca Cima. Preliminary results on Ontology-based Open Data Publishing. In Proceedings of the Thirtieth International Workshop on Description Logics (DL 2017), volume 1879 of CEUR Electronic Workshop Proceedings, 2017.

- Gianluca Cima, Giuseppe De Giacomo, Maurizio Lenzerini, and Antonella Poggi. Querying OWL 2 QL Ontologies under the SPARQL Metamodeling Semantics Entailment Regime. In Proceedings of the Twenty-Fifth Italian Symposium on Advanced Database Systems (SEBD 2017), volume 2037 of CEUR Electronic Workshop Proceedings, page 165, 2017. 


\section{Contents}

1 Introduction 1

1.1 Contributions of the Thesis . . . . . . . . . . . . . 4

1.2 Structure of the Thesis . . . . . . . . . . . . . . . . . 8

\begin{tabular}{|lll}
2 & Theoretical Background & 11
\end{tabular}

2.1 Relational Databases . . . . . . . . . . . . . . . . . . . . . . . 11

2.2 Query Languages and Homomorphism . . . . . . . . . . . . . . . . . 11

$2.3 \quad$ Computational Complexity $\ldots \ldots \ldots \ldots \ldots$

2.4 View-based Query Processing . . . . . . . . . . . . . . . . . . . . . . 14

$2.4 .1 \quad$ Exact View Assumption . . . . . . . . . . . . . . . . . . . . 14

2.4 .2 Sound View Assumption . . . . . . . . . . . . . . . . 15

2.5 Description Logic Ontologies and Knowledge Bases . . . . . . . . . . 16

$2.5 .1 \quad$ DL-Lite $\mathcal{R}_{\mathcal{R}}$ Ontologies: Syntax . . . . . . . . . . . . . 16

$2.5 .2 \quad$ DL-Lite $_{\mathcal{R}}$ Ontologies: Semantics $\ldots \ldots \ldots \ldots \ldots$

$2.5 .3 \quad$ DL-Lite $_{\mathcal{R}}$ Knowledge Bases . . . . . . . . . . . . . . . . . 18

2.6 Ontology-based Data Management . . . . . . . . . . . . . . . . . . 22

2.6 .1 Mapping Specifications . . . . . . . . . . . . . . . . 23

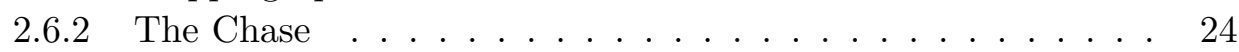

$2.6 .3 \quad$ Semantics and Query Answering . . . . . . . . . . . 24

3 Abstraction in Ontology-based Data Management: Framework 27

3.1 The notion of Source-to-Ontology Rewriting . . . . . . . . . . . . 27

3.2 Computational Problems . . . . . . . . . . . . . . . . . . . 30

3.3 Related Work . . . . . . . . . . . . . . . . . . . . . . . . 31

$3.3 .1 \quad$ A slightly different Semantics . . . . . . . . . . . . . . . 31

3.3.2 $\quad$ Relationship with Ontology-to-Source Rewritings . . . . . . . 32

$3.3 .3 \quad$ Relationship with View-based Query Processing . . . . . . . 32

$3.3 .4 \quad$ Inverse Mapping and/or Reversing the Arrows (Not!)

\begin{tabular}{|lll}
4 & Dealing with Inequalities in Lightweight Description Logics & 39
\end{tabular}

4.1 The Chase for Answering Queries with Inequalities . . . . . . . . . . 42

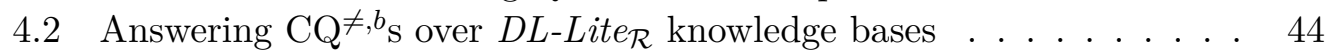

$4.2 .1 \quad$ Queries with Inequalities in Ontology-based Data Management 46

4.2 .2 The case of the Direct Semantics Entailment Regime. . . . . . 47

4.3 Answering $\mathrm{UCQ}^{\neq, b}$ s over $D L$-Lite $e_{\mathcal{R}}$ knowledge bases $\ldots \ldots \ldots$. . . . 50

$4.4 \quad$ Answering $\mathrm{UCQ}^{\neq} \mathrm{S}$ over $D L$-Lite $e_{\mathrm{RDFS}}$ knowledge bases $\ldots . . . . \quad 59$ 
4.5 Answering $\mathrm{UCQ}^{1,{ }_{\mathrm{S}}}$ over DL-Lite $e_{\mathrm{RDFS}}$ knowledge bases . . . . . . . . 64

4.6 Containment of $\mathrm{UCQ}^{\mp} \mathrm{s}$ in Relational Databases $\ldots . . . . . . .69$

4.6 .1 Lower Bounds $\ldots \ldots \ldots \ldots$. . . . . . . . . . . . . . 69

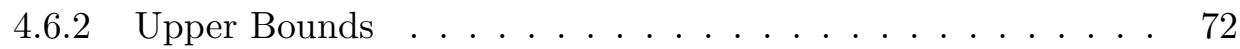

$\begin{array}{|lll|}5 & \text { Complete Source-to-Ontology Rewritings } & \mathbf{7 7}\end{array}$

5.1 Verification Problem . . . . . . . . . . . . . . . . 77

5.2 Computation Problem . . . . . . . . . . . . . . . . . . . 79

5.3 Improving by means of Inequalities . . . . . . . . . . . . . . . . . . 83

$5.4 \quad$ Dropping the UNA $\ldots \ldots \ldots \ldots$. . . . . . . . . . . . . . . . . 89

6 Sound Source-to-Ontology Rewritings 93

6.1 Verification Problem . . . . . . . . . . . . . . . . . . . . 93

6.2 Computation Problem $\ldots \ldots \ldots$. . . . . . . . . . . . . 97

$\begin{array}{lll}7 & \text { Perfect Source-to-Ontology Rewritings } & 113\end{array}$

7.1 Verification Problem . . . . . . . . . . . . . . . . . . . . . 113

7.2 Computation Problem . . . . . . . . . . . . . . . . . . . . 114

8 Sound Source-to-Ontology Rewritings in Restricted Scenarios 115

8.1 Restricted Scenario for UCQJFEs . . . . . . . . . . . . . . . . . . . 119

$8.1 .1 \quad$ Verification Problem . . . . . . . . . . . . . . . . . . . . . . . 119

$8.1 .2 \quad$ Computation Problem . . . . . . . . . . . . . . . . . . 121

8.2 Restricted Scenario for CQJFEs . . . . . . . . . . . . . . . . 128

$8.2 .1 \quad$ Verification Problem . . . . . . . . . . . . . . . . . . . 129

$8.2 .2 \quad$ Computation Problem . . . . . . . . . . . . . . 130

8.3 View-based Query Processing in the presence of Disjunctive Views . 138

9 Non-Monotonic Source-to-Ontology Rewritings 143

9.1 Towards EQL-Lite(UCQ) Abstractions . . . . . . . . . . . . . . . . . 144

9.1.1 The EQL-Lite(UCQ) Query Language . . . . . . . . . . . . . 144

$9.1 .2 \quad$ EQL-Lite(UCQ) Source-to-Ontology Rewritings. . . . . . . . . 147

9.2 On the non-existence of EQL-Lite(UCQ) Source-to-Ontology Rewritings 149

9.3 Source-to-Ontology Rewritings in a fragment of EQL-Lite(UCQ) . . 154

9.4 The case of One-To-One Mappings $\ldots \ldots$. . . . . . . . . . . . . 159

9.4.1 Complete Source-to-Ontology Rewritings . . . . . . . . . . . 160

$9.4 .2 \quad$ Sound Source-to-Ontology Rewritings . . . . . . . . . . . . . 162

$9.4 .3 \quad$ Perfect Source-to-Ontology Rewritings . . . . . . . . . . . . . 167

$\begin{array}{lr}10 \text { Conclusions } & 169\end{array}$

10.1 Discussion $\ldots \ldots \ldots \ldots \ldots$

10.2 Future Work . . . . . . . . . . . . . . . . . . . . 170

\begin{tabular}{ll}
\hline References & 173
\end{tabular} 


\section{Chapter 1}

\section{Introduction}

In many aspects of our society there is growing awareness and consent on the need for data-driven approaches that are resilient, transparent, and fully accountable. It is therefore not surprising that the architecture of many modern Information Systems is based on data services, i.e., services deployed on top of data stores, other services, and/or applications to encapsulate a wide range of data-centric operations Carey et al., 2012. Data services are also used to handle the programming logic for data virtualisation in a cloud-hosted data storage infrastructure, so as to delegate most administrative tasks to the cloud infrastructure, and effectively realising the idea of data-as-a-service Machan, 2009. Furthermore, since data may be obtuse, disorganised, and may not make much sense to most potential users, in order to get value from them, it is reasonable to resort to data services built on top of massive amount of raw data.

However, in order to fulfil the promises and benefits of a data-driven society Pentland, 2013, it is of vital importance to well document and clearly specify the semantics of data services. Effectively documenting data services is indeed a crucial issue for organisations, not only for governing their own data, that often grow rapidly in the current Big Data era Chen et al., 2014, but also for interoperation purposes. Most current techniques manually associate APIs (Application Programming Interfaces) to data services, and describe their intended meaning with ad-hoc methods, often using natural language or complex metadata ${ }^{1}$ Zheng et al. 2013. This is clearly insufficient since such description of data services lack support for a formal semantics, and ergo, they are limited to human consumption only. Contrariwise, data service consumers need access to enhanced metadata, which are both machine-readable and human-readable. These metadata are essential to integrate entities returned from different data services and/or to understand the relationships between various data services, so as to be able to formulate queries and navigate between sets of entities.

In this thesis, we propose a new approach, whose goal is to automatically associate formal semantic descriptions to data services, thus bringing them into compliance with the FAIR guiding principles Wilkinson et al., 2016, i.e., make data services automatically Findable, Accessible, Interoperable, and Reusable (FAIR).

The proposal of the thesis is based on the Ontology-based Data Management

\footnotetext{
${ }^{1}$ As defined in Duval et al., 2002, metadata are "structured data about data".
} 
$\left(O B D M^{2}\right)$ paradigm Lenzerini, 2011, which is an advanced approach to semantic data integration De Giacomo et al., 2018 experimented and used in the practice in the last years (see, e.g., [Antonioli et al., 2014, Kharlamov et al., 2017]). OBDM is a promising attempt to give principles and techniques to effectively govern even modern, complex information systems by providing a unified access to data. An OBDM specification consists of a triple $\Sigma=\langle\mathcal{O}, \mathcal{S}, \mathcal{M}\rangle$, where $\mathcal{O}$ is an ontology expressed in a specific Description Logic language, $\mathcal{S}$, called source schema, is the schema of the data sources forming the data layer of an information system, and $\mathcal{M}$ is a mapping between the source schema and the ontology, i.e., an explicit representation of the correspondence between the data sources and the elements of the ontology. The ontology is a formal logic-based representation of the underlying domain that gives a high-level view of the information contained in the data sources. Thus, the OBDM paradigm provides a means for managing data through the lens of an ontology Lenzerini, 2018, and enables the application of Knowledge Representation and Reasoning principles and techniques to various data management tasks.

But how can we automatically produce a semantic characterisation of a data service, having an OBDM specification available? The idea is to exploit a novel reasoning task over the OBDM specification, which we call abstraction, that works as follows: given a data service expressed as a query $q_{\mathcal{S}}$ over the source schema, automatically derive a query $q_{\mathcal{O}}$ over the ontology that describes "at best" the data service $q_{\mathcal{S}}$ with respect to the underlying OBDM specification $\Sigma=\langle\mathcal{O}, \mathcal{S}, \mathcal{M}\rangle$. Thus, $q_{\mathcal{O}}$ represents an abstraction of the data service represented by $q_{\mathcal{S}}$ in terms of the domain ontology $\mathcal{O}$ through the mapping $\mathcal{M}$. In this way, the query expression $q_{\mathcal{O}}$, together with the elements in the vocabulary of the ontology $\mathcal{O}$, form a basis for annotating the data service represented by the query $q_{\mathcal{S}}$ with suitable metadata expressing its semantics. The next example illustrates this idea.

Example 1.1. Let $\Sigma=\langle\mathcal{O}, \mathcal{S}, \mathcal{M}\rangle$ be the following OBDM specification:

- $\mathcal{O}=\left\{\right.$ IndependentPolitician $\sqsubseteq$ Politician, $\exists$ HasTutor $^{-} \sqsubseteq$ Professor $\}$

- $\mathcal{S}=\left\{s_{1}, s_{2}, s_{3}\right\}$

- $\mathcal{M}=\left\{m_{1}, m_{2}, m_{3}, m_{4}\right\}$, where:

$$
\begin{array}{rrrr}
m_{1}: & s_{1}(x) & \rightarrow & \text { Professor }(x), \\
m_{2}: & s_{1}(x) & \rightarrow & \text { Politician }(x), \\
m_{3}: & s_{2}\left(x_{1}, x_{2}\right) & \rightarrow & \text { HasTutor }\left(x_{1}, x_{2}\right), \\
m_{4}: & s_{3}(x) & \rightarrow & \text { IndependentPolitician }(x) .
\end{array}
$$

Let the data service be expressed as the logical query $q_{\mathcal{S}}=\left\{(x) \mid\left(s_{1}(x)\right) \vee\right.$ $\left.\left(\exists y . s_{2}(y, x) \wedge s_{3}(x)\right)\right\}$ over the source schema $\mathcal{S}$. Conceivably, by inspecting the mapping assertions in $\mathcal{M}$ and the ontology assertions in $\mathcal{O}$, one can argue that the query $q_{\mathcal{O}}$ over the ontology $\mathcal{O}$ that describes at best the data service $q_{\mathcal{S}}$ with respect to the OBDM specification $\Sigma$ is $q_{\mathcal{O}}=\{(x) \mid \operatorname{Professor}(x) \wedge$ Politician $(x)\}$.

\footnotetext{
${ }^{2}$ Throughout the thesis, it is preferred the usage of the acronym OBDM rather than its similar $O B D A$, which stands for Ontology-based Data Access Poggi et al., 2008, because data access is just one aspect, although one of the most important, of the more general notion of data management.
} 
As testified by Poggi et al., 2008, Calvanese et al., 2009: Bienvenu, 2016; Xiao et al., 2018; Ortiz, 2018. (and references therein), most of, if not all, the literature about managing data sources through an ontology deals with users' queries expressed over the ontology, and studies the problem of finding a so-called ontologyto-source rewriting, i.e., a query over the source schema that, once executed over the data, provides the answers to the original query. Here, the problem is reversed, because we start with a source query $q_{\mathcal{S}}$ over the source schema, and we aim at deriving a corresponding query $q_{\mathcal{O}}$ over the ontology $\mathcal{O}$, which we call a source-to ontology rewriting, that is as much close as possible to $q_{\mathcal{S}}$, taking into account the ontology and the mapping. Thus, we deal with a sort of reverse engineering problem, which is novel in the investigation of both OBDM and data integration.

This new notion of source-to-ontology rewriting is also useful in a context strictly related to data services, namely open data publishing. In recent years, both public and private organisations have been faced with the issue of publishing open datd ${ }^{3}$ in particular with the goal of providing data consumers with suitable information to capture the semantics of their published datasets. Current practices for publishing open data, however, focus essentially on providing extensional information (often in very simple forms, such as CSV4 files), and they carry out the task of documenting data mostly by using metadata expressed in natural languages, or in terms of record structures. As a consequence, the semantics of datasets is not formally expressed in a machine-readable form. Only few recent proposals (see, e.g., Rashid et al. 2020 ) provide methodologies to associate formal semantics to datasets by means of machine-readable metadata.

When an OBDM specification is available in an organisation there is an obvious method, called top-down, to publish high-quality, semantically annotated open data that are compliant with the $\mathrm{W}_{3} \mathrm{C}^{5}$ Linked Open Data (LOD) principles Bizer et al., 2009: ( $i$ ) express the dataset to be published in terms of a SPARQL query over the ontology, (ii) compute the certain answers to the query, and (iii) publish the result of the certain answer computation, using the query expression and the elements in the vocabulary of the ontology as a basis for annotating the dataset with suitable metadata expressing its semantics. Unfortunately, in many organisations (for instance, in Public Administration) IT employees are not yet ready to formulate SPARQL queries, rather they may be tempted to directly publish a dataset as the result of the evaluation of a query $q_{\mathcal{S}}$ (over the source schema) over the data of the information system, in any structured form representing it. In order to publish publish both the content and the semantics of the dataset, it is possible to follow a method, called bottom-up, that first derive a source-to-ontology rewriting $q_{\mathcal{O}}$ of $q_{\mathcal{S}}$ and then, using $q_{\mathcal{O}}$, continues with the steps $(i i)$ and $(i i i)$ of the top-down approach.

Besides semantic characterisations of data services and open data publishing, we point out that the reasoning task of abstraction is relevant in other plethora of application scenarios, as for example:

\footnotetext{
${ }^{3}$ According to the Organisation for Economic Co-operation and Development (OECD), open data are "data that can be used by anyone without technical or legal restrictions. The use encompasses both access and reuse" OECD, 2015].

${ }^{4}$ Comma-Separated Values: https://tools.ietf.org/html/rfc4180

${ }^{5}$ World Wide Web Consortium: https://www.w3.org/
} 
- Source profiling: Source profiling Abedjan et al., 2017, Abedjan et al., 2018] is a very general term that has to do with the analysis of raw data for the purpose of understanding the source contents. The task of abstraction arguably provides a semantic-based approach to source profiling, in particular for describing the structure and the content of a data source in terms of the business vocabulary.

- Updating: As noted in Lutz et al., 2018, the concept of realization of source queries, similar to one of the notions studied here, can be used to check whether the mapping provides the right coverage for expressing the relevant data services at the ontology level. If this is not the case, then, probably, the existing data sources and/or the ontology need to be updated Lembo et al., 2017 .

- Explanation of classifiers: Understanding and explaining the decisions made by machine learning algorithms is widely recognized as a very important task for wide and safe adoption of machine learning and data mining technologies (see, e.g., European Union, Parliament and Council, 2016, Goodman and Flaxman, 2017]), especially in high-risk domains, and in dealing with bias. The task of abstraction also moves towards this direction. As an example of its potential usefulness in the explainable machine learning field, suppose to acquire the outcome of a binary classifier over tuples of data sources in the information system, and that an OBDM specification is also available. Then, it is possible to semantically describe the choices taken by such a classifier by means of a query over the domain ontology, and therefore in terms of the elements in the vocabulary of this latter. For instance, as a naive criteria for the semantic description of the classifier, one may require that the answers to the query include all the tuples classified positively, and none of the tuples classified negatively. For a more detailed discussion on this topic, the reader is referred to Croce et al., 2020.

\subsection{Contributions of the Thesis}

This thesis mainly addresses the topic of abstraction in OBDM. The principal contributions can be summarised as follows:

I. We present a formal framework for the reasoning task of abstraction in OBDM. In particular, three semantically well-founded notions are introduced, namely perfect, sound, and complete source-to-ontology rewriting, and two basic computational problems are defined, namely verification and computation. The former verifies whether a given query $q_{\mathcal{O}}$ over the ontology is a perfect (respectively, sound, complete) source-to-ontology rewriting of a data service expressed as a query $q_{\mathcal{S}}$ over the source schema, whereas the latter computes one such source-to-ontology rewriting, provided it exists.

II. Although the ideal notion is the one of perfect source-to-ontology rewriting, we show that there are cases where, with the current OBDM specification, no query over the ontology can precisely characterise the data service at hand. Thus, two further notions are introduced, namely maximally sound and minimally 
complete source-to-ontology rewriting, which intuitively aim at approximating the perfect source-to-ontology rewriting of a data service at best, with the goal of either precision (sound rewriting), or recall (complete rewriting).

III. Before of delving into the computational problems introduced in the framework, we provide a thorough analysis of the implications that the presence of inequalities in queries has in the context of lightweight ontologies, which is a problem that has been rarely addressed. This is necessary because we do study also cases where the target query language for expressing source-to-ontology rewritings allows inequality atoms. In particular, we concentrate on the problem of answering conjunctive queries with inequalities $\left(\mathrm{CQ}^{\neq} \mathrm{s}\right)$ and unions thereof $\left(\mathrm{UCQ}^{\neq} \mathrm{s}\right)$ over DL-Lite $e_{\mathcal{R}}$ knowledge bases (i.e., pairs of ontology and ABox assertions), both with and without the unique name assumption (UNA). Since it is known that the problem is in general undecidable, we explore two alternative strategies for recovering decidability, and especially tractability:

- The first strategy is to weaken the query language by restricting the application of the inequality predicate to either individuals or distinguished variables (variables representing output values) only. The resulting query language is called "UCQs with bounded inequalities" $\left(\mathrm{UCQ}^{\neq, b} \mathrm{~s}\right)$.

- The second strategy is to weaken the ontology language, so as to eliminate all the constructs introducing incomplete information resulting from existentially quantified assertions in the ontology. The outcome is a sublanguage of $D L$-Lite $_{\mathcal{R}}$, called $D L$-Lit $\overrightarrow{\mathrm{RDFS}}_{\mathrm{R}}$.

When the UNA is adopted, we prove that both the problems of answering $\mathrm{UCQ}^{\neq, b_{\mathrm{S}}}$ over $D L$-Lite $e_{\mathcal{R}}$ knowledge bases and answering $\mathrm{UCQ}^{\neq}{ }_{\mathrm{S}}$ over $D L$-Lit $e_{\mathrm{RDFS}}$ knowledge bases are a straightforward generalisation of the well-known problem of answering union of conjunctive queries (UCQs) over $D L$-Lite $e_{\mathcal{R}}$ knowledge bases, i.e., the problems are still in $\mathrm{AC}^{0}$ in data complexity (i.e., with respect to the size of the ABox only) and NP-complete in combined complexity (i.e., with respect to the size of the whole input, including the query). Afterwards, we concentrate on the case when the UNA is not adopted.

For the case of $(\mathrm{U}) \mathrm{CQ}^{\neq, b} \mathrm{~s}$, we show that answering $\mathrm{CQ}^{\neq, b}$ s over DL-Lite $e_{\mathcal{R}}^{\neq}$ knowledge bases has the same computational complexity of the UCQ case, i.e., it is in $\mathrm{AC}^{0}$ in data complexity and NP-complete in combined complexity. However, perhaps surprisingly, answering $\mathrm{UCQ}^{\neq, b_{\mathrm{S}}}$ over $D L$-Lite $\mathcal{R}_{\mathcal{R}}$ knowledge bases is $\Pi_{2}^{p}$-complete in combined complexity. Thus, unless NP $=$ coNP, the presence of union makes the problem of answering queries with inequalities over $D L$-Lite $e_{\mathcal{R}}$ knowledge bases significantly different from the UCQ case.

For the case of $D L$-Lit $\overrightarrow{\mathrm{RDFS}}$, we show that answering $\mathrm{UCQ}^{\neq} \mathrm{S}$ is decidable, and in particular coNP-complete in data complexity, and $\Pi_{2}^{p}$-complete in combined complexity. We also investigate if the number of inequalities in each disjunct plays a role in falling into intractability. We answer positively to this question, by showing that if the query has at most one inequality per disjunct, answering $\mathrm{UCQ}^{\neq} \mathrm{S}$ is PTIME-complete in data complexity, and NP-complete in combined complexity, while it is coNP-hard in data complexity if the query is conjunctive 
and has at most two inequalities. We also show that going from one to two inequalities causes the jump from NP-hardness to $\Pi_{2}^{p}$-hardness in combined complexity for $\mathrm{UCQ}^{\neq} \mathrm{s}$, and we conjecture that this holds already for $\mathrm{CQ}^{\neq} \mathrm{s}$.

We argue that the above results considerably improve our understanding of the implication that the presence of inequalities in queries has in the context of lightweight ontologies. In particular, to the best of our knowledge, our investigation on $D L$-Lit $\overrightarrow{\mathrm{RDFS}}_{\overrightarrow{\mathrm{D}}}$ provides the first results on reasoning with inequalities when querying $D L$-Lite $e_{\mathrm{RDF}}$ knowledge bases.

IV. The mentioned results on answering $\mathrm{CQ}^{\neq, b}$ s over $D L$-Lite $e_{\mathcal{R}}$ knowledge bases allows us to improve the current state-of-the-art on the problem of answering $\mathrm{UCQ}^{\neq}{ }_{\mathrm{s}}$ posed over OWL 2 QL knowledge bases interpreted under the Direct Semantics Entailment Regime (DSER), i.e., the regime usually adopted in the Semantic Web scenarios that slightly differs from the classical First Order Logic (FOL) semantics. In particular, we prove that answering $\mathrm{UCQ}^{{ }^{*}} \mathrm{~s}_{\text {over }}$ OWL 2 QL knowledge bases interpreted under DSER has the same computational complexity of the UCQ case under FOL, i.e., the problem is in $\mathrm{AC}^{0}$ in data complexity and NP-complete in combined complexity.

V. The results on reasoning with inequalities over $D L$-Lit $\overrightarrow{e_{\text {RDFS }}}$ knowledge bases implies new results on containment of queries with inequalities in the database theory setting. Specifically, we prove that the containment problem for $\mathrm{UCQ}^{\neq_{\mathrm{S}}}$ is still $\Pi_{2}^{p}$-hard in general (and therefore $\Pi_{2}^{p}$-complete) and coNP-hard (and therefore coNP-complete) when the containing query is assumed to be fixed, even if the contained query is a $\mathrm{CQ}$ and the containing query is a $\mathrm{UCQ}^{\neq}$with at most two inequality atoms. Furthermore, we prove that by allowing at most one inequality to occur in $\mathrm{UCQ}^{\neq} \mathrm{S}$ makes the computational complexity of the containment problem for $\mathrm{UCQ}^{\neq} \mathrm{s}$ falling from $\Pi_{2}^{p}$-complete down to NP-complete and, when the containing query is assumed to be fixed, from coNP-complete down to PTIME-complete. To the best of our knowledge, this is the first investigation on how the number of inequality atoms affects the computational complexity of the containment problem for $\mathrm{UCQ}^{\neq} \mathrm{s}$.

VI. We study both the verification, and the computation problem for complete, sound, and perfect source-to-ontology rewritings in a very general scenario which uses languages amongst the most popular considered in the literature: $(i)$ the setting for OBDM specifications is such that the ontology language is $D L$-Lite $_{\mathcal{R}}$, the source schemas do not have integrity constraints, and each mapping assertion maps a conjunctive query (CQ) over the source to a $\mathrm{CQ}$ over the ontology (GLAV assertion), and (ii) the query language for expressing both the data service and the source-to-ontology rewriting is the one of UCQs. We show that the verification problem for complete source-to-ontology rewritings is NP-complete, whereas it is $\Pi_{2}^{p}$-complete for both sound and perfect source-to-ontology rewritings.

As for the computation problem, we illustrate an algorithm to compute minimally complete source-to-ontology rewritings of given queries over the source schema (thus proving that they always exist), and an algorithm that, given 
a query over the source schema, it computes a perfect source-to-ontology rewriting if it exists and can be expressed as a UCQ, otherwise it reports that no such UCQ-perfect source-to-ontology rewriting exists. For the case of sound source-to-ontology rewritings, instead, we precisely determine the cases where a maximally sound source-to-ontology rewriting is not guaranteed to exists.

VII. For the case of complete source-to-ontology rewritings, we extend the general scenario by allowing inequalities to occur in the target query language for expressing source-to-ontology rewritings. We first show that $\mathrm{UCQ}^{\neq}{ }_{\mathrm{S}}$ provide better approximated complete source-to-ontology rewritings compared to UCQs, and then we present an algorithm to compute $\mathrm{UCQ}^{\neq}$-minimally complete source-to-ontology rewritings of given UCQs over the source schema, thus proving that they always exist. In this extended scenario, we also study both the verification, and the computation problem for complete source-to-ontology rewritings when the UNA is dropped.

VIII. We single out two restricted scenarios that are still meaningful from the point of view of expressive power, and guarantees the existence of maximally sound source-to-ontology rewritings. In both such restrained scenarios, we consider the setting for OBDM specifications obtained from the general one by limiting the ontology language to DL-Lite RDFS $_{\text {rather than } D L \text {-Lite }}$, and limiting the mapping language to GAV assertions rather than GLAV assertions. The difference between the two restricted scenarios is in the query language allowed for expressing data services, where in the first one, called restricted scenario for CQJFEs, is the class of conjunctive queries with join-free existential variables (CQJFEs), whereas in the second one, called restricted scenario for UCQJFEs, is the class of unions of CQJFEs (UCQJFEs). For both the restricted scenarios, we study both the verification, and the computation problem for sound sourceto-ontology rewritings.

As for the verification problem, we show that it falls from $\Pi_{2}^{p}$-complete down to coNP-complete in the restricted scenario for UCQJFEs, and even further down to tractability (i.e., in PTIME) in the restricted scenario for CQJFEs.

As for the computation problem, we first provide an algorithm to compute maximally sound source-to-ontology rewritings of given UCQJFEs over the source schema (thus proving that they are guaranteed to exists in these restricted scenarios), and then we specialise it for the case of CQJFEs.

IX. We provide a detailed relationship between the notions introduced in this thesis and the usual notions of ontology-to-source rewriting and view-based query rewriting (i.e., rewriting given queries using view definitions). For this latter long-established notion, we also present new interesting results when dealing with UCQ views, also referred as disjunctive views. Specifically, we delineate the precise dividing line between the existence and the non-existence cases of UCQ-maximally sound rewritings of UCQs with respect to disjunctive views, along the dimension of join existential variables occurring in the bodies of the various disjuncts of the given UCQ to be rewritten. 
X. Finally, we carry out an investigation of source-to-ontology rewritings expressed in a non-monotonic query language. In this endeavour, the choice of the nonmonotonic query language is the class of EQL-Lite(UCQ) queries, a particularly well-behaved fragment of $E Q L$ queries. Such a language incorporates a single modal knowledge operator $\mathbf{K}$, which is used to formalise the epistemic state of the OBDM system. As a first contribution, we show how queries of such a non-monotone query language can be rewritten as FOL queries over the source schema to compute certain answers. We then show that EQL-Lite(UCQ) queries provides a better means to compute abstractions of data services compared to the language of UCQs. In particular, there are cases where the perfect source-to-ontology rewriting of a query over the source schema is expressible as a EQL-Lite(UCQ) query, but not as a UCQ. Also, there are cases where a maximally sound source-to-ontology rewriting exists in the class of EQL-Lite(UCQ) queries, but not in the class of UCQs, and cases where a maximally sound (respectively, minimally complete) source-to-ontology rewriting of a query over the source schema is a better approximation than the analogous in the class of UCQs.

On the other hand, similarly to UCQs, we prove that there are cases where no maximally sound source-to-ontology rewriting exists in the class of EQL-Lite(UCQ) queries. Quite surprisingly, we prove that the same holds for minimally complete source-to-ontology rewritings. In order to address the issue of non-expressibility, we explore two special scenarios. In the first one, we limit the query language, and consider a fragment, still non-monotonic, of EQL-Lite(UCQ) queries, called EQL-Lite ${ }^{-}(\mathrm{UCQ})$ queries, where both nested negation and union are not allowed. In the second one, we limit the mapping language, and consider the so-called One-To-One mapping, where each mapping assertion links one source relation to one ontology element. For both scenarios, we address the problem of computing minimally complete, and maximally sound source-to-ontology rewritings of source queries, presenting algorithms whenever possible.

\subsection{Structure of the Thesis}

The thesis is organised in ten chapters, whose content is briefly summarised below:

- Chapter 1 is the current introduction.

- Chapter 2 introduces the relevant theoretical background needed to understand the thesis.

- Chapter 3 illustrates a formal framework for the task of abstraction in OBDM. Here, the various notions of source-to-ontology rewritings are introduced, some associated computational problems are defined, and a detailed comparison with related work is outlined.

- Chapter 4 deals with the problem of answering queries with inequalities over lightweight ontologies. In addition, it presents some interesting implications 
that our technical results have on the problem of answering queries with inequalities in the Semantic Web context, and on the containment problem for queries with inequalities in the relational database theory.

- Chapter 5 studies both the verification, and the computation problem for complete source-to-ontology rewritings by providing algorithms and characterising the complexity of both tasks. For this notion, it further studies the computation problem when the target query language allows inequality atoms.

- Chapter 6 studies both the verification, and the computation problem for sound source-to-ontology rewritings. It first analyses the computational complexity of the verification problem, and then precisely determine the cases where a maximally sound source-to-ontology rewriting is not guaranteed to exists.

- Chapter 7studies both the verification, and the computation problem for perfect source-to-ontology rewritings by providing algorithms and characterising the complexity of both tasks.

- Chapter 8 studies both the verification, and the computation problem for sound source-to-ontology rewritings in two restricted scenarios. For both scenarios, it provides algorithms and complexity results for both the verification, and the computation problem for sound source-to-ontology rewritings. Furthermore, it illustrates new interesting results on view-based query processing, particularly on the problem of rewriting queries using disjunctive views.

- Chapter 9 carries out an investigation of source-to-ontology rewritings when the target query language is non-monotonic. It first argues that non-monotonicity is an important feature when providing abstractions of data services. Then, it determines cases where both maximally sound, and minimally complete sourceto-ontology rewritings are not guaranteed to exist in the chosen non-monotonic query language. Lastly, the chapter studies the computation problem for both maximally sound, and minimally complete source-to-ontology rewritings in two special scenarios.

- Finally, Chapter 10 concludes the thesis with a brief discussion and possible directions for future work. 



\section{Chapter 2}

\section{Theoretical Background}

In this chapter, we recall some basic theoretical notions and related results which will be used in following discussions.

\subsection{Relational Databases}

A relational database schema (or simply schema) $\mathcal{S}$ is a finite set of predicate symbols, each with a specific arity, and a set of integrity constraints. Given a schema $\mathcal{S}$, an $\mathcal{S}$-database $D$ is a finite set of facts $s(\vec{c})$ satisfying all integrity constraints in $\mathcal{S}$, where $s$ is an $n$-ary predicate symbol of $\mathcal{S}$, and $\vec{c}=\left(c_{1}, \ldots, c_{n}\right)$ is an $n$-tuple of constants, each taken from a countable infinite set of symbols denoted by Const.

An incomplete $\mathcal{S}$-database $\mathcal{W}$ is like an $\mathcal{S}$-database, but where also variables are allowed as terms. Formally, it is a finite set of atoms $s(\vec{t})$ over $\mathcal{S}$, where $s$ is an $n$-ary predicate symbol of $\mathcal{S}$, and $\vec{t}=\left(t_{1}, \ldots, t_{n}\right)$ is a $n$-tuple of terms in which $t_{i}$ is either a constant or a variable, for each $i \in[1, n]$. As usual, each variable is taken from a countable infinite set of symbols denoted by $\operatorname{Var}$, where Const $\cap \operatorname{Var}=\emptyset$. We will use variables to represent unknown values [Imielinski and Lipski Jr., 1984], rather than non-existent values [Zaniolo, 1982]. For an incomplete $\mathcal{S}$-database $\mathcal{W}$, we denote by $\operatorname{dom}(\mathcal{W})$ the set of all terms (i.e., constants and variables) occurring in $\mathcal{W}$.

\subsection{Query Languages and Homomorphism}

In its general form, an $\mathcal{L}_{\mathcal{S}}$ query $q$ over a schema $\mathcal{S}$ is a function in a certain query language $\mathcal{L}_{\mathcal{S}}$ that can be evaluated over an $\mathcal{S}$-database $D$ to return a set of answers $q^{D}$, each answer being a tuple of constants.

We assume to deal with databases supporting queries in First-Order Logic (FOL). An FOL query $q$ over a schema $\mathcal{S}$ is a query of the form $q=\{\vec{t} \mid \phi(\vec{x})\}$, also denoted $q(\vec{t})$, where $\vec{t}$, called the target list of $q$, is an $n$-tuple of terms $(\operatorname{ar}(q)=n$, where $\operatorname{ar}(q)$ denotes the arity of $q$ ), and $\phi(\vec{x})$, called the body of $q$, is an FOL formula over the predicates of $\mathcal{S}$ in which all the free variables (i.e., the variables in the tuple $\vec{x}$ ), called the distinguished variables of $q$, occur in $\vec{t}$. As usual, we impose that each variable $x$ occurring in $\vec{t}$ must also appear in some atom of $\phi(\vec{x})$. When $\operatorname{ar}(q)=0$, the query is called boolean. Given an FOL query $q=\left\{\left(t_{1}, \ldots, t_{n}\right) \mid \phi(\vec{x})\right\}$ of arity $n$ and an $n$-tuple of constants $\vec{c}=\left(c_{1}, \ldots, c_{n}\right)$ such that $c_{j}=t_{j}$ for each $j \in[1, n]$ in 
which $t_{j}$ is a constant, we denote by $q(\vec{c})=\{() \mid \phi(\vec{x} / \vec{c})\}$ the boolean FOL query without free variables, in which $\phi(\vec{x} / \vec{c})$ is the FOL sentence obtained from $\phi(\vec{x})$ by replacing all the occurrences of the term $t_{i}$ with the constant $c_{i}$, for each $i \in[1, n]$.

A conjunctive query $(C Q) q$ over a schema $\mathcal{S}$ is an FOL query of the form $q=\{\vec{t} \mid \exists \vec{y} \cdot \phi(\vec{x}, \vec{y})\}$, where $\vec{y}$ is the tuple of existential variables of $q$, each variable occurring in $\vec{y}$ appears in $\phi(\vec{x}, \vec{y}), \vec{x}$ is the tuple of distinguished variables of $q$, and $\phi(\vec{x}, \vec{y})$ is either $\perp(\vec{x}, \vec{y})$, or a finite conjunction of atoms of the form $s\left(t_{1}^{\prime}, \ldots, t_{n}^{\prime}\right)$, where $s$ is an $n$-ary predicate of $\mathcal{S}$, and term $t_{i}^{\prime}$ is either a constant or a variable occurring in $\vec{x}$ or $\vec{y}$, for each $i=[1, n]$. Given a CQ $q=\{\vec{t} \mid \exists \vec{y} \cdot \phi(\vec{x}, \vec{y})\}$, we say that an existential variable $y$ occurring in $\vec{y}$ is a join existential variable of $q$ if it occurs more than once in the atoms of $\phi(\vec{x}, \vec{y})$. In what follows, we also consider a subclass of CQs, namely conjunctive queries with join-free existential variables (CQJFEs). A CQ $q$ is also a CQJFE if there is no join existential variable occurring in $q$.

A conjunctive query with inequalities $\left(C Q^{\neq}\right)$over a schema $\mathcal{S}$ is an expression of the form $q=\{\vec{t} \mid \exists \vec{y} \cdot \phi(\vec{x}, \vec{y})\}$ similar to a CQ, but where $\phi(\vec{x}, \vec{y})$ may additionally contains inequality atoms, i.e., atoms of the form $\neq\left(z_{1}, z_{k}\right)$, also denoted by $z_{i} \neq z_{k}$, where both $z_{i}$ and $z_{k}$ are either constants or variables in $\vec{x}$ or $\vec{y}$. As usual, we impose safeness Abiteboul et al., 1995, i.e., each variable $z$ occurring in an inequality atom also occurs in an atom that is not an inequality atom.

When convenient, we treat tuples as sets, in those cases we implicitly refer to the set of all the terms occurring in the tuple. For a $\mathrm{CQ}^{\neq} q=\{\vec{t} \mid \exists \vec{y} \cdot \phi(\vec{x}, \vec{y})\}$, notice that $\vec{x} \subseteq \vec{t}$ and $\vec{t} \cap \vec{y}=\emptyset$. Furthermore, when convenient, we treat $\mathrm{CQ}^{\neq} \mathrm{s} q$ and their bodies $\phi(\vec{x}, \vec{y})$ as a set of atoms, in those cases we implicitly refer to the set of all the atoms occurring in $\phi(\vec{x}, \vec{y})$ that are not inequality atoms. In particular, for a $\mathrm{CQ}^{\neq} q=\{\vec{t} \mid \exists \vec{y} \cdot \phi(\vec{x}, \vec{y})\}$ over a schema $\mathcal{S}$, we denote $(i)$ by $\mathcal{W}_{q}$ the incomplete $\mathcal{S}$-database associated to $q$, i.e., the set of all atoms over $\mathcal{S}$ occurring in $\phi(\vec{x}, \vec{y})$ that are not inequality atoms, and $(i i)$ by $D_{q}$ (called the freezing of $q$ ) the $\mathcal{S}$-database associated to $q$, i.e., the set of facts over $\mathcal{S}$ obtained from $\mathcal{W}_{q}$ by replacing each variable $v$ occurring in $\mathcal{W}_{q}$ with a different fresh constant denoted by $c_{v}$.

A class of queries laying between CQs and $\mathrm{CQ}^{\neq} \mathrm{S}$ is the class of conjunctive queries with bound inequalities $\left(C Q^{\neq, b} s\right)$. A $\mathrm{CQ}^{\neq, b} q=\{\vec{t} \mid \exists \vec{y} \cdot \phi(\vec{x}, \vec{y})\}$ is a $\mathrm{CQ}^{\neq}$ whose inequality atoms involve only constants or distinguished variables, i.e., for each inequality atom $z_{i} \neq z_{k}$ appearing in $\phi(\vec{x}, \vec{y})$, we have both $z_{i} \notin \vec{y}$ and $z_{k} \notin \vec{y}$.

Another class between CQs and $\mathrm{CQ}^{\neq} \mathrm{S}$ is the class of conjunctive queries with at most $k$ inequalities $\left(C Q^{k, \neq}\right)$. $\mathrm{A} \mathrm{CQ}^{k, \neq}$ is a $\mathrm{CQ}^{\neq}$having at most $k$ inequality atoms.

Finally, a $U C Q^{\neq}$(respectively, $U C Q^{\neq, b}, U C Q^{k, \neq}, U C Q, U C Q J F E$ ) is a union of a finite set of $\mathrm{CQ}^{\neq} \mathrm{s}$ (respectively, $\mathrm{CQ}^{\neq, b} \mathrm{~s}, \mathrm{CQ}^{k, \neq} \mathrm{s}, \mathrm{CQs}$, CQJFEs) with same arity, called its disjuncts.

To define the evaluation of $\mathrm{UCQ}^{{ }^{*}} \mathrm{~s}$ over $\mathcal{S}$-databases, we resort to the notion of homomorphism. Given two (possibly infinite) sets of atoms $\mathcal{W}$ and $\mathcal{W}^{\prime}$, a homomorphism from $\mathcal{W}$ to $\mathcal{W}^{\prime}$ is a function $h: \operatorname{dom}(\mathcal{W}) \rightarrow \operatorname{dom}\left(\mathcal{W}^{\prime}\right)$ for which:

- $h(c)=c$ for each constant $c \in$ Const $\cap \operatorname{dom}(\mathcal{W})$; and

- $h(\mathcal{W}) \subseteq \mathcal{W}^{\prime}$

where $h(\mathcal{W})$ is the image of $\mathcal{W}$ under $h$, i.e., $h(\mathcal{W})=\{h(\alpha) \mid \alpha \in \mathcal{W}\}$ and $h\left(s\left(t_{1}, \ldots, t_{n}\right)\right)=s\left(h\left(t_{1}\right), \ldots, h\left(t_{n}\right)\right)$ for each atom $\alpha=s\left(t_{1}, \ldots, t_{n}\right)$. 
Given a $\mathrm{CQ}^{\neq} q=\{\vec{t} \mid \exists \vec{y} \cdot \phi(\vec{x}, \vec{y})\}$, the evaluation of $q$ over a (possibly infinite) set of atoms $\mathcal{W}$ is the set $q^{\mathcal{W}}$ of tuples of terms $\vec{c}$ such that there exists a homomorphism $h$ from $\mathcal{W}_{q}$ (i.e., the set of all atoms occurring in $\phi(\vec{x}, \vec{y})$ that are not inequality atoms) to $\mathcal{W}$ for which $(i) h\left(z_{i}\right) \neq h\left(z_{k}\right)$ for each inequality atom $z_{i} \neq z_{k}$ occurring in $\phi(\vec{x}, \vec{y})$, and $(i i) h(\vec{t})=\vec{c}$, where $h(\vec{t})=\left(h\left(t_{1}\right), \ldots, h\left(t_{n}\right)\right)$ for a tuple of terms $\vec{t}=\left(t_{1}, \ldots, t_{n}\right)$. In what follows, we also say that this is a homomorphism from $q$ to $\mathcal{W}$ (or also a homomorphism from $\phi(\vec{x}, \vec{y})$ to $\mathcal{W}$ ) with $h(\vec{t})=\vec{c}$, and write $h(q)$ (or also $h(\phi(\vec{x}, \vec{y}))$ ), to actually denote $h\left(\mathcal{W}_{q}\right)$. Finally, the evaluation of a $\mathrm{UCQ}^{\neq} \mathrm{s}$ over a set of atoms $\mathcal{W}$ is simply the union of the evaluation of its disjuncts over $\mathcal{W}$. As an usual convention, for a boolean $\mathrm{CQ}^{\neq} q$, the evaluation of $q$ over a set of atoms $\mathcal{W}$ amounts to $q^{\mathcal{W}}=\{()\}$ (also denoted by $\mathcal{W} \models q$ ) if and only if there is a homomorphism from $\mathcal{W}_{q}$ to $\mathcal{W}$.

Furthermore, given two $\mathrm{CQ}^{\neq} \mathrm{s} q_{1}=\left\{\overrightarrow{t_{1}} \mid \exists \overrightarrow{y_{1}} \cdot \phi_{1}\left(\overrightarrow{x_{1}}, \overrightarrow{y_{1}}\right)\right\}$ and $q_{2}=\left\{\overrightarrow{t_{2}} \mid\right.$ $\left.\exists \overrightarrow{y_{2}} \cdot \phi_{2}\left(\overrightarrow{x_{2}}, \overrightarrow{y_{2}}\right)\right\}$, we say that $h$ is a homomorphism from $q_{2}$ to $q_{1}$ if $h$ is a homomorphism from $\mathcal{W}_{q_{2}}$ to $\mathcal{W}_{q_{1}}$ for which $(i) h\left(z_{i}\right) \neq h\left(z_{k}\right)$ for each inequality atom occurring in $\phi_{2}(\vec{x}, \vec{y})$, and $(i i) h\left(\overrightarrow{t_{2}}\right)=\overrightarrow{t_{1}}$.

Given a schema $\mathcal{S}$ and two queries of the same arity $q_{1}$ and $q_{2}$ over $\mathcal{S}$, we write $q_{1} \sqsubseteq \mathcal{S} q_{2}$ (or simply $q_{1} \sqsubseteq q_{2}$ when $\mathcal{S}$ is clear from the context) if $q_{1}^{D} \subseteq q_{2}^{D}$ for every $\mathcal{S}$-database $D$. Furthermore, we write $q_{1} \equiv_{\mathcal{S}} q_{2}$ (or simply $q_{1} \equiv q_{2}$ when $\mathcal{S}$ is clear from the context) if both $q_{1} \sqsubseteq q_{2}$ and $q_{2} \sqsubseteq q_{1}$ hold, that is, if $q_{1}^{D}=q_{2}^{D}$ for every $\mathcal{S}$-database $D$. When $\mathcal{S}$ is a database schema without integrity constraints, it is well-known that, if both $q_{1}=\left\{\overrightarrow{t_{1}} \mid \exists \overrightarrow{y_{1}} \cdot \phi_{1}\left(\overrightarrow{x_{1}}, \overrightarrow{y_{1}}\right)\right\}$ and $q_{2}=\left\{\overrightarrow{t_{2}} \mid \exists \overrightarrow{y_{2}} \cdot \overrightarrow{\phi_{2}}\left(\overrightarrow{x_{2}}, \overrightarrow{y_{2}}\right)\right\}$ are CQs over $\mathcal{S}$, then $q_{1} \sqsubseteq q_{2}$ if and only if $\overrightarrow{t_{1}} \in q_{2}^{\mathcal{W}_{q_{1}}}$, i.e., if and only if there is a homomorphism $h$ from $q_{2}$ to $\mathcal{W}_{q_{1}}$ with $h\left(\overrightarrow{t_{2}}\right)=\overrightarrow{t_{1}}$ |Chandra and Merlin, 1977, and if both $q_{1}$ and $q_{2}$ are UCQs over $\mathcal{S}$, then $q_{1} \sqsubseteq q_{2}$ if and only if for each disjunct $q$ of $q_{1}$ there is a disjunct $q^{\prime}$ of $q_{2}$ such that $q \sqsubseteq q^{\prime}$ [Sagiv and Yannakakis, 1980.

Given a $\mathrm{CQ}^{\neq} q=\left\{\left(t_{1}, \ldots, t_{n}\right) \mid \exists \vec{y} \cdot \phi(\vec{x}, \vec{y})\right\}$ of arity $n$ and an $n$-tuple of constants $\vec{c}=\left(c_{1}, \ldots, c_{n}\right)$, we denote by $q(\vec{c})=\{() \mid \exists \vec{y} . \phi(\vec{x} / \vec{c}, \vec{y})\}$ the boolean CQ in which the formula $\phi(\vec{x} / \vec{c}, \vec{y})$ corresponds to $\perp(\vec{y})$ in the case that there is some $i \in[1, n]$ for which $t_{i} \neq c_{i}$ and $t_{i}$ is a constant, otherwise $\phi(\vec{x} / \vec{c}, \vec{y})$ is obtained from $\phi(\vec{x}, \vec{y})$ by replacing all the occurrences of the term $t_{i}$ with the constant $c_{i}$, for each $i \in[1, n]$.

Given a $\mathrm{UCQ}^{\neq} q=q_{1} \cup \ldots \cup q_{m}$ of arity $n$ and an $n$-tuple of constants $\vec{c}=$ $\left(c_{1}, \ldots, c_{n}\right)$, we denote by $q(\vec{c})=q_{1}(\vec{c}) \cup \ldots \cup q_{m}(\vec{c})$ the boolean $\mathrm{UCQ}^{\neq}$obtained from $q$ by replacing the disjunct $q_{i}$ with $q_{i}(\vec{c})$, for each $i \in[1, n]$.

\subsection{Computational Complexity}

We assume familiarity with basic notions about computational complexity, as defined in standard textbooks Garey and Johnson, 1979, Papadimitriou, 1994, Arora and Barak, 2009]. In particular, we consider the following complexity classes:

$$
\begin{aligned}
& \mathrm{AC}^{0} \subsetneq \mathrm{LOGSPACE} \subseteq \mathrm{NLOGSPACE} \subseteq \text { PTIME } \subseteq \\
& {\left[\begin{array}{c}
\mathrm{NP} \\
\text { coNP }
\end{array}\right] \subseteq \mathrm{DP} \subseteq \mathrm{PTIme}^{\mathrm{NP}} \subseteq\left[\begin{array}{c}
\Sigma_{2}^{p} \\
\Pi_{2}^{p}
\end{array}\right] \subseteq \mathrm{ExPTime} \subseteq\left[\begin{array}{c}
\text { NExPTime } \\
\text { соNExPTıме }
\end{array}\right]}
\end{aligned}
$$

It is known that $\mathrm{AC}^{0} \subsetneq$ LOGSPACE, for example the undirected graph reachability problem is in LogSpace Reingold, 2008 but not in $\mathrm{AC}^{0}$. The strictness of all the 
other inclusions, as well as whether complexity classes in square brackets coincide, are still open problems. By the time hierarchy theorems Hartmanis and Stearns, 1965 Cook, 1973, however, it is known that PTime $\subsetneq$ ExPTime and NP $\subsetneq$ NExPTime (respectively, coNP $\subsetneq$ CONEXPTIME).

Since readers might be less familiar with the complexity class $\mathrm{AC}^{0}$, we briefly provide a basic intuition about it, and refer to Vollmer, 1999. for its formal definition which is based on the circuit model. Intuitively, a decision problem belongs to $\mathrm{AC}^{0}$ if it can be decided in constant time using a number of processors that is polynomial in the size of the input. A typical decision problem belonging to $\mathrm{AC}^{0}$ is the evaluation of FOL queries over relational databases, where only the database is regarded as the input, and the query is assumed to be fixed Abiteboul et al., 1995.

A decision problem $\mathcal{P}$ is said to be $\mathcal{C}$-hard for a complexity class $\mathcal{C}$, if any decision problem $\mathcal{P}^{\prime} \in \mathcal{C}$ can be reduced to $\mathcal{P}$, and it is said to be $\mathcal{C}$-complete if in addition $\mathcal{P} \in \mathcal{C}$. Most of the reductions presented in this thesis are LOGSPACE reductions.

A LOGSPACE reduction is a reduction computable by a three-tape Turing machine [Turing, 1937] that, with an input written on the read-only input tape, writes its output on the write-only output tape using a number of cells of the (initially-blank) $\mathrm{read} /$ write work tape that is logarithmic in the size of the input.

\subsection{View-based Query Processing}

View-based query processing is a general term denoting several tasks related to the presence of views in database systems. In particular, two notions have been subject to extensive investigations in literature, namely view-based query answering and view-based query rewriting, whose relationship, although widely discussed (see, e.g., Calvanese et al., 2000 Calvanese et al., 2007c), seems to be often ignored.

In view-based query answering, a notion originated with Duschka and Genesereth, 1997, we are given a query, a set of view definitions, and a set of view extensions, and the goal is to compute the so-called certain answers, i.e., the set of tuples satisfying the query in all databases consistent with the views.

In view-based query rewriting, a notion originated with Levy et al., 1995], we are given a query and a set of view definitions, and the goal is to reformulate the query into an expression over the alphabet of the view names that satisfies certain conditions. In this thesis, we are mainly interested to view-based query rewriting.

We denote by $\mathcal{V}=\left\{V_{1}, \ldots, V_{n}\right\}$ and $\mathcal{E}=\left\{E_{1}, \ldots, E_{n}\right\}$ a set of view definitions and view extensions over a schema $\mathcal{S}$, respectively, where, for each $i \in[1, n]$, to the symbol $V_{i}$ it is implicitly associated a query over a schema $\mathcal{S}$, and $E_{i}$ is the view extension of $V_{i}$ (i.e., a set of facts having $V_{i}$ as a predicate with arity the one of the corresponding query). When dealing with (U)CQ view definitions $\mathcal{V}=\left\{V_{1}, \ldots, V_{n}\right\}$, we implicitly assume that the target list (of each disjunct) of the (U)CQ associated to $V_{i}$ does not have repeated variables or constants, for each $i \in[1, n]$.

\subsubsection{Exact View Assumption}

Given a schema $\mathcal{S}$, a set of view definitions $\mathcal{V}=\left\{V_{1}, \ldots, V_{n}\right\}$ over $\mathcal{S}$, and a query $q_{\mathcal{S}}$ over $\mathcal{S}$, the goal is to reformulate $q_{\mathcal{S}}$ into an expression over the view alphabet (called rewriting) that, when evaluated over the set of view extensions $\mathcal{V}(D)=\left\{V_{1}^{D}, \ldots, V_{n}^{D}\right\}$ 
(for a view symbol $V_{i}, V_{i}^{D}$ denotes the evaluation of the query associated to $V_{i}$ over $D$ ), coincide with the evaluation of $q_{\mathcal{S}}$ over $D$ (i.e., $q_{\mathcal{S}}^{D}$ ), for each $\mathcal{S}$-database $D$.

Formally, for a set of view definitions $\mathcal{V}$ and a query $q_{\mathcal{S}}$ over a schema $\mathcal{S}$, we say that a query $q_{\mathcal{V}}$ is an exact rewriting of $q_{\mathcal{S}}$ with respect to $\mathcal{V}$, if $q_{\mathcal{S}}^{D}=q_{\mathcal{V}}{ }^{(D)}$ for each $\mathcal{S}$-database $D$. The underlying decision problem associated to exact rewritings is the expressibility problem: given a set of view definitions $\mathcal{V}$ and a query $q_{\mathcal{S}}$ over a schema $\mathcal{S}$, does there exist an exact rewriting of $q_{\mathcal{S}}$ with respect to $\mathcal{V}: 17$ This happens to be a difficult problem, it is indeed in general undecidable already when both view definitions in $\mathcal{V}$ and queries $q_{\mathcal{S}}$ are CQs Gogacz and Marcinkowski, 2016.

Obviously, one might be interested not in arbitrary exact rewritings, but in those belonging to a certain query language $\mathcal{L}$. Formally, we say that $q \mathcal{V}$ is an $\mathcal{L}$-exact rewriting of a query $q_{\mathcal{S}}$ with respect to a set of view definitions $\mathcal{V}$, if $q \mathcal{V}$ is an exact rewriting of $q_{\mathcal{S}}$ with respect to $\mathcal{V}$ and $q_{\mathcal{V}} \in \mathcal{L}$. When view definitions in $\mathcal{V}$ are CQs and queries $q_{\mathcal{S}}$ are (U)CQs, the problem of deciding whether there exists a (U)CQ-exact rewriting is NP-complete Levy et al., 1995].

\subsubsection{Sound View Assumption}

The setup we have considered so far is that of exact views, in the sense that the extension of each view is precisely the result of evaluating the corresponding view expression over the database. There is, however, at least one other notion of a database being coherent with the view extensions. ${ }^{2}$ namely that of sound views. Specifically, an $\mathcal{S}$-database $D$ is consistent with the set of view extensions $\mathcal{E}=\left\{E_{1}, \ldots, E_{n}\right\}$ with respect to the set of view definitions $\mathcal{V}=\left\{V_{1}, \ldots, V_{n}\right\}$ under the sound view assumption, if $\mathcal{E} \subseteq \mathcal{V}(D)$, i.e., if $E_{i} \subseteq V_{i}^{D}$ for each $i \in[1, n]$.

Given a set of view definitions $\mathcal{V}$ over a schema $\mathcal{S}$, a set of view extensions $\mathcal{E}$, and a query $q_{\mathcal{S}}$ over $\mathcal{S}$, we denote by $\operatorname{cert}_{q_{\mathcal{S}}, \mathcal{V}}^{\mathcal{V}}$ the certain answers of $q_{\mathcal{S}}$ with respect to $\mathcal{V}$ and $\mathcal{E}$, i.e., the set of tuples of constants that are in all the evaluations of $q_{\mathcal{S}}$ over $\mathcal{S}$-databases $D$ consistent with $\mathcal{E}$ with respect to $\mathcal{V}$. Formally:

$$
\operatorname{cert}_{q_{\mathcal{S}}, \mathcal{V}}^{\mathcal{E}}:=\bigcap_{D: \mathcal{E} \subseteq \mathcal{V}(D)} q_{\mathcal{S}}^{D}
$$

Given a set of view definitions $\mathcal{V}$ over a schema $\mathcal{S}$ and a query $q_{\mathcal{S}}$ over $\mathcal{S}$, following the literature terminology, we say that a query $q \mathcal{V}$ over the view alphabet $\mathcal{V}$ is a perfect rewriting of $q_{\mathcal{S}}$ with respect to $\mathcal{V}$, if $q_{\mathcal{V}}^{\mathcal{E}}=\operatorname{cert}_{q_{\mathcal{S}}, \mathcal{V}}^{\mathcal{V}}$ for each view extension $\mathcal{E}$.

Furthermore, we say that a query $q \mathcal{V}$ over the view alphabet $\mathcal{V}$ is a sound rewriting of $q_{\mathcal{S}}$ with respect to $\mathcal{V}$, if $q_{\mathcal{V}}^{\mathcal{E}} \subseteq q_{\mathcal{S}}^{D}$ for each view extension $\mathcal{E}$ and $\mathcal{S}$-database $D$ such that $\mathcal{E} \subseteq \mathcal{V}(D)$. Obviously, the interest is in computing sound rewritings that capture the original query at best. This is formalized by the following notion, where $\mathcal{L}$ is a query language. A query $q \mathcal{V} \in \mathcal{L}$ over the view alphabet $\mathcal{V}$ is an $\mathcal{L}$-maximally sound rewriting of $q_{\mathcal{S}}$ with respect to $\mathcal{V}$, if $q_{\mathcal{V}}$ is a sound rewriting of $q_{\mathcal{S}}$ with respect to $\mathcal{V}$ and there exists no query $q_{\mathcal{V}}^{\prime} \in \mathcal{L}$ such that $(i) q_{\mathcal{V}}^{\prime}$ is a sound rewriting of $q_{\mathcal{S}}$ with

\footnotetext{
${ }^{1}$ In literature, this problem is also referred as determinacy Nash et al., 2010 and losslessness under the exact view assumption Calvanese et al., 2007c.

${ }^{2}$ In fact, a third notion considered in literature is that of complete views, which, however, we do not consider it here.
} 
respect to $\mathcal{V},(i i) q_{\mathcal{V}}^{\mathcal{E}} \subseteq q_{\mathcal{V}}^{\prime} \mathcal{E}$ for each set of view extensions $\mathcal{E}$, and $(i i i) q_{\mathcal{V}}^{\mathcal{E}^{\prime}} \subsetneq q_{\mathcal{V}}^{\prime} \mathcal{E}^{\prime}$ for a set of view extensions $\mathcal{E}^{\prime}$.

We conclude this section with the following renowned positive result for CQ view definitions and UCQ queries.

Theorem 2.1. Levy et al., 1995 Let $\mathcal{V}$ be a set of $C Q$ view definitions over a schema $\mathcal{S}$. For a $U C Q q_{\mathcal{S}}$ over $\mathcal{S}$, it is always possible to compute the UCQ qv which is the union of all CQ-maximally sound rewritings of $q_{\mathcal{S}}$ with respect to $\mathcal{V}$. Moreover:

- $q_{\mathcal{V}}$ is a UCQ-maximally sound rewriting of $q_{\mathcal{S}}$ with respect to $\mathcal{V}$;

- $q_{\mathcal{V}}$ is a perfect rewriting of $q_{\mathcal{S}}$ with respect to $\mathcal{V}$;

- $q_{\mathcal{V}}$ is a UCQ-exact rewriting of $q_{\mathcal{S}}$ wih respect to $\mathcal{V}$, if this latter exists.

Notice, however, that the above theorem is no longer true when view definitions $\mathcal{V}$ are expressed as UCQs rather than CQs Duschka and Genesereth, 1998; Afrati and Chirkova, 2019. Specifically, there are cases where a UCQ-maximally sound rewriting of a $\mathrm{CQ} q_{\mathcal{S}}$ with respect to a set of UCQ view definitions $\mathcal{V}$ does not exist.

\subsection{Description Logic Ontologies and Knowledge Bases}

Description Logics (DLs) are fragments of FOL languages using only unary and binary predicates, called atomic concepts and atomic roles, respectively Baader et al., 2003; Baader et al., 2017. According to Gruber, 1993 Gruber, 2018], an ontology is a formal explicit specification of a shared conceptualisation of a domain of interest. Since DLs are logics specifically designed to represent structure knowledge and to reason about it, they are arguably well-suited to represent ontologies.

In this thesis, a $D L$ ontology (or simply ontology) $\mathcal{O}$ is a TBox ("Terminological Box") expressed in a specific DL, that is, a set of assertions stating general properties of concepts and roles (built according to the syntax of the specific DL) which represents the intensional knowledge of a modeled domain.

Sometimes, we also need to view an ontology $\mathcal{O}$ as a schema. In such cases, we implicitly refer to the finite set of unary and binary predicates corresponding to atomic concepts and atomic roles, respectively, which constitute the alphabet of $\mathcal{O}$.

\subsubsection{DL-Lite R $_{\mathcal{R}}$ Ontologies: Syntax}

We are interested in DL ontologies expressed in DL-Lite $\mathcal{R}_{\mathcal{R}}$, a member of the DL-Lite family 3 Calvanese et al., 2004a; Calvanese et al., 2005; Calvanese et al., 2007b of DLs. Notably, DL-Lite $e_{\mathcal{R}}$ is the logic underpinning OWL 2 QI ${ }^{4}$, i.e., one of the three OWL $2^{5}$ profiles Cuenca Grau et al., 2008; Motik et al., 2012, specifically the one especially designed for efficient query answering. Notice that, in the Semantic Web Berners-Lee et al., 2001 context, OWL 2 is the current W3C recommended standard ontology language.

\footnotetext{
${ }^{3}$ Not to be confused with the DL-Lit $e_{\text {bool }}$ family studied in Artale et al., 2009, a supremum of the $D L$-Lite family in the lattice of DLs.

${ }^{4}$ OWL 2 Query Language: https://www.w3.org/TR/owl2-profiles/\#OWL_2_QL

${ }^{5}$ OWL 2 Web Ontology Language: https://www.w3.org/TR/owl2-profiles/
} 
In $D L$-Lite , $_{\mathcal{R}}$ concepts and roles obey to the following syntax:

$$
B \longrightarrow A|\exists R \quad R \longrightarrow P| P^{-}
$$

where $A, P$, and $P^{-}$denote an atomic concept (atomic concepts include the universal concept $T$ and the bottom concept $\perp$ ), an atomic role, and the inverse of an atomic role, respectively. $R$ denotes a basic role, i.e., a role that is either an atomic role or its inverse. $B$ denotes a basic concept, i.e., a concept that is either an atomic concept or $\exists R$, where this latter is the standard DL construct of unqualified existential quantification on basic roles.

A $D L$-Lite $e_{\mathcal{R}}$ ontology $\mathcal{O}$ is a finite set of assertions of the form:

$$
\begin{aligned}
& B_{1} \sqsubseteq B_{2} \quad R_{1} \sqsubseteq R_{2} \quad \text { (concept/role inclusion assertion) } \\
& B_{1} \sqsubseteq \neg B_{2} \quad R_{1} \sqsubseteq \neg R_{2} \quad \text { (concept/role disjointness assertion) }
\end{aligned}
$$

Without loss of generality, we assume that each $D L$-Lite R $_{\mathcal{R}}$ ontology $\mathcal{O}$ contains an inclusion assertion of the form $A \sqsubseteq \top$ (respectively, $\exists P \sqsubseteq \top$ and $\exists P^{-} \sqsubseteq \top$ ), for each possible atomic concept $A$ (respectively, atomic role $P$ ) in the alphabet of $\mathcal{O}$.

Let $C$ (respectively, $E$ ) denote a general concept (respectively, general role), i.e., a concept (respectively, role) that is either a basic concept (respectively, basic role) or its negation. In principle, one might also include $B_{1} \sqcup B_{2}$ and $R_{1} \sqcup R_{2}$ (respectively, $C_{1} \sqcap C_{2}$ and $E_{1} \sqcap E_{2}$ ) in the constructs for the left-hand side (respectively, right-hand side) of assertions, where $\sqcup$ (respectively, $\sqcap$ ) denotes union (respectively, intersection). Notice, however, that the expressive capabilities of the language would remain the same, since in fact assertions of the form $B_{1} \sqcup B_{2} \sqsubseteq C$ and $R_{1} \sqcup R_{2} \sqsubseteq E$ (respectively, $B \sqsubseteq C_{1} \sqcap C_{2}$ and $R \sqsubseteq E_{1} \sqcap E_{2}$ ) are equivalent to the pair of assertions $B_{1} \sqsubseteq C, B_{2} \sqsubseteq C$ and $R_{1} \sqsubseteq E, R_{2} \sqsubseteq E$ (respectively, $B \sqsubseteq C_{1}, B \sqsubseteq C_{2}$ and $R \sqsubseteq E_{1}, R \sqsubseteq E_{2}$ ).

Similar arguments hold for the additional assertions included in OWL 2 QL, namely symmetry, asymmetry, qualified existential quantification, reflexivity, and irreflexivity. It is immediate to see that symmetry (respectively, asymmetry) of an atomic role $P$ can be expressed by means of the inclusion assertion $P \sqsubseteq P^{-}$(respectively, $\left.P \sqsubseteq \neg P^{-}\right)$. Likewise, a qualified existential quantification of the form $B_{1} \sqsubseteq \exists R . B_{2}$ is equivalent to the assertions $B_{1} \sqsubseteq \exists P_{\text {new }}, P_{\text {new }} \sqsubseteq R$, and $\exists P_{\text {new }}^{-} \sqsubseteq B_{2}$, where $P_{\text {new }}$ is a fresh atomic role. Furthermore, the addition of reflexivity and irreflexivity assertions (which include a form of second-order constructs) does not affect the computational complexity of the basic inference problems (including query answering) Corona et al., 2009 Artale et al., 2009, and such constructs could be easily included with only minor changes in reasoning algorithms.

Observe that $D L$-Lite $e_{\mathcal{R}}$ is an extension of (the DL-like part of) the ontology language RDF $\mathrm{S}^{6}$ Cuenca Grau, 2004, the schema language for RDF7. We will refer to this latter DL ontology language as $D L$-Lite $e_{\mathrm{RDFS}}$. Specifically, a $D L$-Lite RDFS $_{\text {is a }}$ finite set of assertions of the form:

$$
B \sqsubseteq A \quad R_{1} \sqsubseteq R_{2}
$$

\footnotetext{
${ }^{6}$ Resource Description Framework Schema Brickley and Guha, 2014.

${ }^{7}$ Resource Description Framework: https://www.w3.org/RDF/
} 
We will also consider a slight extension of the DL ontology language DL-Lite $e_{\mathrm{RDFS}}$, namely $D L-L i t e_{\mathrm{RDFS}}$, which also allows for the concept/role disjointness assertions expressible in $D L$-Lite . $_{\mathcal{R}}$.

We further note that $D L$-Lite $e_{\mathcal{R}}$ is an extension of $D L$-Lite core $_{\text {e }}$ i.e., the basic logic

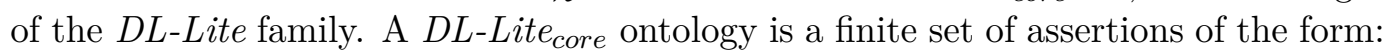

$$
B_{1} \sqsubseteq B_{2} \quad B_{1} \sqsubseteq \neg B_{2}
$$

Observe that $D L$-Lite core $_{\text {con }}$ and $D L$-Lite $e_{\mathrm{RDFS}}$ (respectively, $D L$-Lite $\overrightarrow{\mathrm{RDFS}}_{\text {) }}$ ) are incomparable fragments of $D L-$ Lite $_{\mathcal{R}}$.

\subsubsection{DL-Lite R $_{\mathcal{R}}$ Ontologies: Semantics}

The semantics of DL ontologies is specified through the notion of interpretation: an FOL interpretation $\mathcal{I}$ for an ontology $\mathcal{O}$ is a pair $\mathcal{I}=\left\langle\Delta^{\mathcal{I}},{ }^{\mathcal{I}}\right\rangle$, where the interpretation domain $\Delta^{\mathcal{I}} \subseteq$ Const is a non-empty, possibly infinite set of objects, and the interpretation function. I assigns to each atomic concept $A$ a set of domain objects $A^{\mathcal{I}} \subseteq \Delta^{\mathcal{I}}$, and to each atomic role $P$ a set of pairs of domain objects $P^{\mathcal{I}} \subseteq \Delta^{\mathcal{I}} \times \Delta^{\mathcal{I}}$. For the constructs of $D L$-Lite $\mathcal{R}_{\mathcal{R}}$, the interpretation function extends to other basic concepts, basic roles, and the additional binary predicate $\neq$ as follows:

- $\top^{\mathcal{I}}=\Delta^{\mathcal{I}}$

- $\perp^{\mathcal{I}}=\emptyset$

- $\neq^{\mathcal{I}}=\left\{\left(o, o^{\prime}\right) \mid o, o^{\prime} \in \Delta^{\mathcal{I}} \wedge o \neq o^{\prime}\right\}$. We often write $\left(o, o^{\prime}\right) \in \neq^{\mathcal{I}}$ as $o \neq^{\mathcal{I}} o^{\prime}$.

- $(\exists P)^{\mathcal{I}}=\left\{o \mid \exists o^{\prime} .\left(o, o^{\prime}\right) \in P^{\mathcal{I}}\right\}$

- $\left(P^{-}\right)^{\mathcal{I}}=\left\{\left(o, o^{\prime}\right) \mid\left(o^{\prime}, o\right) \in P^{\mathcal{I}}\right\}$

When convenient, we treat interpretations $\mathcal{I}=\left\langle\Delta^{\mathcal{I}},{ }^{\mathcal{I}}\right\rangle$ for $\mathcal{O}$ as a (possibly infinite) set of facts over $\mathcal{O}$, in those cases we implicitly refer to the set of facts including: for each unary predicate $U$ and for each $e \in \Delta^{\mathcal{I}}$ (respectively, for each binary predicate $B$ and for each pair $e_{1}, e_{2} \in \Delta^{\mathcal{I}}$ ), the fact $U(e)$ (respectively, $\left.B\left(e_{1}, e_{2}\right)\right)$ if and only if $e \in U^{\mathcal{I}}$ (respectively, $\left(e_{1}, e_{2}\right) \in B^{\mathcal{I}}$ ).

We say that an interpretation $\mathcal{I}$ for an ontology $\mathcal{O}$ satisfies a concept inclusion assertion $B_{1} \sqsubseteq B_{2}$ (respectively, role inclusion assertion $R_{1} \sqsubseteq R_{2}$ ) if $B_{1}^{\mathcal{I}} \subseteq B_{2}^{\mathcal{I}}$ (respectively, $R_{1}^{\mathcal{I}} \subseteq R_{2}^{\mathcal{I}}$ ), and it satisfies a concept disjointness assertion $B_{1} \sqsubseteq \neg B_{2}$ (respectively, role disjointness assertion $R_{1} \sqsubseteq \neg R_{2}$ ) if $B_{1}^{\mathcal{I}} \cap B_{2}^{\mathcal{I}}=\emptyset$ (respectively, $R_{1}^{\mathcal{I}} \cap R_{2}^{\mathcal{I}}=\emptyset$ ). Finally, we say that an interpretation $\mathcal{I}$ for an ontology $\mathcal{O}$ satisfies $\mathcal{O}$, denoted by $\mathcal{I} \models \mathcal{O}$, if $\mathcal{I}$ satisfies every assertion in $\mathcal{O}$.

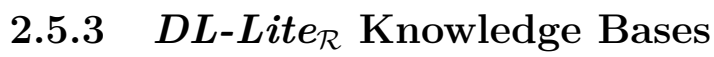

An $\mathcal{L}$ knowledge base $\mathcal{K}$ is a pair $\mathcal{K}=\langle\mathcal{O}, \mathcal{A}\rangle$, where $\mathcal{O}$ is a DL ontology expressed in $\mathcal{L}$, and $\mathcal{A}$ is an ABox ("Assertional Box") for $\mathcal{O}$, i.e., a finite set of membership assertions (or equivalently, a finite set of facts) of the form:

$$
A(a) \quad P(a, b)
$$


where $a, b$ are constants (also known as individuals) in Const, and $A$ and $P$ is an atomic concept and an atomic role, respectively, in the alphabet of $\mathcal{O}$.

The semantics of an $\mathcal{L}$ knowledge base $\mathcal{K}=\langle\mathcal{O}, \mathcal{A}\rangle$ is given in terms of interpretations for $\mathcal{K}$, i.e., FOL interpretations $\mathcal{I}=\left\langle\Delta^{\mathcal{I}},{ }^{\mathcal{I}}\right\rangle$ for $\mathcal{O}$ such that the interpretation function ${ }^{\mathcal{I}}$ further assigns to each individual $a$ occurring in $\mathcal{A}$ a domain object $a^{\mathcal{I}} \in \Delta^{\mathcal{I}}$. Unless otherwise stated, we adopt the so-called unique name assumption (UNA), i.e., we consider only those interpretations $\mathcal{I}$ for which $a^{\mathcal{I}} \neq b^{\mathcal{I}}$ for each pair of individuals $a, b$ occurring in $\mathcal{A}$ with $a \neq b$

An interpretation $\mathcal{I}$ for an $\mathcal{L}$ knowledge base $\mathcal{K}=\langle\mathcal{O}, \mathcal{A}\rangle$ is a model of $\mathcal{K}$, denoted by $\mathcal{I} \models \mathcal{K}$, if $\mathcal{I} \models \mathcal{O}$ and $\mathcal{I} \models \mathcal{A}$, where $\mathcal{I} \models \mathcal{A}$ if, for each membership assertion $A(a)$ (respectively, $P(a, b))$ in $\mathcal{A}$, we have that $a^{\mathcal{I}} \in \mathcal{A}^{\mathcal{I}}$ (respectively, $\left(a^{\mathcal{I}}, b^{\mathcal{I}}\right) \in P^{\mathcal{I}}$ ).

The set of models of an $\mathcal{L}$ knowledge base $\mathcal{K}=\langle\mathcal{O}, \mathcal{A}\rangle$, denoted by $\operatorname{Mod}_{\mathcal{A}}(\mathcal{O})$, is the set of interpretations $\mathcal{I}$ for $\mathcal{K}$ such that $\mathcal{I} \models \mathcal{K}$. An $\mathcal{L}$ knowledge base $\mathcal{K}=\langle\mathcal{O}, \mathcal{A}\rangle$ is said to be satisfiable if $\operatorname{Mod}_{\mathcal{A}}(\mathcal{O}) \neq \emptyset$, unsatisfiable otherwise.

For an $\mathcal{L}$ knowledge base $\mathcal{K}=\langle\mathcal{O}, \mathcal{A}\rangle$, an interpretation $\mathcal{I}=\left\langle\Delta^{\mathcal{I}},{ }^{\mathcal{I}}\right\rangle$ for $\mathcal{K}$, and a $\mathrm{UCQ}^{\neq}$query $q_{\mathcal{O}}$ over $\mathcal{O}$, with a slight abuse of notation, we denote by $q_{\mathcal{O}}^{\mathcal{I}}$ the evaluation of the query $q_{\mathcal{O}}^{\prime}$ over $\mathcal{I}$, where $q_{\mathcal{O}}^{\prime}$ is obtained from $q_{\mathcal{O}}$ by replacing each individual $c$ occurring in $q_{\mathcal{O}}$ with the domain object $c^{\mathcal{I}} \in \Delta^{\mathcal{I}}$ (if defined, i.e., if $c$ occurs in $\mathcal{A}$ ). Furthermore, for a $\mathrm{CQ}^{\neq} q_{\mathcal{O}}$ and an interpretation $\mathcal{I}$, we say that $h$ is a homomorphism from $q_{\mathcal{O}}$ to $\mathcal{I}$, if $h$ is a homomorphism from $\mathcal{W}_{q_{\mathcal{O}}^{\prime}}$ (i.e., the set of all atoms occurring in the body of $q_{\mathcal{O}}^{\prime}$ that are not inequality atoms) to $\mathcal{I}$.

Finally, given an $\mathcal{L}$ knowledge base $\mathcal{K}=\langle\mathcal{O}, \mathcal{A}\rangle$ and a $\mathrm{UCQ}^{\neq} q_{\mathcal{O}}$ over $\mathcal{O}$, we denote by $\operatorname{cert}_{q_{\mathcal{O}}, \mathcal{O}}^{\mathcal{A}}$ the set of certain answers of $q_{\mathcal{O}}$ with respect to $\mathcal{O}$ and $\mathcal{A}$ i.e., the set of tuples of constants $\left(c_{1}, \ldots, c_{n}\right)$ such that $\left(c_{1}^{\mathcal{I}}, \ldots, c_{n}^{\mathcal{I}}\right) \in q_{\mathcal{O}}^{\mathcal{I}}$ for each $\mathcal{I} \in \operatorname{Mod}_{\mathcal{A}}(\mathcal{O})$. For a boolean FOL query $q_{\mathcal{O}}$ over $\mathcal{O}$, we denote by $\mathcal{I}=q_{\mathcal{O}}$ (also by $\left.q_{\mathcal{O}}^{\mathcal{I}}=\{()\}\right)$ the fact that the body of $q_{\mathcal{O}}$, which is a FOL sentence, is true in $\mathcal{I}$, and by $\mathcal{K} \models q_{\mathcal{O}}$ (also by $\operatorname{cert}_{q_{\mathcal{O}} \mathcal{O}}^{\mathcal{A}}=\{()\}$ ) the fact that $\mathcal{I} \models q_{\mathcal{O}}$ for each model $\mathcal{I}$ of $\mathcal{K}$.

Observe that, if $\mathcal{K}=\langle\mathcal{O}, \mathcal{A}\rangle$ is unsatisfiable, then the set of certain answers of any query $q_{\mathcal{O}}$ (over $\mathcal{O}$ ) with respect to $\mathcal{O}$ and $\mathcal{A}$ is trivially the set of all possible tuples of constants occurring in $\mathcal{A}$ whose arity is the one of the query (ex falso sequitur quodlibet), i.e., $\operatorname{cert}_{q_{\mathcal{O}} \mathcal{O}}^{\mathcal{A}}=\left\{\left(x_{1}, \ldots, x_{a r\left(q_{\mathcal{O}}\right)}\right) \mid \top\left(x_{1}, \ldots, x_{a r\left(q_{\mathcal{O}}\right)}\right)\right\}^{\mathcal{I}_{\mathcal{A}}}$, where $\mathrm{T}\left(x_{1}, \ldots, x_{\operatorname{ar}\left(q_{\mathcal{O}}\right)}\right)$ (similarly, $\left.\perp\left(x_{1}, \ldots, x_{\operatorname{ar}\left(q_{\mathcal{O}}\right)}\right)\right)$ is a shortcut denoting the conjunction of atoms $T\left(x_{1}\right) \wedge \ldots \wedge \top\left(x_{a r\left(q_{\mathcal{O}}\right)}\right)$ (similarly, $\left.\perp\left(x_{1}\right) \wedge \ldots \wedge \perp\left(x_{\operatorname{ar}\left(q_{\mathcal{O}}\right)}\right)\right)$, and $\mathcal{I}_{\mathcal{A}}$ is the interpretation for $\mathcal{K}$ obtained as follows:

- $\Delta^{\mathcal{I}_{\mathcal{A}}}$ is composed of all individuals occurring in $\mathcal{A}$;

- $a^{\mathcal{I}_{\mathcal{A}}}=a$, for each individual $a$ occurring in $\mathcal{A}$;

- $A^{\mathcal{I}_{\mathcal{A}}}=\{a \mid A(a) \in \mathcal{A}\}$ for each atomic concept $\mathcal{A}$;

- $P^{\mathcal{I}_{\mathcal{A}}}=\{(a, b) \mid P(a, b) \in \mathcal{A}\}$ for each atomic role $P$.

When we talk about the problem of answering queries belonging to a query language $\mathcal{Q}$ over $\mathcal{L}$ knowledge bases, we implicitly refer to the following decision problem: Given a query $q \in \mathcal{Q}$, an $\mathcal{L}$ knowledge base $\mathcal{K}=\langle\mathcal{O}, \mathcal{A}\rangle$, and a tuple of constants $\vec{c}$, check whether $\vec{c} \in \operatorname{cert}_{q_{\mathcal{O}}, \mathcal{O}}^{\mathcal{A}}$.

From Calvanese et al., 2007b], it is well-known that answering UCQs over $D L$-Lite R $_{\mathcal{R}}$ knowledge bases is FOL-rewritable, i.e., for every UCQ $q$ and every 
$D L$ - Lite $_{\mathcal{R}}$ knowledge base $\mathcal{K}=\langle\mathcal{O}, \mathcal{A}\rangle$, it is possible to compute the set of certain answers $\operatorname{cert}_{q_{\mathcal{O}}, \mathcal{O}}^{\mathcal{A}}$ by first reformulating $q_{\mathcal{O}}$ with respect to $\mathcal{O}$ (obtaining a FOL query over $\mathcal{O}$ ), and then by evaluating the reformulated query over $\mathcal{I}_{\mathcal{A}}$.

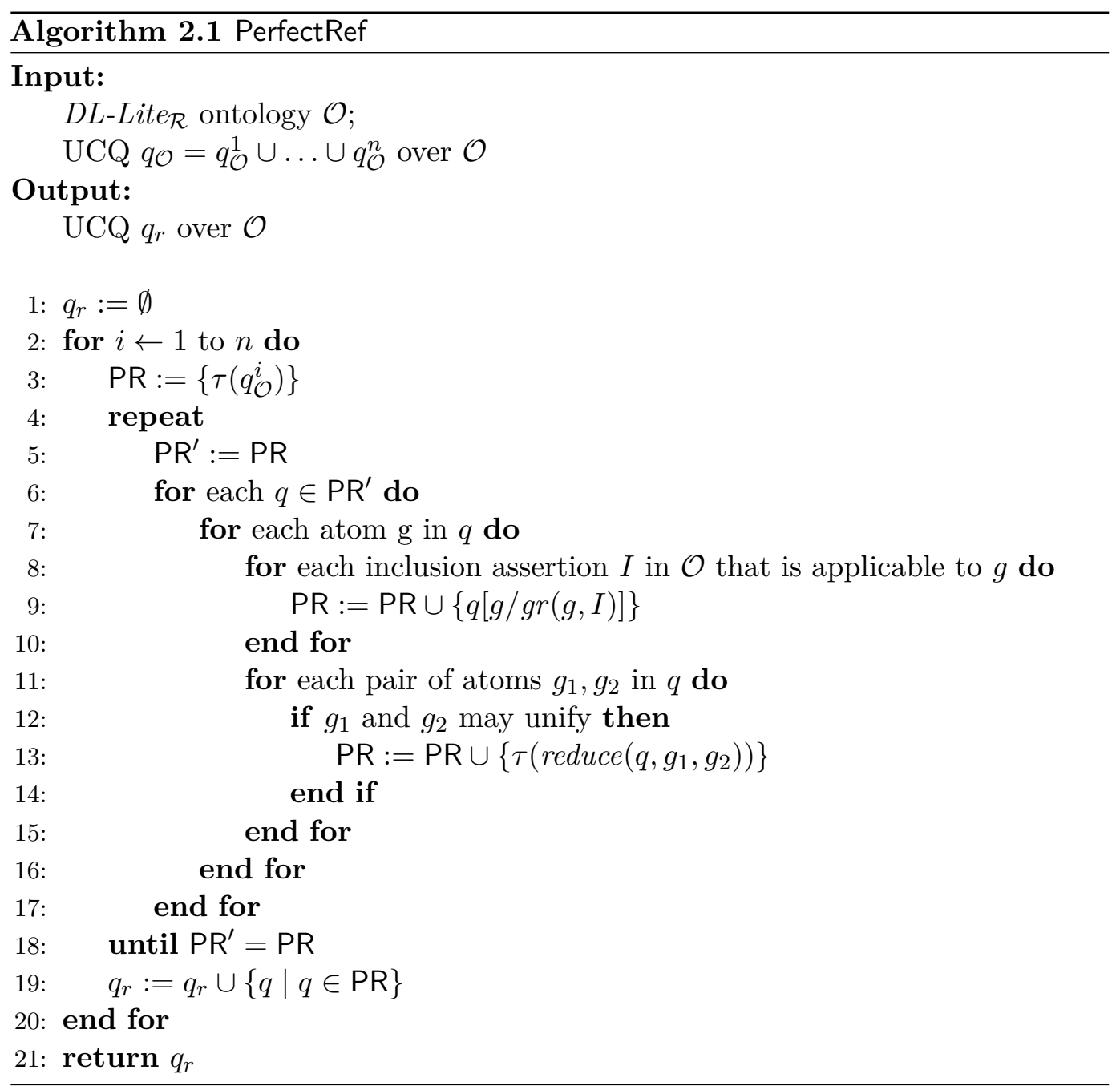

More specifically, for satisfiable $D L$-Lite $e_{\mathcal{R}}$ knowledge bases $\mathcal{K}=\langle\mathcal{O}, \mathcal{A}\rangle$ we have that, if $q_{\mathcal{O}}$ is a UCQ over $\mathcal{O}$, then $\operatorname{PerfectRef}\left(\mathcal{O}, q_{\mathcal{O}}\right)^{\mathcal{I}_{\mathcal{A}}}=\operatorname{cert}_{q_{\mathcal{O}}, \mathcal{O}}^{\mathcal{A}}$, where PerfectRef $\left(\mathcal{O}, q_{\mathcal{O}}\right)$ is the UCQ obtained by executing the algorithm PerfectRef described in Calvanese et al., 2007b and reported above on $\mathcal{O}$ and $q_{\mathcal{O}}$. Note that PerfectRef ignores the disjointness assertions of the input $D L$-Lite $e_{\mathcal{R}}$ ontology. Let "_" represents an existential variable that is not a join existential variable. In the algorithm, $\tau$ is a function that, given a CQ $q$, it returns a CQ obtained by replacing each existential variable that is not a join existential variable with the symbol _. The algorithm uses the notion of applicability of an inclusion assertion to an atom. Specifically, an inclusion assertion $I$ is applicable to an atom $A(x)$ (respectively, $P\left(x_{1}, x_{2}\right)$ ), if the right-hand side of $I$ is $A$ (respectively, if $(i) x_{2}=\ldots$ and the right-hand side of $I$ is $\exists P$; or $(i i) x_{1}=$ _ and the right-hand side of $I$ is $\exists P^{-}$; or (iii) the right-hand side of $I$ is either $P$ or $\left.P^{-}\right)$. Furthermore, $q[g / g r(g, I)]$ denotes the CQ obtained from $q$ by replacing the atom $g$ with a new atom $\operatorname{gr}(g, I)$, where, 
for an inclusion assertion $I$ applicable to $g, \operatorname{gr}(g, I)$ is the atom defined as follows:

- if $g=A(x)$ and $I=A_{1} \sqsubseteq A$, then $\operatorname{gr}(g, I)=A_{1}(x)$;

- if $g=A(x)$ and $I=\exists P \sqsubseteq A$, then $\operatorname{gr}(g, I)=P\left(x,_{-}\right)$;

- if $g=A(x)$ and $I=\exists P^{-} \sqsubseteq A$, then $\operatorname{gr}(g, I)=P\left({ }_{-}, x\right)$;

- if $g=P\left(x,_{-}\right)$and $I=A \sqsubseteq \exists P$, then $g r(g, I)=A(x)$;

- if $g=P\left(x,_{-}\right)$and $I=\exists P_{1} \sqsubseteq \exists P$, then $g r(g, I)=P_{1}\left(x,_{-}\right)$;

- if $g=P\left(x,{ }_{-}\right)$and $I=\exists P_{1}^{-} \sqsubseteq \exists P$, then $\operatorname{gr}(g, I)=P_{1}\left({ }_{-}, x\right)$;

- if $g=P\left({ }_{-}, x\right)$ and $I=A \sqsubseteq \exists P^{-}$, then $\operatorname{gr}(g, I)=A(x)$;

- if $g=P\left({ }_{-}, x\right)$ and $I=\exists P_{1} \sqsubseteq \exists P^{-}$, then $g r(g, I)=P_{1}\left(x,_{-}\right)$;

- if $g=P\left({ }_{-}, x\right)$ and $I=\exists P_{1}^{-} \sqsubseteq \exists P^{-}$, then $g r(g, I)=P_{1}\left({ }_{-}, x\right)$;

- if $g=P\left(x_{1}, x_{2}\right)$ and either $I=P_{1} \sqsubseteq P$ or $I=P_{1}^{-} \sqsubseteq P^{-}$, then $g r(g, I)=$ $P_{1}\left(x_{1}, x_{2}\right)$;

- if $g=P\left(x_{1}, x_{2}\right)$ and either $I=P_{1} \sqsubseteq P^{-}$or $P_{1}^{-} \sqsubseteq P$, then $\operatorname{gr}(g, I)=P_{1}\left(x_{2}, x_{1}\right)$.

Finally, reduce is a function that, given a CQ $q$ and two atoms $g_{1}$ and $g_{2}$, it returns a new CQ obtained by applying the most general unifier between $g_{1}$ and $g_{2}$. In unifying $g_{1}$ and $g_{2}$, each occurrence of the symbol _ is considered a different existential variable. The most general unifier substitutes each symbol in $g_{1}$ with the corresponding argument in $g_{2}$, and vice versa (obviously, if both arguments are _, the resulting argument is still _ ).

Furthermore, a $D L$ - $_{\text {Lite }}$ knowledge base $\mathcal{K}=\langle\mathcal{O}, \mathcal{A}\rangle$ is satisfiable if and only if $\operatorname{cert}_{\mathcal{V}_{\mathcal{O}}, \mathcal{O}^{p}}^{\mathcal{A}}=\emptyset$, where again $\operatorname{cert}_{\mathcal{V}_{\mathcal{O}}, \mathcal{O}^{p}}^{\mathcal{A}}=\operatorname{PerfectRef}\left(\mathcal{O}, \mathcal{V}_{\mathcal{O}}\right)^{\mathcal{I}_{\mathcal{A}}}$. Here, $\mathcal{O}^{p}$ is the $D L$-Lite $\mathcal{R}_{\mathcal{R}}$ ontology obtained from $\mathcal{O}$ by removing the disjointness assertions in $\mathcal{O}$, and $\mathcal{V}_{\mathcal{O}}$ is the violation query for $\mathcal{O}$, i.e., the boolean UCQ obtained by including a disjunct of the form $\left\{() \mid \exists y \cdot A_{1}(y) \wedge A_{2}(y)\right\}$ (respectively, $\left\{() \mid \exists y_{1}, y_{2} \cdot A_{1}\left(y_{1}\right) \wedge R\left(y_{1}, y_{2}\right)\right\}$, $\left\{() \mid \exists y_{1}, y_{2}, y_{3} \cdot R_{1}\left(y_{1}, y_{2}\right) \wedge R_{2}\left(y_{1}, y_{3}\right)\right\}$, and $\left.\left\{() \mid \exists y_{1}, y_{2} \cdot R_{1}\left(y_{1}, y_{2}\right) \wedge R_{2}\left(y_{1}, y_{2}\right)\right\}\right)$ for each disjointness assertion $A_{1} \sqsubseteq \neg A_{2}$ (respectively, $A_{1} \sqsubseteq \neg \exists R$ or $\exists R \sqsubseteq \neg A_{1}$, $\exists R_{1} \sqsubseteq \neg \exists R_{2}$, and $\left.R_{1} \sqsubseteq \neg R_{2}\right)$, where an atom of the form $R\left(y, y^{\prime}\right)$ stands for either $P\left(y, y^{\prime}\right)$ if $R$ denotes an atomic role $P$, or $P\left(y^{\prime}, y\right)$ if $R$ denotes the inverse of an atomic role, i.e., $R=P^{-}$. Furthermore, we denote by $\mathcal{V}_{\mathcal{O}}^{n}$ the UCQ over $\mathcal{O}$ of arity $n$ obtained by adding the target list $\left(x_{1}, \ldots, x_{n}\right)$ and $\top\left(x_{1}, \ldots, x_{n}\right)$ in the body to each disjunct of $\mathcal{V}_{\mathcal{O}}$, where $x_{i}$ is a fresh distinguished variable for each $i \in[1, n]$.

Observe that $D L-$ Lite $_{\mathcal{R}}$ is insensitive to the adoption of the UNA for UCQ answering Artale et al., 2009. Specifically, a $D L$-Lite L $_{\mathcal{R}}$ knowledge base $\mathcal{K}=\langle\mathcal{O}, \mathcal{A}\rangle$ is satisfiable if and only if it is so without the UNA, and the set of certain answers of an UCQ $q$ with respect to $\mathcal{O}$ and $\mathcal{A}$ does not depend on the adoption of the UNA.

We conclude this subsection with a consideration on the computational complexity of the query answering problem. Both with and without the UNA, the problem of answering UCQs over DL-Lite $e_{\mathcal{R}}$ knowledge bases is FOL-rewritable (and therefore in $\mathrm{AC}^{0}$ in data complexity, i.e., the complexity where only the ABox is considered to be the input (Vardi, 1982]), and NP-complete in combined complexity, i.e., the complexity with respect to all inputs of the problem Calvanese et al., 2007b. 


\subsection{Ontology-based Data Management}

According to Lenzerini, 2018, Ontology-based Data Management (OBDM) can be seen as a sophisticated form of Information Integration Lenzerini, 2002, Calvanese and De Giacomo, 2005, Doan et al., 2012, where the usual global schema is replaced by the conceptual model of an application domain, formulated as an ontology.

The OBDM paradigm resorts to a three-level architecture, consisting of the ontology, some existing data sources relevant for an organization, and the mapping between the two. In all this thesis, we assume that data sources are expressed as a unique relational database schema. Note that this is a realistic assumption, since many, nowadays available, off-the-shelf Data Federation/Virtualisation tools can be used to wrap multiple, possibly non-relational, sources, and present them as they were structured according to a single schema.

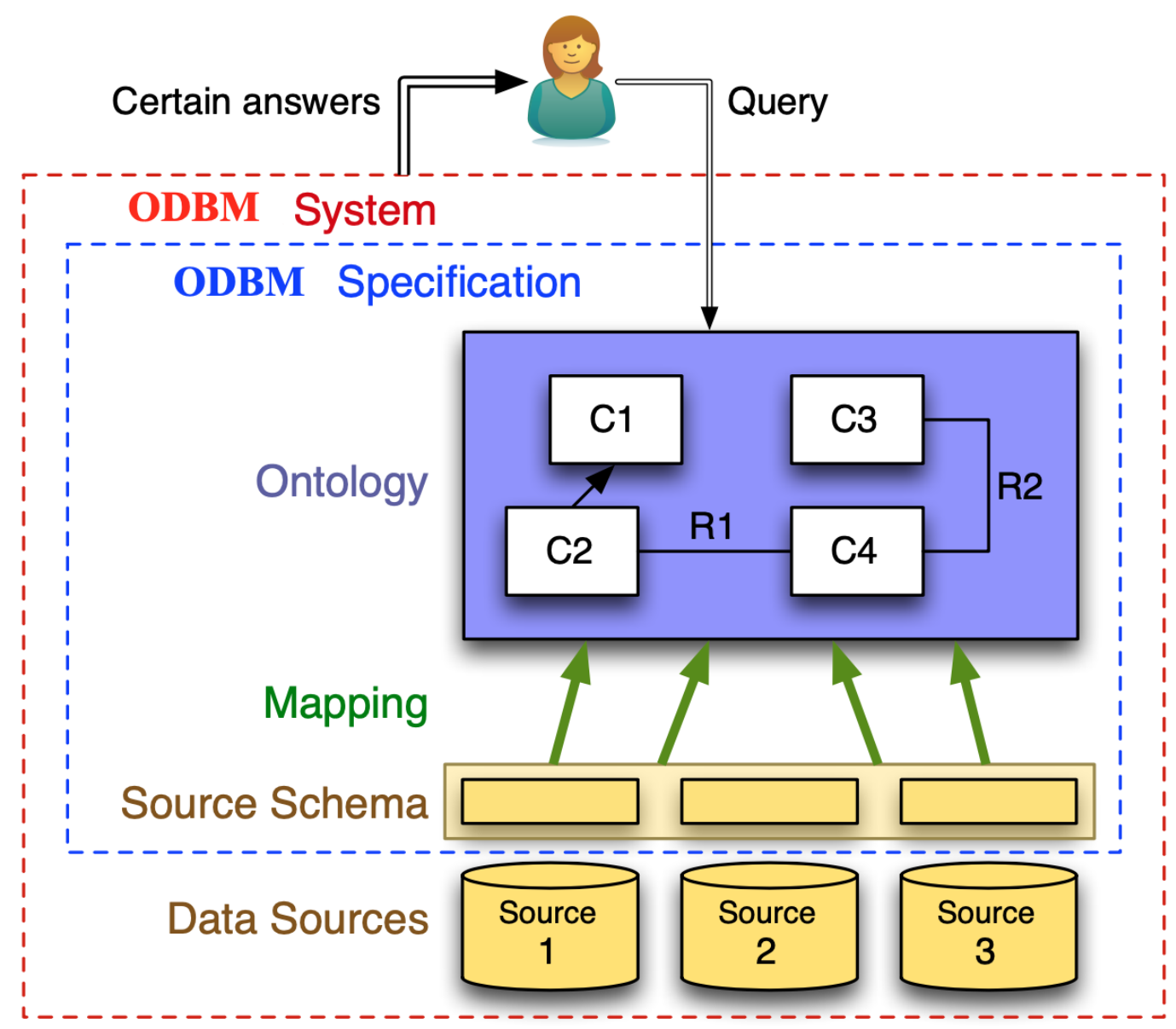

Figure 2.1. OBDM Specification and System

We distinguish between the specification of an OBDM system, and the OBDM system itself (cf. Figure 2.1). From a more formal perspective, an OBDM specification $\Sigma$ determines the intensional level of the system, and it is expressed as a triple $\Sigma=\langle\mathcal{O}, \mathcal{S}, \mathcal{M}\rangle$, where:

- $\mathcal{O}$ is a DL ontology; 
- $\mathcal{S}$ is a relational database schema, also called source schema;

- $\mathcal{M}$ is a mapping, i.e., a finite set of mapping assertions relating $\mathcal{S}$ to $\mathcal{O}$.

An OBDM system is a pair $\langle\Sigma, D\rangle$, where $\Sigma=\langle\mathcal{O}, \mathcal{S}, \mathcal{M}\rangle$ is an OBDM specification, and $D$ is an $\mathcal{S}$-database, also called source database for $\Sigma$.

\subsubsection{Mapping Specifications}

When mapping data sources to ontologies, one should take into account that sources store values (data), whereas instances of atomic concepts (respectively, atomic roles) are objects (respectively, pair of objects), where each object should be denoted by an ad-hoc identifier (a semantical object), not to be confused with any data item. Dealing with this problem, however, would uselessly complicate our technical treatment. Therefore, for ease of exposition, we ignore this problem (known as the impedance mismatch problem (Meseguer and Qian, 1993), and refer to Poggi et al., 2008 for technical details on how to solve it. We notice, however, that all our results can easily carry over in a setting that take into account also such issue.

Commonly, mapping assertions are assumed to be sound, which means that certain patterns over the source schema implies certain patterns at the ontology level. Typically, from a logical point of view, sound mapping assertions constituting a mapping $\mathcal{M}$ relating a schema $\mathcal{S}$ to an ontology $\mathcal{O}$ are of the form $\forall \vec{x} \cdot(\exists \vec{y} \cdot \phi \mathcal{S}(\vec{x}, \vec{y}) \rightarrow$ $\left.\exists \vec{z} \cdot \varphi_{\mathcal{O}}(\vec{x}, \vec{z})\right)$, where $\phi_{\mathcal{S}}(\vec{x}, \vec{y})$ and $\varphi_{\mathcal{O}}(\vec{x}, \vec{z})$ are body of CQs, i.e., finite conjunction of atoms over $\mathcal{S}$ and $\mathcal{O}$, respectively Lenzerini, 2002, Doan et al., 2012. Mapping assertions of the above form are also called GLAV (Global-and-Local-as-View) mapping assertions. Special cases of GLAV mapping assertions are GAV (Global-asView) and LAV (Local-as-View) mapping assertions.

A GAV mapping assertion is a GLAV mapping assertion in which the right-hand side of the implication does not make use of existential variables, i.e., it is an assertion of the form $\forall \vec{x}$. $\left(\exists \vec{y} . \phi_{\mathcal{S}}(\vec{x}, \vec{y}) \rightarrow \varphi_{\mathcal{O}}(\vec{x})\right)$. Furthermore, a GAV mapping assertion is called pure if $\varphi_{\mathcal{O}}(\vec{x})$ is a conjunction of atoms without constants or variables that are repeated more than once in the body of $\varphi_{\mathcal{O}}(\vec{x})$.

A LAV mapping assertion is a GLAV mapping assertion in which the left-hand side of the implication is simply an atom without constants or repeated variables, and all universally quantified variables appear in the right-hand side of the implication, i.e., it is an assertion of the form $\forall \vec{x} \cdot\left(\phi_{\mathcal{S}}(\vec{x}) \rightarrow \exists \vec{z} \cdot \varphi_{\mathcal{O}}(\vec{x}, \vec{z})\right)$, where $\phi_{\mathcal{S}}(\vec{x})$ corresponds to $s\left(x_{1}, \ldots, x_{n}\right)$ with $s$ being an $n$-ary predicate symbol of $\mathcal{S}$, and $x_{1}, \ldots, x_{n}$ being pairwise different variables.

For readability purposes, from now on we will drop universal quantifiers in front of mapping assertions. Finally, we say that a mapping $\mathcal{M}$ is a GLAV (respectively, LAV, GAV, pure GAV) mapping if it consists of a finite set of GLAV (respectively, LAV, GAV, pure GAV) mapping assertions.

Due to the assumption that ontologies contain the atomic concept $T$ in their alphabet (with its obvious semantics), in each mapping $\mathcal{M}$ relating a source schema $\mathcal{S}$ to an ontology $\mathcal{O}$, we tacitly assume that, for each $n$-ary predicate $s \in \mathcal{S}$, the mapping $\mathcal{M}$ contains the following both LAV and pure GAV mapping assertion:

$$
s\left(x_{1}, x_{2}, \ldots, x_{n}\right) \rightarrow \top\left(x_{1}\right) \wedge \top\left(x_{2}\right) \wedge \ldots \wedge \top\left(x_{n}\right) .
$$




\subsubsection{The Chase}

The chase is a fixpoint algorithm typically used to reason about data dependencies [Beeri and Vardi, 1984]. It has been introduced in [Maier et al., 1979], and now it plays a central role in various contexts, e.g., database schema design Bernstein, 1976 Fagin, 1977], query containment [Kolaitis and Vardi, 2000], data exchange [Fagin et al., 2005a; Fagin et al., 2005b; Arenas et al., 2014], etc.

Here, with chase we implicitly refer to the so-called oblivious chase Calì et al., 2013 (also known as the naive chase ten Cate et al., 2009) rather than to the standard chase Fagin et al., 2005a. Formally, given a set of atoms $\mathcal{W}$ over a schema $\mathcal{S}$ and a mapping $\mathcal{M}$ relating $\mathcal{S}$ to an ontology $\mathcal{O}$, the chase of $\mathcal{W}$ with respect to $\mathcal{M}$, denoted by $\mathcal{M}(\mathcal{W})$, is computed as follows: $(i)$ we start with an empty set of atoms $J:=\emptyset$ over $\mathcal{O}$, then $(i i)$ for every GLAV assertion $\exists \vec{y} \cdot \phi_{\mathcal{S}}(\vec{x}, \vec{y}) \rightarrow \exists \vec{z} \cdot \varphi_{\mathcal{O}}(\vec{x}, \vec{z})$ in $\mathcal{M}$ and for every homomorphism $h$ from the set of all atoms occurring in $\phi_{\mathcal{S}}(\vec{x}, \vec{y})$ to $\mathcal{W}$, we add to $J$ the image of the set of all atoms occurring in $\varphi_{\mathcal{O}}(\vec{x}, \vec{z})$ under $h^{\prime}$, that is, $J:=J \cup h^{\prime}\left(\varphi_{\mathcal{O}}(\vec{x}, \vec{z})\right)$, where $h^{\prime}$ extends $h$ by assigning to each variable $z \in \vec{z}$ a different fresh variable in Var still not present in $J$.

With a slight abuse of notation, given a $\mathrm{CQ}^{\neq} q$ over a schema $\mathcal{S}$ and a mapping $\mathcal{M}$ relating schema $\mathcal{S}$ to an ontology $\mathcal{O}$, we denote by $\mathcal{M}(q)$ the conjunction of all the atoms in $\mathcal{M}\left(\mathcal{W}_{q}\right)$, i.e., the conjunction of all the atoms obtained by chasing the incomplete $\mathcal{S}$-database associated to $q$ with respect to $\mathcal{M}$.

\subsubsection{Semantics and Query Answering}

The semantics of an OBDM system $\langle\Sigma, D\rangle$, with $\Sigma=\langle\mathcal{O}, \mathcal{S}, \mathcal{M}\rangle$ an OBDM specification and $D$ an $\mathcal{S}$-database, is given in terms of interpretations $\mathcal{I}$ for $\langle\Sigma, D\rangle$, i.e., interpretations $\mathcal{I}=\left\langle\Delta^{\mathcal{I}},{ }^{\mathcal{I}}\right\rangle$ for $\mathcal{O}$ in which the interpretation function ${ }^{\mathcal{I}}$ further assigns to each constant $a \in \operatorname{dom}(D) \cup \operatorname{con}_{\mathcal{M}}$ a domain object $a^{\mathcal{I}} \in \Delta^{\mathcal{I}}$, where $\operatorname{con}_{\mathcal{M}}$ denotes the set of all constants occurring in $\mathcal{M}$. Unless otherwise stated, we adopt the UNA, i.e., we consider only those interpretations $\mathcal{I}$ for which $a^{\mathcal{I}} \neq b^{\mathcal{I}}$ for each pair of constants $a, b \in \operatorname{dom}(D) \cup \operatorname{con}_{\mathcal{M}}$ with $a \neq b$.

Given an OBDM specification $\Sigma=\langle\mathcal{O}, \mathcal{S}, \mathcal{M}\rangle$, an $\mathcal{S}$-database $D$, an interpretation $\mathcal{I}=\left\langle\Delta^{\mathcal{I}},{ }^{\mathcal{I}}\right\rangle$ for $\langle\Sigma, D\rangle$, and a GLAV mapping assertion $m=\exists \vec{y} \cdot \phi_{\mathcal{S}}(\vec{x}, \vec{y}) \rightarrow$ $\exists \vec{z} \cdot \varphi_{\mathcal{O}}(\vec{x}, \vec{z})$ belonging to a mapping $\mathcal{M}$ relating schema $\mathcal{S}$ to $\mathcal{O}$, we say that the pair $\langle D, \mathcal{I}\rangle$ satisfies $m$ if the following holds: for each homomorphism $h$ from $\phi_{\mathcal{S}}(\vec{x}, \vec{y})$ to $D$, there exists a homomorphism $h^{\prime}$ from $\varphi_{\mathcal{O}}^{h_{\mathcal{I}}}(\vec{x}, \vec{z})$ to $\mathcal{I}$, where $\varphi_{\mathcal{O}}^{h_{\mathcal{I}}}(\vec{x}, \vec{z})$ denotes the set of all atoms occurring in $\varphi_{\mathcal{O}}(\vec{x}, \vec{z})$ obtained by replacing each term $t$, that is either a constant or $t \in \vec{x}$, with $h(t)^{\mathcal{I}}$ (by definition, $h(t)$ is either a constant occurring in $D$ or a constant occurring in $\mathcal{M}$, and therefore $h(t)^{\mathcal{I}}$ is a domain object belonging to $\left.\Delta^{\mathcal{I}}\right)$. Furthermore, we say that $\langle D, \mathcal{I}\rangle$ satisfies a mapping $\mathcal{M}$ relating schema $\mathcal{S}$ to $\mathcal{O}$, denoted by $\langle D, \mathcal{I}\rangle \models \mathcal{M}$, if $\langle D, \mathcal{I}\rangle$ satisfies every assertion $m \in \mathcal{M}$.

We are now ready to formalise the notion of model of an OBDM system. An interpretation $\mathcal{I}$ for an OBDM system $\langle\Sigma, D\rangle$ is a model of $\langle\Sigma, D\rangle$ (also called a model of $\Sigma$ relative to $D$ ), denoted by $\mathcal{I} \models\langle\Sigma, D\rangle$, if $(i) \mathcal{I} \models \mathcal{O}$ and $(i i)\langle D, \mathcal{I}\rangle \models \mathcal{M}$.

The set of models of an OBDM system $\langle\Sigma, D\rangle$, denoted by $\operatorname{Mod}_{D}(\Sigma)$, is the set of interpretations $\mathcal{I}$ for $\langle\Sigma, D\rangle$ such that $\mathcal{I}=\langle\Sigma, D\rangle$. An $\mathcal{S}$-database $D$ is said to be consistent with $\Sigma=\langle\mathcal{O}, \mathcal{S}, \mathcal{M}\rangle$ if $\operatorname{Mod}_{D}(\Sigma) \neq \emptyset$, inconsistent otherwise. 
In OBDM one of the main service of interest is query answering, i.e., computing the certain answers to queries posed over the ontology (cf. Figure 2.1). Given an OBDM specification $\Sigma=\langle\mathcal{O}, \mathcal{S}, \mathcal{M}\rangle$, an $\mathcal{S}$-database $D$, and an $\mathrm{UCQ}^{\neq}$query $q_{\mathcal{O}}$ over $\mathcal{O}$, we denote by $\operatorname{cert}_{q_{\mathcal{O}}, \Sigma}^{D}$ the set of certain answers of $q_{\mathcal{O}}$ with respect to $\Sigma$ and $D$, i.e., the set of tuples of constants $\left(c_{1}, \ldots, c_{n}\right)$ such that $\left(c_{1}^{\mathcal{I}}, \ldots, c_{n}^{\mathcal{I}}\right) \in q_{\mathcal{O}}^{\mathcal{I}}$ for each $\mathcal{I} \in \operatorname{Mod}_{D}(\Sigma)$. Observe that, if $\langle\Sigma, D\rangle$ is unsatisfiable, then the set of certain answers of any query $q_{\mathcal{O}}$ (over $\mathcal{O}$ ) with respect to $\Sigma$ and $D$ is trivially the set of all possible tuples of constants occurring in $D$ whose arity is the one of the query.

Given an OBDM specification $\Sigma=\langle\mathcal{O}, \mathcal{S}, \mathcal{M}\rangle$ and two queries $q_{\mathcal{O}}^{1}, q_{\mathcal{O}}^{2}$ over $\mathcal{O}$, we write $\operatorname{cert}_{q_{\mathcal{O}}^{1}, \Sigma} \sqsubseteq \operatorname{cert}_{q_{\mathcal{O}}^{2}, \Sigma}$ if $\operatorname{cert}_{q_{\mathcal{O}}^{1}, \Sigma}^{D} \subseteq \operatorname{cert}_{q_{\mathcal{O}}^{2}, \Sigma}^{D}$ for each $\mathcal{S}$-database $D$. We also write $\operatorname{cert}_{q_{\mathcal{O}}^{1}, \Sigma} \sqsubset \operatorname{cert}_{q_{\mathcal{O}}^{2}, \Sigma}$ if $(i) \operatorname{cert}_{q_{\mathcal{O}}^{1}, \Sigma} \sqsubseteq \operatorname{cert}_{q_{\mathcal{O}}^{2}, \Sigma}$, and in addition $(i i) \operatorname{cert}_{q_{\mathcal{O}}^{1}, \Sigma}^{D} \subsetneq \operatorname{cert}_{q_{\mathcal{O}}^{2}, \Sigma}^{D}$ for at least an $\mathcal{S}$-database $D$. Finally, we say that $q_{\mathcal{O}}^{1}$ and $q_{\mathcal{O}}^{2}$ are equivalent with respect to $\Sigma$, denoted by $\operatorname{cert}_{q_{\mathcal{O}}^{1}, \Sigma} \equiv \operatorname{cert}_{q_{\mathcal{O}}^{2}, \Sigma}$, if both $\operatorname{cert}_{q_{\mathcal{O}}^{1}, \Sigma} \sqsubseteq \operatorname{cert}_{q_{\mathcal{O}}^{2}, \Sigma}$ and $\operatorname{cert}_{q_{\mathcal{O}}^{2}, \Sigma} \sqsubseteq \operatorname{cert}_{q_{\mathcal{O}}^{1}, \Sigma}$ hold, that is, $\operatorname{cert}_{q_{\mathcal{O}}^{1}, \Sigma}^{D}=\operatorname{cert}_{q_{\mathcal{O}}^{2}, \Sigma}^{D}$ for each $\mathcal{S}$-database $D$.

For an OBDM specification $\Sigma=\langle\mathcal{O}, \mathcal{S}, \mathcal{M}\rangle$ and a query $q_{\mathcal{O}}$ over $\mathcal{O}$, following the literature terminology, we say that a query $q_{\mathcal{S}}$ over $\mathcal{S}$ is a perfect (respectively, sound) $\mathcal{O}$-to-S $\Sigma$-rewriting of $q_{\mathcal{O}}$ if $q_{\mathcal{S}}^{D}=\operatorname{cert}_{q_{\mathcal{O}}, \Sigma}^{D}\left(\right.$ respectively, $\left.q_{\mathcal{S}}^{D} \subseteq \operatorname{cert}_{q_{\mathcal{O}}, \Sigma}^{D}\right)$ for each $\mathcal{S}$-database $D$. The perfect $\mathcal{O}$-to- $\mathcal{S} \Sigma$-rewriting of $q_{\mathcal{O}}$ is denoted by $\mathrm{REW}_{q_{\mathcal{O}}, \Sigma}$. Observe that, by definition, $\operatorname{REW}_{q_{\mathcal{O}}, \Sigma}^{D}=\operatorname{cert}_{q_{\mathcal{O}}, \Sigma}^{D}$ for each $\mathcal{S}$-database $D$.

Let $\Sigma=\langle\mathcal{O}, \mathcal{S}, \mathcal{M}\rangle$ be an OBDM specification where $\mathcal{O}=\emptyset$, i.e., $\mathcal{O}$ has no assertions, and $\mathcal{M}$ is a GLAV mapping. From result of Friedman et al., 1999 Calvanese et al., 2012, it is well-known that, given a UCQ $q_{\mathcal{O}}$ over $\mathcal{O}$, by splitting the GLAV mapping $\mathcal{M}$ into a GAV mapping followed by a LAV mapping over an intermediate alphabet, it is always possible to compute a UCQ over $\mathcal{S}$, denoted by $\operatorname{MapRef}\left(q_{\mathcal{O}}, \mathcal{M}\right)$, such that $\operatorname{MapRef}\left(q_{\mathcal{O}}, \mathcal{M}\right) \equiv \operatorname{REW}_{q_{\mathcal{O}}, \Sigma}$

We conclude this chapter with the following observations for OBDM specifications $\Sigma=\langle\mathcal{O}, \mathcal{S}, \mathcal{M}\rangle$ where $\mathcal{O}$ is a $D L$-Lite $e_{\mathcal{R}}$ ontology and $\mathcal{M}$ is a GLAV mapping:

- Given a UCQ $q_{\mathcal{O}}$ over $\mathcal{O}$, we denote by $\operatorname{PerfRef}_{q_{\mathcal{O}}, \Sigma}$ the UCQ obtained by first executing the algorithm PerfectRef on $\mathcal{O}$ and $q_{\mathcal{O}}$, and then by rewriting the obtained UCQ with respect to mapping $\mathcal{M}$, i.e., $\operatorname{PerfRef}_{q_{\mathcal{O}}, \Sigma}:=$ $\operatorname{MapRef}\left(\operatorname{PerfectRef}\left(\mathcal{O}, q_{\mathcal{O}}\right), \mathcal{M}\right)$. From results of Subsection 2.5.3 and the above observation, it is easy to see that, if $q_{\mathcal{O}}$ is a UCQ over $\mathcal{O}$ of arity $n$, then $\operatorname{REW}_{q_{\mathcal{O}}, \Sigma} \equiv \operatorname{PerfRef}_{q_{\mathcal{O}}, \Sigma} \cup \operatorname{PerfRef}_{\mathcal{V}_{\mathcal{O}}^{n}, \Sigma}$. In other words, if $q_{\mathcal{O}}$ is a UCQ over $\mathcal{O}$, then the UCQ PerfRef $q_{\mathcal{O}}, \Sigma \cup \operatorname{PerfRef}_{\mathcal{V}_{\mathcal{O}}^{n}, \Sigma}$ is the perfect $\mathcal{O}$-to- $\mathcal{S} \Sigma$-rewriting of $q_{\mathcal{O}}$, i.e, $\left(\operatorname{PerfRef}_{q_{\mathcal{O}}, \Sigma} \cup \operatorname{PerfRef}_{\mathcal{V}_{\mathcal{O}}^{n}, \Sigma}\right)^{D}=\operatorname{cert}_{q_{\mathcal{O}}, \Sigma}^{D}$ for every $\mathcal{S}$-database $D$.

- For an $\mathcal{S}$-database $D$, we denote by $\mathcal{C}_{\mathcal{O}}^{\mathcal{M}(D)}$ the canonical structure of $\mathcal{O}$ with respect to $\mathcal{M}$ and $D$, i.e., the (possibly infinite) set of atoms over $\mathcal{O}$ obtained by first chasing $D$ with respect to $\mathcal{M}$, and then by chasing, possibly ad infinitum, the resulting set of atoms $\mathcal{M}(D)$ with respect to $\mathcal{O}$ as described in Calvanese et al., 2007b, Definition 5] but using the alphabet Var of variables whenever a new element is needed in the chase. By combining results of Fagin et al., 2005a Proposition 4.2] with [Calvanese et al., 2007b, Theorem 29], it is well-known that, if $q_{\mathcal{O}}$ is a UCQ over $\mathcal{O}$ and $\vec{c}$ is a tuple of constants, then $\vec{c} \in \operatorname{cert}_{q_{\mathcal{O}}, \Sigma}^{D}$ if and only if $\vec{c} \in q_{\mathcal{O}}^{\mathcal{C}^{\mathcal{M}(D)}}$, i.e., if and only if $\mathcal{C}_{\mathcal{O}}^{\mathcal{M}(D)} \models q_{\mathcal{O}}(\vec{c})$. 



\section{Chapter 3}

\section{Abstraction in Ontology-based Data Management: Framework}

In this chapter, we illustrate a formal framework for abstraction in OBDM. Informally, given a data service expressed as a query $q_{\mathcal{S}}$ over the source schema, the goal is in finding a query $q_{\mathcal{O}}$ over the ontology that represents an abstraction of the data service represented by $q_{\mathcal{S}}$ in terms of the domain ontology through the mapping.

Specifically, we introduce three semantically well-founded notions, namely perfect, sound, and complete source-to-ontology rewriting, and two basic computational problems, namely verification and computation. In what follows, $\Sigma=\langle\mathcal{O}, \mathcal{S}, \mathcal{M}\rangle$ refers to an OBDM specification, and $q_{\mathcal{S}}$ and $q_{\mathcal{O}}$ to queries over the source schema $\mathcal{S}$ and over the ontology $\mathcal{O}$, respectively, of the same arity.

\subsection{The notion of Source-to-Ontology Rewriting}

Intuitively, given a data service expressed as a query $q_{\mathcal{S}}$ over $\mathcal{S}$, we aim at finding the query $q_{\mathcal{O}}$ over $\mathcal{O}$ that semantically characterises $q_{\mathcal{S}}$ with respect to the OBDM specification $\Sigma=\langle\mathcal{O}, \mathcal{S}, \mathcal{M}\rangle$ of the information system. Since the evaluation of queries over $\mathcal{O}$ is based on certain answers, this means that we aim at finding a query over $\mathcal{O}$ whose certain answers with respect to $\Sigma$ and $D$ precisely capture the evaluation of $q_{\mathcal{S}}$ over $D$, for every $\mathcal{S}$-database $D$. Therefore, we are naturally led to the notion of perfect source-to-ontology rewriting.

Definition 3.1. We say that $q_{\mathcal{O}}$ is a perfect $\mathcal{S}$-to- $\mathcal{O} \Sigma$-rewriting of $q_{\mathcal{S}}$, if for every $\mathcal{S}$-database $D, \operatorname{Mod}_{D}(\Sigma) \neq \emptyset$ implies $\operatorname{cert}_{q_{\mathcal{O}}, \Sigma}^{D}=q_{\mathcal{S}}^{D}$. If in addition $q_{\mathcal{O}} \in \mathcal{L}_{\mathcal{O}}$ for a query language $\mathcal{L}_{\mathcal{O}}$, then we say that $q_{\mathcal{O}}$ is an $\mathcal{L}_{\mathcal{O}}$-perfect $\mathcal{S}$-to- $\mathcal{O} \Sigma$-rewriting of $q_{\mathcal{S}}$.

Example 3.1. Let $\Sigma=\langle\mathcal{O}, \mathcal{S}, \mathcal{M}\rangle, q_{\mathcal{S}}$, and $q_{\mathcal{O}}$ be the OBDM specification, the query over $\mathcal{S}$, and the query over $\mathcal{O}$, respectively, illustrated in Example 1.1. One can easily verify that $q_{\mathcal{O}}$ is a CQ-perfect $\mathcal{S}$-to- $\mathcal{O} \Sigma$-rewriting of $q_{\mathcal{S}}$.

The next proposition states that perfect source-to-ontology rewritings are always unique (up to equivalence with respect to the underlying OBDM specification $\Sigma$ ).

Proposition 3.1. If $q_{1}$ and $q_{2}$ are perfect $\mathcal{S}$-to- $\mathcal{O} \Sigma$-rewritings of $q_{\mathcal{S}}$, then they are equivalent with respect to $\Sigma$, i.e., cert $_{q_{1}, \Sigma} \equiv \operatorname{cert}_{q_{2}, \Sigma}$. 
Proof. Following Definition 3.1, since $q_{1}$ and $q_{2}$ are perfect $\mathcal{S}$-to- $\mathcal{O} \Sigma$-rewritings of $q_{\mathcal{S}}$, we have that $\operatorname{cert}_{q_{1}, \Sigma}^{D}=q_{\mathcal{S}}^{D}=\operatorname{cert}_{q_{2}, \Sigma}^{D}$ for all $\mathcal{S}$-databases $D$ consistent with $\Sigma$. For all the $\mathcal{S}$-databases $D$ that are not consistent with $\Sigma$, however, by definition of certain answers, we have that $\operatorname{cert}_{q_{1}, \Sigma}^{D}=\operatorname{cert}_{q_{2}, \Sigma}^{D}$ as well. So, $\operatorname{cert}_{q_{1}, \Sigma}^{D}=\operatorname{cert}_{q_{2}, \Sigma}^{D}$ for all $\mathcal{S}$-databases $D$. Thus, cert $_{q_{1}, \Sigma} \equiv \operatorname{cert}_{q_{2}, \Sigma}$, as required.

The next example shows that perfect source-to-ontology rewritings are not guaranteed to exists, even in trivial cases.

Example 3.2. Let $\Sigma=\langle\mathcal{O}, \mathcal{S}, \mathcal{M}\rangle$ be the following OBDM specification:

- $\mathcal{O}=\{\exists$ WorksFor $\sqsubseteq$ Worker, MathStudent $\sqsubseteq$ Student $\}$

- $\mathcal{S}=\left\{s_{1}, s_{2}, s_{3}, s_{4}, s_{5}\right\}$

- $\mathcal{M}=\left\{m_{1}, m_{2}, m_{3}, m_{4}, m_{5}, m_{6}\right\}$, where:

$$
\begin{array}{rrrl}
m_{1}: & s_{1}(x) & \rightarrow \text { Worker }(x), \\
m_{2}: & s_{1}(x) & \rightarrow \text { Student }(x), \\
m_{3}: & s_{2}\left(x_{1}, x_{2}\right) & \rightarrow & \text { WorksFor }\left(x_{1}, x_{2}\right), \\
m_{4}: & s_{3}(x) & \rightarrow & \text { MathStudent }(x), \\
m_{5}: & s_{1}(x) \wedge s_{4}(x) & \rightarrow & \text { Engineer }(x), \\
m_{6}: & s_{1}\left(x_{1}\right) \wedge s_{5}\left(x_{1}, x_{2}\right) & \rightarrow & \text { PlaysSport }\left(x_{1}, x_{2}\right) .
\end{array}
$$

Let the data service be expressed as the query $q_{\mathcal{S}}=\left\{(x) \mid s_{1}(x)\right\}$ over the source schema $\mathcal{S}$. By inspecting the mapping $\mathcal{M}$ and the ontology $\mathcal{O}$ one can see that, since the certain answers of $q_{\mathcal{O}}^{1}=\{(x) \mid$ Worker $(x)\}$ include also the values stored in the projection on the first component of $s_{2}$, and since the certain answers of $q_{\mathcal{O}}^{2}=\{(x) \mid$ Student $(x)\}$ include also the values stored in $s_{3}$, both queries are too general for exactly characterising $q_{\mathcal{S}}$. On the other hand, queries $q_{\mathcal{O}}^{3}=\{(x) \mid$ Engineer $(x)\}$ and $q_{\mathcal{O}}^{4}=\{(x) \mid \exists y$.PlaysSport $(x, y)\}$ are too specific, and therefore we conclude that no perfect $\mathcal{S}$-to- $\mathcal{O} \Sigma$-rewriting of $q_{\mathcal{S}}$ exists.

In order to cope with the situations illustrated above, we introduce the notions of sound and complete source-to-ontology rewritings, which, intuitively, provide sound and complete approximations of perfect source-to-ontology rewritings, respectively.

Definition 3.2. We say that $q_{\mathcal{O}}$ is a sound $\mathcal{S}$-to- $\mathcal{O} \Sigma$-rewriting of $q_{\mathcal{S}}$, if for every $\mathcal{S}$-database $D, \operatorname{Mod}_{D}(\Sigma) \neq \emptyset$ implies $\operatorname{cert}_{q_{\mathcal{O}}, \Sigma}^{D} \subseteq q_{\mathcal{S}}^{D}$.

Definition 3.3. We say that $q_{\mathcal{O}}$ is a complete $\mathcal{S}$-to- $\mathcal{O} \Sigma$-rewriting of $q_{\mathcal{S}}$, if for every $\mathcal{S}$-database $D, \operatorname{Mod}_{D}(\Sigma) \neq \emptyset$ implies $q_{\mathcal{S}}^{D} \subseteq \operatorname{cert}_{q_{\mathcal{O}}, \Sigma}^{D}$.

We illustrate these notions continuing on the previous example.

Example 3.3. Refer to Example 3.2. Note that $q_{\mathcal{O}}^{1}$ and $q_{\mathcal{O}}^{2}$ are complete $\mathcal{S}$-to- $\mathcal{O}$ $\Sigma$-rewritings of $q_{\mathcal{S}}$, whereas $q_{\mathcal{O}}^{3}$ and $q_{\mathcal{O}}^{4}$ are sound $\mathcal{S}$-to- $\mathcal{O} \Sigma$-rewritings of $q_{\mathcal{S}}$.

Obviously, $q_{\mathcal{O}}$ is a perfect $\mathcal{S}$-to- $\mathcal{O} \Sigma$-rewriting of $q_{\mathcal{S}}$ if and only if $q_{\mathcal{O}}$ is both a sound, and a complete $\mathcal{S}$-to- $\mathcal{O} \Sigma$-rewriting of $q_{\mathcal{S}}$.

As Example 3.3 shows, different sound or complete source-to-ontology rewritings of a query $q_{\mathcal{S}}$ may exist, and therefore it is reasonable to look for the "best" approximations of $q_{\mathcal{S}}$, at least relative to a certain query language $\mathcal{L}_{\mathcal{O}}$ over $\mathcal{O}$. 
Definition 3.4. We say that $q_{\mathcal{O}} \in \mathcal{L}_{\mathcal{O}}$ is an $\mathcal{L}_{\mathcal{O}}$-maximally sound $\mathcal{S}$-to- $\mathcal{O} \Sigma$-rewriting of $q_{\mathcal{S}}$, if $q_{\mathcal{O}}$ is a sound $\mathcal{S}$-to- $\mathcal{O} \Sigma$-rewriting of $q_{\mathcal{S}}$ and there exists no $q^{\prime} \in \mathcal{L}_{\mathcal{O}}$ such that $(i) q^{\prime}$ is a sound $\mathcal{S}$-to- $\mathcal{O} \Sigma$-rewriting of $q_{\mathcal{S}},(i i) \operatorname{cert}_{q_{\mathcal{O}}, \Sigma} \sqsubseteq \operatorname{cert}_{q^{\prime}, \Sigma}$, and $(i i i)$ $\operatorname{cert}_{q_{\mathcal{O}}, \Sigma}^{D} \subsetneq \operatorname{cert}_{q^{\prime}, \Sigma}^{D}$ for an $\mathcal{S}$-database $D$.

Definition 3.5. We say that $q_{\mathcal{O}} \in \mathcal{L}_{\mathcal{O}}$ is an $\mathcal{L}_{\mathcal{O}}$-minimally complete $\mathcal{S}$-to- $\mathcal{O} \Sigma$ rewriting of $q_{\mathcal{S}}$, if $q_{\mathcal{O}}$ is a complete $\mathcal{S}$-to- $\mathcal{O} \Sigma$-rewriting of $q_{\mathcal{S}}$ and there exists no $q^{\prime} \in$ $\mathcal{L}_{\mathcal{O}}$ such that $(i) q^{\prime}$ is a complete $\mathcal{S}$-to- $\mathcal{O}$ s-rewriting of $q_{\mathcal{S}},{ }_{(i i)} \operatorname{cert}_{q^{\prime}, \Sigma} \sqsubseteq \operatorname{cert}_{q_{\mathcal{O}}, \Sigma}$, and $(i i i) \operatorname{cert}_{q^{\prime}, \Sigma}^{D} \subsetneq \operatorname{cert}_{q_{\mathcal{O}, \Sigma}}^{D}$ for an $\mathcal{S}$-database $D$.

We illustrate the above notions by developing on Example 3.2 .

Example 3.4. We refer again to Example 3.2. Observe that neither $q_{\mathcal{O}}^{1}$ nor $q_{\mathcal{O}}^{2}$ are CQ-minimally complete $\mathcal{S}$-to- $\mathcal{O} \Sigma$-rewritings of $q_{\mathcal{S}}$. Indeed, one can verify that the CQ $q_{\mathcal{O}}^{5}=\{(x) \mid \operatorname{Worker}(x) \wedge \operatorname{Student}(x)\}$ is a UCQ-minimally complete $\mathcal{S}$-to- $\mathcal{O}$ $\Sigma$-rewriting of $q_{\mathcal{S}}$. As for queries $q_{\mathcal{O}}^{3}$ and $q_{\mathcal{O}}^{4}$, it is easy to see that they are both CQ-maximally sound $\mathcal{S}$-to- $\mathcal{O} \Sigma$-rewritings of $q_{\mathcal{S}}$, but neither of them is a UCQmaximally sound $\mathcal{S}$-to- $\mathcal{O} \Sigma$-rewriting of $q_{\mathcal{S}}$. Indeed, one can verify that the UCQ $q_{\mathcal{O}}^{6}=q_{\mathcal{O}}^{3} \cup q_{\mathcal{O}}^{4}$ is a UCQ-maximally sound $\mathcal{S}$-to- $\mathcal{O}$ L-rewriting of $q_{\mathcal{S}}$.

As we will see in a next proposition, there are settings for OBDM specifications and query languages $\mathcal{L}_{\mathcal{O}}$ for which it is always the case that, if there exists an $\mathcal{L}_{\mathcal{O}}$-maximally sound (respectively, $\mathcal{L}_{\mathcal{O}}$-minimally complete) $\mathcal{S}$-to- $\mathcal{O} \Sigma$-rewriting of a query $q_{\mathcal{S}}$, then it is unique (up to equivalence w.r.t. $\Sigma$ ). In those cases, it is reasonable to talk about the unique (up to equivalence w.r.t. $\Sigma$ ) $\mathcal{L}_{\mathcal{O}}$-maximally sound (respectively, $\mathcal{L}_{\mathcal{O}}$-minimally complete) $\mathcal{S}$-to- $\mathcal{O} \Sigma$-rewriting of $q_{\mathcal{S}}$.

Definition 3.6. We say that $q_{\mathcal{O}} \in \mathcal{L}_{\mathcal{O}}$ is the unique (up to equivalence w.r.t. $\Sigma$ ) $\mathcal{L}_{\mathcal{O}^{-}}$ maximally sound $\mathcal{S}$-to- $\mathcal{O} \Sigma$-rewriting of $q_{\mathcal{S}}$, if $(i) q_{\mathcal{O}}$ is a sound $\mathcal{S}$-to- $\mathcal{O} \Sigma$-rewriting of $q_{\mathcal{S}}$, and $(i i)$ every $q^{\prime} \in \mathcal{L}_{\mathcal{O}}$ that is a sound $\mathcal{S}$-to- $\mathcal{O} \Sigma$-rewriting of $q_{\mathcal{S}}$ is such that $\operatorname{cert}_{q^{\prime}, \Sigma} \sqsubseteq \operatorname{cert}_{q_{\mathcal{O}}, \Sigma}$.

Definition 3.7. We say that $q_{\mathcal{O}} \in \mathcal{L}_{\mathcal{O}}$ is the unique (up to equivalence w.r.t. $\Sigma$ ) $\mathcal{L}_{\mathcal{O}}$-minimally complete $\mathcal{S}$-to- $\mathcal{O} \Sigma$-rewriting of $q_{\mathcal{S}}$, if $(i) q_{\mathcal{O}}$ is a complete $\mathcal{S}$-to- $\mathcal{O}$ $\Sigma$-rewriting of $q_{\mathcal{S}}$, and $(i i)$ every $q^{\prime} \in \mathcal{L}_{\mathcal{O}}$ that is a complete $\mathcal{S}$-to- $\mathcal{O} \Sigma$-rewriting of $q_{\mathcal{S}}$ is such that $\operatorname{cert}_{q_{\mathcal{O}}, \Sigma} \sqsubseteq \operatorname{cert}_{q^{\prime}, \Sigma}$.

Let us continue on the running example of this section.

Example 3.5. Refer again to Example 3.2, and consider also the queries $q_{\mathcal{O}}^{5}$ and $q_{\mathcal{O}}^{6}$ defined in Example 3.4 . It is straightforward to verify that $q_{\mathcal{O}}^{5}$ (respectively, $q_{\mathcal{O}}^{6}$ ) is the unique (up to equivalence w.r.t. $\Sigma$ ) UCQ-minimally complete (respectively, UCQ-maximally sound) $\mathcal{S}$-to- $\mathcal{O} \Sigma$-rewriting of $q_{\mathcal{S}}$. Furthermore, observe that: $(i)$ since $q_{\mathcal{O}}^{5}$ is a CQ, it is also the unique (up to equivalence w.r.t. $\Sigma$ ) CQ-minimally complete $\mathcal{S}$-to- $\mathcal{O} \Sigma$-rewriting of $q_{\mathcal{S}}$, and $(i i)$ since both $q_{\mathcal{O}}^{3}$ and $q_{\mathcal{O}}^{4}$ are CQ-maximally sound $\mathcal{S}$-to- $\mathcal{O} \Sigma$-rewritings of $q_{\mathcal{S}}$ and they are not equivalent w.r.t. $\Sigma$, we conclude that the unique (up to equivalence w.r.t. $\Sigma$ ) CQ-maximally sound $\mathcal{S}$-to- $\mathcal{O} \Sigma$-rewriting of $q_{\mathcal{S}}$ does not exists. 


\subsection{Computational Problems}

Given the general framework presented in the previous section, it is natural to consider (at least) the following two basic computational problems, for classes $\mathcal{L}_{\mathcal{S}}$ and $\mathcal{L}_{\mathcal{O}}$ of queries over the source schema $\mathcal{S}$ and over the ontology $\mathcal{O}$, respectively:

- Verification: given $\Sigma=\langle\mathcal{O}, \mathcal{S}, \mathcal{M}\rangle, q_{\mathcal{S}}$ over $\mathcal{S}$ such that $q_{\mathcal{S}} \in \mathcal{L}_{\mathcal{S}}$, and $q_{\mathcal{O}}$ over $\mathcal{O}$ such that $q_{\mathcal{O}} \in \mathcal{L}_{\mathcal{O}}$, verify whether $q_{\mathcal{O}}$ is a perfect (respectively, sound, complete) $\mathcal{S}$-to- $\mathcal{O} \Sigma$-rewriting of $q_{\mathcal{S}}$.

- Computation: given $\Sigma=\langle\mathcal{O}, \mathcal{S}, \mathcal{M}\rangle$ and $q_{\mathcal{S}}$ over $\mathcal{S}$ such that $q_{\mathcal{S}} \in \mathcal{L}_{\mathcal{S}}$, compute any perfect (respectively, $\mathcal{L}_{\mathcal{O}}$-perfect, $\mathcal{L}_{\mathcal{O}}$-maximally sound, $\mathcal{L}_{\mathcal{O}}$-minimally complete) $\mathcal{S}$-to- $\mathcal{O} \Sigma$-rewriting of $q_{\mathcal{S}}$, if it exists.

In what follows, if not otherwise stated, we silently refer to the following scenario which use languages amongst the most popular considered in the literature: $(i)$ the setting for OBDM specifications is such that the DL ontology language is DL-Lite , $_{\mathcal{R}}$ the source schemas do not have integrity constraints, and the mapping language follows the GLAV approach; and $(i i)$ both $\mathcal{L}_{\mathcal{S}}$ and $\mathcal{L}_{\mathcal{O}}$ denote the class of UCQs.

Interestingly, in this scenario, we have the following result.

Proposition 3.2. If $q_{1}$ and $q_{2}$ are UCQ-minimally complete (respectively, UCQmaximally sound) $\mathcal{S}$-to- $\mathcal{O} \Sigma$-rewritings of $q_{\mathcal{S}}$, then they are equivalent w.r.t. $\Sigma$.

Proof. We first address the case of UCQ-maximally sound, and then the case of UCQ-minimally complete.

Assume that $q_{1}$ and $q_{2}$ are UCQ-maximally sound $\mathcal{S}$-to- $\mathcal{O} \Sigma$-rewritings of $q_{\mathcal{S}}$ and suppose, for the sake of contradiction, that they are not equivalent w.r.t. $\Sigma$. This implies the existence of two $\mathcal{S}$-databases $D_{1}$ and $D_{2}$ such that $(i) \overrightarrow{c_{1}} \notin \operatorname{cert} t_{q_{1}, \Sigma}^{D_{1}}$ and $\overrightarrow{c_{1}} \in \operatorname{cert}_{q_{2}, \Sigma}^{D_{1}}$ for a tuple of constants $\overrightarrow{c_{1}}$, and $(i i) \overrightarrow{c_{2}} \notin \operatorname{cert}_{q_{2}, \Sigma}^{D_{2}}$ and $\overrightarrow{c_{2}} \in \operatorname{cert}_{q_{1}, \Sigma}^{D_{2}}$ for a tuple of constants $\overrightarrow{c_{2}}$. But then, it can be readily seen that the UCQ $Q=q_{1} \cup q_{2}$ is such that $(i)$ since both $q_{1}$ and $q_{2}$ are sound $\mathcal{S}$-to- $\mathcal{O} \Sigma$-rewritings of $q_{\mathcal{S}}, Q$ is a sound $\mathcal{S}$-to- $\mathcal{O} \Sigma$-rewriting of $q_{\mathcal{S}},(i i)$ for both $i=1$ and $i=2$, we have $\operatorname{cert}_{q_{i}, \Sigma} \sqsubseteq \operatorname{cert}_{Q, \Sigma}$, and (iii) for both $i=1$ and $i=2$, the $\mathcal{S}$-database $D_{i}$ is such that $\operatorname{cert}_{q_{i}, \Sigma}^{D} \subsetneq \operatorname{cert}{ }_{Q, \Sigma}^{D_{i}}$. Therefore, according to Definition 3.4 we have a contradiction on the fact that $q_{1}$ and $q_{2}$ are UCQ-maximally sound $\mathcal{S}$-to- $\mathcal{O} \Sigma$-rewritings of $q_{\mathcal{S}}$, as required.

Assume that $q_{1}$ and $q_{2}$ are UCQ-minimally complete $\mathcal{S}$-to- $\mathcal{O} \Sigma$-rewritings of $q_{\mathcal{S}}$ and suppose, for the sake of contradiction, that they are not equivalent w.r.t. $\Sigma$. This implies the existence of two $\mathcal{S}$-databases $D_{1}$ and $D_{2}$ such that $(i) \overrightarrow{c_{1}} \in \operatorname{cert}_{q_{1}, \Sigma}^{D_{1}}$ and $\overrightarrow{c_{1}} \notin \operatorname{cert}_{q_{2}, \Sigma}^{D_{1}}$ for a tuple of constants $\overrightarrow{c_{1}}$, and $(i i) \overrightarrow{c_{2}} \in \operatorname{cert}_{q_{2}, \Sigma}^{D_{2}}$ and $\overrightarrow{c_{2}} \notin$ $\operatorname{cert}_{q_{1}, \Sigma}^{D_{2}}$ for a tuple of constants $\overrightarrow{c_{2}}$. But then, consider the query $q$ where $\operatorname{cert}_{q, \Sigma}^{D}=$ $\operatorname{cert}_{q_{1}, \Sigma}^{D} \cap \operatorname{cert}_{q_{2}, \Sigma}^{D}$ for every $\mathcal{S}$-database $D$. Obviously, since $q_{1}$ and $q_{2}$ are UCQs, $q$ always exists and can be expressed as a UCQ, too. It can be readily seen that (i) since $q_{1}$ and $q_{2}$ are complete $\mathcal{S}$-to- $\mathcal{O} \Sigma$-rewritings of $q_{\mathcal{S}}, q$ is a complete $\mathcal{S}$-to- $\mathcal{O}$ $\Sigma$-rewriting of $q_{\mathcal{S}}$ as well, (ii) for both $i=1$ and $i=2$, we have $\operatorname{cert}_{q, \Sigma} \sqsubseteq \operatorname{cert}_{q_{i}, \Sigma}$, and $(i i i)$ for both $i=1$ and $i=2$, the $\mathcal{S}$-database $D_{i}$ is such that $\operatorname{cert}_{q, \Sigma}^{D_{i}} \subsetneq \operatorname{cert}_{q_{i}, \Sigma}^{D_{i}}$. Therefore, according to Definition 3.5 we have a contradiction on the fact that $q_{1}$ and $q_{2}$ are UCQ-minimally complete $\mathcal{S}$-to- $\mathcal{O} \Sigma$-rewritings of $q_{\mathcal{S}}$, as required. 


\subsection{Related Work}

In this section, we provide a detailed relationship between the new definitions introduced in Section 3.1 and some literature notions about pertinent subjects that we argue are worth comparing to.

\subsubsection{A slightly different Semantics}

A similar, but not equivalent, notion of perfect source-to-ontology rewriting given in Definition 3.1 is the notion of realization provided in Lutz et al., 2018. Specifically, $q_{\mathcal{O}}$ is a realization of $q_{\mathcal{S}}$ in $\Sigma$ if $\operatorname{cert}_{q_{\mathcal{O}}, \Sigma}^{D}=q_{\mathcal{S}}^{D}$ for every $\mathcal{S}$-database $D$. Observe that, while the latter sanctions that $\operatorname{cert}_{q_{\mathcal{O}, \Sigma}}^{D}=q_{\mathcal{S}}^{D}$ for all $\mathcal{S}$-databases $D$, in Definition 3.1 the condition is limited only to the $\mathcal{S}$-databases $D$ that are consistent with $\Sigma$. The different behaviour between the two notions is highlighted by the following example.

Example 3.6. Let $\Sigma=\langle\mathcal{O}, \mathcal{S}, \mathcal{M}\rangle$ be the following OBDM specification:

- $\mathcal{O}=\{$ Professor $\sqsubseteq \neg$ Student $\}$

- $\mathcal{S}=\left\{s_{1}, s_{2}, s_{3}\right\}$

- $\mathcal{M}=\left\{m_{1}, m_{2}, m_{3}\right\}$, where:

$$
\begin{aligned}
& m_{1}: s_{1}(x) \rightarrow \text { Worker }(x), \\
& m_{2}: s_{2}(x) \rightarrow \text { Professor }(x), \\
& m_{3}: \quad s_{3}(x) \rightarrow \text { Student }(x) .
\end{aligned}
$$

Let the query over $\mathcal{S}$ be $q_{\mathcal{S}}=\left\{(x) \mid s_{1}(x)\right\}$, and let the query over $\mathcal{O}$ be $q_{\mathcal{O}}=$ $\{(x) \mid \operatorname{Worker}(x)\}$. Note that $(i) \operatorname{PerfectRef}\left(\mathcal{O}, q_{\mathcal{O}}\right)=q_{\mathcal{O}}$, and therefore $\operatorname{PerfRef}_{q_{\mathcal{O}}, \Sigma}=$ $\operatorname{MapRef}\left(\operatorname{PerfectRef}\left(\mathcal{O}, q_{\mathcal{O}}\right), \mathcal{M}\right)=\left\{(x) \mid s_{1}(x)\right\}$, and $(i i) \mathcal{V}_{\mathcal{O}}=\{() \mid \exists y \cdot \operatorname{Professor}(y) \wedge$ Student $(y)\}$, and therefore $\operatorname{PerfRef}_{\mathcal{V}_{\mathcal{O}}, \Sigma}=\operatorname{MapRef}\left(\operatorname{PerfectRef}\left(\mathcal{O}, \mathcal{V}_{\mathcal{O}}\right), \mathcal{M}\right)=\{() \mid$ $\left.\exists y . s_{2}(y) \wedge s_{3}(y)\right\}$. Finally, we know that $\operatorname{REW}_{q_{\mathcal{O}}, \Sigma} \equiv \operatorname{PerfRef}_{q_{\mathcal{O}}, \Sigma} \cup \operatorname{PerfRef}_{\mathcal{V}_{\mathcal{O}}^{1}, \Sigma}$, where $\mathcal{V}_{\mathcal{O}}^{1}=\{(x) \mid \exists y$.Professor $(y) \wedge \operatorname{Student}(y) \wedge \top(x)\}$.

Consider now the $\mathcal{S}$-database $D=\left\{s_{1}\left(c_{1}\right), s_{2}\left(c_{2}\right), s_{3}\left(c_{2}\right)\right\}$. We have that $q_{\mathcal{S}}^{D}=$ $\left\{\left(c_{1}\right)\right\}$, whereas, since $D$ is inconsistent with $\Sigma$ (i.e., $\operatorname{Mod}_{D}(\Sigma)=\emptyset$ ), $\operatorname{cert}_{q_{\mathcal{O}}, \Sigma}^{D}=$ PerfRef ${ }_{\mathcal{V}_{\mathcal{O}}^{1}, \Sigma}^{D}$, and therefore the set of certain answers of $q_{\mathcal{O}}$ with respect to $\Sigma$ and $D$ contains all the 1-tuples of constants in $\operatorname{dom}(D)=\left\{c_{1}, c_{2}\right\}$ (hence including the tuple $\left(c_{2}\right)$ ). It follows that, according to the semantics proposed in Lutz et al., 2018 (which ranges over all $\mathcal{S}$-databases), $q_{\mathcal{O}}$ is not a realization of $q_{\mathcal{S}}$ in $\Sigma$, whereas, according to Definition 3.1, $q_{\mathcal{O}}$ is a perfect $\mathcal{S}$-to- $\mathcal{O} \Sigma$-rewriting of $q_{\mathcal{S}}$ because $q_{\mathcal{S}}^{D}=\operatorname{cert}_{q_{\mathcal{O}}, \Sigma}^{D}$ for each $\mathcal{S}$-database $D$ consistent with $\Sigma$.

Notice, however, that the two notions turn out to be in fact equivalent when dealing with OBDM specifications where inconsistencies can not arise. For instance, OBDM specifications of our setting where the DL ontology language DL-Lite $e_{\mathcal{R}}$ is replaced with an ontology language not able to express inconsistencies, such as the DL DL-Lite $e_{\mathrm{RDFS}}$ and the DLs $\mathcal{E} \mathcal{L}$ and $\mathcal{E} \mathcal{L H} \mathcal{I}$ Baader et al., 2005] considered in Lutz et al., 2018. 


\subsubsection{Relationship with Ontology-to-Source Rewritings}

As argued in the introduction, most of (if not all) the literature about managing data sources through an ontology, or more generally, about data integration, assume that the user query is expressed over a global domain schema, and the goal is to find an ontology-to-source rewriting (i.e., a query over the source schema) that captures the original query in the best way, independently from the current source database. The framework just introduced can be therefore seen as a sort of reverse engineering problem, because we start with a source query and we aim at deriving a corresponding query over the ontology, called a source-to-ontology rewriting. Here, we make explicit the relationships between these new notions of source-to-ontology rewritings and the usual notions of ontology-to-source rewritings studied in OBDM.

Theorem 3.1. Let $\Sigma=\langle\mathcal{O}, \mathcal{S}, \mathcal{M}\rangle$ be an $O B D M$ specification, and let $q_{\mathcal{S}}$ and $q_{\mathcal{O}}$ be queries over $\mathcal{S}$ and over $\mathcal{O}$, respectively. We have that:

1. $q_{\mathcal{S}}$ is a sound $\mathcal{O}$-to-S $\mathcal{S}$-rewriting of $q_{\mathcal{O}}$ if and only if $q_{\mathcal{O}}$ is a complete $\mathcal{S}$-to-O $\Sigma$-rewriting of $q_{\mathcal{S}}$;

2. If $q_{\mathcal{S}}$ is a perfect $\mathcal{O}$-to-S $\Sigma$-rewriting of $q_{\mathcal{O}}$, then $q_{\mathcal{O}}$ is a perfect $\mathcal{S}$-to- $\mathcal{O} \Sigma$ rewriting of $q_{\mathcal{S}}$. The converse does not necessarily hold.

Proof. As for 1, by definition $q_{\mathcal{S}}$ is a sound $\mathcal{O}$-to- $\mathcal{S} \Sigma$-rewriting of $q_{\mathcal{O}}$ if and only if $q_{\mathcal{S}}^{D} \subseteq \operatorname{cert}_{q_{\mathcal{O}}, \Sigma}^{D}$ for every $\mathcal{S}$-database $D$. Since for all the $\mathcal{S}$-databases $D$ that are inconsistent with $\Sigma$ the above inclusion trivially holds, this is equivalent to the condition $q_{\mathcal{S}}^{D} \subseteq \operatorname{cert}_{q_{\mathcal{O}}, \Sigma}^{D}$ for every $\mathcal{S}$-database $D$ consistent with $\Sigma$, which is exactly the definition of $q_{\mathcal{O}}$ being a complete $\mathcal{S}$-to- $\mathcal{O}$ $\Sigma$-rewriting of $q_{\mathcal{S}}$ (cf. Definition 3.3).

As for the implication part of 2 , by definition $q_{\mathcal{S}}$ is a perfect $\mathcal{O}$-to- $\mathcal{S} \Sigma$-rewriting of $q_{\mathcal{O}}$ if and only if $q_{\mathcal{S}}^{D}=\operatorname{cert}_{q_{\mathcal{O}}, \Sigma}^{D}$ for every $\mathcal{S}$-database $D$, which obviously implies that $q_{\mathcal{O}}$ is a perfect $\mathcal{S}$-to- $\mathcal{O} \Sigma$-rewriting of $q_{\mathcal{S}}$.

To show that the converse does not necessarily hold, consider $\Sigma=\langle\mathcal{O}, \mathcal{S}, \mathcal{M}\rangle, q_{\mathcal{S}}$, and $q_{\mathcal{O}}$ as described in Example 3.6. In particular, $q_{\mathcal{O}}$ is a perfect $\mathcal{S}$-to- $\mathcal{O} \Sigma$-rewriting of $q_{\mathcal{S}}$, whereas $q_{\mathcal{S}}$ is not a perfect $\mathcal{O}$-to- $\mathcal{S} \Sigma$-rewriting of $q_{\mathcal{O}}$.

Once again, it is easy to see that the converse statement of point 2 of the above theorem becomes in fact true when dealing with ontologies expressed in those DLs where inconsistencies can not arise.

\subsubsection{Relationship with View-based Query Processing}

In order to establish a relationship between the notion of source-to-ontology rewritings and the view-based query processing approach, here we consider OBDM specifications $\Sigma=\langle\mathcal{O}, \mathcal{S}, \mathcal{M}\rangle$ where $\mathcal{O}=\emptyset$ and mapping $\mathcal{M}$ is a pure GAV mapping.

As a first consideration, observe that every pure GAV mapping $\mathcal{M}$ can be equivalently rewritten as a set of pure GAV mapping assertions of the form $\exists \vec{y} \cdot \phi_{\mathcal{S}}(\vec{x}, \vec{y}) \rightarrow \varphi_{\mathcal{O}}(\vec{x})$, where $\varphi_{\mathcal{O}}(\vec{x})$ is simply an atom without constants or repeated variables, i.e., $\varphi_{\mathcal{O}}(\vec{x})$ is either of the form $A(x)$ for an atomic concept $A$ in the alphabet of $\mathcal{O}$, or of the form $P\left(x_{1}, x_{2}\right)$ for an atomic role $P$ in the alphabet of $\mathcal{O}$ and with $x_{1}$ and $x_{2}$ being different variables. 
Example 3.7. Let $\mathcal{M}$ be composed of the following pure GAV mapping assertions:

$$
\begin{array}{ccc}
m_{1}: \quad \exists y_{1}, y_{2} \cdot s_{1}\left(y_{1}, x_{1}, x_{3}\right) \wedge s_{2}\left(x_{3}, x_{2}, y_{2}\right) & \rightarrow P_{1}\left(x_{1}, x_{2}\right) \wedge P_{2}\left(x_{1}, x_{3}\right), \\
m_{2}: & \exists y_{1} \cdot s_{3}\left(x_{1}, x_{2}, y_{1}\right) & \rightarrow P_{2}\left(x_{1}, x_{2}\right) .
\end{array}
$$

Then, $\mathcal{M}$ is equivalent to the following set of pure GAV mapping assertions:

$$
\begin{array}{rrrr}
m_{1}: \quad \exists y_{1}, y_{2}, y_{3} \cdot s_{1}\left(y_{1}, x_{1}, y_{3}\right) \wedge s_{2}\left(y_{3}, x_{2}, y_{2}\right) & \rightarrow P_{1}\left(x_{1}, x_{2}\right), \\
m_{2}: \quad \exists y_{1}, y_{2}, y_{3} \cdot s_{1}\left(y_{1}, x_{1}, x_{2}\right) \wedge s_{2}\left(x_{2}, y_{3}, y_{2}\right) & \rightarrow P_{2}\left(x_{1}, x_{2}\right), \\
m_{3}: & \exists y_{1} \cdot s_{3}\left(x_{1}, x_{2}, y_{1}\right) & \rightarrow & P_{2}\left(x_{1}, x_{2}\right) .
\end{array}
$$

Let $\mathcal{M}$ be a pure GAV mapping relating a schema $\mathcal{S}$ to an ontology $\mathcal{O}$, and let $A$ (respectively, $P$ ) be an atomic concept (respectively, atomic role) in the alphabet of $\mathcal{O}$. We denote by $V_{A}$ (respectively, $V_{P}$ ) the following UCQ over $\mathcal{S}$ :

$$
\begin{gathered}
V_{A}=\left\{(x) \mid \exists \overrightarrow{y_{1}} \cdot \phi_{\mathcal{S}}^{1}\left(x, \overrightarrow{y_{1}}\right)\right\} \cup \ldots \cup\left\{(x) \mid \exists \overrightarrow{y_{l_{A}}} \cdot \phi_{\mathcal{S}}^{l_{A}}\left(x, \overrightarrow{y_{A}}\right)\right\} \\
V_{P}=\left\{\left(x_{1}, x_{2}\right) \mid \exists \overrightarrow{y_{1}} \cdot \phi_{\mathcal{S}}^{1}\left(x_{1}, x_{2}, \overrightarrow{y_{1}}\right)\right\} \cup \ldots \cup\left\{\left(x_{1}, x_{2}\right) \mid \exists \overrightarrow{y_{P}} \cdot \phi_{\mathcal{S}}^{l_{P}}\left(x_{1}, x_{2}, \overrightarrow{y_{P}}\right)\right\},
\end{gathered}
$$

where $\exists \overrightarrow{y_{i}} \cdot \phi_{\mathcal{S}}^{i}\left(x, \overrightarrow{y_{i}}\right) \rightarrow A(x)$ (respectively, $\exists \overrightarrow{y_{i}} \cdot \phi_{\mathcal{S}}^{i}\left(x_{1}, x_{2}, \overrightarrow{y_{i}}\right) \rightarrow P\left(x_{1}, x_{2}\right)$ ) is a mapping assertion in $\mathcal{M}$ for each $i \in\left[1, l_{A}\right]$ (respectively, for each $i \in\left[1, l_{P}\right]$ ). Furthermore, we denote by $\mathcal{V}_{\mathcal{M}}=\left\{V_{A_{1}}, \ldots, V_{A_{n}}, V_{P_{1}}, \ldots, V_{P_{m}}\right\}$ the set of UCQ view definitions over schema $\mathcal{S}$ obtained by associating the UCQ $V_{A}$ (respectively, $V_{P}$ ) to each atomic concept $A$ (respectively, atomic role $P$ ) in the alphabet of $\mathcal{O}$. Finally, given a query $q_{\mathcal{O}}$ over $\mathcal{O}$, we denote by $q_{\mathcal{O}}^{\mathcal{V}_{\mathcal{M}}}$ the query over the view alphabet $\mathcal{V}_{\mathcal{M}}$ obtained by replacing each predicate name $A$ (respectively, $P$ ) with $V_{A}$ (respectively, $V_{P}$ ).

Example 3.8. Let $\mathcal{M}=\left\{m_{1}, m_{2}, m_{3}\right\}$ be the pure GAV mapping defined in Example 3.7, and let $q_{\mathcal{O}}=\left\{(x) \mid \exists y \cdot P_{2}(x, y)\right\}$. We have that $\mathcal{V}_{\mathcal{M}}=\left\{V_{P_{1}}, V_{P_{2}}\right\}$, where $V_{P_{1}}=\left\{(x) \mid \exists y_{1}, y_{2}, y_{3} \cdot s_{1}\left(y_{1}, x_{1}, y_{3}\right) \wedge s_{2}\left(y_{3}, x_{2}, y_{2}\right)\right\}$ and $V_{P_{2}}=\left\{\left(x_{1}, x_{2}\right) \mid\right.$ $\left.\exists y_{1}, y_{2}, y_{3} . s_{1}\left(y_{1}, x_{1}, x_{2}\right) \wedge s_{2}\left(x_{2}, y_{3}, y_{2}\right)\right\} \cup\left\{\left(x_{1}, x_{2}\right) \mid \exists y_{1} . s_{3}\left(x_{1}, x_{2}, y_{1}\right)\right\}$, and $q_{\mathcal{O}} \mathcal{V}_{\mathcal{M}}=$ $\left\{(x) \mid \exists y \cdot V_{P_{2}}(x, y)\right\}$.

We are now ready to draw the correspondence between the notions of source-toontology rewritings introduced in Section 3.1 and the usual notions of rewritings with respect to view definitions (cf. Section 2.4.

Theorem 3.2. Let $\Sigma=\langle\mathcal{O}, \mathcal{S}, \mathcal{M}\rangle$ be an $O B D M$ specification where $\mathcal{O}=\emptyset$ and $\mathcal{M}$ is a pure GAV mapping, and let $q_{\mathcal{S}}$ and $q_{\mathcal{O}}$ be two UCQs over $\mathcal{S}$ and $\mathcal{O}$, respectively. We have that $q_{\mathcal{O}}$ is a perfect (respectively, sound) $\mathcal{S}$-to- $\mathcal{O} \Sigma$-rewriting of $q_{\mathcal{S}}$ if and only if $q_{\mathcal{O}}^{\mathcal{V}_{\mathcal{M}}}$ is an exact (respectively, a sound) rewriting of $q_{\mathcal{S}}$ with respect to $\mathcal{V}_{\mathcal{M}}$.

Proof. The proof is based on the following three observations:

1. By Levy et al., 1995, a UCQ $q$ is an exact (respectively, a sound) rewriting of a UCQ $q_{\mathcal{S}}$ with respect to a set of UCQ view definitions $\mathcal{V}$ if and only if $\exp _{\mathcal{V}}(q) \equiv q_{\mathcal{S}}$ (respectively, $\exp _{\mathcal{V}}(q) \sqsubseteq q_{\mathcal{S}}$ ), where $\exp _{\mathcal{V}}(\cdot)$ is the function that, given a UCQ $q$ over the view alphabet, replace each atom occurring in $q$ by the definition of the views (being careful to use unique variables in place of 
those variables that appear in the bodies of the view but not in the heads of those), and then turning the resulting formula into an equivalent UCQ (e.g., $\exp _{\mathcal{V}_{\mathcal{M}}}\left(q_{\mathcal{O}}^{\mathcal{V}_{\mathcal{M}}}\right)=\left\{(x) \mid \exists y, y_{1}, y_{2}, y_{3} . s_{1}\left(y_{1}, x, y\right) \wedge s_{2}\left(y, y_{3}, y_{2}\right)\right\} \cup\{(x) \mid$ $\left.\exists y, y_{1} \cdot s_{3}\left(x, y, y_{1}\right)\right\}$, where $q_{\mathcal{O}}^{\mathcal{V}_{\mathcal{M}}}$ and $\mathcal{V}_{\mathcal{M}}$ are the query and the set of view definitions, respectively, of Example 3.8).

2. For OBDM specifications $\Sigma=\langle\mathcal{O}, \mathcal{S}, \mathcal{M}\rangle$ with $\mathcal{O}=\emptyset$ and $\mathcal{M}$ a pure GAV mapping, a UCQ $q_{\mathcal{O}}$ is a perfect (respectively, sound) $\mathcal{S}$-to- $\mathcal{O} \Sigma$-rewriting of a $\mathrm{UCQ} q_{\mathcal{S}}$ if and only if $\operatorname{Map} \operatorname{Ref}\left(q_{\mathcal{O}}, \mathcal{M}\right) \equiv q_{\mathcal{S}}$ (respectively, $\operatorname{MapRef}\left(q_{\mathcal{O}}, \mathcal{M}\right) \sqsubseteq$ $\left.q_{\mathcal{S}}\right)$, where $\operatorname{Map} \operatorname{Ref}\left(q_{\mathcal{O}}, \mathcal{M}\right)$ in this case is equivalent to unfolding the query $q_{\mathcal{O}}$ with respect to $\mathcal{M}$ Poggi et al., 2008, i.e., replacing each atom $\alpha$ occurring in $q_{\mathcal{O}}$ by the logical disjunction of all the left-hand sides of mapping assertions in $\mathcal{M}$ having the predicate name $\alpha$ in the right-hand side (being careful to use unique variables in place of those variables that appear in the left-hand side of the mapping assertions but not in the right-hand side of those), and then turning the resulting formula into an equivalent UCQ (e.g., $\operatorname{MapRef}\left(q_{\mathcal{O}}, \mathcal{M}\right)=\{(x) \mid$ $\left.\exists y, y_{1}, y_{2}, y_{3} \cdot s_{1}\left(y_{1}, x, y\right) \wedge s_{2}\left(y, y_{3}, y_{2}\right)\right\} \cup\left\{(x) \mid \exists y, y_{1} . s_{3}\left(x, y, y_{1}\right)\right\}$, where $q_{\mathcal{O}}$ and $\mathcal{M}$ are the query and the pure GAV mapping, respectively, of Example 3.8.

3. By construction, $\operatorname{MapRef}\left(q_{\mathcal{O}}, \mathcal{M}\right)=\exp _{\mathcal{V}_{\mathcal{M}}}\left(q_{\mathcal{O}}^{\mathcal{V}_{\mathcal{M}}}\right)$ for any UCQ $q_{\mathcal{O}}$ over an ontology $\mathcal{O}$ and for any pure GAV mapping $\mathcal{M}$ relating a schema $\mathcal{S}$ to $\mathcal{O}$.

Thus, $q_{\mathcal{O}}$ is a perfect (respectively, sound) $\mathcal{S}$-to- $\mathcal{O} \Sigma$-rewriting of $q_{\mathcal{S}}$ if and only if $\operatorname{MapRef}\left(q_{\mathcal{O}}, \mathcal{M}\right) \equiv q_{\mathcal{S}}$ (respectively, $\left.\operatorname{MapRef}\left(q_{\mathcal{O}}, \mathcal{M}\right) \sqsubseteq q_{\mathcal{S}}\right)$, which, since $\operatorname{MapRef}\left(q_{\mathcal{O}}, \mathcal{M}\right)=\exp _{\mathcal{V}_{\mathcal{M}}}\left(q_{\mathcal{O}}^{\mathcal{V}_{\mathcal{M}}}\right)$, it is so if and only if $\exp _{\mathcal{V}_{\mathcal{M}}}\left(q_{\mathcal{O}} \mathcal{V}_{\mathcal{M}}\right) \equiv q_{\mathcal{S}}$ (respectively, $\exp _{\mathcal{V}_{\mathcal{M}}}\left(q_{\mathcal{O}}^{\mathcal{V}_{\mathcal{M}}}\right) \sqsubseteq q_{\mathcal{S}}$ ), and therefore if and only if $q_{\mathcal{O}}^{\mathcal{V}_{\mathcal{M}}}$ is an exact (respectively, a sound) rewriting of $q_{\mathcal{S}}$ with respect to $\mathcal{V}_{\mathcal{M}}$, as required.

From the sound part of the above theorem, we derive the following corollary.

Corollary 3.1. Let $\Sigma=\langle\mathcal{O}, \mathcal{S}, \mathcal{M}\rangle$ be an $O B D M$ specification where $\mathcal{O}=\emptyset$ and $\mathcal{M}$ is a pure GAV mapping, and let $q_{\mathcal{S}}$ and $q_{\mathcal{O}}$ be two UCQs over $\mathcal{S}$ and $\mathcal{O}$, respectively. We have that $q_{\mathcal{O}}$ is the unique (up to equivalence w.r.t. $\Sigma$ ) UCQ-maximally sound $\mathcal{S}$-to-O $\Sigma$-rewriting of $q_{\mathcal{S}}$ if and only if $q_{\mathcal{O}}^{\mathcal{V}_{\mathcal{M}}}$ is a UCQ-maximally sound rewriting of $q_{\mathcal{S}}$ with respect to $\mathcal{V}_{\mathcal{M}}$.

In order to continue deriving further connections, we explore the following result.

Theorem 3.3. Abiteboul and Duschka, 1998, Duschka and Genesereth, 1998 Let $\mathcal{V}$ be a set of $U C Q$ view definitions over a schema $\mathcal{S}$, and let $q_{\mathcal{S}}$ be a UCQ. If query $q \mathcal{V}$ over the view alphabet $\mathcal{V}$ is a UCQ-maximally sound rewriting of $q_{\mathcal{S}}$ with respect to $\mathcal{V}$, then $q_{\mathcal{V}}$ is a perfect rewriting of $q_{\mathcal{S}}$ with respect to $\mathcal{V}$.

The assumption that the target list of each disjunct of the various view definitions in $\mathcal{V}$ does not have repeated variables or constants is essential for the above theorem to hold. In fact, as shown in Afrati and Chirkova, 2019 and also in the following example, the above theorem does not carry over when this assumption is removed.

Example 3.9. Consider the set $\mathcal{V}=\{V\}$ of a CQ view definition over schema $\mathcal{S}=\{s\}$ and the CQ $q_{\mathcal{S}}$ over $\mathcal{S}$, where $V=\{(x, x) \mid s(x)\}$ and $q_{\mathcal{S}}=\{(x) \mid s(x)\}$. 
It is clear that $q_{\mathcal{V}}=\{(x) \mid V(x, x)\}$ is a UCQ-maximally sound rewriting of $q_{\mathcal{S}}$ with respect to $\mathcal{V}$. Consider, however, the view extension $\mathcal{E}=\{E\}$ with $E=\left\{V\left(c_{1}, c_{2}\right), V\left(c_{3}, c_{3}\right)\right\}$. By construction of the view definition $V$, we have $\left(c_{1}, c_{2}\right) \notin V^{D}$ for each $\mathcal{S}$-database $D$. But then, since $V\left(c_{1}, c_{2}\right) \in \mathcal{E}$, there is no $\mathcal{S}$-database $D$ for which $\mathcal{E} \subseteq \mathcal{V}(D)$. By definition this implies that $\operatorname{cert}_{q_{\mathcal{S}}, \mathcal{V}}^{\mathcal{E}}=\emptyset$, whereas $q_{\mathcal{V}}^{\mathcal{V}}=\left\{\left(c_{3}\right)\right\}$. So, $q_{\mathcal{V}}$ is not a perfect rewriting of $q_{\mathcal{S}}$ with respect to $\mathcal{V}$.

From Corollary 3.1 and the above theorem, it is immediate to derive an interesting correspondence between the two notions under considerations.

Corollary 3.2. Let $\Sigma=\langle\mathcal{O}, \mathcal{S}, \mathcal{M}\rangle$ be an $O B D M$ specification where $\mathcal{O}=\emptyset$ and $\mathcal{M}$ is a pure GAV mapping, and let $q_{\mathcal{S}}$ and $q_{\mathcal{O}}$ be two UCQs over $\mathcal{S}$ and $\mathcal{O}$, respectively. We have that $q_{\mathcal{O}}$ is the unique (up to equivalence w.r.t. $\Sigma$ ) UCQ-maximally sound $\mathcal{S}$-to-O $\mathcal{O} \Sigma$-rewriting of $q_{\mathcal{S}}$ if and only if $q_{\mathcal{O}}{ }^{\mathcal{M}}$ is a perfect rewriting of $q_{\mathcal{S}}$ with respect to $\mathcal{V}_{\mathcal{M}}$.

However, as shown in Duschka and Genesereth, 1998; Afrati and Chirkova, 2019, UCQ-maximally sound rewritings of CQs $q_{\mathcal{S}}$ with respect to UCQ view definitions $\mathcal{V}$ are not guaranteed to exists. The following example gives an intuitive reason of why a UCQ-maximally sound rewriting may not exists for certain choices of $q_{\mathcal{S}}$ and $\mathcal{V}$.

Example 3.10. Consider the set $\mathcal{V}=\left\{V_{1}, V_{2}\right\}$ of UCQ view definitions over schema $\mathcal{S}=\left\{s_{c}, s_{e}\right\}$ and the CQ $q_{\mathcal{S}}$, where:

$$
\begin{aligned}
& V_{1}=\left\{(x) \mid s_{c}(x, \text { Red })\right\} \cup\left\{(x) \mid s_{c}(x, \text { Green })\right\} \\
& V_{2}=\left\{\left(x_{1}, x_{2}\right) \mid s_{e}\left(x_{1}, x_{2}\right)\right\} \\
& q_{\mathcal{S}}=\left\{() \mid \exists y_{1}, y_{2}, y_{3} . s_{e}\left(y_{1}, y_{2}\right) \wedge s_{c}\left(y_{1}, y_{3}\right) \wedge s_{c}\left(y_{2}, y_{3}\right)\right\}
\end{aligned}
$$

Intuitively, source predicate $s_{e}$ contains the edges of a graph and mirror them into $V_{2}$, whereas $V_{1}$ will contain those vertices in $s_{c}$ colored by either Red or Green. Finally, query $q_{\mathcal{S}}$ asks whether there is a pair of vertices connected by an edge and colored with the same color.

The fact that $s_{e}$ may contain the edges of a non-2-colourable graph can be easily detected by certain queries issued over the view alphabet. For instance, the query $q_{\mathcal{V}}^{3}=\left\{() \mid \exists y_{1}, y_{2}, y_{3} \cdot V_{2}\left(y_{1}, y_{2}\right) \wedge V_{2}\left(y_{2}, y_{3}\right) \wedge V_{2}\left(y_{3}, y_{1}\right) \wedge V_{1}\left(y_{1}\right) \wedge V_{1}\left(y_{2}\right) \wedge V_{1}\left(y_{3}\right)\right\}$ if true implies the existence of a triangle (and therefore a non-2-colourable pattern) in the graph represented by source predicates $s_{e}$ and $s_{c}$.

More generally, one can verify that each query $q \mathcal{V}$ over the view alphabet is a sound rewriting of $q_{\mathcal{S}}$ with respect to $\mathcal{V}$ if and only if the graph described by $q \mathcal{V}$ is not 2colourable. Since a graph is not 2-colourable if and only if it contains an odd cycle Asratian et al., 1998, we conclude that the query $q_{\mathcal{V}}^{i}=\left\{() \mid \exists y_{1}, \ldots, y_{i} \cdot V_{2}\left(y_{1}, y_{2}\right) \wedge\right.$ $\left.V_{2}\left(y_{2}, y_{3}\right) \wedge \ldots \wedge V_{2}\left(y_{i-1}, y_{i}\right) \wedge V_{2}\left(y_{i}, y_{1}\right) \wedge V_{1}\left(y_{1}\right) \wedge V_{1}\left(y_{2}\right) \wedge \ldots \wedge V_{1}\left(y_{i}\right)\right\}$ is a sound rewriting of $q_{\mathcal{S}}$ with respect to $\mathcal{V}$, for each odd $i \geq 3$. Furthermore, the following query $q_{\mathcal{V}}$ over the view alphabet is a perfect rewriting of $q_{\mathcal{S}}$ with respect to $\mathcal{V}$ :

$$
q \mathcal{V}=\bigcup_{\text {odd } i \geq 3} q_{\mathcal{V}}^{i}
$$

Obviously, such infinite union is not representable by a UCQ, and therefore we conclude that no UCQ-maximally sound rewriting of $q_{\mathcal{S}}$ with respect to $\mathcal{V}$ exists. 
This result, together with Corollary 3.1, allows us to derive the first negative result on UCQ-maximally sound source-to-ontology rewritings.

Corollary 3.3. UCQ-maximally sound $\mathcal{S}$-to- $\mathcal{O}$ $\Sigma$-rewritings of $C Q s$ q $q_{\mathcal{S}}$ over $\mathcal{S}$ are not guaranteed to exists, even when $O B D M$ specifications $\Sigma=\langle\mathcal{O}, \mathcal{S}, \mathcal{M}\rangle$ are such that $\mathcal{O}=\emptyset$ and $\mathcal{M}$ is a pure GAV mapping.

Proof. Let $\Sigma=\langle\mathcal{O}, \mathcal{S}, \mathcal{M}\rangle$ be the following OBDM specification:

- $\mathcal{O}=\emptyset$

- $\mathcal{S}=\left\{s_{e}, s_{c}\right\}$

- $\mathcal{M}=\left\{m_{1}, m_{2}, m_{3}\right\}$, where:

$$
\begin{array}{rrl}
m_{1}: & s_{c}(x, \text { Red }) & \rightarrow A(x), \\
m_{2}: & s_{c}(x, \text { Green }) & \rightarrow A(x), \\
m_{3}: & s_{e}\left(x_{1}, x_{2}\right) & \rightarrow P\left(x_{1}, x_{2}\right) .
\end{array}
$$

Similarly to Example 3.10 for the CQ $q_{\mathcal{S}}=\left\{() \mid \exists y_{1}, y_{2}, y_{3} \cdot s_{e}\left(y_{1}, y_{2}\right) \wedge s_{c}\left(y_{1}, y_{3}\right) \wedge\right.$ $\left.s_{c}\left(y_{2}, y_{3}\right)\right\}$ no UCQ-maximally sound $\mathcal{S}$-to- $\mathcal{O} \Sigma$-rewriting of $q_{\mathcal{S}}$ exists.

In Example 3.10, a maximally sound rewriting of $q_{\mathcal{S}}$ with respect to $\mathcal{V}$ does exist in the class of Datalog queries, non-2-colourability is indeed expressible in Datalog (see, e.g., Kolaitis and Vardi, 2008 ). Actually, if we modify the above example by considering $\mathcal{V}^{\prime}=\left\{V_{1}^{\prime}, V_{2}^{\prime}\right\}$ where $V_{1}^{\prime}=\left\{(x) \mid s_{c}(x\right.$, Red $\left.)\right\} \cup\left\{(x) \mid s_{c}(x\right.$, Green $\left.)\right\} \cup\{(x) \mid$ $s_{c}(x$, Blue $\left.)\right\}$ and $V_{2}^{\prime}=V_{2}$, we have that a maximally sound rewriting of $q_{\mathcal{S}}$ with respect to $\mathcal{V}^{\prime}$ does not exist even in the class of Datalog queries. This is due to the following facts: $(i)$ Given as input a set of view extensions $\mathcal{E}=\left\{E_{1}, E_{2}\right\}$ where $E_{i}$ is the view extension of $V_{i}^{\prime}$ for both $i=1$ and $i=2$, as shown in van der Meyden, 1993 via a reduction from non-3-colourability, the problem of checking whether $\operatorname{cert}_{q_{\mathcal{S}} \mathcal{V}^{\prime}}^{\mathcal{E}}$ is true is coNP-hard in the size of $\mathcal{E},{ }^{1}$ ( $\left.i i\right)$ Analogously to Theorem 3.3 every maximally sound rewriting of a UCQ $q_{\mathcal{S}}$ with respect to a set of UCQ view definitions $\mathcal{V}$ in the class of Datalog queries is also a perfect rewriting of $q_{\mathcal{S}}$ with respect to $\mathcal{V}$; (iii) Answering Datalog queries over relational databases is in PTIME in data complexity Vardi, 1982. Thus, unless PTIME $=$ NP, a maximally sound rewriting of $q_{\mathcal{S}}$ with respect to $\mathcal{V}^{\prime}$ can not exists in the class of Datalog queries ${ }^{2}$

Lastly, we observe that Example 3.10, as well as the non-existence results provided in Duschka and Genesereth, 1998; Afrati and Chirkova, 2019, makes use of CQs $q_{\mathcal{S}}$ having more than one join existential variable in their body. On the one hand, in Section 6.2 we will strengthen such a result, by showing that it holds even for CQs $q_{\mathcal{S}}$ with only a single join existential variable. On the other hand, in Chapter 8 we will prove that having no join existential variables in the body of UCQs $q_{\mathcal{S}}$ is a sufficient condition that guarantees the existence of UCQ-maximally sound rewritings of $q_{\mathcal{S}}$ with respect to UCQ view definitions $\mathcal{V}$. Thus, due to Theorem 3.3. in such cases the

\footnotetext{
${ }^{1}$ More precisely, given a set of UCQ view definitions $\mathcal{V}$ over a schema $\mathcal{S}$, a set of view extensions $\mathcal{E}$, a UCQ $q_{\mathcal{S}}$ over $\mathcal{S}$, and a tuple of constants $\vec{c}$, the problem of checking whether $\vec{c} \in \operatorname{cert}_{q_{\mathcal{S}}, \mathcal{V}}^{\mathcal{E}}$ is coNP-complete in the size of $\mathcal{E}$ Abiteboul and Duschka, 1998.

${ }^{2}$ Conversely, as shown in Duschka and Genesereth, 1998. Afrati et al., 1999, maximally sound rewritings (which are again perfect rewritings) of UCQs $q_{\mathcal{S}}$ with respect to UCQ view definitions $\mathcal{V}$ always exist in the class of Disjunctive Datalog queries Lobo et al., 1992, Eiter et al., 1997.
} 
perfect rewriting of $q_{\mathcal{S}}$ with respect to $\mathcal{V}$ can be always expressed as a UCQ, exactly as in the case of CQ view definitions LLevy et al., 1995]. We refer to Section 8.3 for a complete discussion on this topic.

\subsubsection{Inverse Mapping and/or Reversing the Arrows (Not!)}

An important concept studied in the data exchange literature is the one of inverse mapping. In a nutshell, given a mapping $\mathcal{M}$ from a schema $\mathcal{S}$ to a schema $\mathcal{O}$, the inverse of $\mathcal{M}$, denoted by $\mathcal{M}^{\prime}$, is a mapping from $\mathcal{O}$ to $\widehat{\mathcal{S}}$ that recovers as much information as possible. Here, $\widehat{\mathcal{S}}=\{\widehat{s} \mid s \in \mathcal{S}\}$, i.e., $\widehat{\mathcal{S}}$ is simply a copy of $\mathcal{S}$. According to Fagin, 2007, $\mathcal{M}^{\prime}$ is an inverse of $\mathcal{M}$ if and only if $\mathcal{M} \circ \mathcal{M}^{\prime} \equiv \widehat{I d}$, where $\mathcal{M} \circ \mathcal{M}^{\prime}$ is the composition formula Fagin et al., 2005c and $\widehat{I d}$ is the identity mapping from $\mathcal{S}$ to $\widehat{\mathcal{S}}$, i.e., $\widehat{I d}=\left\{s\left(x_{1}, \ldots, x_{n}\right) \rightarrow \widehat{s}\left(x_{1}, \ldots, x_{n}\right) \mid s \in \mathcal{S}\right\}$. In other words, $\mathcal{M}^{\prime}$ is an inverse of $\mathcal{M}$ if and only if $\operatorname{Mod}_{\mathcal{M} \circ \mathcal{M}^{\prime}}(D)=\operatorname{Mod}_{\widehat{I d}}(D)=\left\{D^{\prime} \mid \widehat{D} \subseteq D^{\prime}\right\}$ for each $\mathcal{S}$-database $D$, where $\widehat{D}=\left\{\widehat{s}\left(c_{1}, \ldots, c_{n}\right) \mid s\left(c_{1}, \ldots, c_{n}\right) \in D\right\}$, and, for a mapping $\mathcal{M}$ relating schema $\mathcal{S}$ to schema $\widehat{\mathcal{S}}$ and an $\mathcal{S}$-database $D, \operatorname{Mod}_{\mathcal{M}}(D)$ denotes the set of all $\widehat{S}$-databases $D^{\prime}$ for which $\left\langle D, D^{\prime}\right\rangle \models \mathcal{M}$. This notion turns out to be rather restrictive, as it is rare that a schema mapping possesses an inverse.

Example 3.11. Let $\mathcal{M}=\left\{m_{1}, m_{2}, m_{3}\right\}$, where:

$$
\begin{aligned}
& m_{1}: \quad s_{1}(x) \rightarrow A_{1}(x), \\
& m_{2}: \quad s_{1}(x) \wedge s_{3}(x) \rightarrow A_{2}(x), \\
& m_{3}: \quad s_{2}(x) \wedge s_{4}(x) \quad \rightarrow \quad A_{2}(x) .
\end{aligned}
$$

It is straightforward to verify that $\mathcal{M}$ does not have an inverse mapping.

To remedy this situation, (at least) two other notions have been introduced in literature: $(i)$ the notion of quasi-inverse which is a principled relaxation of the notion of inverse. According to Fagin et al., 2008, a mapping $\mathcal{M}^{\prime}$ is a quasi-inverse of $\mathcal{M}$ if and only if for each pair of $\mathcal{S}$-databases $D_{1}$ and $D_{2}$ there are two $\mathcal{S}$-databases $D_{3}$ and $D_{4}$ for which $\operatorname{Mod}_{\mathcal{M}}\left(D_{1}\right)=\operatorname{Mod}_{\mathcal{M}}\left(D_{3}\right), \operatorname{Mod}_{\mathcal{M}}\left(D_{2}\right)=\operatorname{Mod}_{\mathcal{M}}\left(D_{4}\right)$, and $\operatorname{Mod}_{\mathcal{M} \circ \mathcal{M}^{\prime}}\left(D_{3}\right)=\left\{D^{\prime} \mid \widehat{D}_{4} \subseteq D^{\prime}\right\} ;(i i)$ the notion of maximum recovery which is a strict generalisation of the notion of inverse. According to Arenas et al., 2009, $\mathcal{M}^{\prime}$ is a maximum recovery of $\mathcal{M}$ if and only if $\langle D, \widehat{D}\rangle \models \mathcal{M} \circ \mathcal{M}^{\prime}$ for each $\mathcal{S}$-database $D$, and for every mapping $\mathcal{M}^{\prime \prime}$ either $\langle D, \widehat{D}\rangle \not \models \mathcal{M} \circ \mathcal{M}^{\prime \prime}$ for at least an $\mathcal{S}$-database $D$, or $\operatorname{Mod}_{\mathcal{M} \circ \mathcal{M}^{\prime \prime}}(D) \subseteq \operatorname{Mod}_{\mathcal{M} \circ \mathcal{M}^{\prime}}(D)$ for each $\mathcal{S}$-database $D$.

Example 3.12. Let $\mathcal{M}$ be the mapping as defined in Example 3.11, and consider the mapping $\mathcal{M}^{\prime}=\left\{m_{1}^{\prime}, m_{2}^{\prime}, m_{3}^{\prime}\right\}$, where:

$$
\begin{array}{ll}
m_{1}^{\prime}: & A_{1}(x) \rightarrow \widehat{s_{1}}(x), \\
m_{2}^{\prime}: & A_{2}(x) \rightarrow \widehat{s_{1}}(x) \wedge \widehat{s_{3}}(x), \\
m_{3}^{\prime}: & A_{2}(x) \rightarrow \widehat{s_{2}}(x) \wedge \widehat{s_{4}}(x) .
\end{array}
$$

One can see that $\mathcal{M}^{\prime}$ is both a quasi-inverse and a maximum recovery of $\mathcal{M}$.

To figure out a possible correspondence between the notion of source-to-ontology rewriting and the notions of quasi-inverse and maximum recovery, here we consider OBDM specifications $\Sigma=\langle\mathcal{O}, \mathcal{S}, \mathcal{M}\rangle$ where $\mathcal{O}=\emptyset$. 
Due to Proposition 1, one might think that in order to compute a perfect $\mathcal{S}$ to- $\mathcal{O} \Sigma$-rewriting of $q_{\mathcal{S}}$ it is sufficient to compute a mapping $\mathcal{M}^{\prime}$ that is either a quasi-inverse or a maximum recovery of $\mathcal{M}$, and then computing the perfect $\mathcal{O}$-to- $\widehat{S}$ $\Sigma^{\prime}$-rewriting of $\widehat{q_{\mathcal{S}}}$ using the well-known techniques of data integration (cf. Section 2.6). Here, $\widehat{q_{\mathcal{S}}}$ denotes the query obtained from $q_{\mathcal{S}}$ by replacing each predicate name $s \in \mathcal{S}$ with $\widehat{s} \in \widehat{\mathcal{S}}$, and $\Sigma^{\prime}=\left\langle\widehat{S}, \mathcal{M}^{\prime}, \mathcal{O}\right\rangle$ denotes an OBDM specification where $\widehat{S}$ plays the role of an (empty) ontology and $\mathcal{O}$ plays the role of a source schema. We now prove that this is an absolutely wrong belief.

Example 3.13. Let $\Sigma=\langle\mathcal{O}, \mathcal{S}, \mathcal{M}\rangle$ be the following OBDM specification:

- $\mathcal{O}=\emptyset$

- $\mathcal{S}=\left\{s_{1}, s_{2}, s_{3}, s_{4}\right\}$

- $\mathcal{M}=\left\{m_{1}, m_{2}, m_{3}\right\}$ is the mapping defined in Example 3.11

Let, moreover, $\Sigma^{\prime}=\left\langle\widehat{S}, \mathcal{M}^{\prime}, \mathcal{O}\right\rangle$ be the following OBDM specification:

- $\widehat{\mathcal{S}}=\emptyset$

- $\mathcal{O}=\left\{A_{1}, A_{2}\right\}$

- $\mathcal{M}^{\prime}=\left\{m_{1}^{\prime}, m_{2}^{\prime}, m_{3}^{\prime}\right\}$ is as defined in Example 3.12 , i.e., it is both the quasiinverse and the maximum recovery of $\mathcal{M}$.

Let the query over $\mathcal{S}$ be $q_{\mathcal{S}}=\left\{(x) \mid s_{1}(x)\right\}$. We have that $q_{\mathcal{O}}=\left\{(x) \mid A_{1}(x)\right\}$ is the perfect $\mathcal{S}$-to- $\mathcal{O} \Sigma$-rewriting of $q_{\mathcal{S}}$, whereas one can verify that the perfect $\mathcal{O}$-to- $\widehat{S} \Sigma^{\prime}$-rewriting of $\widehat{q_{\mathcal{S}}}$ is the query $q_{\mathcal{O}}^{\prime}=\left\{(x) \mid A_{1}(x)\right\} \cup\left\{(x) \mid A_{2}(x)\right\}$, which is a complete, but not a sound, $\mathcal{S}$-to- $\mathcal{O} \Sigma$-rewriting of $q_{\mathcal{S}}$.

Furthermore, one might think that the perfect $\mathcal{O}$-to- $\widehat{S} \Sigma^{\prime}$-rewriting of $\widehat{q_{\mathcal{S}}}$ always produces a complete $\mathcal{S}$-to- $\mathcal{O} \Sigma$-rewriting of $q_{\mathcal{S}}$. We now prove that this a wrong belief as well. Let the query over $\mathcal{S}$ be $q_{\mathcal{S}}^{\prime}=\left\{(x) \mid s_{1}(x) \wedge s_{2}(x)\right\}$. We have that the query $q_{\mathcal{O}}^{\prime \prime}=\left\{(x) \mid A_{1}(x) \wedge A_{2}(x)\right\} \cup\left\{(x) \mid A_{2}(x)\right\}$ is the perfect $\mathcal{O}$-to- $\widehat{S} \Sigma^{\prime}$-rewriting of $\widehat{q_{\mathcal{S}}^{\prime}}$, but not a complete $\mathcal{S}$-to- $\mathcal{O} \Sigma$-rewriting of $q_{\mathcal{S}}^{\prime}$. In this case, one can verify that the unique (up to equivalence w.r.t. $\Sigma$ ) UCQ-minimally complete $\mathcal{S}$-to- $\mathcal{O} \Sigma$-rewriting of $q_{\mathcal{S}}^{\prime}$ is the query $q_{\mathcal{O}}$ defined above.

We conclude with a very elementary but useful observation. Notice that, in the above example, the quasi-inverse and maximum recovery mapping $\mathcal{M}^{\prime}$ of $\mathcal{M}$ is obtained by simply reversing the arrows of $\mathcal{M}$. Therefore, given an OBDM specification $\Sigma=\langle\mathcal{O}, \mathcal{S}, \mathcal{M}\rangle$, the OBDM specification $\operatorname{rev}(\Sigma)=\langle\widehat{S}, \operatorname{rev}(\mathcal{M}), \mathcal{O}\rangle$ where $\operatorname{rev}(\mathcal{M})$ is the mapping from $\widehat{S}$ to $\mathcal{O}$ obtained by simply reversing the arrows of $\mathcal{M}$ does not help in any way for finding source-to-ontology rewritings. 


\section{Chapter 4}

\section{Dealing with Inequalities in Lightweight Description Logics}

As explained in the introduction, we will see that by allowing inequalities in a certain target query language considered in this thesis provide a better means to compute abstractions of data services compared to the same language without inequalities.

This chapter deals with the problem of adding inequalities to UCQs in the OBDM scenario. Notably, although UCQs constitute the most popular class of queries studied for both databases and ontologies, they have several limitations in expressive power. For instance, suppose we want to retrieve all the triangles in an undirected graph possibly with loops ${ }^{1}$ This property can not be expressed by means of a UCQ, whereas it can be expressed as the following $\mathrm{CQ}^{\neq, b} q=\{(x, y, z) \mid$ edge $(x, y) \wedge \operatorname{edge}(x, z) \wedge \operatorname{edge}(y, z) \wedge x \neq y \wedge x \neq z \wedge y \neq z\}$, where the predicate edge represents the connections between vertices in the graph.

It is worth noting that in OBDM, while answering (U)CQs has been extensively studied, e.g., by establishing bound on the size of rewritings Gottlob et al., 2014, developing optimisation algorithms [Rosati and Almatelli, 2010], implementing engines for real-world applications Calvanese et al., 2011: Civili et al., 2013; Calvanese et al., 2017, etc., the problem of answering (U)CQ ${ }^{\neq} \mathrm{s}$ has been rarely investigated. We start by analysing the case of knowledge bases.

To the best of our knowledge, the basic known facts about such problem can be summarised as follows, which hold regardless of whether the UNA is adopted or not:

- In stark contrast to the UCQ case, answering $\mathrm{UCQ}^{\neq}$s even over DL-Lite knowledge bases is undecidable Gutiérrez-Basulto et al., 2012]. For DL-Lite $e_{\mathcal{R}}$ knowledge bases, undecidability holds already for $\mathrm{CQ}^{\neq} \mathrm{s} \mid$ Gutiérrez-Basulto et al., 2015. Looking at these results, one can easily realise that the main of source of undecidability stems from both the ability of the ontology language to express incomplete information through existential quantifiers, and the possibility of imposing inequalities between existential variables in the query;

- In Gutiérrez-Basulto et al., 2015, it is also proved that for the subclasses of $\mathrm{CQ}^{\neq} \mathrm{s}$ and $\mathrm{UCQ}^{\neq} \mathrm{s}$ named local $C Q^{\neq} s$ and local $U C Q^{\neq} s$, respectively, query answering over $D L$-Lite R $_{\mathcal{R}}$ knowledge bases is decidable, but with a CONEXPTimE

\footnotetext{
${ }^{1}$ In graph theory, a loop is an edge that connects a vertex with itself Bondy and Murty, 2008.
} 
upper bound in data complexity. Furthermore, it is provably intractable (coNPhard in data complexity) already for local $\mathrm{CQ}^{\neq} \mathrm{s}$. Local (U)CQs are special (U)CQs with inequalities, designed in such a way that each inequality atom in the query that contributes to a certain answer with respect to a DL knowledge base has at least one of its terms bound by an individual in the ABox.

The goal of this chapter is then to investigate under which conditions, stronger than local UCQs, tractability of answering queries with inequalities is recovered, or at least the complexity is lowered with respect to the one of local UCQs. The basic idea to achieve this goal is to explore ontology languages and query languages ensuring the following property: each inequality atom $\alpha \neq \beta$ that contributes to the certain answer to a query with respect to a DL knowledge base, it does so with both terms $\alpha$ and $\beta$ bounded by individuals in the ABox. In order to follow this path, we explore two alternative strategies:

1. The first strategy is to consider $\mathrm{UCQ}^{\neq, b} \mathrm{~s}$, which restricts the application of the inequality atoms to either individuals or distinguished variables, i.e., we consider the problem of answering (U)CQ ${ }^{\neq, b}$ s over DL-Lite $\mathcal{R}_{\mathcal{R}}$ knowledge bases. Notice that, although limited, the expressive power of $(\mathrm{U}) \mathrm{CQ}^{\neq, b} \mathrm{~s}$ allow for interesting queries to be expressed such as the one computing the triangles in an undirected graph with loops.

2. The second strategy is to consider DL-Lite $\overrightarrow{\mathrm{RDFS}}$ knowledge bases, which eliminates all the constructs introducing incomplete information resulting from existentially quantified assertions, i.e., we consider the problem of answering $\mathrm{UCQ}^{\neq}{ }_{\mathrm{s}}$ over $D L$-Lite $e_{\mathrm{RDFS}}$ knowledge bases.

As a first consideration, observe that the above problems are straightforward generalisations of the UCQ case when the UNA is adopted. To see this, it is sufficient to consider the slight extension of the algorithm PerfectRef accepting $\mathrm{UCQ}^{\neq}{ }_{\mathrm{S}}$ as input queries rather than UCQs, where the predicate $\neq$ is treated in the reformulation algorithm as an additional atomic role of the input DL-Lite $\mathcal{R}_{\mathcal{R}}$ ontology. From now on, we implicitly refer to PerfectRef as the algorithm described in Subsection 2.5.3 with the above modification. With this discussion in mind, it is straightforward to verify that the computational complexity of the above defined problems remains the same as that of the problem of answering UCQs over DL-Lite $e_{\mathcal{R}}$ knowledge bases.

Theorem 4.1. Both the problem of answering $U C Q^{\neq, b}$ s over DL-Lite knowledge $_{\mathcal{R}}$ bases and the problem of answering $U C Q^{\neq} s$ over DL-Lite $\overrightarrow{R D F S}_{\text {knowledge bases are }}$ FOL-rewritable (and therefore in $A C^{0}$ in data complexity), and NP-complete in combined complexity.

Proof. Let $q$ be a $\mathrm{UCQ}^{\neq, b}$ (respectively, $\mathrm{UCQ}^{\neq}$) over a $D L$-Lite $e_{\mathcal{R}}$ (respectively, $D L$-Lite $\overrightarrow{\mathrm{RDFS}}$ ) knowledge base $\mathcal{K}=\langle\mathcal{O}, \mathcal{A}\rangle$. Analogously to Calvanese et al., 2007b, Theorem 29], since we are adopting the UNA, it is easy to see that $\vec{c} \in \operatorname{cert}_{q, \mathcal{O}}^{\mathcal{A}}$ if and only if there is a disjunct $q^{\prime}=\{\vec{t} \mid \exists \vec{y} \cdot \phi(\vec{x}, \vec{y})\}$ of $q$ for which there is a homomorphism $h$ from $q^{\prime}$ to $\mathcal{C}_{\mathcal{O}}^{\mathcal{A}}$ with $h(\vec{t})=\vec{c}$. Note that $(i) h(\alpha) \neq h(\beta)$ for each inequality atom $\alpha \neq \beta$ occurring in $q^{\prime} ;(i i)$ both $h(\alpha) \neq h(\beta)$ are individuals. 
This is because, in the case that $\mathcal{O}$ is a $D L-L i t e_{\mathcal{R}}$ ontology, $h(\alpha)$ and $h(\beta)$ are necessarily individuals occurring in $\mathcal{A}$ since $q$ is a $\mathrm{UCQ}^{\neq, b}$. In the case that $\mathcal{O}$ is a $D L$-Lite $e_{\mathrm{RDFS}}$ ontology, again, both $h(\alpha)$ and $h(\beta)$ are necessarily individuals occurring in $\mathcal{A}$ since $\mathcal{C}_{\mathcal{O}}^{\mathcal{A}}$ does not introduce any variable in Var. Thus, with the same arguments of [Calvanese et al., 2007b, Lemma 39], we can easily conclude that $\operatorname{cert}_{q, \mathcal{O}}^{\mathcal{A}}=\operatorname{PerfectRef}(\mathcal{O}, q)^{\mathcal{I}_{\mathcal{A}}}$, from which the claim trivially follows.

However, while answering UCQs over $D L$-Lite R $_{\mathcal{R}}$ knowledge bases is insensitive to the adoption of the UNA Artale et al., 2009. (cf. Subsection 2.5.3), the following example shows that this is not anymore the case even when answering $\mathrm{CQ}^{\neq, b}$ s over knowledge bases with empty TBoxes.

Example 4.1. Consider the knowledge base $\mathcal{K}=\langle\mathcal{O}, \mathcal{A}\rangle$, where $\mathcal{O}=\emptyset$ and $\mathcal{A}=$ $\left\{P\left(c_{1}, c_{2}\right)\right\}$. For the $\mathrm{CQ}^{\neq, b} q=\left\{\left(x_{1}, x_{2}\right) \mid P\left(x_{1}, x_{2}\right) \wedge x_{1} \neq x_{2}\right\}$, it is easy to see that the tuple $\left(c_{1}, c_{2}\right)$ is in the certain answers of $q$ with respect to $\mathcal{O}$ and $\mathcal{A}$ when the UNA is adopted, while it is not if the UNA is not adopted. For this latter, consider the model $\mathcal{I}=\left\langle\Delta^{\mathcal{I}}, \cdot^{\mathcal{I}}\right\rangle$ of $\mathcal{K}$ with $\Delta^{\mathcal{I}}=c, c_{1}^{\mathcal{I}}=c_{2}^{\mathcal{I}}=c$, and $P^{\mathcal{I}}=\{(c, c)\}$, which does not satisfy the UNA. We have $q^{\mathcal{I}}=\emptyset$.

All the rest of this chapter is devoted to analyse the computational complexity of the above problems when the UNA is not adopted, as in the OWL 2 web ontology language profiles. We also make a connection on how these new results contribute, on the one hand, to new results on containment of $\mathrm{UCQ}^{\neq} \mathrm{s}$ in the relational databases theory, and, on the other hand, to new results on the Direct Semantics Entailment Regime (DSER) Glimm, 2011. The complexity results are summarised in Figure 4.1

In principle, since the UNA is not adopted, it may be reasonable to include in knowledge bases $\mathcal{K}=\langle\mathcal{O}, \mathcal{A}\rangle$ also $\mathrm{ABox}$ assertions sanctioning the fact that two individuals have to be interpreted as different elements in all possible of models of $\mathcal{K}$, as can be effectively done in OWL 2 QL by means of the DifferentIndividuals assertions. Notice, however, that the expressive capabilities of the DL-Lite $e_{\mathcal{R}}$ and $D L-L i t e_{\mathrm{RDFS}}^{\neg}$ knowledge base languages would remain the same, since in fact each ABox assertion of the form $\alpha=\operatorname{DifferentIndividuals}\left(c_{1}, c_{2}\right)$ is equivalent to the ABox assertions $\operatorname{DIFF}_{1}\left(c_{1}, \alpha\right), \operatorname{DIFF}_{2}\left(c_{2}, \alpha\right)$, where $\alpha$ is a fresh individual and $\operatorname{DIFF}_{1}$, $\mathrm{DIFF}_{2}$ are two atomic roles such that $\mathrm{DIFF}_{1} \sqsubseteq \neg \mathrm{DIFF}_{2}$ is a disjointness assertion of every ontology $\mathcal{O}$. An analogous but different argument (since the ontology size of the equivalent knowledge base is linear on the size of the original ABox) holds also for DL-Lite core knowledge bases. Indeed, each ABox assertion of the form $\alpha=$ DifferentIndividuals $\left(c_{1}, c_{2}\right)$ is equivalent to the set of assertions $A_{\text {new }}^{\alpha_{1}}\left(c_{1}\right)$, $A_{\text {new }}^{\alpha_{2}}\left(c_{2}\right)$, and $A_{\text {new }}^{\alpha_{1}} \sqsubseteq \neg A_{\text {new }}^{\alpha_{2}}$, where $A_{\text {new }}^{\alpha_{1}}$ and $A_{\text {new }}^{\alpha_{2}}$ are fresh atomic concepts.

In what follows on this chapter, unless otherwise stated, we implicitly assume to deal with satisfiable knowledge bases ${ }^{2}$ Moreover, unless otherwise stated and without loss of generality, we consider only boolean $\mathrm{UCQ}^{\neq} \mathrm{s}$. Indeed, given an $n$-ary $\mathrm{UCQ}^{\neq} q_{\mathcal{O}}$, a $D L$-Lite R $_{\mathcal{R}}$ knowledge base $\mathcal{K}=\langle\mathcal{O}, \mathcal{A}\rangle$, and an $n$-tuple $\vec{c}$ of constants, checking whether $\vec{c} \in \operatorname{cert}_{q_{\mathcal{O}}, \mathcal{O}}^{\mathcal{A}}$ is equivalent to checking whether $\mathcal{K} \equiv q_{\mathcal{O}}(\vec{c})$.

\footnotetext{
${ }^{2}$ As discussed in Subsection 2.5.3, for unsatisfiable knowledge bases query answering is trivial. Furthermore, the problem of checking whether a $D L$-Lite $_{\mathcal{R}}$ knowledge base is satisfiable is FOLrewritable (and therefore in $\mathrm{AC}^{0}$ in data complexity) and in PTIME in the size of the ontology.
} 
DL-Lite ${ }_{\mathcal{R}}$

\begin{tabular}{|c||c|c|}
\hline & Data & Combined \\
\hline \hline $\mathrm{CQ}^{\neq, b} \mathrm{~s}$ & in $\mathrm{AC}^{0}$ & NP-c \\
\hline $\mathrm{UCQ}^{\neq, b} \mathrm{~s}$ & in $\mathrm{AC}^{0}$ & $\Pi_{2}^{p}$-c \\
\hline
\end{tabular}

$D L-L i t e_{\mathrm{RDFS}}^{\neg}$

\begin{tabular}{|c||c|c|}
\hline & Data & Combined \\
\hline \hline $\mathrm{UCQ}^{1,{ }_{\mathrm{S}}}$ & PTIME-c & NP-c \\
\hline $\mathrm{UCQ}^{\neq} \mathrm{s}$ & coNP-c & $\Pi_{2}^{p}$-c \\
\hline
\end{tabular}

Figure 4.1. Summary of data and combined complexity results of our considered problems when the UNA is not adopted ("-c" abbreviates "-complete"). The table on the left

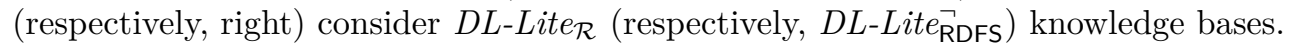

\subsection{The Chase for Answering Queries with Inequalities}

The conceptual tool we use for addressing the problem of answering queries with inequalities over $D L$-Lite $e_{\mathcal{R}}$ knowledge base is an extension of the chase illustrated in [Calvanese et al., 2007b]. Specifically, given a DL-Lite $\mathcal{R}_{\mathcal{R}}$ knowledge base $\mathcal{K}=$ $\langle\mathcal{O}, \mathcal{A}\rangle$, we build a (possibly infinite) set of atoms, starting from $C h^{0}(\mathcal{K}):=\mathcal{A}$, and repeatedly computing $C h^{j+1}(\mathcal{K})$ from $C h^{j}(\mathcal{K})$ by applying suitable rules, where each rule can be applied only if certain conditions hold. In doing so, we make use of the alphabet Var of variables, and follow a deterministic strategy that is fair, i.e., if at some point a rule is applicable, then it will be eventually applied. Finally, we set $\mathcal{I}_{\mathcal{K}}:=\bigcup_{i \geq 0} C h^{i}(\mathcal{K})$. Notice that we make use of the additional binary predicate symbol ineq, which is used to record all inequalities logically implied by $\mathcal{K}$.

The rules we use include all the ones illustrated in Calvanese et al., 2007b Definition 5]. For instance, if $A_{1} \sqsubseteq \exists P \in \mathcal{O}, A_{1}\left(e_{1}\right) \in C h^{j}(\mathcal{K})$, and no $e_{2}$ exists such that $P\left(e_{1}, e_{2}\right) \in C h^{j}(\mathcal{K})$, then we set $C h^{j+1}(\mathcal{K}):=C h^{j}(\mathcal{K}) \cup\left\{P\left(e_{1}, e^{\prime}\right)\right\}$, where $e^{\prime} \in \operatorname{Var}$ does not appear in $C h^{j}(\mathcal{K})$. There are, however, two crucial additions related to the ineq predicate. In what follows, when we say $R\left(e_{1}, e_{2}\right)$ holds in $C h^{j}(\mathcal{K})$, where $R$ is a basic role, we mean $(i) P\left(e_{1}, e_{2}\right) \in C h^{j}(\mathcal{K})$, if $R=P$, or $(i i)$ $P\left(e_{2}, e_{1}\right) \in C h^{j}(\mathcal{K})$, if $R=P^{-}$. Also, when we say that $B\left(e_{1}\right)$ holds in $C h^{j}(\mathcal{K})$, where $B$ is a basic concept, we mean $(i) A\left(e_{1}\right) \in C h^{j}(\mathcal{K})$ if $B=A$, and $(i i) R\left(e_{1}, e_{2}\right)$ holds in $C h^{j}(\mathcal{K})$ for some $e_{2}$, if $B=\exists R$. The additional two rules are as follows:

- if $B_{1} \sqsubseteq \neg B_{2} \in \mathcal{O}, B_{1}\left(e_{1}\right)$ and $B_{2}\left(e_{2}\right)$ hold in $C h^{j}(\mathcal{K})$, and ineq $\left(e_{1}, e_{2}\right) \notin$ $C h^{j}(\mathcal{K})$, then $C h^{j+1}(\mathcal{K}):=C h^{j}(\mathcal{K}) \cup\left\{\right.$ ineq $\left(e_{1}, e_{2}\right)$, ineq $\left.\left(e_{2}, e_{1}\right)\right\} ;$

- if $R_{1} \sqsubseteq \neg R_{2} \in \mathcal{O}$, ineq $\left(e_{1}, e_{2}\right) \notin C h^{j}(\mathcal{K})$, and either $R_{1}\left(e_{3}, e_{1}\right)$ and $R_{2}\left(e_{3}, e_{2}\right)$ hold in $C h^{j}(\mathcal{K})$ or $R_{1}\left(e_{1}, e_{3}\right)$ and $R_{2}\left(e_{2}, e_{3}\right)$ hold in $C h^{j}(\mathcal{K})$, then $C h^{j+1}(\mathcal{K}):=$ $C h^{j}(\mathcal{K}) \cup\left\{\right.$ ineq $\left(e_{1}, e_{2}\right)$, ineq $\left.\left(e_{2}, e_{1}\right)\right\}$.

In other words, for a $D L$-Lite $\mathcal{R}_{\mathcal{R}}$ knowledge base $\mathcal{K}=\langle\mathcal{O}, \mathcal{A}\rangle$, the set of atoms $\mathcal{I}_{\mathcal{K}}$ contains all atoms contained in $\mathcal{C}_{\mathcal{O}}^{\mathcal{A}}$ plus some possible atoms with ineq as predicate, used to record all and only the inequalities logically implied by $\mathcal{K}$.

Obviously, for a $D L$-Lite $e_{\mathcal{R}}$ knowledge base $\mathcal{K}$, the set $\mathcal{I}_{\mathcal{K}}$ can be infinite, due to the presence of existential quantifiers in the right-hand side of inclusion assertions, which, by introducing fresh unknown variables, can trigger an infinite number of rule applications. It is easy to see that, on the contrary, for a $D L$-Lit $e_{\mathrm{RDFS}}$ knowledge base $\mathcal{K}, \mathcal{I}_{\mathcal{K}}$ is finite, and can be computed in polynomial time in the size of $\mathcal{K}$.

We next show that $\mathcal{I}_{\mathcal{K}}$ enjoys some crucial properties. 
Proposition 4.1. If $\mathcal{M}=\left\langle\Delta^{\mathcal{M}}, \cdot \mathcal{M}\right\rangle$ is a model of a DL-Lite $\mathcal{R}_{\mathcal{R}}$ knowledge base $\mathcal{K}$, then there exists a function $\Psi$ from $\operatorname{dom}\left(\mathcal{I}_{\mathcal{K}}\right)$ to $\Delta^{\mathcal{M}}$ such that:

1. for every $e \in \operatorname{dom}\left(\mathcal{I}_{\mathcal{K}}\right)$, if $A(e) \in \mathcal{I}_{\mathcal{K}}$, then $\Psi(e) \in A^{\mathcal{M}}$;

2. for every pair $e_{1}, e_{2} \in \operatorname{dom}\left(\mathcal{I}_{\mathcal{K}}\right)$, if $P\left(e_{1}, e_{2}\right) \in \mathcal{I}_{\mathcal{K}}$, then $\left(\Psi\left(e_{1}\right), \Psi\left(e_{2}\right)\right) \in P^{\mathcal{M}}$;

3. for every pair $e_{1}, e_{2} \in \operatorname{dom}\left(\mathcal{I}_{\mathcal{K}}\right)$, if ineq $\left(e_{1}, e_{2}\right) \in \mathcal{I}_{\mathcal{K}}$, then $\Psi\left(e_{1}\right) \neq \Psi\left(e_{2}\right)$.

Proof. Consider any model $\mathcal{M}$ of $\mathcal{K}=\langle\mathcal{O}, \mathcal{A}\rangle$. By Calvanese et al., 2007b Lemma 28], there exists a function $\Psi$ from $\operatorname{dom}\left(\mathcal{I}_{\mathcal{K}}\right)$ to $\Delta^{\mathcal{M}}$ with $\Psi(c)=c^{\mathcal{M}}$ for each individual $c$ occurring in $\mathcal{A}$ such that both conditions 1 and 2 hold.

As for condition 3 , from the rules applied for building $\mathcal{I}_{\mathcal{K}}$, if two elements $e_{1}, e_{2} \in \operatorname{dom}\left(\mathcal{I}_{\mathcal{K}}\right)$ are such that ineq $\left(e_{1}, e_{2}\right) \in \mathcal{I}_{\mathcal{K}}$, then by definition one of the following two conditions holds: $(i) B_{1} \sqsubseteq \neg B_{2} \in \mathcal{O}$ and $B_{1}\left(e_{1}\right), B_{2}\left(e_{2}\right)$ hold in $\mathcal{I}_{\mathcal{K}}$ (thus, $B_{1}\left(e_{1}\right) \in \mathcal{I}_{\mathcal{K}}$ and $\left.B_{2}\left(e_{2}\right) \in \mathcal{I}_{\mathcal{K}}\right),(i i) R_{1} \sqsubseteq \neg R_{2} \in \mathcal{O}$ and either $R_{1}\left(e_{3}, e_{1}\right)$ and $R_{2}\left(e_{3}, e_{2}\right)$ hold in $\mathcal{I}_{\mathcal{K}}$ (thus, $R_{1}\left(e_{3}, e_{1}\right) \in \mathcal{I}_{\mathcal{K}}$ and $\left.R_{2}\left(e_{3}, e_{2}\right) \in \mathcal{I}_{\mathcal{K}}\right)$, or $R_{1}\left(e_{1}, e_{3}\right)$ and $R_{2}\left(e_{2}, e_{3}\right)$ hold in $\mathcal{I}_{\mathcal{K}}$ (thus, $R_{1}\left(e_{1}, e_{3}\right) \in \mathcal{I}_{\mathcal{K}}$ and $\left.R_{2}\left(e_{2}, e_{3}\right) \in \mathcal{I}_{\mathcal{K}}\right)$.

Suppose by contradiction that $\Psi$ does not satisfy condition 3 , i.e., there are two elements $e_{1}, e_{2} \in \operatorname{dom}\left(\mathcal{I}_{\mathcal{K}}\right)$ such that ineq $\left(e_{1}, e_{2}\right) \in \mathcal{I}_{\mathcal{K}}$ and $\Psi\left(e_{1}\right)=\Psi\left(e_{2}\right)$.

If ineq $\left(e_{1}, e_{2}\right) \in \mathcal{I}_{\mathcal{K}}$ because of $(i)$, then, by condition 1 , we derive $\Psi\left(e_{1}\right) \in B_{1}^{\mathcal{M}}$ and $\Psi\left(e_{2}\right) \in B_{2}^{\mathcal{M}}$, which implies that $\mathcal{M}$ does not satisfy the disjointness assertion $B_{1} \sqsubseteq \neg B_{2}$ of $\mathcal{O}$, thus contradicting the fact that $\mathcal{M}$ is a model of $\mathcal{K}$, as required.

If ineq $\left(e_{1}, e_{2}\right) \in \mathcal{I}_{\mathcal{K}}$ because of $(i i)$, then, by condition 2 , we derive that either $\left(\Psi\left(e_{3}\right), \Psi\left(e_{1}\right)\right) \in R_{1}^{\mathcal{M}}$ and $\left(\Psi\left(e_{3}\right), \Psi\left(e_{2}\right)\right) \in R_{2}^{\mathcal{M}}$, or $\left(\Psi\left(e_{1}\right), \Psi\left(e_{3}\right)\right) \in R_{1}^{\mathcal{M}}$ and $\left(\Psi\left(e_{2}\right), \Psi\left(e_{3}\right)\right) \in R_{2}^{\mathcal{M}}$. Notice, however, that both cases imply that $\mathcal{M}$ does not satisfy the disjointness assertion $R_{1} \sqsubseteq \neg R_{2}$ of $\mathcal{O}$, thus contradicting the fact that $\mathcal{M}$ is a model of $\mathcal{K}$, as required.

Therefore, we conclude that $\Psi$ must be such that for every pair $e_{1}, e_{2} \in \operatorname{dom}\left(\mathcal{I}_{\mathcal{K}}\right)$, if ineq $\left(e_{1}, e_{2}\right) \in \mathcal{I}_{\mathcal{K}}$, then $\Psi\left(e_{1}\right) \neq \Psi\left(e_{2}\right)$.

A reasonable question to ask is whether $\mathcal{I}_{\mathcal{K}}$ is the right tool for query answering. The next theorem provides a positive answer to this question for $\mathrm{CQ}^{\neq, b} \mathrm{~s}$. In what follows, given a $\mathrm{CQ}^{\neq, b} q$ over an ontology $\mathcal{O}, \delta(q)$ denotes the query obtained by replacing each inequality atom $x_{1} \neq x_{2}$ in $q$ with the atom $i n e q\left(x_{1}, x_{2}\right)$.

Theorem 4.2. Let $\vec{c}$ be a tuple of individuals occurring in a satisfiable DL-Lite $\mathcal{R}_{\mathcal{R}}$ knowledge base $\mathcal{K}=\langle\mathcal{O}, \mathcal{A}\rangle$, and let $q$ be a $C Q^{\neq, b}$ over $\mathcal{O}$. We have that $\vec{c} \in \operatorname{cert}_{q, \mathcal{O}}^{\mathcal{A}}$ if and only if $\vec{c} \in \delta(q)^{\mathcal{I}_{\mathcal{K}}}$.

Proof. Consider the boolean query $q(\vec{c})$. By definition, we have $\vec{c} \in \operatorname{cert}_{q, \mathcal{O}}^{\mathcal{A}}$ if and only if $\mathcal{K}=q(\vec{c})$, and $\vec{c} \in \delta(q)^{\mathcal{I}_{\mathcal{K}}}$ if and only if $\mathcal{I}_{\mathcal{K}} \models \delta(q(\vec{c}))$. We now prove that $\mathcal{K} \models q(\vec{c})$ if and only if $\mathcal{I}_{\mathcal{K}} \models \delta(q(\vec{c}))$, thus showing the claim.

"If part:" If $\mathcal{I}_{\mathcal{K}} \models \delta(q(\vec{c}))$, then, using Proposition 4.1, we easily derive that $\mathcal{M} \models q(\vec{c})$ for each model $\mathcal{M}$ of $\mathcal{K}$. Thus, $\mathcal{K}=q(\vec{c})$, as required.

"Only-if part:" Suppose that $\mathcal{I}_{\mathcal{K}} \not \models \delta(q(\vec{c}))$. If in $\delta(q(\vec{c}))$ there is at least an atom, which is not an ineq atom, that is not satisfied by $\mathcal{I}_{\mathcal{K}}$, then, from $\mathcal{I}_{\mathcal{K}}$ itself, we easily obtain a model $\mathcal{M}_{\mathcal{K}}$ of $\mathcal{K}$ such that $\mathcal{M}_{\mathcal{K}} \not \models q(\vec{c})$, and therefore $\mathcal{K} \not \models q(\vec{c})$. 
On the other hand, if $\mathcal{I}_{\mathcal{K}}$ satisfies all atoms of $\delta(q(\vec{c}))$ different from ineq atoms, then there is at least one atom of the form ineq $\left(c_{1}, c_{2}\right)$ in $\delta(q(\vec{c}))$ that is false in $\mathcal{I}_{\mathcal{K}}$. Observe that, since $q(\vec{c})$ is a boolean $\mathrm{CQ}^{\neq, b}$, both $c_{1}$ and $c_{2}$ must be individuals.

Consider the interpretation $\mathcal{M}^{\prime}=\left\langle\Delta^{\mathcal{M}}, . \mathcal{M}\right\rangle$ for $\mathcal{K}$ obtained from $\mathcal{I}_{\mathcal{K}}$ as follows: (i) $\Delta^{\mathcal{M}}=$ Const; $(i i) c^{\mathcal{M}^{\prime}}=c$, for each individual $c$ occurring in $\mathcal{A}$ such that $c \neq c_{1}$ and $c \neq c_{2} ;(i i i) c_{1}^{\mathcal{M}^{\prime}}=c_{2}^{\mathcal{M}^{\prime}}=c^{\prime}$, where $c^{\prime} \in$ Const is a fresh constant not occurring in $\mathcal{A}$; and $(i v)$ the extensions of atomic concepts and atomic roles properly follows atoms of $\mathcal{I}_{\mathcal{K}}$, but where each variable $v \in$ Var occurring in $\mathcal{I}_{\mathcal{K}}$ is replaced everywhere with a fresh constant $c_{v} \in$ Const. Since ineq $\left(c_{1}, c_{2}\right) \notin \mathcal{I}_{\mathcal{K}}$, we have that $\mathcal{M}^{\prime}$ is a model of $\mathcal{K}$. In proof, if $\mathcal{M}^{\prime}$ does not satisfy a disjointness assertion, then, by simple induction on the steps applied for building $\mathcal{I}_{\mathcal{K}}$, we would easily derive a contradiction on the fact that ineq $\left(c_{1}, c_{2}\right) \notin \mathcal{I}_{\mathcal{K}}$.

But then, $\mathcal{M}^{\prime}$ is a model of $\mathcal{K}$ such that $c_{1}^{\mathcal{M}^{\prime}}=c_{2}^{\mathcal{M}^{\prime}}$. Since, however, $c_{1} \neq c_{2}$ is an inequality atom occurring in the body of $q(\vec{c})$, we derive $\mathcal{M}^{\prime} \not \models q(\vec{c})$. Therefore, $\mathcal{M}^{\prime}$ is a model of $\mathcal{K}$ for which $\mathcal{M}^{\prime} \not \models q(\vec{c})$. Thus, $\mathcal{K} \not \models q(\vec{c})$, as required.

\subsection{Answering $\mathrm{CQ}^{\neq, b}$ s over $\mathrm{DL}$ - Lite $_{\mathcal{R}}$ knowledge bases}

In this section, we first consider the problem of answering conjunctive queries with bounded inequalities $\left(\mathrm{CQ}^{\neq, b_{\mathrm{s}}}\right.$ ) over satisfiable $D L$-Lite $\mathcal{R}_{\mathcal{R}}$ knowledge bases, and then we discuss how these results carry over on the one hand to the OBDM scenario, and, on the other hand, to the case of the DSER, which is the semantics usually adopted in the Semantic Web scenarios that slightly differs from the classical FOL semantics.

We start by introducing some notations. Given an inequality atom $x_{1} \neq x_{2}$ and a disjointness assertion $\gamma$, we denote by $\rho\left(x_{1} \neq x_{2}, \gamma\right)$ the following open formula:

- $\rho\left(x_{1} \neq x_{2}, A_{1} \sqsubseteq \neg A_{2}\right)=A_{1}\left(x_{1}\right) \wedge A_{2}\left(x_{2}\right)$,

- $\rho\left(x_{1} \neq x_{2}, A \sqsubseteq \neg \exists R\right)=\rho\left(x_{1} \neq x_{2}, \exists R \sqsubseteq \neg A\right)=\exists y \cdot A\left(x_{1}\right) \wedge R\left(x_{2}, y\right)$, where $y$ is a fresh existential variable,

- $\rho\left(x_{1} \neq x_{2}, \exists R_{1} \sqsubseteq \neg \exists R_{2}\right)=\exists y_{1}, y_{2} \cdot R_{1}\left(x_{1}, y_{1}\right) \wedge R_{2}\left(x_{2}, y_{2}\right)$, where $y_{1}$ and $y_{2}$ are fresh different existential variables, and

- $\rho\left(x_{1} \neq x_{2}, R_{1} \sqsubseteq \neg R_{2}\right)=\exists y \cdot R_{1}\left(x_{1}, y\right) \wedge R_{2}\left(x_{2}, y\right) \vee \exists y \cdot R_{1}\left(y, x_{1}\right) \wedge R_{2}\left(y, x_{2}\right)$, where $y$ is a fresh existential variable,

where an atom of the form $R(x, y)$ stands for either $P(x, y)$ if $R$ denotes an atomic role $P$, or $P(y, x)$ if $R$ denotes the inverse of an atomic role, i.e., $R=P^{-}$.

Example 4.2. Consider the atom $x \neq c$ and the DL-Lite $\mathcal{R}_{\mathcal{R}}$ disjointness assertion $P_{1} \sqsubseteq \neg P_{2}$. Then, we have that $\rho\left(x \neq c, P_{1} \sqsubseteq \neg P_{2}\right)=\exists y \cdot P_{1}(x, y) \wedge P_{2}(c, y) \vee$ $\exists y \cdot P_{1}(y, x) \wedge P_{2}(y, c)$.

Given an inequality atom $x_{1} \neq x_{2}$ and a $D L$-Lite $\mathcal{R}_{\mathcal{R}}$ ontology $\mathcal{O}$, we denote by $\sigma\left(x_{1} \neq x_{2}, \mathcal{O}\right)$ the following disjunction of atoms:

$$
\bigvee_{i=1}^{m}\left(\rho\left(x_{1} \neq x_{2}, \gamma_{i}\right) \vee \rho\left(x_{2} \neq x_{1}, \gamma_{i}\right)\right)
$$

where $\gamma_{1}, \ldots, \gamma_{m}$ are all the disjointness assertions of the $D L$-Lite $\mathcal{R}_{\mathcal{R}}$ ontology $\mathcal{O}$. 
Example 4.3. Consider the atom $x \neq c$ and the DL-Lite $e_{\mathcal{R}}$ ontology $\mathcal{O}=\{A \sqsubseteq$ $\left.\exists P, P_{1} \sqsubseteq \neg P_{2}, P_{3} \sqsubseteq P_{2}\right\}$. We have that $\sigma(x \neq c, \mathcal{O})=\exists y \cdot P_{1}(x, y) \wedge P_{2}(c, y) \vee$ $\exists y \cdot P_{1}(y, x) \wedge P_{2}(y, c) \vee \exists y \cdot P_{1}(c, y) \wedge P_{2}(x, y) \vee \exists y \cdot P_{1}(y, c) \wedge P_{2}(y, x)$.

Finally, for a $\mathrm{CQ}^{\neq} q$ over a $D L$-Lite $e_{\mathcal{R}}$ ontology $\mathcal{O}$, we denote by $\lambda(q, \mathcal{O})$ the query obtained from $q$ by replacing every inequality atom $x_{1} \neq x_{2}$ with $\sigma\left(x_{1} \neq x_{2}, \mathcal{O}\right)$, and then turning the resulting formula into an equivalent UCQ.

Example 4.4. Consider the $\mathrm{CQ}^{\neq} q=\left\{(x) \mid \exists y_{1} \cdot P\left(x, y_{1}\right) \wedge x \neq c\right\}$ over the ontology $\mathcal{O}$ of Example 4.3 . We have that $\lambda(q, \mathcal{O})=\left\{q_{1}, q_{2}, q_{3}, q_{4}\right\}$, where

- $q_{1}=\left\{(x) \mid \exists y_{1}, y_{2} . P\left(x, y_{1}\right) \wedge P_{1}\left(x, y_{2}\right) \wedge P_{2}\left(c, y_{2}\right)\right\}$.

- $q_{2}=\left\{(x) \mid \exists y_{1}, y_{2} \cdot P\left(x, y_{1}\right) \wedge P_{1}\left(y_{2}, x\right) \wedge P_{2}\left(y_{2}, c\right)\right\}$.

- $q_{3}=\left\{(x) \mid \exists y_{1}, y_{2} \cdot P\left(x, y_{1}\right) \wedge P_{1}\left(c, y_{2}\right) \wedge P_{2}\left(x, y_{2}\right)\right\}$.

- $q_{4}=\left\{(x) \mid \exists y_{1}, y_{2} . P\left(x, y_{1}\right) \wedge P_{1}\left(y_{2}, c\right) \wedge P_{2}\left(y_{2}, x\right)\right\}$.

The next important proposition, whose proof relies on Calvanese et al., 2007b Theorem 29] and on Theorem 4.2 states that computing cert $_{q, \mathcal{O}}^{\mathcal{\mathcal { O }}}$, for a given DL-Lite $\mathcal{R}_{\mathcal{R}}$ knowledge base $\mathcal{K}=\langle\mathcal{O}, \mathcal{A}\rangle$ and $\mathrm{CQ}^{\neq, b} q$ over $\mathcal{O}$, can be reduced to computing the certain answers of the UCQ $\lambda(q, \mathcal{O})$ with respect to $\mathcal{O}$ and $\mathcal{A}$.

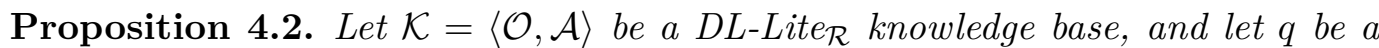
$C Q^{\neq, b}$ over $\mathcal{O}$. Then, we have that $\operatorname{cert}_{q, \mathcal{O}}^{\mathcal{A}}=\operatorname{cert}_{\lambda(q, \mathcal{O}), \mathcal{O}}^{\mathcal{A}}$.

Proof. Let $\vec{c}$ be any tuple of individuals occurring in $\mathcal{A}$. By definition, we have $\vec{c} \in \operatorname{cert}_{q, \mathcal{O}}^{\mathcal{A}}$ if and only if $\mathcal{K}=q(\vec{c})$. Analogously, $\vec{c} \in \operatorname{cert}_{\lambda(q, \mathcal{O}), \mathcal{O}}^{\mathcal{A}}$ if and only if $\mathcal{K} \models \lambda(q, \mathcal{O})(\vec{c})$. We now prove that $\mathcal{K} \models q(\vec{c})$ if and only if $\mathcal{K} \models \lambda(q, \mathcal{O})(\vec{c})$.

"If part:" If $\mathcal{K} \models \lambda(q, \mathcal{O})(\vec{c})$, then, due to Calvanese et al., 2007b. Theorem 29], we derive that there is a boolean CQ $q^{\prime}$ in the boolean UCQ $\lambda(q, \mathcal{O})(\vec{c})$ for which $\mathcal{I}_{\mathcal{K}} \models q^{\prime}$. Observe that, by construction, each boolean CQ $q^{\prime}$ in the boolean UCQ $\lambda(q, \mathcal{O})(\vec{c})$ is such that $q^{\prime}=\left\{() \mid \alpha_{1} \wedge \ldots \wedge \alpha_{n} \wedge \beta_{1} \wedge \beta_{1}^{\prime} \wedge \ldots \wedge \beta_{m} \wedge \beta_{m}^{\prime}\right\}$, where $\alpha_{i}$ s are atoms that were not inequality atoms in the boolean CQ $q(\vec{c})$, whereas the pair of atoms $\left(\beta_{i}, \beta_{i}^{\prime}\right)$ s are obtained after replacing an inequality atom of the form $c_{1} \neq c_{2}$ with $\sigma\left(c_{1} \neq c_{2}, \mathcal{O}\right)$. Since $q(\vec{c})$ is a boolean CQ, both $c_{1}$ and $c_{2}$ must be individuals.

Notice, however, that since $\mathcal{I}_{\mathcal{K}} \models \beta_{i} \wedge \beta_{i}^{\prime}$, by construction of $\sigma\left(c_{1} \neq c_{2}, \mathcal{O}\right)$ and the additional rules applied for building $\mathcal{I}_{\mathcal{K}}$ (the ones regarding the ineq predicate), we easily derive that ineq $\left(c_{1}, c_{2}\right) \in \mathcal{I}_{\mathcal{K}}$. Moreover, since this is true for each pair of atoms $\left(\beta_{i}, \beta_{i}^{\prime}\right)$ for $i \in[1, m]$, we further derive that $\delta(q(\vec{c}))$ is such that $\mathcal{I}_{\mathcal{K}} \models \delta(q(\vec{c}))$, thus implying that $\vec{c} \in \delta(q)^{\mathcal{I}_{\mathcal{K}}}$. By virtue of Theorem 4.2 we derive that $\vec{c} \in \operatorname{cert}_{q, \mathcal{O}}^{\mathcal{A}}$, and therefore $\mathcal{K} \models q(\vec{c})$, as required.

"Only-if part:" If $\mathcal{K} \models q(\vec{c})$, then, by virtue of Theorem 4.2 , we derive that $\mathcal{I}_{\mathcal{K}} \models \delta(q(\vec{c}))$. Consider each atom of the form ineq $\left(c_{1}, c_{2}\right)$ occurring in $\delta(q(\vec{c}))$. Observe that, since $\delta(q(\vec{c}))$ is a boolean CQ, both $c_{1}$ and $c_{2}$ must be individuals. From the premises of the additional rules for building $\mathcal{I}_{\mathcal{K}}$ (the ones regarding the ineq predicate), we easily derive that the formula $\sigma\left(c_{1} \neq c_{2}, \mathcal{O}\right)$ is true when evaluated over $\mathcal{I}_{\mathcal{K}}$, i.e., $\mathcal{I}_{\mathcal{K}} \models \sigma\left(c_{1} \neq c_{2}, \mathcal{O}\right)$. Therefore, it is easy to see that $\mathcal{I}_{\mathcal{K}} \models q^{\prime}$ for at least a boolean CQ $q^{\prime}$ in $\lambda(q, \mathcal{O})(\vec{c})$. Thus, due to Calvanese et al., 2007b, Theorem 29], we derive $\mathcal{K} \models \lambda(q, \mathcal{O})(\vec{c})$, as required. 
Let NoUNAPerfectRef denotes the algorithm that, given in input a $D L$-Lite R $_{\mathcal{R}}$ ontology $\mathcal{O}$ and a $\mathrm{CQ}^{\neq, b} q$ over $\mathcal{O}$, it returns the UCQ PerfectRef $(\mathcal{O}, \lambda(q, \mathcal{O}))$. By combining the above proposition with Calvanese et al., 2007b, Lemma 39], we immediately derive the following result.

Corollary 4.1. Let $\mathcal{O}$ be a $D L$-Lite $e_{\mathcal{R}}$ ontology, and let $q$ be a $C Q^{\neq, b}$ over $\mathcal{O}$. For every $A B o x \mathcal{A}$ such that the $D L$-Lite $e_{\mathcal{R}}$ knowledge base $\mathcal{K}=\langle\mathcal{O}, \mathcal{A}\rangle$ is satisfiable, we have $\operatorname{cert}_{q, \mathcal{O}}^{\mathcal{A}}=\operatorname{NoUNAPerfectRef}(\mathcal{O}, q)^{\mathcal{I}_{\mathcal{A}}}$.

Example 4.5. Consider the $D L$-Lite $e_{\mathcal{R}}$ knowledge base $\mathcal{K}=\langle\mathcal{O}, \mathcal{A}\rangle$, where $\mathcal{O}$ is the $D L$-Lite $\mathcal{R}_{\mathcal{R}}$ ontology described in Example 4.3 and $\mathcal{A}=\left\{A\left(c_{1}\right), P_{1}\left(c_{2}, c\right), P_{3}\left(c_{2}, c\right)\right\}$. Let, moreover, $q$ be the $\mathrm{CQ}^{\neq}$described in Example 4.4 . NoUNAPerfectRef $(\mathcal{O}, q)$ contains, among others, the following CQ:

$$
q_{5}=\left\{(x) \mid \exists y_{2} \cdot A(x) \wedge P_{1}\left(y_{2}, c\right) \wedge P_{3}\left(y_{2}, x\right)\right\},
$$

which is obtained from the CQ $q_{4}$ of $\lambda(q, \mathcal{O})$ described in Example 4.4 by first applying $\tau$ to $q_{4}$, thus obtaining $\left\{(x) \mid \exists y_{2} . P\left(x,{ }_{-}\right) \wedge P_{1}\left(y_{2}, c\right) \wedge P_{2}\left(y_{2}, x\right)\right\}$, and then by applying the inclusion assertions $A \sqsubseteq \exists P$ and $P_{3} \sqsubseteq P_{2}$ to atoms $P\left(x,{ }_{-}\right)$ and $P_{2}\left(y_{2}, x\right)$, respectively. Obviously, $q_{5}^{\mathcal{I}_{\mathcal{A}}}=\left\{\left(c_{1}\right)\right\}$. One can easily verify that $\left(c_{1}\right) \in \operatorname{cert}_{q, \mathcal{O}}^{\mathcal{A}}$ (more specifically, $\operatorname{cert}_{q, \mathcal{O}}^{\mathcal{A}}=\left\{\left(c_{1}\right)\right\}$ ), as expected.

The next theorem shows that, even when the UNA is not adopted, answering $\mathrm{CQ}^{\neq, b}$ s over $D L$-Lite $e_{\mathcal{R}}$ knowledge bases has exactly the same computational complexity of answering UCQs over DL-Lite $e_{\mathcal{R}}$ knowledge bases.

Theorem 4.3. Answering $C Q^{\neq, b}$ s over DL-Lite $e_{\mathcal{R}}$ knowledge bases is FOL-rewritable (and therefore in $A C^{0}$ in data complexity) and NP-complete in combined complexity.

Proof. FOL-rewritability is a direct consequence of Corollary 4.1 .

The membership in NP in combined complexity can be easily proven with a version of the NoUNAPerfectRef algorithm that nondeterministically guess a CQ of the final UCQ NoUNAPerfectRef $(\mathcal{O}, q)$, which can be generated after a polynomial number of transformations of the initial $\mathrm{CQ}^{\neq, b} q$. Finally, NP-hardness already follows from CQ evaluation over relational databases Abiteboul et al., 1995.

\subsubsection{Queries with Inequalities in Ontology-based Data Manage- ment}

We now briefly discuss how the above results behave in the context of the OBDM scenario when the UNA is not adopted. Let $\Sigma=\langle\mathcal{O}, \mathcal{S}, \mathcal{M}\rangle$ be an OBDM specification where $\mathcal{O}$ is a $D L$-Lite $e_{\mathcal{R}}$ ontology and $\mathcal{M}$ is a GLAV mapping. Clearly, since DL-Lite $\mathcal{R}_{\mathcal{R}}$ is insensitive to the adoption of the UNA for UCQ answering, given a UCQ $q_{\mathcal{O}}$ over $\mathcal{O}$ of arity $n$, it is not hard to verify that $\operatorname{PerfRef}_{q_{\mathcal{O}}, \Sigma} \cup \operatorname{PerfRef}_{\mathcal{V}_{\mathcal{O}}^{n}, \Sigma}$ is the perfect $\mathcal{O}$-to- $\mathcal{S} \Sigma$-rewriting of $q_{\mathcal{O}}$ even when the UNA is not adopted, where $\mathcal{V}_{\mathcal{O}}$ is the violation query for $\mathcal{O}$ and $\operatorname{PerfRef}_{q, \Sigma}:=\operatorname{MapRef}(\operatorname{PerfectRef}(\mathcal{O}, q), \mathcal{M})$ for a UCQ $q$ over $\mathcal{O}$ (cf. Section 2.6).

Let us now turn to the case of $\mathrm{CQ}^{\neq, b}$ s. Given a $\mathrm{CQ}^{\neq, b} q_{\mathcal{O}}$ over $\mathcal{O}$ of arity $n$, we denote by NoUNAPerfRef $q_{\mathcal{O}}, \Sigma$ the UCQ over $\mathcal{S}$ obtained by first executing the algorithm NoUNAPerfectRef on $\mathcal{O}$ and $q_{\mathcal{O}}$, and then by rewriting the obtained UCQ with respect to mapping $\mathcal{M}$, i.e., NoUNAPerfRef $q_{\mathcal{O}, \Sigma}:=\operatorname{MapRef}\left(\operatorname{NoUNAPerfectRef}\left(\mathcal{O}, q_{\mathcal{O}}\right), \mathcal{M}\right)$. 
Theorem 4.4. Let $\Sigma=\langle\mathcal{O}, \mathcal{S}, \mathcal{M}\rangle$ be an $O B D M$ specification where $\mathcal{O}$ is a $D L-$ Lite $_{\mathcal{R}}$ ontology and $\mathcal{M}$ is a GLAV mapping. When the UNA is not adopted, given a $C Q^{\neq, b}$ over $\mathcal{O}$, we have that NoUNAPerfRef $q_{\mathcal{O}, \Sigma} \cup \operatorname{PerfRef}_{\mathcal{V}_{\mathcal{O}}^{n}, \Sigma}$ is the perfect $\mathcal{O}$-to-S $\Sigma$-rewriting of $q_{\mathcal{O}}$, i.e., (NoUNAPerfRef $\left.q_{\mathcal{O}, \Sigma} \cup \operatorname{PerfRef}_{\mathcal{V}_{\mathcal{O}}^{n}, \Sigma}\right)^{D}=$ cert $_{q_{\mathcal{O}}, \Sigma}^{D}$, for every $\mathcal{S}$-database $D$.

Proof. Let $D$ be an $\mathcal{S}$-database. If $D$ is inconsistent with $\Sigma$, i.e., $\operatorname{Mod}_{D}(\Sigma)=\emptyset$, then PerfRef $\mathcal{V}_{\mathcal{O}, \Sigma}^{D}=\operatorname{cert}_{q_{\mathcal{O}}, \Sigma}^{D}$, which corresponds to the set of all possible tuples of constants occurring in $D$ whose arity is the one of the query.

On the other hand, if $D$ is consistent with $\Sigma$ (and therefore $\operatorname{PerfRef}_{\mathcal{V}_{\mathcal{O}}^{n}, \Sigma}^{D}=$ $\emptyset)$, then, due to Corollary 4.1. we have $\operatorname{cert} t_{q_{\mathcal{O}}, \Sigma}^{D}=\operatorname{cert}_{q^{\prime}, \Sigma^{\prime}}^{D}$, where $q^{\prime}=$ NoUNAPerfectRef $\left(\mathcal{O}, q_{\mathcal{O}}\right)$ and $\Sigma^{\prime}=\langle\emptyset, \mathcal{S}, \mathcal{M}\rangle$. But then, since $q^{\prime}$ is a UCQ and the ontology of $\Sigma^{\prime}=\langle\emptyset, \mathcal{S}, \mathcal{M}\rangle$ contains no assertions, $\operatorname{cert}_{q^{\prime}, \Sigma^{\prime}}^{D}=\operatorname{MapRef}\left(q^{\prime}, \mathcal{M}\right)^{D}$ (cf. Section 2.6. It follows that $\operatorname{cert}_{q_{\mathcal{O}}, \Sigma}^{D}=\operatorname{Map} \operatorname{Ref}\left(\operatorname{NoUNAPerfectRef}\left(\mathcal{O}, q_{\mathcal{O}}\right), \mathcal{M}\right)^{D}$, i.e., $\operatorname{cert}_{q_{\mathcal{O}}, \Sigma}^{D}=$ NoUNAPerfRef ${ }_{q_{\mathcal{O}}, \mathcal{M}}^{D}$, as required.

\subsubsection{The case of the Direct Semantics Entailment Regime}

The de facto standard query language for the Semantic Web is SPARQI ${ }^{3}$ As defined in a W3C standard specification Glimm and Ogbuji, 2013, the query language SPARQL 1.1 [Harris and Seaborne, 2013] (i.e., the current version of SPARQL) relies on the notion of SPARQL entailment regime, which defines:

1. the syntax and the semantics of assertions of the queried knowledge base;

2. the syntax of conjunctive queries considered legal for the regime;

3. the semantics of such queries, i.e., what are the answers to a query.

As for 1, we assume to deal with (satisfiable) OWL 2 QL (equivalently, DL-Lite $\mathcal{R}$ ) knowledge bases without the UNA. As for 2, we now consider general $\mathrm{UCQ}^{\neq} \mathrm{s}$. Finally, as for 3, we follow the OWL 2 QL DSER, which is the most typical SPARQL Entailment Regime for OWL 2 QL (equivalently, $D L$-Lite $\mathcal{R}$ ) knowledge bases.

Specifically, given a $D L$-Lite $\mathcal{R}_{\mathcal{R}}$ knowledge base $\mathcal{K}=\langle\mathcal{O}, \mathcal{A}\rangle$ and a $\mathrm{CQ}^{\neq} q=$ $\left\{\left(t_{1}, \ldots, t_{n}\right) \mid \exists y_{1}, \ldots, y_{m} . \phi(\vec{x}, \vec{y})\right\}$ over $\mathcal{O}$, DSER defines the answers to $q$ with respect to $\mathcal{O}$ and $\mathcal{A}$, denoted by $D S c e r t_{q, \mathcal{O}}^{\mathcal{A}}$, as follows: an $n$-tuple of individuals $\vec{c}=\left(c_{1}, \ldots, c_{n}\right)$ (with $c_{j}=t_{j}$ for each $j \in[1, n]$ in which $t_{j}$ is a constant) is in $D \operatorname{Scert}_{q, \mathcal{O}}^{\mathcal{A}}$ if and only if there exists an $m$-tuple of individuals $\overrightarrow{c^{\prime}}=\left(c_{1}^{\prime}, \ldots, c_{n}^{\prime}\right)$ for which $\mathcal{K}=q\left(\vec{c}, \overrightarrow{c^{\prime}}\right)$, where $q\left(\vec{c}, \overrightarrow{c^{\prime}}\right)=\left\{() \mid \phi\left(\vec{x} / \vec{c}, \vec{y} / \overrightarrow{c^{\prime}}\right)\right\}$ denotes the boolean $\mathrm{CQ}^{\neq}$in which $\phi\left(\vec{x} / \vec{c}, \vec{y} / \overrightarrow{c^{\prime}}\right)$ is the body obtained from $\phi(\vec{x}, \vec{y})$ by replacing all the occurrences of the term $t_{i}$ (respectively, $y_{i}$ ) with the individual $c_{i}$ (respectively, $c_{i}^{\prime}$ ), for each $i \in[1, n]$ (respectively, $i \in[1, m]$ ). In other words, for an $n$-tuple of individuals $\vec{c}$, in contrast to classical logic, existential variables $\vec{y}$, although projected out from the final answers, are required to be bound to the same $m$-tuple of constants $\overrightarrow{c^{\prime}}$, in every model of $\mathcal{K}$.

Example 4.6. Let us recall Example 4.5. We have that $\left(c_{1}\right) \notin D S c e r t_{q, \mathcal{O}}^{\mathcal{A}}$, since there is no $y_{1}$ for which $P\left(c, y_{1}\right)$ is known to hold.

\footnotetext{
${ }^{3}$ SPARQL Protocol and RDF Query Language: https://www.w3.org/2001/sw/wiki/SPARQL
} 
Furthermore, given a $D L$ - Lite $_{\mathcal{R}}$ knowledge base $\mathcal{K}=\langle\mathcal{O}, \mathcal{A}\rangle$ and a $\mathrm{UCQ}^{\neq} q=$ $q_{1} \cup \ldots \cup q_{l}$ over $\mathcal{O}$, again differently from the classical logic, we have $D S c e r t_{q, \mathcal{O}}^{\mathcal{A}}=$ $D S c e r t q_{q_{1}, \mathcal{O}}^{\mathcal{A}} \cup \ldots \cup \operatorname{DScert} q_{q_{l}, \mathcal{O}}^{\mathcal{A}}$.

Example 4.7. Consider the knowledge base $\mathcal{K}=\langle\mathcal{O}, \mathcal{A}\rangle$, where $\mathcal{O}=\emptyset$ and $\mathcal{A}=$ $\{P(a, b)\}$. For the boolean $\mathrm{UCQ}^{\neq, b} Q=q_{1} \cup q_{2}$ over $\mathcal{O}$, where $q_{1}=\{() \mid P(a, a)\}$ and $q_{2}=\{() \mid a \neq b\}$, it is easy to see that both $\operatorname{DScert}_{q_{1}, \mathcal{O}}^{\mathcal{A}}=\emptyset$ and $D S c e r t_{q_{2}, \mathcal{O}}^{\mathcal{A}}=\emptyset$ hold. For the former, consider a model $\mathcal{M}$ of $\mathcal{K}$ in which $a^{\mathcal{M}} \neq b^{\mathcal{M}}$. For the latter, consider a model $\mathcal{M}$ of $\mathcal{K}$ in which $a^{\mathcal{M}}=b^{\mathcal{M}}$. Therefore, $\operatorname{DScert}_{Q, \mathcal{O}}^{\mathcal{A}}=\emptyset$ by definition.

Notice, however, that $\mathcal{K} \models Q$. Indeed, for any model $\mathcal{M}$ of $\mathcal{K}, a^{\mathcal{M}} \neq b^{\mathcal{M}}$ implies $\mathcal{M} \models q_{2}$, whereas $a^{\mathcal{M}}=b^{\mathcal{M}}$ implies $\mathcal{M}=q_{1}$. Thus, cert $_{Q, \mathcal{O}}^{\mathcal{A}}=\{()\}$.

We now present the algorithm DSERPerfectRef for answering $\mathrm{UCQ}^{\neq} \mathrm{S}$ over $D L-$ Lite $_{\mathcal{R}}$ knowledge bases under the DSER semantics. Given in input a DL-Lite P $_{\mathcal{R}}$ ontology $\mathcal{O}$ and a $\mathrm{UCQ}^{\neq} q$ over $\mathcal{O}$, DSERPerfectRef returns a reformulated UCQ.

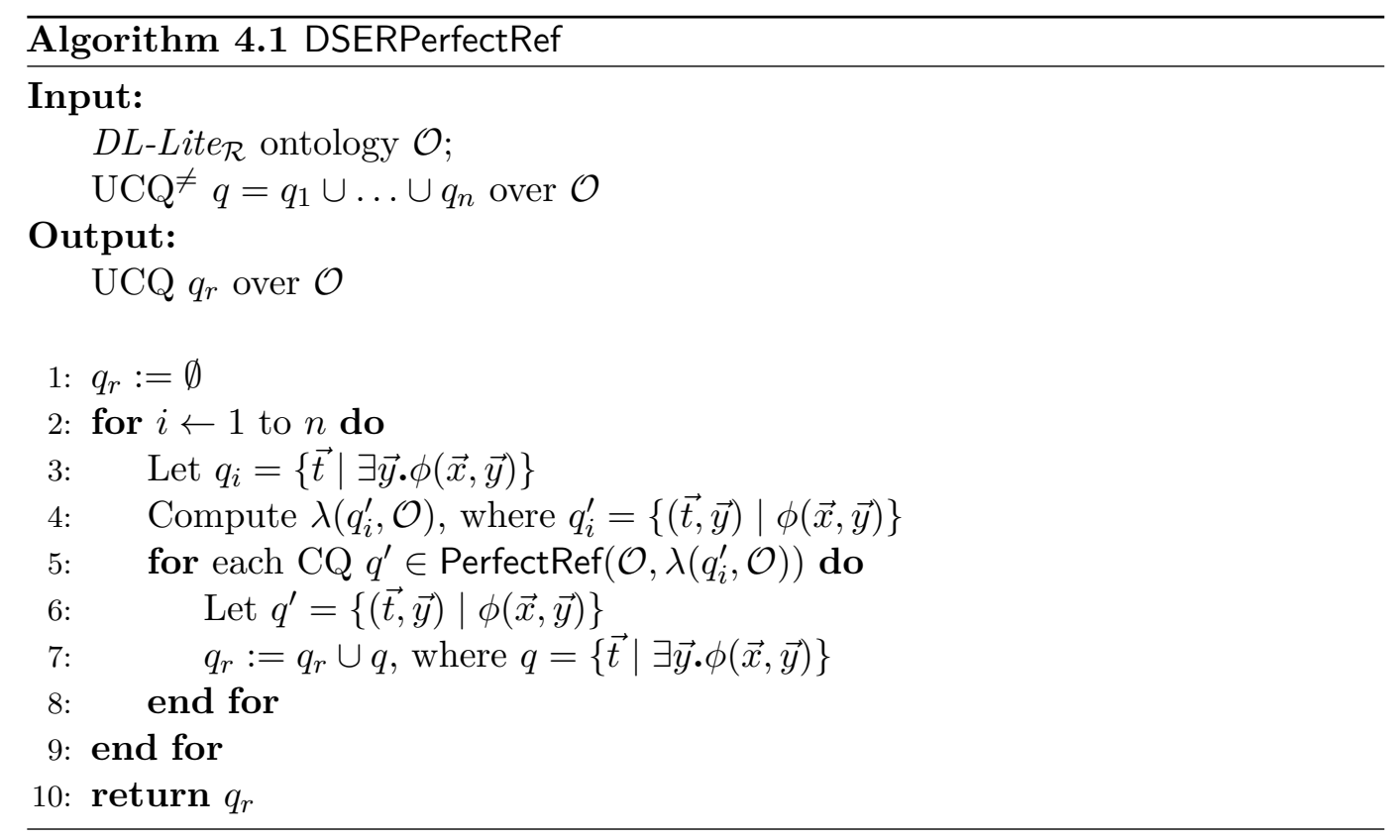

Roughly speaking, according to the meaning of the union operator under DSER, the algorithm treats each CQ of $q$ separately. In particular, for each CQ $q_{i}=\{\vec{t} \mid$ $\exists \vec{y} . \phi(\vec{x}, \vec{y})\}$ of $q$, according to the meaning of the existential quantifiers under DSER, the algorithm consider the CQ $q_{i}^{\prime}=\{(\vec{t}, \vec{y}) \mid \phi(\vec{x}, \vec{y})\}$, i.e., the query obtained from $q$ in which existential variables $\vec{y}$ become distinguished (and therefore bound). After that, it rewrites inequality atoms through the function $\lambda$ to capture only those inequalities logically implied. Finally, for each CQ in $\operatorname{PerfectRef}\left(\mathcal{O}, \lambda\left(q_{i}^{\prime}, \mathcal{O}\right)\right)$, the algorithm projects those variables that were originally existential variables.

Example 4.8. Consider the $D L$-Lite $e_{\mathcal{R}}$ knowledge base $\mathcal{K}=\langle\mathcal{O}, \mathcal{A}\rangle$, where

- $\mathcal{O}=\{$ Male $\sqsubseteq \neg F e m a l e$, Pregnant $\sqsubseteq$ Female, Employee $\sqsubseteq \exists$ WorksFor $\}$ 
$\{$ WorksFor(John, HOPE), WorksFor(Cleo, HOPE), Employee(Alice),

- $\mathcal{A}=$ Parent(John, Bill), Parent(John, Wendy), Parent(Alice, Bill), Parent(Alice, Wendy), Parent(Cleo, Alex), Parent(Cleo, Alexander), Male(Bill), Pregnant(Wendy) \}.

Consider also the following $\mathrm{CQ}^{\neq} q$ over $\mathcal{O}$ asking for those people working for some company and having at least two children:

$q=\left\{(x) \mid \exists y_{1}, y_{2}, y_{3}\right.$. WorksFor $\left.\left(x, y_{1}\right) \wedge \operatorname{Parent}\left(x, y_{2}\right) \wedge \operatorname{Parent}\left(x, y_{3}\right) \wedge y_{2} \neq y_{3}\right\}$.

As a first step, DSERPerfectRef $\left(\mathcal{O}, q_{\mathcal{O}}\right)$ computes the UCQ $\lambda\left(q^{\prime}, \mathcal{O}\right)$, where $q^{\prime}=$ $\left\{\left(x, y_{1}, y_{2}, y_{3}\right) \mid \operatorname{WorksFor}\left(x, y_{1}\right) \wedge \operatorname{Parent}\left(x, y_{2}\right) \wedge \operatorname{Parent}\left(x, y_{3}\right) \wedge y_{2} \neq y_{3}\right\}$. Note that $\lambda\left(q^{\prime}, \mathcal{O}\right)$ contains, among others, the CQ $\left\{\left(x, y_{1}, y_{2}, y_{3}\right) \mid \operatorname{WorksFor}\left(x, y_{1}\right) \wedge\right.$ $\left.\operatorname{Parent}\left(x, y_{2}\right) \wedge \operatorname{Parent}\left(x, y_{3}\right) \wedge \operatorname{Male}\left(y_{2}\right) \wedge \operatorname{Female}\left(y_{3}\right)\right\}$.

Then, PerfectRef $\left(\mathcal{O}, \lambda\left(q^{\prime}, \mathcal{O}\right)\right)$ computes, among others, the CQ $\left\{\left(x, y_{1}, y_{2}, y_{3}\right) \mid\right.$ WorksFor $\left.\left(x, y_{1}\right) \wedge \operatorname{Parent}\left(x, y_{2}\right) \wedge \operatorname{Parent}\left(x, y_{3}\right) \wedge \operatorname{Male}\left(y_{2}\right) \wedge \operatorname{Pregnant}\left(y_{3}\right)\right\}$. Note that, since $y_{1}$ is a distinguished variable in $q^{\prime}$, the inclusion assertion Employee $\sqsubseteq \exists$ WorksFor is not used to rewrite $q^{\prime}$ with respect to $\mathcal{O}$.

As a last step, the algorithm returns the UCQ which contains, among others, the CQ $q_{1}=\left\{(x) \mid \exists y_{1}, y_{2}, y_{3}\right.$.WorksFor $\left(x, y_{1}\right) \wedge \operatorname{Parent}\left(x, y_{2}\right) \wedge \operatorname{Parent}\left(x, y_{3}\right) \wedge$ $\left.\operatorname{Male}\left(y_{2}\right) \wedge \operatorname{Pregnant}\left(y_{3}\right)\right\}$. Observe that $q_{1}^{\mathcal{I}_{\mathcal{A}}}=\{(\mathrm{John})\}$. One can indeed verify that $D S \operatorname{sert} t_{q, \mathcal{O}}^{\mathcal{A}}=\{(\mathrm{John})\}$, as expected. Finally, note that $(\mathrm{Cleo}) \notin \operatorname{DScert}_{q, \mathcal{O}}^{\mathcal{A}}$ and (Alice) $\notin D S c e r t_{q, \mathcal{O}}^{\mathcal{A}}$ since Alex and Alexander are not known to be different individuals and it is not known which is the project Alice works for, respectively.

From the results given previously for $\mathrm{CQ}^{\neq, b} \mathrm{~s}$, and from the considerations of how the algorithm capture the DSER, we immediately derive the following result.

Proposition 4.3. Let $\mathcal{O}$ be a DL-Lite $e_{\mathcal{R}}$ ontology, and let $q$ be a $U C Q^{\neq}$over $\mathcal{O}$. For

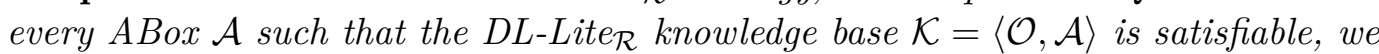
have $D$ Scert ${ }_{q, \mathcal{O}}^{\mathcal{A}}=D S E R P e r f e c t R e f(\mathcal{O}, q)^{\mathcal{I}_{\mathcal{A}}}$.

We also get that answering $\mathrm{UCQ}^{\neq} \mathrm{s}$ over $D L$-Lite R $_{\mathcal{R}}$ knowledge bases under DSER has exactly the same computational complexity of answering UCQs over DL-Lite $\mathcal{R}_{\mathcal{R}}$ knowledge bases.

Theorem 4.5. Answering $U C Q^{\neq} s$ over DL-Lite $e_{\mathcal{R}}$ knowledge bases under DSER is FOL-rewritable (and therefore in $A C^{0}$ in data complexity) and NP-complete in combined complexity.

Related to this problem is the result in Gutiérrez-Basulto et al., 2015], which shows that answering even $\mathrm{CQ}^{\neq} \mathrm{s}$ over $D L$ - Lite $_{\mathcal{R}}$ knowledge bases is in general undecidable. We point out, however, that such a negative result is due to the fact that existential variables are assigned the standard logical meaning, which is not the case in DSER. To the best of our knowledge, only few works Kontchakov et al., 2014 Gottlob and Pieris, 2015; Poggi, 2016; Arenas et al., 2018 have investigated the problem of answering queries over DL-Lite $\mathcal{R}_{\mathcal{R}}$ knowledge bases under DSER. With the only exception of Poggi, 2016] (which shows that the problem of answering $\mathrm{UCQ}^{{ }^{*}} \mathrm{~S}$ over $D L$-Lite $e_{\mathcal{R}}$ knowledge bases under DSER can be reduced to the evaluation of a 
Datalog program, and therefore is in PTIME in data complexity and in ExPTIME in combined complexity), none of the other works consider queries possibly containing inequalities. In fact, in Gottlob and Pieris, 2015] it is even claimed that inequalities cannot occur within legal OWL 2 QL basic graph patterns, which is a strong assumption, because inequalities can be expressed by means of DifferentIndividuals atoms.

\subsection{Answering $\mathrm{UCQ}^{\neq, b}$ s over $\boldsymbol{D L}$ - Lite $_{\mathcal{R}}$ knowledge bases}

In this section, we consider the problem of answering $\mathrm{UCQ}^{\neq, b_{\mathrm{S}}}$ over satisfiable $D L$-Lite R $_{\mathcal{R}}$ knowledge bases. The next example shows that, differently from the UCQ case, the certain answers of $\mathrm{UCQ}^{\neq, b_{\mathrm{S}}}$ over $D L$-Lite $\mathcal{R}_{\mathcal{R}}$ knowledge bases is not necessarily equivalent to the union of the certain answers of the singles CQs.

Example 4.9. Recall Example 4.7, where $\mathcal{K}=\langle\mathcal{O}, \mathcal{A}\rangle$ with $\mathcal{O}=\emptyset$ and $\mathcal{A}=$ $\{P(a, b)\}$. For the boolean $\mathrm{UCQ}^{\neq, b} Q=q_{1} \cup q_{2}$ over $\mathcal{O}$, where $q_{1}=\{() \mid P(a, a)\}$ and $q_{2}=\{() \mid a \neq b\}$, it is easy to see that both $\mathcal{K} \not \forall q_{1}$ and $\mathcal{K} \not \neq q_{2}$. For the former, consider a model $\mathcal{M}$ of $\mathcal{K}$ in which $a^{\mathcal{M}} \neq b^{\mathcal{M}}$. For the latter, consider a model $\mathcal{M}$ of $\mathcal{K}$ in which $a^{\mathcal{M}}=b^{\mathcal{M}}$. Notice, however, that $\mathcal{K} \models Q$. Indeed, for any model $\mathcal{M}$ of $\mathcal{K}, a^{\mathcal{M}} \neq b^{\mathcal{M}}$ implies $\mathcal{M} \models q_{2}$, and $a^{\mathcal{M}}=b^{\mathcal{M}}$ implies $\mathcal{M} \models q_{1}$.

The above example also tells us that answering $\mathrm{UCQ}^{\neq, b}$ s over $D L$-Lite $e_{\mathcal{R}}$ knowledge bases $\mathcal{K}=\langle\mathcal{O}, \mathcal{A}\rangle$ can not be done simply using the interpretation $\mathcal{I}_{\mathcal{K}}$. Indeed, one can verify that $\delta(Q)=\delta\left(q_{1}\right) \cup \delta\left(q_{2}\right)$ is such that $\mathcal{I}_{\mathcal{K}} \not \models \delta(Q)$, whereas $\mathcal{K} \models Q$.

The conceptual tool we use for addressing the problem of answering queries with inequalities over $D L$-Lite $\mathcal{R}_{\mathcal{R}}$ knowledge base is the notion of equivalence relation 4 Specifically, we now introduce the notions of $e$-satisfiability and $e$-entailment for an equivalence relation $e$. In what follows, for an equivalence relation $e$, we write $c_{1} \sim_{e} c_{2}$ to actually denote $\left(c_{1}, c_{2}\right) \in e$.

Definition 4.1. Let $\mathcal{K}=\langle\mathcal{O}, \mathcal{A}\rangle$ be a knowledge base, $e$ be an equivalence relation on a set $I$ of individuals occurring in $\mathcal{A}$, and $\mathcal{M}$ be a model of $\mathcal{K}$. Then, we say that $\mathcal{M}$ is an e-model of $\mathcal{K}$, denoted by $\mathcal{M}=_{e} \mathcal{K}$, if for any pair of individuals $c_{1}, c_{2}$ occurring in $I$, we have that $c_{1}^{\mathcal{M}}=c_{2}^{\mathcal{M}}$ if and only if $c_{1} \sim_{e} c_{2}$. Furthermore, we say that $\mathcal{K}$ is $e$-satisfiable if it has an $e$-model.

Definition 4.2. Let $\mathcal{K}=\langle\mathcal{O}, \mathcal{A}\rangle$ be a knowledge base, $q$ be a boolean query over $\mathcal{O}$, and $e$ be an equivalence relation on a set $I$ of individuals occurring in $\mathcal{A}$. Then, we say that $\mathcal{K}$ e-entails $q$, denoted by $\mathcal{K} \models_{e} q$, if $\mathcal{M} \models q$ for each $e$-model $\mathcal{M}$ of $\mathcal{K}$.

Observe that, for a knowledge base $\mathcal{K}=\langle\mathcal{O}, \mathcal{A}\rangle$, if $e$ is the equivalence relation on the set of all individuals occurring in $\mathcal{A}$ such that $e=\{(c, c) \mid c$ occurs in $\mathcal{A}\}$ (respectively, $e$ is the equivalence relation on the empty set of individuals, i.e., $e=\{\}$ ), then the notions of $e$-satisfiability and $e$-entailment coincide with the usual notion of satisfiability and entailment, respectively, when the UNA is adopted (respectively, when the UNA is not adopted).

\footnotetext{
${ }^{4}$ An equivalence relation $e$ on a set of individuals $I$ is a binary relation over $I$ that is reflexive, symmetric, and transitive.
} 
In what follows, for a knowledge base $\mathcal{K}=\langle\mathcal{O}, \mathcal{A}\rangle$ and an equivalence relation $e$, we denote by $\mathcal{K}_{e}=\left\langle\mathcal{O}_{e}, \mathcal{A}_{e}\right\rangle$ the knowledge base obtained from $\mathcal{K}$ by adding $e$ in the alphabet of $\mathcal{O}$ as a new atomic role (obtaining the ontology $\mathcal{O}_{e}$ ), and $\mathcal{A}_{e}$ is the ABox for $\mathcal{O}_{e}$ such that $\mathcal{A}_{e}=\mathcal{A} \cup$ e, i.e., $\mathcal{A}_{e}=\mathcal{A} \cup\left\{e\left(c_{1}, c_{2}\right) \mid\left(c_{1}, c_{2}\right) \in e\right\}$. Moreover, for a query $q$ over an ontology $\mathcal{O}$, we denote by $I_{q}$ the set of all individuals occurring in $q$.

We now prove that, in principle, the notion of e-entailment can be used for the problem of answering $\mathrm{UCQ}^{\neq, b_{\mathrm{S}}}$ over $D L$-Lite $e_{\mathcal{R}}$ knowledge bases.

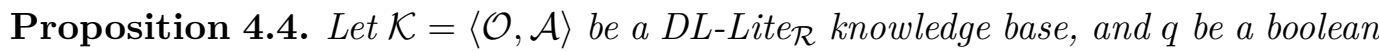
$U C Q^{\neq, b}$. We have that $\mathcal{K} \not q$ if and only if there exists an equivalence relation e on $I_{q}$ such that $\mathcal{K} \forall_{e} q$.

Proof. "If part:" Suppose that there exists an equivalence relation $e$ on $I_{q}$ such that $\mathcal{K} \models_{e} q$. This implies that there is an $e$-model $\mathcal{M}$ of $\mathcal{K}$ such that $\mathcal{M} \forall q$. Since $\mathcal{M}$ is an $e$-model of $\mathcal{K}$, then $\mathcal{M}$ is also a model of $\mathcal{K}$. Thus, $\mathcal{K} \not \forall q$, as required.

"Only-if part:" Suppose that $\mathcal{K} \not \models q$. It follows that there is a model $\mathcal{M}$ of $\mathcal{K}$ for which $\mathcal{M} \not q$. Consider now the equivalence relation $e$ on $I_{q}$ given by the model $\mathcal{M}$, that is, $\left(c_{1}, c_{2}\right) \in e$ if and only if $c_{1}^{\mathcal{M}}=c_{2}^{\mathcal{M}}$ and $c_{1}, c_{2} \in I_{q}$.

Let $\mathcal{I}$ be the interpretation for $\mathcal{K}$ similar to $\mathcal{M}$ but, for each individual $c \notin I_{q}$, we set $c^{\mathcal{I}}=c$ and replace each occurrence of the object $c^{\mathcal{M}}$ with $c^{\mathcal{I}}=c$. Since $\mathcal{M} \not \models q$, it can be easily verified that $\mathcal{I} \not \models q$ as well. In particular, since $c^{\mathcal{I}}=c^{\mathcal{M}}$ for each $c \in I_{q}$ (i.e., for each individual appearing in the query), the evaluation of each inequality atom of $q$ (since $q$ is a boolean $\mathrm{UCQ}^{\neq, b}$, the inequality atoms are only between individuals) over $\mathcal{I}$ and $\mathcal{M}$ coincide. Notice, moreover, that since $\mathcal{M}$ is a model of $\mathcal{K}$, we have that $\mathcal{I}$ is a model of $\mathcal{K}$ as well, and therefore an $e$-model of $\mathcal{K}$ by construction. So, $\mathcal{I}$ is an $e$-model of $\mathcal{K}$ such that $\mathcal{I} \not \models q$, and therefore $\mathcal{K} \forall_{e} q$.

To conclude the proof, observe that $e$ is an equivalence relation on the set of individuals $I_{q}$ such that $\mathcal{K} \forall_{e} q$, as required.

The above proposition suggests a nondeterministic algorithm for the problem of answering $\mathrm{UCQ}^{\neq, b}$ s over $D L$-Lite R $_{\mathcal{R}}$ knowledge bases, which consists in guessing an equivalence relation $e$ on $I_{q}$, and then checking whether $\mathcal{K} \not \nvdash_{e} q$, where $\mathcal{K}=\langle\mathcal{O}, \mathcal{A}\rangle$ and $q$ are the input $D L$-Lite $e_{\mathcal{R}}$ knowledge base and $\mathrm{UCQ}^{\neq, b}$ over $\mathcal{O}$, respectively. So, for the case of $D L$-Lite $e_{\mathcal{R}}$ knowledge bases $\mathcal{K}=\langle\mathcal{O}, \mathcal{A}\rangle$, we now study the problems of $(i)$ checking whether $\mathcal{K}$ is $e$-satisfiable for an arbitrary equivalence relation $e$ on a set $I$ of individuals of $\mathcal{A}$; $(i i)$ checking whether $\mathcal{K} \models_{e} q$ for a boolean $\mathrm{UCQ}^{\neq, b} q$ over $\mathcal{O}$ and an arbitrary equivalence relation $e$ on $I_{q}$ such that $\mathcal{K}$ is $e$-satisfiable. Specifically, we next show that the computational complexity of both the above problems remains the same of the case when $e$ simulates the adoption of the UNA.

We start with the $e$-satisfiability check. We assume to deal with satisfiable knowledge bases (notice that $e$-satisfiability always implies satisfiability, and therefore, in case of unsatisfiable knowledge bases, $e$-satisfiability becomes trivial).

Proposition 4.5. Let $\mathcal{K}=\langle\mathcal{O}, \mathcal{A}\rangle$ be a DL-Lite $\mathcal{R}_{\mathcal{R}}$ knowledge base and e be an equivalence relation on a set $I$ of individuals occurring in $\mathcal{A}$. The problem of checking whether $\mathcal{K}$ is e-satisfiable is in $A C^{0}$ in the size of $\mathcal{A}$ and e, and in PTIME in the size of $\mathcal{O}$. 
Proof. Let $\mathcal{V}_{\mathcal{O}}^{e}$ be the $e$-violation query for $\mathcal{O}$ obtained from $\mathcal{V}_{\mathcal{O}}$ by adding the following disjuncts over the signature of $\mathcal{O}_{e}$ :

- $\left\{() \mid \exists y_{1}, y_{2} \cdot A_{1}\left(y_{1}\right) \wedge A_{2}\left(y_{2}\right) \wedge e\left(y_{1}, y_{2}\right)\right\}$ for each disjointness assertion of the form $A_{1} \sqsubseteq \neg A_{2}$ belonging to $\mathcal{O}$,

- $\left\{() \mid \exists y_{1}, y_{2}, y_{3} \cdot A\left(y_{1}\right) \wedge R\left(y_{2}, y_{3}\right) \wedge e\left(y_{1}, y_{2}\right)\right\}$ for each disjointness assertion of the form $A \sqsubseteq \neg \exists R$ or of the form $\exists R \sqsubseteq \neg A$ belonging to $\mathcal{O}$,

- $\left\{() \mid \exists y_{1}, y_{2}, y_{3}, y_{4} \cdot R_{1}\left(y_{1}, y_{3}\right) \wedge R_{2}\left(y_{2}, y_{4}\right) \wedge e\left(y_{1}, y_{2}\right)\right\}$ for each disjointness assertion of the form $\exists R_{1} \sqsubseteq \neg \exists R_{2}$ belonging to $\mathcal{O}$,

- $\left\{() \mid \exists y_{1}, y_{2}, y_{3}, y_{4} \cdot R_{1}\left(y_{1}, y_{3}\right) \wedge R_{2}\left(y_{2}, y_{4}\right) \wedge e\left(y_{1}, y_{2}\right) \wedge e\left(y_{3}, y_{4}\right)\right\}$ for each disjointness assertion of the form $R_{1} \sqsubseteq \neg R_{2}$ belonging to $\mathcal{O}$,

where an atom of the form $R\left(y, y^{\prime}\right)$ stands for either $P\left(y, y^{\prime}\right)$ if $R$ denotes an atomic role $P$, or $P\left(y^{\prime}, y\right)$ if $R$ denotes the inverse of an atomic role, i.e., $R=P^{-}$.

Intuitively, for checking $e$-satisfiability it is sufficient to check whether the equivalence relation $e$ contradicts a disjointness assertion of $\mathcal{O}$. We now prove that $\mathcal{K}$ is $e$-satisfiable if and only if $\mathcal{K}_{e} \not \models \mathcal{V}_{\mathcal{O}}^{e}$.

If $\mathcal{K}_{e} \models q$ for some disjunct $q$ of $\mathcal{V}_{\mathcal{O}}^{e}$, then it is easy to see that there are at least two individuals $c_{1} \sim_{e} c_{2}$ of $I$ such that $\mathcal{I} \forall \mathcal{K}$ in each interpretation $\mathcal{I}$ with $c_{1}^{\mathcal{I}}=c_{2}^{\mathcal{I}}$, and therefore $\mathcal{K}$ is not $e$-satisfiable, as required. Conversely, if $\mathcal{K}_{e} \not \forall \mathcal{V}_{\mathcal{O}}^{e}$, then from $\mathcal{I}_{\mathcal{K}}$ it is possible to obtain a model $\mathcal{M}$ where $c_{1}^{\mathcal{M}}=c_{2}^{\mathcal{M}}$ for each $\left(c_{1}, c_{2}\right) \in e$. It is easy to see that $\mathcal{M} \models_{e} \mathcal{K}$, and therefore $\mathcal{K}$ is $e$-satisfiable.

Finally, observe that $\mathcal{V}_{\mathcal{O}}^{e}$ can be constructed in PTime in the size of $\mathcal{O}$, and, as usual, checking whether $\mathcal{K}_{e} \not \neq \mathcal{V}_{\mathcal{O}}^{e}$ is FOL-rewritable (and therefore in $\mathrm{AC}^{0}$ in the size of $\mathcal{A}_{e}$ ) and in PTIME in the size of $\mathcal{O}_{e}$, thus showing the claim.

We now focus on the problem of checking whether $\mathcal{K} \models_{e} q$ for a $D L$-Lite $\mathcal{R}$ knowledge base $\mathcal{K}=\langle\mathcal{O}, \mathcal{A}\rangle$, boolean $\mathrm{UCQ}^{\neq, b}$ over $\mathcal{O}$, and equivalence relation $e$ on $I_{q}$. To this aim, we provide algorithm EquivalenceRelationRef that, given in input a boolean $\mathrm{UCQ}^{\neq, b} q$ over $\mathcal{O}$ and an equivalence relation $e$ on $I_{q}$, it reformulates $q$ according to $e$ and returns a UCQ over $\mathcal{O}_{e}$. In the algorithm, we assume that boolean queries may contain false and true atoms in their body, with their obvious semantics. Furthermore, $\mathfrak{P}(\cdot)$ is a function returning the power set of a given set as input. Finally, ej $(\cdot)$ is the function that takes a $\mathrm{CQ} q$ as input, and returns the set of all existential variables of $q$ that are join existential variables, i.e., the existential variables $y$ such that $m_{y} \geq 2$, where $m_{y}$ denotes the number of occurrences of the variable $y$ in the body of $q$.

Intuitively, the algorithm first reformulates the given $\mathrm{UCQ}^{\neq, b} q$ into a $\mathrm{UCQ}$ by directly evaluating each inequality atom $c_{1} \neq c_{2}$ based on the equivalence relation $e$ (notice that, since $q$ is a boolean $\mathrm{UCQ}^{\neq, b}$, each inequality atom $c_{1} \neq c_{2}$ is such that both $c_{1}$ and $c_{2}$ are individuals).

Afterwards, for each possible set $\mathcal{Y} \in \mathfrak{P}\left(\operatorname{ej}\left(q_{i}\right)\right)$ of join existential variables occurring in the CQ $q_{i}$, the algorithm reformulates $q_{i}$ by allowing that different occurrences $y^{1}, y^{2}, \ldots, y^{m_{y}}$ of the same existential variable $y \in \mathcal{Y}$ may be possibly mapped to also distinct individuals, with the proviso that such distinct individuals belong to the same equivalence class in $e$ (which is captured by $e\left(z, z^{\prime}\right)$ atoms). 


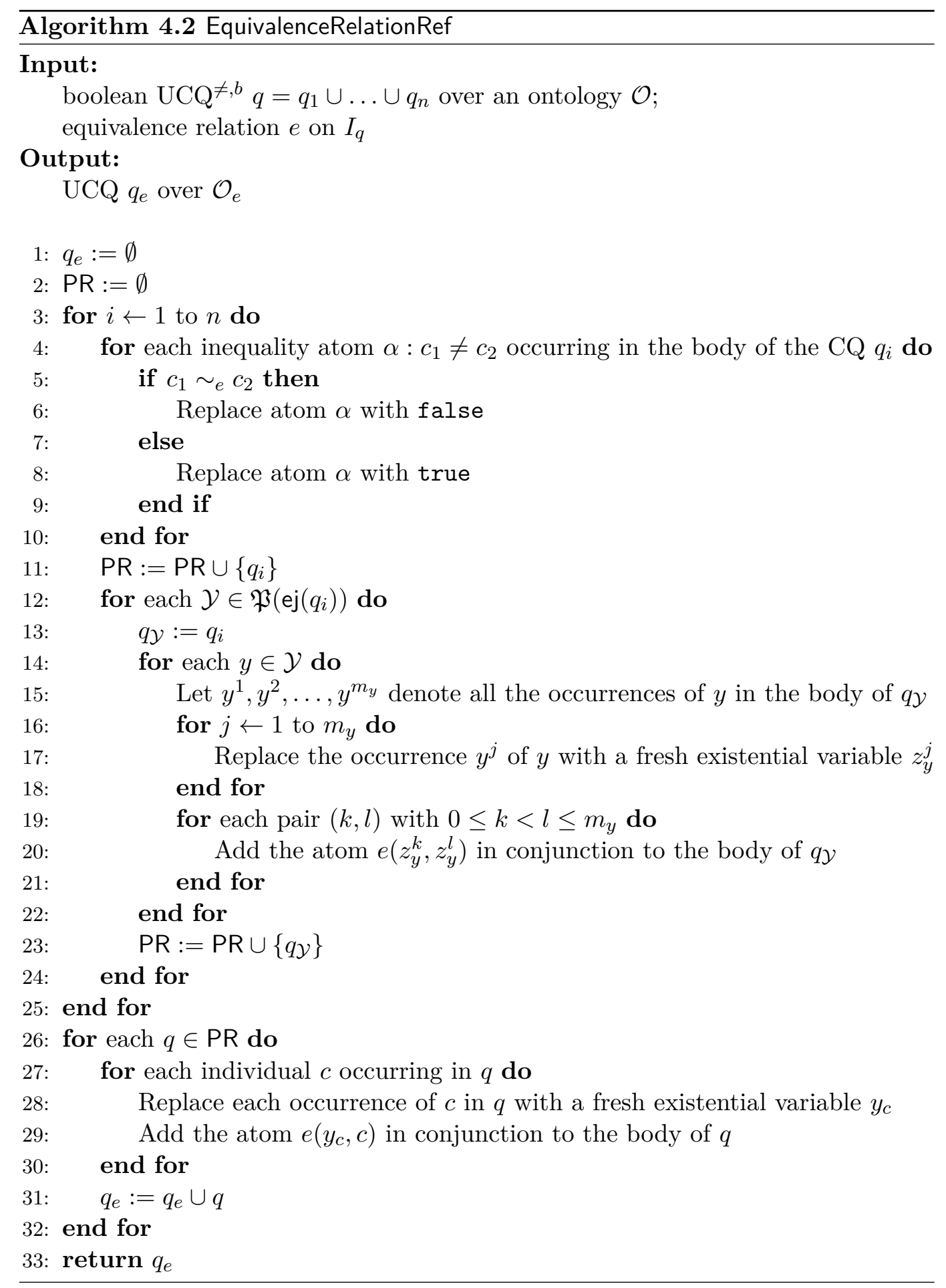

An analogous consideration holds for the various individuals occurring in queries, where in the last steps of the algorithm each CQ $q$ is further reformulated to allow that an existential variable $y_{c}$ may match an individual $c^{\prime}$ that is not necessarily the original individual $c$ of $q$, but it is such that $c \sim_{e} c^{\prime}$, i.e., an individual that is in the same equivalence class of $c$ in $e$. 
Example 4.10. Consider the knowledge base $\mathcal{K}=\langle\mathcal{O}, \mathcal{A}\rangle$, where $\mathcal{O}=\emptyset$, and $\mathcal{A}=\{$ Author(Nicky), Author(Nicolas), Cited(Nicolas, Nicky), Cited(Nicky, Nicolas) $\}$. Consider also the following $\mathrm{UCQ}^{\neq, b}$ over $\mathcal{O}$ asking for the pair of authors that either are known to be different, or have been cited by the same author:

$$
\begin{aligned}
q^{\prime}=\quad & \left\{\left(x_{1}, x_{2}\right) \mid \text { Author }\left(x_{1}\right) \wedge \text { Author }\left(x_{2}\right) \wedge x_{1} \neq x_{2}\right\} \cup \\
& \left\{\left(x_{1}, x_{2}\right) \mid \exists y . \text { Cited }\left(y, x_{1}\right) \wedge \operatorname{Cited}\left(y, x_{2}\right)\right\} .
\end{aligned}
$$

Suppose we want to check whether (Nicky, Nicolas) $\in \operatorname{cert}_{q^{\prime}, \mathcal{O}}^{\mathcal{A}}$, i.e., whether $\mathcal{K} \models q$, where $q=\{() \mid$ Author(Nicky), Author(Nicolas) $\wedge$ Nicky $\neq$ Nicolas $\} \cup$ $\{() \mid \exists y$.Cited $(y, \operatorname{Nicky}) \wedge \operatorname{Cited}(y$, Nicolas $)\}$. By exploiting Proposition 4.4, we consider the two possible equivalence relation on $I_{q}$ :

- $e_{1}=\{($ Nicky, Nicky), (Nicolas, Nicolas $)\}$;

- $e_{2}=\{($ Nicky, Nicky), (Nicolas, Nicolas), (Nicky, Nicolas), (Nicolas, Nicky) $\}$.

As for $e_{1}$, we easily derive that $\mathcal{K} \models_{e_{1}} q$ because $\mathrm{Nicky}^{\mathcal{I}} \neq$ Nicolas $^{\mathcal{I}}$ in each $e_{1}$-model $\mathcal{I}$ of $\mathcal{K}$. Moreover, one can easily verify that $\mathcal{K}_{e_{1}} \models q_{e_{1}}$ for $q_{e_{1}}=$ EquivalenceRelation $\operatorname{Ref}\left(q, e_{1}\right)$, since the inequality atom Nicky $\neq$ Nicolas of the first disjunct of $q$ is replaced with the atom true in $q_{e_{1}}$.

As for $e_{2}$, we have $\mathcal{K}=e_{e_{2}} q$ as well, since in each model $\mathcal{I}$ of $\mathcal{K}$ for which Nicky $^{\mathcal{I}}=$ Nicolas $^{\mathcal{I}}=o \in \Delta^{\mathcal{I}}$ we have that object $o$ has been cited by itself. Moreover, observe that the second disjunct of $q_{e_{2}}=$ EquivalenceRelation $\operatorname{Ref}\left(q, e_{2}\right)$ is

$$
\begin{aligned}
&\left\{() \mid \quad \exists z_{y}^{1}, z_{y}^{2}, y_{\text {Nicky }}, y_{\text {Nicolas }} \cdot \operatorname{Cited}\left(z_{y}^{1}, y_{\text {Nicky }}\right) \wedge \operatorname{Cited}\left(z_{y}^{2}, y_{\text {Nicolas }}\right) \wedge\right. \\
&\left.e\left(z_{y}^{1}, z_{y}^{2}\right) \wedge e\left(y_{\text {Nicky }}, \text { Nicky }\right) \wedge e\left(y_{\text {Nicolas }}, \operatorname{Nicolas}\right)\right\} .
\end{aligned}
$$

One can easily verify that, as expected, $\mathcal{K}_{e_{2}} \models q_{e_{2}}$ with the bindings for the above disjunct $z_{y}^{1}=y_{\text {Nicolas }} \rightarrow$ Nicolas, and $z_{y}^{2}=y_{\text {Nicky }} \rightarrow$ Nicky.

We conclude that $\mathcal{K} \models q$, and thus (Nicky, Nicolas) $\in \operatorname{cert}_{q^{\prime}, \mathcal{O}}^{\mathcal{A}}$.

The next crucial proposition states that checking whether $\mathcal{K} \models_{e} q$, for a given $D L$-Lite $e_{\mathcal{R}}$ knowledge base $\mathcal{K}=\langle\mathcal{O}, \mathcal{A}\rangle$, boolean $\mathrm{UCQ}^{\neq, b} q$ over $\mathcal{O}$, and equivalence relation $e$ on $I_{q}$ such that $\mathcal{K}$ is $e$-satisfiable, can be reduced to checking whether $\mathcal{K}_{e} \models q_{e}$, where $q_{e}$ is the UCQ returned by EquivalenceRelation $\operatorname{Ref}(q, e)$.

Proposition 4.6. Let $\mathcal{K}=\langle\mathcal{O}, \mathcal{A}\rangle$ be a DL-Lite $\mathcal{R}_{\mathcal{R}}$ knowledge base, q be a boolean $U C Q^{\neq, b}$ over $\mathcal{O}$, and $e$ be an equivalence relation $e$ on $I_{q}$ such that $\mathcal{K}$ is esatisfiable. We have that $\mathcal{K} \models_{e} q$ if and only if $\mathcal{K}_{e} \models q_{e}$, where $q_{e}$ is the $U C Q$ EquivalenceRelation Ref $(q, e)$.

Proof. "If part:" Suppose that $\mathcal{K}_{e} \models q_{e}$. Since $\mathcal{K}$ is $e$-satisfiable by assumption (and therefore $\mathcal{K}_{e}$ is a satisfiable $D L-L i t e_{\mathcal{R}}$ knowledge base) and $q_{e}$ is a UCQ, due to Calvanese et al., 2007b. Theorem 29], there is a disjunct $q \mathcal{Y}$ of $q_{e}$ for which there is a homomorphism $h$ from $q \mathcal{Y}$ to $\mathcal{C}_{\mathcal{O}_{e}}^{\mathcal{A}_{e}}$, where $\mathcal{C}_{\mathcal{O}_{e}}^{\mathcal{A}_{e}}$ is the canonical structure of $\mathcal{O}_{e}$ with respect to $\mathcal{A}_{e}$, and $q \mathcal{Y}$ is a $\mathrm{CQ}$ obtained from some disjunct $q_{i}$ of $q$ by reformulating it according to $e$ (thus removing inequality atoms) and the set $\mathcal{Y} \in \mathfrak{P}\left(\mathrm{ej}\left(q_{i}\right)\right)$ of join 
existential variables of $q_{i}$. We now prove that $\mathcal{M} \models q_{i}$ for each $e$-model $\mathcal{M}$ of $\mathcal{K}$, thus implying that $\mathcal{K} \models_{e} q$.

Consider any $e$-model $\mathcal{M}$ of $\mathcal{K}$, and let $\psi$ be the function satisfying conditions 1 and 2 of Proposition 4.1. As a first consideration, note that each possible inequality atom of $q$ (which is only between individuals) is evaluated exactly as in the algorithm over the model $\mathcal{M}$. Furthermore, even if two variables $z_{y}^{k}$ and $z_{y}^{l}$ of $q \mathcal{Y}$ that replace an existential variable $y \in \mathcal{Y}$ of $q_{i}$ may match two distinct individuals in $\mathcal{C}_{\mathcal{O}_{e}}^{\mathcal{A}_{e}}$ under $h$ (i.e., $\left.h\left(z_{y}^{k}\right) \neq h\left(z_{y}^{l}\right)\right)$, the facts that $e\left(h\left(z_{y}^{k}\right), h\left(z_{y}^{l}\right)\right) \in \mathcal{C}_{\mathcal{O}_{e}}^{\mathcal{A}_{e}}$ and that $\mathcal{M}$ is an $e$-model of $\mathcal{K}$ implies that $z_{y}^{k^{\mathcal{M}}}=z_{y}^{l}{ }^{\mathcal{M}}$. An analogous consideration holds also for variables $y_{c}$ of $q \mathcal{Y}$ replacing all occurrences of individuals $c \in I_{q}$ of $q_{i}$.

But then, consider the function $f_{\mathcal{M}}$ with $(i) f_{\mathcal{M}}(y)=\psi\left(h\left(z_{y}^{i}\right)\right)$ for an arbitrary $i \in\left[1, m_{y}\right]$, for each $y \in \mathcal{Y},(i i) f_{\mathcal{M}}(y)=\psi(h(y))$, for each $y \notin \mathcal{Y}$, and (iii) $f(c)=\psi\left(h\left(y_{c}\right)\right)$, for each individual $c \in I_{q_{i}}$. It is not hard to see that $f_{\mathcal{M}}$ consists in a homomorphism from $q_{i}$ to $\mathcal{M}$, and therefore $\mathcal{M} \models q_{i}$ as required.

"Only-if part:" Suppose that $\mathcal{K} \models_{e} q$. Consider the DL-Lite $e_{\mathcal{R}}$ knowledge base $\mathcal{K}^{\prime}=\left\langle\mathcal{O}, \mathcal{A}^{\prime}\right\rangle$ and the $\mathrm{UCQ}^{\neq, b} q^{\prime}$, where $\mathcal{A}^{\prime}$ and $q^{\prime}$ are the ABox and the query, respectively, obtained from $\mathcal{A}$ and $q$ by replacing each individual $c \in I_{q}$ with a fresh individual denoting its equivalence class in $e$. Obviously, since $\mathcal{K} \models_{e} q$, we have that $\mathcal{K}^{\prime} \models q^{\prime}$. In fact, there is a homomorphism $h$ from some disjunct $q_{i}^{\prime}$ of $q^{\prime}$ to $\mathcal{C}_{\mathcal{O}}^{\mathcal{A}^{\prime}}$ with $h(c)=c$ for each individual $c \in I_{q^{\prime}}$, and therefore $h\left(c_{1}\right) \neq h\left(c_{2}\right)$ for each inequality atom $c_{1} \neq c_{2}$ of $q_{i}^{\prime}$.

Let now $\mathcal{Y} \in \mathfrak{P}\left(\mathrm{ej}\left(q_{i}\right)\right)$ be the set of join existential variables of $q_{i}^{\prime}$ for which $y \in \mathcal{Y}$ if and only if $h(y)$ is one of the fresh individual introduced in $\mathcal{A}^{\prime}$. It is not hard to ascertain that, if we reformulate the disjunct $q_{i}$ of $q$ with respect to $\mathcal{Y}$ as described in the algorithm, then we obtain a CQ $q \mathcal{Y}$ for which there is a homomorphism from $q \mathcal{Y}$ to $\mathcal{C}_{\mathcal{O}_{e}}^{\mathcal{A}_{e}}$, thus implying that $\mathcal{K}_{e} \models q \mathcal{Y}$. It follows that $\mathcal{K}_{e}=q_{e}$, as required.

Based on this results, we are now ready to characterise the computational complexity of checking whether $\mathcal{K} \models_{e} q$ for a $D L$-Lite $e_{\mathcal{R}}$ knowledge base $\mathcal{K}=\langle\mathcal{O}, \mathcal{A}\rangle$, boolean $\mathrm{UCQ}^{\neq, b} q$, and equivalence relation $e$ on $I_{q}$.

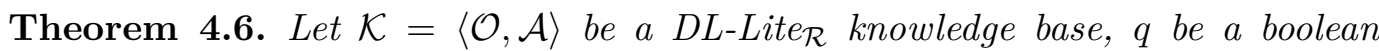
$U C Q^{\neq, b}$ over $\mathcal{O}$, and e be an equivalence relation e on $I_{q}$. The problem of checking whether $\mathcal{K} \models{ }_{e} q$ is $N P$-complete, and in $A C^{0}$ in the size of $\mathcal{A}$ and $e$.

Proof. Checking whether $\mathcal{K} \models_{e} q$ can be done by first checking whether $\mathcal{K}$ is $e$ satisfiable, and then, by exploiting Proposition 4.6. checking whether $\mathcal{K}_{e} \models q_{e}$, where $q_{e}$ is the UCQ returned by EquivalenceRelation $\operatorname{Ref}(q, e)$. Notice that, due to Proposition 4.5 the $e$-satisfiability check can be done in $\mathrm{AC}^{0}$ in the size of $\mathcal{A}$ and $e$, and in polynomial time in the size of $\mathcal{O}$. Moreover, as usual, the problem of checking whether $\mathcal{K}_{e}=q_{e}$ for a $D L$-Lite $\mathcal{R}_{\mathcal{R}}$ knowledge base $\mathcal{K}_{e}=\left\langle\mathcal{O}_{e}, \mathcal{A}_{e}\right\rangle$ and a UCQ $q_{e}$ is in $\mathrm{AC}^{0}$ in the size of $\mathcal{A}_{e}$ and in NP in combined complexity.

Thus, as for the claim of the theorem, the membership in $\mathrm{AC}^{0}$ in the size of $\mathcal{A}$ and $e$ is a direct consequence of Propositions 4.5 and 4.6, whereas the membership in NP in the size of the input can be easily proven by considering a version of the EquivalenceRelationRef algorithm that nondeterministically guess a disjunct $q_{i}$ of $q$ and a subset $\mathcal{Y} \in \mathfrak{P}\left(\operatorname{ej}\left(q_{i}\right)\right)$, and then with a further NP-step checks whether the reformulated $q_{i}$ according to $\mathcal{Y}$ is logically implied by $\mathcal{K}_{e}$. 
Finally, NP-hardness follows from CQ evaluation over relational databases.

With the above result at hand, and by recalling Proposition 4.4 , we are ready to show an upper bound for our problem. Notably, answering $\mathrm{UCQ}^{\neq, b} \mathrm{~S}$ over $D$ L-Lite R $_{\mathcal{R}}$ knowledge bases has the same data complexity of the UCQs and $\mathrm{CQ}^{\neq, b}$ s cases.

Theorem 4.7. Answering $U C Q^{\neq, b}$ s over DL-Lite $e_{\mathcal{R}}$ knowledge bases is in $A C^{0}$ in data complexity and in $\Pi_{2}^{p}$ in combined complexity.

Proof. For a $D L$-Lite $_{\mathcal{R}}$ knowledge base $\mathcal{K}=\langle\mathcal{O}, \mathcal{A}\rangle$ and a $\mathrm{UCQ}^{\neq, b} q$ over $\mathcal{O}$, we now show how to decide $\mathcal{K} \not \models q$ in $\mathrm{AC}^{0}$ in data complexity and in $\Sigma_{2}^{p}$ in combined complexity, thus proving the claim. Due to Proposition 4.4 $\mathcal{K} \not \models q$ can be decided as follows:

1. Guess an equivalence relation $e$ on $I_{q}$;

2. If $\mathcal{K} \not \models_{e} q$, return true (i.e., $\mathcal{K} \not \models q$ ), otherwise return false (i.e., $\mathcal{K} \models q$ ),

where, due to Theorem 4.6, this last step can be done in $\mathrm{AC}^{0}$ in the size of $\mathcal{A}$ and $e$, and in coNP in the size of $\mathcal{A}, e$, and $q$.

So, we easily derive a nondeterministic algorithm deciding $\mathcal{K} \not q$ that first requires an NP step in the size of $q$ in order to guess an equivalence relation $e$ on $I_{q}$ (observe that the equivalence relation is only on the set $I_{q}$ of individuals occurring in $q$, and thus such step is constant time in the size of $\mathcal{A}$ ). Finally, with a single call to an coNP-oracle, the algorithm checks whether $\mathcal{K} \nvdash_{e} q$, where, as already said, this check can be done in $\mathrm{AC}^{0}$ in the size of $\mathcal{A}$ and $e$.

We conclude the analysis of the considered problem of this section by establishing that the $\Pi_{2}^{p}$ combined complexity upper bound is tight. Thus, unless the polynomial hierarchy collapses to the first level, answering $\mathrm{UCQ}^{\neq, b}$ s over $D L$-Lite R $_{\mathcal{R}}$ knowledge bases does not have the same combined complexity of the UCQs and $\mathrm{CQ}^{\neq, b}$ s cases.

Theorem 4.8. Answering $U C Q^{\neq, b}$ s over DL-Lite $\mathcal{R}_{\mathcal{R}}$ knowledge bases is $\Pi_{2}^{p}$-hard in combined complexity.

Proof. The proof is by a LOGSPACE reduction from the $\forall \exists$-CNF problem, which is $\Pi_{2}^{p}$-complete Stockmeyer, 1976. $\forall \exists-\mathrm{CNF}$ is the problem of deciding, given a 3-CNF formula $F=c_{1} \wedge \ldots \wedge c_{p}$ on a set of variables $X=\left\{x_{1}, \ldots, x_{m}\right\} \cup$ $Y=\left\{y_{1}, \ldots, y_{n}\right\}$ such that the variables in $X$ (respectively, $Y$ ) are universally (respectively, existentially) quantified, whether $F$ is true, i.e., whether for each possible truth assignment to the variables in $X$, there exists a truth assignment to the variables in $Y$ that satisfies $F$. Each clause $c_{i}$ is a disjunction of three literals, where each literal is either a variable $z \in X \cup Y$ or its negated. For $i=1, \ldots, p$, we denote by $z_{i, 1}, z_{i, 2}, z_{i, 3}$ the first, the second, and the third, respectively, variable appearing (either positive or negated) in clause $c_{i}$.

Let $F$ be an instance of the $\forall \exists$-CNF problem. We now construct a $D L$-Lite $e_{\mathcal{R}}$ knowledge base $\mathcal{K}_{F}=\left\langle\mathcal{O}, \mathcal{A}_{F}\right\rangle$ and a $\mathrm{UCQ}^{\neq, b} Q_{F}$.

For each clause $c_{i}$ of $F$, observe that there are exactly seven satisfying truth assignment for the clause $c_{i}$. As individuals of $\mathcal{A}_{F}$ we have 0,1 , an individual $x_{i}$ for each $i \in[1, m]$, and an individual $A_{i, k}$ for each $i \in[1, p]$ and for each $k \in[1,7]$. 
Intuitively, $A_{i, k}$ denotes the $k$-th satisfying truth assignment $\left\{v_{k, 1}, v_{k, 2}, v_{k, 3}\right\}$ for the clause $c_{i}$, where, for $j \in[1,3]$, value $v_{k, j}$ corresponds to the truth assignment (i.e., either 0 or 1 ) given to the variable $z_{i, j}$. Then, the $\mathrm{ABox} \mathcal{A}_{F}$ is defined as follows:

$$
\begin{aligned}
\mathcal{A}_{F}= & \left\{P_{i, 1}\left(A_{i, k}, v_{k, 1}\right), P_{i, 2}\left(A_{i, k}, v_{k, 2}\right), P_{i, 3}\left(A_{i, k}, v_{k, 3}\right) \mid i \in[1, p] \text { and } k \in[1,7]\right\} \cup \\
& \left\{H_{i}\left(x_{i}\right) \mid i \in[1, m]\right\},
\end{aligned}
$$

where $(i)$ for each $i \in[1, p]$ and for each $j \in[1,3], P_{i, j}$ is an atomic role in the alphabet of $\mathcal{O}$, and $(i i)$ for each universally quantified variable $x_{i} \in X, H_{i}$ is an atomic concept in the alphabet of $\mathcal{O}$. Finally, the knowledge base $\mathcal{K}_{F}$ is defined as the pair $\mathcal{K}_{F}=\left\langle\mathcal{O}, \mathcal{A}_{F}\right\rangle$, where the ontology $\mathcal{O}$ contains no axioms, i.e., $\mathcal{O}=\emptyset$.

As for the $\mathrm{UCQ}^{\neq, b}$, let $Q_{F}=q_{1} \cup \ldots \cup q_{m} \cup q_{F}$, where $q_{i}=\left\{\left(x_{1}, \ldots, x_{m}\right) \mid\right.$ $\left.H_{1}\left(x_{1}\right) \wedge \ldots \wedge H_{m}\left(x_{m}\right) \wedge x_{i} \neq 0 \wedge x_{i} \neq 1\right\}$ for each $i \in[1, m]$, and $q_{F}$ is

$$
\begin{aligned}
&\left\{\left(x_{1}, \ldots, x_{m}\right) \mid \exists\right. a_{1}, \ldots, a_{p}, y_{1}, \ldots, y_{n} . \\
&\left.\bigwedge_{i \in[1, p]}\left(P_{i, 1}\left(a_{i}, z_{i, 1}\right) \wedge P_{i, 2}\left(a_{i}, z_{i, 2}\right) \wedge P_{i, 3}\left(a_{i}, z_{i, 3}\right)\right) \wedge \bigwedge_{i \in[1, m]}\left(H_{i}\left(x_{i}\right)\right)\right\} .
\end{aligned}
$$

Observe that $\mathcal{O}=\emptyset$ is fixed, whereas both $\mathcal{A}_{F}$ and $Q_{F}$ can be constructed in LogSpace from $F$. To illustrate the reduction, consider the formula $F=$ $\forall x_{1}, x_{2} \cdot \exists y_{1}, y_{2} \cdot\left(\left(y_{1} \vee y_{2} \vee x_{1}\right) \wedge\left(\neg y_{1} \vee \neg y_{2} \vee \neg x_{2}\right)\right)$. In this case, the reduction produces the knowledge base $\mathcal{K}_{F}=\left\langle\mathcal{O}, \mathcal{A}_{F}\right\rangle$, where $\mathcal{O}=\emptyset$ and $\mathcal{A}_{F}$ contains the assertions $\left\{H_{1}\left(x_{1}\right), H_{2}\left(x_{2}\right)\right\}$ in union to all the the assertions involving atomic roles $P_{i, j}$ for $i \in[1,2]$ and $j \in[1,3]$, which, for ease of exposition, are visualised as the extension of each atomic role $P_{i, j}$ in Figure 4.2 .

\begin{tabular}{|c|c|c|c|c|c|}
\hline$P_{1,1}$ & $P_{1,2}$ & $P_{1,3}$ & $P_{2,1}$ & $P_{2,2}$ & $P_{2,3}$ \\
\hline$A_{1,1}, 0$ & $A_{1,1}, 0$ & $A_{1,1}, 1$ & $A_{2,1}, 0$ & $A_{2,1}, 0$ & $A_{2,1}, 0$ \\
$A_{1,2}, 0$ & $A_{1,2}, 1$ & $A_{1,2}, 0$ & $A_{2,2}, 0$ & $A_{2,2}, 0$ & $A_{2,2}, 1$ \\
$A_{1,3}, 0$ & $A_{1,3}, 1$ & $A_{1,3}, 1$ & $A_{2,3}, 0$ & $A_{2,3}, 1$ & $A_{2,3}, 0$ \\
$A_{1,4}, 1$ & $A_{1,4}, 0$ & $A_{1,4}, 0$ & $A_{2,4}, 0$ & $A_{2,4}, 1$ & $A_{2,4}, 1$ \\
$A_{1,5}, 1$ & $A_{1,5}, 0$ & $A_{1,5}, 1$ & $A_{2,5}, 1$ & $A_{2,5}, 0$ & $A_{2,5}, 0$ \\
$A_{1,6}, 1$ & $A_{1,6}, 1$ & $A_{1,6}, 0$ & $A_{2,6}, 1$ & $A_{2,6}, 0$ & $A_{2,6}, 1$ \\
$A_{1,7}, 1$ & $A_{1,7}, 1$ & $A_{1,7}, 1$ & $A_{2,7}, 1$ & $A_{2,7}, 1$ & $A_{2,7}, 0$ \\
\hline
\end{tabular}

Figure 4.2. Extension of atomic roles $P_{i, j}$. Each row $A_{i, k}, v_{k, j}$ (with $v_{k, j}$ being either 0 or 1 ) of an atomic role $P_{i, j}$ has to be read as the ABox assertion $P_{i, j}\left(A_{i, k}, v_{k, j}\right)$

Finally, the query $Q_{F}$ produced by the reduction contains the following disjuncts:

$$
\begin{gathered}
q_{1}=\left\{\left(x_{1}, x_{2}\right) \mid H_{1}\left(x_{1}\right) \wedge H_{2}\left(x_{2}\right) \wedge x_{1} \neq 0 \wedge x_{1} \neq 1\right\} \cup \\
q_{2}=\left\{\left(x_{1}, x_{2}\right) \mid H_{1}\left(x_{1}\right) \wedge H_{2}\left(x_{2}\right) \wedge x_{2} \neq 0 \wedge x_{2} \neq 1\right\} \cup \\
q_{F}=\left\{\left(x_{1}, x_{2}\right) \mid \exists a_{1}, a_{2}, y_{1}, y_{2} .\right. \\
P_{1,1}\left(a_{1}, y_{1}\right) \wedge P_{1,2}\left(a_{1}, y_{2}\right) \wedge P_{1,3}\left(a_{1}, x_{1}\right) \wedge P_{2,1}\left(a_{2}, y_{1}\right) \wedge P_{2,2}\left(a_{2}, y_{2}\right) \wedge \\
\left.P_{2,3}\left(a_{2}, x_{2}\right) \wedge H_{1}\left(x_{1}\right) \wedge H_{2}\left(x_{2}\right)\right\} .
\end{gathered}
$$

Informally, any model $\mathcal{I}$ of $\mathcal{K}_{F}$ for which both $x_{i}^{\mathcal{I}} \neq 0^{\mathcal{I}}$ and $x_{i}^{\mathcal{I}} \neq 1^{\mathcal{I}}$ hold for some $i \in[1, m]$ is such that the tuple $\left(x_{1}^{\mathcal{I}}, \ldots, x_{m}^{\mathcal{I}}\right) \in q_{i}^{\mathcal{I}}$, and therefore $\left(x_{1}^{\mathcal{I}}, \ldots, x_{m}^{\mathcal{I}}\right) \in Q_{F}^{\mathcal{I}}$. 
Conversely, if this is not the case, then we can see the model $\mathcal{I}$ as a truth assignment to the universally quantified variables of $F$. Consider now the query $q_{F}$. For each $i \in[1, m]$, because of the presence of atom $H_{i}\left(x_{i}\right)$ and the ABox assertion $H\left(x_{i}\right)$, the distinguished variable $x_{i}$ of $q_{F}$ is either $1^{\mathcal{I}}$ or $0^{\mathcal{I}}$. Then, given a truth assignment to the universally quantified variables of $F$, the query $q_{F}$ asks whether there exists a truth assignment to the existentially quantified variables that satisfies formula $F$.

More formally, let $F$ be any instance of the of the $\forall \exists-C N F$ problem. We now prove that $\left(x_{1}, \ldots, x_{m}\right) \in \operatorname{cert}_{Q_{F}, \mathcal{O}}^{\mathcal{A}_{F}}$ if and only if $F$ is true.

"If part:" Suppose that $F$ is true, that is, for every possible truth assignment to the variables in $X$, there exists a truth assignment to the variables in $Y$ that satisfies $F$. Consider any possible model $\mathcal{I}$ for $\mathcal{K}_{F}$. If both $x_{i}^{\mathcal{I}} \neq 0^{\mathcal{I}}$ and $x_{i}^{\mathcal{I}} \neq 1^{\mathcal{I}}$ for some $i \in[1, m]$, then it is straightforward to verify that $\left(x_{1}^{\mathcal{I}}, \ldots, x_{m}^{\mathcal{I}}\right) \in q_{i}^{\mathcal{I}}$. Then, let individual $x_{i}$ be such that either $x_{i}^{\mathcal{I}}=0^{\mathcal{I}}$ or $x_{i}^{\mathcal{I}}=1^{\mathcal{I}}$, for each $i \in[1, m]$. We can see such interpretation $\mathcal{I}$ as a truth assignment to the variables in $X$. Specifically, let $V_{X}=\left\{v_{x_{1}}, \ldots, v_{x_{m}}\right\}$ be the truth values given by $\mathcal{I}$ to the variables in $X$, i.e., $v_{x_{i}}=1$ if $x_{i}^{\mathcal{I}}=1^{\mathcal{I}}$, otherwise (i.e., $x_{i}^{\mathcal{I}}=0^{\mathcal{I}}$ ) $v_{x_{i}}=0$.

Consider the partial function $h$ from variables of $q_{F}$ to $\mathcal{I}$ such that $h\left(x_{i}\right)=x_{i}^{\mathcal{I}}$. Since for every truth assignment to the variables in $X$ there exists a truth assignment to the variables in $Y$ that satisfies $F$, let $V_{Y}=\left\{v_{y_{1}}, \ldots, v_{y_{n}}\right\}$ be such truth assignment for the variables in $Y$. For each $i \in[1, p]$, consider the following extension of $h$ : $h\left(y_{i}\right)=v_{y_{i}}^{\mathcal{I}}$ and $h\left(a_{i}\right)=A_{i, k}^{\mathcal{I}}$ for an arbitrary $k \in[1,7]$ satisfying $(i)\left(A_{i, k}^{\mathcal{I}}, h\left(z_{i, 1}\right)\right) \in$ $P_{i, 1}^{\mathcal{I}},(i i)\left(A_{i, k}^{\mathcal{I}}, h\left(z_{i, 2}\right)\right) \in P_{i, 2}^{\mathcal{I}}$, and $(i i i)\left(A_{i, k}^{\mathcal{I}}, h\left(z_{i, 3}\right)\right) \in P_{i, 3}^{\mathcal{I}}$. Observe that, since by assumption clause $c_{i}$ is satisfied under the truth assignment $V=V_{X} \cup V_{Y}$, at least one individual $A_{i, k}$ for some $k \in[1,7]$ must exists by construction.

But then, it can be easily verified that $h$ is a homomorphism from $q_{F}$ to $\mathcal{I}$ such that $h(\vec{x})=\left(x_{1}^{\mathcal{I}}, \ldots, x_{m}^{\mathcal{I}}\right)$, thus implying $\left(x_{1}^{\mathcal{I}}, \ldots, x_{m}^{\mathcal{I}}\right) \in q_{F}^{\mathcal{I}}$. It follows that, for each possible model $\mathcal{I}$ for $\mathcal{K}_{F}$, either $\left(x_{1}^{\mathcal{I}}, \ldots, x_{m}^{\mathcal{I}}\right) \in q_{i}^{\mathcal{I}}$ for some $i \in[1, m]$, or $\left(x_{1}^{\mathcal{I}}, \ldots, x_{m}^{\mathcal{I}}\right) \in q_{F}^{\mathcal{I}}$. Thus, $\left(x_{1}, \ldots, x_{m}\right) \in \operatorname{cert}_{Q_{F}, \mathcal{O}}^{\mathcal{A}_{F}}$, as required.

"Only-if part:" Suppose, for the sake of contradiction, that $F$ is not true, that is, there exists a truth assignment to the variables in $X$ such that every possible truth assignment to the variables in $Y$ does not satisfy $F$. Let $V_{X}=\left\{v_{1}, \ldots, v_{m}\right\}$ be the assignment to the variables in $X$ ( $v_{i}$ is either 1 or 0 , for each $i \in[1, m]$ ) that makes $F$ not satisfiable. Consider the model $\mathcal{I}$ of $\mathcal{K}_{F}$ such that $(i) \Delta^{\mathcal{I}}=$ $\{0,1\} \cup\left\{A_{i, k} \mid i \in[1, p]\right.$ and $\left.k \in[1,7]\right\},(i i) x_{i}^{\mathcal{I}}=v_{i}$ for each $i \in[1, m], 0^{\mathcal{I}}=0$, $1^{\mathcal{I}}=1$, and $A_{i, k}^{\mathcal{I}}=A_{i, k}$ for each $i \in[1, p]$ and for each $k \in[1,7]$, and $(i i i) P_{i, j}^{\mathcal{I}}=P_{i, j}^{\mathcal{I}_{A}}$ for each $i \in[1, p]$ and for each $j \in[1,3]$, and $H_{i}^{\mathcal{I}}=\left\{v_{i}\right\}$ for each $i \in[1, m]$.

For each $i \in[1, m]$, since individual $x_{i}$ is such that either $x_{i}^{\mathcal{I}}=1^{\mathcal{I}}$ or $x_{i}^{\mathcal{I}}=0^{\mathcal{I}}$, we have $q_{i}^{\mathcal{I}}=\emptyset$. Moreover, due to the fact that, for each $i \in[1, m]$, we have $H_{i}^{\mathcal{I}}=\left\{v_{i}\right\}$ and $H_{i}\left(x_{i}\right)$ is an atom of $q_{F}$, every possible homomorphism $h$ from $q_{F}$ to $\mathcal{I}$ must be such that $h\left(x_{i}\right)=v_{i}$ for each $i \in[1, m]$. Since, however, by assumption $F$ is not satisfiable when replacing variables $x_{i}$ s with truth values $v_{i}$ s, by construction of $q_{F}$ and $\mathcal{I}$ we easily conclude that $q_{F}^{\mathcal{I}}=\emptyset$. It follows that, for the model $\mathcal{I}$ of $\mathcal{K}_{F}$, we have $\left(x_{1}^{\mathcal{I}}, \ldots, x_{m}^{\mathcal{I}}\right) \notin Q_{F}^{\mathcal{I}}$. Thus, $\left(x_{1}, \ldots, x_{m}\right) \notin \operatorname{cert}_{Q_{F}, \mathcal{O}}^{\mathcal{A}_{F}}$, as required.

Finally, observe that the same proof works even for boolean $\mathrm{UCQ}^{\neq, b} \mathrm{~s}$. To see this, it is sufficient to consider the boolean $\mathrm{UCQ}^{\neq, b} Q_{F}^{\prime}$ similar to $Q_{F}$, but where each disjunct has an empty target list and the distinguished variable $x_{i}$ is replaced 
with the corresponding individual $x_{i}$ of the $\mathrm{ABox} \mathcal{A}_{F}$, for each $i \in[1, m]$.

Corollary 4.2. Answering $U C Q^{\neq, b}$ s over DL-Lite $e_{\mathcal{R}}$ knowledge bases is in $A C^{0}$ in data complexity and $\Pi_{2}^{p}$-complete in combined complexity.

By looking at the proof of the above theorem, one can see that $\Pi_{2}^{p}$-hardness holds already for $\mathrm{UCQ}^{\neq, b_{\mathrm{S}}}$ having at most two inequalities per disjunct and for knowledge bases having an empty ontology (i.e., an ontology without assertions).

\subsection{Answering $\mathrm{UCQ}^{\neq} \mathrm{s}$ over $D$ L-Lite $\overrightarrow{\mathrm{RDFS}}^{-}$knowledge bases}

In this section, we study the problem of answering $\mathrm{UCQ}^{\neq} \mathrm{S}$ over satisfiable $D L-L i t \overrightarrow{\mathrm{RDFS}}_{\overrightarrow{\mathrm{R}}}$ knowledge bases. Theorem 4.2 tells us that the certain answers to a $\mathrm{CQ}^{\neq, b} q$ over a $D L-L i t e_{\mathrm{RDFS}}^{\vec{c}}$ knowledge base $\mathcal{K}$ are the tuples of individuals $\vec{c}$ such that $\vec{c} \in \delta(q)^{\mathcal{I}_{\mathcal{K}}}$. The next example shows that the problem drastically changes as soon as we consider general $\mathrm{CQ}^{\neq} \mathrm{S}$.

Example 4.11. Consider the $D L$-Lite $\overrightarrow{\mathrm{RDFS}}$ knowledge base $\mathcal{K}=\langle\mathcal{O}, \mathcal{A}\rangle$, where $\mathcal{O}=\left\{A_{1} \sqsubseteq \neg A_{2}\right\}$ and $\mathcal{A}=\left\{A_{1}\left(a_{1}\right), A_{2}\left(a_{2}\right), P\left(b, c_{1}\right), P\left(b, c_{2}\right), P\left(c_{1}, a_{1}\right), P\left(c_{2}, a_{2}\right)\right\}$. For the boolean $\mathrm{CQ}^{\neq} q=\left\{() \mid \exists y_{1}, y_{2}, y_{3} \cdot P\left(y_{1}, y_{2}\right) \wedge P\left(y_{1}, y_{3}\right) \wedge y_{2} \neq y_{3}\right\}$, we have that $\mathcal{I}_{\mathcal{K}} \not \models \delta(q)$ because ineq $\left(c_{1}, c_{2}\right) \notin \mathcal{I}_{\mathcal{K}}$ (regarding the ineq predicate, only ineq $\left(a_{1}, a_{2}\right)$ and ineq $\left(a_{2}, a_{1}\right)$ are in $\left.\mathcal{I}_{\mathcal{K}}\right)$. Notice, however, that $\mathcal{K} \models q$. Indeed, in each model $\mathcal{M}$ with $c_{1}^{\mathcal{M}} \neq c_{2}^{\mathcal{M}}$, we have $\mathcal{M}=q$ with the bindings $y_{1}, y_{2}, y_{3} \rightarrow b, c_{1}, c_{2}$, whereas, in each model $\mathcal{M}$ with $c_{1}^{\mathcal{M}}=c_{2}^{\mathcal{M}}$, we have $\mathcal{M} \models q$ with the bindings $y_{1}, y_{2}, y_{3} \rightarrow c_{1}, a_{1}, a_{2}$.

The above example provides a hint on how to design an algorithm for our problem. Intuitively, given a $D L$ - it $_{\text {RDFS }}^{\overrightarrow{\mathrm{R}}}$ knowledge base $\mathcal{K}=\langle\mathcal{O}, \mathcal{A}\rangle$ and a boolean $\mathrm{UCQ}^{\neq}$ $q$ over $\mathcal{O}$, we can check whether $\mathcal{K} \forall q$ by simply guessing an equivalence relation $e$ on the set $I$ of individuals occurring in $\mathcal{A}$ for which $\mathcal{K} \not \nvdash_{e} q$.

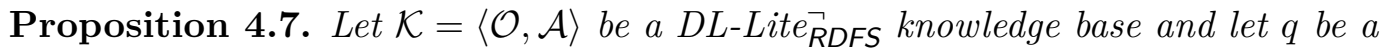
boolean $U C Q^{\neq}$over $\mathcal{O}$. We have that $\mathcal{K} \forall q$ if and only if there exists an equivalence relation $e$ on a set I of individuals occurring in $\mathcal{A}$ such that $\mathcal{K} \nvdash_{e} q$.

Proof. "If part:" Same as in the proof of the "If part" of Proposition 4.4

"Only-if part:" Suppose that $\mathcal{K} \not \models q$. It follows that there exists a model $\mathcal{M}$ of $\mathcal{K}$ such that $\mathcal{M} \not \forall q$. Consider the equivalence relation $e$ on the set $I$ of all individuals of $\mathcal{A}$ such that, for any pair $c_{1}, c_{2}$ of individuals of $\mathcal{A},\left(c_{1}, c_{2}\right) \in e$ if and only if $c_{1}^{\mathcal{M}}=c_{2}^{\mathcal{M}}$. By definition, we have that $\mathcal{M}$ is an $e$-model of $\mathcal{K}$ for which $\mathcal{M} \not \forall q$. Thus, $e$ is the equivalence relation such that $\mathcal{K} \forall_{e} q$, as required.

From the above result, we can derive upper bounds in both data and combined complexity for the problem of answering $\mathrm{UCQ}^{\neq}{ }_{\mathrm{S}}$ over $D L$-Lit $e_{\mathrm{RDFS}}^{\neg}$ knowledge bases.

Theorem 4.9. Answering $U C Q^{\neq} s$ over $D L$-Lite $\overrightarrow{R D F S}$ ontologies is in coNP in data complexity and in $\Pi_{2}^{p}$ in combined complexity. 
Proof. For a $D L$-Lite $\overrightarrow{\mathrm{RDFS}}$ knowledge base $\mathcal{K}=\langle\mathcal{O}, \mathcal{A}\rangle$ and a $\mathrm{UCQ}^{\neq} q$ over $\mathcal{O}$, we now show how to decide $\mathcal{K} \not q$ in NP in data complexity and in $\Sigma_{2}^{p}$ in combined complexity, thus proving the claim. Due to Proposition 4.7, $\mathcal{K} \not \models q$ can be decided as follows:

1. Guess an equivalence relation $e$ on the set $I$ of all individuals of $\mathcal{A}$;

2. If $\mathcal{K} \not \models_{e} q$, return true (i.e., $\mathcal{K} \not \models q$ ), otherwise return false (i.e., $\mathcal{K} \models q$ ),

where, due to the fact that we are considering $D L$-Lite $\overrightarrow{\text { RDFS }}$ knowledge base (and thus, $\mathcal{I}_{\mathcal{K}}$ is finite and does not introduce any variable), this last step can be done by simply computing $\mathcal{I}_{\mathcal{K}}$ and, after replacing each occurrence of every individual $c$ in $\mathcal{I}_{\mathcal{K}}$ and in $q$ with a new object denoting its equivalence class in $e$, we can check whether the resulting $\mathcal{I}_{\mathcal{K}}$ and $q$ are such that $(i) \mathcal{I}_{\mathcal{K}} \not \forall \delta(q)$ and $(i i)$ there is no object $o$ for which $\operatorname{ineq}(o, o) \in \mathcal{I}_{\mathcal{K}}$ (i.e., $\mathcal{K}$ is $e$-satisfiable).

So, we easily derive a nondeterministic algorithm deciding $\mathcal{K} \not \models q$ that first requires an NP step in the size of $\mathcal{A}$ in order to guess an equivalence relation $e$. Then, it requires a polynomial time step for computing $\mathcal{I}_{\mathcal{K}}$, replacing all individuals $c$ in $\mathcal{I}_{\mathcal{K}}$ and $q$ with a new object denoting its equivalence class in $e$, and check whether there is no object $o$ for which ineq $(o, o) \in \mathcal{I}_{\mathcal{K}}$. Finally, with a single call to an coNP-oracle, the algorithm checks whether the resulting $\mathcal{I}_{\mathcal{K}}$ and $q$ are such that $\mathcal{I}_{\mathcal{K}} \not \models \delta(q)$. Observe that this last check can be done in $\mathrm{AC}^{0}$ in the size of $\mathcal{A}$.

We now provide matching lower bounds for both data and combined complexity, showing that they hold already for the case of $\mathrm{CQ}^{\neq} \mathrm{s}$. We start with data complexity.

Theorem 4.10. Answering $C Q^{f} s$ over DL-Lite $\overrightarrow{R D F S}$ knowledge bases is coNP-hard in data complexity.

Proof. The proof is by a LOGSPACE reduction from the data complexity version of the problem of answering $\mathrm{CQ}^{f_{\text {s }}}$ over a data exchange setting Fagin et al., 2005a), known to be coNP-hard (in fact, coNP-complete) already for boolean $\mathrm{CQ}^{2, \neq_{\mathrm{S}}}$ |Madry, 2005. Since the target schema $T$ of the problem considered in Madry, 2005 uses only binary predicates and has no target constraints (i.e., $T$ has no assertions), and since it uses a set of LAV source-to-target dependencies, such problem can be reformulated in the OBDM scenario as follows: there exists an OBDM specification $\Sigma=\langle T, \mathcal{S}, \mathcal{M}\rangle$ with $T=\emptyset$ and $\mathcal{M}$ being a LAV mapping, and a boolean $\mathrm{CQ}^{2, \neq}$ $q$ over $T$ such that, given an $\mathcal{S}$-database $D$, checking whether $\operatorname{cert}_{q, \Sigma}^{D}$ is true (i.e., $\left.\operatorname{cert}_{q, \Sigma}^{D}=\{()\}\right)$ when the UNA is adopted is in general a coNP-hard problem.

Let $D$ be an $\mathcal{S}$-database. We now construct a $D L$-Lite ${ }_{\mathrm{RDFS}}$ knowledge base $\mathcal{K}_{D}=\left\langle\mathcal{O}, \mathcal{A}_{D}\right\rangle$, where $\mathcal{O}=\left\{P_{1} \sqsubseteq \neg P_{2}\right\}$ with the alphabet being composed of all the binary predicates (equivalently, atomic roles) of the target schema $T$ plus the two fresh atomic roles $P_{1}$ and $P_{2}$. Note that the above $\mathrm{CQ}^{2, \neq} q$ is also a query over $\mathcal{O}$.

In order to construct $\mathcal{A}_{D}$, we consider $\mathcal{M}(D)$, i.e., the chase of $D$ with respect to $\mathcal{M}$. Specifically, from $\mathcal{M}(D)$ we obtain a set of ABox assertions by replacing each variable $v \in$ Var occurring in $\mathcal{M}(D)$ with a different fresh individual denoted by $c_{v}$. Furthermore, for each pair of constants $c_{1}, c_{2}$ occurring in $D$ with $c_{1} \neq c_{2}$, $\mathcal{A}_{D}$ contains the ABox assertions $P_{1}\left(\alpha_{c_{1}, c_{2}}, c_{1}\right)$ and $P_{2}\left(\alpha_{c_{1}, c_{2}}, c_{2}\right)$, where $\alpha_{c_{1}, c_{2}}$ is a 
fresh individual. Intuitively, such assertions simulates the UNA but only on the individuals that were in $D$.

Observe that both $\mathcal{O}$ and $q$ do not depend on $D$, which is the only input of the (data complexity version of the) problem we are reducing from. Finally, since $\mathcal{M}$ is fixed, the ABox $\mathcal{A}_{D}$ can be constructed in LOGSpaCE from $D$ Arenas et al., 2010.

Let $D$ be any $\mathcal{S}$-database. We now prove that $\mathcal{K}_{D} \models q$ (when the UNA is not adopted) if and only if $\operatorname{cert}_{q, \Sigma}^{D}=\{()\}$ (when the UNA is adopted).

"If part:" Suppose that $\mathcal{K}_{D} \not \models q$, i.e., there is a model $\mathcal{I}$ of $\mathcal{K}_{D}$ for which $\mathcal{I} \not \neq q$. By construction, however, since $\mathcal{I}$ is a model of $\mathcal{K}_{D}$, we have $c_{1}^{\mathcal{I}} \neq c_{2}^{\mathcal{I}}$ for each pair of individuals $c_{1}, c_{2}$ occurring in $D$ with $c_{1} \neq c_{2}$. But then, from $\mathcal{I}$ it is straightforward to obtain a model $\mathcal{I}^{\prime} \in \operatorname{Mod}_{D}(\Sigma)$ satisfying the UNA for which $\mathcal{I}^{\prime} \not \models q$. Thus, $\operatorname{cert}_{q, \Sigma}^{D}=\emptyset$, as required.

"Only-if part:" Suppose that $\operatorname{cert}_{q, \Sigma}^{D}=\emptyset$ when the UNA is adopted. It follows that there is a model $\mathcal{I}^{\prime}=\left\langle\Delta^{\mathcal{I}^{\prime}}, \mathcal{I}^{\prime}\right\rangle$ of $\Sigma$ relative to $D$ that satisfies the UNA for which $\mathcal{I}^{\prime} \not \models q$. By Fagin et al., 2005a, Theorem 3.3], there exists a function $\psi$ from $\operatorname{dom}\left(\mathcal{C}_{\mathcal{O}}^{\mathcal{M}(D)}\right)$ to $\Delta^{\mathcal{I}^{\prime}}$ satisfying conditions 1 and 2 of Proposition 4.1 such that $\psi(c)=c^{\mathcal{I}^{\prime}}$ for each constant $c \in \operatorname{dom}(D)$.

Consider the interpretation $\mathcal{I}=\left\langle\Delta^{\mathcal{I}},{ }^{\mathcal{I}}\right\rangle$ for $\mathcal{K}_{D}$, where $\Delta^{\mathcal{I}}=\Delta^{\mathcal{I}^{\prime}} \cup\left\{\alpha_{c_{1}, c_{2}} \mid\right.$ $\left.c_{1}, c_{2} \in \operatorname{dom}(D) \wedge c_{1} \neq c_{2}\right\}$, and ${ }^{\mathcal{I}}$ extends ${ }^{\mathcal{I}^{\prime}}$ by $(i)$ assigning to each individual $c_{v}$ of $\mathcal{A}_{D}$ that replace a variable $v$ in $\mathcal{M}(D)$ the image of $v$ under $\psi$, i.e., $c_{v}^{\mathcal{I}}=\psi(v),(i i)$ assigning $\alpha_{c_{1}, c_{2}}^{\mathcal{I}}=\alpha_{c_{1}, c_{2}}$ to each introduced individual $\alpha_{c_{1}, c_{2}}$ in $\mathcal{A}_{D}$, and (iii) for both $i=1$ and $i=2$, assigning to atomic role $P_{i}$ the set $P_{i}^{\mathcal{I}}=\left\{(\alpha, c) \mid P_{i}(\alpha, c) \in \mathcal{A}_{D}\right\}$.

Since $\mathcal{I}^{\prime} \in \operatorname{Mod}_{D}(\Sigma)$ and satisfies the UNA (i.e., $c_{1}^{\mathcal{I}} \neq c_{2}^{\mathcal{I}}$ for each pair of individuals $c_{1}, c_{2}$ occurring in $D$ with $c_{1} \neq c_{2}$ ) by assumption, we derive that $\mathcal{I}$ is a model of $\mathcal{K}_{D}$. Furthermore, since $\mathcal{I}^{\prime} \not \models q$, we have that $\mathcal{I} \not \models q$ as well. It follows that $\mathcal{K}_{D} \not \models q$, as required.

Finally, we point out that the same result holds even for the problem of answering $\mathrm{UCQ}^{\neq}{ }_{\mathrm{S}}^{\mathrm{s}}$ over $D L-L i t e_{\mathrm{RDFS}}$ knowledge bases (in particular, even when ontologies have no assertions). In particular, observe that it is sufficient to apply the following changes to the above reduction: $(i)$ ontology $\mathcal{O}^{\prime}$ has the same alphabet of $\mathcal{O}$ but has no assertions, i.e., $\mathcal{O}^{\prime}=\emptyset$, and $(i i)$ the query issued over the ontology $\mathcal{O}^{\prime}$ is the fixed UCQ $q^{\prime}=q \cup q_{P}$, where $q$ is the $\mathrm{CQ}^{2, \neq}$ as in the above reduction and $q_{P}$ is the CQ $\left\{() \mid \exists y_{1}, y_{2} \cdot P_{1}\left(y_{1}, y_{2}\right) \wedge P_{2}\left(y_{1}, y_{2}\right)\right\}$, which intuitively asks whether two constants $c_{1}, c_{2}$ occurring in $D$ with $c_{1} \neq c_{2}$ are interpreted as the same domain object.

Let $D$ be any $\mathcal{S}$-database. Using similar arguments as the ones given above, it is easy to see that $\mathcal{K}_{D}^{\prime} \models q^{\prime}$ (when the UNA is not adopted) if and only if $\operatorname{cert}_{q, \Sigma}^{D}=\{()\}$ (when the UNA is adopted), where $\mathcal{K}_{D}^{\prime}$ is the DL-Lite RDFs knowledge base $\mathcal{K}_{D}^{\prime}=\left\langle\mathcal{O}^{\prime}, \mathcal{A}_{D}\right\rangle$.

The proof of the above theorem has two interesting implications: $(i)$ coNPhardness in data complexity holds even for $\mathrm{CQ}^{2,{ }_{\mathrm{s}}}$; $(i i)$ Answering $\mathrm{UCQ}^{2,{ }_{\mathrm{S}}}$ (in particular, the union of a $\mathrm{CQ}^{2, \neq}$ and of a $\mathrm{CQ}$ ) over knowledge bases with empty ontologies is coNP-hard in data complexity, too.

Interestingly, implication (ii) corrects an erroneous statement in Rosati, 2007, Theorem 11], where it is claimed that answering $\mathrm{UCQ}^{\neq} \mathrm{s}$ over $D L$-Lite $e_{\mathrm{RDFS}}$ knowledge bases is in LOGSPACE in data complexity regardless of whether the UNA is adopted 
or not. It turns out that, unless LOGSPACE $=\mathrm{NP}$, this latter statement is true only when the UNA is adopted (cf. beginning of this chapter).

We now provide the matching lower bound for combined complexity.

Theorem 4.11. Answering $C Q^{\neq} s$ over DL-Lite $\overrightarrow{R D F S}$ knowledge bases is $\Pi_{2}^{p}$-hard in combined complexity.

Proof. The proof is by a LoGSpace reduction from the containment problem for conjunctive queries with inequalities, known to be $\Pi_{2}^{p}$-hard (in fact, $\Pi_{2}^{p}$-complete) even when restricted to boolean $\mathrm{CQ}^{\neq} \mathrm{s}$ over a schema with all predicates having arity at most two Kolaitis et al., 1998. The containment problem for conjunctive queries with inequalities is the problem of deciding, given two $\mathrm{CQ}^{\neq} \mathrm{S} q_{1}, q_{2}$ over the same schema $\mathcal{S}$, whether $q_{1} \sqsubseteq q_{2}$, i.e., whether $q_{1}^{D} \subseteq q_{2}^{D}$ for each $\mathcal{S}$-database $D$.

Let $q_{1}, q_{2}$ be two boolean $\mathrm{CQ}^{\neq}$s over the same database schema $\mathcal{S}$ with all the predicates of $\mathcal{S}$ having arity at most 2 . We define a $D L$-Lite $e_{\mathrm{RDFS}}$ knowledge base $\mathcal{K}_{q_{1}}=\left\langle\mathcal{O}, \mathcal{A}_{q_{1}}\right\rangle$ where $\mathcal{O}=\left\{P_{1} \sqsubseteq \neg P_{2}\right\}$ and the alphabet of $\mathcal{O}$ is composed of all the binary predicates (equivalently, atomic roles) and unary predicates (equivalently, atomic concepts) of the schema $\mathcal{S}$, plus the two fresh atomic roles $P_{1}$ and $P_{2}$.

To construct $\mathcal{A}_{q_{1}}$, we consider the freezing of $q_{1}$, i.e., the set of facts obtained from the body of $q_{1}$ by replacing each variable in $v$ with a fresh individual $c_{v}$.

Specifically, $\mathcal{A}_{q_{1}}$ is such that $(i)$ every non-inequality atom occurring in the body of $q_{1}$ becomes an ABox assertion of $\mathcal{A}$ (after replacing variables $v$ with its corresponding individual $c_{v}$ ); (ii) for each inequality atom $z_{1} \neq z_{2}$ occurring in $q_{1}$, we have the ABox assertions $P_{1}\left(\alpha_{z_{1}, z_{2}}, z_{1}^{\prime}\right)$ and $P_{2}\left(\alpha_{z_{1}, z_{2}}, z_{2}^{\prime}\right)$, where $\alpha_{z_{1}, z_{2}}$ is a fresh individual, and for both $i=1$ and $i=2$, if $z_{i}$ is a variable $v$, then $z_{i}^{\prime}=c_{v}$, otherwise (i.e., $z_{i}$ is a constant), $z_{i}^{\prime}=z_{i}$; and finally, (iii) for each pair $c_{1}, c_{2}$ of constants occurring in $q_{1}$ with $c_{1} \neq c_{2}$, we have the ABox assertions $P_{1}\left(\alpha_{c_{1}, c_{2}}, c_{1}\right)$ and $P_{2}\left(\alpha_{c_{1}, c_{2}}, c_{2}\right)$, where $\alpha_{z_{1}, z_{2}}$ is a fresh individual. Intuitively, such assertions simulates the UNA on the individuals that were in $q_{1}$ and on the terms $t_{1} \neq t_{2}$ that were inequality atoms in (the freezing of) $q_{1}$.

We now prove that $q_{1} \sqsubseteq q_{2}$ if and only if $\mathcal{K}_{q_{1}} \models q_{2}$.

"If part:" Suppose that $q_{1} \nsubseteq q_{2}$, i.e., there exists an $\mathcal{S}$-database $D$ for which $D \models q_{1}$ and $D \forall \neq q_{2}$. Consider the homomorphism $h$ from $q_{1}$ to $D$ (at least one exists because $D \models q_{1}$ ), and let $\mathcal{I}$ be an interpretation for $\mathcal{K}_{q_{1}}$ such that (i) $\Delta^{\mathcal{I}}=\operatorname{dom}(D) \cup\{\alpha \mid$ for each introduced individual $\alpha\} ;(i i) c^{\mathcal{I}}=c$ for each constant $c$ occurring in $q_{1}$, and $\alpha^{\mathcal{I}}=\alpha$ for each introduced individual $\alpha$; (iii) $c_{v}^{\mathcal{I}}=h(v)$ for each variable $v$ occurring in $q_{1} ;(i v)$ for both $i=1$ and $i=2$, $P_{i}^{\mathcal{I}}=\left\{(\alpha, c) \mid P_{i}(\alpha, c) \in \mathcal{A}_{q_{1}}\right\}$; and $(v)$ the extension in $\mathcal{I}$ of each atomic role and atomic concept of $\mathcal{O}$ corresponding to a relation in $\mathcal{S}$ is the same as the corresponding relation in $D$.

Since $h\left(z_{1}\right) \neq h\left(z_{2}\right)$ for each inequality atom $z_{1} \neq z_{2}$ of $q_{1}$, we have that $\mathcal{I}$ is a model of $\mathcal{K}_{q_{1}}$ by construction. Moreover, since $D \not \models q_{2}$, we derive $\mathcal{I} \not \models q_{2}$ as well. But then, $\mathcal{I}$ is a model of $\mathcal{K}_{q_{1}}$ for which $\mathcal{I} \not \models q_{2}$. It follows that $\mathcal{K}_{q_{1}} \not \forall q_{2}$, as required.

"Only-if part:" Suppose that $\mathcal{K}_{q_{1}} \not \models q_{2}$, i.e., there exists a model $\mathcal{I}$ of $\mathcal{K}_{q_{1}}$ for which $\mathcal{I} \forall q_{2}$. Since $\mathcal{I}$ is a model of $\mathcal{K}_{q_{1}}$, by construction we have that $z_{1}^{\prime \mathcal{I}} \neq z_{2}^{\prime \mathcal{I}}$ for each inequality atom $z_{1} \neq z_{2}$ occurring in $q_{1}$, where for both $i=1$ and $i=2$ if $z_{i}$ is a variable $v$, then $z_{i}^{\prime}=c_{v}$, otherwise (i.e., $z_{i}$ is a constant), $z_{i}^{\prime}=z_{i}$. 
Consider now the $\mathcal{S}$-database $D$ similar to $\mathcal{I}$ where each unary (resp., binary) predicate in $D$ has the same extension of the corresponding atomic concept (resp., role) in $\mathcal{I}$, but, for every constant $c$ of $q_{1}$, every occurrence of the object $c^{\mathcal{I}}$ is replaced with the constant $c$ in $D$ (observe that, since $\mathcal{I}$ is a model of $\mathcal{K}_{q_{1}}, c_{1}^{\mathcal{I}} \neq c_{2}^{\mathcal{I}}$ for each pair $c_{1}, c_{2}$ of constants occurring in $q_{1}$ with $c_{1} \neq c_{2}$ ). Obviously, since $\mathcal{I} \not \models q_{2}$, we have that $D \not q_{2}$ as well.

Furthermore, let $h$ be a function from variables and constants of $q_{1}$ to constants of $D$ such that $(i) h(c)=c$ for each constant $c$ of $q_{1}$; and $(i i) h(v)=c_{v}^{\mathcal{I}}$ for each variable $v$ of $q_{1}$. It is straightforward to verify that $h$ is a homomorphism from $q_{1}$ to $D$. But then, the $\mathcal{S}$-database $D$ is such that $D \models q_{1}$ and $D \not \models q_{2}$. It follows that $q_{1} \nsubseteq q_{2}$, as required.

Corollary 4.3. Answering $U C Q^{\neq} s$ over DL-Lite $\overrightarrow{R D F S}_{\text {knowledge bases is coNP- }}$ complete in data complexity and $\Pi_{2}^{p}$-complete in combined complexity.

By looking at the proof of the above theorem, one can see that the output of the reduction produces a $\mathrm{CQ}^{\neq}$whose number of inequalities is equal to the number of inequalities of the input query $q_{2}$, and therefore is not fixed a priori.

To the best of our knowledge, it is not known whether checking $q_{1} \sqsubseteq q_{2}$ is $\Pi_{2}^{p}$-hard even if $q_{2}$ uses a fixed number of inequalities. More generally, it is thus natural to ask which is the minimum number of inequalities in $\mathrm{CQ}^{\neq} \mathrm{s}$ that makes the problem $\Pi_{2}^{p}$-hard in combined complexity. Similarly to the case of the coNP-hardness result in data complexity, we conjecture that such number is two.

Conjecture 4.1. Answering $C Q^{2, \neq}$ s over DL-Lit $\vec{R}_{R F S}$ knowledge bases is $\Pi_{2}^{p}$-hard in combined complexity.

Even though we have not been able to prove this conjecture, interestingly, the proof of Theorem 4.8 shows that $\Pi_{2}^{p}$-hardness holds for $\mathrm{UCQ}^{2, \neq, b}$ s (i.e., $\mathrm{UCQ}^{2, \neq} \mathrm{s}$ with bounded inequalities) over knowledge bases with empty ontologies. Notice, however, that the illustrated $\mathrm{UCQ}^{2, \neq, b}$ does not have a fixed number of disjuncts.

Actually, with a slight adaptation of such proof, we now prove that the same result holds even for $\mathrm{UCQ}^{2, \neq} \mathrm{s}$ that are the union of a fixed (but not bounded) $\mathrm{CQ}^{2, \neq}$ and a $\mathrm{CQ}$ without inequalities.

Theorem 4.12. Answering $U C Q^{2, \neq} s$ over knowledge bases with empty ontologies is $\Pi_{2}^{p}$-hard in data complexity.

Proof. Consider the following changes to the reduction illustrated in the proof of Theorem 4.8 .

- The ontology $\mathcal{O}$ contains the additional atomic concept $B$ in its alphabet;

- For each $i \in[1, m]$, the ABox $\mathcal{A}_{F}$ additionally contains the ABox assertion $B\left(x_{i}\right)$ (recall that $x_{i}$ is the individual corresponding to the universally quantified variable $\left.x_{i}\right)$;

- The boolean $\mathrm{UCQ}^{2, \neq} Q_{F}$ is the union of a fixed $\mathrm{CQ}^{2, \neq} q$ and of $q_{F}$, where:

$$
-q=\{() \mid \exists y \cdot B(y) \wedge y \neq 0 \wedge y \neq 1\}
$$


$-q_{F}$ is the boolean version of the CQ defined in the reduction of the proof of Theorem 4.8 .

$$
\begin{aligned}
& \left\{() \mid \exists a_{1}, \ldots, a_{p}, y_{1}, \ldots, y_{n} .\right. \\
& \left.\quad \bigwedge_{i \in[1, p]}\left(P_{i, 1}\left(a_{i}, z_{i, 1}\right) \wedge P_{i, 2}\left(a_{i}, z_{i, 2}\right) \wedge P_{i, 3}\left(a_{i}, z_{i, 3}\right)\right) \wedge \bigwedge_{i \in[1, m]}\left(H_{i}\left(x_{i}\right)\right)\right\} .
\end{aligned}
$$

Intuitively, through the possibility of involving existential variables in inequality atoms, the $\mathrm{CQ}^{2, \neq} q$ encases the behaviour of the $\mathrm{UCQ}^{\neq, b} q_{1} \cup \ldots \cup q_{m}$ that asks whether some individual $x_{i}$ (corresponding to the universally quantified variable $x_{i}$ of formula $F$ ) in a possible interpretation $\mathcal{I}$ is such that $x_{i}^{\mathcal{I}} \neq 0^{\mathcal{I}}$ and $x_{i}^{\mathcal{I}} \neq 1^{\mathcal{I}}$.

Let $F$ be any instance of the of the $\forall \exists$-CNF problem. Using analogous arguments to the ones provided in the proof of Theorem 4.8 , it is easy to see that $F$ is true if and only if $\mathcal{K}_{F} \mid=Q_{F}$, where $\mathcal{K}_{F}=\left\langle\mathcal{O}, \mathcal{A}_{F}\right\rangle$ and $Q_{F}$ are the knowledge base and the query, respectively, obtained by modifying the reduction illustrated in the proof of Theorem 4.8 as explained above.

Observe that the hardness results of Theorems 4.10 and 4.11 do not hold if we replace $D L$-Lite $\overrightarrow{\mathrm{RDFS}}$ with $D L$-Lite $e_{\mathrm{RDFS}}$. This is due to the inability of the

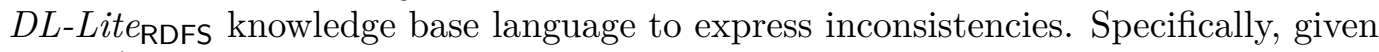

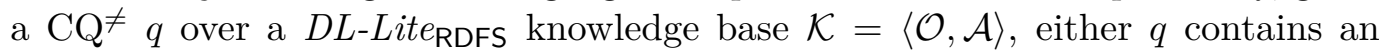
inequality atom, and therefore $\mathcal{K} \forall \neq q$ trivially holds, or $q$ is a CQ. This clearly proves that answering $\mathrm{CQ}^{f_{\mathrm{s}}}$ over $D L$-Lite $\mathrm{RDF}$ knowledge bases has the same computational complexity of answering CQs over the same language (cf. beginning of this chapter).

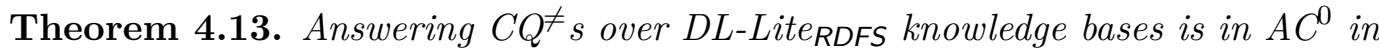
data complexity and NP-complete in combined complexity.

As already noticed, if we move to consider $\mathrm{UCQ}^{\neq} \mathrm{s}$ over $D L$-Lite $e_{\mathrm{RDF}}$ knowledge bases, the adaptation illustrated in the reduction of the proof of Theorem 4.10 and Theorem 4.12 show a jump from $\mathrm{AC}^{0}$ to coNP for data complexity and from NP to $\Pi_{2}^{p}$ in combined complexity, respectively.

Theorem 4.14. Answering $U C Q^{\neq} s$ over DL-Lite ${ }_{R D F S}$ knowledge bases is coNPcomplete in data complexity and $\Pi_{2}^{p}$-complete in combined complexity. Both the hardness results already hold for $U C Q^{2, \neq} s$ over knowledge bases with empty ontologies.

To fully complete the picture of the problem of answering $\mathrm{UCQ}^{f_{\mathrm{S}}}$ over $D L-L i t \overrightarrow{\mathrm{RDFS}}^{-}$knowledge bases, it remains to study the case of $\mathrm{UCQ}^{1,{ }_{\mathrm{s}}} \mathrm{s}$, i.e., UCQs having at most one inequality per disjunct. We do so in the next section.

\subsection{Answering $\mathrm{UCQ}^{1, \neq} \mathrm{S}$ over $D L$-Lite $e_{\mathrm{RDFS}}^{\urcorner}$knowledge bases}

In what follows, without loss of generality, we assume that each $\mathrm{UCQ}^{1, \neq} q$ is written as $q=q_{1} \cup q_{2}$, where $q_{2}$ is a UCQ with no inequalities and $q_{1}$ is a $\mathrm{UCQ}^{1, \neq}$ having exactly one inequality per disjunct.

In principle, for answering $\mathrm{UCQ}^{1, \neq_{\mathrm{S}}}$ over $D L$-Lite $\overrightarrow{\mathrm{RDFS}}_{\mathrm{R}}$ knowledge bases, it is possible to use the algorithm provided in [Fagin et al., 2005a, Theorem 5.12] in the 
context of data exchange. Notice, however, that the running time of this algorithm would be polynomial in the size of the ABox but exponential in the size of the query. On the contrary, by elaborating on the idea of [Fagin et al., 2005a, Theorem 5.12], we are able to prove that the problem is in PTIME in data complexity and in NP in combined complexity. We start with the following definition and algorithm.

Definition 4.3. Let $\mathcal{K}=\langle\mathcal{O}, \mathcal{A}\rangle$ be a $D L$-Lite $\overrightarrow{\mathrm{RDFS}}$ knowledge base, $q$ be a boolean $\mathrm{UCQ}^{1, \neq}$ over $\mathcal{O}$, and $F=\left[f_{1}, \ldots, f_{m}\right]$ be a list of functions from the set of variables and individuals occurring in $q$ to the set of individuals occurring in $\mathcal{A}$. We say that $F$ is a good sequence with respect to $\mathcal{K}$ and $q$ if $\operatorname{Check} \operatorname{Good}(\mathcal{K}, q, F)$ returns true.

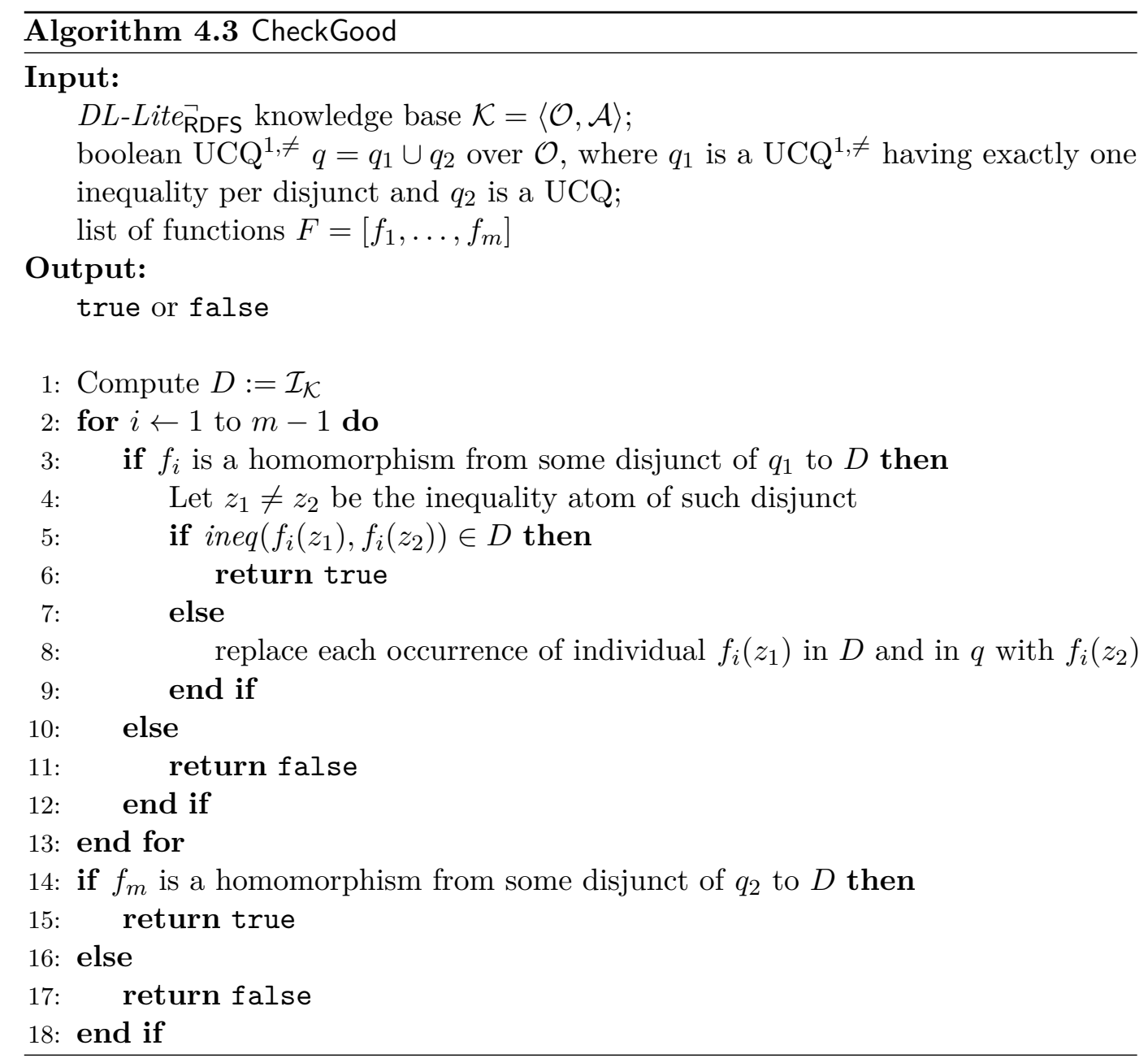

Roughly speaking, starting from a set of facts $D:=\mathcal{I}_{\mathcal{K}}$ over $\mathcal{O}$ (notice that $\mathcal{I}_{\mathcal{K}}$ does not introduce variables), in each step $i$ from 1 to $m-1$ such that $f_{i}$ is a homomorphism from a disjunct $q^{\prime}$ of $q_{1}$ to $D$, the algorithm CheckGood replaces everywhere the individual $f_{i}\left(z_{1}\right)$ with the individual $f_{i}\left(z_{2}\right)$, to consider the models in which $f_{i}\left(z_{1}\right)=f_{i}\left(z_{2}\right)$, where $z_{1} \neq z_{2}$ is the only inequality atom occurring in the body of $q^{\prime}$. Indeed, since $f_{i}$ is a homomorphism from $q^{\prime}$ to $D, q_{1}$ is true in those models where $f_{i}\left(z_{1}\right) \neq f_{i}\left(z_{2}\right)$. 
Afterwards, the algorithm sanctions that $F$ is a good sequence if and only if either it is not possible to equate two individuals without contradicting an ineq fact of $D$, or the resulting $D$ and $q_{2}$ are such that $D \models q_{2}$.

Using the above notion of good sequence, it is possible to derive the following characterisation $\left(n_{\mathcal{A}}\right.$ denotes the number of individuals occurring in the $\mathrm{ABox} \mathcal{A}$ ).

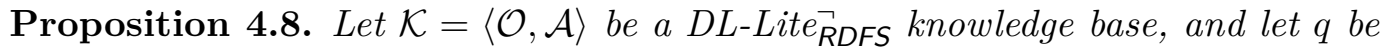
a boolean $U C Q^{1, \neq}$ over $\mathcal{O}$. We have that $\mathcal{K} \models q$ if and only if there exists a list $F=\left[f_{1}, \ldots, f_{m}\right]$ of functions, with $m \leq n_{\mathcal{A}}$, such that $F$ is a good sequence with respect to $\mathcal{K}$ and $q$.

Proof. "If part:" Suppose $F=\left[f_{1}, \ldots, f_{m}\right]$ (with $m \leq n_{\mathcal{A}}$ ) is a good sequence with respect to $\mathcal{K}$ and $q$, i.e., CheckGood $(\mathcal{K}, q, F)$ returns true. It follows that, after possibly applying $l \leq m-1$ equalities between individuals on $D$ and $q$ (where $D$ starts from $\mathcal{I}_{\mathcal{K}}$ ), either $l \leq m-2$ and $f_{l+1}$ is a homomorphism from a disjunct $q^{\prime}$ of $q_{1}$ to the resulting set of facts $D$ such that ineq $\left(f_{l+1}\left(z_{1}\right), f_{l+1}\left(z_{2}\right)\right) \in D$ (where $z_{1} \neq z_{2}$ is the only inequality atom in the body of $q^{\prime}$ ), or $l=m-1$ and $f_{m}$ is a homomorphism from a disjunct of $q_{2}$ to $D$.

In both cases, consider each homomorphism $f_{i}$ from some of the disjuncts of $q_{1}$ to $D$, for $i=[1, l]$. It is easy to see that all models $\mathcal{I}$ of $\mathcal{K}$ in which $f_{i}\left(z_{1}\right)^{\mathcal{I}} \neq f_{i}\left(z_{2}\right)^{\mathcal{I}}$ (where $z_{1} \neq z_{2}$ is the inequality atom of the disjunct $q^{\prime}$ of $q_{1}$ for which $f_{i}$ is a homomorphism to $D$ ) is such that $\mathcal{I} \models q_{1}$, and therefore $\mathcal{I} \models q$. So, at each iteration the algorithm equates $f_{i}\left(z_{1}\right)$ and $f_{i}\left(z_{2}\right)$ in $D$ to consider all the other possible models in which $f_{i}\left(z_{1}\right)^{\mathcal{I}}=f_{i}\left(z_{2}\right)^{\mathcal{I}}$.

If $f_{l+1}$ is a homomorphism from a disjunct $q^{\prime}$ of $q_{1}$ to the resulting set of facts $D$ such that ineq $\left(f_{l+1}\left(z_{1}\right), f_{l+1}\left(z_{2}\right)\right) \in D$ (where $z_{1} \neq z_{2}$ is the only inequality atom in the body of $q^{\prime}$ ), due to Proposition 4.1. we derive that there is no models $\mathcal{I}$ in which $f_{i}\left(z_{1}\right)^{\mathcal{I}}=f_{i}\left(z_{2}\right)^{\mathcal{I}}$. It trivially follows that $\mathcal{K} \models q_{1}$, and therefore $\mathcal{O} \models q$.

Finally, in the case that $f_{m}$ is a homomorphism from a disjunct of $q_{2}$ to the resulting set of facts $D$, due to the above considerations, it can be easily proven that each model $\mathcal{I}$ of $\mathcal{K}$ is such that either $\mathcal{I} \models q_{1}$, or $\mathcal{I} \models q_{2}$. Thus, $\mathcal{K} \models q$, as required.

"Only-if part:" Suppose there is no such good sequence $F$ with respect to $\mathcal{K}$ and $q$, and consider the set of facts obtained in the following way starting from $D:=\mathcal{I}_{\mathcal{K}}$ : for each possible homomorphism $h$ from a disjunct of $q_{1}$ to $D$, replace each occurrence of $h\left(z_{1}\right)$ in $D$ and in $q$ with $h\left(z_{2}\right)$, where $z_{1} \neq z_{2}$ is the inequality atom of the disjunct of $q_{1}$ for which $h$ is a homomorphism to $D$.

Since there are $n_{\mathcal{A}}$ individuals in the $\operatorname{ABox} \mathcal{A}$, and so also in $D$, there can be at most $n_{\mathcal{A}}-1$ of such homomorphisms. Indeed, after applying $n_{\mathcal{A}}-1$ replacing as described above, the resulting set of facts $D$ would contain only one individual.

For the resulting set of facts $D$ and query $q$ observe that $(i)$ it is never the case that ineq $\left(h\left(z_{1}\right), h\left(z_{2}\right)\right) \in D$ for some homomorphism $h$; and $(i i)$ there is no disjunct of $q_{2}$ for which there is a homomorphism to $D$. In proof, if either point $(i)$ or point (ii) is not true, then we would easily derive a contradiction on the fact that there exists no good sequence $F$ with respect to $\mathcal{K}$ and $q$.

But then, by construction $D$ is such that $D \forall q_{1}$ and $D \not \models q_{2}$, and therefore $D \forall \forall$. From the resulting $D$, moreover, it is immediate to construct a model $\mathcal{I}_{D}$ of $\mathcal{K}$ such that $\mathcal{I}_{D} \not \models q$. It follows that $\mathcal{K} \not \models q$, as required. 
We are now ready to establish upper bound results for the problem of answering $\mathrm{UCQ}^{1,{ }_{\mathrm{s}}}$ over $D L$-Lite $\overrightarrow{\mathrm{RDFS}}_{\mathrm{f}}$ knowledge bases.

Theorem 4.15. Answering $U C Q^{1, \neq} s$ over DL-Lite $\overrightarrow{R D F S}$ knowledge bases is in PTIME in data complexity and in NP (and therefore NP-complete) in combined complexity.

Proof. Due to Proposition 4.8 , checking whether $\mathcal{K} \models q$ for a $D L$-Lite $\overrightarrow{\mathrm{RDFS}}$ knowledge base $\mathcal{K}=\langle\mathcal{O}, \mathcal{A}\rangle$ and $\mathrm{UCQ}^{1, \neq} q$ can be done as follows:

- Guess a list $F=\left[f_{1}, \ldots, f_{m}\right]$ of $m \leq n_{\mathcal{A}}$ functions from the set of variables and individuals occurring in $q$ to the set of individuals occurring in $\mathcal{A}$;

- Check whether $F$ is a good sequence with respect to $\mathcal{K}$ and $q$,

where checking whether $F$ is a good sequence with respect to $\mathcal{K}$ and $q$ can be done by means of the above described CheckGood algorithm.

So, we easily derive a nondeterministic algorithm deciding $\mathcal{K} \models q$ that first requires an NP step in order to guess the list $F=\left[f_{1}, \ldots, f_{m}\right]$ with $m \leq n_{\mathcal{A}}$ of functions. This can be done in polynomial time in the size of $\mathcal{A}$. Then, by exploiting the CheckGood algorithm, we check whether $F$ is a good sequence with respect to $\mathcal{K}$ and $q$ using: $(i)$ a polynomial time step in the size of $\mathcal{K}$ for computing $D:=\mathcal{I}_{\mathcal{K}}$ (ii) for each $i \in[1, m-1]$, a polynomial time step for checking whether $f_{i}$ is a homomorphism from a disjunct of $q_{1}$ to $D$, ineq $\left(f_{i}\left(z_{1}\right), f_{i}\left(z_{2}\right)\right) \notin D$ (where $z_{1} \neq z_{2}$ is the inequality atom of such a disjunct), and for replacing each occurrence of $f_{i}\left(z_{1}\right)$ with $f_{i}\left(z_{2}\right)$; finally, (iii) another polynomial time step for checking whether $f_{m}$ is a homomorphism from some disjunct of the UCQ $q_{2}$ to the resulting $D$.

While NP-hardness in combined complexity trivially follows from CQ evaluation over relational databases, we now provide a matching lower bound for data complexity, showing that it holds already for the case of $\mathrm{CQ}^{1, \neq} \mathrm{s}$.

Theorem 4.16. Answering $C Q^{1, \neq}$ s over DL-Lite $\overrightarrow{R D F S}$ knowledge bases is PTIMEhard in data complexity.

Proof. The proof is by a LoGSpace reduction from the entailment problem for HORN-3CNF, known to be PTime-complete [Börger et al., 1997]. Given a set of formulas $F=\left\{f_{1}, \ldots, f_{m}\right\}$ on a set of propositional variables $A=\left\{a_{1}, \ldots, a_{n}\right\}$ where $f_{i}$ is either of the form $f_{i}=\left(a_{j} \wedge a_{k} \rightarrow a_{h}\right)$ or of the form $f_{i}=\left(\top \rightarrow a_{h}\right)$, for each $i=[1, n]$, and given a propositional variable $a_{w} \in A$, the entailment problem for HORN-3CNF is the problem of deciding whether $F=a_{w}$.

Let $H, P_{1}$, and $P_{2}$ be three atomic roles, and let $B_{1}$ and $B_{2}$ be two atomic concepts. We define the following fixed $D L$-Lite $\overrightarrow{\mathrm{RDFS}}$ ontology $\mathcal{O}$ and boolean $\mathrm{CQ}^{1, \neq} q$ over $\mathcal{O}$ with $\mathcal{O}=\left\{B_{1} \sqsubseteq \neg B_{2}\right\}$ and $q=\left\{() \mid \exists y_{1}, y_{2} \cdot H\left(y_{1}, y_{2}\right) \wedge P_{1}\left(y_{2}, t\right) \wedge P_{2}\left(y_{2}, t\right) \wedge y_{1} \neq t\right\}$, where $t$ is an individual.

Given a HORN-3CNF formula $F$, we construct an ABox $\mathcal{A}_{F}$ as follows: $(i)$ for each formula $f_{i} \in F$ of the form $f_{i}=\left(a_{j} \wedge a_{k} \rightarrow a_{h}\right)$, we include the ABox assertions $H\left(a_{h}, f_{i}\right), P_{1}\left(f_{i}, a_{j}\right)$, and $P_{2}\left(f_{i}, a_{k}\right)$, where $a_{h}, f_{i}, a_{j}$, and $a_{k}$ are individuals of $\mathcal{A} ;(i i)$ for each formula $f_{i} \in F$ of the form $f_{i}=\left(\top \rightarrow a_{h}\right)$, we include the ABox assertions $H\left(a_{h}, f_{i}\right), P_{1}\left(f_{i}, t\right)$, and $P_{2}\left(f_{i}, t\right)$, where $a_{h}, f_{i}$ and $t$ are individuals of $\mathcal{A}$; and, $(i i i)$ 
we include the $\mathrm{ABox}$ assertions $B_{1}\left(a_{w}\right)$ and $B_{2}(t)$, stating that individuals $a_{w}$ and $t$ have to be interpreted as different elements in each possible model of $\mathcal{K}_{F}=\left\langle\mathcal{O}, \mathcal{A}_{F}\right\rangle$.

Observe that $\mathcal{O}$ and $q$ do not depend on the inputs of the entailment problem for HORN-3CNF, while $\mathcal{A}_{F}$ can be constructed in LoGSPACE from them.

We now prove that $\mathcal{K}_{F}=q$ if and only if $F \models a_{w}$, where $\mathcal{K}_{F}$ is the DL-Lite $\overrightarrow{\text { RDFS }}$ knowledge base $\mathcal{K}_{F}=\left\langle\mathcal{O}, \mathcal{A}_{F}\right\rangle$.

"If part:" Suppose that $F \models a_{w}$. Then, it is possible to derive $a_{w}$ using the following inference rules:

- $F \models a_{h}$ for each formula of the form $\left(T \rightarrow a_{h}\right)$ occurring in $F$;

- if $F \models a_{j}, F \models a_{k}$, and $\left(a_{j} \wedge a_{k} \rightarrow a_{h}\right)$ is a formula in $F$, then $F \models a_{h}$.

For each propositional variable $a_{h} \in A$ such that $F \models a_{h}$ we now show, by induction on the length of the derivation of $a_{h}$ from $F$, that $a_{h}^{\mathcal{I}}=t^{\mathcal{I}}$ in each interpretation $\mathcal{I}$ satisfying the ABox assertions in $\mathcal{A}_{F}$ and such that $\mathcal{I} \not \models q$.

Base case (l=0): Let $f_{i} \in F$ be a formula of the form $f_{i}=\left(\top \rightarrow a_{h}\right)$. Then, in the ABox $\mathcal{A}_{F}$ there are the assertions $H\left(a_{h}, f_{i}\right), P_{1}\left(f_{i}, t\right)$, and $P_{2}\left(f_{i}, t\right)$. It is easy to see that every interpretation $\mathcal{I}$ satisfying such assertions is such that either $\mathcal{I} \models q$, or $a_{h}^{\mathcal{I}}=t^{\mathcal{I}}$. It follows that, for each propositional variable $a_{h}$ such that $\left(\top \rightarrow a_{h}\right) \in F$ and for each interpretation $\mathcal{I}$ satisfying the ABox assertions in $\mathcal{A}_{F}$ and such that $\mathcal{I} \not \models q$, we have $a_{h}^{\mathcal{I}}=t^{\mathcal{I}}$, as required.

Inductive step: Let $f_{i} \in F$ be a formula of the form $f_{i}=\left(a_{j} \wedge a_{k} \rightarrow a_{h}\right)$, where both propositional variables $a_{j}$ and $a_{k}$ are derived from $F$ at length $\lambda \leq l-1$. By the inductive hypothesis, we have that $a_{j}^{\mathcal{I}}=a_{k}^{\mathcal{I}}=t^{\mathcal{I}}$ in each interpretation $\mathcal{I}$ satisfying the ABox assertions in $\mathcal{A}_{F}$ and such that $\mathcal{I} \not \models q$. Since, however, $H\left(a_{h}, f_{i}\right)$, $P_{1}\left(f_{i}, a_{j}\right)$, and $P_{2}\left(f_{i}, a_{k}\right)$ are assertions occurring in $\mathcal{A}_{F}$, for such interpretations $\mathcal{I}$ we have $\left(a_{h}^{\mathcal{I}}, f_{i}^{\mathcal{I}}\right) \in H^{\mathcal{I}},\left(f_{i}^{\mathcal{I}}, a_{j}^{\mathcal{I}}=t^{\mathcal{I}}\right) \in P_{1}^{\mathcal{I}}$, and $\left(f_{i}^{\mathcal{I}}, a_{k}^{\mathcal{I}}=t^{\mathcal{I}}\right) \in P_{2}^{\mathcal{I}}$. Thus, for each propositional variable $a_{h}$ derived at length $l$ and for each interpretation $\mathcal{I}$ satisfying the ABox assertions in $\mathcal{A}_{F}$ and such that $\mathcal{I} \not \models q$, we derive that $a_{h}^{\mathcal{I}}=t^{\mathcal{I}}$, as required.

Finally, due to the fact that $F \models a_{w}$ by assumption, we have that $a_{w}^{\mathcal{I}}=t^{\mathcal{I}}$ in each interpretation $\mathcal{I}$ satisfying the ABox assertions in $\mathcal{A}_{F}$ and such that $\mathcal{I} \not \models q$. But then, since both $B_{1}\left(a_{w}\right)$ and $B_{2}(t)$ are assertions in $\mathcal{A}_{F}$, those interpretations $\mathcal{I}$ do not satisfy the ontology assertion $B_{1} \sqsubseteq \neg B_{2}$, and therefore they are not models of $\mathcal{K}_{F}$. Therefore, every model $\mathcal{I}$ of $\mathcal{K}_{F}$ is such that $\mathcal{I} \models q$. Thus, $\mathcal{K}_{F}=q$, as required.

"Only-if part:" Suppose that $F \not \forall a_{w}$. Let $V \subset A$ be the set of propositional variables $a_{h} \in A$ such that $F \models a_{h}$. Consider the interpretation $\mathcal{I}=\left\langle\Delta^{\mathcal{I}},{ }^{\mathcal{I}}\right\rangle$ with $\Delta^{\mathcal{I}}=\left\{a_{h} \mid a_{h} \in A \backslash V\right\} \cup\left\{f_{i} \mid f_{i} \in F\right\} \cup\{t\}$, where $(i) t^{\mathcal{I}}=t ;(i i) f_{i}^{\mathcal{I}}=f$, for each formula $f_{i} \in F ;($ iii $) a_{h}^{\mathcal{I}}=t^{\mathcal{I}}=t$, for each $a_{h} \in V ;(i v) a_{h}^{\mathcal{I}}=a_{h}$, for each $a_{h} \in A \backslash V$; (v) $H^{\mathcal{I}}=\left\{\left(a_{h}^{\mathcal{I}}, f_{i}\right) \mid f_{i}=\left(a_{j} \wedge a_{k} \rightarrow a_{h}\right) \in F\right\} \cup\left\{\left(a_{h}^{\mathcal{I}}, f_{i}\right) \mid f_{i}=\left(\top \rightarrow a_{h}\right) \in F\right\}$, $P_{1}^{\mathcal{I}}=\left\{\left(f_{i}, a_{j}^{\mathcal{I}}\right) \mid f_{i}=\left(a_{j} \wedge a_{k} \rightarrow a_{h}\right) \in F\right\} \cup\left\{\left(f_{i}, t\right) \mid f_{i}=\left(\top \rightarrow a_{h}\right) \in F\right\}$, and $P_{2}^{\mathcal{I}}=\left\{\left(f_{i}, a_{k}^{\mathcal{I}}\right) \mid f_{i}=\left(a_{j} \wedge a_{k} \rightarrow a_{h}\right) \in F\right\} \cup\left\{\left(f_{i}, t\right) \mid f_{i}=\left(\top \rightarrow a_{h}\right) \in F\right\} ;$ and $(v i)$ $B_{1}^{\mathcal{I}}=\left\{a_{w}^{\mathcal{I}}\right\}$ and $B_{2}^{\mathcal{I}}=\left\{t^{\mathcal{I}}=t\right\}$.

It is straightforward to verify that $\mathcal{I} \not \models q$. Moreover, since $F \not \forall a_{w}$, we have $a_{w}^{\mathcal{I}} \neq t^{\mathcal{I}}=t$, and therefore $\mathcal{I}$ is a model of $\mathcal{K}$. It follows that $\mathcal{K}_{F} \not \models q$, as required.

Finally, observe that the same proof works even by removing the ontology assertion $B_{1} \sqsubseteq \neg B_{2}$ (i.e., with an empty ontology $\mathcal{O}$ ), but adding the fixed boolean 
disjunct $\left\{() \mid \exists y \cdot B_{1}(y) \wedge B_{2}(y)\right\}$ to the query $q$, and therefore answering $\mathrm{UCQ}^{1, \neq_{\mathrm{s}}}$

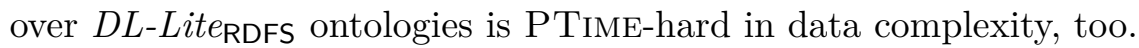

Corollary 4.4. Answering $U C Q^{1, \neq} s$ over DL-Lite $\overrightarrow{R D F S}$ knowledge bases is PTIMEcomplete in data complexity and NP-complete in combined complexity.

Again, from the proof of the above theorem we can derive interesting observations: (i) since the result holds even for ontologies having disjointness assertions only between concepts, the theorem strengthens the PTime-hardness result of GutiérrezBasulto et al., 2015, Theorem 15] for the case of DL-Lite core $_{\text {knowledge bases when }}$ the UNA is not adopted; $(i i)$ answering $\mathrm{UCQ}^{1, \neq_{\mathrm{S}}}$ over $D L$-Lite $e_{\mathrm{RDFS}}$ knowledge bases is PTIme-hard in data complexity, too.

We conclude this section with an observation for DL-Lite $e_{\text {RDFS }}$ knowledge bases. If we move to consider $\mathrm{UCQ}^{1,{ }_{\mathrm{s}}}$ rather than $\mathrm{CQ}^{\neq} \mathrm{s}$, we only have a jump from $\mathrm{AC}^{0}$ to PTime for data complexity, while the combined complexity remains the same.

Theorem 4.17. Answering $U C Q^{1, \neq} s$ over DL-Lite $R D F S$ knowledge bases is PTIMEcomplete in data complexity and NP-complete in combined complexity. Both the hardness results already hold for knowledge bases with empty ontologies.

\subsection{Containment of $\mathrm{UCQ}^{\neq} \mathrm{s}$ in Relational Databases}

The results presented in Sections 4.4 and 4.5 have some interesting implications in the context of containment of UCQs with inequalities in relational databases Klug, 1988 van der Meyden, 1997; Kolaitis et al., 1998; Koutris et al., 2017.

The containment problem for $\mathrm{UCQ}^{\neq} \mathrm{s}$ has been shown to be in $\Pi_{2}^{p}$ and conjectured to be $\Pi_{2}^{p}$-complete in Klug, 1988. Such conjecture has been then confirmed to be true in van der Meyden, 1997. Later on, Kolaitis et al., 1998 has studied the impact on the computational complexity of some syntactic and structural conditions for such problem. Specifically, the problem of checking whether $q^{\prime} \sqsubseteq q$ remains $\Pi_{2}^{p}$-hard even when restricted to queries $q^{\prime}$ and $q$ such that all database predicates have arity at most two and every database predicate occurs at most three times in the body of $q^{\prime}$. Finally, note that the problem of checking whether $q^{\prime} \sqsubseteq q$ is coNP-complete in the size of $q^{\prime}$, i.e., when the query $q$ is assumed to be fixed.

To the best of our knowledge, however, it has never been investigated how the number of inequality atoms affects the computational complexity of this problem. We do so in the remaining of this section.

\subsubsection{Lower Bounds}

In order to derive some interesting lower bound results, we start by proving that the problem of checking whether $\mathcal{K} \models q$, for a $D L$-Lite $\overrightarrow{\mathrm{RDFS}}$ knowledge base and boolean $\mathrm{UCQ}^{\neq} q$ over $\mathcal{O}$, can be polynomially reduced to the problem of checking whether $q_{\mathcal{K}}^{\prime} \sqsubseteq q$, where $q_{\mathcal{K}}^{\prime}$ is a $\mathrm{CQ}^{\neq}$.

Proposition 4.9. Let $\mathcal{K}=\langle\mathcal{O}, \mathcal{A}\rangle$ be a DL-Lite $\overrightarrow{R D F S}$ knowledge base, and let $q$ be a boolean $U C Q^{\neq}$over $\mathcal{O}$. The problem of checking whether $\mathcal{K} \models q$ is polynomially reducible to the problem of checking whether $q_{\mathcal{K}}^{\prime} \sqsubseteq q$, where $q_{\mathcal{K}}^{\prime}$ is a boolean $C Q^{\neq}$. 
Proof. Without loss of generality, we can assume that $q$ contains no constants in the bodies of its disjuncts. If this is not the case, then for each constant $c$ occurring in a disjunct $q^{\prime}$ of $q$, we add the assertion $A_{c}(c)$ to the $\mathrm{ABox} \mathcal{A}$, the atom $A_{c}\left(y_{c}\right)$ in conjunction to the body of $q^{\prime}$, and replace each occurrence of $c$ in the body of $q^{\prime}$ with $y_{c}$, where $A_{c}$ and $y_{c}$ are a fresh atomic concept and a fresh existential variable, respectively. It is straightforward to verify that query entailment is preserved with this polynomial time transformation.

Given a $D L$-Lit $\overrightarrow{\mathrm{RDFS}}$ knowledge base $\mathcal{K}=\langle\mathcal{O}, \mathcal{A}\rangle$, we construct a $\mathrm{CQ}^{\neq} q_{\mathcal{K}}^{\prime}$ by means of the following polynomial time steps:

1. We compute $\mathcal{I}_{\mathcal{K}}$

2. We consider the set of atoms $S$ obtained from $\mathcal{I}_{\mathcal{K}}$ in the following way: for every individual $c$ occurring in $\mathcal{I}_{\mathcal{K}}$, we replace each occurrence of $c$ in $\mathcal{I}_{\mathcal{K}}$ with a fresh existential variable $v_{c}$;

3. We replace each atom of the form $i n e q\left(v_{c_{1}}, v_{c_{2}}\right) \in S$ with the inequality atom $v_{c_{1}} \neq v_{c_{2}}$

4. We set $q_{\mathcal{K}}^{\prime}:=\left\{() \mid \exists \vec{y} \cdot S_{q}(\vec{y})\right\}$, where $S_{q}(\vec{y})$ denotes the conjunction of all atoms occurring in the resulting set of atoms $S$.

We now prove that $q_{\mathcal{K}}^{\prime} \sqsubseteq q$ if and only if $\mathcal{K} \models q$, thus showing the claim.

"If part:" Suppose that $q_{\mathcal{K}}^{\prime} \nsubseteq q$. Since both $q_{\mathcal{K}}^{\prime}$ and $q$ are queries over the schema $\mathcal{O}$, it follows that there is a set of facts $D$ over $\mathcal{O}$ for which $D \not \models q$ and $D \models q_{\mathcal{K}}^{\prime}$. Since $D=q_{\mathcal{K}}^{\prime}$, there is a homomorphism $h$ from $q_{\mathcal{K}}^{\prime}$ to $D$ (and therefore $h\left(v_{c_{1}}\right) \neq h\left(v_{c_{2}}\right)$ for each inequality atom $v_{c_{1}} \neq v_{c_{2}}$ occurring in the body of $\left.q_{\mathcal{K}}^{\prime}\right)$. Consider the interpretation $\mathcal{I}=\left\langle\Delta^{\mathcal{I}},{ }^{\mathcal{I}}\right\rangle$ for $\mathcal{K}$ with $(i) \Delta^{\mathcal{I}}=\operatorname{dom}(D),(i i) c^{\mathcal{I}}=h\left(v_{c}\right)$, for each constant $c$ occurring in $\mathcal{A}$, and finally (iii) the extension of each atomic atomic concept and atomic role is the same as in $D$.

Clearly, since $D \not \models q$ and $D \models q_{\mathcal{K}}^{\prime}$, we have $\mathcal{I} \not \models q$ and $\mathcal{I} \models q_{\mathcal{K}}^{\prime}$ as well. Moreover, since $h\left(v_{c_{1}}\right) \neq h\left(v_{c_{2}}\right)$ for each inequality atom $v_{c_{1}} \neq v_{c_{2}}$ occurring in the body of $q_{\mathcal{K}}^{\prime}$, we have $c_{1}^{\mathcal{I}} \neq c_{2}^{\mathcal{I}}$ for each atom of the form $\operatorname{ineq}\left(c_{1}, c_{2}\right) \in \mathcal{I}_{\mathcal{K}}$. This, together with the fact that $\mathcal{I} \models q_{\mathcal{K}}^{\prime}$, implies that $\mathcal{I}$ is a model of $\mathcal{K}$. Thus, $\mathcal{I}$ is a model of $\mathcal{K}$ such that $\mathcal{I} \not \models q$. It follows that $\mathcal{K} \not \forall q$, as required.

"Only-if part:" Suppose that $\mathcal{K} \not=q$, i.e., there is a model $\mathcal{I}=\left\langle\Delta^{\mathcal{I}},{ }^{\mathcal{I}}\right\rangle$ of $\mathcal{K}$ for which $\mathcal{I} \not \models q$. Consider the function $h$ from the variables occurring in $q_{\mathcal{K}}^{\prime}$ to $\Delta^{\mathcal{I}}$ such that $h\left(v_{c}\right)=c^{\mathcal{I}}$, for each variable $v_{c}$ of $q_{\mathcal{K}}^{\prime}$ (note that $v_{c}$ is the variable that has replaced the constant $c$ of $\mathcal{A}$ in the step 2 of the reduction).

Since $\mathcal{I}$ is a model of $\mathcal{K}$, it is straightforward to verify that $h$ consists in a homomorphism from $q_{\mathcal{K}}^{\prime}$ to $\mathcal{I}$ (and therefore $h\left(v_{c_{1}}\right) \neq h\left(v_{c_{2}}\right)$ for each inequality atom $v_{c_{1}} \neq v_{c_{2}}$ occurring in the body of $\left.q_{\mathcal{K}}^{\prime}\right)$, and so $\mathcal{I} \models q_{\mathcal{K}}^{\prime}$. From the facts that $\mathcal{I} \not \models q$ and $\mathcal{I} \models q_{\mathcal{K}}^{\prime}$, we can easily obtain a set of facts $D_{\mathcal{I}}$ over $\mathcal{O}$ for which $D_{\mathcal{I}} \mid \models q$ and $D_{\mathcal{I}} \models q_{\mathcal{K}}^{\prime}$. It follows that $q_{\mathcal{K}}^{\prime} \nsubseteq q$, as required.

Observe that $(i)$ if $\mathcal{K}$ is a $D L$-Lite RDFS $_{\text {knowledge base rather than a } D L-L i t e_{\mathrm{RDFS}}}$ knowledge base, then the query $q_{\mathcal{K}}^{\prime}$ produced by the above illustrated reduction contains no inequality atoms because $\mathcal{I}_{\mathcal{K}}$ contains no atoms with ineq as predicate, and therefore $q_{\mathcal{K}}^{\prime}$ is a boolean $\mathrm{CQ}$ rather than a boolean $\mathrm{CQ}^{\neq}$; and $(i i)$ if $\mathcal{K}=\langle\mathcal{O}, \mathcal{A}\rangle$ 
is a $D L-L i t e_{\mathrm{RDFS}}$ knowledge base such that $\mathcal{O}$ contains no inclusion assertions, then the above illustrated reduction is in fact a LOGSPACE reduction. From these observations, we immediately derive the following two corollaries.

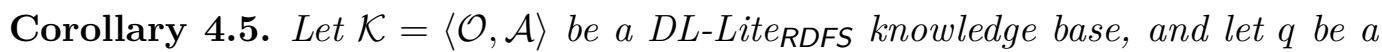
boolean $U C Q^{\neq}$over $\mathcal{O}$. The problem of checking whether $\mathcal{K} \models q$ is polynomially reducible to the problem of checking whether $q_{\mathcal{K}}^{\prime} \sqsubseteq q$, where $q_{\mathcal{K}}^{\prime}$ is a boolean $C Q$.

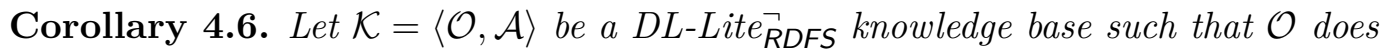
not have inclusion assertions, and let $q$ be a boolean $U C Q^{\neq}$over $\mathcal{O}$. The problem of checking whether $\mathcal{K} \models q$ is LOGSPACE reducible to the problem of checking whether $q_{\mathcal{K}}^{\prime} \sqsubseteq q$, where $q_{\mathcal{K}}^{\prime}$ is a boolean $C Q^{\neq}$.

Using results of Section 4.4, we are now ready to derive interesting lower bounds. Let the containment problem for $\mathrm{UCQ}^{\neq} \mathrm{s}$ be the following decision problem: given two $\mathrm{UCQ}^{\neq} \mathrm{s} q^{\prime}, q$ over the same schema $\mathcal{S}$, check whether $q^{\prime} \sqsubseteq q$.

Theorem 4.18. The containment problem for $U C Q^{\neq} s$ is $\Pi_{2}^{p}$-hard (and therefore $\Pi_{2}^{p}$-complete) already when (i) both the input queries $q^{\prime}$ and $q$ are boolean and every database predicate have arity at most two, (ii) $q^{\prime}$ is a $C Q^{\neq}$(respectively, $C Q$ ), and (iii) $q$ is a $C Q^{\neq}$(respectively, $U C Q^{2, \neq}$ which is the union of a fixed $C Q^{2, \neq}$ and of a $C Q$ without inequalities).

Proof. By looking at the proof of Theorem 4.11 (respectively, Theorem 4.12), one realises that checking whether $\mathcal{K} \models q$ for a given $D L$-Lit $\overrightarrow{\mathrm{RDFS}}_{\overrightarrow{\mathrm{R}}}$ (respectively,

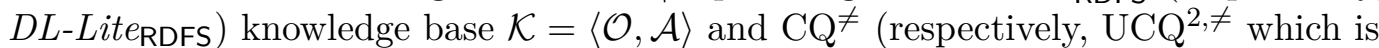
the union of a fixed $\mathrm{CQ}^{2, \neq}$ and of a given $\mathrm{CQ}$ without inequalities) $q$ is in general $\Pi_{2}^{p}$-hard. Since due to Proposition 4.9 (respectively, Corollary 4.5 ) this problem is polynomially reducible to checking whether $q_{\mathcal{K}}^{\prime} \sqsubseteq q$, where $q_{\mathcal{K}}^{\prime}$ is a $\mathrm{CQ}^{\neq}$(respectively, $\mathrm{CQ}$ ), then the claim trivially follows.

We also conjecture a stronger version of the above result, which turns out to be valid as soon as Conjecture 4.1 is verified.

Conjecture 4.2. The containment problem for $U C Q^{\neq} s$ is $\Pi_{2}^{p}$-hard (and therefore $\Pi_{2}^{p}$-complete) already when (i) both the input queries $q^{\prime}$ and $q$ are boolean and every databases predicate have arity at most two, (ii) $q^{\prime}$ is a $C Q^{\neq}$, and (iii) $q$ is a $C Q^{2, \neq}$.

As for the complexity of the containment problem for $\mathrm{UCQ}^{{ }^{*} \mathrm{~s}}$ when the containing query $q$ is assumed to be fixed, we derive the following lower bounds.

Theorem 4.19. When the containing query $q$ is assumed to be fixed, the containment problem for $U C Q^{\neq} s$ is coNP-hard (and therefore coNP-complete) already when (i) both the input query $q^{\prime}$ and the fixed query $q$ are boolean and every database predicate have arity at most two, (ii) $q^{\prime}$ is a $C Q^{\neq}$(respectively, $C Q$ ), and (iii) $q$ is a $C Q^{2, \neq}$ (respectively, $U C Q^{2, \neq}$ which is the union of a $C Q^{2, \neq}$ and of a $C Q$ without inequalities).

Proof. By looking at the proof of Theorem 4.10, one realises that there exists a $\mathrm{CQ}^{2, \neq}$ (respectively, $\mathrm{UCQ}^{2, \neq}$ which is the union of a $\mathrm{CQ}^{2, \neq}$ and of a $\mathrm{CQ}$ without inequalities) $q$ such that checking whether $\mathcal{K}=q$ for a given $D L$-Lit $e_{\mathrm{RDFS}}^{\neg}$ (respectively, $D L$-Lite $e_{\mathrm{RDFS}}$ ) knowledge base $\mathcal{K}=\langle\mathcal{O}, \mathcal{A}\rangle$ is in general coNP-hard. Since 
due to Proposition 4.9 (respectively, Corollary 4.5) this problem is polynomially reducible to checking whether $q_{\mathcal{K}}^{\prime} \sqsubseteq q$, where $q_{\mathcal{K}}^{\prime}$ is a $\mathrm{CQ}^{\neq}$(respectively, CQ), then the claim trivially follows.

As for the case of the containment for queries having only one inequality per disjunct, we have the following lower bound.

Theorem 4.20. When the containing query $q$ is assumed to be fixed, the containment problem for $U C Q^{1, \neq} s$ is PTIME-hard already when the input query $q^{\prime}$ is a $C Q^{1, \neq}$ (respectively, $C Q$ ) and the fixed query $q$ is a $C Q^{1, \neq}$ (respectively, $U C Q^{1, \neq}$ which is the union of a $C Q^{1, \neq}$ and of a $C Q$ without inequalities).

Proof. By looking at the proof of Theorem 4.16, one realises that there exists a $\mathrm{CQ}^{1, \neq}$ (respectively, $\mathrm{UCQ}^{1, \neq}$ which is the union of a $\mathrm{CQ}^{1, \neq}$ and of a $\mathrm{CQ}$ without inequalities) $q$ such that checking whether $\mathcal{K} \models q$ for a given $D L$-Lit $\overrightarrow{\mathrm{RDFS}}_{\overrightarrow{\mathrm{Q}}}$ (respectively, $D L$-Lite RDFS $_{\text {S }} \mathcal{K}=\langle\mathcal{O}, \mathcal{A}\rangle$ with $\mathcal{O}$ having no inclusion assertions is in general PTime-hard. Since due to Corollary 4.6 (respectively, the combination of Corollary 4.6 and Corollary 4.5 this problem is LOGSPACE reducible to checking whether $q_{\mathcal{K}}^{\prime} \sqsubseteq q$, where $q_{\mathcal{K}}^{\prime}$ is a $\mathrm{CQ}^{1, \neq}$ (respectively, CQ), then the claim trivially follows.

\subsubsection{Upper Bounds}

By exploiting again the close connection between answering $\mathrm{UCQ}^{\neq}{ }_{\mathrm{S}}$ over $D L-L i t e_{\mathrm{RDFS}}$ knowledge bases and the containment problem for $\mathrm{UCQ}^{\neq} \mathrm{s}$, we can prove new upper bound complexity results for the containment problem for $\mathrm{UCQ}^{1,{ }_{\mathrm{s}}} \mathrm{s}$. We follow a presentation path very similar to the one of Section 4.5. and start with the following definition and CheckFContains algorithm.

Definition 4.4. Let $q^{\prime}$ be a $\mathrm{CQ}^{\neq}, q$ be a $\mathrm{UCQ}^{1, \neq}$, and $F=\left[f_{1}, \ldots, f_{m}\right]$ be a list of functions from the set of variables and constants occurring in $q$ to the set of variables and constants occurring in $q^{\prime}$. We say that $F$ is a good sequence with respect to $q^{\prime}$ and $q$ if CheckFContains $\left(q^{\prime}, q, F\right)$ returns true.

In the algorithm, for a $\mathrm{CQ}^{\neq} q^{\prime}$ and two terms $f_{i}\left(z_{1}\right)$ and $f_{i}\left(z_{2}\right)$ of $q^{\prime}$, the query $q_{f_{i}\left(z_{1}\right)}^{\prime f_{i}\left(z_{2}\right)}$ denotes the $\mathrm{CQ}^{\neq}$obtained from $q^{\prime}$ in the following way: if one among $f_{i}\left(z_{1}\right)$ and $f_{i}\left(z_{2}\right)$ is a constant, then the variable is replaced everywhere in $q^{\prime}$ by the constant; if both are variables, then one is replaced everywhere in $q^{\prime}$ by the other.

Roughly speaking, consider each step $i$ from 1 to $m-1$ such that $f_{i}$ is a homomorphism from a disjunct $q^{\prime \prime}$ of $q$. Clearly, if conditions of step 3 are satisfied, then we trivially have that $q^{\prime} \sqsubseteq q^{\prime \prime}$ and the algorithm CheckFContains returns true. If not, the algorithm replaces everywhere in $q^{\prime}$ one of the two terms among $f_{i}\left(z_{1}\right)$ and $f_{i}\left(z_{2}\right)$ with the other, to consider a "representative database" of $q^{\prime}$ in which $f_{i}\left(z_{1}\right)=f_{i}\left(z_{2}\right)$, where $z_{1} \neq z_{2}$ is the inequality atom occurring in the body of $q^{\prime \prime}$.

Thus, the algorithm returns true if and only if either it is not possible to equate two terms without contradicting an inequality atom of $q^{\prime}$ (or because they are both constants), or the resulting $q^{\prime}$ is such that $q^{\prime} \sqsubseteq q_{2}$.

Using the above notion of good sequence, it is possible to derive the following characterisation $\left(n_{q^{\prime}}\right.$ denotes the number of terms occurring in the query $\left.q^{\prime}\right)$. 


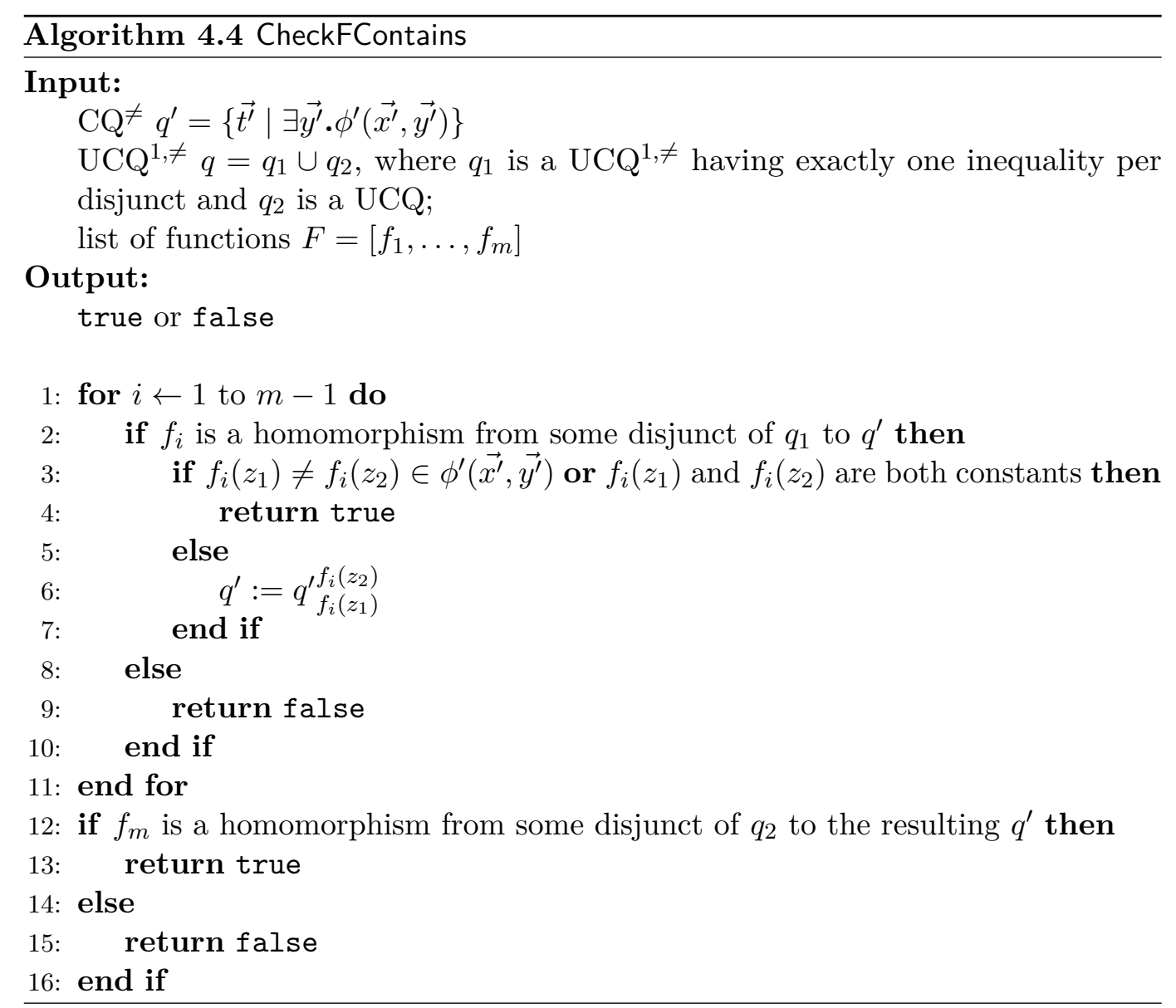

Proposition 4.10. Let $q^{\prime}$ and $q$ be a $C Q^{\neq}$and a $U C Q^{1, \neq}$, respectively. We have that $q^{\prime} \sqsubseteq q$ if and only if there exists a list $F=\left[f_{1}, \ldots, f_{m}\right]$ of functions, with $m \leq n_{q^{\prime}}$, such that $F$ is a good sequence with respect to $q^{\prime}$ and $q$.

Proof. "If part:" Suppose $F=\left[f_{1}, \ldots, f_{m}\right]$ (with $m \leq n_{q^{\prime}}$ ) is a good sequence with respect to $q^{\prime}$ and $q$, i.e., CheckFContains $\left(q^{\prime}, q, F\right)$ returns true. It follows that, after possibly applying $l \leq m-1$ equalities between terms on $q^{\prime}$, either $l \leq m-2$ and $f_{l+1}$ is a homomorphism satisfying conditions of steps 2 and 3 of the algorithm, or $l=m-1$ and $f_{m}$ is a homomorphism from some disjunct of $q_{2}$ to $q^{\prime}$.

In both cases, consider each homomorphism $f_{i}$ from some of the disjuncts of $q_{1}$ to $q^{\prime}$, for $i=[1, l]$. It is easy to see that all databases $D$ and tuples of constants $\vec{c}$ for which there is a homomorphism $h$ from $q^{\prime}$ to $D$ with $h\left(\overrightarrow{t^{\prime}}\right)=\vec{c}$ (thus $\vec{c} \in q^{\prime D}$ ) and in addition with $h\left(f_{i}\left(z_{1}\right)\right) \neq h\left(f_{i}\left(z_{2}\right)\right)$ is such that $\vec{c} \in q^{\prime \prime D}$ as well, where $z_{1} \neq z_{2}$ is the only inequality atom of the disjunct $q^{\prime \prime}$ of $q_{1}$ for which $f_{i}$ is a homomorphism to $q^{\prime}$. So, at each iteration the algorithm equates $f_{i}\left(z_{1}\right)$ and $f_{i}\left(z_{2}\right)$ in $q^{\prime}$ to consider all the other possible representative databases in which $f_{i}\left(z_{1}\right)=f_{i}\left(z_{2}\right)$.

If $f_{l+1}$ is a homomorphism from a disjunct $q^{\prime \prime}$ of $q_{1}$ to the resulting $q^{\prime}$ such that either $f_{l+1}\left(z_{1}\right) \neq f_{l+1}\left(z_{2}\right)$ is an inequality atom of $q^{\prime}$ (where $z_{1} \neq z_{2}$ is the only inequality atom of $\left.q^{\prime \prime}\right)$ or $f_{l+1}\left(z_{1}\right)$ and $f_{l+1}\left(z_{2}\right)$ are both constants, then we clearly have $q^{\prime} \sqsubseteq q_{1}$, and therefore $q^{\prime} \sqsubseteq q$. 
Finally, in the case that $f_{m}$ is a homomorphism from a disjunct of $q_{2}$ to the resulting $q^{\prime}$, due to the above considerations, it can be easily proven that each database $D$ is such that either $q^{\prime D} \subseteq q_{1}{ }^{D}$, or ${q^{\prime}}^{D} \subseteq q_{2}{ }^{D}$. Thus, $q^{\prime} \sqsubseteq q$, as required.

"Only-if part:" Suppose there is no such good sequence $F$ with respect to $q^{\prime}$ and $q$, and consider the following changes to the $\mathrm{CQ}^{\neq} q^{\prime}$ : for each possible homomorphism $h$ from a disjunct of $q_{1}$ to $q^{\prime}$, we replace everywhere in $q^{\prime}$ one of the two terms $h\left(z_{1}\right)$ and $h\left(z_{2}\right)$ with the other, as described in step 6 of the algorithm, where $z_{1} \neq z_{2}$ is the inequality atom of the disjunct of $q_{1}$ for which $h$ is a homomorphism to $q^{\prime}$.

Since there are $n_{q^{\prime}}$ terms in the $\mathrm{CQ}^{\neq} q^{\prime}$, there can be at most $n_{q^{\prime}}-1$ of such homomorphisms. Indeed, after applying $n_{q^{\prime}}-1$ replacing as described above, the resulting $q^{\prime}$ would contain only one term.

For the $\mathrm{CQ}^{\neq} q^{\prime}$ and $\mathrm{UCQ}^{1, \neq} q=q_{1} \cup q_{2}$ we have that $(i)$ there is no homomorphism $h$ from a disjunct of $q_{1}$ to $q^{\prime}$ with either $h\left(z_{1}\right) \neq h\left(z_{2}\right)$, or $h\left(z_{1}\right)$ and $h\left(z_{2}\right)$ being both constants, where $z_{1} \neq z_{2}$ is the inequality atom of the disjunct for which $h$ is a homomorphism to $q^{\prime}$; and (ii) there is no disjunct of $q_{2}$ for which there is a homomorphism to $q^{\prime}$. In proof, if either point $(i)$ or point $(i i)$ is not true, then we would easily derive a contradiction on the fact that there exists no good sequence $F$ with respect to $q^{\prime}$ and $q$.

Consider now the freezing of the resulting $\mathrm{CQ}^{\neq} q^{\prime}$, i.e., the set of facts $D_{q^{\prime}}$, here denoted by $D$ for the sake of readability, corresponding to the set of all atoms occurring in the body of $q^{\prime}$ that are not inequality atoms, but where each variable $v$ is replaced with a different fresh constant $c_{v}$. Let, moreover, $\overrightarrow{c^{\prime}}$ be the tuple of constants obtained from the target list $\overrightarrow{t^{\prime}}$ of the resulting $q^{\prime}$ after replacing each distinguished variable $v$ with the constant $c_{v}$.

Clearly, $D$ and $\overrightarrow{c^{\prime}}$ are such that $\overrightarrow{c^{\prime}} \in q^{\prime D}$ by construction. Furthermore, due to the facts that $q^{\prime}$ and $q$ are such that both the above points $(i)$ and $(i i)$ hold, we derive that $\overrightarrow{c^{\prime}} \notin q^{D}$. Thus, $q^{D} \nsubseteq q^{D}$, and therefore $q^{\prime} \nsubseteq q$, as required.

For UCQs with no inequalities, it is known that query containment is in NP (in particular, NP-complete), and is in PTime when the containing query $q$ is assumed to be fixed. We are now ready to show that the same holds even when the contained query $q^{\prime}$ is a $\mathrm{UCQ}^{\neq}$and the containing query $q$ is a $\mathrm{UCQ}^{1, \neq}$.

Theorem 4.21. The containment problem for $U C Q^{\neq} s$ when the containing query $q$ is a $U C Q^{1, \neq}$ is in NP (and therefore NP-complete). Moreover, it is in PTIME when the containing $U C Q^{1, \neq} q$ is assumed to be fixed.

Proof. Given a $\mathrm{UCQ}^{\neq} q^{\prime}$ and $\mathrm{UCQ}^{1, \neq} q$, observe that $q^{\prime} \sqsubseteq q$ if and only if $q^{\prime \prime} \sqsubseteq q$ for each disjunct $q^{\prime \prime}$ of $q^{\prime}$, where $q^{\prime \prime}$ is a $\mathrm{CQ}^{\neq}$.

Due to Proposition 4.10, checking whether $q^{\prime \prime} \sqsubseteq q$ for a $\mathrm{CQ}^{\neq} q^{\prime \prime}$ and $\mathrm{UCQ}^{1, \neq} q$ can be done as follows:

- Guess a list $F=\left[f_{1}, \ldots, f_{m}\right]$ of $m \leq n_{q^{\prime \prime}}$ functions from the set of variables and constants occurring in $q$ to the set of variables and constants occurring in $q^{\prime \prime}$

- Check whether $F$ is a good sequence with respect to $q^{\prime \prime}$ and $q$, 
where checking whether $F$ is a good sequence with respect to $q^{\prime \prime}$ and $q$ can be done by means of the above described CheckFContains algorithm.

So, we easily derive a nondeterministic algorithm deciding $q^{\prime} \sqsubseteq q$. With an NP step, for each disjunct $q^{\prime \prime}$ of $q^{\prime}$, we guess a list $F_{q^{\prime \prime}}=\left[f_{1}, \ldots, f_{m}\right]$ with $m \leq n_{q^{\prime \prime}}$ of functions from the set of variables and constants occurring in $q$ to the set of variables and constants occurring in $q^{\prime \prime}$. Notice that this can be done in polynomial time in the size of $q^{\prime}$. Then, for each disjunct $q^{\prime \prime}$ of $q$, by exploiting the CheckFContains algorithm we check whether $F_{q^{\prime \prime}}$ is a good sequence with respect to $q^{\prime \prime}$ and $q$ using: $(i)$ for each $i \in[1, m-1]$, a polynomial time step for checking whether $f_{i}$ is a homomorphism from a disjunct of $q_{1}$ to $q^{\prime \prime}$, and for replacing everywhere in $q^{\prime \prime}$ one of the two terms among $f_{i}\left(z_{1}\right)$ and $f_{i}\left(z_{2}\right)$ with the other; finally, $(i i)$ another polynomial time step for checking whether $f_{m}$ is a homomorphism from some disjunct of the UCQ $q_{2}$ to the resulting $\mathrm{CQ}^{\neq} q^{\prime \prime}$.

Notice, however, that when the containing query is assumed to be fixed, the containment problem for UCQs with no inequalities can be solved even in $\mathrm{AC}^{0}$ (it is indeed sufficient to evaluate the containing query $q$ over the database associated to the contained query $q^{\prime}$ [Chandra and Merlin, 1977]). On the other hand, as shown in Theorem 4.20, the containment problem for $\mathrm{UCQ}^{1, \neq_{\mathrm{S}}}$ is PTIME-hard when the containing query is assumed to be fixed. Since $\mathrm{AC}^{0} \subset$ PTIme, this makes the two problems significantly different. We conclude the chapter by characterising the computational complexity of the containment problem for $\mathrm{UCQ}^{1,{ }_{\mathrm{s}}}$, which can be obtained by combining Theorem 4.20 with the above theorem.

Corollary 4.7. The containment problem for $U C Q^{\neq} s$ when the containing query $q$ is a $U C Q^{1, \neq}$ is NP-complete. Moreover, it is PTIME-complete when the containing query $q$ is assumed to be fixed. 



\section{Chapter 5}

\section{Complete Source-to-Ontology Rewritings}

In this chapter, we study both the verification, and the computation problem for complete source-to-ontology rewritings. In what follows, given a syntactic object $x$ such as a UCQ, an ontology, or a mapping, we denote by $\sigma(x)$ its size, i.e., the number of symbols needed to write it, with names of predicates, variables, etc. counting as one.

\subsection{Verification Problem}

Suppose we want to check whether $q_{\mathcal{O}}$ is a complete $\mathcal{S}$-to- $\mathcal{O} \Sigma$-rewriting of $q_{\mathcal{S}}$. Obviously, if $q_{\mathcal{S}}$ is contained in $\operatorname{PerfRef}_{q_{\mathcal{O}}, \Sigma}$, then for every $\mathcal{S}$-database $D$ consistent with $\Sigma$, we have that $q_{\mathcal{S}}^{D} \subseteq \operatorname{cert}_{q_{\mathcal{O}}, \Sigma}^{D}$, and therefore the answer is positive. If $q_{\mathcal{S}}$ is not contained in $\operatorname{PerfRef}_{q_{\mathcal{O}}, \Sigma}$, however, it might be the case that $q_{\mathcal{O}}$ is still a complete $\mathcal{S}$-to- $\mathcal{O} \Sigma$-rewriting of $q_{\mathcal{S}}$, in particular in the case where the non-emptiness of the answers of $q_{\mathcal{S}}$ over $D$ reveals the presence of inconsistencies. From this observation, we can easily derive the following characterisation.

Lemma 5.1. $q_{\mathcal{O}}$ is a complete $\mathcal{S}$-to-O $\mathcal{O}$ L-rewriting of $q_{\mathcal{S}}$ if and only if $q_{\mathcal{S}} \sqsubseteq$ $\left(\operatorname{PerfRef}_{q_{\mathcal{O}}, \Sigma} \cup \operatorname{PerfRef}_{\mathcal{V}_{\mathcal{O}}^{n}, \Sigma}\right)$, where $n=\operatorname{ar}\left(q_{\mathcal{O}}\right)=\operatorname{ar}\left(q_{\mathcal{S}}\right)$.

Proof. "Only-if part:" Suppose that $q_{\mathcal{O}}$ is a complete $\mathcal{S}$-to- $\mathcal{O} \Sigma$-rewriting of $q_{\mathcal{S}}$. By definition, we have that for every $\mathcal{S}$-database $D$ either $D$ is not consistent with $\Sigma$, or $q_{\mathcal{S}}^{D} \subseteq \operatorname{cert}_{q_{\mathcal{O}} \Sigma \Sigma}^{D}$. In the former case, we have $\operatorname{PerfRef}_{\mathcal{V}_{\mathcal{O}}, \Sigma}^{D}=\{()\}$, which obviously implies that $q_{\mathcal{S}}^{D} \subseteq \operatorname{PerfRef}_{\mathcal{V}_{\mathcal{O}}^{n}, \Sigma}^{D}$. In the latter case, since $D$ is consistent with $\Sigma$, we have that $\operatorname{cert}_{q_{\mathcal{O}}, \Sigma}^{D}=\operatorname{PerfRef}_{q_{\mathcal{O}}, \Sigma}^{D}$. Therefore, we have that $q_{\mathcal{S}}^{D} \subseteq$ $\left(\operatorname{PerfRef}_{q_{\mathcal{O}}, \Sigma} \cup \operatorname{PerfRef}_{\mathcal{V}_{\mathcal{O}}^{n}, \Sigma}\right)^{D}$ for every $\mathcal{S}$-database $D$, as required.

"If part:" Suppose, for the sake of contradiction, that $q_{\mathcal{O}}$ is not a complete $\mathcal{S}$-to- $\mathcal{O} \Sigma$-rewriting of $q_{\mathcal{S}}$, that is, there exists an $\mathcal{S}$-database $D$ consistent with $\Sigma$ such that $q_{\mathcal{S}}^{D} \nsubseteq \operatorname{cert}_{q_{\mathcal{O}}, \Sigma}^{D}$. Since $D$ is consistent with $\Sigma$, we have $(i) \operatorname{PerfRef}_{\mathcal{V}_{\mathcal{O}, \Sigma}}^{D}=\emptyset$, which implies that $(i) \operatorname{PerfRef}_{\mathcal{V}_{\mathcal{O}}^{n}, \Sigma}^{D}=\emptyset$ and $(i i) \operatorname{cert}_{q_{\mathcal{O}}, \Sigma}^{D}=\operatorname{PerfRef}_{q_{\mathcal{O}}, \Sigma}^{D}$. Therefore, for the $\mathcal{S}$-database $D$, we have that $q_{\mathcal{S}}^{D} \nsubseteq\left(\operatorname{PerfRef}_{q_{\mathcal{O}}, \Sigma} \cup \operatorname{PerfRef}_{\mathcal{V}_{\mathcal{O}}^{n}, \Sigma}\right)^{D}$. Thus, $q_{\mathcal{S}} \nsubseteq\left(\operatorname{PerfRef}_{q_{\mathcal{O}}, \Sigma} \cup \operatorname{PerfRef}_{\mathcal{V}_{\mathcal{O}}^{n}, \Sigma}\right)$, as required. 
The following theorem characterises the computational complexity of the verification problem for complete source-to-ontology rewritings.

Theorem 5.1. The verification problem for complete source-to-ontology rewritings is NP-complete.

Proof. As for the upper bound, by virtue of Lemma 5.1, it is sufficient to show how to check the containment $q_{\mathcal{S}} \sqsubseteq\left(\operatorname{PerfRef}_{q_{\mathcal{O}}, \Sigma} \cup \operatorname{PerfRef}_{\mathcal{V}_{\mathcal{O}}^{n}, \Sigma}\right)$ in NP, where $n=\operatorname{ar}\left(q_{\mathcal{O}}\right)$. In particular, for every disjunct $q$ of $q_{\mathcal{S}},(i)$ we guess a query $q^{\prime}$ over $\mathcal{O}$ with the same arity of $q_{\mathcal{O}}$ and size at most the maximum between $\sigma\left(q_{\mathcal{O}}\right)$ and $\sigma\left(\mathcal{V}_{\mathcal{O}}^{n}\right)$, a sequence $\rho$ of ontology assertions, a query $q^{\prime \prime}$ over $\mathcal{S}$ with the same arity of $q_{\mathcal{O}}$ and size at most $\sigma(\mathcal{M}) \times \sigma\left(q^{\prime}\right)$, and a function $\phi$ from the variables of $q^{\prime \prime}$ to the variables of $q$, and $(i i)$ we check in polynomial time whether we can rewrite either $q_{\mathcal{O}}$ or $\mathcal{V}_{\mathcal{O}}^{n}$ into $q^{\prime}$ through $\rho, q^{\prime \prime}$ is in $\operatorname{Map} \operatorname{Ref}\left(q^{\prime}, \mathcal{M}\right)$, and $\phi$ is a homomorphism from $q^{\prime \prime}$ to $q$.

As for the lower bound, the proof of NP-hardness is by a LOGSPACE reduction from the 3-colourability problem, which is NP-complete Garey et al., 1976. 3colourability is the problem of deciding, given an undirected graph $G=(V, E)$ with no loops, whether $G$ is 3 -colourable, i.e., whether there exists a function $f: V \rightarrow\{R, G, B\}$ such that $f\left(y_{i}\right) \neq f\left(y_{j}\right)$ for each $\left(y_{i}, y_{j}\right) \in E$.

Let $\Sigma=\langle\mathcal{O}, \mathcal{S}, \mathcal{M}\rangle$ be an OBDM specification as follows: ontology $\mathcal{O}$ contains no axioms, i.e., $\mathcal{O}=\emptyset$, schema $\mathcal{S}$ involves a binary predicate $E$ and three unary predicates $s_{R}, s_{G}$, and $s_{B}$, and finally the mapping $\mathcal{M}=\left\{m_{1}, m_{2}, m_{3}, m_{4}\right\}$ with:

$$
\begin{array}{rrl}
m_{1}: & E\left(x_{1}, x_{2}\right) & \rightarrow P\left(x_{1}, x_{2}\right), \\
m_{2}: & s_{R}(x) & \rightarrow R(x), \\
m_{3}: & s_{G}(x) & \rightarrow G(x), \\
m_{4}: & s_{B}(x) & \rightarrow B(x),
\end{array}
$$

where $R, G$, and $B$ are atomic concepts, whereas $P$ is an atomic role.

Let $V=\left(y_{1}, y_{2}, \ldots, y_{n}\right)$, then we define a boolean $\mathrm{CQ} q_{\mathcal{O}}$ over $\mathcal{O}$ as follows:

$$
\begin{aligned}
q_{\mathcal{O}}=\left\{\left(x_{R}, x_{G}, x_{B}\right) \mid \exists y_{1}, \ldots, y_{n} \cdot R\left(x_{R}\right) \wedge G\left(x_{G}\right) \wedge B\left(x_{B}\right) \wedge\right. & \left.\wedge \bigwedge_{\left(y_{i}, y_{j}\right) \in E}\left(P\left(y_{i}, y_{j}\right) \wedge P\left(y_{j}, y_{i}\right)\right)\right\} .
\end{aligned}
$$

Also, let $q_{\mathcal{S}}$ be the boolean CQJFE over $\mathcal{S}$ defined as follows: $q_{\mathcal{S}}=\left\{\left(x_{R}, x_{G}, x_{B}\right) \mid\right.$ $s_{R}\left(x_{R}\right) \wedge s_{G}\left(x_{G}\right) \wedge s_{B}\left(x_{B}\right) \wedge E\left(x_{R}, x_{G}\right) \wedge E\left(x_{G}, x_{R}\right) \wedge E\left(x_{R}, x_{B}\right) \wedge E\left(x_{B}, x_{R}\right) \wedge$ $\left.E\left(x_{B}, x_{G}\right) \wedge E\left(x_{G}, x_{B}\right)\right\}$.

Observe that $\Sigma=\langle\mathcal{O}, \mathcal{S}, \mathcal{M}\rangle$ and $q_{\mathcal{S}}$ do not depend on the input of the 3COLOURABILITY problem (i.e., $G=(V, E)$ ), whereas $q_{\mathcal{O}}$ can be constructed in LOGSPACE from it.

We now show that $G$ is 3-colourable if and only if $q_{\mathcal{O}}$ is a complete $\mathcal{S}$-to- $\mathcal{O}$ $\Sigma$-rewriting of $q_{\mathcal{S}}$. To begin observe that $\mathcal{V}_{\mathcal{O}} \equiv \perp$, and hence, for each $\mathcal{S}$-database $D$, we have that $\operatorname{cert}_{q_{\mathcal{O}}, \Sigma}^{D}=\operatorname{PerfRef}_{q_{\mathcal{O}}, \Sigma}^{D}$, where PerfRef ${ }_{q_{\mathcal{O}}, \Sigma}$ is the CQ over $\mathcal{S}$ :

$$
\begin{aligned}
\left\{\left(x_{R}, x_{G}, x_{B}\right) \mid \exists y_{1}, \ldots, y_{n} . s_{R}\left(x_{R}\right) \wedge s_{G}\left(x_{G}\right) \wedge s_{B}\left(x_{B}\right) \wedge\right. \\
\left.\wedge \bigwedge_{\left(y_{i}, y_{j}\right) \in E}\left(E\left(y_{i}, y_{j}\right) \wedge E\left(y_{j}, y_{i}\right)\right)\right\} .
\end{aligned}
$$


"Only-if part:" Suppose that $G$ is 3-colourable, that is, there exists a function $f: V \rightarrow\{R, G, B\}$ such that $f\left(y_{i}\right) \neq f\left(y_{j}\right)$ for each $\left(y_{i}, y_{j}\right) \in E$. But then, consider the function $h$ from the variables of $\operatorname{PerfRef}_{q_{\mathcal{O}}, \Sigma}$ to the variables of $q_{\mathcal{S}}$ such that $h\left(x_{R}\right)=x_{R}, h\left(x_{G}\right)=x_{G}, h\left(x_{B}\right)=x_{B}$, and, for each $i=1, \ldots, n$ :

$$
h\left(y_{i}\right)= \begin{cases}x_{R}, & \text { if } f\left(y_{i}\right)=R \\ x_{G}, & \text { if } f\left(y_{i}\right)=G \\ x_{B}, & \text { if } f\left(y_{i}\right)=B\end{cases}
$$

It can be readily seen that $h$ consists in a homomorphism from PerfRef $q_{\mathcal{O}}, \Sigma$ to $q_{\mathcal{S}}$. It follows that $q_{\mathcal{S}} \sqsubseteq \operatorname{PerfRef}_{q_{\mathcal{O}}, \Sigma}$ which, due to Lemma 5.1. implies that $q_{\mathcal{O}}$ is a complete $\mathcal{S}$-to- $\mathcal{O} \Sigma$-rewriting of $q_{\mathcal{S}}$.

"If part:" Suppose that $G$ is not 3-colourable, that is, every possible function $f: V \rightarrow\{R, G, B\}$ is such that $f\left(y_{i}\right)=f\left(y_{j}\right)$ for some $\left(y_{i}, y_{j}\right) \in E$. It is not hard to see that this implies that there exists no homomorphism from $\operatorname{PerfRef}_{q_{\mathcal{O}}, \Sigma}$ to $q_{\mathcal{S}}$ Therefore, since $\mathcal{V}_{\mathcal{O}} \equiv \perp$, we have that $q_{\mathcal{S}} \nsubseteq\left(\operatorname{PerfRef}_{q_{\mathcal{O}}, \Sigma} \cup \operatorname{PerfRef}_{\mathcal{V}_{\mathcal{O}}^{3}, \Sigma}\right)$ which, due to Lemma 5.1, implies that $q_{\mathcal{O}}$ is not a complete $\mathcal{S}$-to- $\mathcal{O} \Sigma$-rewriting of $q_{\mathcal{S}}$.

Note that the result of NP-hardness already holds when $\Sigma=\langle\mathcal{O}, \mathcal{S}, \mathcal{M}\rangle$ is fixed (i.e., it does not depend on the input of the reduction) with $\mathcal{O}$ containing no axioms and $\mathcal{M}$ being both a pure GAV mapping and a LAV mapping, $q_{\mathcal{S}}$ is a fixed CQJFE, and finally $q_{\mathcal{O}}$ is a $\mathrm{CQ}$.

\subsection{Computation Problem}

We now provide the algorithm MinimallyComplete for computing UCQ-minimally complete source-to-ontology rewritings.

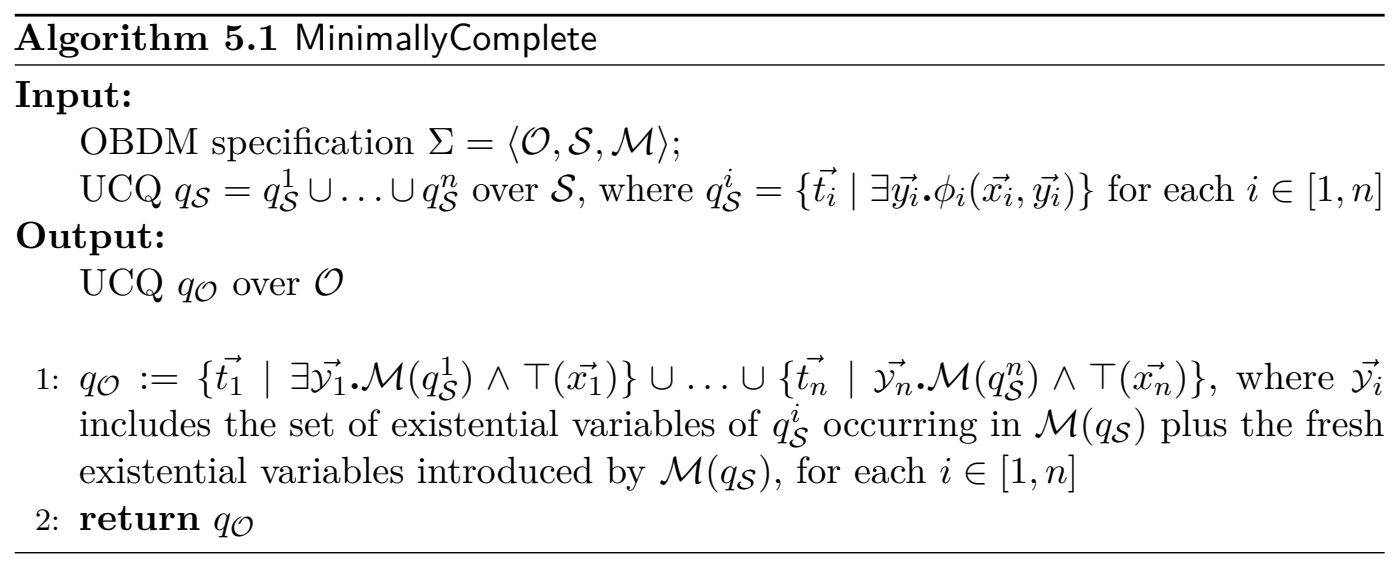

Informally, for each disjunct $q_{\mathcal{S}}^{i}$ of $q_{\mathcal{S}}$, the algorithm obtains a CQ by simply chasing (the incomplete $\mathcal{S}$-database associated to) $q_{\mathcal{S}}^{i}$ with respect to $\mathcal{M}$, using $T$ to bind the distinguished variables that are not involved in the application of $\mathcal{M}$ to $q_{\mathcal{S}}^{i}$. Finally, the output query $q_{\mathcal{O}}$ is the union of all such CQs.

Example 5.1. Let $\Sigma=\langle\mathcal{O}, \mathcal{S}, \mathcal{M}\rangle$ be the following OBDM specification:

- $\mathcal{O}=\emptyset$ 
- $\mathcal{S}=\left\{s_{1}, s_{2}, s_{3}\right\}$

- $\mathcal{M}=\left\{m_{1}, m_{2}, m_{3}, m_{4}\right\}$, where:

$$
\begin{array}{rrrl}
m_{1}: & s_{1}(x) & \rightarrow \exists z \cdot P_{1}(x, z) \wedge A_{1}(z), \\
m_{2}: & \exists y \cdot s_{2}\left(x_{1}, y\right) \wedge s_{2}\left(y, x_{2}\right) & \rightarrow P_{2}\left(x_{1}, x_{2}\right), \\
m_{3}: & \exists y \cdot s_{1}\left(c_{1}\right) \wedge s_{3}(x, y) & \rightarrow P_{3}\left(x, c_{2}\right), \\
m_{4}: & \exists y \cdot s_{3}\left(x_{1}, x_{2}\right) \wedge s_{2}\left(x_{2}, y\right) & \rightarrow P_{4}\left(x_{1}, x_{2}\right) .
\end{array}
$$

Let the data service be expressed as the following UCQ $q_{\mathcal{S}}$ over $\mathcal{S}$ :

$$
\begin{aligned}
& \left\{\left(x_{1}, x_{2}\right) \mid \exists y_{1}, y_{2} . s_{1}\left(x_{1}\right) \wedge s_{2}\left(x_{1}, y_{1}\right) \wedge s_{2}\left(y_{2}, x_{2}\right)\right\} \cup \\
& \left\{\left(x_{1}, c_{3}\right) \mid \exists y_{1}, y_{2} \cdot s_{1}\left(c_{1}\right) \wedge s_{3}\left(x_{1}, y_{1}\right) \wedge s_{2}\left(y_{1}, y_{2}\right)\right\} .
\end{aligned}
$$

One can verify that MinimallyComplete $\left(\Sigma, q_{\mathcal{S}}\right)$ returns the UCQ $q_{\mathcal{O}}$ :

$$
\begin{aligned}
& \left\{\left(x_{1}, x_{2}\right) \mid \exists y_{3} \cdot P_{1}\left(x_{1}, y_{3}\right) \wedge A_{1}\left(y_{3}\right) \wedge \top\left(x_{2}\right)\right\} \cup \\
& \left\{\left(x_{1}, c_{3}\right) \mid \exists y_{1}, y_{3} \cdot P_{1}\left(c_{1}, y_{3}\right) \wedge A_{1}\left(y_{3}\right) \wedge P_{3}\left(x_{1}, c_{2}\right) \wedge P_{4}\left(x_{1}, y_{1}\right)\right\}
\end{aligned}
$$

which corresponds to the unique (up to equivalence w.r.t. $\Sigma$ ) UCQ-minimally complete $\mathcal{S}$-to- $\mathcal{O} \Sigma$-rewriting of $q_{\mathcal{S}}$.

The following theorem establishes termination and correctness of the MinimallyComplete algorithm.

Theorem 5.2. MinimallyComplete $\left(\Sigma, q_{\mathcal{S}}\right)$ terminates and returns the unique (up to equivalence w.r.t. $\Sigma$ ) UCQ-minimally complete $\mathcal{S}$-to-O $\Sigma$-rewriting of $q_{\mathcal{S}}$.

Proof. Termination of the algorithm easily follows from the termination of the chase of a source instance (possibly containing variables) with respect to a GLAV mapping, or, equivalently, with respect to a set of source-to-target tgds [Fagin et al., 2005a .

As for the correctness, we first show that the computed $q_{\mathcal{O}}=q_{\mathcal{O}}^{1} \cup \ldots \cup q_{\mathcal{O}}^{n}$, with $q_{\mathcal{O}}^{i}=\left\{\overrightarrow{t_{i}} \mid \exists \overrightarrow{\mathcal{Y}_{i}} \cdot \mathcal{M}\left(q_{\mathcal{S}}^{i}\right) \wedge \top\left(\overrightarrow{x_{i}}\right)\right\}$ for every $i \in[1, n]$, is a complete $\mathcal{S}$-to- $\mathcal{O} \Sigma$-rewriting of $q_{\mathcal{S}}$. By construction, for every $i \in[1, n]$, the $\mathrm{CQ} q_{\mathcal{S}}^{i}$ corresponds to, or it is contained in, a disjunct of $\operatorname{MapRef}\left(q_{\mathcal{O}}^{i}, \mathcal{M}\right)$. Thus, $q_{\mathcal{S}}^{i} \sqsubseteq \operatorname{PerfRef}_{q_{\mathcal{O}}^{i}, \Sigma}$ holds for every $i \in[1, n]$. It follows that $q_{\mathcal{S}} \sqsubseteq \operatorname{PerfRef}_{q_{\mathcal{O}}, \Sigma}$ which, due to Lemma 5.1, implies that $q_{\mathcal{O}}$ is a complete $\mathcal{S}$-to- $\mathcal{O} \Sigma$-rewriting of $q_{\mathcal{S}}$. We now show that $q_{\mathcal{O}}$ is actually the unique (up to equivalence w.r.t. $\Sigma$ ) UCQ-minimally complete $\mathcal{S}$-to- $\mathcal{O} \Sigma$-rewriting of $q_{\mathcal{S}}$, that is, each UCQ $q_{\mathcal{O}}^{\prime}$ that is a complete $\mathcal{S}$-to- $\mathcal{O} \Sigma$-rewriting of $q_{\mathcal{S}}$ is such that $\operatorname{cert}_{q_{\mathcal{O}}, \Sigma} \sqsubseteq \operatorname{cert}_{q_{\mathcal{O}}^{\prime}, \Sigma}$ (cf. Definition 3.7). We do this by way of contradiction.

Let $q_{\mathcal{O}}^{\prime}$ be a UCQ such that $\operatorname{cert}_{q_{\mathcal{O}}, \Sigma} \nsubseteq \operatorname{cert}_{q_{\mathcal{O}}^{\prime}, \Sigma}$, that is, there exists an $\mathcal{S}$ database $D$ consistent with $\Sigma$ such that $\operatorname{cert}_{q_{\mathcal{O}}, \Sigma}^{D} \not \subset \operatorname{cert}_{q_{\mathcal{O}}^{\prime}, \Sigma}^{D}$. It follows that there is a tuple of constant $\vec{c}=\left(c_{1}, \ldots, c_{m}\right)$ such that $\vec{c} \notin \operatorname{cert}_{q_{\mathcal{O}}^{\prime}, \Sigma}^{D}$, but $\vec{c} \in \operatorname{cert}_{q_{\mathcal{O}}, \Sigma}^{D}$, i.e., $\vec{c} \in \operatorname{cert}_{q_{\mathcal{O}}^{i}, \Sigma}^{D}$ for some $i \in[1, n]$. Consider now the freezing of $q_{\mathcal{S}}^{i}=\left\{\overrightarrow{t_{i}} \mid \exists y_{i} \cdot \phi_{i}\left(\vec{x}_{i}, \vec{y}_{i}\right)\right\}$, i.e., the set $D_{q_{\mathcal{S}}^{i}}$ (here denoted by $D_{i}$ ) of all facts over $\mathcal{S}$ obtained from $\phi_{i}\left(\overrightarrow{x_{i}}, \overrightarrow{y_{i}}\right)$ by replacing each variable $v \in \overrightarrow{x_{i}} \cup \overrightarrow{y_{i}}$ with a different fresh constant denoted by $c_{v}$. Let, moreover, $\overrightarrow{c^{i}}$ be the freezed tuple of constants $\overrightarrow{c^{i}}=\left(c_{1}^{i}, \ldots, c_{m}^{i}\right)$ where, for each $j \in[1, m], c_{j}^{i}=t_{j}$ if $t_{j}$ is a constant, and $c_{j}^{i}=c_{x}$ if $t_{j}=x$. We now prove 
that $\overrightarrow{c^{i}} \in q_{\mathcal{S}}^{i D_{i}}$ and $\overrightarrow{c^{i}} \notin \operatorname{cert}_{q_{\mathcal{O}}^{\prime}, \Sigma}^{D_{i}}$, thus showing that $q_{\mathcal{O}}^{\prime}$ is not a complete $\mathcal{S}$-to- $\mathcal{O}$ $\Sigma$-rewriting of $q_{\mathcal{S}}$.

Obviously, $\overrightarrow{c^{i}} \in q_{\mathcal{S}}^{i^{D}}{ }^{i}$ trivially holds. Consider $\mathcal{C}_{\mathcal{O}}^{\mathcal{M}(D)}$, i.e., the canonical structure of $\mathcal{O}$ with respect to $\mathcal{M}$ and $D$. Since $\vec{c} \in \operatorname{cert}_{q_{\mathcal{O}}^{i}, \Sigma}^{D}$, there exists a homomorphism $h$ from $q_{\mathcal{O}}^{i}$ to $\mathcal{C}_{\mathcal{O}}^{\mathcal{M}(D)}$ for which $h\left(\overrightarrow{t_{i}}\right)=\vec{c}$. Furthermore, due to the facts that $\mathcal{M}$ is a GLAV mapping and $\mathcal{O}$ is a DL-Lite $\mathcal{R}_{\mathcal{R}}$ ontology, and due to the fact that $\vec{c}$ is in the evaluation of $q_{\mathcal{O}}^{i}$ over $\mathcal{C}_{\mathcal{O}}^{\mathcal{M}(D)}$ (i.e., $\vec{c} \in q_{\mathcal{O}}^{i}{ }^{\mathcal{C}_{\mathcal{O}}^{\mathcal{M}}(D)}$ ), by construction of $q_{\mathcal{O}}^{i}$ and $D_{i}$ it is easy to see the existence of a function $f$ from $\mathcal{C}_{\mathcal{O}}^{\mathcal{M}\left(D_{i}\right)}$ to $\mathcal{C}_{\mathcal{O}}^{\mathcal{M}(D)}$ for which (i) $f(c)=h(c)=c$ for each constant $c$ occurring in $q_{\mathcal{O}}^{i}$, (ii) $f\left(c_{v}\right)=h(v)$ for each variable $v \in \overrightarrow{x_{i}} \cup \overrightarrow{y_{i}}$ of $q_{\mathcal{S}}^{i}$ occurring in $\mathcal{M}\left(q_{\mathcal{S}}^{i}\right)$, and $(i i i) f\left(\mathcal{C}_{\mathcal{O}}^{\mathcal{M}\left(D_{i}\right)}\right) \subseteq \mathcal{C}_{\mathcal{O}}^{\mathcal{M}(D)}$, where $f\left(\mathcal{C}_{\mathcal{O}}^{\mathcal{M}\left(D_{i}\right)}\right)$ is the image of $\mathcal{C}_{\mathcal{O}}^{\mathcal{M}\left(D_{i}\right)}$ under $f$. Observe that $f\left(\overrightarrow{c^{i}}\right)=\vec{c}$, and, since $D$ is consistent with $\Sigma, D_{i}$ is consistent with $\Sigma$ as well.

Due to the existence of this function $f$ and the assumption that $\vec{c} \notin \operatorname{cert}_{q_{\mathcal{O}}^{\prime}, \Sigma}^{D}$, we derive that there is no disjunct $q^{\prime}=\left\{\overrightarrow{t^{\prime}} \mid \exists y^{\prime} \cdot \phi^{\prime}\left(\overrightarrow{x^{\prime}}, \overrightarrow{y^{\prime}}\right)\right\}$ of $q_{\mathcal{O}}^{\prime}$ for which there is a homomorphism $h^{\prime}$ from $q^{\prime}$ to $\mathcal{C}_{\mathcal{O}}^{\mathcal{M}\left(D_{i}\right)}$ such that $h^{\prime}\left(\overrightarrow{t^{\prime}}\right)=\overrightarrow{c^{i}}$ (otherwise, the composition function $f \circ h^{\prime}$ would result in a homomorphism from $q^{\prime}$ to $\mathcal{C}_{\mathcal{O}}^{\mathcal{M}(D)}$ such that $f\left(h^{\prime}\left(\overrightarrow{t^{\prime}}\right)\right)=\vec{c}$, and therefore this would contradict the assumption that $\left.\vec{c} \notin \operatorname{cert}_{q_{\mathcal{O}}^{\prime}, \Sigma}^{D}\right)$. Thus, $\overrightarrow{c^{i}} \notin \operatorname{cert}_{{q_{\mathcal{O}}^{\prime}, \Sigma}^{\prime}}^{D_{i}}$ as well. To conclude the proof, observe that $D_{i}$ is an $\mathcal{S}$-database consistent with $\Sigma$ for which $\overrightarrow{c^{i}} \in q_{\mathcal{S}}^{i^{D_{i}}}$ (and so $\overrightarrow{c^{i}} \in q_{\mathcal{S}}^{D_{i}}$ ) and $\overrightarrow{c^{i}} \notin \operatorname{cert}_{q_{\mathcal{O}}^{\prime}, \Sigma}^{D_{i}}$, thus implying that $q_{\mathcal{O}}^{\prime}$ is not a complete $\mathcal{S}$-to- $\mathcal{O} \Sigma$-rewriting of $q_{\mathcal{S}}$.

The following result is an immediate consequence of the above theorem.

Corollary 5.1. The unique (up to equivalence w.r.t. $\Sigma$ ) UCQ-minimally complete $\mathcal{S}$-to-O $\Sigma$-rewriting of $q_{\mathcal{S}}$ always exists. Furthermore, if $q_{\mathcal{S}}$ is a $C Q$, then it can be expressed as a $C Q$ as well.

Regarding the cost of the MinimallyComplete algorithm, we observe that, essentially, it applies the chase of each (possibly incomplete source instance associated to the) disjunct of $q_{\mathcal{S}}$ via the conjunction of atoms that appear in the left-hand side of the assertions in $\mathcal{M}$. This results in a running time that does not depend on $\mathcal{O}$ and $\mathcal{S}$, is exponential in $\sigma(\mathcal{M})$, and only polynomial in $\sigma\left(q_{\mathcal{S}}\right)$.

Notice, however, that if $\mathcal{M}$ is a LAV mapping, then the application of the chase is feasible in polynomial time even in $\sigma(\mathcal{M})$ (indeed, in this case there is no conjunction of atoms to evaluate when applying the chase), and therefore the running time of the algorithm becomes polynomial in the size of the input of the problem.

Conversely, even in the case of pure GAV mappings, next we show that a polynomial time algorithm for computing UCQ-minimally complete source-to-ontology rewritings already of CQJFEs would imply a polynomial time algorithm for checking whether $q_{1} \sqsubseteq q_{2}$, where $q_{1}$ is a CQJFE and $q_{2}$ is a CQ. Since we also show that this latter problem is NP-hard, it turns out that, unless PTIME $=\mathrm{NP}$, the computation problem for complete source-to-ontology rewritings can not be solved in polynomial time, even in the case of pure GAV mappings $\mathcal{M}$ and CQJFEs $q_{\mathcal{S}}$.

We start by proving the following lemma. 
Lemma 5.2. If $q_{1}$ is a UCQJFE and $q_{2}$ is a UCQ, then the problem of checking whether $q_{1} \sqsubseteq q_{2}$ is NP-complete.

Proof. The membership in NP follows from the membership in NP of the more general case of containment between UCQs Sagiv and Yannakakis, 1980.

We now show that the problem is NP-hard even when $q_{1}$ is a boolean CQJFE, $q_{2}$ is a boolean CQ, and both $q_{1}$ and $q_{2}$ use only binary predicates. We follow a proof strategy that is similar to the reduction illustrated in Aho et al., 1979. In particular, we provide a LOGSPACE reduction from the 3-CNF problem, which is NP-complete Karp, 1972. 3-CNF is the problem of deciding, given a CNF formula $F=c_{1} \wedge \ldots \wedge c_{m}$ on a set of variables $Y=\left\{y_{1}, \ldots, y_{n}\right\}$, whether $F$ is satisfiable, that is, whether there exists a truth assignment $V=\left\{v_{1}, \ldots, v_{n}\right\}$ to the variables in $Y$ that satisfies $F$. Each clause $c_{i}$ is a disjunction of three literals, where each literal is either a variable $x \in X$ or its negated. For $i=1, \ldots, m$, we denote by $z_{i, 1}, z_{i, 2}$, and $z_{i, 3}$ the first, the second, and the third, respectively, variable appearing (either positive or negated) in clause $c_{i}$.

Let $F$ be an instance of the 3 -CNF problem. We now define a boolean CQJFE $q_{1}$ and a boolean CQ $q_{2}$, where both queries use only binary predicates.

As for $q_{1}$, it is the conjunction of the atoms appearing in its body: for each clause $c_{i}$ of $F$, and for each of the seven satisfying truth assignments $A_{i, k}=\left\{v_{1}, v_{2}, v_{3}\right\}$ for $c_{i}$ (where, for each $k=1, \ldots, 7, A_{i, k}$ is a constant, and, for each $j=1,2,3$, $v_{j}$ is either the constant 1 or 0$)$, the body of $q_{1}$ contains the atoms $s_{i, 1}\left(A_{i, k}, v_{1}\right)$, $s_{i, 2}\left(A_{i, k}, v_{2}\right)$, and $s_{i, 3}\left(A_{i, k}, v_{3}\right)$.

As for $q_{2}$, it is the conjunction of the atoms appearing in its body: for each clause $c_{i}$ of $F$, the body of $q_{2}$ contains the atoms $s_{i, 1}\left(a_{i}, z_{i, 1}\right), s_{i, 2}\left(a_{i}, z_{i, 2}\right)$, and $s_{i, 3}\left(a_{i}, z_{i, 3}\right)$, where $a_{i}$ denotes a fresh existential variable.

To illustrate the reduction, consider the formula $F=\left(y_{1} \vee y_{2} \vee \neg y_{3}\right) \wedge\left(\neg y_{1} \vee\right.$ $\left.y_{2} \vee \neg y_{4}\right)$. In this case, the reduction produces the following boolean CQJFE

$$
\begin{aligned}
q_{1}=\{() \mid & \alpha_{1}\left(A_{1,1}, 0,0,0\right) \wedge \alpha_{1}\left(A_{1,2}, 0,1,0\right) \wedge \alpha_{1}\left(A_{1,3}, 0,1,1\right) \wedge \alpha_{1}\left(A_{1,4}, 1,0,0\right) \wedge \\
& \alpha_{1}\left(A_{1,5}, 1,0,1\right) \wedge \alpha_{1}\left(A_{1,6}, 1,1,0\right) \wedge \alpha_{1}\left(A_{1,7}, 1,1,1\right) \wedge \alpha_{2}\left(A_{2,1}, 0,0,0\right) \wedge \\
& \alpha_{2}\left(A_{2,2}, 0,0,1\right) \wedge \alpha_{2}\left(A_{2,3}, 0,1,0\right) \wedge \alpha_{2}\left(A_{2,4}, 0,1,1\right) \wedge \alpha_{2}\left(A_{2,5}, 1,0,0\right) \wedge \\
& \left.\alpha_{2}\left(A_{2,6}, 1,1,0\right) \wedge \alpha_{2}\left(A_{2,7}, 1,1,1\right)\right\}
\end{aligned}
$$

where an atom of the form $\alpha_{i}(x, y, z, w)$ is a shortcut for the conjunction of atoms $s_{i, 1}(x, y) \wedge s_{i, 2}(x, z) \wedge s_{i, 3}(x, w)$.

Moreover, the reduction produces the following boolean CQ

$$
\begin{aligned}
q_{2}=\left\{() \mid \exists a_{1}, a_{2}, y_{1}, y_{2}, y_{3}, y_{4} \cdot s_{1,1}\left(a_{1}, y_{1}\right)\right. & \wedge s_{1,2}\left(a_{1}, y_{2}\right) \wedge s_{1,3}\left(a_{1}, y_{3}\right) \wedge \\
s_{2,1}\left(a_{2}, y_{1}\right) & \left.\wedge s_{2,2}\left(a_{2}, y_{2}\right) \wedge s_{2,3}\left(a_{2}, y_{4}\right)\right\}
\end{aligned}
$$

With the same arguments given in Aho et al., 1979, it is possible to prove that every truth assignment satisfying formula $F$ consists in a homomorphism from $q_{2}$ to $q_{1}$ and vice versa.

In particular, from every truth assignment $V=\left\{v_{1}, \ldots, v_{n}\right\}$ to the variables in $Y$ that satisfies $F$ it is possible to derive a homomorphism $h$ from $q_{2}$ to $q_{1}$ in the following way: $(i) h\left(x_{j}\right)=v_{j}$ for each $j \in[1, n]$, and $(i i) h\left(a_{i}\right)=A_{i, k}$ for each $i \in[1, m]$, where 
$k \in[1,7]$ is an arbitrary value for which $\alpha_{i}\left(A_{i, k}, h\left(z_{i, 1}\right), h\left(z_{i, 2}\right), h\left(z_{i, 3}\right)\right)$ is an atom of $q_{1}$ (observe that, since by assumption clause $c_{i}$ is satisfied under truth assignment $V$, at least one constant $A_{i, k}$ for some $k \in[1,7]$ must exists by construction).

Also, from every homomorphism $h$ from $q_{2}$ to $q_{1}$, the truth assignment $V=$ $\left\{h\left(y_{1}\right), \ldots, h\left(y_{n}\right)\right\}$ satisfies formula $F$ by construction.

From the above considerations, it follows that $F$ is satisfiable if and only if $q_{1} \sqsubseteq q_{2}$. To conclude the proof, we observe that both the boolean CQJFE $q_{1}$ and the boolean CQ $q_{2}$ can be constructed in LOGSPACE from $F$.

Proposition 5.1. Assuming PTime $\subsetneq N P$, the computation problem for complete source-to-ontology rewritings can not be solved in polynomial time.

Proof. Let $q_{1}=\left\{() \mid \exists \overrightarrow{y_{1}} \cdot \psi\left(\overrightarrow{y_{1}}\right)\right\}$ and $q_{2}=\left\{() \mid \exists \overrightarrow{y_{2}} \cdot \phi\left(\overrightarrow{y_{2}}\right)\right\}$ be a boolean CQJFE and a boolean $\mathrm{CQ}$, respectively. We define an OBDM specification $\Sigma=\langle\mathcal{O}, \mathcal{S}, \mathcal{M}\rangle$ as follows: ontology $\mathcal{O}$ contains no axioms, schema $\mathcal{S}$ is composed of all predicates involved in $\psi\left(\overrightarrow{y_{1}}\right)$ and in $\phi\left(\overrightarrow{y_{2}}\right)$ plus an additional fresh unary predicate $s$, and finally the mapping $\mathcal{M}$ comprises only the following pure GAV mapping assertion:

$$
\exists \overrightarrow{y_{2}} \cdot s(x) \wedge \phi\left(\overrightarrow{y_{2}}\right) \rightarrow A(x),
$$

where $A$ is an atomic concept of $\mathcal{O}$.

We also define a boolean CQJFE over $\mathcal{S}: q_{\mathcal{S}}=\left\{() \mid \exists \overrightarrow{y_{1}} \cdot \exists y \cdot s(y) \wedge \psi\left(\overrightarrow{y_{1}}\right)\right\}$, where $y$ denotes a fresh existential variable occurring neither in $\overrightarrow{y_{1}}$ nor in $\overrightarrow{y_{2}}$.

Note that the unique (up to equivalence w.r.t. $\Sigma$ ) UCQ-minimally complete $\mathcal{S}$-to- $\mathcal{O} \Sigma$-rewriting of $q_{\mathcal{S}}$ is either the query $q_{\mathcal{O}}=\{() \mid \exists y \cdot A(y)\}$ if it is a complete $\mathcal{S}$-to- $\mathcal{O} \Sigma$-rewriting of $q_{\mathcal{S}}$, or the query $q_{\mathcal{O}}^{\prime}=\{() \mid \exists y \cdot \top(y)\}$.

Specifically, we now prove that $q_{\mathcal{O}}$ is the unique (up to equivalence w.r.t. $\Sigma$ ) UCQminimally complete $\mathcal{S}$-to- $\mathcal{O} \Sigma$-rewriting if and only if $q_{1} \sqsubseteq q_{2}$. Due to Lemma 5.1, $q_{\mathcal{O}}$ is a complete $\mathcal{S}$-to- $\mathcal{O} \Sigma$-rewriting of $q_{\mathcal{S}}$ if and only if $q_{\mathcal{S}} \sqsubseteq \operatorname{PerfRef}_{q_{\mathcal{O}}, \Sigma} \cup \operatorname{PerfRef}_{\mathcal{V}_{\mathcal{O}}, \Sigma}$. Since $\mathcal{V}_{\mathcal{O}} \equiv \perp$, in this case we have that $q_{\mathcal{O}}$ is a complete $\mathcal{S}$-to- $\mathcal{O} \Sigma$-rewriting of $q_{\mathcal{S}}$ if and only if $q_{\mathcal{S}} \sqsubseteq \operatorname{PerfRef}_{q_{\mathcal{O}}, \Sigma}$. Notice, however, that PerfRef $\operatorname{Ro}_{q_{\mathcal{O}}, \Sigma}=\{() \mid$ $\left.\exists \overrightarrow{y_{2}} \cdot \exists y \cdot s(y) \wedge \phi\left(\overrightarrow{y_{2}}\right)\right\}$, and therefore $q_{\mathcal{S}} \sqsubseteq \operatorname{PerfRef}_{q_{\mathcal{O}}, \Sigma}$ if and only if $q_{1} \sqsubseteq q_{2}$.

We have reduced the problem of checking whether $q_{1} \sqsubseteq q_{2}$ for a boolean CQJFE $q_{1}$ and a boolean CQ $q_{2}$ to the problem of computing the unique (up to equivalence w.r.t. $\Sigma$ ) UCQ-minimally complete $\mathcal{S}$-to- $\mathcal{O} \Sigma$-rewriting of a CQJFE $q_{\mathcal{S}}$, where both $\Sigma=\langle\mathcal{O}, \mathcal{S}, \mathcal{M}\rangle$ and $q_{\mathcal{S}}$ can be constructed in LOGSPACE from $q_{1}$ and $q_{2}$.

Thus, a polynomial time algorithm for computing UCQ-minimally complete source-to-ontology rewritings of CQJFEs $q_{\mathcal{S}}$ would imply a polynomial time algorithm for checking whether $q_{1} \sqsubseteq q_{2}$, where $q_{1}$ is a CQJFE and $q_{2}$ is a CQ. Since by Lemma 5.2 we know that this latter containment problem is in general NP-hard, it follows that, unless PTIME $=\mathrm{NP}$, the computation problem for complete source-toontology rewritings can not be solved in polynomial time, even in the case of pure GAV mappings $\mathcal{M}$ and CQJFEs $q_{\mathcal{S}}$.

\subsection{Improving by means of Inequalities}

In this section, we consider an extension of the scenario introduced in Section 3.2 in which $\mathcal{L}_{\mathcal{O}}$ is the class of $\mathrm{UCQ}^{\neq} \mathrm{s}$ rather than UCQs. Specifically, we tackle the problem of computing $\mathrm{UCQ}^{\neq}$-minimally complete source-to-ontology rewritings. 
The next example shows that the usage of inequalities is an interesting feature to consider when providing abstractions of data services. In general, $\mathrm{UCQ}^{\neq}{ }_{\mathrm{s}}$ provide better approximated complete source-to-ontology rewritings compared to UCQs.

Example 5.2. Let $\Sigma=\langle\mathcal{O}, \mathcal{S}, \mathcal{M}\rangle$ be the following OBDM specification:

- $\mathcal{O}=\emptyset$

- $\mathcal{S}=\left\{s_{1}, s_{2}\right\}$

- $\mathcal{M}=\left\{m_{1}, m_{2}, m_{3}\right\}$, where:

$$
\begin{aligned}
& m_{1}: s_{1}\left(x_{1}, x_{2}\right) \quad \rightarrow \quad P\left(x_{1}, x_{2}\right), \\
& m_{2}: \quad s_{1}(x, x) \rightarrow A(x), \\
& m_{3}: s_{2}\left(x_{1}, x_{2}\right) \quad \rightarrow \quad P\left(x_{1}, x_{2}\right) .
\end{aligned}
$$

Let the query over $\mathcal{S}$ be the CQ $q_{\mathcal{S}}=\left\{\left(x_{1}, x_{2}\right) \mid s_{1}\left(x_{1}, x_{2}\right)\right\}$.

Then, MinimallyComplete $\left(\Sigma, q_{\mathcal{S}}\right)$ returns the CQ $q_{\mathcal{O}}=\left\{\left(x_{1}, x_{2}\right) \mid P\left(x_{1}, x_{2}\right)\right\}$, which is the unique (up to equivalence w.r.t. $\Sigma$ ) UCQ-minimally complete $\mathcal{S}$-to- $\mathcal{O}$ $\Sigma$-rewriting of $q_{\mathcal{S}}$. Consider, however, the $\mathrm{UCQ}^{\neq} q_{\mathcal{O}}^{\prime}=\left\{\left(x_{1}, x_{2}\right) \mid P\left(x_{1}, x_{2}\right) \wedge x_{1} \neq\right.$ $\left.x_{2}\right\} \cup\{(x, x) \mid P(x, x) \wedge A(x)\}$. Consider any $\mathcal{S}$-database $D$ and any tuple $\left(c_{1}, c_{2}\right) \in q_{\mathcal{S}}^{D}$. If $c_{1} \neq c_{2}$, then $\left(c_{1}, c_{2}\right) \in \operatorname{cert}_{q_{\mathcal{O}}^{\prime}, \Sigma}^{D}$ because of the first disjunct. Conversely, if $c_{1}=c_{2}$, then $\left(c_{1}, c_{2}\right) \in \operatorname{cert}_{q_{\mathcal{O}}^{\prime}, \Sigma}^{D}$ because of the second disjunct. This clearly proves that $q_{\mathcal{O}}^{\prime}$ is a complete $\mathcal{S}$-to- $\mathcal{O} \Sigma$-rewriting of $q_{\mathcal{S}}$.

Furthermore, notice that $\operatorname{cert}_{q_{\mathcal{O}}^{\prime}, \Sigma} \sqsubset \operatorname{cert}_{q_{\mathcal{O}, \Sigma}}$ holds. On the one hand, $\operatorname{cert}_{q_{\mathcal{O}}^{\prime}, \Sigma} \sqsubseteq$ cert $_{q_{\mathcal{O}}, \Sigma}$ is trivially true. On the other hand, for the $\mathcal{S}$-database $D=\left\{s_{2}(c, c)\right\}$, we have $\operatorname{cert}_{q_{\mathcal{O}}^{\prime}, \Sigma}^{D}=\emptyset$, whereas $\operatorname{cert}_{q_{\mathcal{O}}, \Sigma}^{D}=\{(c, c)\}$, and therefore $\operatorname{cert}_{q_{\mathcal{O}}^{\prime}, \Sigma}^{D} \subsetneq \operatorname{cert}_{q_{\mathcal{O}}, \Sigma}^{D}$. Thus, $q_{\mathcal{O}}^{\prime}$ is a better complete approximation of $q_{\mathcal{S}}$ (w.r.t. $\Sigma$ ) compared to $q_{\mathcal{O}}$.

The above example also suggests an algorithm for computing $\mathrm{UCQ}^{\neq}$-minimally complete source-to-ontology rewritings. Informally, before of chasing (the incomplete $\mathcal{S}$-database associated to) the various disjuncts $q_{\mathcal{S}}^{i}$ of $q_{\mathcal{S}}$ with respect to $\mathcal{M}$, the basic idea is to first compute the so-called inequality saturation of $q_{\mathcal{S}}$ with respect to $\operatorname{conL}_{\mathcal{M}}$, and only after chasing each (incomplete $\mathcal{S}$-database associated to the) disjunct of the obtained query. Here, for a mapping $\mathcal{M}$, conL $\mathrm{M}_{\mathcal{M}}$ denotes the set of all constants occurring in the left-hand side of mapping assertions in $\mathcal{M}$.

Roughly speaking, the inequality saturation of a UCQ $q_{\mathcal{S}}$ with respect to a set of constants con is a $\mathrm{UCQ}^{\neq}$obtained by replacing each $\mathrm{CQ} q_{\mathcal{S}}^{i}$ of $q_{\mathcal{S}}$ with an equivalent $\mathrm{UCQ}^{\neq}$. This latter is obtained from $q_{\mathcal{S}}^{i}$ by computing a $\mathrm{CQ}^{\neq}$for each possible unification between the terms in $\operatorname{ter}\left(q_{\mathcal{S}}^{i}\right) \cup$ con, and then imposing inequalities between the syntactically different remaining variables. Here, for a CQ $q$, $\operatorname{ter}(q)$ denotes the set of all terms (i.e., constants and variables) occurring in $q$.

We now present the algorithm Saturate that, given a UCQ $Q$ and a set of constants con, returns a logically equivalent $\mathrm{UCQ}^{\neq}$representing the inequality saturation of $Q$ with respect to con. Essentially, the core of the algorithm is the one illustrated in Lembo et al., 2015], but extended in order to deal with a set of constants con given as input. We include below the algorithm for the sake of completeness.

In the algorithm, two terms $t_{1}$ and $t_{2}$ are compatible if $t_{1}$ and $t_{2}$ denote distinct terms and at least one of them is a variable. Furthermore, for a query $q, q\left[t_{1} / t_{2}\right]$ 
denotes the query obtained from $q$ by replacing every occurrence (even in the target list) of the term $t_{1}$ in $q$ with the term $t_{2}$ (obviously, if one of the two terms is a constant, then we always assume that $t_{2}$ is the constant and $t_{1}$ is the variable).

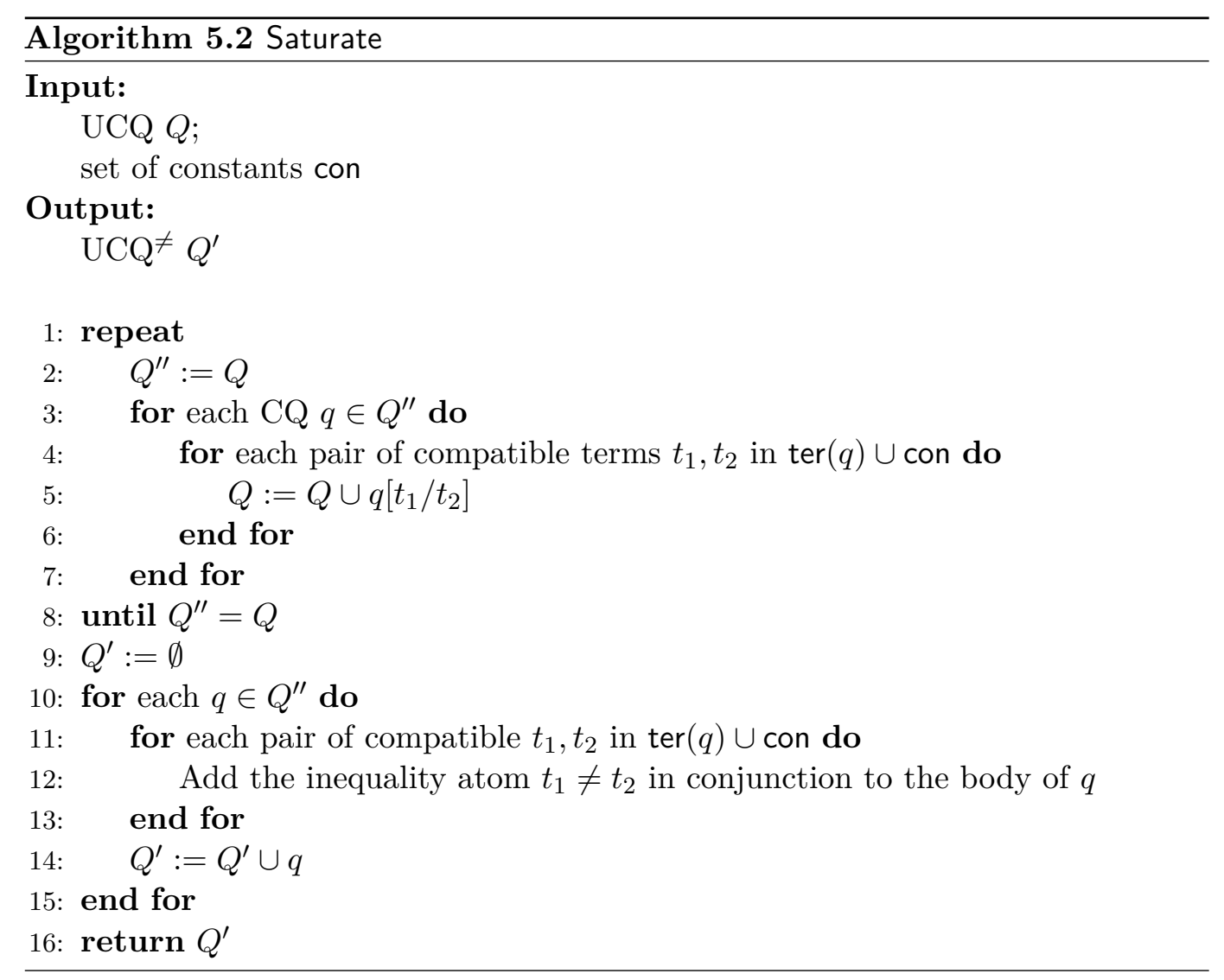

For a UCQ $Q$ and a set of constants con, Saturate $(Q$, con) first computes the UCQ $Q^{\prime \prime}$ by unifying compatible terms in each query $q \in Q$ in all possible ways. Then, for any query $q \in Q^{\prime \prime}$ and for each pair of terms $t_{1}$ and $t_{2}$ that are syntactically different and compatible, it adds the inequality atom $t_{1} \neq t_{2}$ to the body of $q$.

The following example illustrates an execution of the Saturate algorithm.

Example 5.3. Let $q_{\mathcal{S}}$ be the UCQ $q_{\mathcal{S}}=q_{\mathcal{S}}^{1} \cup q_{\mathcal{S}}^{2}$, where $q_{\mathcal{S}}^{1}=\left\{(x) \mid \exists y . s_{1}(x, y)\right\}$ and $q_{\mathcal{S}}^{2}=\left\{(x) \mid s_{2}\left(x, c_{2}\right)\right\}$, and let con $=\left\{c_{1}\right\}$. One can verify that Saturate $\left(q_{\mathcal{S}}\right.$, con $)$ returns the $\mathrm{UCQ}^{\neq} Q^{\prime}=q_{\mathcal{S}}^{1,1} \cup q_{\mathcal{S}}^{1,2} \cup q_{\mathcal{S}}^{1,3} \cup q_{\mathcal{S}}^{1,4} \cup q_{\mathcal{S}}^{1,5} \cup q_{\mathcal{S}}^{2,1} \cup q_{\mathcal{S}}^{2,2} \cup q_{\mathcal{S}}^{2,3}$, where:

- $q_{\mathcal{S}}^{1,1}=\left\{(x) \mid \exists y \cdot s_{1}(x, y) \wedge x \neq y \wedge x \neq c_{1} \wedge y \neq c_{1}\right\}$

- $q_{\mathcal{S}}^{1,2}=\left\{(x) \mid s_{1}(x, x) \wedge x \neq c_{1}\right\}$

- $q_{\mathcal{S}}^{1,3}=\left\{\left(c_{1}\right) \mid \exists y \cdot s_{1}\left(c_{1}, y\right) \wedge y \neq c_{1}\right\}$

- $q_{\mathcal{S}}^{1,4}=\left\{(x) \mid s_{1}\left(x, c_{1}\right) \wedge x \neq c_{1}\right\}$;

- $q_{\mathcal{S}}^{1,5}=\left\{\left(c_{1}\right) \mid s_{1}\left(c_{1}, c_{1}\right)\right\}$;

- $q_{\mathcal{S}}^{2,1}=\left\{(x) \mid s_{2}\left(x, c_{2}\right) \wedge x \neq c_{1} \wedge x \neq c_{2}\right\}$; 
- $q_{\mathcal{S}}^{2,2}=\left\{\left(c_{1}\right) \mid s_{2}\left(c_{1}, c_{2}\right)\right\}$

- $q_{\mathcal{S}}^{2,3}=\left\{\left(c_{2}\right) \mid s_{2}\left(c_{2}, c_{2}\right)\right\}$

We are now ready to focus on the problem of computing $\mathrm{UCQ}^{\neq}$-minimally complete source-to-ontology rewritings, and present algorithm MinimallyCompletelneq.

In the algorithm, we write each $\mathrm{CQ}^{\neq} q$ as $q=\{\vec{t} \mid \exists \vec{y} \cdot \phi(\vec{x}, \vec{y}) \wedge \xi(\vec{x}, \vec{y})\}$, where $\phi(\vec{x}, \vec{y})$ is the conjunction of the atoms occurring in the body of $q$ that are not inequality atoms, whereas $\xi(\vec{x}, \vec{y})$ is the conjunction of all the inequality atoms occurring in the body of $q$. Furthermore, $\mathcal{M}(q)$ for a $\mathrm{CQ}^{\neq} q$ is computed by simply ignoring the inequality atoms of $q$, and thus treating it as a CQ. Finally, $\xi(\vec{x}, \overrightarrow{\mathcal{Y}})$ is the conjunction of the inequality atoms obtained from $\xi(\vec{x}, \vec{y})$ by removing all those atoms of the form $y \neq t$ and $t \neq y$ in which $y$ is an existential variable occurring in $\vec{y}$ but not in $\overrightarrow{\mathcal{Y}}$ (i.e., not in $\mathcal{M}(q)$ ), and $t$ is any other possible term.

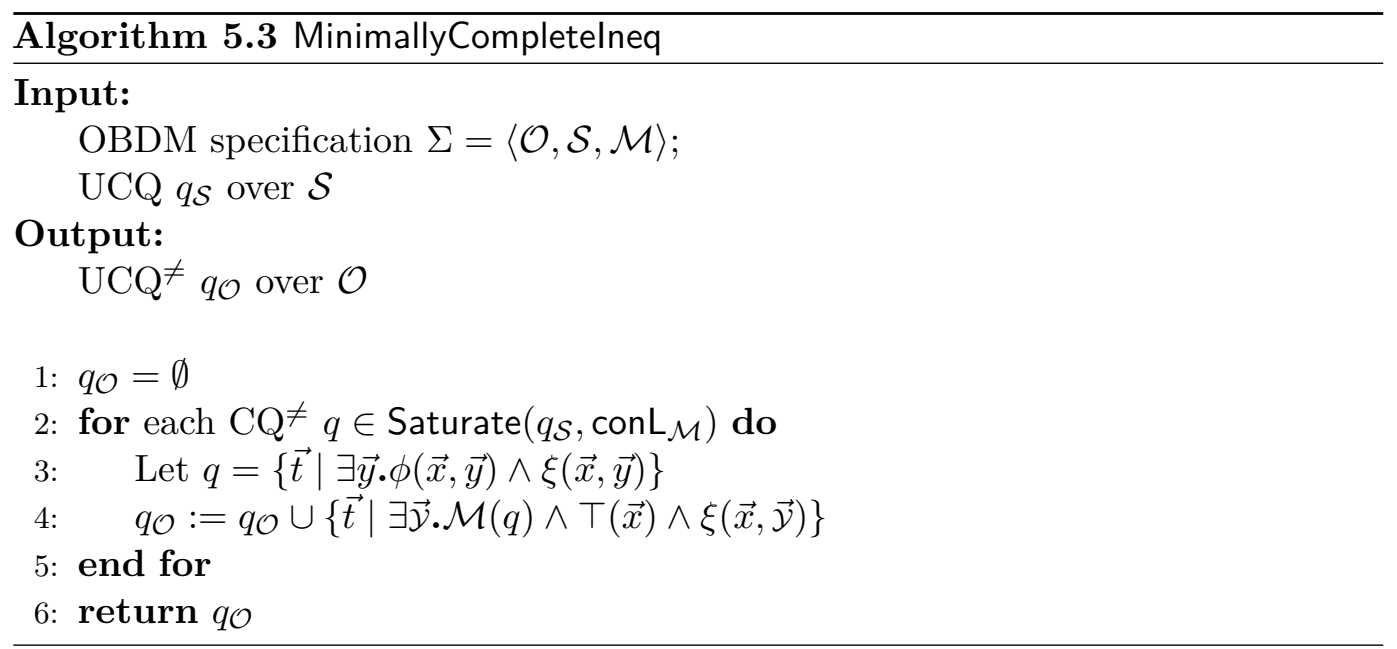

Informally, the algorithm first computes the inequality saturation of $q_{\mathcal{S}}$ with respect to $\operatorname{con} L_{\mathcal{M}}$. Then, for each $\mathrm{CQ}^{\neq} q \in \operatorname{Saturate}\left(q_{\mathcal{S}}, \operatorname{conL} \mathrm{M}_{\mathcal{M}}\right)$ the algorithm obtains a $\mathrm{CQ}^{\neq}$by chasing (the incomplete $\mathcal{S}$-database associated to) the conjunctive part of $q$ with respect to $\mathcal{M}$, using $\top$ to bind the distinguished variables that are not involved in the application of $\mathcal{M}$, and adding the inequality atoms of $q$ to the so obtained query, where the inequality atoms involving existential variables not appearing in $\mathcal{M}(q)$ are removed. Finally, the output $\mathrm{UCQ}^{\neq} q_{\mathcal{O}}$ is the union of all such $\mathrm{CQ}^{\neq} \mathrm{s}$.

Example 5.4. Let $\Sigma=\langle\mathcal{O}, \mathcal{S}, \mathcal{M}\rangle$ be the following OBDM specification:

- $\mathcal{O}=\emptyset$

- $\mathcal{S}=\left\{s_{1}, s_{2}, s_{3}\right\}$

- $\mathcal{M}=\left\{m_{1}, m_{2}, m_{3}, m_{4}, m_{5}, m_{6}\right\}$, where: 


$$
\begin{aligned}
& m_{1}: \exists y \cdot s_{1}(x, y) \rightarrow \text { AdvertisingCompany }(x), \\
& m_{2}: \quad s_{1}\left(c_{1}, x\right) \rightarrow \text { PublicCompany }(x), \\
& m_{3}: \quad s_{2}\left(x_{1}, x_{2}\right) \rightarrow \operatorname{Controls}\left(x_{1}, x_{2}\right), \\
& m_{4}: \quad s_{2}(x, x) \rightarrow \text { SelfHoldingCompany }(x), \\
& m_{5}: \quad s_{3}\left(x_{1}, x_{2}\right) \rightarrow \operatorname{Controls}\left(x_{1}, x_{2}\right) ; \\
& m_{6}: \quad s_{4}(x) \rightarrow \text { AdvertisingCompany }(x) \text {. }
\end{aligned}
$$

Let $q_{\mathcal{S}}$ be the UCQ illustrated in Example 5.3. Since $\operatorname{conL}_{\mathcal{M}}=\left\{c_{1}\right\}$, Saturate $\left(q_{\mathcal{S}}, \operatorname{conL}_{\mathcal{M}}\right)$ is the $\mathrm{UCQ}^{\neq} Q^{\prime}=q_{\mathcal{S}}^{1,1} \cup q_{\mathcal{S}}^{1,2} \cup q_{\mathcal{S}}^{1,3} \cup q_{\mathcal{S}}^{1,4} \cup q_{\mathcal{S}}^{1,5} \cup q_{\mathcal{S}}^{2,1} \cup q_{\mathcal{S}}^{2,2} \cup q_{\mathcal{S}}^{2,3}$ illustrated in Example 5.3. One can therefore verify that MinimallyCompletelneq $\left(\Sigma, q_{\mathcal{S}}\right)$ returns the $\mathrm{UCQ}^{\neq} q_{\mathcal{O}}=q_{\mathcal{O}}^{1,1} \cup q_{\mathcal{O}}^{1,2} \cup q_{\mathcal{O}}^{1,3} \cup q_{\mathcal{O}}^{1,4} \cup q_{\mathcal{O}}^{1,5} \cup q_{\mathcal{O}}^{2,1} \cup q_{\mathcal{O}}^{2,2} \cup q_{\mathcal{O}}^{2,3}$, where:

- $q_{\mathcal{O}}^{1,1}=q_{\mathcal{O}}^{1,2}=\left\{(x) \mid\right.$ AdvertisingCompany $\left.(x) \wedge x \neq c_{1}\right\}$

- $q_{\mathcal{O}}^{1,3}=\left\{\left(c_{1}\right) \mid \exists y\right.$.AdvertisingCompany $\left.\left(c_{1}\right) \wedge \operatorname{PublicCompany}(y) \wedge y \neq c_{1}\right\}$

- $q_{\mathcal{O}}^{1,4}=\left\{(x) \mid\right.$ AdvertisingCompany $\left.(x) \wedge x \neq c_{1}\right\}$;

- $q_{\mathcal{O}}^{1,5}=\left\{\left(c_{1}\right) \mid \operatorname{AdvertisingCompany}\left(c_{1}\right) \wedge \operatorname{PublicCompany}\left(c_{1}\right)\right\}$

- $q_{\mathcal{O}}^{2,1}=\left\{(x) \mid \operatorname{Controls}\left(x, c_{2}\right) \wedge x \neq c_{1} \wedge x \neq c_{2}\right\}$;

- $q_{\mathcal{O}}^{2,2}=\left\{\left(c_{1}\right) \mid \operatorname{Controls}\left(c_{1}, c_{2}\right)\right\}$

- $q_{\mathcal{S}}^{2,3}=\left\{\left(c_{2}\right) \mid \operatorname{Controls}\left(c_{2}, c_{2}\right) \wedge \operatorname{SelfHoldingCompany}\left(c_{2}\right)\right\}$,

which corresponds to the unique (up to equivalence w.r.t. $\Sigma$ ) $\mathrm{UCQ}^{\neq}$-minimally complete $\mathcal{S}$-to- $\mathcal{O} \Sigma$ rewriting of $q_{\mathcal{S}}$.

The following theorem establishes termination and correctness of the MinimallyCompletelneq algorithm.

Theorem 5.3. MinimallyCompletelneq $\left(\Sigma, q_{\mathcal{S}}\right)$ terminates and returns the unique (up to equivalence w.r.t. $\Sigma) U C Q^{\neq}$-minimally complete $\mathcal{S}$-to- $\mathcal{O} \Sigma$-rewriting of $q_{\mathcal{S}}$.

Proof. Termination of the algorithm follows from $(i)$ the termination of the chase of a source instance (possibly containing variables) with respect to a GLAV mapping; (ii) the termination of Saturate $\left(q_{\mathcal{S}}, \operatorname{con}_{\mathcal{M}}\right)$, which is guaranteed by the fact that $q_{\mathcal{S}}$ is a disjunction of a finite number of CQs in which there is a finite number of atoms and terms, and the fact that $\operatorname{con}_{\mathcal{M}}$ is a set of a finite number of constants.

As for the correctness, we first show that the computed $q_{\mathcal{O}}$ is a complete $\mathcal{S}$-to- $\mathcal{O}$ $\Sigma$-rewriting of $q_{\mathcal{S}}$. Let $\vec{c}=\left(c_{1}, \ldots, c_{n}\right)$ be any tuple of constants such that $\vec{c} \in q_{\mathcal{S}}^{D}$. We now prove that $\vec{c} \in \operatorname{cert}_{q_{\mathcal{O}}, \Sigma}^{D}$. Clearly, $\vec{c} \in q_{\mathcal{S}}^{D}$ implies the presence of a $\mathrm{UCQ}^{\neq}$ $q \in \operatorname{Saturate}\left(q_{\mathcal{S}}, \operatorname{conL}_{\mathcal{M}}\right)$ where $q=\{\vec{t} \mid \exists \vec{y} . \phi(\vec{x}, \vec{y}) \wedge \xi(\vec{x}, \vec{y})\}$ for which $\vec{c} \in q^{D}$, i.e., there is a homomorphism $h$ from the set of atoms occurring in $\phi(\vec{x}, \vec{y})$ to $D$ for which (i) $h(\vec{t})=\vec{c}$ and $(i i) h(\alpha) \neq h(\beta)$ for each inequality atom $\alpha \neq \beta$ occurring in $\xi(\vec{x}, \vec{y})$.

Consider now the $\mathrm{CQ}^{\neq} q^{\prime}=\{\vec{t} \mid \exists \overrightarrow{\mathcal{Y}} . \mathcal{M}(q) \wedge \top(\vec{x}) \wedge \xi(\vec{x}, \overrightarrow{\mathcal{Y}})\}$, which is by construction a disjunct of $q_{\mathcal{O}}$. Clearly, due to the existence of $h$, there must be a homomorphism $h^{\prime}$ from the set of all atoms occurring in $\mathcal{M}(q)$ and $\top(\vec{x})$ to $\mathcal{M}(D)$ for which $h^{\prime}(t)=h(t)$ for each term $t$ occurring in $q$. This implies that $(i) h^{\prime}(\vec{t})=\vec{c}$; 
(ii) $h^{\prime}(y)$ is a constant for each existential variable of $q^{\prime}$ which was also in $q$; and (iii) since atoms in $\xi(\vec{x}, \overrightarrow{\mathcal{y}})$ are a subset of those in $\xi(\vec{x}, \vec{y})$, we have $h^{\prime}(\alpha) \neq h^{\prime}(\beta)$ for each inequality atom $\alpha \neq \beta$ occurring in $\xi(\vec{x}, \overrightarrow{\mathcal{Y}})$, where both $h^{\prime}(\alpha)$ and $h^{\prime}(\beta)$ are constants due to $(i i)$. Since we are adopting the UNA, we have that $\left(h^{\prime}(\alpha)\right)^{\mathcal{I}} \neq\left(h^{\prime}(\beta)\right)^{\mathcal{I}}$ for each inequality atom $\alpha \neq \beta$ occurring in $\xi(\vec{x}, \overrightarrow{\mathcal{Y}})$ and for each model $\mathcal{I} \in \operatorname{Mod}_{D}(\Sigma)$.

Due to the existence of homomorphism $h^{\prime}$ from atoms occurring in $\mathcal{M}(q)$ and $\top(\vec{x})$ to $\mathcal{M}(D)$ satisfying $(i),(i i)$, and (iii), and the above observation regarding the UNA, we derive that $\left(c_{1}^{\mathcal{I}}, \ldots, c_{n}^{\mathcal{I}}\right) \in q^{\mathcal{I}}$ for each model $\mathcal{I} \in \operatorname{Mod}_{D}(\Sigma)$. Therefore, by definition of certain answers, $\vec{c} \in \operatorname{cert}_{q^{\prime}, \Sigma}^{D}$. Thus, $\vec{c} \in \operatorname{cert}_{q_{\mathcal{O}, \Sigma},}^{D}$, as required.

We now show that $q_{\mathcal{O}}$ is actually the unique (up to equivalence w.r.t. $\Sigma$ ) $\mathrm{UCQ}^{\neq}$minimally complete $\mathcal{S}$-to- $\mathcal{O} \Sigma$-rewriting of $q_{\mathcal{S}}$, that is, each $\mathrm{UCQ}^{\neq} q_{\mathcal{O}}^{\prime}$ that is a complete $\mathcal{S}$-to- $\mathcal{O} \Sigma$-rewriting of $q_{\mathcal{S}}$ is such that $\operatorname{cert}_{q_{\mathcal{O}}, \Sigma} \sqsubseteq \operatorname{cert}_{q_{\mathcal{O}}^{\prime}, \Sigma}$ (cf. Definition 3.7). We do this by way of contradiction.

Let $q_{\mathcal{O}}^{\prime}$ be a $\mathrm{UCQ}^{\neq}$such that $\operatorname{cert}_{q_{\mathcal{O}}, \Sigma} \nsubseteq \operatorname{cert}_{q_{\mathcal{O}}^{\prime}, \Sigma}$, that is, there exists an $\mathcal{S}$ database $D$ consistent with $\Sigma$ such that $\operatorname{cert}_{q_{\mathcal{O}}, \Sigma}^{D} \nsubseteq \operatorname{cert}_{q_{\mathcal{O}}^{\prime}, \Sigma}^{D}$. It follows that there is a tuple of constant $\vec{c}=\left(c_{1}, \ldots, c_{n}\right)$ such that $\vec{c} \notin \operatorname{cert}_{q_{\mathcal{O}}^{\prime}, \Sigma}^{D}$, but $\vec{c} \in \operatorname{cert}_{q_{\mathcal{O}}, \Sigma}^{D}$. By the definition of certain answers, there exists a model $\mathcal{I} \in \operatorname{Mod}_{D}(\Sigma)$ such that $\left(c_{1}^{\mathcal{I}}, \ldots, c_{n}^{\mathcal{I}}\right) \notin q_{\mathcal{O}}^{\prime} \mathcal{I}$, whereas $\left(c_{1}^{\mathcal{I}}, \ldots, c_{n}^{\mathcal{I}}\right) \in q_{\mathcal{O}}^{\mathcal{I}}$. We now show the existence of an $\mathcal{S}$-database $D^{\prime}$ for which $(i) \vec{c} \in q_{\mathcal{S}}^{D^{\prime}}$, and $(i i) \mathcal{I} \in \operatorname{Mod}_{D^{\prime}}(\Sigma)$ (and therefore $\left.\vec{c} \notin \operatorname{cert}_{q_{\mathcal{O}}^{\prime}, \Sigma}^{D^{\prime}}\right)$, thus proving that $q_{\mathcal{O}}^{\prime}$ is not a complete $\mathcal{S}$-to- $\mathcal{O} \Sigma$-rewriting of $q_{\mathcal{S}}$.

Since $\left(c_{1}^{\mathcal{I}}, \ldots, c_{n}^{\mathcal{I}}\right) \in q_{\mathcal{O}}^{\mathcal{I}}$, there is a disjunct in $q_{\mathcal{O}}$ of the form $\{\vec{t} \mid \exists \overrightarrow{\mathcal{Y}} \cdot \mathcal{M}(q) \wedge$ $\top(\vec{x}) \wedge \xi(\vec{x}, \overrightarrow{\mathcal{Y}})\}$ for which there is a homomorphism $h$ from atoms occurring in $\mathcal{M}(q)$ and $\top(\vec{x})$ to $\mathcal{I}$ satisfying $(i) h(\vec{t})=\vec{c}$ and $(i i) h(\alpha) \neq h(\beta)$ for each inequality atom $\alpha \neq \beta$ occurring in $\xi(\vec{x}, \overrightarrow{\mathcal{Y}})$. Observe that $q \in \operatorname{Saturate}\left(q_{\mathcal{S}}, \operatorname{conL} \mathrm{M}_{\mathcal{M}}\right)$ is a $\mathrm{CQ}^{\neq}$of the form $\{\vec{t} \mid \exists \vec{y} . \phi(\vec{x}, \vec{y}) \wedge \xi(\vec{x}, \vec{y})\}$. Consider the $\mathcal{S}$-database $D^{\prime}=h^{\prime}(\phi(\vec{x}, \vec{y}))$, where $h^{\prime}$ extends $h$ by assigning a different fresh constant to each existential variable $y \in \vec{y}$ not occurring in $\overrightarrow{\mathcal{Y}}$ (i.e., not occurring in $\mathcal{M}(q)$ ).

Clearly, we have that $\vec{c} \in q^{D^{\prime}}$. Furthermore, due to the existence of the homomorphism $h$ from atoms occurring in $\mathcal{M}(q)$ and $\top(\vec{x})$ to $\mathcal{I}$ and by construction of $D^{\prime}$, we have $\left\langle D^{\prime}, \mathcal{I}\right\rangle \models \mathcal{M}$. Notice that this implies $\mathcal{I} \in \operatorname{Mod}_{D^{\prime}}(\Sigma)$. Indeed, on the one hand $\left\langle D^{\prime}, \mathcal{I}\right\rangle \models \mathcal{M}$, and, on the other hand, $\mathcal{I} \models \mathcal{O}$ due to the initial assumption that $\mathcal{I} \in \operatorname{Mod}_{D}(\Sigma)$. But then, $\vec{c} \in q^{D^{\prime}}$ for a query $q \in \operatorname{Saturate}\left(q_{\mathcal{S}}\right.$, conL $\left.L_{\mathcal{M}}\right)$ implies that $\vec{c} \in q_{\mathcal{S}}^{D^{\prime}}$, and $\left(c_{1}^{\mathcal{I}}, \ldots, c_{n}^{\mathcal{I}}\right) \notin q_{\mathcal{O}}^{\prime}{ }^{\mathcal{I}}$ for a model $\mathcal{I} \in \operatorname{Mod}_{D^{\prime}}(\Sigma)$ implies that $\vec{c} \notin \operatorname{cert}_{q_{\mathcal{O}}^{\prime}, \Sigma}^{D^{\prime}}$ It follows that, for the $\mathcal{S}$-database $D^{\prime}$ consistent with $\Sigma, \vec{c} \in q^{D^{\prime}}$ and $\vec{c} \notin \operatorname{cert}_{q_{\mathcal{O}}^{\prime}, \Sigma}^{D^{\prime}}$. Thus, $q_{\mathcal{O}}^{\prime}$ is not a complete $\mathcal{S}$-to- $\mathcal{O} \Sigma$-rewriting of $q_{\mathcal{S}}$, as required.

The following result is an immediate consequence of the above theorem.

Corollary 5.2. The unique (up to equivalence w.r.t. $\Sigma$ ) $U C Q^{\neq}$-minimally complete $\mathcal{S}$-to- $\mathcal{O} \Sigma$-rewriting of $q_{\mathcal{S}}$ always exists.

Differently from the MinimallyComplete algorithm, the running time of the MinimallyCompletelneq algorithm becomes exponential in $\sigma\left(q_{\mathcal{S}}\right)$. This is due to the computation of the inequality saturation of $q_{\mathcal{S}}$ with respect to con $L_{\mathcal{M}}$, which is done by means of the Saturate algorithm that, in general, even for a CQ $Q$ and an empty set of constants as input, it produces a number of queries that is exponential 
in the number of terms occurring in $Q$. As a result, the MinimallyCompletelneq algorithm returns, in general, a $\mathrm{UCQ}^{\neq}$having exponentially many disjuncts with respect to the number of terms occurring in $q_{\mathcal{S}}$. Finally, we point out that it is straightforward to construct cases where the number of disjuncts of the unique (up to equivalence w.r.t. $\Sigma$ ) $\mathrm{UCQ}^{\neq}$-minimally complete $\mathcal{S}$-to- $\mathcal{O} \Sigma$-rewriting of $q_{\mathcal{S}}$ is necessarily exponential in the number of terms occurring in $q_{\mathcal{S}}$.

\subsection{Dropping the UNA}

Let us now study complete source-to-ontology rewritings when the UNA is not adopted. Clearly, since DL-Lite $\mathcal{R}_{\mathcal{R}}$ is insensitive to the adoption of the UNA for UCQ answering, from Theorem 5.2 we easily derive the following corollary.

Corollary 5.3. When the UNA is not adopted, MinimallyComplete $\left(\Sigma, q_{\mathcal{S}}\right)$ terminates and returns the unique (up to equivalence w.r.t. $\Sigma$ ) UCQ-minimally complete $\mathcal{S}$-to- $\mathcal{O}$ $\Sigma$-rewriting of $q_{\mathcal{S}}$.

Differently from the UCQ case, the next example shows that a $\mathrm{UCQ}^{\neq} q_{\mathcal{O}}$ that is a complete $\mathcal{S}$-to- $\mathcal{O} \Sigma$-rewriting of $q_{\mathcal{S}}$ when the UNA is adopted is not necessarily so when the UNA is not adopted.

Example 5.5. Let $\Sigma=\langle\mathcal{O}, \mathcal{S}, \mathcal{M}\rangle, q_{\mathcal{S}}$, and $q_{\mathcal{O}}^{\prime}$ be the OBDM specification, the $\mathrm{CQ}$ over $\mathcal{S}$, and the $\mathrm{UCQ}^{\neq}$over $\mathcal{O}$, respectively, illustrated in Example 5.2 .

Consider the $\mathcal{S}$-database $D=\left\{s_{1}\left(c_{1}, c_{2}\right)\right\}$, and the interpretation $\mathcal{I}$ for $\langle\Sigma, D\rangle$ with $c_{1}^{\mathcal{I}}=c_{2}^{\mathcal{I}}=c, P^{\mathcal{I}}=\{(c, c)\}$, and $A^{\mathcal{I}}=\emptyset$. Clearly, by definition of mapping satisfaction (cf. Subsection 2.6.3), we have $\langle D, \mathcal{I}\rangle \models \mathcal{M}$. Since $\mathcal{I} \models \mathcal{O}$ trivially holds because $\mathcal{O}=\emptyset$, we have $\mathcal{I} \in \operatorname{Mod}_{D}(\Sigma)$ (when the UNA is not adopted). Notice, however, that $q_{\mathcal{O}}^{\prime}{ }^{\mathcal{I}}=\emptyset$ because the inequality atom of the first disjunct is not satisfied. Thus, $\left(c_{1}, c_{2}\right) \notin \operatorname{cert}_{q_{\mathcal{O}}^{\prime}, \Sigma}^{D}$, whereas $\left(c_{1}, c_{2}\right) \in q_{\mathcal{S}}^{D}$. Therefore, when the UNA is not adopted, $q_{\mathcal{O}}^{\prime}$ is not a complete $\mathcal{S}$-to- $\mathcal{O} \Sigma$-rewriting of $q_{\mathcal{S}}$.

As we will see, the unique (up to equivalence w.r.t. $\Sigma$ ) $\mathrm{UCQ}^{\neq}$-minimally complete $\mathcal{S}$-to- $\mathcal{O} \Sigma$-rewriting of $q_{\mathcal{S}}$ is the UCQ $q_{\mathcal{O}}$ illustrated again in Example 5.2 .

Quite interestingly, the next theorem proves that, when the UNA is not adopted, for each OBDM specification $\Sigma=\langle\mathcal{O}, \mathcal{S}, \mathcal{M}\rangle$ and query $q_{\mathcal{S}}$ of our scenario under consideration, the unique (up to equivalence w.r.t. $\Sigma$ ) $\mathrm{UCQ}^{\neq}$-minimally complete $\mathcal{S}$-to- $\mathcal{O} \Sigma$-rewriting of $q_{\mathcal{S}}$ always coincides with the unique (up to equivalence w.r.t. $\Sigma$ ) UCQ-minimally complete $\mathcal{S}$-to- $\mathcal{O} \Sigma$-rewriting of $q_{\mathcal{S}}$.

Theorem 5.4. When the UNA is not adopted, MinimallyComplete $\left(\Sigma, q_{\mathcal{S}}\right)$ terminates and returns the unique (up to equivalence w.r.t. $\Sigma$ ) $U C Q^{f}$-minimally complete $\mathcal{S}$-to- $\mathcal{O}$ $\Sigma$-rewriting of $q_{\mathcal{S}}$.

Proof. Termination is already proven in Theorem 5.2 .

The fact that the computed query $q_{\mathcal{O}}$ is a complete $\mathcal{S}$-to- $\mathcal{O} \Sigma$-rewriting of $q_{\mathcal{S}}$ when the UNA is not adopted follows from the facts that it is so when the UNA is adopted (cf. Theorem 5.2) and the insensitiveness of the UNA for UCQ answering. We now prove that, when the UNA is not adopted, $q_{\mathcal{O}}$ is actually the unique (up to equivalence w.r.t. $\Sigma$ ) $\mathrm{UCQ}^{\neq}$-minimally complete $\mathcal{S}$-to- $\mathcal{O} \Sigma$-rewriting of $q_{\mathcal{S}}$, 
that is, each $\mathrm{UCQ}^{\neq} q_{\mathcal{O}}^{\prime}$ that is a complete $\mathcal{S}$-to- $\mathcal{O} \Sigma$-rewriting of $q_{\mathcal{S}}$ is such that cert $_{q_{\mathcal{O}}, \Sigma} \sqsubseteq \operatorname{cert}_{q_{\mathcal{O}}^{\prime}, \Sigma}$ (cf. Definition 3.7). We do this by way of contradiction.

Let $q_{\mathcal{O}}^{\prime}$ be a $\mathrm{UCQ}^{\neq}$such that $\operatorname{cert}_{q_{\mathcal{O}}, \Sigma} \nsubseteq \operatorname{cert}_{q_{\mathcal{O}}^{\prime}, \Sigma}$, that is, there exists an $\mathcal{S}$ database $D$ consistent with $\Sigma$ such that $\operatorname{cert}_{q_{\mathcal{O}}, \Sigma}^{D} \not \operatorname{cert}_{q_{\mathcal{O}}^{\prime}, \Sigma}^{D}$. It follows that there is a tuple of constant $\vec{c}=\left(c_{1}, \ldots, c_{m}\right)$ such that $\vec{c} \notin \operatorname{cert}_{q_{\mathcal{O}}^{\prime}, \Sigma}^{D}$, but $\vec{c} \in \operatorname{cert}_{q_{\mathcal{O}}, \Sigma}^{D}$. By the definition of certain answers, there exists a model $\mathcal{I}=\left\langle\Delta^{\mathcal{I}},{ }^{\mathcal{I}}\right\rangle \in \operatorname{Mod}_{D}(\Sigma)$ such that $\left(c_{1}^{\mathcal{I}}, \ldots, c_{m}^{\mathcal{I}}\right) \notin q_{\mathcal{O}}^{\prime} \mathcal{I}$, whereas $\left(c_{1}^{\mathcal{I}}, \ldots, c_{n}^{\mathcal{I}}\right) \in q_{\mathcal{O}}^{\mathcal{I}}$. We now show the existence of an $\mathcal{S}$-database $D^{\prime}$ for which $(i) \vec{c} \in q_{\mathcal{S}}^{D^{\prime}}$, and $(i i) \mathcal{I} \in \operatorname{Mod}_{D^{\prime}}(\Sigma)$ (and therefore $\left.\vec{c} \notin \operatorname{cert}_{q_{\mathcal{O}}^{\prime}, \Sigma}^{D^{\prime}}\right)$, thus proving that $q_{\mathcal{O}}^{\prime}$ is not a complete $\mathcal{S}$-to- $\mathcal{O} \Sigma$-rewriting of $q_{\mathcal{S}}$.

Since $\left(c_{1}^{\mathcal{I}}, \ldots, c_{m}^{\mathcal{I}}\right) \in q_{\mathcal{O}}^{\mathcal{I}}$, there is a homomorphism $h$ from a CQ $q_{\mathcal{O}}^{i}$ of $q_{\mathcal{O}}$ for some $i \in[1, n]$ to $\mathcal{I}$, where $q_{\mathcal{O}}^{i}=\left\{\overrightarrow{t_{i}} \mid \exists \overrightarrow{y_{i}} \cdot \mathcal{M}\left(q_{\mathcal{S}}^{i}\right) \wedge \top\left(\overrightarrow{x_{i}}\right)\right\}$. Consider now the freezing of $q_{\mathcal{S}}^{i}=\left\{\overrightarrow{t_{i}} \mid \exists y_{i} \cdot \phi_{i}\left(\vec{x}_{i}, \vec{y}_{i}\right)\right\}$, i.e., the set $D_{q_{\mathcal{S}}^{i}}$ (here denoted by $D^{\prime}$ ) of all facts over $\mathcal{S}$ obtained from $\phi_{i}\left(\overrightarrow{x_{i}}, \overrightarrow{y_{i}}\right)$ by replacing each variable $v \in \overrightarrow{x_{i}} \cup \overrightarrow{y_{i}}$ with a fresh constant denoted by $c_{v}$. Let, moreover, $\overrightarrow{c^{\prime}}$ be the freezed tuple of constants $\overrightarrow{c^{\prime}}=\left(c_{1}^{\prime}, \ldots, c_{m}^{\prime}\right)$ where, for each $j \in[1, m], c_{j}^{\prime}=t_{j}$ if $t_{j}$ is a constant, and $c_{j}^{\prime}=c_{x}$ if $t_{j}=x$.

Let $\mathcal{I}^{\prime}=\left\langle\Delta^{\mathcal{I}},{ }^{\mathcal{I}^{\prime}}\right\rangle$ be the interpretation for $\left\langle\Sigma, D^{\prime}\right\rangle$ with the same domain of $\mathcal{I}$ and the interpretation function $\cdot^{\mathcal{I}^{\prime}}$ such that $(i) c^{\mathcal{I}^{\prime}}=c^{\mathcal{I}}$ for each constant $c$ occurring in $q_{\mathcal{S}}^{i} ;($ ii $) c_{\mathcal{Y}}^{\mathcal{I}^{\prime}}=h(\mathcal{Y})$, for each existential variable $\mathcal{Y} \in \overrightarrow{\mathcal{Y}_{i}}$ occurring in $q_{\mathcal{O}}^{i} ; c_{x}^{\mathcal{I}^{\prime}}=h(x)$ for each distinguished variable $x \in \overrightarrow{x_{i}}$ occurring in $q_{\mathcal{S}}^{i} ;(i v) c_{y}^{\mathcal{I}^{\prime}}$ is any object of $\Delta^{\mathcal{I}}$, for each existential variable $y \in \overrightarrow{y_{i}}$ not occurring in $\mathcal{M}\left(q_{\mathcal{S}}^{i}\right)$; and $(v)$ the extensions of atomic concepts and atomic roles of $\mathcal{O}$ is the same as in the interpretation $\mathcal{I}$. Observe that $\left(c_{1}^{\prime \mathcal{I}^{\prime}}, \ldots, c_{m}^{\prime}{ }^{\prime}\right)=\left(c_{1}^{\mathcal{I}}, \ldots, c_{m}^{\mathcal{I}}\right)$.

Obviously, $\overrightarrow{c^{\prime}} \in q_{\mathcal{S}}^{i} D^{\prime}$ trivially holds. Furthermore, due to the existence of the homomorphism $h$ from atoms occurring in $\mathcal{M}\left(q_{\mathcal{S}}^{i}\right)$ and $\top(\vec{x})$ to $\mathcal{I}$, and by construction of $D^{\prime}$ and $\mathcal{I}^{\prime}$, we have $\left\langle D^{\prime}, \mathcal{I}^{\prime}\right\rangle \models \mathcal{M}$. Notice that this implies $\mathcal{I}^{\prime} \in \operatorname{Mod}_{D^{\prime}}(\Sigma)$. Indeed, on the one hand $\left\langle D^{\prime}, \mathcal{I}^{\prime}\right\rangle \models \mathcal{M}$, and, on the other hand, $\mathcal{I}^{\prime} \models \mathcal{O}$ due to the initial assumption that $\mathcal{I} \in \operatorname{Mod}_{D}(\Sigma)$ and the fact that $\mathcal{I}$ and $\mathcal{I}^{\prime}$ have the same extensions of atomic concepts and atomic roles. But then, $\left(c_{1}^{\mathcal{I}}, \ldots, c_{m}^{\mathcal{I}}\right) \notin q_{\mathcal{O}}^{\prime}{ }^{\mathcal{I}}$ implies $\left(c_{1}^{\prime \mathcal{I}^{\prime}}, \ldots, c_{m}^{\prime}{ }^{\mathcal{I}^{\prime}}\right) \notin q_{\mathcal{O}}^{\prime \mathcal{I}^{\prime}}$ since $\mathcal{I}$ and $\mathcal{I}^{\prime}$ have the same extension of atomic concepts and atomic roles and constants of $q_{\mathcal{S}}^{i}$ are interpreted in the same way in $\mathcal{I}$ and $\mathcal{I}^{\prime}$. Thus, $\left(c_{1}^{\prime \mathcal{I}^{\prime}}, \ldots, c_{m}^{\prime} \mathcal{I}^{\prime}\right) \notin q_{\mathcal{O}}^{\prime \mathcal{I}^{\prime}}$ for a model $\mathcal{I}^{\prime} \in \operatorname{Mod}_{D^{\prime}}(\Sigma)$ implies $\overrightarrow{c^{\prime}} \notin \operatorname{cert} t_{q_{\mathcal{O}}^{\prime}, \Sigma}^{D^{\prime}}$.

It follows that, for the $\mathcal{S}$-database $D^{\prime}$ consistent with $\Sigma$, we have $\overrightarrow{c^{\prime}} \in q_{\mathcal{S}}^{i} D^{\prime}$ (and therefore $\overrightarrow{c^{\prime}} \in q_{\mathcal{S}}^{D^{\prime}}$ ) and $\overrightarrow{c^{\prime}} \notin \operatorname{cert} t_{q_{\mathcal{O}}^{\prime}, \Sigma}^{D^{\prime}}$. This allows us to conclude that $q_{\mathcal{O}}^{\prime}$ is not a complete $\mathcal{S}$-to- $\mathcal{O} \Sigma$-rewriting of $q_{\mathcal{S}}$, as required.

The following result is an immediate consequence of the above theorem.

Corollary 5.4. When the UNA is not adopted, the unique (up to equivalence w.r.t. $\Sigma) U C Q^{\neq}$-minimally complete $\mathcal{S}$-to- $\mathcal{O} \Sigma$-rewriting of $q_{\mathcal{S}}$ always exists and can be expressed as a UCQ. Furthermore, if $q_{\mathcal{S}}$ is a $C Q$, then it can be expressed as a $C Q$ as well.

Using techniques developed on Chapter 4, we conclude this chapter with a result on a particular instance of the verification problem when the UNA is not adopted. 
Theorem 5.5. When the UNA is not adopted, the problem of checking whether a $C Q^{\neq, b} q_{\mathcal{O}}$ is a complete $\mathcal{S}$-to-O $\Sigma$-rewriting of a $U C Q q_{\mathcal{S}}$ is NP-complete.

Proof. Observe that Theorem 5.1 holds regardless of whether the UNA is adopted or not. Thus, NP-hardness trivially follows from the NP-hardness result of Theorem 5.1 which holds even when $q_{\mathcal{O}}$ is a CQ.

Notice that, due to Theorem 4.4, when the UNA is not adopted, for a $\mathrm{CQ}^{\neq, b} q_{\mathcal{O}}$ over $\mathcal{O}$ of arity $n$, the UCQ NoUNAPerfRef $q_{\mathcal{O}}, \Sigma \cup \operatorname{PerfRef}_{\mathcal{V}_{\mathcal{O}}^{n}, \Sigma}$ over $\mathcal{S}$ is the perfect $\mathcal{O}$-to- $\mathcal{S} \Sigma$-rewriting of $q_{\mathcal{O}}$, i.e., $\operatorname{cert}_{q_{\mathcal{O}}, \Sigma}^{D}=\left(\operatorname{NoUNAPerfRef}_{q_{\mathcal{O}}, \Sigma} \cup \operatorname{PerfRef}_{\mathcal{V}_{\mathcal{O}}^{n}, \Sigma}\right)^{D}$ for each $\mathcal{S}$-database $D$. So, analogously to Lemma 5.1, it is easy to see that, when the UNA is not adopted, $q_{\mathcal{O}}$ is a complete $\mathcal{S}$-to- $\mathcal{O} \Sigma$-rewriting of $q_{\mathcal{S}}$ if and only if $q_{\mathcal{S}} \sqsubseteq\left(\right.$ NoUNAPerfRef $\left._{q_{\mathcal{O}}, \Sigma} \cup \operatorname{PerfRef}_{\mathcal{V}_{\mathcal{O}}^{n}, \Sigma}\right)$, where $n=\operatorname{ar}\left(q_{\mathcal{O}}\right)=\operatorname{ar}\left(q_{\mathcal{S}}\right)$.

As for the upper bound, it is therefore sufficient to show how to check the containment $q_{\mathcal{S}} \sqsubseteq\left(\right.$ NoUNAPerfRef $\left._{q_{\mathcal{O}}, \Sigma} \cup \operatorname{PerfRef}_{\mathcal{V}_{\mathcal{O}}^{n}, \Sigma}\right)$ in NP. For this, it is enough to slightly extend the nondeterministic algorithm illustrated in the upper bound proof of Theorem 5.1 in the following way: $(i)$ we replace the guessed sequence $\rho$ of ontology assertions with the sequence $\rho^{\prime}$, where $\rho^{\prime}$ extends $\rho$ by possibly including some disjointness assertions of $\mathcal{O}$ used to rewrite the inequality atoms of $q_{\mathcal{O}} ;(i i)$ Before of checking in polynomial time whether we can apply $\rho$, we preliminarily check, again in polynomial time, whether we can rewrite inequality atoms of $q_{\mathcal{O}}$ through the additional assertions of $\rho^{\prime}$ (obtaining so a CQ in $\lambda\left(q_{\mathcal{O}}, \mathcal{O}\right)$, cf. Section 4.2). 



\section{Chapter 6}

\section{Sound Source-to-Ontology Rewritings}

In this chapter, we study both the verification, and the computation problem for sound source-to-ontology rewritings.

\subsection{Verification Problem}

We recall that, for an $\mathcal{S}$-database $D$ consistent with $\Sigma$, PerfRef ${ }_{q_{\mathcal{O}}, \Sigma}^{D}$ computes exactly $\operatorname{cert}_{q_{\mathcal{O}}, \Sigma}^{D}$. So, intuitively, checking whether $q_{\mathcal{O}}$ is a sound $\mathcal{S}$-to- $\mathcal{O} \Sigma$-rewriting of $q_{\mathcal{S}}$ means checking whether for all $\mathcal{S}$-databases $D$, either $\operatorname{Mod}_{D}(\Sigma)=\emptyset$ or $\operatorname{PerfRef}_{q_{\mathcal{O}}, \Sigma}^{D} \subseteq$ $q_{\mathcal{S}}^{D}$. From this observation, we easily derive the following characterisation.

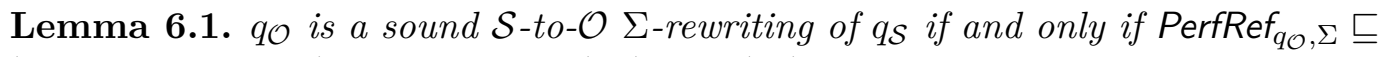
$\left(q_{\mathcal{S}} \cup\right.$ PerfRef $\left._{\mathcal{V}_{\mathcal{O}}^{n}, \Sigma}\right)$, where $n=\operatorname{ar}\left(q_{\mathcal{O}}\right)=\operatorname{ar}\left(q_{\mathcal{S}}\right)$.

Proof. "Only-if part:" Suppose that $q_{\mathcal{O}}$ is a sound $\mathcal{S}$-to- $\mathcal{O} \Sigma$-rewriting of $q_{\mathcal{S}}$. By definition, we have that for every $\mathcal{S}$-database $D$ either $D$ is not consistent with $\Sigma$, or $\operatorname{cert}_{q_{\mathcal{O}}, \Sigma}^{D} \subseteq q_{\mathcal{S}}^{D}$. In the former case, we have $\operatorname{PerfRef}_{\mathcal{V}_{\mathcal{O}}, \Sigma}^{D}=\{()\}$, which obviously implies that $\operatorname{PerfRef}_{q_{\mathcal{O}}, \Sigma}^{D} \subseteq \operatorname{PerfRef}_{\mathcal{V}_{\mathcal{O}}^{n}, \Sigma}^{D}$. In the latter case, since $D$ is consistent with $\Sigma$, we have that $\operatorname{cert}_{q_{\mathcal{O}, \Sigma}}^{D}=\operatorname{PerfRef}_{q_{\mathcal{O}}, \Sigma}^{D}$. Therefore, we have that PerfRef $q_{q_{\mathcal{O}}, \Sigma}^{D} \subseteq\left(q_{\mathcal{S}} \cup \operatorname{PerfRef}_{\mathcal{V}_{\mathcal{O}}^{n}, \Sigma}\right)^{D}$ for every $\mathcal{S}$-database $D$, as required.

"If part:" Suppose, for the sake of contradiction, that $q_{\mathcal{O}}$ is not a sound $\mathcal{S}$-to- $\mathcal{O}$ $\Sigma$-rewriting of $q_{\mathcal{S}}$, that is, there exists an $\mathcal{S}$-database $D$ consistent with $\Sigma$ such that $\operatorname{cert}_{q_{\mathcal{O}}, \Sigma}^{D} \nsubseteq q_{\mathcal{S}}^{D}$. Since $D$ is consistent with $\Sigma$, we have $(i) \operatorname{PerfRef}_{\mathcal{V}_{\mathcal{O}, \Sigma}}^{D}=\emptyset$, which implies that $(i) \operatorname{PerfRef}_{\mathcal{V}_{\mathcal{O}}^{n}, \Sigma}^{D}=\emptyset$ and $(i i) \operatorname{cert}_{q_{\mathcal{O}, \Sigma}}^{D}=\operatorname{PerfRef}_{q_{\mathcal{O}}, \Sigma}^{D}$. Therefore, for the $\mathcal{S}$-database $D$, we have that $\operatorname{PerfRef}_{q_{\mathcal{O}}, \Sigma}^{D} \nsubseteq\left(q_{\mathcal{S}} \cup \operatorname{PerfRef}_{\mathcal{V}_{\mathcal{O}}^{n}, \Sigma}\right)^{D}$. Thus, $\operatorname{PerfRef}_{q_{\mathcal{O}}, \Sigma} \nsubseteq q_{\mathcal{S}} \cup \operatorname{PerfRef}_{\mathcal{V}_{\mathcal{O}}^{n}, \Sigma}$, as required.

The following theorem characterises the computational complexity of the verification problem for sound source-to-ontology rewritings.

Theorem 6.1. The verification problem for sound source-to-ontology rewritings is $\Pi_{2}^{p}$-complete. 
Proof. As for the upper bound, by virtue of Lemma 6.1, it is sufficient to show how to check the containment $\operatorname{PerfRef}_{\mathcal{O}_{\mathcal{O}}, \Sigma} \sqsubseteq\left(q_{\mathcal{S}} \cup \operatorname{PerfRef}_{\mathcal{V}_{\mathcal{O}}^{n}, \Sigma}\right)$ in $\Pi_{2}^{p}$, where $n=\operatorname{ar}\left(q_{\mathcal{O}}\right)$. In particular, checking whether $\operatorname{PerfRef}_{q_{\mathcal{O}}, \Sigma} \nsubseteq\left(q_{\mathcal{S}} \cup \operatorname{PerfRef}_{\mathcal{V}_{\mathcal{O}}^{n}, \Sigma}\right)$ can be done in $\Sigma_{2}^{p}$ in the following way: $(i)$ we guess a CQ $q_{1}$ over $\mathcal{S}$ with the same arity of $q_{\mathcal{O}}$ and size at most $\sigma(\mathcal{M}) \times \sigma\left(q_{\mathcal{O}}\right)$, and $(i i)$ with an NP-oracle, similarly to what described in Theorem 5.1, we first check whether $q_{1}$ corresponds to, or it is contained in, a disjunct of PerfRef $q_{\mathcal{O}}, \Sigma$, i.e., whether $q_{1} \sqsubseteq \operatorname{PerfRef}_{q_{\mathcal{O}}, \Sigma}$, and then whether $q_{1} \nsubseteq\left(q_{\mathcal{S}} \cup \operatorname{PerfRef}_{\mathcal{V}_{\mathcal{O}}^{n}, \Sigma}\right)$, again using the method mentioned in Theorem 5.1

As for the lower bound, the proof of $\Pi_{2}^{p}$-hardness is by a LOGSPACE reduction from the $\forall \exists-\mathrm{CNF}$ problem, which is $\Pi_{2}^{p}$-complete Stockmeyer, 1976]. $\forall \exists-\mathrm{CNF}$ is the problem of deciding, given a $3-\mathrm{CNF}$ formula $F=c_{1} \wedge \ldots \wedge c_{p}$ on a set of variables $Y=\left\{y_{1}, \ldots, y_{m}\right\} \cup X=\left\{x_{1}, \ldots, x_{n}\right\}$ such that the variables in $Y$ (respectively, $X$ ) are universally (respectively, existentially) quantified, whether $F$ is true, i.e., whether for each truth assignment to the variables in $Y$, there exists a truth assignment to the variables in $X$ that satisfies $F$. Each clause $c_{i}$ is a disjunction of three literals, where each literal is either a variable $z \in Y \cup X$ or its negated. For $i=1, \ldots, p$, we denote by $z_{i, 1}, z_{i, 2}, z_{i, 3}$ the first, the second, and the third, respectively, variable appearing (either positive or negated) in clause $c_{i}$.

We follow a proof strategy that is similar to Millstein et al., 2003, Theorem 3.3], in which the reduction can be seen as an extension of the one provided in Lemma 5.2 .

In particular, we define an OBDM specification $\Sigma=\langle\mathcal{O}, \mathcal{S}, \mathcal{M}\rangle$ with $\mathcal{O}$ containing no axioms, and $\mathcal{S}$ and $\mathcal{M}$ as follows. For each clause $c_{i}$ of $F$, schema $\mathcal{S}$ contains three binary predicates, namely $s_{i, 1}, s_{i, 2}$, and $s_{i, 3}$. Intuitively, the first argument of all three predicates represents the clause $c_{i}$, while, for each $j=1,2,3$, the second argument of predicate $s_{i, j}$ represents the variable $z_{i, j}$. Additionally, $\mathcal{S}$ also contains $m$ unary predicates $e_{i}$, one for each universally quantified variable $y_{i}$, and two further unary predicates, namely zero and one. The mapping $\mathcal{M}$ is composed of two parts.

The first part simply mirrors each relation $s_{i, j}$ into the atomic role $R_{i, j}$ of $\mathcal{O}$ through the mapping assertion $s_{i, j}\left(x_{1}, x_{2}\right) \rightarrow R_{i, j}\left(x_{1}, x_{2}\right)$, for each $i=1, \ldots, n$ and for each $j=1,2,3$.

The second part of mapping $\mathcal{M}$ mirrors each relation $e_{i}$ into the atomic concept $W_{i}$ of $\mathcal{O}$ through the mapping assertion $e_{i}(x) \rightarrow W_{i}(x)$, for each $i=1, \ldots, m$. Finally, in the second part of $\mathcal{M}$ there are two further mapping assertions representing the possible truth value (either 0 or 1 ) for the universally quantified variables in $Y$ : $z e r o(x) \rightarrow H_{1}(x, 0) \wedge \ldots \wedge H_{m}(x, 0)$, and one $(x) \rightarrow H_{1}(x, 1) \wedge \ldots \wedge H_{m}(x, 1)$, where 0 and 1 are constants, and, for each $i=1, \ldots, m, H_{i}$ is an atomic role of $\mathcal{O}$.

We define the CQ $q_{\mathcal{S}}$ as the conjunction of the atoms appearing in its body: $(i)$ for each clause $c_{i}$ of $F$, the body of $q_{\mathcal{S}}$ contains the atoms $s_{i, 1}\left(a_{i}, z_{i, 1}\right), s_{i, 2}\left(a_{i}, z_{i, 2}\right)$, and $s_{i, 3}\left(a_{i}, z_{i, 3}\right)$, where $a_{i}$ denotes a fresh existential variable, and (ii) for each universally quantified variable $y_{i} \in Y$, there is also the atom $e_{i}\left(y_{i}\right)$.

Analogously, we define the $\mathrm{CQ} q_{\mathcal{O}}$ as the conjunction of the atoms appearing in its body: $(i)$ for each clause $c_{i}$ of $F$, and for each of the seven satisfying truth assignments $A_{i, k}=\left\{v_{1}, v_{2}, v_{3}\right\}$ for $c_{i}$ (where, for each $k=1, \ldots, 7, A_{i, k}$ is a constant, and, for each $j=1,2,3, v_{j}$ is either the constant 1 or the constant 0$)$, the body of $q_{\mathcal{O}}$ contains the atoms $R_{i, 1}\left(A_{i, k}, v_{1}\right), R_{i, 2}\left(A_{i, k}, v_{2}\right)$, and $R_{i, 3}\left(A_{i, k}, v_{3}\right)$, and $(i i)$ for each universally quantified variable $y_{i} \in Y$, there are also the atoms $W_{i}\left(y_{i}\right)$ and $H_{i}\left(u_{i}, y_{i}\right)$, where $u_{i}$ denotes a fresh existential variable. 
Observe that $\Sigma=\langle\mathcal{O}, \mathcal{S}, \mathcal{M}\rangle, q_{\mathcal{S}}$, and $q_{\mathcal{O}}$ can be constructed in LOGSPACE from $F$, where $\mathcal{O}=\emptyset, \mathcal{M}$ is both a GAV mapping and a LAV mapping, and both $q_{\mathcal{S}}$ and $q_{\mathcal{O}}$ are boolean CQs.

To illustrate the reduction, we use the following formula: $F=\left(x_{1} \vee x_{2} \vee\right.$ $\left.y_{1}\right) \wedge\left(\neg x_{1} \vee \neg x_{2} \vee \neg y_{2}\right)$. In this case, the reduction would produce the mapping $\mathcal{M}$ composed of the following mapping assertions:

$$
\begin{aligned}
s_{1,1}\left(x_{1}, x_{2}\right) & \rightarrow R_{1,1}\left(x_{1}, x_{2}\right), \\
s_{1,2}\left(x_{1}, x_{2}\right) & \rightarrow R_{1,2}\left(x_{1}, x_{2}\right), \\
s_{1,3}\left(x_{1}, x_{2}\right) & \rightarrow R_{1,3}\left(x_{1}, x_{2}\right), \\
s_{2,1}\left(x_{1}, x_{2}\right) & \rightarrow R_{2,1}\left(x_{1}, x_{2}\right), \\
s_{2,2}\left(x_{1}, x_{2}\right) & \rightarrow R_{2,2}\left(x_{1}, x_{2}\right), \\
s_{2,3}\left(x_{1}, x_{2}\right) & \rightarrow R_{2,3}\left(x_{1}, x_{2}\right), \\
e_{1}(x) & \rightarrow W_{1}(x), \\
e_{2}(x) & \rightarrow W_{2}(x), \\
\text { zero }(x) & \rightarrow H_{1}(x, 0) \wedge H_{2}(x, 0), \\
\text { one }(x) & \rightarrow H_{1}(x, 1) \wedge H_{2}(x, 1),
\end{aligned}
$$

and the following CQs $q_{\mathcal{S}}$ and $q_{\mathcal{O}}$ :

$$
\begin{gathered}
q_{\mathcal{S}}=\left\{() \mid \exists a_{1}, a_{2}, x_{1}, x_{2}, y_{1}, y_{2} \cdot s_{1,1}\left(a_{1}, x_{1}\right) \wedge s_{1,2}\left(a_{1}, x_{2}\right) \wedge s_{1,3}\left(a_{1}, y_{1}\right) \wedge\right. \\
s_{2,1}\left(a_{2}, x_{1}\right) \wedge s_{2,2}\left(a_{2}, x_{2}\right) \wedge s_{2,3}\left(a_{2}, y_{2}\right) \wedge \\
\left.e_{1}\left(y_{1}\right) \wedge e_{2}\left(y_{2}\right)\right\} ; \\
q_{\mathcal{O}}=\left\{() \mid \exists u_{1}, u_{2}, y_{1}, y_{2} \cdot \beta_{1}\left(A_{1,1}, 0,0,1\right) \wedge \beta_{1}\left(A_{1,2}, 0,1,0\right) \wedge \beta_{1}\left(A_{1,3}, 0,1,1\right) \wedge\right. \\
\beta_{1}\left(A_{1,4}, 1,0,0\right) \wedge \beta_{1}\left(A_{1,5}, 1,0,1\right) \wedge \beta_{1}\left(A_{1,6}, 1,1,0\right) \wedge \\
\beta_{1}\left(A_{1,7}, 1,1,1\right) \wedge \beta_{2}\left(A_{2,1}, 0,0,0\right) \wedge \beta_{2}\left(A_{2,2}, 0,0,1\right) \wedge \\
\\
\beta_{2}\left(A_{2,3}, 0,1,0\right) \wedge \beta_{2}\left(A_{2,4}, 0,1,1\right) \wedge \beta_{2}\left(A_{2,5}, 1,0,0\right) \wedge \\
\\
\beta_{2}\left(A_{2,6}, 1,0,1\right) \wedge \beta_{2}\left(A_{2,7}, 1,1,0\right) \wedge \\
\\
\left.W_{1}\left(y_{1}\right) \wedge H_{1}\left(u_{1}, y_{1}\right) \wedge W_{2}\left(y_{2}\right) \wedge H_{2}\left(u_{2}, y_{2}\right)\right\},
\end{gathered}
$$

where an atom of the form $\beta_{i}(x, y, z, w)$ stands for the conjunction of atoms $R_{i, 1}(x, y) \wedge R_{i, 2}(x, z) \wedge R_{i, 3}(x, w)$.

Observe that $\mathcal{V}_{\mathcal{O}} \equiv \perp$ and therefore, for each $\mathcal{S}$-database $D$, we have that $\operatorname{cert}_{q_{\mathcal{O}, \Sigma}}^{D}=\operatorname{PerfRef}_{q_{\mathcal{O}}, \Sigma}^{D}$. Note, moreover, that in all the cases where $F$ is without universally quantified variables, i.e., a 3 -CNF instance of the more general $\forall \exists$-CNF problem, in $q_{\mathcal{S}}$ there are no atoms of the form $e_{i}\left(y_{i}\right)$, and in $q_{\mathcal{O}}$ there are no atoms of the form $W_{i}\left(y_{i}\right)$ and $H_{i}\left(u_{i}, y_{i}\right)$. In such cases, PerfRef $q_{\mathcal{O}, \Sigma}$ corresponds to the CQ $q$ over $\mathcal{S}$ obtained from $q_{\mathcal{O}}$ by unfolding atoms $R_{i, j}(x, y)$ with $s_{i, j}(x, y)$. Using the same arguments provided in Lemma 5.2 it can be shown that $F$ is satisfiable if and only if $q \sqsubseteq q_{\mathcal{S}}$, thus implying that this is a valid reduction from the 3 -CNF problem to the problem of checking whether $q_{\mathcal{O}}$ is a sound $\mathcal{S}$-to- $\mathcal{O} \Sigma$-rewriting of $q_{\mathcal{S}}$.

We now address the more general case when $F$ is a $\forall \exists$-CNF formula, and prove that $F$ is true if and only if $q_{\mathcal{O}}$ is a sound $\mathcal{S}$-to- $\mathcal{O} \Sigma$-rewriting of $q_{\mathcal{S}}$.

"Only-if part:" Suppose that $F$ is true, that is, for every truth assignment to the variables in $Y$, there exists a truth assignment to the variables in $X$ that 
satisfies $F$. Note that every disjunct of $\operatorname{PerfRef}_{q_{\mathcal{O}}, \Sigma}$ (totally, $2^{m}$ ) corresponds to an assignment to the variables in $Y$ depending on the choice done for unfolding the atoms $H_{1}\left(u_{1}, y_{1}\right), \ldots, H_{m}\left(u_{m}, y_{m}\right)$, where each $H_{i}\left(u_{i}, y_{i}\right)$ is unfolded either with the atom zero $\left(u_{i}\right)$ (thus, forcing $y_{i}=0$ ), or with the atom one $\left(u_{i}\right)$ (thus, forcing $y_{i}=1$ ). This implies that in each disjunct of PerfRef $q_{\mathcal{O}}, \Sigma$ appears either the atom $e_{i}(0)$ or the atom $e_{i}(1)$, for each $i=1, \ldots, m$.

For instance, in the running example, if the atom $H_{1}\left(u_{1}, y_{1}\right)$ is unfolded with $z e r o\left(u_{1}\right) \wedge y_{1}=0$ and the atom $H_{2}\left(u_{2}, y_{2}\right)$ is unfolded with one $\left(u_{1}\right) \wedge y_{2}=1$, then the disjunct of PerfRef $q_{\mathcal{O}}, \Sigma$ obtained is the following CQ: $\left\{() \mid \exists u_{1}, u_{2} \cdot \beta_{1}\left(A_{1,1}, 0,0,1\right) \wedge\right.$ $\beta_{1}\left(A_{1,2}, 0,1,0\right) \wedge \beta_{1}\left(A_{1,3}, 0,1,1\right) \wedge \beta_{1}\left(A_{1,4}, 1,0,0\right) \wedge \beta_{1}\left(A_{1,5}, 1,0,1\right) \wedge \beta_{1}\left(A_{1,6}, 1,1,0\right) \wedge$ $\beta_{1}\left(A_{1,7}, 1,1,1\right) \wedge \beta_{2}\left(A_{2,1}, 0,0,0\right) \wedge \beta_{2}\left(A_{2,2}, 0,0,1\right) \wedge \beta_{2}\left(A_{2,3}, 0,1,0\right) \wedge \beta_{2}\left(A_{2,4}, 0,1,1\right) \wedge$ $\beta_{2}\left(A_{2,5}, 1,0,0\right) \wedge \beta_{2}\left(A_{2,6}, 1,0,1\right) \wedge \beta_{2}\left(A_{2,7}, 1,1,0\right) \wedge e_{1}(0) \wedge$ zero $\left(u_{1}\right) \wedge e_{2}(1) \wedge$ one $\left.\left(u_{2}\right)\right\}$, where an atom of the form $\beta_{i}(x, y, z, w)$ stands for the conjunction of atoms $s_{i, 1}(x, y) \wedge$ $s_{i, 2}(x, z) \wedge s_{i, 3}(x, w)$.

Since, however, for every possible truth assignment to the variables in $Y$ there exists an assignment to the variables in $X$ that satisfies $F$, it can be readily seen that this is equivalent to the fact that there is a homomorphism from $q_{\mathcal{S}}$ to each possible disjunct of $\operatorname{PerfRef}_{q_{\mathcal{O}}, \Sigma}$. It follows that $\operatorname{PerfRef}_{q_{\mathcal{O}}, \Sigma} \sqsubseteq q_{\mathcal{S}}$ which, due to Lemma 6.1 implies that $q_{\mathcal{O}}$ is a sound $\mathcal{S}$-to- $\mathcal{O} \Sigma$-rewriting of $q_{\mathcal{S}}$.

"If part:" Suppose that $F$ is not true, that is, there exists a truth assignment to the variables in $Y$ such that every possible truth assignment to the variables in $X$ does not satisfy $F$. Let $V=\left\{v_{1}, \ldots, v_{m}\right\}$ be the assignment to the variables in $Y=\left\{y_{1}, \ldots, y_{m}\right\}$ that makes $F$ not satisfiable. Let, moreover, $q$ be the disjunct of PerfRef $q_{\mathcal{O}}, \Sigma$ obtained by unfolding, for each $i=1, \ldots, m$, the atom $H_{i}\left(u_{i}, y_{i}\right)$ of $q_{\mathcal{O}}$ with zero $\left(u_{i}\right) \wedge y_{i}=0$ if $v_{i}$ is 0 , and with one $\left(u_{i}\right) \wedge y_{i}=1$ otherwise (i.e., $v_{i}=1$ ). Since $F$ is not satisfiable when substituting variable $y_{i}$ with value $v_{i}$ for each $i=1, \ldots, m$, this implies that there is no homomorphism from $q_{\mathcal{S}}$ to $q$. It follows that PerfRef $q_{\mathcal{O}}, \Sigma \nsubseteq q_{\mathcal{S}}$, and, since $\mathcal{V}_{\mathcal{O}} \equiv \perp$, we also have that $\operatorname{PerfRef}_{q_{\mathcal{O}}, \Sigma} \nsubseteq$ $\left(q_{\mathcal{S}} \cup\right.$ PerfRef $\left._{\mathcal{V}_{\mathcal{O}}, \Sigma}\right)$ which, due to Lemma 6.1, implies that $q_{\mathcal{O}}$ is not a sound $\mathcal{S}$-to-O $\Sigma$-rewriting of $q_{\mathcal{S}}$.

As a last consideration, we observe that the same proof works even with a reduction where $\mathcal{M}$ is a pure GAV mapping but not a LAV mapping, rather than both a GAV mapping and a LAV mapping as in the above case. In particular, it is straightforward to verify that for this it is sufficient to apply the following changes at the above reduction:

- for each $i=1, \ldots, m$, now consider $e_{i}$ as a binary predicate;

- replace the second part of the mapping $\mathcal{M}$ by including, for each $i=1, \ldots, m$, the following two pure GAV mapping assertions: $e_{i}(x, 0) \rightarrow W_{i}(x)$ and $e_{i}(x, 1) \rightarrow W_{i}(x)$

- for each $i=1, \ldots, m$, replace in $q_{\mathcal{S}}$ the atom $e_{i}\left(y_{i}\right)$ with the atom $e_{i}\left(u_{i}, y_{i}\right)$, where $u_{i}$ is a fresh existential variable;

- replace in $q_{\mathcal{O}}$ the conjunction of atoms $W_{i}\left(y_{i}\right) \wedge H_{i}\left(u_{i}, y_{i}\right)$ with the atom $W_{i}\left(u_{i}\right)$. 
Note that the result of $\Pi_{2}^{p}$-hardness already holds when both $q_{\mathcal{S}}$ and $q_{\mathcal{O}}$ are boolean CQs, and $\Sigma=\langle\mathcal{O}, \mathcal{S}, \mathcal{M}\rangle$ is such that the ontology $\mathcal{O}$ contains no axioms and $\mathcal{M}$ is either both a GAV mapping and a LAV mapping, or a pure GAV mapping.

It is an interesting open problem to derive the computational complexity of the verification problem for sound source-to-ontology rewritings when $\mathcal{M}$ is both a pure GAV mapping and a LAV mapping.

\subsection{Computation Problem}

We now address the problem of computing UCQ-maximally sound source-to-ontology rewritings. Our main result is that there are many cases where UCQ-maximally sound source-to-ontology rewritings are not guaranteed to exist.

In order to illustrate the result, from the general scenario introduced in Section 3.2, we introduce two restricted ones, namely restricted scenario for CQJFEs and restricted scenario for UCQJFEs. In both such restrained scenarios, the setting for OBDM specifications is obtained from the general one by limiting the DL ontology

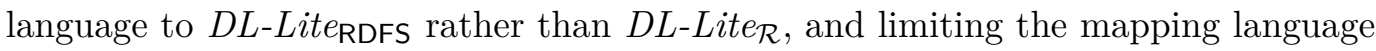
to follow the pure GAV approach rather than the GLAV approach.

The difference between the two restricted scenarios is in the query language $\mathcal{L}_{\mathcal{S}}$ allowed for expressing data services, where in the former $\mathcal{L}_{\mathcal{S}}$ denotes the class of CQJFEs, whereas in the latter $\mathcal{L}_{\mathcal{S}}$ denotes the class of UCQJFEs.

We now show that, surprisingly, as soon as we try to expand the restricted scenario for CQJFEs either by extending its associated specific setting for OBDM specifications, or by extending the query language $\mathcal{L}_{\mathcal{S}}$ to CQs rather than CQJFEs, we lose the guarantee of the existence of UCQ-maximally sound source-to-ontology rewritings of queries over $\mathcal{S}$.

Theorem 6.2. UCQ-maximally sound source-to-ontology rewritings of a query $q_{\mathcal{S}}$ may not exist if we extend the restricted scenario for CQJFEs with one of the following features:

1. $q_{\mathcal{S}}$ expressed in a fragment of CQs going beyond CQJFEs.

2. disjointness assertions in the ontology;

3. inclusion assertions of the form $B \sqsubseteq \exists R$ in the ontology, where $B$ is a basic concept and $R$ is a basic role;

4. LAV mapping assertions appearing in the mapping, even without joins involving existential variables in the right-hand side;

5. non-pure GAV mapping assertions appearing in the mapping.

Proof. 1 This case already follows from the proof of Corollary 3.3. We now prove a stronger version, where only one existential variable occurs in the body of the CQ $q_{\mathcal{S}}$. Let $\Sigma=\langle\mathcal{O}, \mathcal{S}, \mathcal{M}\rangle$ be the following OBDM specification of the restricted setting:

- $\mathcal{O}=\emptyset$

- $\mathcal{S}=\left\{s_{1}, s_{2}, s_{3}, s_{4}, s_{5}\right\}$ 
- $\mathcal{M}=\left\{m_{1}, m_{2}, m_{3}, m_{4}\right\}$, where:

$$
\begin{aligned}
& m_{1}: \quad s_{1}(x) \rightarrow A_{1}(x), \\
& m_{2}: \quad s_{2}\left(x_{1}\right) \wedge s_{3}\left(x_{1}, x_{2}\right) \quad \rightarrow \quad P\left(x_{1}, x_{2}\right), \\
& m_{3}: \quad s_{1}\left(x_{2}\right) \wedge s_{5}\left(x_{1}, x_{2}\right) \rightarrow P\left(x_{1}, x_{2}\right), \\
& m_{4}: \quad s_{2}(x) \wedge s_{4}(x) \quad \rightarrow \quad A_{2}(x) .
\end{aligned}
$$

Let $q_{\mathcal{S}}$ be the following boolean CQ over $\mathcal{S}: q_{\mathcal{S}}=\left\{() \mid \exists y \cdot s_{1}(y) \wedge s_{2}(y)\right\}$.

Observe that the CQ $q_{\mathcal{O}}^{\prime}=\left\{() \mid \exists y_{1}, y_{2} \cdot A_{1}\left(y_{1}\right) \wedge A_{2}\left(y_{2}\right)\right\}$ is not a sound $\mathcal{S}$-to- $\mathcal{O}$ $\Sigma$-rewriting of $q_{\mathcal{S}}$, because the query $q_{\mathcal{S}}^{\prime}=\left\{() \mid \exists y_{1}, y_{2} \cdot s_{1}\left(y_{1}\right) \wedge s_{2}\left(y_{2}\right) \wedge s_{4}\left(y_{2}\right)\right\}$ is a disjunct of PerfRef $q_{q_{\mathcal{O}}^{\prime}, \Sigma}$ (in fact, the only one) such that $q_{\mathcal{S}}^{\prime} \nsubseteq q_{\mathcal{S}}$ (cf. Lemma 6.1).

In order to continue the proof, we now introduce a pattern for an infinite number of CQs over $\mathcal{O}$ and related technical lemmata. Specifically, for every $i \geq 0$, let $q_{\mathcal{O}}^{i}$ be the following $\mathrm{CQ}$ over $\mathcal{O}$ :

- if $i=0$, then

$$
q_{\mathcal{O}}^{0}=\left\{() \mid \exists y_{0} \cdot A_{1}\left(y_{0}\right) \wedge A_{2}\left(y_{0}\right)\right\}
$$

- if $i \geq 1$, then

$$
q_{\mathcal{O}}^{i}=\left\{() \mid \exists y_{0}, \ldots, y_{i} \cdot A_{1}\left(y_{0}\right) \wedge\left(\bigwedge_{j=0}^{j=i-1} P\left(y_{j}, y_{j+1}\right)\right) \wedge A_{2}\left(y_{i}\right)\right\} .
$$

Lemma 6.2. For every $i \geq 0$, we have that $q_{\mathcal{O}}^{i}$ is a sound $\mathcal{S}$-to-O $\mathcal{O}$-rewriting of $q_{\mathcal{S}}$.

Proof. As for $q_{\mathcal{O}}^{0}$, its perfect $\mathcal{O}$-to- $\mathcal{S} \Sigma$-rewriting is the CQ $\operatorname{PerfRef}_{q_{\mathcal{O}}^{0}, \Sigma}=\{() \mid$ $\left.\exists y_{0} . s_{1}\left(y_{0}\right) \wedge s_{2}\left(y_{0}\right) \wedge s_{4}\left(y_{0}\right)\right\}$, which is clearly contained in $q_{\mathcal{S}}$. Thus, due to Lemma 6.1 we can conclude that $q_{\mathcal{O}}^{0}$ is a sound $\mathcal{S}$-to- $\mathcal{O} \Sigma$-rewriting of $q_{\mathcal{S}}$.

Consider now $q_{\mathcal{O}}^{i}$, for each $i \geq 0$. Observe that $\operatorname{PerfRef}_{q_{\mathcal{O}}^{i}, \Sigma}$ is a union of CQs, where the body of each CQ contains: $(i)$ the atom $s_{1}\left(y_{0}\right)$ originating from $A_{1}\left(y_{0}\right)$; $(i i)$ the conjunction of atoms $s_{2}\left(y_{i}\right) \wedge s_{4}\left(y_{i}\right)$ originating from $A_{2}\left(y_{i}\right)$; and (iii) for every $j \in[0, i-1]$, either the conjunction of atoms $s_{2}\left(y_{j}\right) \wedge s_{3}\left(y_{j}, y_{j+1}\right)$ or the conjunction of atoms $s_{1}\left(y_{j+1}\right) \wedge s_{5}\left(y_{j}, y_{j+1}\right)$ originating from $P\left(y_{j}, y_{j+1}\right)$ using the mapping assertions $m_{2}$ and $m_{3}$, respectively. It follows that each disjunct of $\operatorname{PerfRef}_{q_{\mathcal{O}}^{i}, \Sigma}$ contains the conjunction of atoms $s_{1}\left(y_{k}\right) \wedge s_{2}\left(y_{k}\right)$, for at least one $k \in[0, i]$. This implies that each disjunct of $\operatorname{PerfRef}_{q_{\mathcal{O}}^{i}, \Sigma}$ is contained in $q_{\mathcal{S}}$, and therefore $\operatorname{PerfRef}_{q_{\mathcal{O}}^{i}, \Sigma} \sqsubseteq q_{\mathcal{S}}$. Thus, due to Lemma 6.1, we can conclude that $q_{\mathcal{O}}^{i}$ is a sound $\mathcal{S}$-to- $\mathcal{O} \Sigma$-rewriting of $q_{\mathcal{S}}$.

Lemma 6.3. For every pair of natural numbers $i, k \geq 0$ with $i \neq k$, we have that both $\operatorname{cert}_{q_{\mathcal{O}}^{i}, \Sigma} \nsubseteq \operatorname{cert}_{q_{\mathcal{O}}^{k}, \Sigma}$ and $\operatorname{cert}_{q_{\mathcal{O}}^{k}, \Sigma} \nsubseteq \operatorname{cert}_{q_{\mathcal{O}}^{i}, \Sigma}$ hold.

Proof. Let $i, k>0$ (the case in which either $i$ or $k$ is 0 can be proven analogously) be any pair of natural numbers such that $i \neq k$, and consider the CQs $q_{\mathcal{O}}^{i}$ and $q_{\mathcal{O}}^{k}$. To prove the claim, it is sufficient to exhibit a disjunct $q_{\mathcal{S}}^{i}$ of $\operatorname{PerfRef}_{q_{\mathcal{O}}^{i}, \Sigma}$ and a disjunct $q_{\mathcal{S}}^{k}$ of PerfRef ${ }_{q_{\mathcal{O}}^{k}, \Sigma}$ such that both $q_{\mathcal{S}}^{i} \nsubseteq \operatorname{PerfRef}_{q_{\mathcal{O}}^{k}, \Sigma}$ and $q_{\mathcal{S}}^{k} \nsubseteq \operatorname{PerfRef}_{q_{\mathcal{O}}^{i}, \Sigma}$ hold. Let $q_{\mathcal{S}}^{i}$ (respectively, $q_{\mathcal{S}}^{k}$ ) be the disjunct of PerfRef $q_{q_{\mathcal{O}}^{i}, \Sigma}$ (respectively, PerfRef $q_{q_{\mathcal{O}}^{k}, \Sigma}$ ) obtained by unfolding the atom $A_{1}\left(y_{0}\right)$ with $s_{1}\left(y_{0}\right)$, the atom $A_{2}\left(y_{i}\right)$ (respectively, $A_{2}\left(y_{k}\right)$ ) 
with $s_{2}\left(y_{i}\right) \wedge s_{4}\left(y_{i}\right)$ (respectively, $\left.s_{2}\left(y_{k}\right) \wedge s_{4}\left(y_{k}\right)\right)$, and all the atoms $P\left(y_{j}, y_{j+1}\right)$, for $j \in[0, i-1]$ (respectively, $j \in[0, k-1]$ ), with $s_{2}\left(y_{j}\right) \wedge s_{3}\left(y_{j}, y_{j+1}\right)$. It is immediate to see that each possible other disjunct $q_{\mathcal{S}}^{k^{\prime}}$ (respectively, $q_{\mathcal{S}}^{i^{\prime}}$ ) of PerfRef $q_{q_{\mathcal{O}}^{k}, \Sigma}$ (respectively, PerfRef $_{q_{\mathcal{O}}^{i}, \Sigma}$ ) different from $q_{\mathcal{S}}^{k}$ (respectively, $q_{\mathcal{S}}^{i}$ ) is such that $q_{\mathcal{S}}^{i} \not q_{\mathcal{S}}^{k^{\prime}}$ (respectively, $q_{\mathcal{S}}^{k} \nsubseteq q_{\mathcal{S}}^{i^{\prime}}$ ), because $q_{\mathcal{S}}^{k^{\prime}}$ (respectively, $q_{\mathcal{S}}^{i^{\prime}}$ ) contains at least an atom with $s_{5}$ as predicate name, whereas $q_{\mathcal{S}}^{i}$ (respectively, $q_{\mathcal{S}}^{k}$ ) does not.

Thus, in order to prove that $q_{\mathcal{S}}^{i} \nsubseteq \operatorname{PerfRef}_{q_{\mathcal{O}}^{k}, \Sigma}$ (respectively, $\left.q_{\mathcal{S}}^{k} \nsubseteq \operatorname{PerfRef}_{q_{\mathcal{O}}^{i}, \Sigma}\right)$ hold, it is enough to show that $q_{\mathcal{S}}^{i} \nsubseteq q_{\mathcal{S}}^{k}$ (respectively, $q_{\mathcal{S}}^{k} \nsubseteq q_{\mathcal{S}}^{i}$ ) hold. Consider the disjuncts $q_{\mathcal{S}}^{i}$ and $q_{\mathcal{S}}^{k}$ obtained as described above, that is:

$$
\begin{gathered}
q_{\mathcal{S}}^{i}=\left\{() \mid \exists y_{0}, \ldots, y_{i} \cdot s_{1}\left(y_{0}\right) \wedge\left(\bigwedge_{j=0}^{j=i-1} s_{2}\left(y_{j}\right) \wedge s_{3}\left(y_{j}, y_{j+1}\right)\right) \wedge s_{2}\left(y_{i}\right) \wedge s_{4}\left(y_{i}\right)\right\} \\
q_{\mathcal{S}}^{k}=\left\{() \mid \exists y_{0}, \ldots, y_{k} . s_{1}\left(y_{0}\right) \wedge\left(\bigwedge_{j=0}^{j=k-1} s_{2}\left(y_{j}\right) \wedge s_{3}\left(y_{j}, y_{j+1}\right)\right) \wedge s_{2}\left(y_{k}\right) \wedge s_{4}\left(y_{k}\right)\right\}
\end{gathered}
$$

Let $k>i$ (the case $i>k$ is specular). Since $(i)$ both queries are rooted at $s_{1}\left(y_{0}\right)$; and $(i i)$ in $q_{\mathcal{S}}^{i}$ there is the conjunction of atoms $s_{2}\left(y_{i}\right) \wedge s_{4}\left(y_{i}\right)$ whereas in $q_{\mathcal{S}}^{k}$ there is $s_{2}\left(y_{i}\right)$ but not $s_{4}\left(y_{i}\right)$, one can easily verify that this implies $q_{\mathcal{S}}^{k} \nsubseteq q_{\mathcal{S}}^{i}$. On the other hand, since $(i)$ both queries are rooted at $s_{1}\left(y_{0}\right)$; and $(i i)$ in $q_{\mathcal{S}}^{k}$ there is the conjunction of atoms $s_{3}\left(y_{i}, y_{i+1}\right) \wedge s_{2}\left(y_{i+1}\right)$ which is not occurring in $q_{\mathcal{S}}^{i}$, one can easily verify that this implies $q_{\mathcal{S}}^{i} \nsubseteq q_{\mathcal{S}}^{k}$. It follows that both $q_{\mathcal{S}}^{k} \nsubseteq q_{\mathcal{S}}^{i}$ and $q_{\mathcal{S}}^{i} \nsubseteq q_{\mathcal{S}}^{k}$ hold, as required.

Before going further, we introduce a fair assumption. In what follows, without loss of generality, when we say that a CQ $q_{\mathcal{O}}^{\prime}$ over $\mathcal{O}$ is $(i)$ a sound $\mathcal{S}$-to- $\mathcal{O} \Sigma$-rewriting of $q_{\mathcal{S}}$, and $(i i)$ the body of $q_{\mathcal{O}}^{\prime}$ is a conjunction of $n$ atoms, we implicitly assume that all the $n$ atoms occurring in the body of $q_{\mathcal{O}}^{\prime}$ are relevant, i.e., if we remove any atom from the body of $q_{\mathcal{O}}^{\prime}$, then we obtain a CQ that is not a sound $\mathcal{S}$-to- $\mathcal{O} \Sigma$-rewriting of $q_{\mathcal{S}}$. Clearly, when seeking for (U)CQ-maximally sound source-to-ontology rewritings of queries over the source schema, one can always limit the attention to only (unions of) CQs whose body is the conjunction of relevant atoms. Furthermore, for the given $\Sigma$ and $q_{\mathcal{S}}$, we can limit the attention to only CQs with no constants in their body.

Lemma 6.4. For every $n \geq 2$, if a $C Q q_{\mathcal{O}}^{\prime}$ over $\mathcal{O}$ is a sound $\mathcal{S}$-to- $\mathcal{O} \Sigma$-rewriting of $q_{\mathcal{S}}$ and the body of $q_{\mathcal{O}}^{\prime}$ is the conjunction of $n$ atoms, then $q_{\mathcal{O}}^{\prime} \equiv q_{\mathcal{O}}^{i}$, where $i=n-2$.

Proof. We prove the claim by induction on the number of atoms $n$.

Base step $(n=2)$ : Let $q_{\mathcal{O}}^{\prime}$ be a sound $\mathcal{S}$-to- $\mathcal{O} \Sigma$-rewriting of $q_{\mathcal{S}}$ whose body is the conjunction of only two atoms. Since $q_{\mathcal{O}}^{\prime}$ is a sound $\mathcal{S}$-to- $\mathcal{O} \Sigma$-rewriting of $q_{\mathcal{S}}$, it must be the case that each disjunct $q^{\prime}$ of $\operatorname{PerfRef}_{q_{\mathcal{O}}^{\prime}, \Sigma}$ is such that $q^{\prime} \sqsubseteq q_{\mathcal{S}}$. This implies that in the body of each $q^{\prime}$ there must be at least a conjunction of atoms of the form $s_{1}\left(y^{\prime}\right) \wedge s_{2}\left(y^{\prime}\right)$ for some existential variable $y^{\prime}$. Furthermore, since by assumption the body of $q_{\mathcal{S}}^{\prime}$ is the conjunction of only two atoms, by inspecting the mapping assertions in $\mathcal{M}$, one can easily verify that the only possibility for $q_{\mathcal{O}}^{\prime}$ to be a sound $\mathcal{S}$-to- $\mathcal{O}$ L-rewriting of $q_{\mathcal{S}}$ is that $q_{\mathcal{O}}^{\prime}=\left\{() \mid \exists y^{\prime} . A_{1}\left(y^{\prime}\right) \wedge A_{2}\left(y^{\prime}\right)\right\}$ for some existential variable $y^{\prime}$. It follows that $q_{\mathcal{O}}^{\prime} \equiv q_{\mathcal{O}}^{0}$, as required. 
Inductive step: Let $q_{\mathcal{O}}^{\prime}$ be a sound $\mathcal{S}$-to- $\mathcal{O} \sum$-rewriting of $q_{\mathcal{S}}$ whose body is the conjunction of $n$ atoms. We now prove that $q_{\mathcal{O}}^{\prime} \equiv q_{\mathcal{O}}^{i}$, where $i=n-2$. To start, let $\overline{q_{\mathcal{O}}}$ be the CQ obtained from $q_{\mathcal{O}}^{\prime}$ by removing one atom $F$ from the body of $q_{\mathcal{O}}^{\prime}$. Due to the fair assumption that the body $q_{\mathcal{O}}^{\prime}$ is the conjunction of only relevant atoms, we get that $\overline{q_{\mathcal{O}}}$ is not a sound $\mathcal{S}$-to- $\mathcal{O} \Sigma$-rewriting of $q_{\mathcal{S}}$. Let the removed atom be of the form $F=P\left(z, z^{\prime}\right)$ (the cases $F=A(z)$ and $F=B\left(z^{\prime}\right)$ are easier and can be proven following a similar line of reasoning) for some existential variables $z, z^{\prime}$ not necessarily distinct, and consider the two unfoldings of the atom $P\left(z, z^{\prime}\right)$, namely $s_{2}(z) \wedge s_{3}\left(z, z^{\prime}\right)$ and $s_{1}\left(z^{\prime}\right) \wedge s_{5}\left(z, z^{\prime}\right)$. Since $q_{\mathcal{O}}^{\prime}$ is a sound $\mathcal{S}$-to- $\mathcal{O} \Sigma$-rewriting of $q_{\mathcal{S}}$ whereas $\overline{q_{\mathcal{O}}}$ is not, by construction we have that each possible disjunct of both $\operatorname{PerfRef}_{q_{\mathcal{O}}^{\prime}, \Sigma}$ and PerfRef $\overline{\overline{q_{\mathcal{O}}}, \Sigma}$ contains both the atom $s_{1}(z)$ and the atom $s_{2}\left(z^{\prime}\right)$ (if not, we easily get a contradiction on the fact that $q_{\mathcal{O}}^{\prime}$ is a sound $\mathcal{S}$-to- $\mathcal{O} \Sigma$-rewriting of $q_{\mathcal{S}}$ ). Consider now the following two queries:

- Let $q_{\mathcal{O}}^{l}$ be the CQ obtained from $q_{\mathcal{O}}^{\prime}$ by $(i)$ removing the atom $A_{2}\left(z^{\prime \prime}\right)$ (for some existential variable $z^{\prime \prime}$, such atom must occur in the body of $q_{\mathcal{O}}^{\prime}$, otherwise we easily get a contradiction on the fact that $q_{\mathcal{O}}^{\prime}$ is a sound $\mathcal{S}$-to- $\mathcal{O} \Sigma$-rewriting of $q_{\mathcal{S}}$ ), and ( $\left.i i\right)$ replacing the above discussed atom $P\left(z, z^{\prime}\right)$ with the atom $A_{2}(z)$.

- Let $q_{\mathcal{O}}^{r}$ be the CQ obtained from $q_{\mathcal{O}}^{\prime}$ by $(i)$ removing the atom $A_{1}\left(z^{\prime \prime}\right)$ (for some existential variable $z^{\prime \prime}$, such atom must occur in the body of $q_{\mathcal{O}}^{\prime}$, otherwise we easily get a contradiction on the fact that $q_{\mathcal{O}}^{\prime}$ is a sound $\mathcal{S}$-to- $\mathcal{O} \Sigma$-rewriting of $\left.q_{\mathcal{S}}\right)$, and (ii) replacing the above discussed atom $P\left(z, z^{\prime}\right)$ with the atom $A_{1}\left(z^{\prime}\right)$.

Since as discussed above each disjunct of PerfRef $\operatorname{Ron}_{\overline{q_{\mathcal{O}}}, \Sigma}$ contains the atom $s_{1}(z)$ (respectively, $s_{2}\left(z^{\prime}\right)$ ), we get that $q_{\mathcal{O}}^{l}$ (respectively, $q_{\mathcal{O}}^{r}$ ) is a sound $\mathcal{S}$-to- $\mathcal{O} \Sigma$-rewriting of $q_{\mathcal{S}}$ because the unfolding of the atom $A_{2}(z)$ (respectively, $\left.A_{1}\left(z^{\prime}\right)\right)$ is $s_{2}(z) \wedge s_{4}(z)$ (respectively, $s_{1}\left(z^{\prime}\right)$ ), and therefore there is the join $s_{1}(z) \wedge s_{2}(z)$ (respectively, $\left.s_{1}\left(z^{\prime}\right) \wedge s_{2}\left(z^{\prime}\right)\right)$ in each disjunct of $\operatorname{PerfRef}_{q_{\mathcal{O}}^{l}, \Sigma}$ (respectively, $\left.\operatorname{PerfRef}_{q_{\mathcal{O}}^{r}, \Sigma}\right)$.

Notice, however, that both $q_{\mathcal{O}}^{l}$ and $q_{\mathcal{O}}^{r}$ are CQs over $\mathcal{O}$ whose body is the conjunction of at most $n-1$ atoms. Since they are both sound $\mathcal{S}$-to- $\mathcal{O} \Sigma$-rewritings of $q_{\mathcal{S}}$, by the inductive hypothesis, we derive that

$$
\begin{gathered}
q_{\mathcal{O}}^{l} \equiv\left\{() \mid \exists y_{0}^{l}, \ldots, y_{k+1}^{l}, z \cdot A_{1}\left(y_{0}^{l}\right) \wedge\left(\bigwedge_{j=0}^{j=k} P\left(y_{j}^{l}, y_{j+1}^{l}\right)\right) \wedge P\left(y_{j+1}^{l}, z\right) \wedge A_{2}(z)\right\} ; \\
q_{\mathcal{O}}^{r} \equiv\left\{() \mid \exists z^{\prime}, y_{0}^{r}, \ldots, y_{m+1}^{r} \cdot A_{1}\left(z^{\prime}\right) \wedge P\left(z^{\prime}, y_{0}^{r}\right) \wedge\left(\bigwedge_{j=0}^{j=m} P\left(y_{0}^{r}, y_{j+1}^{r}\right)\right) \wedge A_{2}\left(y_{m+1}^{r}\right)\right\},
\end{gathered}
$$

where $0 \leq k, m \leq i-1$ (we recall that $i=n-2$ ). By conjoining $q_{\mathcal{O}}^{l}$ with $q_{\mathcal{O}}^{r}$ and replacing the conjunction $A_{2}(z) \wedge A_{1}\left(z^{\prime}\right)$ with the original atom $P\left(z, z^{\prime}\right)$, we get that

$$
\begin{aligned}
q_{\mathcal{O}}^{\prime} \equiv\left\{() \mid \exists y_{0}^{l}, \ldots, y_{k+1}^{l}, z, z^{\prime}, y_{0}^{r}, \ldots, y_{m+1}^{r} \cdot A_{1}\left(y_{0}^{l}\right) \wedge\left(\bigwedge_{j=0}^{j=k} P\left(y_{j}^{l}, y_{j+1}^{l}\right)\right) \wedge P\left(y_{j+1}^{l}, z\right) \wedge\right. \\
\left.\wedge P\left(z, z^{\prime}\right) \wedge P\left(z^{\prime}, y_{0}^{r}\right) \wedge\left(\bigwedge_{j=0}^{j=m} P\left(y_{0}^{r}, y_{j+1}^{r}\right)\right) \wedge A_{2}\left(y_{m+1}^{r}\right)\right\},
\end{aligned}
$$

where, since by assumption the body of $q_{\mathcal{O}}^{\prime}$ is the conjunction of $n$ relevant atoms, one can easily verify that $q_{\mathcal{O}}^{\prime} \equiv q_{\mathcal{O}}^{i}$, as required. 
Using the above lemmata, we are now able to prove that no UCQ-maximally sound $\mathcal{S}$-to- $\mathcal{O} \Sigma$-rewriting of $q_{\mathcal{S}}$ exists. Suppose, for the sake of contradiction, the existence of a UCQ $q_{\mathcal{O}}^{\prime}=q_{\mathcal{O}}^{\prime}{ }^{1} \cup \ldots \cup q_{\mathcal{O}}^{\prime}{ }^{n}$ which is a UCQ-maximally sound $\mathcal{S}$-to- $\mathcal{O}$ $\Sigma$-rewriting of $q_{\mathcal{S}}$. For every $j \in[1, n]$, let $k^{j}$ be the number of atoms occurring in the body of the CQ $q_{\mathcal{O}}^{\prime}{ }^{j}$. By Lemma 6.4, we derive that $q_{\mathcal{O}}^{\prime}{ }^{j} \equiv q_{\mathcal{O}}^{k_{j}-2}$, for each $j \in[1, n]$. It follows that $q_{\mathcal{O}}^{\prime} \equiv q_{\mathcal{O}}^{\prime \prime}$, where $q_{\mathcal{O}}^{\prime \prime}=\bigcup_{j \in[1, n]} q_{\mathcal{O}}^{k_{j}-2}$. Consider now the query $q_{\mathcal{O}}^{\prime \prime \prime}=q_{\mathcal{O}}^{\prime \prime} \cup q_{\mathcal{O}}^{l}$, where $l \geq 0$ is an arbitrary number such that $l \neq k_{j}-2$ for each $j \in[1, n]$. Observe that, by Lemma 6.3, we have that $\operatorname{cert}_{q_{\mathcal{O}}^{l}, \Sigma} \nsubseteq \operatorname{cert}_{q_{\mathcal{O}}{ }_{k_{j}-2}, \Sigma}$, for each $j \in[1, n]$. This implies that $\operatorname{cert}_{q_{\mathcal{O}}^{\prime \prime}, \Sigma} \sqsubset \operatorname{cert}_{q_{\mathcal{O}}^{\prime \prime \prime}, \Sigma}$, and therefore, since $q_{\mathcal{O}}^{\prime} \equiv q_{\mathcal{O}}^{\prime \prime}$, we also have that $\operatorname{cert}_{q_{\mathcal{O}}^{\prime}, \Sigma} \sqsubset \operatorname{cert}_{q_{\mathcal{O}}^{\prime \prime}, \Sigma}$. Furthermore, since by Lemma 6.2 each disjunct of $q_{\mathcal{O}}^{\prime \prime \prime}$ is a sound $\mathcal{S}$-to- $\mathcal{O} \Sigma$-rewriting of $q_{\mathcal{S}}$, we can finally conclude that $q_{\mathcal{O}}^{\prime \prime \prime}$ is a sound $\mathcal{S}$-to- $\mathcal{O} \Sigma$-rewriting of $q_{\mathcal{S}}$ such that $\operatorname{cert}_{q_{\mathcal{O}}^{\prime}, \Sigma} \sqsubset \operatorname{cert}_{q_{\mathcal{O}}^{\prime \prime \prime}, \Sigma}$. Thus, following Definition 3.4 this is clearly a contradiction on the fact that $q_{\mathcal{O}}^{\prime}$ is a UCQ-maximally sound $\mathcal{S}$-to-O $\Sigma$-rewriting of $q_{\mathcal{S}}$, as required.

For a more accurate characterisation about the illustrated OBDM specification $\Sigma$ and $\mathrm{CQ} q_{\mathcal{S}}$, let us define an infinite union of CQs $q_{\mathcal{O}}$ over $\mathcal{O}$ as follows:

$$
q_{\mathcal{O}}=\bigcup_{i \geq 0} q_{\mathcal{O}}^{i}
$$

From the foregoing lemmata and considerations, one can immediately derive that $q_{\mathcal{O}}$ is a maximally sound $\mathcal{S}$-to- $\mathcal{O} \Sigma$-rewriting of $q_{\mathcal{S}}$ in the class of Datalog queries.

2 Consider the following OBDM specification $\Sigma=\langle\mathcal{O}, \mathcal{S}, \mathcal{M}\rangle$, where:

- $\mathcal{O}=\left\{A_{1} \sqsubseteq \neg A_{1}, A_{2} \sqsubseteq \neg A_{2}\right\}$

- $\mathcal{S}=\left\{s_{1}, s_{2}, s_{3}, s_{4}\right\}$

- $\mathcal{M}=\left\{m_{1}, m_{2}, m_{3}, m_{4}, m_{5}, m_{6}, m_{7}, m_{8}\right\}$, where:

$$
\begin{array}{lrll}
m_{1}: & s_{1}\left(x_{1}, x_{2}\right) & \rightarrow P_{1}\left(x_{1}, x_{2}\right), \\
m_{2}: & s_{2}\left(x_{1}, x_{2}\right) & \rightarrow P_{1}\left(x_{1}, x_{2}\right), \\
m_{3}: & s_{2}\left(x_{1}, x_{2}\right) & \rightarrow P_{2}\left(x_{1}, x_{2}\right), \\
m_{4}: & s_{3}\left(x_{1}, x_{2}\right) & \rightarrow P_{2}\left(x_{1}, x_{2}\right), \\
m_{5}: & s_{3}\left(x_{1}, x_{2}\right) & \rightarrow P_{3}\left(x_{1}, x_{2}\right), \\
m_{6}: & s_{4}\left(x_{1}, x_{2}\right) & \rightarrow P_{3}\left(x_{1}, x_{2}\right), \\
m_{7}: & \exists y_{1}, y_{2} \cdot s_{2}\left(y_{1}, x\right) \wedge s_{3}\left(x, y_{2}\right) & \rightarrow A_{1}(x), \\
m_{8}: & \exists y_{1}, y_{2} \cdot s_{2}\left(x, y_{1}\right) \wedge s_{4}\left(y_{2}, x\right) & \rightarrow A_{2}(x) .
\end{array}
$$

Let $q_{\mathcal{S}}$ be the following CQJFE over $\mathcal{S}: q_{\mathcal{S}}=\left\{\left(x_{1}, x_{2}\right) \mid s_{1}\left(x_{1}, x_{2}\right)\right\}$.

Since $\mathcal{O}$ contains the disjointness assertions $A_{1} \sqsubseteq \neg A_{1}$ and $A_{2} \sqsubseteq \neg A_{2}$, the violation query for $\mathcal{O}$ is $\mathcal{V}_{\mathcal{O}}=\left\{() \mid \exists y \cdot A_{1}(y)\right\} \cup\left\{() \mid \exists y \cdot A_{2}(y)\right\}$, and therefore $\mathcal{V}_{\mathcal{O}}^{2}=\left\{\left(x_{1}, x_{2}\right) \mid \exists y \cdot A_{1}(y) \wedge \top\left(x_{1}, x_{2}\right)\right\} \cup\left\{() \mid \exists y \cdot A_{2}(y) \wedge \top\left(x_{1}, x_{2}\right)\right\}$. By looking at the mapping assertions $m_{7}$ and $m_{8}$ occurring in $\mathcal{M}$, this means that each query of arity 2 over $\mathcal{S}$ that has a join either between the second component of $s_{2}$ and the first component of $s_{3}$, or between the first component of $s_{2}$ and the second component of $s_{4}$ is contained in $\operatorname{PerfRef}_{\mathcal{V}_{\mathcal{O}}^{2}, \Sigma}$. 
Observe that the CQ $q_{\mathcal{O}}^{\prime}=\left\{\left(x_{1}, x_{2}\right) \mid P_{1}\left(x_{1}, x_{2}\right)\right\}$ is not a sound $\mathcal{S}$-to- $\mathcal{O} \Sigma$ rewriting of $q_{\mathcal{S}}$, because the query $q_{\mathcal{S}}^{\prime}=\left\{\left(x_{1}, x_{2}\right) \mid s_{2}\left(x_{1}, x_{2}\right)\right\}$ is a disjunct of PerfRef $_{q_{\mathcal{O}}, \Sigma}$ such that $q_{\mathcal{S}}^{\prime} \nsubseteq\left(q_{\mathcal{S}} \cup\right.$ PerfRef $\left._{\mathcal{V}_{\mathcal{O}}^{2}, \Sigma}\right)$ (cf. Lemma 6.1).

In order to continue the proof, we now introduce a pattern for an infinite number of CQs over $\mathcal{O}$ and related technical lemmata. Specifically, for every $i \geq 0$, let $q_{\mathcal{O}}^{i}$ be the following $\mathrm{CQ}$ over $\mathcal{O}$ :

- if $i=0$, then $q_{\mathcal{O}}^{0}$ is the following CQ:

$$
\left\{\left(x_{1}, x_{2}\right) \mid P_{1}\left(x_{1}, x_{2}\right) \wedge P_{3}\left(x_{2}, x_{1}\right)\right\} .
$$

- if $i=1$, then $q_{\mathcal{O}}^{1}$ is the following CQ:

$$
\left\{\left(x_{1}, x_{2}\right) \mid \exists y_{1} \cdot P_{1}\left(x_{1}, x_{2}\right) \wedge P_{2}\left(x_{2}, y_{1}\right) \wedge P_{3}\left(y_{1}, x_{1}\right)\right\} .
$$

- if $i \geq 2$, then $q_{\mathcal{O}}^{i}$ is the following CQ:

$$
\left\{\left(x_{1}, x_{2}\right) \mid \exists y_{1}, \ldots, y_{i} \cdot P_{1}\left(x_{1}, x_{2}\right) \wedge P_{2}\left(x_{2}, y_{1}\right) \wedge\left(\bigwedge_{j=1}^{j=i-1} P_{2}\left(y_{j}, y_{j+1}\right)\right) \wedge P_{3}\left(y_{i}, x_{1}\right)\right\} .
$$

Lemma 6.5. For every $i \geq 0$, we have that $q_{\mathcal{O}}^{i}$ is a sound $\mathcal{S}$-to-O $\Sigma$-rewriting of $q_{\mathcal{S}}$.

Proof. Due to Lemma 6.1 it is enough to show that PerfRef $q_{q_{\mathcal{O}}^{i}, \Sigma} \sqsubseteq\left(q_{\mathcal{S}} \cup \operatorname{PerfRef}_{\mathcal{V}_{\mathcal{O}}^{2}, \Sigma}\right)$, i.e., each disjunct $q_{\mathcal{S}}^{\prime}$ of $\operatorname{PerfRef}_{q_{\mathcal{O}}^{i}, \Sigma}$ is such that either $q_{\mathcal{S}}^{\prime} \sqsubseteq q_{\mathcal{S}}$ or $q_{\mathcal{S}}^{\prime} \sqsubseteq \operatorname{PerfRef}_{\mathcal{V}_{\mathcal{O}}^{2}, \Sigma}$, for every $i \geq 0$.

For each $i \geq 0$, the body of $q_{\mathcal{O}}^{i}$ contains the atom $P_{1}\left(x_{1}, x_{2}\right)$ which, by looking at the mapping assertions in $\mathcal{M}$, can be unfolded with either the atom $s_{1}\left(x_{1}, x_{2}\right)$ or the atom $s_{2}\left(x_{1}, x_{2}\right)$. Half of the disjuncts of $\operatorname{PerfRef}_{q_{\mathcal{O}}^{i}, \Sigma}$ (the ones in which $P_{1}\left(x_{1}, x_{2}\right)$ is unfolded with $\left.s_{1}\left(x_{1}, x_{2}\right)\right)$ are therefore easily contained in the CQJFE $q_{\mathcal{S}}=\left\{\left(x_{1}, x_{2}\right) \mid s_{1}\left(x_{1}, x_{2}\right)\right\}$. To prove that $\operatorname{PerfRef}_{q_{\mathcal{O}}^{i}, \Sigma} \sqsubseteq\left(q_{\mathcal{S}} \cup \operatorname{PerfRef}_{\mathcal{V}_{\mathcal{O}}^{2}, \Sigma}\right)$, we now show that the other half of the disjuncts of $\operatorname{PerfRef}_{q_{\mathcal{O}}^{i}, \Sigma}$ (the ones in which the atom $P_{1}\left(x_{1}, x_{2}\right)$ is unfolded with $\left.s_{2}\left(x_{1}, x_{2}\right)\right)$ are contained in $\operatorname{PerfRef}_{\mathcal{V}_{\mathcal{O}}^{2}, \Sigma}$.

As for $q_{\mathcal{O}}^{0}$, consider the disjuncts of PerfRef ${ }_{q_{\mathcal{O}}^{0}, \Sigma}$ in which the atom $P_{1}\left(x_{1}, x_{2}\right)$ is unfolded with the atom $s_{2}\left(x_{1}, x_{2}\right)$, namely $q_{\mathcal{S}}^{\prime}{ }^{1}=\left\{\left(x_{1}, x_{2}\right) \mid s_{2}\left(x_{1}, x_{2}\right) \wedge s_{3}\left(x_{2}, x_{1}\right)\right\}$ and $q_{\mathcal{S}}^{\prime 2}=\left\{\left(x_{1}, x_{2}\right) \mid s_{2}\left(x_{1}, x_{2}\right) \wedge s_{4}\left(x_{2}, x_{1}\right)\right\}$. Since in $q_{\mathcal{S}}^{\prime 1}$ there is the join between the second component of $s_{2}$ and the first component of $s_{3}$, and since in $q_{\mathcal{S}}^{\prime 2}$ there is the join between the first component of $s_{2}$ and the second component of $s_{4}$, we derive that they are both contained in $\operatorname{PerfRef}_{\mathcal{V}_{\mathcal{O}}^{2}, \Sigma}$, as required.

As for $q_{\mathcal{O}}^{1}$, consider the disjuncts of $\operatorname{PerfRef}_{q_{\mathcal{O}}^{1}, \Sigma}$ in which the atom $P_{1}\left(x_{1}, x_{2}\right)$ is unfolded with the atom $s_{2}\left(x_{1}, x_{2}\right)$, namely $q_{\mathcal{S}}^{\prime}{ }^{1}=\left\{\left(x_{1}, x_{2}\right) \mid \exists y_{1} \cdot s_{2}\left(x_{1}, x_{2}\right) \wedge\right.$ $\left.s_{2}\left(x_{2}, y_{1}\right) \wedge s_{3}\left(y_{1}, x_{1}\right)\right\}, q_{\mathcal{S}}^{\prime 2}=\left\{\left(x_{1}, x_{2}\right) \mid \exists y_{1} . s_{2}\left(x_{1}, x_{2}\right) \wedge s_{2}\left(x_{2}, y_{1}\right) \wedge s_{4}\left(y_{1}, x_{1}\right)\right\}$, $q_{\mathcal{S}}^{\prime 3}=\left\{\left(x_{1}, x_{2}\right) \mid \exists y_{1} . s_{2}\left(x_{1}, x_{2}\right) \wedge s_{3}\left(x_{2}, y_{1}\right) \wedge s_{3}\left(y_{1}, x_{1}\right)\right\}$ and $q_{\mathcal{S}}^{\prime 4}=\left\{\left(x_{1}, x_{2}\right) \mid\right.$ $\left.\exists y_{1} . s_{2}\left(x_{1}, x_{2}\right) \wedge s_{3}\left(x_{2}, y_{1}\right) \wedge s_{4}\left(y_{1}, x_{1}\right)\right\}$. One can verify that in each disjunct $q_{\mathcal{S}}^{\prime}$, for $i \in[1,4]$, there is the join either between the second component of $s_{2}$ and the first component of $s_{3}$, or between the first component of $s_{2}$ and the second component of $s_{4}$, and therefore they are contained in $\operatorname{PerfRef}_{\mathcal{V}_{\mathcal{O}}^{2}, \Sigma}$, as required. 
Let us now consider $q_{\mathcal{O}}^{i}=\left\{\left(x_{1}, x_{2}\right) \mid \exists y_{1}, \ldots, y_{i} \cdot P_{1}\left(x_{1}, x_{2}\right) \wedge P_{2}\left(x_{2}, y_{1}\right) \wedge\right.$ $\left.\left(\bigwedge_{j=1}^{j=i-1} P_{2}\left(y_{j}, y_{j+1}\right)\right) \wedge P_{3}\left(y_{i}, x_{1}\right)\right\}$, for any $i \geq 2$, and its disjuncts PerfRef $q_{\mathcal{O}}^{i}, \Sigma$ over $\mathcal{S}$ in which the atom $P_{1}\left(x_{1}, x_{2}\right)$ is unfolded with the atom $s_{2}\left(x_{1}, x_{2}\right)$. Consider first all the possible disjuncts where at least an atom of the form $P_{2}\left(z, z^{\prime}\right)$ occurring in $q_{\mathcal{O}}^{i}$ is unfolded with the atom $s_{3}\left(z, z^{\prime}\right)$. We now prove that they are all contained in PerfRef $\mathcal{V}_{\mathcal{O}}^{2}, \Sigma$. If $P_{2}\left(x_{1}, y_{1}\right)$ is unfolded with the atom $s_{3}\left(x_{2}, y_{1}\right)$, then there is the presence of $s_{2}\left(x_{1}, x_{2}\right) \wedge s_{3}\left(x_{2}, y_{1}\right)$ (i.e., a join between the second component of $s_{2}$ and the first component of $\left.s_{3}\right)$. Analogously, let the atom $P_{2}\left(x_{2}, y_{1}\right)$ be unfolded with the atom $s_{2}\left(x_{2}, y_{1}\right)$ but the atom $P_{2}\left(y_{1}, y_{2}\right)$ be unfolded with the atom $s_{3}\left(y_{1}, y_{2}\right)$. Again, there is a join between the second component of $s_{2}$ and the first component of $s_{3}$, this time on the variable $y_{1}$. Now, let $k \in[2, i-1]$ be the number such that the atom $P_{2}\left(y_{k}, y_{k+1}\right)$ is unfolded with the atom $s_{3}\left(y_{k}, y_{k+1}\right)$ but all the atoms $P_{2}\left(y_{l}, y_{l+1}\right)$, for $l<k$, are unfolded with $s_{2}\left(y_{l}, y_{l+1}\right)$. Once again, there is a join between the second component of $s_{2}$ and the first component of $s_{3}$, this time on the variable $y_{k}$

To conclude the proof, it remains to address the case of the other disjuncts in which all the atoms of the form $P_{2}\left(z, z^{\prime}\right)$ occurring in $q_{\mathcal{O}}^{i}$ are unfolded with $s_{3}\left(z, z^{\prime}\right)$ In particular, note that such disjuncts contain both the atom $s_{2}\left(x_{1}, x_{2}\right)$ (obtained from $\left.P_{1}\left(x_{1}, x_{2}\right)\right)$ and the atom $s_{2}\left(y_{i-1}, y_{i}\right)$ (obtained from $P_{2}\left(y_{i-1}, y_{i}\right)$ ). There are two possible cases: either the atom $P_{3}\left(y_{i}, x_{1}\right)$ is unfolded with $s_{3}\left(y_{i}, x_{1}\right)$ or with $s_{4}\left(y_{i}, x_{1}\right)$. In the former case, there is a join between the second component of $s_{2}$ and the first component of $s_{3}$, this time on the variable $y_{i}$. In the latter case, there is a join between the first component of $s_{2}$ and the second component of $s_{4}$. Thus, we can conclude that all the disjuncts of $q_{\mathcal{O}}^{i}$ in which the atom $P_{1}\left(x_{1}, x_{2}\right)$ is unfolded with the atom $s_{2}\left(x_{1}, x_{2}\right)$ are contained in $\operatorname{PerfRef}_{\mathcal{V}_{\mathcal{O}}^{2}, \Sigma}$, as required.

Lemma 6.6. For every pair of natural numbers $i, k \geq 0$ with $i \neq k$, we have that both $\operatorname{cert}_{q_{\mathcal{O}}^{i}, \Sigma} \nsubseteq \operatorname{cert}_{q_{\mathcal{O}}^{k}, \Sigma}$ and $\operatorname{cert}_{q_{\mathcal{O}}^{k}, \Sigma} \nsubseteq \operatorname{cert}_{q_{\mathcal{O}}^{i}, \Sigma}$ hold.

Proof. Let $i, k \geq 2$ (the case in which either $i$ or $k$ is less than 2 can be proven analogously) be any pair of natural numbers such that $i \neq k$, and consider the CQs $q_{\mathcal{O}}^{i}$ and $q_{\mathcal{O}}^{k}$. To prove the claim, it is sufficient to exhibit a disjunct $q_{\mathcal{S}}^{i}$ of $\operatorname{PerfRef}_{q_{\mathcal{O}}^{i}, \Sigma}$ and a disjunct $q_{\mathcal{S}}^{k}$ of $\operatorname{PerfRef}_{q_{\mathcal{O}}^{k}, \Sigma}$ such that both $q_{\mathcal{S}}^{i} \nsubseteq$ PerfRef $\left._{q_{\mathcal{O}}^{k}, \Sigma} \cup \operatorname{PerfRef}_{\mathcal{V}_{\mathcal{O}}^{2}, \Sigma}\right)$ and $q_{\mathcal{S}}^{k} \nsubseteq\left(\operatorname{PerfRef}_{q_{\mathcal{O}}^{i}, \Sigma} \cup \operatorname{PerfRef}_{\mathcal{V}_{\mathcal{O}}^{2}, \Sigma}\right)$ hold. Let $q_{\mathcal{S}}^{i}$ (respectively, $q_{\mathcal{S}}^{k}$ ) be the disjunct obtained by unfolding the atom $P_{1}\left(x_{1}, x_{2}\right)$ with $s_{1}\left(x_{1}, x_{2}\right)$, the atom $P_{3}\left(y_{i}, x_{1}\right)$ (respectively, $P_{3}\left(y_{k}, x_{1}\right)$ ) with $s_{3}\left(y_{i}, x_{1}\right)$ (respectively, $s_{3}\left(y_{k}, x_{1}\right)$ ), and all the atoms $P\left(y_{j}, y_{j+1}\right)$, for $j \in[1, i-1]$ (respectively, $j \in[1, k-1]$ ), with $s_{3}\left(y_{j}, y_{j+1}\right)$. Some immediate observations follow: $(i)$ both $q_{\mathcal{S}}^{i} \nsubseteq \operatorname{PerfRef}_{\mathcal{V}_{\mathcal{O}}^{2}, \Sigma}$ and $q_{\mathcal{S}}^{k} \nsubseteq \operatorname{PerfRef}_{\mathcal{V}_{\mathcal{O}}^{2}, \Sigma}$ hold; (ii) all the disjuncts $q_{\mathcal{S}}^{k^{\prime}}$ (respectively, $q_{\mathcal{S}}^{i^{\prime}}$ ) of $\operatorname{PerfRef}_{q_{\mathcal{O}}^{k}, \Sigma}$ (respectively, $\operatorname{PerfRef}_{q_{\mathcal{O}}^{i}, \Sigma}$ ) obtained by unfolding the atom $P_{1}\left(x_{1}, x_{2}\right)$ with $s_{2}\left(x_{1}, x_{2}\right)$ are such that $q_{\mathcal{S}}^{i} \nsubseteq q_{\mathcal{S}}^{k^{\prime}}$ (respectively, $q_{\mathcal{S}}^{k} \nsubseteq q_{\mathcal{S}}^{i^{\prime}}$ ), because $q_{\mathcal{S}}^{k^{\prime}}$ (respectively, $q_{\mathcal{S}}^{i^{\prime}}$ ) contains the atom $s_{2}\left(x_{1}, x_{2}\right)$, whereas $q_{\mathcal{S}}^{i}$ (respectively, $q_{\mathcal{S}}^{k}$ ) does not; (iii) all the disjuncts $q_{\mathcal{S}}^{k^{\prime}}$ (respectively, $q_{\mathcal{S}}^{i^{\prime}}$ ) of PerfRef $q_{\mathcal{O}}^{k}, \Sigma$ (respectively, PerfRef $q_{q_{\mathcal{O}}^{i}, \Sigma}$ ) obtained by unfolding the atom $P_{3}\left(y_{k}, x_{1}\right)$ (respectively, $\left.P_{3}\left(y_{i}, x_{1}\right)\right)$ with $s_{2}\left(y_{k}, x_{1}\right)$ (respectively, $\left.s_{2}\left(y_{i}, x_{1}\right)\right)$ are such that $q_{\mathcal{S}}^{i} \nsubseteq$ $q_{\mathcal{S}}^{k^{\prime}}$ (respectively, $q_{\mathcal{S}}^{k} \nsubseteq q_{\mathcal{S}}^{i^{\prime}}$ ), because $q_{\mathcal{S}}^{k^{\prime}}$ (respectively, $q_{\mathcal{S}}^{i^{\prime}}$ ) contains the atom $s_{2}\left(y_{k}, x_{1}\right)$ (respectively, $s_{2}\left(y_{i}, x_{1}\right)$ ), whereas $q_{\mathcal{S}}^{i}$ (respectively, $q_{\mathcal{S}}^{k}$ ) does not; $(i v)$ all the disjuncts $q_{\mathcal{S}}^{k^{\prime}}$ (respectively, $q_{\mathcal{S}}^{i^{\prime}}$ ) of PerfRef $q_{q_{\mathcal{O}}^{k}, \Sigma}$ (respectively, PerfRef $q_{\mathcal{O}}^{i, \Sigma}$ ) obtained by unfolding 
the atom $P_{2}\left(y_{j}, y_{j+1}\right)$, for some $j \in[1, k-1]$ (respectively, $j \in[1, i-1]$ ), with $s_{2}\left(y_{j}, y_{j+1}\right)$ are such that $q_{\mathcal{S}}^{i} \nsubseteq q_{\mathcal{S}}^{k^{\prime}}$ (respectively, $q_{\mathcal{S}}^{k} \nsubseteq q_{\mathcal{S}}^{i^{\prime}}$ ), because $q_{\mathcal{S}}^{k^{\prime}}$ (respectively, $\left.q_{\mathcal{S}}^{i^{\prime}}\right)$ contains the atom $s_{2}\left(y_{j}, y_{j+1}\right)$, whereas $q_{\mathcal{S}}^{i}$ (respectively, $\left.q_{\mathcal{S}}^{k}\right)$ does not.

It follows that, in order to prove that $q_{\mathcal{S}}^{i} \nsubseteq$ PerfRef $_{q_{\mathcal{O}}^{k}, \Sigma}$ (respectively, $q_{\mathcal{S}}^{k} \nsubseteq$ $\operatorname{PerfRef}_{q_{\mathcal{O}}^{i}, \Sigma}$ ) hold, it is enough to show that $q_{\mathcal{S}}^{i} \nsubseteq q_{\mathcal{S}}^{k}$ (respectively, $q_{\mathcal{S}}^{k} \nsubseteq q_{\mathcal{S}}^{i}$ ) hold. Consider the disjuncts $q_{\mathcal{S}}^{i}$ and $q_{\mathcal{S}}^{k}$ obtained as described above, that is:

$$
\begin{aligned}
& q_{\mathcal{S}}^{i}=\left\{() \mid \exists y_{1}, \ldots, y_{i} \cdot s_{2}\left(x_{1}, x_{2}\right) \wedge s_{3}\left(x_{2}, y_{1}\right) \wedge\left(\bigwedge_{j=1}^{j=i-1} s_{2}\left(y_{j}, y_{j+1}\right)\right) \wedge s_{3}\left(y_{i}, x_{1}\right)\right\} \\
& q_{\mathcal{S}}^{k}=\left\{() \mid \exists y_{1}, \ldots, y_{k} \cdot s_{2}\left(x_{1}, x_{2}\right) \wedge s_{3}\left(x_{2}, y_{1}\right) \wedge\left(\bigwedge_{j=1}^{j=k-1} s_{2}\left(y_{j}, y_{j+1}\right)\right) \wedge s_{3}\left(y_{k}, x_{1}\right)\right\}
\end{aligned}
$$

One can easily verify that, if either $i>k$ or $k>i$ hold, then both $q_{\mathcal{S}}^{i} \nsubseteq q_{\mathcal{S}}^{k}$ and $q_{\mathcal{S}}^{k} \nsubseteq q_{\mathcal{S}}^{i}$ hold, as required.

Lemma 6.7. If a $C Q q_{\mathcal{O}}^{\prime}$ over $\mathcal{O}$ is a sound $\mathcal{S}$-to- $\mathcal{O} \Sigma$-rewriting of $q_{\mathcal{S}}$, then $q_{\mathcal{O}}^{\prime} \sqsubseteq q_{\mathcal{O}}^{i}$, for some $i \geq 0$.

Proof. Let a CQ $q_{\mathcal{O}}^{\prime}=\left\{\left(t_{1}, t_{2}\right) \mid \exists \overrightarrow{y^{\prime}} \cdot \phi\left(t_{1}, t_{2}, \overrightarrow{y^{\prime}}\right)\right\}$ be a sound $\mathcal{S}$-to- $\mathcal{O} \Sigma$-rewriting of $q_{\mathcal{S}}$, where the target list $\left(t_{1}, t_{2}\right)$ comprises two (not necessarily distinct) terms $t_{1}$ and $t_{2}$. Clearly, since $q_{\mathcal{O}}^{\prime}$ is a sound $\mathcal{S}$-to- $\mathcal{O} \Sigma$-rewriting of $q_{\mathcal{S}}$, by inspecting the mapping assertions in $\mathcal{M}$, it is easy to see that $\phi\left(t_{1}, t_{2}, \overrightarrow{y^{\prime}}\right)$ must contain the atom $P_{1}\left(t_{1}, t_{2}\right)$ (unless $\phi\left(t_{1}, t_{2}, \overrightarrow{y^{\prime}}\right) \equiv \perp$, from which the claim trivially follows). Due to the mapping assertion $m_{2}$, however, there are disjuncts of $\operatorname{PerfRef}_{q_{\mathcal{O}}^{\prime}, \Sigma}$ that are not contained in $q_{\mathcal{S}}$, because the atom $P_{1}\left(t_{1}, t_{2}\right)$ can be unfolded with $s_{2}\left(t_{1}, t_{2}\right)$. It follows that the only possibility for $q_{\mathcal{O}}^{\prime}$ to be a sound $\mathcal{S}$-to- $\mathcal{O} \Sigma$-rewriting is that those disjuncts must be contained in $\operatorname{PerfRef}_{\mathcal{V}_{\mathcal{O}}^{2}, \Sigma}$, i.e., they must have a join either between the second component of $s_{2}$ and the first component of $s_{3}$, or between the first component of $s_{2}$ and the second component of $s_{4}$.

By inspecting again the mapping, one can verify that at least an atom of the form $P_{3}\left(t_{i}, t_{1}\right)$, for some term $t_{i}$, must occur in $\phi\left(t_{1}, t_{2}, \overrightarrow{y^{\prime}}\right)$ (without loss of generality, we can assume that only one of such atom occurs in $\left.\phi\left(t_{1}, t_{2}, \overrightarrow{y^{\prime}}\right)\right)$. Indeed, if this is not the case, then the disjunct $q_{\mathcal{S}}^{\prime}$ of $\operatorname{PerfRef}_{q_{\mathcal{O}}^{\prime}, \Sigma}$ in which the atom $P_{1}\left(t_{1}, t_{2}\right)$ is unfolded with $s_{2}\left(t_{1}, t_{2}\right)$, all the atoms of the form $P_{2}\left(z, z^{\prime}\right)$ (for any pair of terms $z, z^{\prime}$ ) are unfolded with $s_{2}\left(z, z^{\prime}\right)$, and all the atoms of the form $P_{3}\left(z_{i}, z^{\prime}\right)$ (for any pair of terms $z_{i}, z^{\prime}$, where $\left.z^{\prime} \neq t_{1}\right)$ are unfolded with $s_{4}\left(z_{i}, z^{\prime}\right)$ is such that $q_{\mathcal{S}}^{\prime} \nsubseteq q_{\mathcal{S}} \cup \operatorname{PerfRef}_{\mathcal{V}_{\mathcal{O}}^{2}, \Sigma}$, thus contradicting the fact that $q_{\mathcal{O}}^{\prime}$ is a sound $\mathcal{S}$-to- $\mathcal{O} \Sigma$-rewriting of $q_{\mathcal{S}}$. Observe that there are two possible cases for the atom $P_{3}\left(t_{i}, t_{1}\right)$ : either $t_{i}=t_{2}$ or not. In the former case, we trivially have that $q_{\mathcal{O}}^{\prime} \sqsubseteq q_{\mathcal{O}}^{0}$, as required. Thus, in what follows, we explore the latter case. In the case that there is also an atom of the form $P_{2}\left(t_{2}, t_{i}\right)$ in the body $\phi\left(t_{1}, t_{2}, \overrightarrow{y^{\prime}}\right)$ of $q_{\mathcal{O}}^{\prime}$, one can easily verify that $q_{\mathcal{O}}^{\prime} \sqsubseteq q_{\mathcal{O}}^{1}$, as required.

Let us now consider the case in which $t_{i} \neq t_{2}$ and $P_{2}\left(t_{2}, t_{i}\right)$ does not occur. Specifically, we prove that in the body $\phi\left(t_{1}, t_{2}, \overrightarrow{y^{\prime}}\right)$ of $q_{\mathcal{O}}^{\prime}$ there must necessarily exist a conjunction of atoms of the form $P_{2}\left(t_{2}, z_{1}\right) \wedge P_{2}\left(z_{1}, z_{2}\right) \wedge \ldots \wedge P_{2}\left(z_{i-2}, z_{i-1}\right) \wedge P_{2}\left(z_{i-1}, t_{i}\right)$ for some terms $z_{1}, z_{2}, \ldots, z_{i-1}$ and for some number $i \geq 2$. Suppose, for the sake 
of contradiction, that such conjunction of atoms does not occur in the body of $q_{\mathcal{O}}^{\prime}$, i.e., at least one of the following two conditions hold: $(i)$ the atom $P_{2}\left(t_{2}, z_{1}\right)$ for some term $z_{1}$ is missing; ( $\left.i i\right)$ for all the possible conjunction of atoms of the form $P_{2}\left(t_{2}, z_{1}\right) \wedge P_{2}\left(z_{1}, z_{2}\right) \wedge \ldots \wedge P_{2}\left(z_{i-2}, z_{i-1}\right) \wedge P_{2}\left(z_{i-2}, z_{i-1}\right)$ for terms $z_{1}, z_{2}, \ldots, z_{i-1}$ and for a number $i \geq 2$, the atom $P_{2}\left(z_{i-1}, t_{i}\right)$ is missing in $\phi\left(t_{1}, t_{2}, \overrightarrow{y^{\prime}}\right)$.

If $(i)$ holds, then consider the disjunct $q_{\mathcal{S}}^{\prime}$ of PerfRef $_{q_{\mathcal{O}}^{\prime}, \Sigma}$ obtained by unfolding the atom $P_{1}\left(t_{1}, t_{2}\right)$ with $s_{2}\left(t_{1}, t_{2}\right)$, all the atoms of the form $P_{2}\left(z, z^{\prime}\right)$ (for any pair of terms $z, z^{\prime}$ with $\left.z \neq t_{2}\right)$ with $s_{3}\left(z, z^{\prime}\right)$, and the atom $P_{3}\left(t_{i}, t_{1}\right)$ with $s_{3}\left(t_{i}, t_{1}\right)$. Obviously, we have that $q_{\mathcal{S}}^{\prime} \nsubseteq q_{\mathcal{S}}$ and, moreover, since $s_{3}\left(t_{2}, z\right)$ does not occur in the body of $q_{\mathcal{S}}^{\prime}$ for any term $z$, we easily get that $q_{\mathcal{S}}^{\prime} \nsubseteq \operatorname{PerfRef}_{\mathcal{V}_{\mathcal{O}}^{2}, \Sigma}$. Thus, $q_{\mathcal{S}}^{\prime} \nsubseteq q_{\mathcal{S}} \cup \operatorname{PerfRef}_{\mathcal{V}_{\mathcal{O}}^{2}, \Sigma}$ which contradicts the fact that $q_{\mathcal{O}}^{\prime}$ is a sound $\mathcal{S}$-to- $\mathcal{O} \Sigma$-rewriting of $q_{\mathcal{S}}$.

If $(i i)$ holds, then consider the disjunct $q_{\mathcal{S}}^{\prime}$ of $\operatorname{PerfRef}_{q_{\mathcal{O}}^{\prime}, \Sigma}$ obtained by unfolding the atom $P_{1}\left(t_{1}, t_{2}\right)$ with $s_{2}\left(t_{1}, t_{2}\right)$, all the possible conjunction of atoms of the form $P_{2}\left(t_{2}, z_{1}\right) \wedge P_{2}\left(z_{1}, z_{2}\right) \wedge \ldots \wedge P_{2}\left(z_{i-2}, z_{i-1}\right)$, for terms $z_{1}, z_{2}, \ldots, z_{i-1}$ and for a number $i \geq 2$, with $s_{2}\left(t_{2}, z_{1}\right) \wedge s_{2}\left(z_{1}, z_{2}\right) \wedge \ldots \wedge s_{2}\left(z_{i-2}, z_{i-1}\right)$, all the other possible atoms $P_{2}\left(z, z^{\prime}\right)$ (for any pair of terms $z, z^{\prime}$ ) not included in the previous case with $s_{3}\left(z, z^{\prime}\right)$, and the atom $P_{3}\left(t_{i}, t_{1}\right)$ with $s_{3}\left(t_{i}, t_{1}\right)$. Obviously, we have that $q_{\mathcal{S}}^{\prime} \nsubseteq q_{\mathcal{S}}$ and, moreover, since $s_{2}\left(z, t_{i}\right)$ does not occur in the body of $q_{\mathcal{S}}^{\prime}$ for any term $z$, one can easily verify that $q_{\mathcal{S}}^{\prime} \nsubseteq$ PerfRef $_{\mathcal{V}_{\mathcal{O}}^{2}, \Sigma}$. Thus, $q_{\mathcal{S}}^{\prime} \nsubseteq q_{\mathcal{S}} \cup$ PerfRef $_{\mathcal{V}_{\mathcal{O}}^{2}, \Sigma}$, which contradicts the fact that $q_{\mathcal{O}}^{\prime}$ is a sound $\mathcal{S}$-to- $\mathcal{O} \Sigma$-rewriting of $q_{\mathcal{S}}$.

As a consequence of the above considerations, we derive that the body of each $q_{\mathcal{O}}^{\prime}$ that is a sound $\mathcal{S}$-to- $\mathcal{O} \Sigma$-rewriting must contains the conjunction of atoms $P_{1}\left(t_{1}, t_{2}\right) \wedge P_{2}\left(t_{2}, z_{1}\right) \wedge P_{2}\left(z_{1}, z_{2}\right) \wedge \ldots \wedge P_{2}\left(z_{i-2}, z_{i-1}\right) \wedge P_{2}\left(z_{i-1}, t_{i}\right) \wedge P_{3}\left(t_{i}, t_{1}\right)$, where $\left(t_{1}, t_{2}\right)$ is the target list of $q_{\mathcal{O}}^{\prime}$ and $i$ is a number such that $i \geq 2$. But then, we easily get that $q_{\mathcal{O}}^{\prime}$ is such that $q_{\mathcal{O}}^{\prime} \sqsubseteq q_{\mathcal{O}}^{i}$, as required.

Using the above lemmata, we are now able to prove that no UCQ-maximally sound $\mathcal{S}$-to- $\mathcal{O} \Sigma$-rewriting of $q_{\mathcal{S}}$ exists. Suppose, for the sake of contradiction, the existence of a UCQ $q_{\mathcal{O}}^{\prime}=q_{\mathcal{O}}^{\prime}{ }^{1} \cup \ldots \cup q_{\mathcal{O}}^{\prime}{ }^{n}$ which is a UCQ-maximally sound $\mathcal{S}$-to- $\mathcal{O} \Sigma$-rewriting of $q_{\mathcal{S}}$. For each $j \in[1, n]$, by Lemma 6.7, we know the existence of a number $i_{j}$ for which $q_{\mathcal{O}}^{\prime} \sqsubseteq q_{\mathcal{O}}^{i_{j}}$. It follows that $q_{\mathcal{O}}^{\prime} \sqsubseteq q_{\mathcal{O}}^{\prime \prime}$, where $q_{\mathcal{O}}^{\prime \prime}=\bigcup_{j \in[1, n]} q_{\mathcal{O}}^{i_{j}}$. Consider now the query $q_{\mathcal{O}}^{\prime \prime \prime}=q_{\mathcal{O}}^{\prime \prime} \cup q_{\mathcal{O}}^{l}$, where $l \geq 0$ is an arbitrary number such that $l \neq i_{j}$ for each $j \in[1, n]$. Observe that, by Lemma 6.6, we have that $\operatorname{cert}_{q_{\mathcal{O}}^{l}, \Sigma} \nsubseteq \operatorname{cert}_{q_{\mathcal{O}}, \Sigma}$, for each $j \in[1, n]$. This implies that $\operatorname{cert}_{q_{\mathcal{O}}^{\prime \prime}, \Sigma} \sqsubset \operatorname{cert}_{q_{\mathcal{O}}^{\prime \prime \prime}, \Sigma}$, and therefore, since $q_{\mathcal{O}}^{\prime} \sqsubseteq q_{\mathcal{O}}^{\prime \prime}$, we also have that $\operatorname{cert}_{q_{\mathcal{O}}^{\prime}, \Sigma} \sqsubset \operatorname{cert}_{q_{\mathcal{O}}^{\prime \prime \prime}, \Sigma}$. Furthermore, since by Lemma 6.5 each disjunct of $q_{\mathcal{O}}^{\prime \prime \prime}$ is a sound $\mathcal{S}$-to- $\mathcal{O} \Sigma$-rewriting of $q_{\mathcal{S}}$, we can finally conclude that $q_{\mathcal{O}}^{\prime \prime \prime}$ is a sound $\mathcal{S}$-to- $\mathcal{O}$ इ-rewriting such that $\operatorname{cert}_{q_{\mathcal{O}}^{\prime}, \Sigma} \sqsubset \operatorname{cert}_{q_{\mathcal{O}}^{\prime \prime \prime}, \Sigma}$. Thus, following Definition 3.4 . this is clearly a contradiction on the fact that $q_{\mathcal{O}}^{\prime}$ is a UCQ-maximally sound $\mathcal{S}$-to- $\mathcal{O} \Sigma$-rewriting of $q_{\mathcal{S}}$, as required.

For a more accurate characterisation about the illustrated OBDM specification $\Sigma$ and CQJFE $q_{\mathcal{S}}$, let us define an infinite union of CQs $q_{\mathcal{O}}$ over $\mathcal{O}$ as follows:

$$
q_{\mathcal{O}}=\bigcup_{i \geq 0} q_{\mathcal{O}}^{i}
$$

From the foregoing lemmata and considerations, one can immediately derive that $q_{\mathcal{O}}$ is a maximally sound $\mathcal{S}$-to- $\mathcal{O} \Sigma$-rewriting of $q_{\mathcal{S}}$ in the class of Datalog queries. 
3 Let $\Sigma=\langle\mathcal{O}, \mathcal{S}, \mathcal{M}\rangle$ be the following OBDM specification:

- $\mathcal{O}=\{A \sqsubseteq \exists P\}$

- $\mathcal{S}=\left\{s_{1}, s_{2}\right\}$

- $\mathcal{M}=\left\{m_{1}, m_{2}\right\}$, where:

$$
\begin{aligned}
& m_{1}: s_{1}\left(x_{1}, x_{2}\right) \quad \rightarrow \quad P\left(x_{1}, x_{2}\right), \\
& m_{2}: \quad s_{2}(x) \quad \rightarrow \quad A(x) .
\end{aligned}
$$

Let $q_{\mathcal{S}}$ be the following CQJFE over $\mathcal{S}: q_{\mathcal{S}}=\left\{(x) \mid \exists y \cdot s_{1}(x, y)\right\}$.

Observe that the CQ $q_{\mathcal{O}}^{\prime}=\{(x) \mid \exists y . P(x, y)\}$ is not a sound $\mathcal{S}$-to- $\mathcal{O} \Sigma$-rewriting of $q_{\mathcal{S}}$, because the query $q_{\mathcal{S}}^{\prime}=\left\{(x) \mid s_{2}(x)\right\}$ is a disjunct of PerfRef $q_{\mathcal{O}}^{\prime}, \Sigma$ (obtained by rewriting $q_{\mathcal{O}}^{\prime}$ with the ontology assertion $A \sqsubseteq \exists P$, thus obtaining the CQ $\{(x) \mid A(x)\}$, and then by unfolding this latter with respect to $\mathcal{M})$ such that $q_{\mathcal{S}}^{\prime} \nsubseteq q_{\mathcal{S}}$.

In order to continue the proof, we now introduce two patterns for an infinite number of CQs over $\mathcal{O}$ and related technical lemmata. The first one ranges over natural numbers. Specifically, for every $i \geq 0$, let $q_{\mathcal{O}}^{i}$ be the following CQ over $\mathcal{O}$ :

- if $i=0$, then

$$
q_{\mathcal{O}}^{0}=\left\{(x) \mid \exists y_{0}, y_{1} \cdot P\left(x, y_{0}\right) \wedge P\left(y_{0}, y_{1}\right)\right\}
$$

- if $i \geq 1$, then

$$
\begin{aligned}
q_{\mathcal{O}}^{i}=\left\{(x) \mid \exists y_{0}, \ldots, y_{2 i+1}\right. & P\left(x, y_{0}\right) \wedge\left(\bigwedge_{i=1}^{i} P\left(y_{2 i-1}, y_{2 i-2}\right) \wedge P\left(y_{2 i-1}, y_{2 i}\right)\right) \wedge \\
& \left.\wedge P\left(y_{2 i}, y_{2 i+1}\right)\right\}
\end{aligned}
$$

For instance, with $i=3$, we have $q_{\mathcal{O}}^{3}=\left\{(x) \mid \exists y_{0}, \ldots, y_{7} \cdot P\left(x, y_{0}\right) \wedge P\left(y_{1}, y_{0}\right) \wedge\right.$ $\left.P\left(y_{1}, y_{2}\right) \wedge P\left(y_{3}, y_{2}\right) \wedge P\left(y_{3}, y_{4}\right) \wedge P\left(y_{5}, y_{4}\right) \wedge P\left(y_{5}, y_{6}\right) \wedge P\left(y_{6}, y_{7}\right)\right\}$.

The second one ranges over natural numbers and constants $c \in$ Const. Specifically, for every constant $c \in$ Const and for every $i \geq 0$, let $q_{\mathcal{O}}^{c, i}$ be the following CQ over $\mathcal{O}$ :

- if $i=0$, then

$$
q_{\mathcal{O}}^{c, 0}=\{(x) \mid P(x, c)\}
$$

- if $i=1$, then

$$
q_{\mathcal{O}}^{c, 1}=\left\{(x) \mid \exists y_{0}, y_{1} \cdot P\left(x, y_{0}\right) \wedge P\left(y_{1}, y_{0}\right) \wedge P\left(y_{1}, c\right)\right\}
$$

- if $i \geq 2$, then

$$
\begin{aligned}
q_{\mathcal{O}}^{c, i}=\left\{(x) \mid \exists y_{0}, \ldots, y_{2 i-1}\right. & P\left(x, y_{0}\right) \wedge\left(\bigwedge_{i=1}^{i-1} P\left(y_{2 i-1}, y_{2 i-2}\right) \wedge P\left(y_{2 i-1}, y_{2 i}\right)\right) \wedge \\
& \left.\wedge P\left(y_{2 i-1}, y_{2 i-2}\right) \wedge P\left(y_{2 i-1}, c\right)\right\}
\end{aligned}
$$

For instance, with $i=3$ and any $c \in$ Const, we have $q_{\mathcal{O}}^{c, 3}=\{(x)$ $\left.\exists y_{0}, \ldots, y_{7} \cdot P\left(x, y_{0}\right) \wedge P\left(y_{1}, y_{0}\right) \wedge P\left(y_{1}, y_{2}\right) \wedge P\left(y_{3}, y_{2}\right) \wedge P\left(y_{3}, y_{4}\right) \wedge P\left(y_{5}, y_{4}\right) \wedge P\left(y_{5}, c\right)\right\}$. 
Before delving into the technical lemmata, we introduce some considerations on the rewriting of each of these above illustrated CQs with respect to the ontology $\mathcal{O}$. Consider $q_{\mathcal{O}}^{0}$ (respectively, $q_{\mathcal{O}}^{c, 0}$ for some constant $c \in$ Const). Since the existential variable $y_{0}$ is in join because of the occurrence of the atom $P\left(y_{0}, y_{1}\right)$ (respectively, the atom $P(x, c)$ has a constant in its second argument), when rewriting $q_{\mathcal{O}}^{0}$ (respectively, $q_{\mathcal{O}}^{c, 0}$ ) with respect to $\mathcal{O}$ by means of the PerfectRef algorithm, the inclusion assertion $A \sqsubseteq \exists P$ is not applicable to the atom $P\left(x, y_{0}\right)$ (respectively, $\left.P(x, c)\right)$. Thus, $\operatorname{PerfectRef}\left(\mathcal{O}, q_{\mathcal{O}}^{0}\right)=q_{\mathcal{O}}^{0}\left(\right.$ respectively, $\left.\operatorname{PerfectRef}\left(\mathcal{O}, q_{\mathcal{O}}^{c, 0}\right)=q_{\mathcal{O}}^{c, 0}\right)$.

Consider now $q_{\mathcal{O}}^{1}$ (respectively, $q_{\mathcal{O}}^{c, 1}$ for some constant $c \in$ Const). Here, the existential variable $y_{0}$ of the atom $P\left(x, y_{0}\right)$ is in join because of the occurrence of the atom $P\left(y_{1}, y_{0}\right)$, and therefore the inclusion assertion $A \sqsubseteq \exists P$ is not applicable to the atom $P\left(x, y_{0}\right)$. Furthermore, the existential variable $y_{2}$ of the atom $P\left(y_{1}, y_{2}\right)$ is in join because of the occurrence of the atom $P\left(y_{2}, y_{3}\right)$ (respectively, the atom $P\left(y_{1}, c\right)$ has a constant in its second argument), and therefore the inclusion assertion $A \sqsubseteq \exists P$ is not applicable either to both the atoms $P\left(y_{1}, y_{2}\right)$ and $P\left(y_{2}, y_{3}\right)$ (respectively, to the atom $P\left(y_{1}, c\right)$ ). Notice, however, that when rewriting $q_{\mathcal{O}}^{1}$ (respectively, $q_{\mathcal{O}}^{c, 1}$ ) with respect to $\mathcal{O}$, the PerfectRef algorithm produces a new $\mathrm{CQ}$ obtained by unifying $P\left(x, y_{0}\right)$ and $P\left(y_{1}, y_{0}\right)$, i.e., it produces a new CQ in which $x=y_{1}$. One can easily verify that such a new $\mathrm{CQ}$ is logically equivalent to $q_{\mathcal{O}}^{0}$ (respectively, $q_{\mathcal{O}}^{c, 0}$ ), and therefore the inclusion assertion $A \sqsubseteq \exists P$ is not applicable to this new CQ, too. Thus, $\operatorname{PerfectRef}\left(\mathcal{O}, q_{\mathcal{O}}^{1}\right)=q_{\mathcal{O}}^{1} \cup \operatorname{PerfectRef}\left(\mathcal{O}, q_{\mathcal{O}}^{0}\right)=q_{\mathcal{O}}^{1} \cup q_{\mathcal{O}}^{0}$ (respectively, $\left.\operatorname{PerfectRef}\left(\mathcal{O}, q_{\mathcal{O}}^{c, 1}\right)=q_{\mathcal{O}}^{c, 1} \cup \operatorname{PerfectRef}\left(\mathcal{O}, q_{\mathcal{O}}^{c, 0}\right)=q_{\mathcal{O}}^{c, 1} \cup q_{\mathcal{O}}^{c, 0}\right)$.

Let us consider the CQ $q_{\mathcal{O}}^{i}$ (respectively, $q_{\mathcal{O}}^{c, i}$ for some constant $c \in$ Const), for any natural number $i \geq 2$. As before, the existential variable $y_{0}$ of the atom $P\left(x, y_{0}\right)$ is in join because of the occurrence of the atom $P\left(y_{1}, y_{0}\right)$, and therefore the inclusion assertion $A \sqsubseteq \exists P$ is not applicable to the atom $P\left(x, y_{0}\right)$. Furthermore, for each even $j \in[2,2 i-2]$, the existential variable $y_{j}$ is in join because occurs in the second argument of both the atom $P\left(y_{j-1}, y_{j}\right)$ and the atom $P\left(y_{j+1}, y_{j}\right)$, and therefore the inclusion assertion $A \sqsubseteq \exists P$ is not applicable either to both the atoms $P\left(y_{j-1}, y_{j}\right)$ and $P\left(y_{j+1}, y_{j}\right)$. Finally, the existential variable $y_{2 i}$ of the atom $P\left(y_{2 i-1}, y_{2 i}\right)$ is in join because of the occurrence of the atom $P\left(y_{2 i}, y_{2 i+1}\right)$ (respectively, the atom $P\left(y_{2 i-1}, c\right)$ has a constant in its second argument), and therefore, once again, the inclusion assertion $A \sqsubseteq \exists P$ is not applicable either to both the atoms $P\left(y_{2 i-1}, y_{2 i}\right)$ and $P\left(y_{2 i}, y_{2 i+1}\right)$ (respectively, to the atom $P\left(y_{2 i-1}, c\right)$ ). Note that when rewriting $q_{\mathcal{O}}^{i}$ (respectively, $q_{\mathcal{O}}^{c, i}$ ) with respect to $\mathcal{O}$, the PerfectRef algorithm, by means of the unification step, considers the following set of equalities $E=\left\{x=y_{1}, y_{1}=y_{3}, y_{3}=y_{5}, \ldots, y_{2 i-3}=y_{2 i-1}\right\}$. Specifically, for each set of equalities $e$ in the power set of $E$ (i.e., for each $e \in \mathfrak{P}(E)$ ), a new $\mathrm{CQ}$ is obtained from $q_{\mathcal{O}}^{i}$ in which all the equalities in $e$ are applied. One can easily verify that each CQ obtained in this way is logically equivalent to $q_{\mathcal{O}}^{i-|e|}$ (respectively, $q_{\mathcal{O}}^{c, i-|e|}$ ), where $|e|$ is the cardinality of $e$ (i.e., the number of equalities in $e$ ). So, with a trivial induction on $i$, it can be proven that the inclusion assertion $A \sqsubseteq \exists P$ is not applicable to these new CQs, too. Thus, for any $i \geq 2$, we have $\operatorname{PerfectRef}\left(\mathcal{O}, q_{\mathcal{O}}^{i}\right)=q_{\mathcal{O}}^{i} \cup \operatorname{PerfectRef}\left(\mathcal{O}, q_{\mathcal{O}}^{i-1}\right)=q_{\mathcal{O}}^{i} \cup q_{\mathcal{O}}^{i-1} \cup \operatorname{PerfectRef}\left(\mathcal{O}, q_{\mathcal{O}}^{i-2}\right)=$ $\ldots=q_{\mathcal{O}}^{i} \cup q_{\mathcal{O}}^{i-1} \cup q_{\mathcal{O}}^{i-2} \cup \ldots \cup q_{\mathcal{O}}^{0}$ (respectively, $\operatorname{PerfectRef}\left(\mathcal{O}, q_{\mathcal{O}}^{c, i}\right)=$ $q_{\mathcal{O}}^{c, i} \cup \operatorname{PerfectRef}\left(\mathcal{O}, q_{\mathcal{O}}^{c, i-1}\right)=q_{\mathcal{O}}^{c, i} \cup q_{\mathcal{O}}^{c, i-1} \cup \operatorname{PerfectRef}\left(\mathcal{O}, q_{\mathcal{O}}^{c, i-2}\right)=\ldots=$ $\left.q_{\mathcal{O}}^{c, i} \cup q_{\mathcal{O}}^{c, i-1} \cup q_{\mathcal{O}}^{c, i-2} \cup \ldots \cup q_{\mathcal{O}}^{c, 0}\right)$. 
Lemma 6.8. For every $i \geq 0$ and for every constant $c \in$ Const, we have that both $q_{\mathcal{O}}^{i}$ and $q_{\mathcal{O}}^{c, i}$ are sound $\mathcal{S}$-to- $\mathcal{O}$ $\Sigma$-rewritings of $q_{\mathcal{S}}$.

Proof. Consider any $i \geq 0$ and any constant $c \in$ Const. From the above considerations, when rewriting the CQs $q_{\mathcal{O}}^{i}$ and $q_{\mathcal{O}}^{c, i}$ with respect to the ontology $\mathcal{O}$, the PerfectRef algorithm never applies the inclusion assertion $A \sqsubseteq \exists P$. So, each possible CQ in $\operatorname{PerfectRef}\left(\mathcal{O}, q_{\mathcal{O}}^{i}\right)$ and each possible CQ in $\operatorname{Perfect\operatorname {Ref}}\left(\mathcal{O}, q_{\mathcal{O}}^{c, i}\right)$ will contain the atom $P\left(x, y_{0}\right)$ (or the atom $P(x, c)$ in $\operatorname{PerfectRef}\left(\mathcal{O}, q_{\mathcal{O}}^{c, 0}\right)$ if $\left.i=0\right)$. By construction of the mapping $\mathcal{M}$, since any atom of the form $P\left(z, z^{\prime}\right)$ (for any pair of not necessarily distinct terms $\left.z, z^{\prime}\right)$ can be unfolded only with $s_{1}\left(z, z^{\prime}\right)$, we derive that each possible CQ in $\operatorname{PerfRef}_{q_{\mathcal{O}}^{i}, \Sigma}$ and each possible CQ in $\operatorname{PerfRef}_{q_{\mathcal{O}}^{c, i}, \Sigma}$ will contain the atom $s_{1}\left(x, y_{0}\right)$ (or the atom $s_{1}(x, c)$ in $\operatorname{PerfRef}_{q_{\mathcal{O}}^{c, 0}, \mathcal{O}}$ if $i=0$ ). Thus, both PerfRef $q_{q_{\mathcal{O}}^{i}, \Sigma} \sqsubseteq q_{\mathcal{S}}$ and $\operatorname{PerfRef}_{q_{\mathcal{O}}^{c, i}, \Sigma} \sqsubseteq q_{\mathcal{S}}$ trivially hold, and therefore, due to Lemma 6.1. we derive that both $q_{\mathcal{O}}^{i}$ and $q_{\mathcal{O}}^{c, i}$ are sound $\mathcal{S}$-to- $\mathcal{O} \Sigma$-rewritings of $q_{\mathcal{S}}$, as required.

Lemma 6.9. For every $i \geq 0$ and for every constant $c \in$ Const, we have that both $\operatorname{cert}_{q_{\mathcal{O}}^{i}, \Sigma} \sqsubset \operatorname{cert}_{q_{\mathcal{O}}^{i+1, \Sigma}}$ and $\operatorname{cert}_{q_{\mathcal{O}}^{c, i}, \Sigma} \sqsubset \operatorname{cert}_{q_{\mathcal{O}}^{c, i+1}, \Sigma}$ hold.

Proof. Let $i \geq 0$ be any natural number, and consider the CQs $q_{\mathcal{O}}^{i}$ and $q_{\mathcal{O}}^{i+1}$ (respectively, the CQs $q_{\mathcal{O}}^{c, i}$ and $q_{\mathcal{O}}^{c, i+1}$ for any constant $c \in$ Const). As already observed, we have $q_{\mathcal{O}}^{i} \in \operatorname{PerfectRef}\left(\mathcal{O}, q_{\mathcal{O}}^{i+1}\right)$ (respectively, $\left.q_{\mathcal{O}}^{c, i} \in \operatorname{PerfectRef}\left(\mathcal{O}, q_{\mathcal{O}}^{c, i+1}\right)\right)$. So, any possible disjunct of PerfRef $q_{q_{\mathcal{O}}^{i}, \Sigma}$ (respectively, PerfRef ${ }_{q_{\mathcal{O}}^{c, i}, \Sigma}$ ) occurs also in $\operatorname{PerfRef}_{q_{\mathcal{O}}^{i+1}, \Sigma}$ (respectively, PerfRef ${ }_{q_{\mathcal{O}}^{c, i+1}, \Sigma}$ ). It follows that $\operatorname{cert}_{q_{\mathcal{O}}^{i}, \Sigma} \sqsubseteq \operatorname{cert}_{q_{\mathcal{O}}^{i+1, \Sigma}}$ (respectively, $\left.\operatorname{cert}_{q_{\mathcal{O}}^{c, i}, \Sigma} \sqsubseteq \operatorname{cert}_{q_{\mathcal{O}}^{c, i+1}, \Sigma}\right)$. In order to prove that actually $\operatorname{cert}_{q_{\mathcal{O}}^{i}, \Sigma} \sqsubset \operatorname{cert}_{q_{\mathcal{O}}^{i+1}, \Sigma}$ (respectively, $\left.\operatorname{cert}_{q_{\mathcal{O}}^{c, i}, \Sigma} \sqsubset \operatorname{cert}_{q_{\mathcal{O}}^{c, i+1}, \Sigma}\right)$ hold, it is therefore enough to exhibit a disjunct $q_{\mathcal{S}}^{i+1}$ of $\operatorname{PerfRef}_{q_{\mathcal{O}}^{i+1, \Sigma}}$ (respectively, PerfRef ${ }_{q_{\mathcal{O}}^{c, i+1}, \Sigma}$ ) for which $q_{\mathcal{S}}^{i+1} \nsubseteq$ PerfRef $_{q_{\mathcal{O}}^{i}, \Sigma}$ (respectively, $\left.q_{\mathcal{S}}^{i+1} \nsubseteq \operatorname{PerfRef}_{q_{\mathcal{O}}^{c, i}, \Sigma}\right)$. As already observed, all the possible CQs in $\operatorname{PerfectRef}\left(\mathcal{O}, q_{\mathcal{O}}^{i}\right)$ (respectively, $\operatorname{Perfect} \operatorname{Ref}\left(\mathcal{O}, q_{\mathcal{O}}^{c, i}\right)$ ) are obtained by applying some equalities to the variables occurring in $q_{\mathcal{O}}^{i}$ (respectively, $q_{\mathcal{O}}^{c, i}$ ).

Consider now the CQ $q_{\mathcal{O}}^{i+1}$ (respectively, $q_{\mathcal{O}}^{c, i+1}$ ). From the above consideration, one can easily verify that, for each possible CQ $q_{\mathcal{O}}^{\prime}$ in $\operatorname{Perfect\operatorname {Ref}}\left(\mathcal{O}, q_{\mathcal{O}}^{i}\right)$ (respectively, $\left.\operatorname{PerfectRef}\left(\mathcal{O}, q_{\mathcal{O}}^{c, i}\right)\right)$, there is no homomorphism from $q_{\mathcal{O}}^{\prime}$ to $q_{\mathcal{O}}^{i+1}$ (respectively, $q_{\mathcal{O}}^{c, i+1}$ ). This is because the variable $x$ occurring in both CQs under examination is a distinguished variable, and therefore every candidate homomorphism $h$ must have $h(x)=x$. It follows that $q_{\mathcal{O}}^{i+1} \nsubseteq \operatorname{PerfectRef}\left(\mathcal{O}, q_{\mathcal{O}}^{i}\right)\left(\right.$ respectively, $\left.q_{\mathcal{O}}^{c, i+1} \nsubseteq \operatorname{PerfectRef}\left(\mathcal{O}, q_{\mathcal{O}}^{c, i}\right)\right)$.

Furthermore, as already observed, the inclusion assertion $A \sqsubseteq \exists P$ is never applied, and thus no atom with predicate name $A$ occurs in the body of the CQs of $\operatorname{PerfectRef}\left(\mathcal{O}, q_{\mathcal{O}}^{i+1}\right)$ (respectively, $\operatorname{PerfectRef}\left(\mathcal{O}, q_{\mathcal{O}}^{c, i+1}\right)$ ). So, all the disjuncts of PerfRef $q_{\mathcal{O}}^{i+1, \Sigma}$ (respectively, PerfRef $\left.{ }_{q_{\mathcal{O}}^{c, i+1}, \Sigma}\right)$ are obtained from the CQs in $\operatorname{PerfectRef}\left(\mathcal{O}, q_{\mathcal{O}}^{i+1}\right)$ (respectively, $\left.\operatorname{PerfectRef}\left(\mathcal{O}, q_{\mathcal{O}}^{c, i+1}\right)\right)$ by just unfolding the atoms in their body, which amounts to replace each predicate name $P$ with $s_{1}$.

It follows that the disjunct $q_{\mathcal{S}}^{i+1}$ of $\operatorname{PerfRef}_{q_{\mathcal{O}}^{i+1}, \Sigma}$ (respectively, PerfRef ${ }_{q_{\mathcal{O}}^{c, i+1}, \Sigma}$ ) obtained from the CQ $q_{\mathcal{O}}^{i+1}$ (respectively, $q_{\mathcal{O}}^{c, i+1}$ ) by unfolding each atom in its body is such that $q_{\mathcal{S}}^{i+1} \nsubseteq \operatorname{PerfRef}_{q_{\mathcal{O}}^{i}, \Sigma}$ (respectively, $q_{\mathcal{S}}^{i+1} \nsubseteq \operatorname{PerfRef}_{q_{\mathcal{O}}^{c, i}, \Sigma}$ ), as required. 
Lemma 6.10. If a $C Q q_{\mathcal{O}}^{\prime}$ is a sound $\mathcal{S}$-to- $\mathcal{O}$ $\Sigma$-rewriting of $q_{\mathcal{S}}$, then, for some $i \geq 0$, either $q_{\mathcal{O}}^{\prime} \sqsubseteq q_{\mathcal{O}}^{i}$, or $q_{\mathcal{O}}^{\prime} \sqsubseteq q_{\mathcal{O}}^{c, i}$ for some constant $c \in$ Const.

Proof. Let a CQ $q_{\mathcal{O}}^{\prime}=\left\{(t) \mid \exists \overrightarrow{y^{\prime}} \cdot \phi\left(t, \overrightarrow{y^{\prime}}\right)\right\}$ be a sound $\mathcal{S}$-to- $\mathcal{O} \sum$-rewriting of $q_{\mathcal{S}}$, where the target list is composed of a single term $t$ (i.e., either a distinguished variable or a constant). Clearly, since $q_{\mathcal{O}}^{\prime}$ is a sound $\mathcal{S}$-to- $\mathcal{O} \Sigma$-rewriting of $q_{\mathcal{S}}$, by inspecting the ontology $\mathcal{O}$ and the mapping $\mathcal{M}$, it is easy to see that $\phi\left(t, \overrightarrow{y^{\prime}}\right)$ must contain an atom of the form $P\left(t, t_{0}\right)$ for a term $t_{0}$ (unless $\phi\left(t, \overrightarrow{y^{\prime}}\right) \equiv \perp$, from which the claim trivially follows). Without loss of generality, we can assume that only one of such atom occurs in $\phi\left(t, \overrightarrow{y^{\prime}}\right)$. There are three possible different cases for the term $t_{0}:(i)$ $t_{0}$ is a constant; (ii) $t_{0}$ is an existential variable and $t_{0}=t ;(i i i) t_{0}$ is an existential variable and $t_{0} \neq t$. In case $(i)$, we trivially have that $q_{\mathcal{O}}^{\prime} \sqsubseteq q_{\mathcal{O}}^{t_{0}, 0}$. On the other hand, in case $(i i)$, we trivially have that $q_{\mathcal{O}}^{\prime} \sqsubseteq q_{\mathcal{O}}^{0}$. So, in what follows we explore case (iii)

By induction on natural numbers $i \geq 1$, we now prove that either one among $q_{\mathcal{O}}^{\prime} \sqsubseteq q_{\mathcal{O}}^{i}$ or $q_{\mathcal{O}}^{\prime} \sqsubseteq q_{\mathcal{O}}^{c, i}$ for some constant $c \in$ Const holds, or $\phi\left(t, \overrightarrow{y^{\prime}}\right)$ contains a conjunction of atom of the form $P\left(t_{2 i-1}, t_{2 i-2}\right) \wedge P\left(t_{2 i-1}, t_{2 i}\right)$ where $t_{2 i}$ is an existential variable and $t_{2 i} \neq t_{2 i-1}$.

Base step $(i=1)$ : Observe that the variable $t_{0}$ of the atom $P\left(t, t_{0}\right)$ must be in join with some other atom. Indeed, if not, $\operatorname{PerfectRef}\left(\mathcal{O}, q_{\mathcal{O}}^{\prime}\right)$ can apply the inclusion assertion $A \sqsubseteq \exists P$ to the atom $P\left(t, t_{0}\right)$ of $q_{\mathcal{O}}^{\prime}$, thus producing a new $\mathrm{CQ}$ in which there is the atom $A(t)$ instead of $P\left(t, t_{0}\right)$, and when rewriting this new CQ with respect to $\mathcal{M}$, the result is a disjunct of $\operatorname{PerfRef}_{q_{\mathcal{O}}^{\prime}, \Sigma}$ that is not contained in $q_{\mathcal{S}}$, thus contradicting the fact that $q_{\mathcal{O}}^{\prime}$ is a sound $\mathcal{S}$-to- $\mathcal{O} \Sigma$-rewriting of $q_{\mathcal{S}}$.

There are two possible cases: either there is an atom of the form $P\left(t_{0}, t_{1}\right)$ for some term $t_{1}$, or there is a conjunction of atoms of the form $P\left(t_{1}, t_{0}\right) \wedge P\left(t_{1}, t_{2}\right)$ for some pair of terms $t_{1}, t_{2}$. Indeed, if otherwise only the atom $P\left(t_{1}, t_{0}\right)$ occurs (and $P\left(t_{1}, t_{2}\right)$ for some term $t_{2}$ does not occur), the same reasoning delineated above applies, after that the unification step of $\operatorname{Perfect\operatorname {Ref}}\left(\mathcal{O}, q_{\mathcal{O}}^{\prime}\right)$ unifies term $t_{1}$ with term $t$, thus reducing the conjunction of atoms $P\left(t, t_{0}\right) \wedge P\left(t_{1}, t_{0}\right)$ to only $P\left(t, t_{0}\right)$.

In the former case, we trivially have that $q_{\mathcal{O}}^{\prime} \sqsubseteq q_{\mathcal{O}}^{0}$, and therefore $q_{\mathcal{O}}^{\prime} \sqsubseteq q_{\mathcal{O}}^{1}$ (observe that $q_{\mathcal{O}}^{l} \sqsubseteq q_{\mathcal{O}}^{m}$ for any pair of natural numbers $l, m \geq 0$ with $l \leq m$ ). In the latter case, there are three possibilities: $(i) t_{2}$ is a constant; $(i i) t_{2}$ is an existential variable and $t_{2}=t_{1} ;($ iii $) t_{2}$ is an existential variable and $t_{2} \neq t_{1}$. In case $(i)$ we trivially have that $q_{\mathcal{O}}^{\prime} \sqsubseteq q_{\mathcal{O}}^{t_{2}, 1}$. On the other hand, in case $(i i)$, we have that $q_{\mathcal{O}}^{\prime} \sqsubseteq q_{\mathcal{O}}^{1}$ with the homomorphism $h$ from $q_{\mathcal{O}}^{1}$ to $q_{\mathcal{O}}^{\prime}$ in which $h(x)=t, h\left(y_{0}\right)=t_{0}$ $h\left(y_{1}\right)=y_{1}$, and $h\left(y_{2}\right)=h\left(y_{3}\right)=t_{1}=t_{2}$. In case $(i i i)$, there is the conjunction of atoms $P\left(t_{2 i-1}, t_{2 i-2}\right) \wedge P\left(t_{2 i-1}, t_{2 i}\right)$ where $t_{2 i}$ is an existential variable and $t_{2 i} \neq t_{2 i-1}$. So, the base step of the induction is verified.

Inductive step: By the inductive hypothesis, we derive that either there is a natural number $k \leq i-1$ for which one among $q_{\mathcal{O}}^{\prime} \sqsubseteq q_{\mathcal{O}}^{k}$ or $q_{\mathcal{O}}^{\prime} \sqsubseteq q_{\mathcal{O}}^{c, k}$ for some constant $c \in$ Const holds, or $\phi\left(t, \overrightarrow{y^{\prime}}\right)$ contains a conjunction of atoms of the form $\bigwedge_{j=1}^{i-1} P\left(t_{2 j-1}, t_{2 j-2}\right) \wedge P\left(t_{2 j-1}, t_{2 j}\right)$ where, for each $j \in[1, i-1]$, term $t_{2 j}$ is an existential variable and $t_{2 j} \neq t_{2 j-1}$. Consider the former case. As already observed, for any $i \geq 0$ and for any $k \leq i-1$, the query $q_{\mathcal{O}}^{k}$ (respectively, $q_{\mathcal{O}}^{c, k}$ for any constant $c \in$ Const) can be obtained from $q_{\mathcal{O}}^{i}$ (respectively, $q_{\mathcal{O}}^{c, i}$ ) by applying some set of equalities between the variables in $q_{\mathcal{O}}^{i}$ (respectively, $q_{\mathcal{O}}^{c, i}$ ). This implies that $q_{\mathcal{O}}^{k} \sqsubseteq q_{\mathcal{O}}^{i}$ 
(respectively, $\left.q_{\mathcal{O}}^{c, k} \sqsubseteq q_{\mathcal{O}}^{c, i}\right)$. It follows that, if one among $q_{\mathcal{O}}^{\prime} \sqsubseteq q_{\mathcal{O}}^{k}$ or $q_{\mathcal{O}}^{\prime} \sqsubseteq q_{\mathcal{O}}^{c, k}$ for some constant $c \in$ Const holds, then, since $q_{\mathcal{O}}^{k} \sqsubseteq q_{\mathcal{O}}^{i}$ (respectively, $q_{\mathcal{O}}^{c, k} \sqsubseteq q_{\mathcal{O}}^{c, i}$ ), we have that either one among $q_{\mathcal{O}}^{\prime} \sqsubseteq q_{\mathcal{O}}^{i}$ or $q_{\mathcal{O}}^{\prime} \sqsubseteq q_{\mathcal{O}}^{c, i}$ holds as well.

Consider the latter case. Observe that the variable $t_{2 i-2}$ of the atom $P\left(t_{2 i-3}, t_{2 i-2}\right)$ must be in join with some other atom. Indeed, if not, then $\operatorname{PerfectRef}\left(\mathcal{O}, q_{\mathcal{O}}^{\prime}\right)$ can apply the inclusion assertion $A \sqsubseteq \exists P$ to the atom $P\left(t, t_{0}\right)$ of $q_{\mathcal{O}}^{\prime}$ after unifying the variables $t$ and $t_{2 j-1}$ for each $j \in[1, i-1]$ (i.e., after applying the set of equalities $e=\left\{t=t_{1}, t_{1}=t_{3}, \ldots, t_{2 i-5}=t_{2 i-3}\right\}$ ). In this way, a new CQ is produced in which there is the atom $A(t)$ instead of $P\left(t, t_{0}\right)$ (notice that this would happen because, for each $j \in[0, i-1]$, term $t_{2 j}$ is an existential variable such that $t_{2 j} \neq t_{2 j-1}$ ), and when rewriting this new CQ with respect to $\mathcal{M}$, the result is a disjunct of PerfRef $q_{q_{\mathcal{O}}^{\prime}, \Sigma}$ that is not contained in $q_{\mathcal{S}}$, thus contradicting the fact that $q_{\mathcal{O}}^{\prime}$ is a sound $\mathcal{S}$-to- $\mathcal{O} \Sigma$-rewriting of $q_{\mathcal{S}}$. We can now proceed in a very similar way to the base step case $(i=1)$. We write it for the sake of completeness.

There are two possible cases: either there is an atom $P\left(t_{2 i-2}, t_{2 i-1}\right)$ for some term $t_{2 i-1}$, or there is a conjunction of atoms $P\left(t_{2 i-1}, t_{2 i-2}\right) \wedge P\left(t_{2 i-1}, t_{2 i}\right)$ for some pair of terms $t_{2 i-1}, t_{2 i}$. In the former case, one can verify that, by construction, $q_{\mathcal{O}}^{\prime} \sqsubseteq q_{\mathcal{O}}^{i-1}$, and therefore $q_{\mathcal{O}}^{\prime} \sqsubseteq q_{\mathcal{O}}^{i}$. In the latter case, there are three possibilities: $(i) t_{2 i}$ is a constant; $(i i) t_{2 i}$ is an existential variable and $t_{2 i}=t_{2 i-1}$; (iii) $t_{2 i}$ is an existential variable and $t_{2 i} \neq t_{2 i-1}$. In case $(i)$, we trivially have that $q_{\mathcal{O}}^{\prime} \sqsubseteq q_{\mathcal{O}}^{t_{2 i}, i}$. On the other hand, in case $(i i)$, we have that $q_{\mathcal{O}}^{\prime} \sqsubseteq q_{\mathcal{O}}^{i}$ with the homomorphism $h$ from $q_{\mathcal{O}}^{i}$ to $q_{\mathcal{O}}^{\prime}$ in which $h(x)=t, h\left(y_{j}\right)=t_{j}$ for each $j \in[0,2 i-1]$, and $h\left(y_{2 i}\right)=h\left(y_{2 i+1}\right)=t_{2 i-1}=t_{2 i}$. In case $(i i i)$, there is the conjunction of atoms $P\left(t_{2 i-1}, t_{2 i-2}\right) \wedge P\left(t_{2 i-1}, t_{2 i}\right)$ where $t_{2 i}$ is an existential variable and $t_{2 i} \neq t_{2 i-1}$. So, even the induction step is verified.

Since by assumption $q_{\mathcal{O}}^{\prime}$ is a CQ, and so its body consists of a finite set of atoms, from the above induction we derive that there must be a natural number $i$ for which one among $q_{\mathcal{O}}^{\prime} \sqsubseteq q_{\mathcal{O}}^{i}$ or $q_{\mathcal{O}}^{\prime} \sqsubseteq q_{\mathcal{O}}^{c, i}$ for some constant $c \in$ Const holds, as required.

Using the above lemmata, we are now able to prove that no UCQ-maximally sound $\mathcal{S}$-to- $\mathcal{O} \Sigma$-rewriting of $q_{\mathcal{S}}$ exists. Suppose, for the sake of contradiction, the existence of a UCQ $q_{\mathcal{O}}^{\prime}=q_{\mathcal{O}}^{\prime}{ }^{1} \cup \ldots \cup q_{\mathcal{O}}^{\prime}{ }^{n}$ which is a UCQ-maximally sound $\mathcal{S}$-to- $\mathcal{O} \Sigma$-rewriting of $q_{\mathcal{S}}$. For each $j \in[1, n]$, by Lemma 6.10, we know the existence of a number $i_{j}$ for which either $q_{\mathcal{O}}^{\prime}{ }^{j} \sqsubseteq q_{\mathcal{O}}^{i_{j}}$, or $q_{\mathcal{O}}^{\prime}{ }^{j} \sqsubseteq q_{\mathcal{O}}^{c, i_{j}}$ for some constant $c \in$ Const holds. It follows that $q_{\mathcal{O}}^{\prime} \sqsubseteq q_{\mathcal{O}}^{\prime \prime}=q_{\mathcal{O}}^{\prime \prime}{ }^{1} \cup \ldots \cup q_{\mathcal{O}}^{\prime \prime}{ }^{n}$, where, for each $j \in[1, n]$, the CQ $q_{\mathcal{O}}^{\prime \prime}{ }^{j}$ corresponds to $q_{\mathcal{O}}^{i_{j}}$ if $q_{\mathcal{O}}^{\prime}{ }^{j} \sqsubseteq q_{\mathcal{O}}^{i_{j}}$, and to $q_{\mathcal{O}}^{c, i_{j}}$ if $q_{\mathcal{O}}^{\prime}{ }^{j} \sqsubseteq q_{\mathcal{O}}^{c, i_{j}}$. Consider now the query $q_{\mathcal{O}}^{\prime \prime \prime}=q_{\mathcal{O}}^{\prime \prime \prime}{ }^{1} \cup \ldots \cup q_{\mathcal{O}}^{\prime \prime \prime n}$ where, for each $j \in[1, n]$, the CQ $q_{\mathcal{O}}^{\prime \prime \prime j}$ corresponds to $q_{\mathcal{O}}^{i_{j}+1}$ if $q_{\mathcal{O}}^{\prime} \sqsubseteq q_{\mathcal{O}}^{i_{j}}$, and to $q_{\mathcal{O}}^{c, i_{j}+1}$ if $q_{\mathcal{O}}^{\prime}{ }^{j} \sqsubseteq q_{\mathcal{O}}^{c, i_{j}}$. Observe that, by Lemma 6.9. we have $\operatorname{cert}_{q_{\mathcal{O}}^{\prime \prime}, \Sigma} \sqsubset \operatorname{cert}_{q_{\mathcal{O}}^{\prime \prime \prime}, \Sigma}$ for each $j \in[1, n]$. This implies that $\operatorname{cert}_{q_{\mathcal{O}}^{\prime \prime}, \Sigma} \sqsubset \operatorname{cert}_{q_{\mathcal{O}}^{\prime \prime \prime}, \Sigma}$, and therefore, since $q_{\mathcal{O}}^{\prime} \sqsubseteq q_{\mathcal{O}}^{\prime \prime}$, we also have that $\operatorname{cert}_{q_{\mathcal{O}}^{\prime}, \Sigma} \sqsubset$ cert $_{q_{\mathcal{O}}^{\prime \prime \prime}, \Sigma}$. Furthermore, since by Lemma 6.8 each disjunct of $q_{\mathcal{O}}^{\prime \prime \prime}$ is a sound $\mathcal{S}$-to- $\mathcal{O} \Sigma$-rewriting of $q_{\mathcal{S}}$, we can finally conclude that $q_{\mathcal{O}}^{\prime \prime \prime}$ is a sound $\mathcal{S}$-to- $\mathcal{O} \Sigma$-rewriting of $q_{\mathcal{S}}$ such that cert $_{q_{\mathcal{O}}^{\prime}, \Sigma} \sqsubset$ cert $_{q_{\mathcal{O}}^{\prime \prime \prime}, \Sigma}$. Thus, following Definition 3.4 this is clearly a contradiction on the fact that $q_{\mathcal{O}}^{\prime}$ is a UCQ-maximally sound $\mathcal{S}$-to- $\mathcal{O} \Sigma$-rewriting of $q_{\mathcal{S}}$, as required.

Two interesting considerations follow:

(i) For a more accurate characterisation about the illustrated OBDM specification $\Sigma$ and CQ $q_{\mathcal{S}}$, we refer the reader to Chapter 9 (specifically to Section 9.4), where 
we will see that in this case even a perfect $\mathcal{S}$-to- $\mathcal{O} \Sigma$-rewriting of $q_{\mathcal{S}}$ does exist, and can be expressed in a target query language equipped with an epistemic operator.

(ii) We point out that the same negative result applies also to CQJFEs $q_{\mathcal{S}}$ with no existential variables at all in their body (i.e., the so-called full conjunctive queries). To see this, it is sufficient to consider a slight modification of the above illustrated OBDM specification $\Sigma=\langle\mathcal{O}, \mathcal{S}, \mathcal{M}\rangle$ and CQJFE $q_{\mathcal{S}}$. Specifically, let $\Sigma_{f}=\left\langle\mathcal{O}_{f}, \mathcal{S}_{f}, \mathcal{M}_{f}\right\rangle$ be the following OBDM specification:

- $\mathcal{O}_{f}=\mathcal{O}=\{A \sqsubseteq \exists P\}$

- $\mathcal{S}_{f}=\left\{s_{1}, s_{2}, s_{3}\right\}$

- $\mathcal{M}_{f}=\left\{m_{1}, m_{2}\right\}$, where:

$$
\begin{array}{rrrl}
m_{1}: & s_{1}\left(x_{1}\right) \wedge s_{3}\left(x_{1}, x_{2}\right) & \rightarrow & P\left(x_{1}, x_{2}\right), \\
m_{2}: & s_{2}(x) & \rightarrow A(x) .
\end{array}
$$

Consider now the full conjunctive query $q_{\mathcal{S}}^{f}=\left\{(x) \mid s_{1}(x)\right\}$. One can easily verify that all the steps in the above proof remain valid even if we replace the OBDM specification $\Sigma$ with $\Sigma_{f}$ and the query $q_{\mathcal{S}}$ with $q_{\mathcal{S}}^{f}$. Thus, the above proof also shows that no UCQ-maximally sound $\mathcal{S}_{f}$-to- $\mathcal{O}_{f} \Sigma_{f}$-rewriting of $q_{\mathcal{S}}^{f}$ exists.

4 The proof is based on the idea that an assertion of the form $A \sqsubseteq \exists P$ entailed by a $D L$-Lite R $_{\mathcal{R}}$ ontology $\mathcal{O}$ can be simulated using LAV mapping assertions. In our case, consider the OBDM specification $\Sigma=\langle\mathcal{O}, \mathcal{S}, \mathcal{M}\rangle$ illustrated in the proof of point 3 , and let $\Sigma^{\prime}=\left\langle\mathcal{O}^{\prime}, \mathcal{S}, \mathcal{M}^{\prime}\right\rangle$ be the OBDM specification in which $\mathcal{O}^{\prime}=\emptyset\left(\mathcal{O}^{\prime}\right.$ and $\mathcal{O}$ share the same alphabet) and $\mathcal{M}^{\prime}=\mathcal{M} \cup\left\{m_{3}: s_{2}(x) \rightarrow \exists y \cdot P(x, y)\right\}$. It can be readily seen that $\Sigma^{\prime}$ is query-preserving with respect to $\Sigma$ Calì et al., 2002 Lenzerini, 2002, that is, $\operatorname{cert}_{q, \Sigma}^{D}=\operatorname{cert}_{q, \Sigma^{\prime}}^{D}$ for every query $q$ over $\mathcal{O}$ (equivalently, over $\mathcal{O}^{\prime}$ ) and for every $\mathcal{S}$-database $D$.

Therefore, a formal proof of this case can be obtained from the above proof of point 3 by replacing the OBDM specification $\Sigma$ with the OBDM specification $\Sigma^{\prime}$.

Furthermore, it is clear that the same reasoning can be done with the OBDM specification $\Sigma_{f}$, and thus also in this case the same negative result applies also when the queries over $\mathcal{S}$ are full conjunctive queries.

5 Let $\Sigma=\langle\mathcal{O}, \mathcal{S}, \mathcal{M}\rangle$ be the following OBDM specification:

- $\mathcal{O}=\emptyset$

- $\mathcal{S}=\left\{s_{1}, s_{2}\right\}$

- $\mathcal{M}=\left\{m_{1}, m_{2}\right\}$, where:

$$
\begin{aligned}
& m_{1}: s_{1}\left(x_{1}, x_{2}\right) \rightarrow P\left(x_{1}, x_{2}\right), \\
& m_{2}: \quad s_{2}(x) \quad \rightarrow \quad P(x, x) .
\end{aligned}
$$

Let $q_{\mathcal{S}}$ be the following boolean CQJFE over $\mathcal{S}: q_{\mathcal{S}}=\left\{\left(x_{1}, x_{2}\right) \mid s_{1}\left(x_{1}, x_{2}\right)\right\}$.

Observe that the CQ $q_{\mathcal{O}}^{\prime}=\left\{\left(x_{1}, x_{2}\right) \mid P\left(x_{1}, x_{2}\right)\right\}$ is not a sound $\mathcal{S}$-to- $\mathcal{O} \Sigma$ rewriting of $q_{\mathcal{S}}$, because the query $q_{\mathcal{S}}^{\prime}=\left\{(x, x) \mid s_{2}(x)\right\}$ is a disjunct of PerfRef $q_{q_{\mathcal{O}}^{\prime}, \Sigma}$ such that $q_{\mathcal{S}}^{\prime} \nsubseteq q_{\mathcal{S}}$. 
Lemma 6.11. If a $C Q q_{\mathcal{O}}$ is a sound $\mathcal{S}$-to- $\mathcal{O}$ $\Sigma$-rewriting of $q_{\mathcal{S}}$, then either $q_{\mathcal{O}} \equiv \perp$ or the target list of $q_{\mathcal{O}}$ does not include any distinguished variable.

Proof. We prove the claim by contradiction. Let $q_{\mathcal{O}}=\left\{\left(t_{1}, t_{2}\right) \mid \exists \vec{y} \cdot \phi\left(t_{1}, t_{2}, \vec{y}\right)\right\}$ be a $\mathrm{CQ}$ over $\mathcal{O}$ such that $\phi\left(t_{1}, t_{2}, \vec{y}\right) \not \equiv \perp$, i.e., $\phi\left(t_{1}, t_{2}, \vec{y}\right)$ is a conjunction of atoms with $P$ as predicate name, and at least one among $t_{1}$ and $t_{2}$ (which are not necessarily different) is a distinguished variable. There are two possible cases: either $P\left(t_{1}, t_{2}\right)$ is an atom occurring in $\phi\left(t_{1}, t_{2}, \vec{y}\right)$, or not.

In the former case, consider the disjunct $q_{\mathcal{S}}^{\prime}=\left\{(t, t) \mid \exists \vec{y} \cdot \phi^{\prime}(t, \vec{y})\right\}$ of $\operatorname{PerfRef}_{q_{\mathcal{O}}, \Sigma}$ obtained by unfolding $(i)$ each atom of the form $P(z, z)$ for a term $z$ with $s_{2}(z),(i i)$ each atom $P\left(t_{1}, t_{2}\right)$ with $s_{2}(t)$, thus imposing the equality $t_{1}=t_{2}$ (where $t=t_{i}$ if $t_{i}$ is a constant for $i=1$ or $i=2$, otherwise $t$ denotes a fresh term), and (iii) each remaining atom $P\left(z_{1}, z_{2}\right)$ for distinct terms $z_{1}$ and $z_{2}$ such that either $z_{1} \neq t_{1}$ or $z_{2} \neq t_{2}$ with $s_{1}\left(z_{1}, z_{2}\right)$. Observe that, since by assumption one among $t_{1}$ and $t_{2}$ is a distinguished variable, such equality is never an equality between different constant.

Consider now each possible function $f$ from terms of $q_{\mathcal{S}}$ to $q_{\mathcal{S}}^{\prime}$ for which $f\left(x_{1}\right)=t$ and $f\left(x_{2}\right)=t$. By construction, in $q_{\mathcal{S}}^{\prime}$ there is no atom of the form $s_{1}(t, t)$, and therefore $s_{1}\left(f\left(x_{1}\right), f\left(x_{2}\right)\right)$ does not occur in the body of $q_{\mathcal{S}}^{\prime}$, where $s_{1}\left(x_{1}, x_{2}\right)$ is the atom occurring in $q_{\mathcal{S}}$. It follows that there is no homomorphism from $q_{\mathcal{S}}$ to $q_{\mathcal{S}}^{\prime}$ which, due to [Chandra and Merlin, 1977], implies that $q_{\mathcal{S}}^{\prime} \nsubseteq q_{\mathcal{S}}$. Since $q_{\mathcal{S}}^{\prime}$ is a disjunct of $\operatorname{PerfRef}_{q_{\mathcal{O}}, \Sigma}$ such that $q_{\mathcal{S}}^{\prime} \nsubseteq q_{\mathcal{S}}$, we get that PerfRef $q_{\mathcal{O}}, \Sigma \nsubseteq q_{\mathcal{S}}$ which, due to Lemma 6.1, implies that $q_{\mathcal{O}}$ is not a sound $\mathcal{S}$-to- $\mathcal{O} \Sigma$-rewriting of $q_{\mathcal{S}}$, as required.

In the latter case, consider the disjunct $q_{\mathcal{S}}^{\prime}=\left\{\left(t_{1}, t_{2}\right) \mid \exists \vec{y} . \phi^{\prime}\left(t_{1}, t_{2}, \vec{y}\right)\right\}$ obtained by unfolding each atom $P\left(z_{1}, z_{2}\right)$ occurring in $q_{\mathcal{O}}$ with $s_{1}\left(z_{1}, z_{2}\right)$. Since $P\left(t_{1}, t_{2}\right)$ never occurs in the body of $q_{\mathcal{O}}$, we have that each possible function $f$ from terms of $q_{\mathcal{S}}$ to $q_{\mathcal{S}}^{\prime}$ for which $f\left(x_{1}\right)=t_{1}$ and $f\left(x_{2}\right)=t_{2}$ is such that $s_{1}\left(f\left(x_{1}\right), f\left(x_{2}\right)\right)$ does not occur in the body of $q_{\mathcal{S}}^{\prime}$. With the same arguments given in the previous case, we obtain that $q_{\mathcal{O}}$ is not a sound $\mathcal{S}$-to- $\mathcal{O} \Sigma$-rewriting of $q_{\mathcal{S}}$, as required.

From the above lemma, we easily derive that each possible candidate CQmaximally sound $\mathcal{S}$-to- $\mathcal{O} \Sigma$-rewriting of $q_{\mathcal{S}}$ is of the form $\left\{\left(c_{1}, c_{2}\right) \mid P\left(c_{1}, c_{2}\right)\right\}$, where $c_{1}, c_{2}$ are constants in Const. With this observation at hand, we are now ready to prove that no UCQ-maximally sound $\mathcal{S}$-to- $\mathcal{O} \Sigma$-rewriting of $q_{\mathcal{S}}$ exists. Suppose, for the sake of contradiction, the existence of a UCQ $q_{\mathcal{O}}^{\prime}=q_{\mathcal{O}}^{\prime}{ }^{1} \cup \ldots \cup q_{\mathcal{O}}^{\prime}{ }^{n}$ which is a UCQ-maximally sound $\mathcal{S}$-to- $\mathcal{O} \Sigma$-rewriting of $q_{\mathcal{S}}$. Consider now the query $q_{\mathcal{O}}^{\prime \prime}=$ $q_{\mathcal{O}}^{\prime} \cup q_{\mathcal{O}}^{\prime}{ }^{n+1}$, where $q_{\mathcal{O}}^{\prime}{ }^{n+1}=\left\{\left(c, c^{\prime}\right) \mid P\left(c, c^{\prime}\right)\right\}$ with $c, c^{\prime}$ being distinct fresh constants of Const not occurring anywhere else in the disjuncts of $q_{\mathcal{O}}^{\prime}$. Obviously, we have that cert $_{q_{\mathcal{O}}^{\prime}{ }^{n+1}, \Sigma} \not \operatorname{cert}_{q_{\mathcal{O}}^{\prime}, \Sigma}$, for each $i \in[1, n]$. This implies that $\operatorname{cert}_{q_{\mathcal{O}}^{\prime}, \Sigma} \sqsubset \operatorname{cert}_{q_{\mathcal{O}}^{\prime \prime}, \Sigma}$. Furthermore, since $q_{\mathcal{O}}^{\prime}{ }^{n+1}$ is a sound $\mathcal{S}$-to- $\mathcal{O} \Sigma$-rewriting of $q_{\mathcal{S}}$, we can finally conclude that $q_{\mathcal{O}}^{\prime \prime}$ is a sound $\mathcal{S}$-to- $\mathcal{O} \Sigma$-rewriting of $q_{\mathcal{S}}$ such that $\operatorname{cert}_{q_{\mathcal{O}}^{\prime}, \Sigma} \sqsubset \operatorname{cert}_{q_{\mathcal{O}}^{\prime \prime} \Sigma}$. Thus, following Definition 3.4 , this is clearly a contradiction on the fact that $q_{\mathcal{O}}^{\prime}$ is a UCQ-maximally sound $\mathcal{S}$-to- $\mathcal{O} \Sigma$-rewriting of $q_{\mathcal{S}}$, as required.

For a more accurate characterisation about the illustrated OBDM specification $\Sigma$ and $\mathrm{CQ} q_{\mathcal{S}}$, the $\mathrm{CQ}^{\neq} q_{\mathcal{O}}=\left\{\left(x_{1}, x_{2}\right) \mid P\left(x_{1}, x_{2}\right) \wedge x_{1} \neq x_{2}\right\}$ over $\mathcal{O}$ is a sound $\mathcal{S}$-to- $\mathcal{O} \Sigma$-rewriting of $q_{\mathcal{S}}$. More precisely, one can verify that $q_{\mathcal{O}}$ is the (unique up to equivalence w.r.t. $\Sigma) \mathrm{UCQ}^{\neq}$-maximally sound $\mathcal{S}$-to- $\mathcal{O} \Sigma$-rewriting of $q_{\mathcal{S}}$. 


\section{Chapter 7}

\section{Perfect Source-to-Ontology Rewritings}

With the results of previous chapters at hand, we now study both the verification, and the computation problem for perfect source-to-ontology rewritings.

\subsection{Verification Problem}

We remind the reader that, by definition, $q_{\mathcal{O}}$ is a perfect $\mathcal{S}$-to- $\mathcal{O} \Sigma$ rewriting of $q_{\mathcal{S}}$ if and only if it is both a sound, and a complete $\mathcal{S}$-to- $\mathcal{O} \Sigma$-rewriting of $q_{\mathcal{S}}$. Thus, by combining Lemma 6.1 and Lemma 5.1, we immediately get the following.

Corollary 7.1. $q_{\mathcal{O}}$ is a perfect $\mathcal{S}$-to-O $\mathcal{O} \Sigma$-rewriting of $q_{\mathcal{S}}$ if and only if both $\operatorname{PerfRe}_{q_{\mathcal{O}}, \Sigma} \sqsubseteq\left(q_{\mathcal{S}} \cup \operatorname{PerfRef}_{\mathcal{V}_{\mathcal{O}}^{n}, \Sigma}\right)$ and $q_{\mathcal{S}} \sqsubseteq\left(\operatorname{PerfRef}_{q_{\mathcal{O}}, \Sigma} \cup \operatorname{PerfRe}_{\mathcal{V}_{\mathcal{O}}^{n}, \Sigma}\right)$ hold, where $n=\operatorname{ar}\left(q_{\mathcal{O}}\right)=\operatorname{ar}\left(q_{\mathcal{S}}\right)$.

As already discussed in Subsection 3.3.1, for OBDM specifications where inconsistencies can not arise, the notion of perfect source-to-ontology rewriting coincides with the notion of realization considered in Lutz et al., 2018. Since the problem of checking whether $q_{\mathcal{O}}$ is a realization of $q_{\mathcal{S}}$ in $\Sigma$ is in general $\Pi_{2}^{p}$-hard even when $\mathcal{O}$ contains no assertions (i.e., $\mathcal{O}=\emptyset), \mathcal{M}$ is a pure GAV mapping, and both $q_{\mathcal{S}}$ and $q_{\mathcal{O}}$ are boolean CQs Lutz et al., 2018, Theorem 11], and since in those cases the two notions are equivalent, such lower bound applies also to our notion.

The following theorem characterises the computational complexity of the verification problem for perfect source-to-ontology rewritings.

Theorem 7.1. The verification problem for perfect source-to-ontology rewritings is $\Pi_{2}^{p}$-complete.

Proof. As for the upper bound, by virtue of Corollary 7.1, it is sufficient to show how to check the following two containments in $\Pi_{2}^{p}$, where $n=\operatorname{ar}\left(q_{\mathcal{O}}\right)=\operatorname{ar}\left(q_{\mathcal{S}}\right):(i)$ $\operatorname{PerfRef}_{q_{\mathcal{O}}, \Sigma} \sqsubseteq\left(q_{\mathcal{S}} \cup \operatorname{PerfRef}_{\mathcal{Y}_{\mathcal{O}}^{n}, \Sigma}\right)$, which holds if and only if $q_{\mathcal{O}}$ is a sound $\mathcal{S}$-to- $\mathcal{O}$ $\Sigma$-rewriting of $q_{\mathcal{S}}$; and $(i i) q_{\mathcal{S}} \sqsubseteq\left(\operatorname{PerfRef}_{q_{\mathcal{O}}, \Sigma} \cup \operatorname{PerfRef}_{\mathcal{V}_{\mathcal{O}}^{n}, \Sigma}\right)$, which holds if and only if $q_{\mathcal{O}}$ is a complete $\mathcal{S}$-to- $\mathcal{O} \Sigma$-rewriting of $q_{\mathcal{S}}$. By Theorem 6.1, the former can be verified in $\Pi_{2}^{p}$, and, by Theorem 5.1 , the latter can be verified even in NP.

As for the lower bound, it follows from Lutz et al., 2018, Theorem 11]. 
We leave as an interesting open problem the question of whether the computational complexity of the verification problem for perfect source-to-ontology rewritings decreases or not when $\mathcal{M}$ is a LAV mapping.

\subsection{Computation Problem}

As for computation, consider any OBDM specification $\Sigma=\langle\mathcal{O}, \mathcal{S}, \mathcal{M}\rangle$ and UCQ $q_{\mathcal{S}}$ over $\mathcal{S}$. We have that $(i)$ the unique (up to equivalence w.r.t. $\Sigma$ ) UCQ-minimally complete $\mathcal{S}$-to- $\mathcal{O} \Sigma$-rewriting of $q_{\mathcal{S}}$ always exists and can be computed by means of the MinimallyComplete algorithm (cf. Theorem 5.2); and (ii) by construction (see Definition 3.5), either this latter is also a sound, and therefore a perfect, $\mathcal{S}$-to- $\mathcal{O}$ $\Sigma$-rewriting of $q_{\mathcal{S}}$, or no UCQ-perfect $\mathcal{S}$-to- $\mathcal{O} \Sigma$-rewriting of $q_{\mathcal{S}}$ exists.

With these observations at hand, we can easily derive the algorithm Perfect together with its termination and correctness.

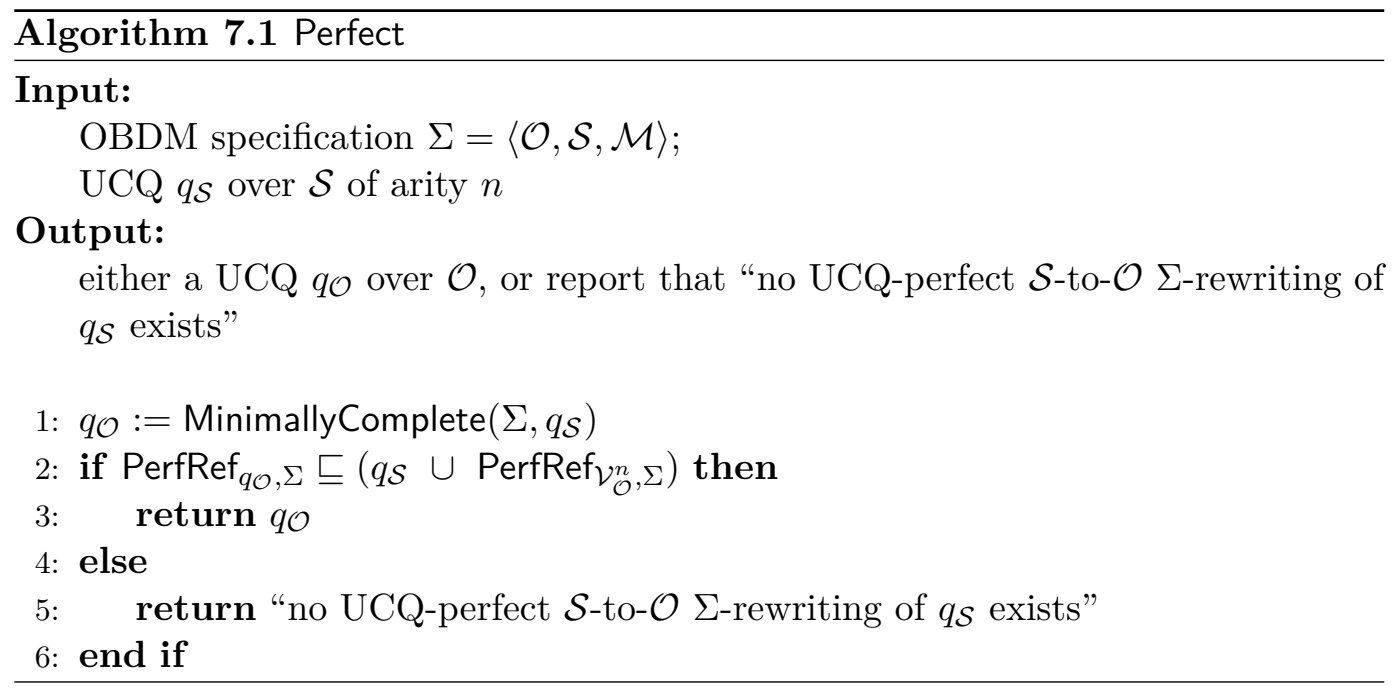

Essentially, the algorithm computes the unique (up to equivalence w.r.t. $\Sigma$ ) UCQ-minimally complete $\mathcal{S}$-to- $\mathcal{O} \Sigma$-rewriting of $q_{\mathcal{S}}$ using the MinimallyComplete algorithm (cf. Section 5.2), and then checks whether this latter is also a sound, and therefore a perfect, $\mathcal{S}$-to- $\mathcal{O} \Sigma$-rewriting of $q_{\mathcal{S}}$. Notice that this last step is always deterministically feasible in exponential time (cf. Theorem 6.1). Finally, observe that the overall running time of the algorithm is exponential in the size of the input.

Theorem 7.2. $\operatorname{Perfect}\left(\Sigma, q_{\mathcal{S}}\right)$ terminates and returns the perfect $\mathcal{S}$-to- $\mathcal{O} \Sigma$-rewriting of $q_{\mathcal{S}}$ if it exists and can be expressed as a UCQ, otherwise it reports that no $U C Q$-perfect $\mathcal{S}$-to- $\mathcal{O} \Sigma$-rewriting of $q_{\mathcal{S}}$ exists.

Furthermore, as a straightforward consequence of Corollary 5.1, we also get the following interesting result.

Corollary 7.2. The UCQ-perfect $\mathcal{S}$-to- $\mathcal{O} \Sigma$-rewriting of a $C Q q_{\mathcal{S}}$ either does not exists, or it can be expressed as a $C Q$ as well. 


\section{Chapter 8}

\section{Sound Source-to-Ontology Rewritings in Restricted Scenarios}

We now deal with the restricted scenarios mentioned in Section 6.2. Before delving into the technical part, we observe that, despite their limitations, the expressive power of these scenarios is enough for various meaningful applications. Indeed, several popular ontologies are expressible in the DL DL-Lite RDFS $_{\text {, e.g., SKOS }}^{1}$ Miles and Pérez-Agüera, 2007, Miles and Bechhofer, 2009] and Dublin Core ${ }^{2}$ Weibel et al., 1998, and the form of pure GAV mapping is exactly the one originally defined in the literature of data integration Lenzerini, 2002].

Furthermore, the class of (U)CQJFEs captures data services expressible in the famous (U)SPJ (Union, Select, Project, Join) fragment of Relational Algebra Codd, 1970], with the only limitation that joining variables must appear in the final projection of the USPJ Relational Algebra query, i.e., they appear in the target list of the equivalent UCQ. Notice that ( $i$ ) CQJFEs extends the class of Full Conjunctive Queries with the possibility of having non-join existential variables occurring in their body. This latter is a well-known class of queries studied for various optimisation in the relational database theory (see, e.g., Beame et al., 2014; Koutris et al., 2016; Ketsman and Suciu, 2017]); and (ii) such fragment is precisely the one needed for all tasks related to source profiling Abedjan et al., 2017; Abedjan et al., 2018.

In both the restricted scenarios, observe that the DL ontology language adopted in

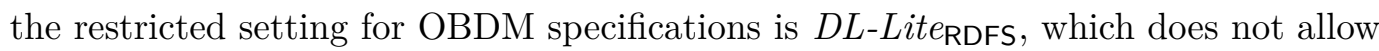
for disjointness axioms. It follows that, for any OBDM specification $\Sigma=\langle\mathcal{O}, \mathcal{S}, \mathcal{M}\rangle$ in this restricted setting, since $\mathcal{O}$ is a $D L$-Lite $e_{\text {RDF }}$ ontology, we have that $\mathcal{V}_{\mathcal{O}} \equiv \perp$. This has two further implications that are worth mentioning:

- Each $\mathcal{S}$-database $D$ is consistent with $\Sigma$, and therefore all the results we will present hold even according to the semantics proposed in Lutz et al., 2018];

- For each UCQ $q_{\mathcal{O}}$ over $\mathcal{O}$, the UCQ PerfRef $q_{\mathcal{O}}, \Sigma$ over $\mathcal{S}$ is the perfect $\mathcal{O}$-to- $\mathcal{S}$ $\Sigma$-rewriting of $q_{\mathcal{O}}$, that is, $\operatorname{cert}_{q_{\mathcal{O}, \Sigma}}^{D}=\operatorname{PerfRef}_{q_{\mathcal{O}}, \Sigma}^{D}$ for each $\mathcal{S}$-database $D$.

\footnotetext{
${ }^{1}$ Simple Knowledge Organization System: https://www.w3.org/2004/02/skos/

${ }^{2}$ http://dublincore.org/
} 
With the above considerations at hand, we easily get a refinement of Corollary 7.1 and Lemmata 6.1 and 5.1 in this restricted setting for OBDM specifications.

Corollary 8.1. In the setting for OBDM specifications of the restricted scenarios, $q_{\mathcal{O}}$ is a perfect (respectively, sound, complete) $\mathcal{S}$-to-O $\mathrm{O}$-rewriting of $q_{\mathcal{S}}$ if and only if $q_{\mathcal{S}} \equiv \operatorname{PerfRef}_{q_{\mathcal{O}}, \Sigma}$ (respectively, $\left.\operatorname{PerfRe}_{q_{\mathcal{O}}, \Sigma} \sqsubseteq q_{\mathcal{S}}, q_{\mathcal{S}} \sqsubseteq \operatorname{PerfRe}_{q_{\mathcal{O}}, \Sigma}\right)$.

Let us now introduce some further properties of this restricted setting. Given an OBDM specification $\Sigma=\langle\mathcal{O}, \mathcal{S}, \mathcal{M}\rangle$ of the restricted setting and an atom $\beta$ over $\mathcal{O}$, we denote by $\rho(\beta, \Sigma)$ the disjunction of conjunctions obtained by first unfolding $\beta$ with respect to $\mathcal{O}$, and then by unfolding the resulting formula with respect to $\mathcal{M}$.

The unfolding of an atom $\beta$ with respect to a DL-Lite RDFS ontology $\mathcal{O}$ is the disjunction of atoms $\lambda(\beta, \mathcal{O})$ defined as follows (see also Cima et al., 2020b):

$$
\begin{aligned}
& \lambda(A(t), \mathcal{O})=\bigvee_{A^{\prime}: \mathcal{O} \models A^{\prime} \sqsubseteq A} A^{\prime}(t) \vee \bigvee_{P: \mathcal{O} \models \exists P \sqsubseteq A}(\exists y \cdot P(t, y)) \vee \bigvee_{P: \mathcal{O} \models \exists P^{-} \sqsubseteq A}(\exists y \cdot P(y, t)), \\
& \lambda\left(P\left(t_{1}, t_{2}\right), \mathcal{O}\right)=\bigvee_{E: \mathcal{O} \models E \sqsubseteq P} E\left(t_{1}, t_{2}\right) \vee \bigvee_{E: \mathcal{O} \models E^{-} \sqsubseteq P} E\left(t_{2}, t_{1}\right),
\end{aligned}
$$

where $y$ denotes a fresh existential variable, $A$ and $A^{\prime}$ denote atomic concepts, and $P$ and $E$ denote atomic roles. Finally, $\mathcal{O} \models B \sqsubseteq A$ for a basic role $B$ and atomic concept $A$ (respectively, $\mathcal{O} \models R \sqsubseteq P$ for a basic role $R$ and atomic role $P$ ) hold if $B^{\mathcal{I}} \subseteq A^{\mathcal{I}}$ (respectively, $R^{\mathcal{I}} \subseteq P^{\mathcal{I}}$ ) in each interpretation $\mathcal{I}$ for $\mathcal{O}$ such that $\mathcal{I}=\mathcal{O}$.

The unfolding of a formula $\lambda(\beta, \mathcal{O})$ for an atom $\beta$ over an ontology $\mathcal{O}$ with respect to a pure GAV mapping $\mathcal{M}$ relating a schema $\mathcal{S}$ to $\mathcal{O}$ is obtained by replacing each atom $\beta^{\prime}$ occurring in $\lambda(\beta, \mathcal{O})$ with the logical disjunction of all the conjunctions of atoms over $\mathcal{S}$ corresponding to the left-hand sides of mapping assertions in $\mathcal{M}$ having the predicate name $\beta^{\prime}$ in the right-hand side (being careful to use unique variables in place of those variables that appear in the left-hand side of the mapping assertions but not in the right-hand side of those).

Example 8.1. Let $\Sigma=\langle\mathcal{O}, \mathcal{S}, \mathcal{M}\rangle$ be the following OBDM specification:

- $\mathcal{O}=\left\{\exists P_{2} \sqsubseteq A\right\}$

- $\mathcal{S}=\left\{s_{1}, s_{2}, s_{3}\right\}$

- $\mathcal{M}=\left\{m_{1}, m_{2}, m_{3}, m_{4}\right\}$, where:

$$
\begin{array}{rrrl}
m_{1}: & s_{1}\left(x_{1}, x_{2}\right) & \rightarrow & P_{1}\left(x_{1}, x_{2}\right), \\
m_{2}: & \exists y . s_{1}\left(x_{1}, y\right) \wedge s_{2}\left(y, x_{2}\right) & \rightarrow & P_{1}\left(x_{1}, x_{2}\right), \\
m_{3}: & \exists y . s_{2}(c, x) \wedge s_{3}(x, y) & \rightarrow A(x), \\
m_{4}: & s_{3}\left(x_{1}, x_{2}\right) & \rightarrow & P_{2}\left(x_{1}, x_{2}\right) .
\end{array}
$$

Consider the atoms $\beta_{1}=P_{1}(y, x)$ and $\beta_{2}=A(x)$ over $\mathcal{O}$. We have $\lambda\left(\beta_{1}, \mathcal{O}\right)=\beta_{1}$ and $\lambda\left(\beta_{2}, \mathcal{O}\right)=\beta_{2} \vee\left(\exists y_{2} \cdot P_{2}\left(x, y_{2}\right)\right)$. Thus, $\rho\left(\beta_{1}, \Sigma\right)=\left(s_{1}(y, x)\right) \vee\left(\exists y_{1} \cdot s_{1}\left(y, y_{1}\right) \wedge\right.$ $\left.s_{2}\left(y_{1}, x\right)\right)$, whereas $\rho\left(\beta_{2}, \Sigma\right)=\left(\exists y_{3} \cdot s_{2}(c, x) \wedge s_{3}\left(x, y_{3}\right)\right) \vee\left(\exists y_{2} \cdot s_{3}\left(x, y_{2}\right)\right)$. 
Finally, since $D L$-Lite RDFS $_{\text {ontologies }} \mathcal{O}$ contain no assertions with $\exists R$ occurring in the right-hand side for a basic role $R$, and since pure GAV mappings do not allow for repetitions of variables or constants in the right-hand side of mapping assertions, given an OBDM specification $\Sigma=\langle\mathcal{O}, \mathcal{S}, \mathcal{M}\rangle$ of the restricted setting and a $\mathrm{CQ}$ $q_{\mathcal{O}}=\{\vec{t} \mid \exists \vec{y} \cdot \phi(\vec{x}, \vec{y})\}$ over $\mathcal{O}$, it can be readily seen that $\operatorname{PerfRef}_{\mathcal{O}_{\mathcal{O}}, \Sigma}$ is equivalent to turning the following logical query into an equivalent UCQ over $\mathcal{S}$ :

$$
\left\{\vec{t} \mid \exists \vec{y} \cdot \bigwedge_{\beta \in \phi(\vec{x}, \vec{y})} \rho(\beta, \Sigma)\right\}
$$

Example 8.2. Let $\Sigma=\langle\mathcal{O}, \mathcal{S}, \mathcal{M}\rangle$ be the the OBDM specification illustrated in Example 8.1. Consider the CQ $q_{\mathcal{O}}=\left\{(x) \mid \exists y \cdot P_{1}(y, x) \wedge A(x)\right\}$ over $\mathcal{O}$, and let $\beta_{1}=P_{1}(y, x)$ and $\beta_{2}=A(x)$. Then, $\operatorname{PerfRef}_{q_{\mathcal{O}}, \Sigma}$ can be obtained by turning the logical query $\left\{(x) \mid \exists y \cdot \rho\left(\beta_{1}, \Sigma\right) \wedge \rho\left(\beta_{2}, \Sigma\right)\right\}=\left\{(x) \mid \exists y \cdot\left(\left(s_{1}(y, x)\right) \vee\left(\exists y_{1} . s_{1}\left(y, y_{1}\right) \wedge\right.\right.\right.$ $\left.\left.\left.s_{2}\left(y_{1}, x\right)\right)\right) \wedge\left(\left(\exists y_{3} \cdot s_{2}(c, x) \wedge s_{3}\left(x, y_{3}\right)\right) \vee\left(\exists y_{2} \cdot s_{3}\left(x, y_{2}\right)\right)\right)\right\}$ into an equivalent UCQ over schema $\mathcal{S}$, thus obtaining $\operatorname{PerfRef}_{q_{\mathcal{O}}, \Sigma}=q_{\mathcal{S}}^{1} \cup q_{\mathcal{S}}^{2} \cup q_{\mathcal{S}}^{3} \cup q_{\mathcal{S}}^{4}$, where:

- $q_{\mathcal{S}}^{1}=\left\{(x) \mid \exists y, y_{3} \cdot s_{1}(y, x) \wedge s_{2}(c, x) \wedge s_{3}\left(x, y_{3}\right)\right\}$;

- $q_{\mathcal{S}}^{2}=\left\{(x) \mid \exists y, y_{2} . s_{1}(y, x) \wedge s_{3}\left(x, y_{2}\right)\right\}$;

- $q_{\mathcal{S}}^{3}=\left\{(x) \mid \exists y, y_{1}, y_{3} . s_{1}\left(y, y_{1}\right) \wedge s_{2}\left(y_{1}, x\right) \wedge s_{2}(c, x) \wedge s_{3}\left(x, y_{3}\right)\right\}$

- $q_{\mathcal{S}}^{4}=\left\{(x) \mid \exists y, y_{2} . s_{1}\left(y, y_{1}\right) \wedge s_{2}\left(y_{1}, x\right) \wedge s_{3}\left(x, y_{2}\right)\right\}$.

Before studying sound source-to-ontology rewritings in the restricted scenarios, we introduce some preliminary crucial notions and results for the class of queries $\mathcal{L}_{\mathcal{S}}=$ UCQJFEs used for expressing data services. Furthermore, from now on, for ease of exposition, we assume that all the queries over source schemas do not involve atoms $T$ and $\perp$. We point out, however, that everything can be generalised in a straightforward manner to include also $T$ and $\perp$ as possible atoms.

Definition 8.1. Let $q=\{\vec{t} \mid \exists \vec{y} \cdot \phi(\vec{x}, \vec{y})\}$ be a CQ over a schema $\mathcal{S}$, and let $\alpha_{1}=$ $s\left(t_{1,1}, \ldots, t_{1, n}\right)$ and $\alpha_{2}=s\left(t_{2,1}, \ldots, t_{2, n}\right)$ be two atoms over $\mathcal{S}$, where $\alpha_{2} \in \phi(\vec{x}, \vec{y})$ We say that $\alpha_{1}$ instantiates $\alpha_{2}$, if the following holds for each $i \in[1, n]:$ if $t_{2, i}$ is a distinguished variable or a constant, then $t_{2, i}=t_{1, i}$.

Example 8.3. Consider the following CQs $q_{1}=\left\{(x) \mid s_{1}(c, x, x) \wedge s_{2}(c, x)\right\}$ and $q_{2}=$ $\left\{(x) \mid \exists y . s_{1}(c, x, y) \wedge s_{2}(x, x)\right\}$ over a schema $\mathcal{S}$. Let $\alpha_{1}=s_{1}(c, x, x), \alpha_{2}=s_{2}(c, x)$, $\alpha_{3}=s_{1}(c, x, y)$, and $\alpha_{4}=s_{2}(x, x)$. We have that $\alpha_{1}$ instantiates $\alpha_{3}$, whereas $\alpha_{2}$ does not instantiate $\alpha_{4}$.

Clearly, given atoms $\alpha_{1}$ and $\alpha_{2}$ occurring in the bodies of CQs $q_{1}$ and $q_{2}$, respectively, checking whether $\alpha_{1}$ instantiates $\alpha_{2}$ can be done in polynomial time. Based on this observation, the following lemmata shows that checking whether a UCQ $q_{1}$ is contained in a UCQJFE $q_{2}$ can be done in polynomial time as well.

Lemma 8.1. Let $q_{1}$ and $q_{2}$ be a $C Q$ and a CQJFE, respectively, over a schema $\mathcal{S}$ with the same target list. We have that $q_{1} \sqsubseteq q_{2}$ if and only if for each atom $\alpha_{2}$ of $q_{2}$ there exists an atom $\alpha_{1}$ of $q_{1}$ such that $\alpha_{1}$ instantiates $\alpha_{2}$. 
Proof. "Only-if part:" Suppose that $q_{1} \sqsubseteq q_{2}$, that is, there exists a homomorphism from $q_{2}$ to $q_{1}$. But then, since $q_{1}$ and $q_{2}$ have the same target list, and since $q_{2}$ is a CQJFE, by construction it can be readily seen that for each atom $\alpha_{2}$ of $q_{2}$ there is at least an atom $\alpha_{1}$ of $q_{1}$ such that $\alpha_{1}$ instantiates $\alpha_{2}$.

"If part:" Suppose that for each atom $\alpha_{2}$ of $q_{2}$ there exists an atom $\alpha_{1}$ of $q_{1}$ such that $\alpha_{1}$ instantiates $\alpha_{2}$. Let $h$ be the function from the terms of $q_{2}$ to the terms of $q_{1}$ such that $(i) h(c)=c$, for each constant $c$ appearing in $q_{2},(i i) h(x)=x$, for every distinguished variable $x$, and finally $($ iii $) h(y)=t$ for every existential variable $y$ occurring in $q_{2}$, where if $y$ occurs as $k$-th argument of atom $\alpha_{2}$ (since $q_{2}$ is a CQJFE, only one occurrence of $y$ exists), then $t$ is the $k$-th argument of the atom $\alpha_{1}$ that instantiates $\alpha_{2}$ (which exists by assumption). Since $q_{2}$ is a CQJFE, and since $q_{2}$ and $q_{1}$ have the same target list, we derive that $h$ consists in a homomorphism from $q_{2}$ to $q_{1}$. It follows that $q_{1} \sqsubseteq q_{2}$, as required.

Let the containment problem for UCQJFEs be the following decision problem: given a UCQ $q^{\prime}$ and a UCQJFE $q$ over the same schema $\mathcal{S}$, check whether $q^{\prime} \sqsubseteq q$.

Lemma 8.2. The containment problem for UCQJFEs is in PTIME.

Proof. To begin, observe that for each pair of UCQs $q_{1}, q_{2}$ we have $q_{1} \sqsubseteq q_{2}$ if and only if for each disjunct $q^{\prime}$ of $q_{1}$ there exists a disjunct $q$ of $q_{2}$ such that $q^{\prime} \sqsubseteq q$ Sagiv and Yannakakis, 1980. It is therefore sufficient to show that, given a CQ $q^{\prime}=\left\{\overrightarrow{t^{\prime}} \mid \exists \overrightarrow{y^{\prime}} \cdot \phi^{\prime}\left(\overrightarrow{x^{\prime}}, \overrightarrow{y^{\prime}}\right)\right\}$ and a CQJFE $q=\{\vec{t} \mid \exists \vec{y} \cdot \phi(\vec{x}, \vec{y})\}$ not necessarily with the same target lists, checking whether $q^{\prime} \sqsubseteq q$ can be done in polynomial time.

If $q^{\prime}$ and $q$ do not have the same target list, i.e., $\overrightarrow{t^{\prime}}=\left(t_{1}^{\prime}, \ldots, t_{n}^{\prime}\right) \neq \vec{t}=\left(t_{1}, \ldots, t_{n}\right)$, then consider the function $f$ from the set of terms in the target list of $q$ to the set of terms in the target list of $q^{\prime}$ with $f\left(t_{i}\right)=t_{i}^{\prime}$, for each $i \in[1, n]$. Formally, since repetitions of terms in target lists is allowed, $f$ might give rise to a multivalued

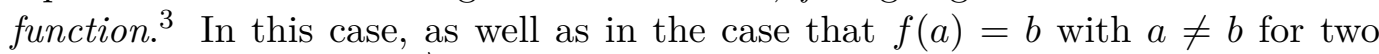
constants $a \in \vec{t}$ and $b \in \overrightarrow{t^{\prime}}$, it is straightforward to verify that $q^{\prime} \nsubseteq q$ trivially holds. Indeed, in those cases there can be no homomorphism from $q$ to $q^{\prime}$ by construction.

Consider the query $q^{\prime \prime}$ obtained in polynomial time from $q$ by replacing every occurrence of term $t_{i}$ in $q$ (even in the target list) with term $f\left(t_{i}\right)=t_{i}^{\prime}$, for each $i \in[1, n]$. Observe that now $q^{\prime \prime}$ is a CQJFE with target list $\overrightarrow{t^{\prime}}$, i.e., the same target list of $q^{\prime}$. By virtue of Lemma 8.1. we can now check in polynomial time whether $q^{\prime} \sqsubseteq q^{\prime \prime}$, where, if the answer is yes, then clearly $q^{\prime} \sqsubseteq q$ as well; otherwise, it can be readily seen that $D_{q^{\prime}}$ (i.e., the freezing of $q^{\prime}$ ) is a database witnessing that $q^{\prime} \nsubseteq q$.

From the above considerations, it is immediate to derive a polynomial time algorithm for checking whether a UCQ $q_{1}$ is contained in a UCQJFE $q_{2}$.

In what follows in this chapter, unless otherwise stated, we assume that OBDM specifications are expressed in the restricted setting for OBDM specifications mentioned in Section 6.2, i.e., the DL ontology language is DL-Lite $e_{\mathrm{RDFS}}$ and the mapping language follows the pure GAV approach. Furthermore, unless otherwise stated, we assume that the query language $\mathcal{L}_{\mathcal{S}}$ for expressing data services is the one of UCQJFEs and CQJFEs in Section 8.1 and Section 8.2, respectively.

\footnotetext{
${ }^{3}$ In mathematics, a multivalued function (also known as multiple-valued function Knopp, 1996) $f: A \rightarrow B$ is similar to a function, but it may associate more than one possible element $y \in B$ to each element $x \in A$.
} 


\subsection{Restricted Scenario for UCQJFEs}

In this section, we study both the verification, and the computation problem for sound source-to-ontology rewritings in the restricted scenario for UCQJFEs. We recall that, while the language $\mathcal{L}_{\mathcal{S}}$ of queries over source schemas $\mathcal{S}$ is the one of UCQJFEs, the language $\mathcal{L}_{\mathcal{O}}$ for queries over ontologies $\mathcal{O}$ remains the one of UCQs.

\subsubsection{Verification Problem}

The following theorem characterises the computational complexity of the verification problem for sound source-to-ontology rewritings in the restricted scenario for UCQJFEs.

Theorem 8.1. In the restricted scenario for UCQJFEs, the verification problem is coNP-complete.

Proof. As for the upper bound, by virtue of Corollary 8.1, it is sufficient to show how to check the containment PerfRef $_{q_{\mathcal{O}}, \Sigma} \sqsubseteq q_{\mathcal{S}}$ in coNP. In particular, checking whether $\operatorname{PerfRef}_{q_{\mathcal{O}}, \Sigma} \nsubseteq q_{\mathcal{S}}$ can be done in NP in the following way: $(i)$ we guess a query $q^{\prime}$ over $\mathcal{O}$ with the same arity of $q_{\mathcal{O}}$ and size at most $\sigma\left(q_{\mathcal{O}}\right)$, a sequence $\rho$ of ontology assertions, and a query $q^{\prime \prime}$ over $\mathcal{S}$ with the same arity of $q_{\mathcal{O}}$ and size at most $\sigma(\mathcal{M}) \times \sigma\left(q^{\prime}\right)$, and (ii) likewise to Theorem 5.1. we check in polynomial time first whether we can rewrite $q_{\mathcal{O}}$ into $q^{\prime}$ through $\rho$, and then whether $q^{\prime \prime}$ is in $\operatorname{MapRef}\left(q^{\prime}, \mathcal{M}\right)$. Finally, we check whether $q^{\prime \prime} \nsubseteq q_{S}$, which, since $q_{\mathcal{S}}$ is a UCQJFE, by virtue of Lemma 8.2, this last step can be done in polynomial time as well.

As for the lower bound, the proof of coNP-hardness is by a LOGSPACE reduction from the VALIDITY problem, which is coNP-complete (see, e.g., Papadimitriou, 1994). VALIDITY is the problem of deciding, given a 3 -DNF formula $F=c_{1} \vee \ldots \vee c_{m}$ on a set of variables $X=\left\{x_{1}, \ldots, x_{n}\right\}$, whether $F$ is valid, i.e., whether $F$ is satisfied by every possible truth assignment to the variables in $X$. Each clause $c_{i}$ is a conjunction of three literals, where each literal is either a variable $x_{i} \in X$ or its negated.

We define an OBDM specification $\Sigma=\langle\mathcal{O}, \mathcal{S}, \mathcal{M}\rangle$ with $\mathcal{O}$ containing no axioms, and $\mathcal{S}$ and $\mathcal{M}$ as follows: for each variable $x_{i} \in X$, schema $\mathcal{S}$ comprises two unary relations $s_{i_{T}}$ and $s_{i_{F}}$, and a further unary relation $s_{i}^{\prime}$. Finally, for each variable $x_{i} \in X$, the mapping $\mathcal{M}$ includes the following three mapping assertions:

- $s_{i_{T}}(x) \rightarrow A_{i}(x)$,

- $s_{i_{F}}(x) \rightarrow A_{i}(x)$,

- $s_{i}^{\prime}(x) \rightarrow B_{i}(x)$,

where each $A_{i}$ and $B_{i}$ are fresh atomic concepts, for each $i \in[1, n]$.

Intuitively, while each $s_{i}^{\prime}$ is simply mirrored to $B_{i}$, the possible unfoldings of an atom $A_{i}\left(x_{i}\right)$ (which are $s_{i_{T}}\left(x_{i}\right)$ and $s_{i_{F}}\left(x_{i}\right)$, respectively) correspond to the possible truth values (true and false, respectively) for the variable $x_{i}$.

We define the UCQJFE over $\mathcal{S}$ as $q_{\mathcal{S}}=q_{1} \cup \ldots \cup q_{m}$, where, for each $i \in[1, m]$, the target list of $q_{i}$ is $\vec{x}=\left(x_{1}, \ldots, x_{n}\right)$ and the body of $q_{i}$ has the conjunction of atoms $s_{1}^{\prime}\left(x_{1}\right) \wedge \ldots \wedge s_{n}^{\prime}\left(x_{n}\right)$ in conjunction to the conjunction of atoms associated 
to the clause $c_{i}$ of $F$, where a positive literal $x_{i}$ is replaced with the atom $s_{i_{T}}\left(x_{i}\right)$, whereas a negative literal $\neg x_{i}$ is replaced with the atom $s_{i_{F}}\left(x_{i}\right)$.

Finally, we define the CQJFE over $\mathcal{O}$ as $q_{\mathcal{O}}=\left\{\vec{x} \mid B_{1}\left(x_{1}\right) \wedge \ldots \wedge B_{n}\left(x_{n}\right) \wedge\right.$ $\left.A_{1}\left(x_{1}\right) \wedge \ldots \wedge A_{n}\left(x_{n}\right)\right\}$.

Observe that $\Sigma=\langle\mathcal{O}, \mathcal{S}, \mathcal{M}\rangle, q_{\mathcal{S}}$, and $q_{\mathcal{O}}$ can be constructed in LOGSPACE from $F$, where $\mathcal{O}=\emptyset$ and $\mathcal{M}$ is both a pure GAV and a LAV mapping.

To illustrate the reduction, we use the following formula: $F=\left(x_{1} \wedge x_{2} \wedge\right.$ $\left.\neg x_{3}\right) \bigvee\left(\neg x_{1} \wedge x_{2} \wedge \neg x_{4}\right)$. In this case, the reduction would produce the mapping $\mathcal{M}$ composed of the following mapping assertions:

$$
\begin{aligned}
s_{1_{T}}(x) & \rightarrow A_{1}(x), \\
s_{1_{F}}(x) & \rightarrow A_{1}(x), \\
s_{2_{T}}(x) & \rightarrow A_{2}(x), \\
s_{2_{F}}(x) & \rightarrow A_{2}(x), \\
s_{3_{T}}(x) & \rightarrow A_{3}(x), \\
s_{3_{F}}(x) & \rightarrow A_{3}(x), \\
s_{4_{T}}(x) & \rightarrow A_{4}(x), \\
s_{4_{F}}(x) & \rightarrow A_{4}(x), \\
s_{1}^{\prime}(x) & \rightarrow B_{1}(x), \\
s_{2}^{\prime}(x) & \rightarrow B_{2}(x), \\
s_{3}^{\prime}(x) & \rightarrow B_{3}(x), \\
s_{4}^{\prime}(x) & \rightarrow B_{4}(x),
\end{aligned}
$$

the following UCQJFE $q_{\mathcal{S}}=\left\{\left(x_{1}, x_{2}, x_{3}, x_{4}\right) \mid s_{1}^{\prime}\left(x_{1}\right) \wedge s_{2}^{\prime}\left(x_{2}\right) \wedge s_{3}^{\prime}\left(x_{3}\right) \wedge s_{4}^{\prime}\left(x_{4}\right) \wedge\right.$ $\left.s_{1_{T}}\left(x_{1}\right) \wedge s_{2_{T}}\left(x_{2}\right) \wedge s_{3_{F}}\left(x_{3}\right)\right\} \cup\left\{\left(x_{1}, x_{2}, x_{3}, x_{4}\right) \mid s_{1}^{\prime}\left(x_{1}\right) \wedge s_{2}^{\prime}\left(x_{2}\right) \wedge s_{3}^{\prime}\left(x_{3}\right) \wedge s_{4}^{\prime}\left(x_{4}\right) \wedge\right.$ $\left.s_{1_{F}}\left(x_{1}\right) \wedge s_{2_{T}}\left(x_{2}\right) \wedge s_{4_{F}}\left(x_{4}\right)\right\}$ over $\mathcal{S}$, and the following CQJFE $q_{\mathcal{O}}=\left\{\left(x_{1}, x_{2}, x_{3}, x_{4}\right)\right.$ $\left.B_{1}\left(x_{1}\right) \wedge B_{2}\left(x_{2}\right) \wedge B_{3}\left(x_{3}\right) \wedge B_{4}\left(x_{4}\right) \wedge A_{1}\left(x_{1}\right) \wedge A_{2}\left(x_{2}\right) \wedge A_{3}\left(x_{3}\right) \wedge A_{4}\left(x_{4}\right)\right\}$ over $\mathcal{O}$.

We now prove that formula $F$ is valid if and only if $q_{\mathcal{O}}$ is a sound $\mathcal{S}$-to- $\mathcal{O}$ $\Sigma$-rewriting of $q_{\mathcal{S}}$.

"Only-if part:" Suppose that formula $F$ is valid, that is, $F$ is satisfied by every possible truth assignment to the variables in $X$. It follows that, for any possible choice for unfolding the atoms $A_{i}\left(x_{i}\right)$ for $i=1, \ldots, n$ (which can be equivalently seen as an assignment $V=\left\{v_{1}, \ldots, v_{n}\right\}$ to the variables in $X=\left\{x_{1}, \ldots, x_{n}\right\}$ ), the query over $\mathcal{S}$ obtained is such that all the atoms also appear in a disjunct $q_{j}$ of $q_{\mathcal{S}}$ for some $j \in[1, m]$ (equivalently, at least one clause $c_{j}$ for some $j \in[1, m]$ is satisfied under the truth assignment $V$ ). It follows that $\operatorname{PerfRef}_{q_{\mathcal{O}}, \Sigma} \sqsubseteq q_{\mathcal{S}}$ which, due to Corollary 8.1, implies that $q_{\mathcal{O}}$ is a sound $\mathcal{S}$-to- $\mathcal{O}$ $\Sigma$-rewriting of $q_{\mathcal{S}}$.

"If part:" Suppose that formula $F$ is not valid, that is, there exists a truth assignment $V=\left\{v_{1}, \ldots, v_{n}\right\}$ to the variables in $X=\left\{x_{1}, \ldots, x_{n}\right\}$ that does not satisfy $F$. Consider now the disjunct $q$ of $\operatorname{PerfRef}_{q_{\mathcal{O}}, \Sigma}$ obtained by unfolding atom $A_{i}\left(x_{i}\right)$ of $q_{\mathcal{O}}$ with atom $s_{i_{T}}\left(x_{i}\right)$ if $v_{i}=1$, and with atom $s_{i_{F}}\left(x_{i}\right)$ otherwise (i.e., $v_{i}=0$ ), for each $i \in[1, n]$. As a result, for each disjunct $q^{\prime}$ of $q_{\mathcal{S}}$, there is at least one atom of $q^{\prime}$ not occurring in $q$. In proof, if there exists some disjunct $q_{j}$ of $q_{\mathcal{S}}$ such that every atom of $q_{j}$ appears also in $q$, then the clause $c_{j}$ corresponding to disjunct $q_{j}$ is satisfied under the truth assignment $V$, which would contradict the fact that $F$ is not satisfied under such truth assignment.

This implies that, for each disjunct $q_{j}$ of $q_{\mathcal{S}}$, there is no homomorphism from $q_{j}$ 
to $q$. It follows that PerfRef $q_{\mathcal{O}}, \Sigma \nsubseteq q_{\mathcal{S}}$ which, due to Corollary 8.1 implies that $q_{\mathcal{O}}$ is not a sound $\mathcal{S}$-to- $\mathcal{O} \Sigma$-rewriting of $q_{\mathcal{S}}$.

Note that $(i)$ the coNP upper bound holds even when ontologies $\mathcal{O}$ are expressed in a fragment of $D L-L i e_{\mathcal{R}}$ that does not admit disjointness assertions (thus, a more expressive language of $D L$-Lite $e_{\mathrm{RDF}}$ ) and mappings $\mathcal{M}$ are GLAV mappings (rather than pure GAV mappings), and (ii) the coNP lower bound already holds when $q_{\mathcal{O}}$ is a CQJFE, both $q_{\mathcal{S}}$ and $q_{\mathcal{O}}$ do not have existential variables, and $\Sigma=\langle\mathcal{O}, \mathcal{S}, \mathcal{M}\rangle$ is such that $\mathcal{O}$ contains no axioms, and $\mathcal{M}$ is both a pure GAV mapping and a LAV mapping. In the following section, we will see that the computational complexity of the verification problem further decreases when $q_{\mathcal{S}}$ is restricted to be a CQJFE.

\subsubsection{Computation Problem}

We now address the computation problem by providing an algorithm to compute UCQ-maximally sound source-to-ontology rewritings, thus proving that UCQmaximally sound source-to-ontology rewritings are guaranteed to exist in the restricted scenario for UCQJFEs.

Let us first introduce some preliminary notions. For a mapping $\mathcal{M}$, we denote by $\gamma(\mathcal{M})$ the number of mapping assertions occurring in $\mathcal{M}$. For a UCQ $q_{\mathcal{S}}$, we denote by $\eta\left(q_{\mathcal{S}}\right)$ the sum of the number of atoms occurring in the body of the various disjuncts of $q_{\mathcal{S}}$. Then, for a mapping $\mathcal{M}$ and a UCQ $q_{\mathcal{S}}$, we define bound $\left(\mathcal{M}, q_{\mathcal{S}}\right)$ as:

$$
\operatorname{bound}\left(\mathcal{M}, q_{\mathcal{S}}\right)=\sum_{i=0}^{\eta\left(q_{\mathcal{S}}\right)} \gamma(\mathcal{M})^{i}
$$

The next crucial lemma shows that, given any OBDM specification $\Sigma=\langle\mathcal{O}, \mathcal{S}, \mathcal{M}\rangle$ and any UCQJFE $q_{\mathcal{S}}$ over $\mathcal{S}$, we can always limit our attention to CQs over $\mathcal{O}$ having at most bound $\left(\mathcal{M}, q_{\mathcal{S}}\right)$ atoms occurring in their bodies when seeking for CQ-maximally sound $\mathcal{S}$-to- $\mathcal{O} \Sigma$-rewritings of $q_{\mathcal{S}}$.

Lemma 8.3. Let $\Sigma=\langle\mathcal{O}, \mathcal{S}, \mathcal{M}\rangle$ be an OBDM specification, and let $q_{\mathcal{S}}$ be a UCQJFE over $\mathcal{S}$. If a $C Q q_{\mathcal{O}}$ over $\mathcal{O}$ is a sound $\mathcal{S}$-to- $\mathcal{O} \Sigma$-rewriting of $q_{\mathcal{S}}$, then there exists a $C Q q_{\mathcal{O}}^{\prime}$ over $\mathcal{O}$ with same target list of $q_{\mathcal{O}}$ such that (i) the body of $q_{\mathcal{O}}^{\prime}$ is the conjunction of at most bound $\left(\mathcal{M}, q_{\mathcal{S}}\right)$ atoms occurring in the body of $q_{\mathcal{O}}$ (and therefore, cert $\left._{q_{\mathcal{O}}, \Sigma} \sqsubseteq \operatorname{cert}_{q_{\mathcal{O}}^{\prime}, \Sigma}\right)$, and (ii) $q_{\mathcal{O}}^{\prime}$ is a sound $\mathcal{S}$-to-O $\mathcal{O}$-rewriting of $q_{\mathcal{S}}$ as well.

Proof. Since $q_{\mathcal{O}}$ is a sound $\mathcal{S}$-to- $\mathcal{O} \Sigma$-rewriting of $q_{\mathcal{S}}$, due to Corollary 8.1, we have that $\operatorname{PerfRef}_{q_{\mathcal{O}}, \Sigma} \sqsubseteq q_{\mathcal{S}}$, that is, each disjunct of $\operatorname{PerfRef}_{q_{\mathcal{O}}, \Sigma}$ is contained in some disjunct of $q_{\mathcal{S}}$. In particular, without loss of generality, we can assume that each disjunct $q$ of $q_{\mathcal{S}}$ is such that there is some disjunct $r$ of $\operatorname{PerfRef}_{\mathcal{O}_{\mathcal{O}}, \Sigma}$ for which $r \sqsubseteq q$. In fact, the other disjuncts of $q_{\mathcal{S}}$ that do not satisfy the above condition can simply be discarded, and the resulting $q_{\mathcal{S}}$ will remain such that $\operatorname{PerfRef}_{q_{\mathcal{O}}, \Sigma} \sqsubseteq q_{\mathcal{S}}$.

Let $n$ denote the arity of $q_{\mathcal{O}}$ and $q_{\mathcal{S}}$, and let the target list of $q_{\mathcal{O}}$ be $\vec{t}=\left(t_{1}, \ldots, t_{n}\right)$. Without loss of generality, we can assume that the target list of each disjunct $q$ of $q_{\mathcal{S}}$ is the same as $q_{\mathcal{O}}$, i.e., $\vec{t}=\left(t_{1}, \ldots, t_{n}\right)$. Indeed, if this is not the case, then each disjunct $q^{\prime}$ of $q_{\mathcal{S}}$ with target list $t^{\prime}=\left(t_{1}^{\prime}, \ldots, t_{n}^{\prime}\right) \neq \vec{t}$ can be replaced with the (equivalent or more specific) disjunct $q$ obtained from $q^{\prime}$ by replacing everywhere 
(even in the target list) term $t_{i}^{\prime}$ with term $t_{i}$, for $i \in[1, n]$. Notice that, since the target list of each disjunct in $\operatorname{PerfRef}_{q_{\mathcal{O}}, \Sigma}$ is the same as $q_{\mathcal{O}}$ (this is because $\mathcal{M}$ is composed of pure GAV mapping assertions), i.e., $\vec{t}$, and since by assumption there exists some disjunct $r$ of $\operatorname{PerfRef}_{q_{\mathcal{O}}, \Sigma}$ for which $r \sqsubseteq q^{\prime}$, when applying this replacement it is never the case that for some pair of numbers $j, k \in[1, n]$ with $j \neq k$ we have $t_{j}^{\prime}=t_{k}^{\prime}$ but $t_{j} \neq t_{k}$, or that $t_{j}^{\prime}$ is a constant whereas $t_{j}$ is a distinguished variable for some number $j \in[1, n]$ (otherwise, this would be easily a contradiction to the fact that $\left.r \sqsubseteq q^{\prime}\right)$.

We now show by induction on $\eta\left(q_{\mathcal{S}}\right)$ (i.e., the sum of the number of atoms occurring in the body of the various disjuncts of $\left.q_{\mathcal{S}}\right)$ the existence of $m \leq \operatorname{bound}\left(\mathcal{M}, q_{\mathcal{S}}\right)$ atoms $\beta_{1}, \ldots, \beta_{m}$ occurring in the body of $q_{\mathcal{O}}$ for which the CQ $q_{\mathcal{O}}^{\prime}=\{\vec{t} \mid$ $\left.\exists \vec{y} . \beta_{1} \wedge \ldots \wedge \beta_{m}\right\}$ is a sound $\mathcal{S}$-to- $\mathcal{O} \sum$-rewriting of $q_{\mathcal{S}}$, thus proving the claim. We do this by exploiting Lemma 8.1. Specifically, consider each CQ $r$ that is a disjunct of $\operatorname{PerfRef}_{q_{\mathcal{O}}, \Sigma}$. We know that there is a CQJFE $q$ that is a disjunct of $q_{\mathcal{S}}$ for which $r \sqsubseteq q$, and, moreover, since $r$ and $q$ have the same target list, by Lemma 8.1 we know that for each atom $\alpha_{2}$ of $q$ there exists an atom $\alpha_{1}$ of $r$ such that $\alpha_{1}$ instantiates $\alpha_{2}$.

Base step $\left(\eta\left(q_{\mathcal{S}}\right)=1\right)$ : In this case, $q_{\mathcal{S}}$ is a single CQJFE whose body consists of only one atom $\alpha$. So, there must exists at least an atom $\beta$ in the body of $q_{\mathcal{O}}$ for which every possible disjunct of $\rho(\beta, \Sigma)$ contains at least an atom that instantiates $\alpha$. Indeed, if this is not the case, then the disjunct $r$ of $\operatorname{PerfRef}_{q_{\mathcal{O}}, \Sigma}$ obtained by unfolding each atom $\beta^{\prime}$ of $q_{\mathcal{O}}$ with a disjunct of $\rho\left(\beta^{\prime}, \Sigma\right)$ which contains no atom that instantiates $\alpha$ would be such that $r \nsubseteq q_{\mathcal{S}}$, because, as a result, there would be no atom in $r$ that instantiates $\alpha$ (cf. Lemma 8.1). Clearly, the fact that $r \nsubseteq q_{\mathcal{S}}$ for a disjunct $r$ of $\operatorname{PerfRef}_{q_{\mathcal{O}}, \Sigma}$ would also imply that $\operatorname{PerfRef}_{q_{\mathcal{O}}, \Sigma} \nsubseteq q_{\mathcal{S}}$ which, by Corollary 8.1, in turn would imply that $q_{\mathcal{O}}$ is not a sound $\mathcal{S}$-to- $\mathcal{O} \Sigma$-rewriting of $q_{\mathcal{S}}$, which would be a contradiction on the initial assumption.

Thus, such atom $\beta$ in the body of $q_{\mathcal{O}}$ must exists. But then, by exploiting Lemma 8.1, it is trivial to see that the CQ $q_{\mathcal{O}}^{\prime}=\{\vec{t} \mid \exists \vec{y} \cdot \beta\}$ is a sound $\mathcal{S}$-to- $\mathcal{O}$ $\Sigma$-rewriting of $q_{\mathcal{S}}$, as required.

Inductive step: We start with the following observation. Since each disjunct $r$ of $\operatorname{PerfRef}_{q_{\mathcal{O}}, \Sigma}$ is such that there exists a disjunct $q$ of $q_{\mathcal{S}}$ for which $r \sqsubseteq q$ (or equivalently, by Lemma 8.1, there exists a disjunct $q$ of $q_{\mathcal{S}}$ for which for each atom $\alpha$ of $q$ there is an atom of $r$ that instantiates $\alpha$ ), there must exists at least one atom $\beta$ of $q_{\mathcal{O}}$ such that in every disjunct of $\rho(\beta, \Sigma)$ there is at least an atom that instantiates some atom occurring in the various disjuncts of $q_{\mathcal{S}}$. Indeed, if this is not the case, then the disjunct $r$ of $\operatorname{PerfRef}_{q_{\mathcal{O}}, \Sigma}$ obtained by unfolding each atom $\beta^{\prime}$ of $q_{\mathcal{O}}$ with a disjunct of $\rho\left(\beta^{\prime}, \Sigma\right)$ which contains no atom that instantiates some atom of $q_{\mathcal{S}}$ would be trivially such that $r \nsubseteq q_{\mathcal{S}}$ due to Lemma 8.1. As explained previously, the fact that $r \nsubseteq q_{\mathcal{S}}$ for a disjunct $r$ of $\operatorname{PerfRef}_{q_{\mathcal{O}}, \Sigma}$ would also imply that $\operatorname{PerfRef}_{q_{\mathcal{O}}, \Sigma} \nsubseteq q_{\mathcal{S}}$ which, by Corollary 8.1 in turn would imply that $q_{\mathcal{O}}$ is not a sound $\mathcal{S}$-to- $\mathcal{O} \Sigma$-rewriting of $q_{\mathcal{S}}$, which would be a contradiction on the initial assumption.

So, there must be (at least) one atom $\beta$ in the body of $q_{\mathcal{O}}$ such that in every disjunct of $\rho(\beta, \Sigma)$ there is at least one atom that instantiates some atom occurring in the various disjuncts of $q_{\mathcal{S}}$. In particular, consider $\rho(\beta, \Sigma)$ for such atom $\beta$. For each disjunct $\theta_{i}$ of $\rho(\beta, \Sigma)$, let $q_{\mathcal{S}}^{\theta_{i}}$ be the UCQJFE obtained from $q_{\mathcal{S}}$ by removing all the atoms $\alpha$ such that an atom of $\theta_{i}$ instantiates $\alpha$. Notice that, since each 
disjunct $\theta_{i}$ of $\rho(\beta, \Sigma)$ instantiates some atom of $q_{\mathcal{S}}$, each UCQJFE $q_{\mathcal{S}}^{\theta_{i}}$ is such that (i) $\eta\left(q_{\mathcal{S}}^{\theta_{i}}\right) \leq \eta\left(q_{\mathcal{S}}\right)-1$ (i.e., there is at least an atom of $q_{\mathcal{S}}$ not occurring anymore in $\left.q_{\mathcal{S}}^{\theta_{i}}\right)$, and $(i i) q_{\mathcal{O}}$ is a sound $\mathcal{S}$-to- $\mathcal{O} \Sigma$-rewriting of $q_{\mathcal{S}}^{\theta_{i}}$, due to the facts that $q_{\mathcal{S}} \sqsubseteq q_{\mathcal{S}}^{\theta_{i}}$ clearly holds and the initial assumption that $q_{\mathcal{O}}$ is a sound $\mathcal{S}$-to- $\mathcal{O} \Sigma$-rewriting of $q_{\mathcal{S}}$.

By the inductive hypothesis, for each disjunct $\theta_{i}$ of $\rho(\beta, \Sigma)$, there are atoms $\beta_{1}^{\theta_{i}}, \beta_{2}^{\theta_{i}}, \ldots, \beta_{p_{i}}^{\theta_{i}}$ for which $q_{\mathcal{O}}^{\theta_{i}}=\left\{\vec{t} \mid \exists \overrightarrow{y_{i}} \cdot \beta_{1}^{\theta_{i}} \wedge \beta_{2}^{\theta_{i}} \wedge \ldots \wedge \beta_{p_{i}}^{\theta_{i}}\right\}$ is a sound $\mathcal{S}$-to- $\mathcal{O}$ $\Sigma$-rewriting of $q_{\mathcal{S}}^{\theta_{i}}$, where $p_{i} \leq \operatorname{bound}\left(\mathcal{M}, q_{\mathcal{S}}^{\theta_{i}}\right)$, i.e., $p_{i} \leq 1+\lambda(\mathcal{M})^{1}+\lambda(\mathcal{M})^{2}+\ldots+$ $\lambda(\mathcal{M})^{\eta\left(q_{\mathcal{S}}\right)-1}$. But then, consider the following CQ:

$q_{\mathcal{O}}^{\prime}=\left\{\vec{t} \mid \exists \vec{y} . \beta \wedge \beta_{1}^{\theta_{1}} \wedge \beta_{2}^{\theta_{1}} \wedge \ldots \wedge \beta_{p_{1}}^{\theta_{1}} \wedge \beta_{1}^{\theta_{2}} \wedge \beta_{2}^{\theta_{2}} \wedge \ldots \wedge \beta_{p_{2}}^{\theta_{2}} \wedge \ldots \wedge \beta_{1}^{\theta_{k}} \wedge \beta_{2}^{\theta_{k}} \wedge \ldots \wedge \beta_{p_{k}}^{\theta_{k}}\right\}$

It is not hard to ascertain that $q_{\mathcal{O}}^{\prime}$ is a sound $\mathcal{S}$-to- $\mathcal{O} \Sigma$-rewriting of $q_{\mathcal{S}}$, where $k$ is the number of disjuncts in $\rho(\beta, \Sigma)$, and $p_{i} \leq 1+\lambda(\mathcal{M})^{1}+\lambda(\mathcal{M})^{2}+\ldots+\lambda(\mathcal{M})^{\eta\left(q_{\mathcal{S}}\right)-1}$ for each $i \in[1, k]$. In proof, consider each disjunct $\theta_{i}$ of $\rho(\beta, \Sigma)$ for $i \in[1, k]$. Since the CQ $q_{\mathcal{O}}^{\theta_{i}}$ is a sound $\mathcal{S}$-to- $\mathcal{O} \Sigma$-rewriting of $q_{\mathcal{S}}^{\theta_{i}}$, by Lemma 8.1. we derive that for each possible disjunct $r^{\theta_{i}}$ obtained by turning in disjunctive normal form the formula $\rho\left(\beta_{1}^{\theta_{i}}, \Sigma\right) \wedge \rho\left(\beta_{2}^{\theta_{i}}, \Sigma\right) \wedge \ldots \wedge \rho\left(\beta_{p_{i}}^{\theta_{i}}, \Sigma\right)$ there is a disjunct $q^{\theta_{i}}$ of $q_{\mathcal{S}}^{\theta_{i}}$ for which for each atom $\alpha$ of $q^{\theta_{i}}$ there is an atom of $r^{\theta_{i}}$ that instantiates $\alpha$. This, together with the fact that all the atoms $\alpha$ occurring in $q_{\mathcal{S}}$ and not occurring in $q_{\mathcal{S}}^{\theta_{i}}$ are such that there is an atom of $\theta_{i}$ that instantiates $\alpha$, allows us to derive that each disjunct $r_{\wedge}^{\theta_{i}}$ in the formula $\theta_{i} \wedge \rho\left(\beta_{1}^{\theta_{i}}, \Sigma\right) \wedge \rho\left(\beta_{2}^{\theta_{i}}, \Sigma\right) \wedge \ldots \wedge \rho\left(\beta_{p_{i}}^{\theta_{i}}, \Sigma\right)$ turned in disjunctive normal form is such that there is a disjunct $q$ of $q_{\mathcal{S}}$ for which for each atom $\alpha$ of $q$ there is an atom of $r_{\wedge}^{\theta_{i}}$ that instantiates $\alpha$. Since this is true for each disjunct $\theta_{i}$ of $\rho(\beta, \Sigma)$, and since for each $i \in[1, k]$ the conjunction of atoms $\beta_{1}^{\theta_{i}} \wedge \beta_{2}^{\theta_{i}} \wedge \ldots \wedge \beta_{p_{i}}^{\theta_{i}}$ occurs in the body of the $\mathrm{CQ} q_{\mathcal{O}}^{\prime}$, we easily derive that for each possible disjunct $r^{\prime}$ of $q_{\mathcal{O}}^{\prime}$ there is a disjunct $q$ of $q_{\mathcal{S}}$ for which for each atom $\alpha$ of $q$ there is an atom of $r^{\prime}$ that instantiates $\alpha$. Thus, by Lemma 8.1, it follows that $q_{\mathcal{O}}^{\prime}$ is a sound $\mathcal{S}$-to- $\mathcal{O}$ $\Sigma$-rewriting of $q_{\mathcal{S}}$, as required.

To conclude the proof, observe that the number of disjuncts in $\rho(\beta, \Sigma)$ is at most $k \leq \lambda(\mathcal{M})$, and therefore, since $p_{i} \leq 1+\lambda(\mathcal{M})^{1}+\lambda(\mathcal{M})^{2}+\ldots+\lambda(\mathcal{M})^{\eta\left(q_{\mathcal{S}}\right)-1}$ for each $i \in[1, k]$, we derive that the number of atoms occurring in the body of the CQ $q_{\mathcal{O}}^{\prime}$ is at most $1+\lambda(\mathcal{M})^{1}+\lambda(\mathcal{M})^{2}+\ldots+\lambda(\mathcal{M})^{\eta\left(q_{\mathcal{S}}\right)}$, as required.

By relying on the above lemma, we immediately derive the following enumerative algorithm MaximallySoundUCQJFEs for computing UCQ-maximally sound sourceto-ontology rewritings in the restricted scenario for UCQJFEs.

Informally, the algorithm simply enumerates all the possible CQs over $\mathcal{O}$ with at most bound $\left(\mathcal{M}, q_{\mathcal{S}}\right)$ atoms occurring in their bodies and possibly involving constants occurring in $q_{\mathcal{S}}$ and $\mathcal{M}$ as terms. Then, it returns the union of all and only the ones that are sound $\mathcal{S}$-to- $\mathcal{O} \Sigma$-rewritings of $q_{\mathcal{S}}$. Notice that checking whether a $\mathrm{CQ} q_{\mathcal{O}}$ is a sound $\mathcal{S}$-to- $\mathcal{O} \Sigma$-rewriting of $q_{\mathcal{S}}$ can be always done deterministically in exponential time (cf. Theorem 8.1).

Example 8.4. Let $\Sigma=\langle\mathcal{O}, \mathcal{S}, \mathcal{M}\rangle$ be the following OBDM specification:

- $\mathcal{O}=\emptyset$

- $\mathcal{S}=\left\{s_{1}, s_{2}, s_{3}, s_{4}, s_{5}\right\}$ 


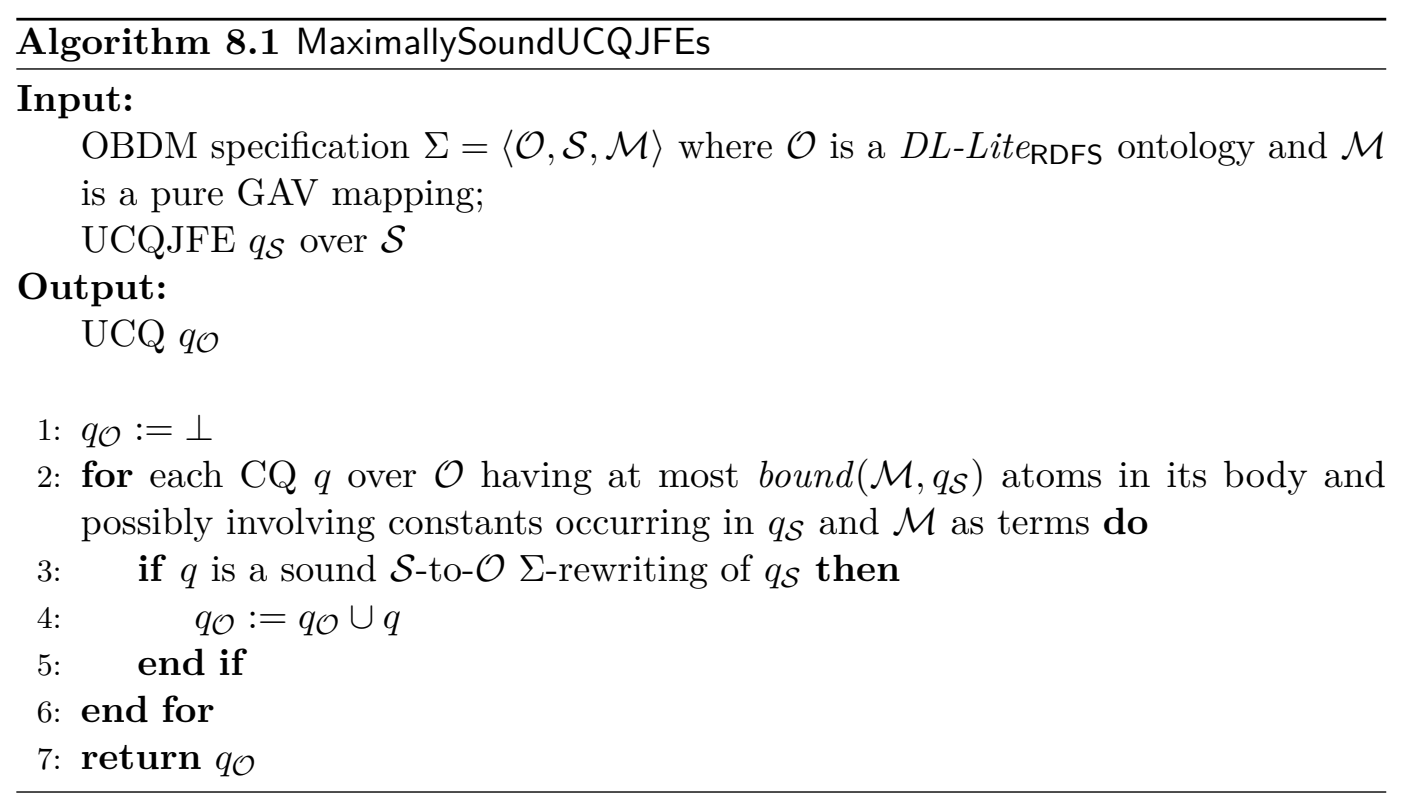

- $\mathcal{M}=\left\{m_{1}, m_{2}, m_{3}, m_{4}, m_{5}, m_{6}, m_{7}, m_{8}\right\}$, where:

$$
\begin{array}{rll}
m_{1}: & s_{1}\left(x_{1}, x_{2}\right) & \rightarrow P_{1}\left(x_{1}, x_{2}\right), \\
m_{2}: & s_{3}\left(x_{1}, x_{2}\right) & \rightarrow P_{1}\left(x_{1}, x_{2}\right), \\
m_{3}: & \exists y \cdot s_{2}(x, y) & \rightarrow A_{1}(x), \\
m_{4}: & s_{4}\left(x, c_{3}\right) & \rightarrow A_{1}(x), \\
m_{5}: & s_{1}\left(x_{1}, x_{2}\right) \wedge s_{3}\left(x_{1}, x_{2}\right) & \rightarrow P_{2}\left(x_{1}, x_{2}\right), \\
m_{6}: & \exists y . s_{5}\left(x_{1}, x_{2}\right) \wedge s_{2}\left(x_{2}, y\right) \wedge s_{4}\left(x_{2}, y\right) & \rightarrow P_{2}\left(x_{1}, x_{2}\right), \\
m_{7}: & s_{1}(x, x) \wedge s_{2}\left(x, c_{2}\right) & \rightarrow A_{2}(x), \\
m_{8}: & s_{3}\left(x_{1}, c_{1}\right) \wedge s_{4}\left(c_{1}, x_{2}\right) & \rightarrow P_{3}\left(x_{1}, x_{2}\right) .
\end{array}
$$

For the UCQJFE $q_{\mathcal{S}}=\left\{\left(x_{1}, x_{2}\right) \mid \exists y . s_{1}\left(x_{1}, x_{2}\right) \wedge s_{2}\left(x_{2}, y\right)\right\} \cup\left\{\left(x_{1}, x_{2}\right) \mid\right.$ $\left.\exists y . s_{3}\left(x_{1}, x_{2}\right) \wedge s_{4}\left(x_{2}, y\right)\right\}$, the unique (up to equivalence w.r.t. $\Sigma$ ) UCQ-maximally sound $\mathcal{S}$-to- $\mathcal{O} \Sigma$-rewriting of $q_{\mathcal{S}}$ is the query $q_{\mathcal{O}}=\left\{\left(x_{1}, x_{2}\right) \mid P_{1}\left(x_{1}, x_{2}\right) \wedge A_{1}\left(x_{2}\right) \wedge\right.$ $\left.P_{2}\left(x_{1}, x_{2}\right)\right\} \cup\left\{(x, x) \mid A_{2}(x)\right\} \cup\left\{\left(x, c_{1}\right) \mid \exists y \cdot P_{3}(x, y)\right\}$. Indeed one can verify that, on the one hand, each disjunct of $q_{\mathcal{O}}$ is a sound $\mathcal{S}$-to- $\mathcal{O} \Sigma$-rewriting of $q_{\mathcal{S}}$, and, on the other hand, each possible CQ $q^{\prime}$ over $\mathcal{O}$ being a sound $\mathcal{S}$-to- $\mathcal{O} \Sigma$-rewriting of $q_{\mathcal{S}}$ is such that $\operatorname{cert}_{q^{\prime}, \Sigma} \sqsubseteq \operatorname{cert}_{q, \Sigma}$ for some disjunct $q$ of $q_{\mathcal{O}}$.

Furthermore, one can easily check that MaximallySoundUCQJFEs $\left(\Sigma, q_{\mathcal{S}}\right)$ returns a UCQ which is equivalent (w.r.t. $\Sigma$ ) to $q_{\mathcal{O}}$, in fact it contains all disjuncts of $q_{\mathcal{O}}$.

The following theorem establishes termination and correctness of the MaximallySoundUCQJFEs algorithm.

Theorem 8.2. In the restricted scenario for UCQJFEs, MaximallySoundUCQJFEs $\left(\Sigma, q_{\mathcal{S}}\right)$ terminates and returns the unique (up to equivalence w.r.t. $\Sigma$ ) UCQ-maximally sound $\mathcal{S}$-to- $\mathcal{O}$ इ-rewriting of $q_{\mathcal{S}}$.

Proof. Termination of the algorithm easily follows from the fact that it just enumerates all possible CQs over $\mathcal{O}$ with a certain bound on the number of atoms occurring in their bodies, and involving only constants occurring in $q_{\mathcal{S}}$ and $\mathcal{M}$. 
As for the correctness, we first point out that the computed UCQ $q_{\mathcal{O}}$ is clearly a sound $\mathcal{S}$-to- $\mathcal{O} \Sigma$-rewriting of $q_{\mathcal{S}}$. Indeed, by construction, $q_{\mathcal{O}}$ is a UCQ whose disjuncts are sound $\mathcal{S}$-to- $\mathcal{O} \Sigma$-rewritings of $q_{\mathcal{S}}$. We now show that $q_{\mathcal{O}}$ is actually the unique (up to equivalence w.r.t. $\Sigma$ ) UCQ-maximally sound $\mathcal{S}$-to- $\mathcal{O} \Sigma$-rewriting of $q_{\mathcal{S}}$, that is, each UCQ $q_{\mathcal{O}}^{\prime}$ that is a sound $\mathcal{S}$-to- $\mathcal{O} \Sigma$-rewriting of $q_{\mathcal{S}}$ is such that $\operatorname{cert}_{q_{\mathcal{O}}^{\prime}, \Sigma} \sqsubseteq \operatorname{cert}_{q_{\mathcal{O}}, \Sigma}$ (cf. Definition 3.6). We do this by way of contradiction.

Let $q_{\mathcal{O}}^{\prime}$ be a UCQ such that $\operatorname{cert}_{q_{\mathcal{O}}^{\prime}, \Sigma} \nsubseteq \operatorname{cert}_{q_{\mathcal{O}}, \Sigma}$, that is, there exists an $\mathcal{S}$ database $D$ consistent with $\Sigma$ such that $\operatorname{cert}_{q_{\mathcal{O}}^{\prime}, \Sigma}^{D} \not \subset \operatorname{cert}_{q_{\mathcal{O}}, \Sigma}^{D}$. It follows that there is a tuple of constants $\vec{c}=\left(c_{1}, \ldots, c_{n}\right)$ such that $\vec{c} \in \operatorname{cert}_{q_{\mathcal{O}}^{\prime}, \Sigma}^{D}$ but, at the same time, $\vec{c} \notin \operatorname{cert}_{q_{\mathcal{O}}, \Sigma}^{D}$. Consider now $\mathcal{C}_{\mathcal{O}}^{\mathcal{M}(D)}$, i.e., the canonical structure of $\mathcal{O}$ with respect to $\mathcal{M}$ and $D$ (cf. Subsection 2.6.3). Notice that, since $\mathcal{M}$ is a GAV mapping and

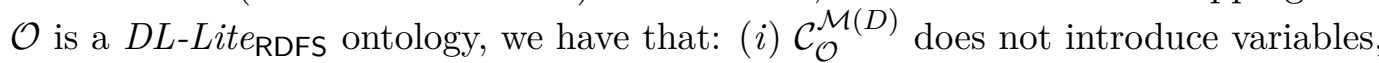
and therefore we can see it as a set of facts $\mathcal{C}_{\mathcal{O}}^{\mathcal{M}(D)}=\left\{\beta_{1}, \ldots, \beta_{m}\right\}$ over $\mathcal{O}$; and $(i i)$ $\operatorname{cert}_{q_{\mathcal{O}}, \Sigma}^{D}=q_{\mathcal{O}}^{\mathcal{C}_{\mathcal{O}}^{\mathcal{M}(D)}}$ for each $\mathcal{S}$-database $D$. We now exhibit an $\mathcal{S}$-database $D^{\prime}$ for which $(i) \vec{c} \notin q_{\mathcal{S}}^{D^{\prime}}$, and $(i i) \mathcal{C}_{\mathcal{O}}^{\mathcal{M}(D)} \subseteq \mathcal{C}_{\mathcal{O}}^{\mathcal{M}\left(D^{\prime}\right)}$. To this aim, we exploit the boolean query $q_{\beta}$ over $\mathcal{O}$ associated to $\mathcal{C}_{\mathcal{O}}^{\mathcal{M}(D)}$, i.e., the following boolean CQ:

$$
q_{\beta}=\left\{() \mid \beta_{1} \wedge \ldots \wedge \beta_{m}\right\},
$$

and all its possible unfoldings $r$ over $\mathcal{S}$. In particular, there are two possible cases: either every disjunct $r$ of $\operatorname{PerfRef}_{q_{\beta}, \Sigma}$ is such that $\vec{c} \in q_{\mathcal{S}}^{D_{r}}$, or not. We recall that, for a $\mathrm{CQ} r$ over $\mathcal{S}, D_{r}$ denotes the $\mathcal{S}$-database associated to $r$, i.e., the set of facts over $\mathcal{S}$ occurring in the body of $r$ in which each existential variable $v$ is replaced by a different fresh constant $c_{v}$.

In the former case, let $q$ be the CQ over $\mathcal{O}$ in which the target list is initially $\vec{c}=\left(c_{1}, \ldots, c_{n}\right)$ and the body is the same as $q_{\beta}$, i.e., $q=\left\{\vec{c} \mid \beta_{1} \wedge \ldots \wedge \beta_{n}\right\}$. Then, consider the following changes to $q$ : for each constant $c$ occurring in $q$ (either in the body or in the target list) but occurring neither in $q_{\mathcal{S}}$ nor in $\mathcal{M}$, replace $c$ everywhere (even in the target list) by a distinguished variable $x_{c}$ if $c_{i}=c$ for some $i \in[1, n]$ (i.e., if $c$ occurs in the target list of $q$ ), and by an existential variable $y_{c}$ otherwise.

Obviously, by construction, we have $\vec{c} \in q^{\mathcal{C}_{\mathcal{O}}^{\mathcal{M}(D)}}$. Furthermore, due to the fact that $\mathcal{M}$ is a pure GAV mapping and the fact that $\mathcal{O}$ contains no assertions with $\exists R$ in the right-hand side for a basic role $R$, each possible disjunct $r_{q}$ of $\operatorname{PerfRef}_{q, \Sigma}$ has a corresponding disjunct $r$ of $\operatorname{PerfRef}_{q_{\beta}, \Sigma}$ in which the body of $r$ is obtained from the body of $r_{q}$ by replacing the distinguished variables $x_{c}$ (respectively, the existential variable $y_{c}$ ) occurring in $q$ with constant $c$. Notice that, by assumption, each disjunct $r$ of $\operatorname{PerfRef}_{q_{\beta}, \Sigma}$ is such that $\vec{c} \in q_{\mathcal{S}}^{D_{r}}$, i.e., for each disjunct $r$ of PerfRef $q_{\beta}, \Sigma$ there is a disjunct $q_{\mathcal{S}}^{\prime}$ of $q_{\mathcal{S}}$ for which $\vec{c} \in q_{\mathcal{S}}^{\prime D_{r}}$. Since $q_{\mathcal{S}}$ is a UCQJFE, by exploiting Lemma 8.1, it is not hard to ascertain that this implies that for each disjunct $r$ of $\operatorname{PerfRe}_{q_{\beta}, \Sigma}$ there is a disjunct $q_{\mathcal{S}}^{\prime}$ of $q_{\mathcal{S}}$ for which for each atom $\alpha$ of $q_{\mathcal{S}}^{\prime}$ there is an atom of $r$ that instantiates $\alpha$. By construction of $q$, however, the above property holds even if we replace $q_{\beta}$ with $q$, i.e., for each disjunct $r_{q}$ of $\operatorname{PerfRef}_{q, \Sigma}$ there is a disjunct $q_{\mathcal{S}}^{\prime}$ of $q_{\mathcal{S}}$ for which for each atom $\alpha$ of $q_{\mathcal{S}}^{\prime}$ there is an atom of $r_{q}$ that instantiates $\alpha$. Thus, by Lemma 8.1, we derive that $q$ is a sound $\mathcal{S}$-to- $\mathcal{O} \Sigma$-rewriting of $q_{\mathcal{S}}$. But then, due to Lemma 8.3 from $q$ it is possible to derive a CQ $q^{\prime}$ with same 
target list as $q$ but whose body is the conjunction of at most bound $\left(\mathcal{M}, q_{\mathcal{S}}\right)$ atoms occurring in $q$ and such that $q^{\prime}$ is a sound $\mathcal{S}$-to- $\mathcal{O} \Sigma$-rewriting of $q_{\mathcal{S}}$. By construction of the algorithm, however, it can be readily seen that such a CQ $q^{\prime}$ is a disjunct of $q_{\mathcal{O}}$. Two considerations are now in order: $(i)$ since $q^{\prime}$ has the same target list as $q$, and since its body is constituted only by a subset of the atoms of $q$, the fact that $\vec{c} \in q^{\mathcal{C}_{\mathcal{O}}^{\mathcal{M}(D)}}$ implies $\vec{c} \in q^{\mathcal{C}_{\mathcal{O}}^{\mathcal{M}(D)}}$ as well, and $(i i)$ since $\vec{c} \in q^{\prime \mathcal{C}_{\mathcal{O}}^{\mathcal{M}(D)}}$ and since $q^{\prime}$ is a disjunct of $q_{\mathcal{O}}$, we have $\vec{c} \in q_{\mathcal{O}} \mathcal{C}^{\mathcal{M}(D)}$. Thus, since in this setting for OBDM specifications $\operatorname{cert}_{q_{\mathcal{O}}, \Sigma}^{D}=q_{\mathcal{O}}^{\mathcal{C}_{\mathcal{O}}^{\mathcal{M}(D)}}$ for each UCQ $q_{\mathcal{O}}$ and $\mathcal{S}$-database $D$, we derive that $\vec{c} \in \operatorname{cert}_{q_{\mathcal{O}}, \Sigma}^{D}$, which is a contradiction on the initial assumption that $\vec{c} \notin \operatorname{cert}_{q_{\mathcal{O}}, \Sigma}^{D}$. It follows that the former case just considered is not possible because it leads to a contradiction. Therefore, we consider only the latter case.

Consider the latter case, that is, there is a disjunct $r$ of $\operatorname{PerfRef}_{q_{\beta}, \Sigma}$ for which $\vec{c} \notin q_{\mathcal{S}}^{D_{r}}$. Observe that the $\mathcal{S}$-database $D^{\prime}$ that we are seeking is $D^{\prime}=D_{r}$. Indeed, since $\mathcal{M}$ is a pure GAV mapping and $\mathcal{O}$ is a DL-Lite $\mathrm{RDFS}$ ontology, and thus contains no assertions with $\exists R$ in the right-hand side for a basic role $R$, and since $r$ is a disjunct of $\operatorname{PerfRe}_{q_{\beta}, \Sigma}$ (i.e., the body of $r$ is a way for unfolding all the facts occurring in $\mathcal{C}_{\mathcal{O}}^{\mathcal{M}(D)}$ ), it is easy to verify that $\mathcal{C}_{\mathcal{O}}^{\mathcal{M}\left(D^{\prime}\right)}$ is such that $\mathcal{C}_{\mathcal{O}}^{\mathcal{M}(D)} \subseteq \mathcal{C}_{\mathcal{O}}^{\mathcal{M}\left(D^{\prime}\right)}$, where $D^{\prime}=D_{r}$. Notice that $\vec{c} \in \operatorname{cert} t_{q_{\mathcal{O}}^{\prime}, \Sigma}^{D}$ holds by assumption, and therefore $\vec{c} \in q_{\mathcal{O}}^{\prime} \mathcal{C}^{\mathcal{M}(D)}$. Furthermore, since $\mathcal{C}_{\mathcal{O}}^{\mathcal{M}(D)} \subseteq \mathcal{C}_{\mathcal{O}}^{\mathcal{M}\left(D^{\prime}\right)}$ and $q_{\mathcal{O}}$ is a UCQ, we trivially derive that $\vec{c} \in q_{\mathcal{O}}^{\prime} \mathcal{C}_{\mathcal{O}}^{\mathcal{M}\left(D^{\prime}\right)}$ as well, which, in turn, implies that $\vec{c} \in \operatorname{cert} t_{q_{\mathcal{O}}^{\prime}, \Sigma}^{D^{\prime}}$.

To complete the proof, consider the $\mathcal{S}$-database $D^{\prime}$. We have that, on the one hand, $\vec{c} \notin q_{\mathcal{S}}^{D^{\prime}}$, and, on the other hand, $\vec{c} \in \operatorname{cert}_{q_{\mathcal{O}}^{\prime}, \Sigma}^{D^{\prime}}$. It follows that $q_{\mathcal{O}}^{\prime}$ is not a sound $\mathcal{S}$-to- $\mathcal{O} \Sigma$-rewriting of $q_{\mathcal{S}}$, as required.

Regarding the cost of the algorithm, we observe that the overall running time is exponential in the size of the input. Notice, moreover, that CQs over $\mathcal{O}$ may have an exponential number of atoms with respect to $\eta\left(q_{\mathcal{S}}\right)$. Next we prove that $(i)$ unless PTIME $=\mathrm{NP}$, the computation problem for sound source-to-ontology rewritings can not be solved in polynomial time, even in the restricted scenario for UCQJFEs; and (ii) there exists OBDM specifications $\Sigma$ and UCQJFEs $q_{\mathcal{S}}$ for which the unique (up to equivalence w.r.t. $\Sigma$ ) UCQ-maximally sound $\mathcal{S}$-to- $\mathcal{O} \Sigma$-rewriting of $q_{\mathcal{S}}$ is a $\mathrm{CQ}$ whose number of atoms is necessarily exponential with respect to $\eta\left(q_{\mathcal{S}}\right)$.

Proposition 8.1. Assuming PTIME $\subsetneq N P$, the computation problem for sound source-to-ontology rewritings in the restricted scenario for UCQJFEs can not be solved in polynomial time.

Proof. Let $F$ be a 3 -DNF formula on a set of variables $X=\left\{x_{1}, \ldots, x_{n}\right\}$. Consider the OBDM specification $\Sigma=\langle\mathcal{O}, \mathcal{S}, \mathcal{M}\rangle$, the UCQJFE $q_{\mathcal{S}}$, and the CQ $q_{\mathcal{O}}$ constructed from $F$ as illustrated in the reduction of the lower bound proof of Theorem 8.1.

In this case, it is straightforward to verify that the unique (up to equivalence w.r.t. $\Sigma$ ) UCQ-maximally sound $\mathcal{S}$-to- $\mathcal{O} \Sigma$-rewriting of $q_{\mathcal{S}}$ is either the CQ $q_{\mathcal{O}}$ if it is a sound $\mathcal{S}$-to- $\mathcal{O} \Sigma$-rewriting of $q_{\mathcal{S}}$, or the $\mathrm{CQ} q_{\mathcal{O}}^{\prime}=\left\{\left(x_{1}, \ldots, x_{n}\right) \mid \perp\left(x_{1}, \ldots, x_{n}\right)\right\}$ otherwise. Specifically, as shown in that coNP-hardness proof of Theorem 8.1, $q_{\mathcal{O}}$ is a sound $\mathcal{S}$-to- $\mathcal{O} \Sigma$-rewriting of $q_{\mathcal{S}}$ if and only formula $F$ is valid, and therefore, due to 
the above observation, $q_{\mathcal{O}}$ is the unique (up to equivalence w.r.t. $\Sigma$ ) UCQ-maximally sound $\mathcal{S}$-to- $\mathcal{O} \Sigma$-rewriting of $q_{\mathcal{S}}$ if and only if formula $F$ is valid.

We have therefore reduced the problem of checking the validity of a 3-DNF formula $F$ to the problem of computing the unique (up to equivalence w.r.t. $\Sigma$ ) UCQmaximally sound $\mathcal{S}$-to- $\mathcal{O} \Sigma$-rewriting of a UCQJFE $q_{\mathcal{S}}$, where both $\Sigma=\langle\mathcal{O}, \mathcal{S}, \mathcal{M}\rangle$ and $q_{\mathcal{S}}$ can be constructed in LOGSPACE from $F$.

Thus, even in the restricted scenario for UCQJFEs, a polynomial time algorithm for computing UCQ-maximally sound source-to-ontology rewritings of UCQJFEs would imply a polynomial time algorithm for checking whether a 3-DNF formula is valid. Since this latter problem is known to be in general coNP-hard, it follows that, unless PTime $=\mathrm{NP}$, the computation problem for sound source-to-ontology rewritings can not be solved in polynomial time, even in the restricted scenario for UCQJFEs.

Proposition 8.2. In the restricted scenario for UCQJFEs, there are OBDM specifications $\Sigma$ and UCQJFEs $q_{\mathcal{S}}$ for which the unique (up to equivalence w.r.t. $\Sigma$ )

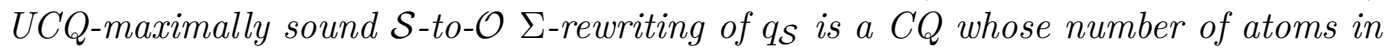
its body is necessarily exponential with respect to $\eta\left(q_{\mathcal{S}}\right)$.

Proof. We provide here a small example showing the main reason of why the number of atoms in the body of the unique (up to equivalence w.r.t. the OBDM specification $\Sigma=\langle\mathcal{O}, \mathcal{S}, \mathcal{M}\rangle)$ UCQ-maximally sound $\mathcal{S}$-to- $\mathcal{O} \Sigma$-rewriting of a UCQJFE $q_{\mathcal{S}}$ may be exponential with respect to $\eta\left(q_{\mathcal{S}}\right)$.

Let $\Sigma=\langle\mathcal{O}, \mathcal{S}, \mathcal{M}\rangle$ be the following OBDM specification:

- $\mathcal{O}=\emptyset$

- $\mathcal{S}=\left\{s_{1}, s_{2}, s_{3}, s_{4}\right\}$

- $\mathcal{M}=\left\{m_{1}, m_{2}, m_{3}, m_{4}, m_{5}, m_{6}, m_{7}, m_{8}, m_{9}, m_{10}, m_{11}, m_{12}\right\}$, where:

$$
\begin{aligned}
& m_{1}: s_{1}(x) \rightarrow A_{1}(x), \\
& m_{2}: s_{2}(x) \rightarrow A_{1}(x), \\
& m_{3}: s_{1}(x) \rightarrow A_{2}(x), \\
& m_{4}: s_{3}(x) \rightarrow A_{2}(x), \\
& m_{5}: \quad s_{1}(x) \rightarrow A_{3}(x), \\
& m_{6}: s_{4}(x) \rightarrow A_{3}(x), \\
& m_{7}: s_{2}(x) \rightarrow A_{4}(x), \\
& m_{8}: s_{3}(x) \rightarrow A_{4}(x), \\
& m_{9}: s_{2}(x) \rightarrow A_{5}(x), \\
& m_{10}: s_{4}(x) \rightarrow A_{5}(x), \\
& m_{11}: s_{3}(x) \rightarrow A_{6}(x), \\
& m_{12}: s_{4}(x) \rightarrow A_{6}(x) .
\end{aligned}
$$

Let $q_{\mathcal{S}}$ be the following UCQJFE over $\mathcal{S}: q_{\mathcal{S}}=\left\{(x) \mid s_{1}(x) \wedge s_{2}(x) \wedge s_{3}(x)\right\} \cup\{(x) \mid$ $\left.s_{1}(x) \wedge s_{2}(x) \wedge s_{4}(x)\right\} \cup\left\{(x) \mid s_{1}(x) \wedge s_{3}(x) \wedge s_{4}(x)\right\} \cup\left\{(x) \mid s_{2}(x) \wedge s_{3}(x) \wedge s_{4}(x)\right\}$. One can verify that the $\mathrm{CQ} q_{\mathcal{O}}=\left\{(x) \mid A_{1}(x) \wedge A_{2}(x) \wedge A_{3}(x) \wedge A_{4}(x) \wedge A_{5}(x) \wedge A_{6}(x)\right\}$ is a sound $\mathcal{S}$-to- $\mathcal{O} \Sigma$-rewriting of $q_{\mathcal{S}}$, and, moreover, every possible CQ $q_{\mathcal{O}}^{\prime}$ whose body contains only a strict subset of the atoms occurring in the body of $q_{\mathcal{O}}$ is such that $q_{\mathcal{O}}^{\prime}$ is not a sound $\mathcal{S}$-to- $\mathcal{O} \Sigma$-rewriting of $q_{\mathcal{S}}$. Thus, it follows that $q_{\mathcal{O}}$ is the 
unique (up to equivalence w.r.t. $\Sigma$ ) UCQ-maximally sound $\mathcal{S}$-to- $\mathcal{O} \Sigma$-rewriting of $q_{\mathcal{S}}$ (in fact, one can verify that $q_{\mathcal{O}}$ is the output of MaximallySoundUCQJFEs $\left(\Sigma, q_{\mathcal{S}}\right)$ ). More precisely, one can verify that $q_{\mathcal{O}}$ is the perfect $\mathcal{S}$-to- $\mathcal{O} \Sigma$-rewriting of $q_{\mathcal{S}}$.

Let denote by $|\mathcal{S}|$ the number of source predicates occurring in the source schema $\mathcal{S}$, and by $\chi(\mathcal{M})$ the number of times that an atomic concept $A_{i}$ in the alphabet of the ontology $\mathcal{O}$ appears in the right-hand side of mapping assertions in $\mathcal{M}$. By generalising the above construction, one can see that it is always possible to compose OBDM specifications $\Sigma=\langle\mathcal{O}, \mathcal{S}, \mathcal{M}\rangle$ and UCQJFEs $q_{\mathcal{S}}$ for which $(i)$ $\eta\left(q_{\mathcal{S}}\right)=|\mathcal{S}|^{2}-|\mathcal{S}|$, and $(i i)$ the number of atoms occurring in the body of the CQ corresponding to the unique (up to equivalence w.r.t. $\Sigma$ ) UCQ-maximally sound $\mathcal{S}$-to- $\mathcal{O} \Sigma$-rewriting of $q_{\mathcal{S}}$ is necessarily equal to $\frac{|\mathcal{S}| !}{(|\mathcal{S}|-\chi(\mathcal{M})) ! \cdot \chi(\mathcal{M}) !}$ (and therefore, an exponential number of atoms with respect to $\left.\eta\left(q_{\mathcal{S}}\right)\right)$.

In the next section, we will see that the bound on the number of atoms of Lemma 8.3 becomes polynomial (rather than exponential) with respect to $\eta\left(q_{\mathcal{S}}\right)$ when queries $q_{\mathcal{S}}$ are CQJFEs (rather than unions thereof).

\subsection{Restricted Scenario for CQJFEs}

In this section, we study both the verification, and the computation problem for sound source-to-ontology rewritings in the restricted scenario for CQJFEs. We recall that, while the language $\mathcal{L}_{\mathcal{S}}$ of queries over source schemas $\mathcal{S}$ is the one of CQJFEs, the language $\mathcal{L}_{\mathcal{O}}$ for queries over ontologies $\mathcal{O}$ remains the one of UCQs.

Before going into details, we introduce the notion of covering.

Definition 8.2. Let $\Sigma=\langle\mathcal{O}, \mathcal{S}, \mathcal{M}\rangle$ be an OBDM specification, $\beta$ be an atom over $\mathcal{O}$, and $\alpha$ be an atom occurring in the body of a CQ $q_{\mathcal{S}}=\{\vec{t} \mid \exists \vec{y} \cdot \phi(\vec{x}, \vec{y})\}$ over $\mathcal{S}$. We say that $\beta \Sigma$-covers $\alpha$, if the following holds: in each disjunct of $\rho(\beta, \Sigma)$ there is at least an atom $\alpha^{\prime}$ such that $\alpha^{\prime}$ instantiates $\alpha$.

Example 8.5. Let $\Sigma=\langle\mathcal{O}, \mathcal{S}, \mathcal{M}\rangle$ be the following OBDM specification:

- $\mathcal{O}=\left\{P_{1} \sqsubseteq P_{2}\right\}$

- $\mathcal{S}=\left\{s_{1}, s_{2}\right\}$

- $\mathcal{M}=\left\{m_{1}, m_{2}\right\}$, where:

$$
\begin{array}{lll}
m_{1}: & s_{1}\left(x_{1}, x_{2}, x_{1}\right) \wedge s_{2}\left(x_{1}, x_{2}\right) \rightarrow P_{1}\left(x_{1}, x_{2}\right), \\
m_{2}: & s_{1}\left(x_{1}, x_{2}, c_{1}\right) \wedge s_{2}\left(x_{2}, x_{2}\right) \rightarrow P_{2}\left(x_{1}, x_{2}\right) .
\end{array}
$$

Consider the query $q_{\mathcal{S}}=\left\{(x) \mid \exists y . s_{1}\left(c_{2}, x, y\right) \wedge s_{2}(x, x)\right\}$ over $\mathcal{S}$, and the atom $\beta=P_{2}\left(c_{2}, x\right)$ over $\mathcal{O}$. Let $\alpha_{1}=s_{1}\left(c_{2}, x, y\right)$ and $\alpha_{2}=s_{2}(x, x)$. Note that $\rho(\beta, \Sigma)=$ $\left(s_{1}\left(c_{2}, x, c_{1}\right) \wedge s_{2}(x, x)\right) \vee\left(s_{1}\left(c_{2}, x, c_{2}\right) \wedge s_{2}\left(c_{2}, x\right)\right)$. Thus, we have that $\beta \Sigma$-covers $\alpha_{1}$, whereas $\beta$ does not $\Sigma$-cover $\alpha_{2}$. This latter is because in the disjunct $\left(s_{1}\left(c_{2}, x, c_{2}\right) \wedge\right.$ $\left.s_{2}\left(c_{2}, x\right)\right)$ of $\rho(\beta, \Sigma)$ there is no atom $\alpha^{\prime}$ such that $\alpha^{\prime}$ instantiates $\alpha_{2}$.

Obviously, for an OBDM specification $\Sigma=\langle\mathcal{O}, \mathcal{S}, \mathcal{M}\rangle$, an atom $\beta$ over $\mathcal{O}$, and an atom $\alpha$ over $\mathcal{S}$, checking whether $\beta \Sigma$-covers $\alpha$ can be done in polynomial time. 


\subsubsection{Verification Problem}

We start by proving the following lemma, which will be used to prove that the verification problem in this setting can be solved in polynomial time.

Lemma 8.4. Let $\Sigma=\langle\mathcal{O}, \mathcal{S}, \mathcal{M}\rangle$ be an $O B D M$ specification, and let $q_{\mathcal{S}}$ and $q_{\mathcal{O}}$ be a $C Q J F E$ over $\mathcal{S}$ and a $C Q$ over $\mathcal{O}$, respectively, with the same target list. We have

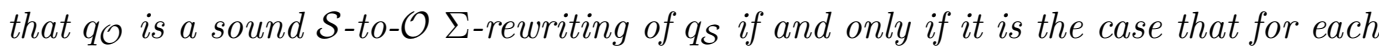
atom $\alpha$ of $q_{\mathcal{S}}$ there exists an atom $\beta$ of $q_{\mathcal{O}}$ such that $\beta \Sigma$-covers $\alpha$.

Proof. "Only-if part:" Suppose, for the sake of contradiction, that there exists an atom $\alpha$ of $q_{\mathcal{S}}$ such that for no atom $\beta$ of $q_{\mathcal{O}}$ it is the case that $\beta \Sigma$-covers $\alpha$. Let $q^{\prime}$ be the disjunct of $\operatorname{PerfRef}_{q_{\mathcal{O}}, \Sigma}$ obtained by unfolding each atom $\beta$ of $q_{\mathcal{O}}$ with the disjunct of $\rho(\beta, \Sigma)$ in which there is no atom $\alpha^{\prime}$ that instantiates $\alpha$. For each atom $\beta$ of $q_{\mathcal{O}}$, there is at least one disjunct among the ones of $\rho(\beta, \Sigma)$ that satisfies this condition, otherwise, following Definition 8.2 , we would trivially derive a contradiction on the fact that $\beta$ does not $\Sigma$-cover $\alpha$.

Thus, the disjunct $q^{\prime}$ of $\operatorname{PerfRef}_{q_{\mathcal{O}}, \Sigma}$ contains no atom that instantiates the atom $\alpha$ of $q_{\mathcal{S}}$, and therefore, due to Lemma 8.1, this implies that $q^{\prime} \nsubseteq q_{\mathcal{S}}$. It follows that $\operatorname{PerfRef}_{q_{\mathcal{O}}, \Sigma} \nsubseteq q_{\mathcal{S}}$, which, due to Corollary 8.1, in turn implies that $q_{\mathcal{O}}$ is not a sound $\mathcal{S}$-to- $\mathcal{O} \Sigma$-rewriting of $q_{\mathcal{S}}$, as required.

"If part:" Since for each atom $\alpha$ of $q_{\mathcal{S}}$ there is an atom $\beta$ such that $\beta \Sigma$-covers $\alpha$, we trivially derive that each possible disjunct $q^{\prime}$ of $\operatorname{PerfRef}_{q_{\mathcal{O}}, \Sigma}$ satisfies the following condition: for each atom $\alpha$ of $q_{\mathcal{S}}$, there is an atom $\alpha^{\prime}$ of $q^{\prime}$ such that $\alpha^{\prime}$ instantiates $\alpha$. Due to Lemma 8.1. it follows that that $q^{\prime} \sqsubseteq q$. Since this is true for each possible disjunct $q^{\prime}$ of $\operatorname{PerfRe}_{q_{\mathcal{O}}, \Sigma}$, we further derive that $\operatorname{PerfRef}_{q_{\mathcal{O}}, \Sigma} \sqsubseteq q_{\mathcal{S}}$, which, due to Corollary 8.1. implies that $q_{\mathcal{O}}$ is a sound $\mathcal{S}$-to- $\mathcal{O} \Sigma$-rewriting of $q_{\mathcal{S}}$, as required.

Based on the above lemma, the following theorem proves that, in the restricted scenario for CQJFEs, the verification problem for sound source-to-ontology rewritings can be solved in polynomial time.

Theorem 8.3. In the restricted scenario for CQJFEs, the verification problem is in PTime.

Proof. Due to Corollary 8.1, it is sufficient to show how to check the containment PerfRef $_{q_{\mathcal{O}}, \Sigma} \sqsubseteq q_{\mathcal{S}}$ in polynomial time, where $q_{\mathcal{O}}$ and $q_{\mathcal{S}}$ are a UCQ over $\mathcal{O}$ and a CQJFE over $\mathcal{S}$, respectively. To start, note that by construction $q_{\mathcal{O}}$ is a sound $\mathcal{S}$-to- $\mathcal{O}$ $\Sigma$-rewriting of $q_{\mathcal{S}}$ if and only if each disjunct $q$ of $q_{\mathcal{O}}$ is a sound $\mathcal{S}$-to- $\mathcal{O} \Sigma$-rewriting of $q_{\mathcal{S}}$, i.e., $\operatorname{PerfRef}_{q_{\mathcal{O}}, \Sigma} \sqsubseteq q_{\mathcal{S}}$ if and only if $\operatorname{PerfRef}_{q, \Sigma} \sqsubseteq q_{\mathcal{S}}$ for each disjunct $q$ of $q_{\mathcal{O}}$. It is therefore enough to show that, given a CQ $q=\left\{\overrightarrow{t^{\prime}} \mid \exists \overrightarrow{y^{\prime}} \cdot \phi^{\prime}\left(\overrightarrow{x^{\prime}}, \overrightarrow{y^{\prime}}\right)\right\}$ over $\mathcal{O}$ and a CQJFE $q_{\mathcal{S}}=\{\vec{t} \mid \exists \vec{y} \cdot \phi(\vec{x}, \vec{y})\}$ over $\mathcal{S}$, checking whether PerfRef $q, \Sigma \sqsubseteq q_{\mathcal{S}}$ can be done in polynomial time.

We assume that every atom $\beta$ of $q$ appears in the right-hand side of some mapping assertion in $\mathcal{M}$, otherwise we trivially have that $\operatorname{PerfRef}_{q, \Sigma} \equiv \perp$, and therefore $q$ is a sound $\mathcal{S}$-to- $\mathcal{O} \Sigma$-rewriting of $q_{\mathcal{S}}$. Furthermore, if $q$ and $q_{\mathcal{S}}$ do not have the same target list, i.e., $\overrightarrow{t^{\prime}}=\left(t_{1}^{\prime}, \ldots, t_{n}^{\prime}\right) \neq \vec{t}=\left(t_{1}, \ldots, t_{n}\right)$, then consider the function $f$ from the set of terms in the target list of $q$ to the set of terms in the target list of $q_{\mathcal{S}}$ with $f\left(t_{i}\right)=t_{i}^{\prime}$, for each $i \in[1, n]$. Formally, since repetitions of terms in target lists is 
allowed, $f$ might give rise to a multivalued function. In this case, as well as in the case that $f(a)=b$ with $a \neq b$ for two constants $a \in \vec{t}$ and $b \in \overrightarrow{t^{\prime}}$, it is straightforward to verify that $\operatorname{PerfRef}_{q, \Sigma} \nsubseteq q_{\mathcal{S}}$ trivially holds. Indeed, in those cases there can be no homomorphism from $q_{\mathcal{S}}$ to the disjuncts of $\operatorname{PerfRef}_{q, \Sigma}$ by construction.

Consider the query $q_{\mathcal{S}}^{\prime}$ obtained in polynomial time from $q_{\mathcal{S}}$ by replacing every occurrence of the term $t_{i}$ in $q_{\mathcal{S}}$ (even in the target list) with the term $f\left(t_{i}\right)=t_{i}^{\prime}$, for each $i \in[1, n]$. Observe that now $q_{\mathcal{S}}^{\prime}$ is a CQJFE having the same target list $\overrightarrow{t^{\prime}}$ of $q$. By virtue of Lemma 8.4, we can now check whether $q$ is a sound $\mathcal{S}$-to- $\mathcal{O} \Sigma$-rewriting of $q_{\mathcal{S}}^{\prime}$ by checking whether it is the case that for each atom $\alpha$ of $q^{\prime}$ there exists an atom $\beta$ of $q$ such that $\beta \Sigma$-covers $\alpha$. This can be clearly done in polynomial time.

Obviously, if $q$ is a sound $\mathcal{S}$-to- $\mathcal{O} \Sigma$-rewriting of $q_{\mathcal{S}}^{\prime}$, then we trivially have that $q$ is sound $\mathcal{S}$-to- $\mathcal{O} \Sigma$-rewriting of $q_{\mathcal{S}}$ as well. Conversely, if $q$ is not a sound $\mathcal{S}$-to- $\mathcal{O}$ $\Sigma$-rewriting of $q_{\mathcal{S}}^{\prime}$, then there is a disjunct $r$ of $\operatorname{PerfRef}_{q, \Sigma}$ such that $r \nsubseteq q^{\prime}$. But then, it can be readily seen that $D_{r}$ (i.e., the freezing of $r$ ) is the database witnessing that $r \nsubseteq q_{\mathcal{S}}$. It follows that, for the $\mathcal{S}$-database $D_{r}$, we have $\operatorname{cert}_{q, \Sigma}^{D_{r}} \nsubseteq q_{\mathcal{S}}^{D_{r}}$, which implies that $q$ is not a sound $\mathcal{S}$-to- $\mathcal{O} \Sigma$-rewriting of $q_{\mathcal{S}}$ as well.

From the above considerations, it is immediate to derive a polynomial time algorithm for checking whether a UCQ $q_{\mathcal{O}}$ over $\mathcal{O}$ is a sound $\mathcal{S}$-to- $\mathcal{O} \Sigma$-rewriting of a CQJFE $q_{\mathcal{S}}$ over $\mathcal{S}$.

\subsubsection{Computation Problem}

As for the computation problem, we now provide an algorithm for computing UCQmaximally sound source-to-ontology rewritings which avoids the enumeration of all possible CQs over $\mathcal{O}$ of a certain bound as algorithm MaximallySoundUCQJFEs in the previous section does. The computation of the returned UCQ over $\mathcal{O}$ is rather guided by the atoms occurring in the input query $q_{\mathcal{S}}$, in a very similar fashion to the bucket algorithm Levy et al., 1996 used for rewriting queries using views.

Specifically, by exploiting Lemma 8.4, the idea is as follows: for each atom $\alpha_{i}$ occurring in the body of $q_{\mathcal{S}}$, we compute a set $B_{i}$ containing all the atoms $\beta$ over $\mathcal{O}$ such that $\beta \Sigma$-cover $\alpha$. Then, disjuncts of the final UCQ $q_{\mathcal{O}}$ over $\mathcal{O}$ are constructed by simply selecting atoms from each set $B_{i}$ and conjoining them.

There is, however, a preliminary issue to solve in order to apply this simple idea: let $\Sigma=\langle\mathcal{O}, \mathcal{S}, \mathcal{M}\rangle$ be an OBDM specification, and let $\alpha$ be an atom of a $\mathrm{CQ}$ $q_{\mathcal{S}}=\{\vec{t} \mid \exists \vec{y} \cdot \phi(\vec{x}, \vec{y})\}$ over $\mathcal{S}$. It might happen that an atom $\beta$ over $\mathcal{O}$ does not $\Sigma$-cover $\alpha$, but $\beta \Sigma$-covers $\alpha^{\prime}$ if some equalities are applied in the target list of $q_{\mathcal{S}}$, where $\alpha^{\prime}$ denotes the atom obtained from $\alpha$ after applying such equalities. The next example shows this complication:

Example 8.6. Let $\Sigma=\langle\mathcal{O}, \mathcal{S}, \mathcal{M}\rangle$ be the following OBDM specification:

- $\mathcal{O}=\emptyset$

- $\mathcal{S}=\{s\}$

- $\mathcal{M}=\left\{m_{1}, m_{2}\right\}$, where:

$m_{1}: \quad s(x, x) \quad \rightarrow \quad A(x)$,

$m_{2}: s\left(x, c_{1}\right) \rightarrow A^{\prime}(x)$. 
Consider the CQJFE $q_{\mathcal{S}}=\left\{\left(x_{1}, x_{2}\right) \mid s\left(x_{1}, x_{2}\right)\right\}$ over $\mathcal{S}$. Observe that there is no atom $\beta$ over $\mathcal{O}$ such that $\beta$ L-covers $s\left(x_{1}, x_{2}\right)$.

Notice that, however, if we consider more specific queries obtained by applying some equalities to the query $q_{\mathcal{S}}$, such as $q_{\mathcal{S}}^{1}=\left\{\left(x_{1}, x_{1}\right) \mid s\left(x_{1}, x_{1}\right)\right\}$ (obtained by applying the equality $\left.x_{1}=x_{2}\right)$ and $q_{\mathcal{S}}^{2}=\left\{\left(x_{1}, c_{1}\right) \mid s\left(x_{1}, c_{1}\right)\right\}$ (obtained by applying the equality $\left.x_{2}=c_{1}\right)$, then we get that atom $A\left(x_{1}\right) \Sigma$-covers $s\left(x_{1}, x_{1}\right)$ and atom $A^{\prime}\left(x_{1}\right)$ $\Sigma$-covers $s\left(x_{1}, c_{1}\right)$. As a result, due to Lemma 8.4 queries $q_{\mathcal{O}}^{1}=\left\{\left(x_{1}, x_{1}\right) \mid A\left(x_{1}\right)\right\}$ and $q_{\mathcal{O}}^{2}=\left\{\left(x_{1}, c_{1}\right) \mid A^{\prime}\left(x_{1}\right)\right\}$ are a sound $\mathcal{S}$-to- $\mathcal{O} \sum$-rewriting of $q_{\mathcal{S}}^{1}$ and $q_{\mathcal{S}}^{2}$, respectively It follows that, since by construction $q_{\mathcal{S}}^{i} \sqsubseteq q_{\mathcal{S}}$ for both $i=1$ and $i=2$, both $q_{\mathcal{O}}^{1}$ and $q_{\mathcal{O}}^{2}$ are sound $\mathcal{S}$-to- $\mathcal{O}$ $\Sigma$-rewritings of $q_{\mathcal{S}}$ as well.

Furthermore, when applying equalities, we have to take into account not only constants occurring in mapping assertions $\mathcal{M}$, but also constants occurring in the body of the input query. Consider indeed the CQJFE $q_{\mathcal{S}}^{\prime}=\left\{(x) \mid s\left(x, c_{2}\right)\right\}$ over $\mathcal{S}$. Observe that there is no atom $\beta$ over $\mathcal{O}$ such that $\beta \Sigma$-covers $s\left(x, c_{2}\right)$. Notice, however, that if we consider the query $q_{\mathcal{S}}^{3}=\left\{\left(c_{2}\right) \mid s\left(c_{2}, c_{2}\right)\right\}$ (obtained by applying the equality $x=c_{2}$ to $\left.q_{\mathcal{S}}^{\prime}\right)$, then we get that atom $A\left(c_{2}\right) \Sigma$-covers $s\left(c_{2}, c_{2}\right)$. As a result, due to Lemma 8.4. the query $q_{\mathcal{O}}^{3}=\left\{\left(c_{2}\right) \mid A\left(c_{2}\right)\right\}$ is a sound $\mathcal{S}$-to- $\mathcal{O} \Sigma$-rewriting of $q_{\mathcal{S}}^{3}$, and therefore a sound $\mathcal{S}$-to- $\mathcal{O} \Sigma$-rewriting of $q_{\mathcal{S}}^{\prime}$ since $q_{\mathcal{S}}^{3} \sqsubseteq q_{\mathcal{S}}^{\prime}$.

Therefore, before of applying the idea described above for computing UCQmaximally sound source-to-ontology rewritings, we first have to compute the head completion of $q_{\mathcal{S}}$ with respect to $\operatorname{con}_{\mathcal{M}} \cup \operatorname{con}_{q_{\mathcal{S}}}$, where $\operatorname{con}_{\mathcal{M}}$ (respectively, $\operatorname{con}_{q_{\mathcal{S}}}$ ) denote the set of all constants occurring in $\mathcal{M}$ (respectively, $q_{\mathcal{S}}$ ).

Roughly speaking, the head completion of a CQ $q_{\mathcal{S}}=\{\vec{t} \mid \exists \vec{y} \cdot \phi(\vec{x}, \vec{y})\}$ with respect to a set of constants con is an equivalent UCQ in which each disjunct is computed by considering a possible unification between the terms in $\vec{t} \cup$ con.

We now present the algorithm HeadCompletion that, given a CQ $q$ and a set of constants con, returns a logically equivalent UCQ representing the head completion of $q$ with respect to con.

In the algorithm, two terms $t_{1}$ and $t_{2}$ are compatible if $t_{1}$ and $t_{2}$ denote distinct terms and at least one of them is a variable. Furthermore, for a query $q, q\left[t_{1} / t_{2}\right]$ denotes the query obtained from $q$ by replacing every occurrence (even in the target list) of the term $t_{1}$ in $q$ with the term $t_{2}$ (obviously, if one of the two terms is a constant, then we always assume that $t_{2}$ is the constant and $t_{1}$ is the variable).

For a CQ $q$ and a set of constants con, HeadCompletion( $q$, con) computes the equivalent UCQ $Q$ obtained by unifying compatible terms of the target list of $q$, and of the set of constants con, in all possible ways.

The following example illustrates an execution of the HeadCompletion algorithm.

Example 8.7. Let $q_{\mathcal{S}}$ be the following CQ $q_{\mathcal{S}}=\left\{\left(x_{1}, x_{2}\right) \mid \exists y \cdot s_{1}\left(x_{1}, c_{2}, y\right) \wedge\right.$ $\left.s_{2}\left(x_{1}, x_{2}\right)\right\}$, and let con be the following set of constants con $=\left\{c_{1}, c_{2}\right\}$. One can verify that HeadCompletion $\left(q_{\mathcal{S}}\right.$, con) returns the UCQ $Q=\bigcup_{1 \leq i \leq 10} q_{\mathcal{S}}^{i}$, where:

- $q_{\mathcal{S}}^{1}=\left\{\left(x_{1}, x_{2}\right) \mid \exists y \cdot s_{1}\left(x_{1}, c_{2}, y\right) \wedge s_{2}\left(x_{1}, x_{2}\right)\right\} ;$

- $q_{\mathcal{S}}^{2}=\left\{\left(x_{1}, x_{1}\right) \mid \exists y \cdot s_{1}\left(x_{1}, c_{2}, y\right) \wedge s_{2}\left(x_{1}, x_{1}\right)\right\}$

- $q_{\mathcal{S}}^{3}=\left\{\left(x_{1}, c_{1}\right) \mid \exists y \cdot s_{1}\left(x_{1}, c_{2}, y\right) \wedge s_{2}\left(x_{1}, c_{1}\right)\right\} ;$ 


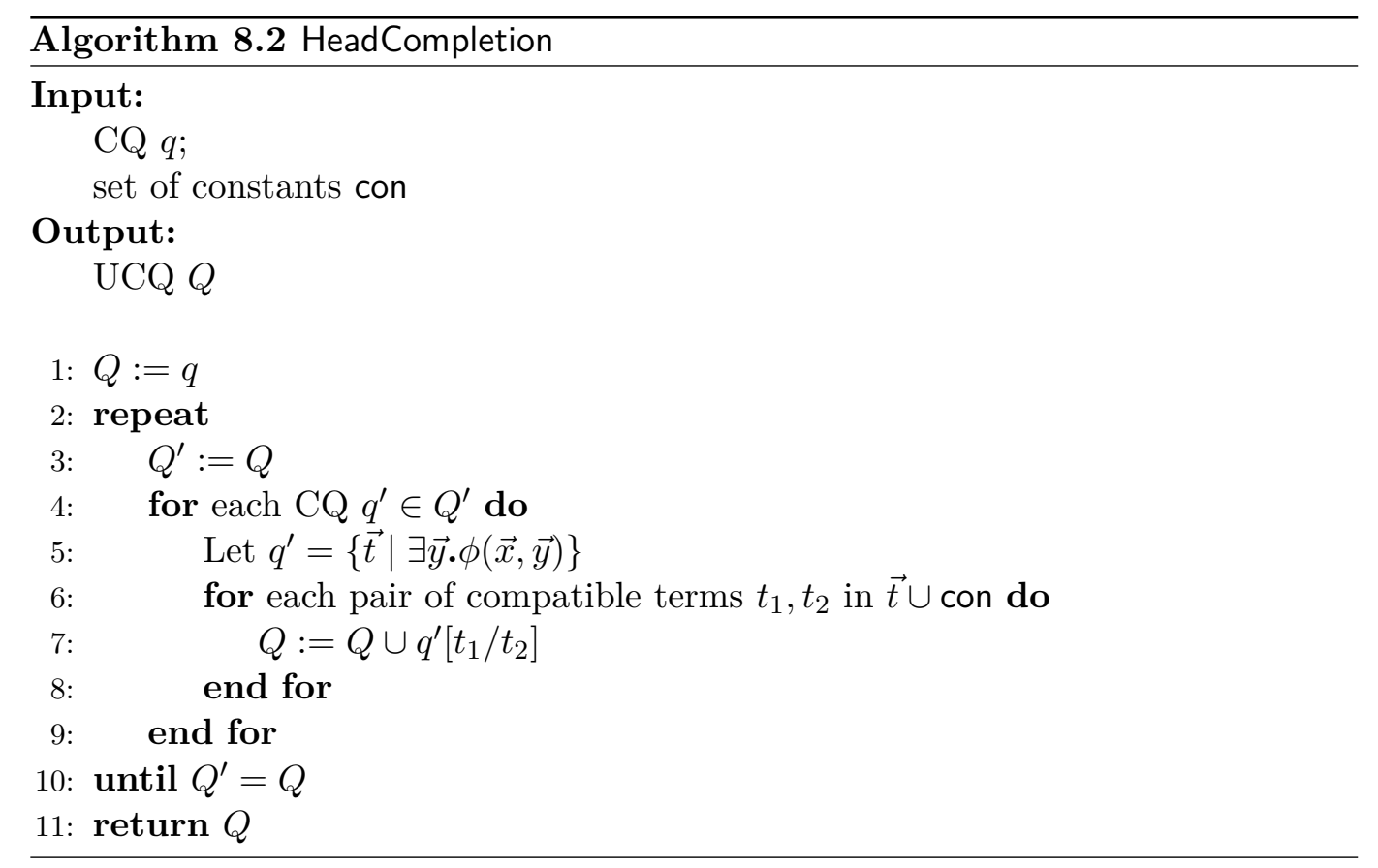

- $q_{\mathcal{S}}^{4}=\left\{\left(x_{1}, c_{2}\right) \mid \exists y \cdot s_{1}\left(x_{1}, c_{2}, y\right) \wedge s_{2}\left(x_{1}, c_{2}\right)\right\}$

- $q_{\mathcal{S}}^{5}=\left\{\left(c_{1}, x_{2}\right) \mid \exists y \cdot s_{1}\left(c_{1}, c_{2}, y\right) \wedge s_{2}\left(c_{1}, x_{2}\right)\right\}$;

- $q_{\mathcal{S}}^{6}=\left\{\left(c_{2}, x_{2}\right) \mid \exists y \cdot s_{1}\left(c_{2}, c_{2}, y\right) \wedge s_{2}\left(c_{2}, x_{2}\right)\right\}$;

- $q_{\mathcal{S}}^{7}=\left\{\left(c_{1}, c_{2}\right) \mid \exists y \cdot s_{1}\left(c_{1}, c_{2}, y\right) \wedge s_{2}\left(c_{1}, c_{2}\right)\right\} ;$

- $q_{\mathcal{S}}^{8}=\left\{\left(c_{1}, c_{1}\right) \mid \exists y \cdot s_{1}\left(c_{1}, c_{2}, y\right) \wedge s_{2}\left(c_{1}, c_{1}\right)\right\} ;$

- $q_{\mathcal{S}}^{9}=\left\{\left(c_{2}, c_{1}\right) \mid \exists y . s_{1}\left(c_{2}, c_{2}, y\right) \wedge s_{2}\left(c_{2}, c_{1}\right)\right\}$;

- $q_{\mathcal{S}}^{10}=\left\{\left(c_{2}, c_{2}\right) \mid \exists y . s_{1}\left(c_{2}, c_{2}, y\right) \wedge s_{2}\left(c_{2}, c_{2}\right)\right\}$;

We are now ready to focus on the problem of computing UCQ-maximally sound source-to-ontology rewritings in the restricted scenario for CQJFEs, and present algorithm MaximallySoundCQJFEs.

Informally, the algorithm first computes the head completion of $q_{\mathcal{S}}$ with respect to con, where con $=\operatorname{con}_{\mathcal{M}} \cup \operatorname{con}_{q_{\mathcal{S}}}$. Subsequently, for each possible CQ $q \in$ HeadCompletion $\left(q_{\mathcal{S}}\right.$, con $)$, the algorithm proceeds in two main steps. In the first step, for each atom $\alpha_{i}$ occurring in the body of $q$, it is computed the set $B_{i}$ of relevant atoms over $\mathcal{O}$, where $\beta \Sigma$-covers $\alpha_{i}$ for each $\beta \in B_{i}$.

In the second step, for each possible combination which includes a single atom from every set $B_{i}$ (i.e., for each possible tuple of the Cartesian product $B_{1} \times \ldots \times$ $\left.B_{\eta\left(q_{\mathcal{S}}\right)}\right)$, the CQ with the same target list of $q$ and body the conjunction of those atoms is added as a disjunct of the final returned UCQ $q_{\mathcal{O}}$ over $\mathcal{O}$.

Example 8.8. Let $\Sigma=\langle\mathcal{O}, \mathcal{S}, \mathcal{M}\rangle$ be the following OBDM specification:

- $\mathcal{O}=\emptyset$ 


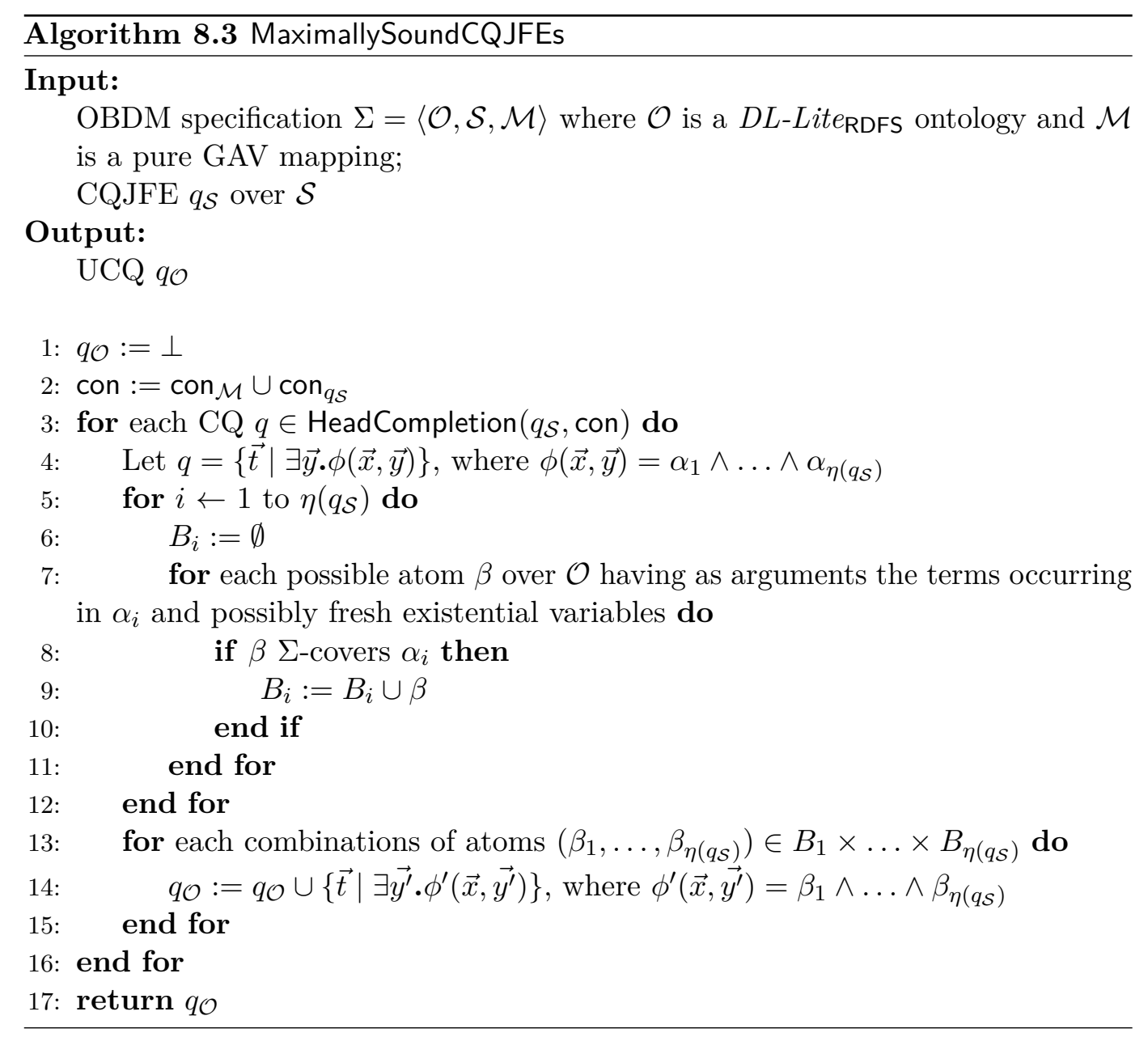

- $\mathcal{S}=\left\{s_{1}, s_{2}, s_{3}, s_{4}, s_{5}, s_{6}\right\}$

- $\mathcal{M}=\left\{m_{1}, m_{2}, m_{3}, m_{4}, m_{5}, m_{6}\right\}$

$$
\begin{aligned}
& m_{1}: \quad \exists y . s_{1}\left(x_{1}, x_{2}, y\right) \quad \rightarrow \quad P_{1}\left(x_{1}, x_{2}\right), \\
& m_{2}: \quad s_{2}\left(x_{1}, x_{2}\right) \quad \rightarrow \quad P_{2}\left(x_{1}, x_{2}\right) \text {, } \\
& m_{3}: \quad s_{3}\left(x_{1}, x_{2}\right) \quad \rightarrow \quad P_{2}\left(x_{1}, x_{2}\right), \\
& m_{4}: \quad \exists y \cdot s_{2}(x, x) \wedge s_{4}(x, y) \quad \rightarrow \quad A_{1}(x), \\
& m_{5}: \quad \exists y \cdot s_{2}(x, x) \wedge s_{4}(x, y) \quad \rightarrow \quad A_{2}(x), \\
& m_{6}: \quad s_{2}\left(x_{1}, c_{1}\right) \wedge s_{6}\left(x_{1}, x_{2}\right) \quad \rightarrow \quad P_{3}\left(x_{1}, x_{2}\right)
\end{aligned}
$$

Let $q_{\mathcal{S}}$ be the CQJFE illustrated in Example 8.7. As a first step, the algorithm computes HeadCompletion $\left(q_{\mathcal{S}}\right.$, con) which, since $\operatorname{con}_{\mathcal{M}}=\left\{c_{1}\right\}$ and $\operatorname{con}_{q_{\mathcal{S}}}=\left\{c_{2}\right\}$, it turns out to be the UCQ $Q=\bigcup_{1 \leq i \leq 10} q_{\mathcal{S}}^{i}$ illustrated in Example 8.7, where $\operatorname{con}=\operatorname{con}_{\mathcal{M}} \cup \operatorname{con}_{q_{\mathcal{S}}}=\left\{c_{1}, c_{2}\right\}$.

Then, for each $i \in[1,10]$, the algorithm processes query $q_{\mathcal{S}}^{i}$ to add possible CQs that are sound $\mathcal{S}$-to- $\mathcal{O} \Sigma$-rewritings of $q_{\mathcal{S}}^{i}$ (and therefore of $q_{\mathcal{S}}$ ). We point out that, for every $j=[1,4,5,6,7]$, the resulting atom $\alpha_{2}^{j}$ with predicate name $s_{2}$ in the body of $q_{\mathcal{S}}^{j}$ is such that $B_{2}^{j}=\emptyset$, i.e., there is no atom $\beta$ for which $\beta \Sigma$-covers $\alpha_{2}^{j}$, and therefore no disjunct is added for those queries. 
As for the query $q_{\mathcal{S}}^{2}=\left\{\left(x_{1}, x_{1}\right) \mid \exists y \cdot s_{1}\left(x_{1}, c_{2}, y\right) \wedge s_{2}\left(x_{1}, x_{1}\right)\right\}$, we have $B_{1}^{2}=$ $\left\{P_{1}\left(x_{1}, c_{2}\right)\right\}$ and $B_{2}^{2}=\left\{A_{1}\left(x_{1}\right), A_{2}\left(x_{1}\right)\right\}$. Thus, the CQs $q_{\mathcal{O}}^{1}=\left\{\left(x_{1}, x_{1}\right) \mid P_{1}\left(x_{1}, c_{2}\right) \wedge\right.$ $\left.A_{1}\left(x_{1}\right)\right\}$ and $q_{\mathcal{O}}^{2}=\left\{\left(x_{1}, x_{1}\right) \mid P_{1}\left(x_{1}, c_{2}\right) \wedge A_{1}\left(x_{1}\right)\right\}$ are disjuncts of the final UCQ $q_{\mathcal{O}}$.

As for the query $q_{\mathcal{S}}^{3}=\left\{\left(x_{1}, c_{1}\right) \mid \exists y \cdot s_{1}\left(x_{1}, c_{2}, y\right) \wedge s_{2}\left(x_{1}, c_{1}\right)\right\}$, we have $B_{1}^{3}=$ $\left\{P_{1}\left(x_{1}, c_{2}\right)\right\}$ and $B_{2}^{3}=\left\{P_{3}\left(x, y^{\prime}\right)\right\}$. Thus, the CQ $q_{\mathcal{O}}^{3}=\left\{\left(x_{1}, c_{1}\right) \mid \exists y^{\prime} \cdot P_{1}\left(x_{1}, c_{1}\right) \wedge\right.$ $\left.P_{3}\left(x_{1}, y^{\prime}\right)\right\}$ is a disjunct of the final UCQ $q_{\mathcal{O}}$.

For the queries $q_{\mathcal{S}}^{j}$ with $j=[8,9,10]$, we observe that all disjuncts over $\mathcal{O}$ generated by the algorithm are subsumed (w.r.t. $\Sigma$ ) by $q_{\mathcal{O}}^{i}$ for some $i=[1,2,3]$. As a conclusion, one can verify that MaximallySoundCQJFEs $\left(\Sigma, q_{\mathcal{S}}\right)$ returns a UCQ that is equivalent (w.r.t. $\Sigma$ ) to $q_{\mathcal{O}}=q_{\mathcal{O}}^{1} \cup q_{\mathcal{O}}^{2} \cup q_{\mathcal{O}}^{3}$, which is the unique (up to equivalence w.r.t. $\Sigma)$ UCQ-maximally sound $\mathcal{S}$-to- $\mathcal{O} \Sigma$-rewriting of $q_{\mathcal{S}}$.

The following theorem establishes termination and correctness of the MaximallySoundCQJFES algorithm.

Theorem 8.4. In the restricted scenario for CQJFEs, MaximallySoundCQJFEs $\left(\Sigma, q_{\mathcal{S}}\right)$ terminates and returns the unique (up to equivalence w.r.t. $\Sigma$ ) UCQ-maximally sound $\mathcal{S}$-to- $\mathcal{O}$ इ-rewriting of $q_{\mathcal{S}}$.

Proof. Termination of the algorithm easily follows from the termination of the HeadCompletion algorithm, and the fact that checking whether an atom $\beta$ over $\mathcal{O}$ $\Sigma$-covers an atom $\alpha$ over $\mathcal{S}$ can be done in finite time (actually, in polynomial time).

As for the correctness, we first show that the computed $q_{\mathcal{O}}$ is a sound $\mathcal{S}$-to- $\mathcal{O}$ $\Sigma$-rewriting of $q_{\mathcal{S}}$. By construction, each disjunct $\left\{\vec{t} \mid \exists \overrightarrow{y^{\prime}} \cdot \phi^{\prime}\left(\vec{x}, \overrightarrow{y^{\prime}}\right)\right\}$ of $q_{\mathcal{O}}$ satisfies the following condition: there is a query $q \in \operatorname{HeadCompletion}\left(q_{\mathcal{S}}\right.$, con) with target list $\vec{t}$ such that for each atom $\alpha_{i}$ of $q$ there is an atom $\beta_{i}$ of $\phi\left(\vec{x}, \overrightarrow{y^{\prime}}\right)$ that $\Sigma$-covers $\alpha_{i}$, where $\operatorname{con}=\operatorname{con}_{\mathcal{M}} \cup \operatorname{con}_{q_{\mathcal{S}}}$. Due to Lemma 8.4, this implies that $\left\{\vec{t} \mid \exists \overrightarrow{y^{\prime}} \cdot \phi^{\prime}\left(\vec{x}, \overrightarrow{y^{\prime}}\right)\right\}$ is a sound $\mathcal{S}$-to- $\mathcal{O} \Sigma$-rewriting of a query $q \in$ HeadCompletion $\left(q_{\mathcal{S}}\right.$, con). Since for each query $q \in$ HeadCompletion $\left(q_{\mathcal{S}}\right.$, con $)$ we trivially have that $q \sqsubseteq q_{\mathcal{S}}$, we derive that $\left\{\vec{t} \mid \exists \overrightarrow{y^{\prime}} \cdot \phi^{\prime}\left(\vec{x}, \overrightarrow{y^{\prime}}\right)\right\}$ is a sound $\mathcal{S}$-to- $\mathcal{O} \sum$-rewriting of $q_{\mathcal{S}}$ as well. Furthermore, since the above condition is true for each disjunct $\left\{\vec{t} \mid \exists \overrightarrow{y^{\prime}} \cdot \phi^{\prime}\left(\vec{x}, \overrightarrow{y^{\prime}}\right)\right\}$ of $q_{\mathcal{O}}$, it follows that the computed $q_{\mathcal{O}}$ is a sound $\mathcal{S}$-to- $\mathcal{O} \Sigma$-rewriting of $q_{\mathcal{S}}$. We now show that $q_{\mathcal{O}}$ is actually the unique (up to equivalence w.r.t. $\Sigma$ ) UCQ-maximally sound $\mathcal{S}$-to- $\mathcal{O} \Sigma$-rewriting of $q_{\mathcal{S}}$, that is, each UCQ $q_{\mathcal{O}}^{\prime}$ that is a sound $\mathcal{S}$-to- $\mathcal{O} \Sigma$-rewriting of $q_{\mathcal{S}}$ is such that $\operatorname{cert}_{q_{\mathcal{O}}^{\prime}, \Sigma} \sqsubseteq \operatorname{cert}_{q_{\mathcal{O}}, \Sigma}$ (cf. Definition 3.6). We do this by way of contradiction.

Let $q_{\mathcal{O}}^{\prime}$ be a UCQ such that $\operatorname{cert}_{q_{\mathcal{O}}^{\prime}, \Sigma} \nsubseteq \operatorname{cert}_{q_{\mathcal{O}}, \Sigma}$, that is, there exists an $\mathcal{S}$ database $D$ consistent with $\Sigma$ such that $\operatorname{cert}_{q_{\mathcal{O}}^{\prime}, \Sigma}^{D} \not \operatorname{cert}_{q_{\mathcal{O}}, \Sigma}^{D}$. It follows that there is a tuple of constants $\vec{c}=\left(c_{1}, \ldots, c_{n}\right)$ such that $\vec{c} \in \operatorname{cert}_{q_{\mathcal{O}}^{\prime}, \Sigma}^{D}$ but, at the same time, $\vec{c} \notin \operatorname{cert}_{q_{\mathcal{O}}, \Sigma}^{D}$. If $\vec{c} \notin q_{\mathcal{S}}^{D}$, then $q_{\mathcal{O}}^{\prime}$ is trivially not a sound $\mathcal{S}$-to- $\mathcal{O} \Sigma$-rewriting of $q_{\mathcal{S}}$, and we are done. Therefore, we assume that $\vec{c} \in q_{\mathcal{S}}^{D}$. Specifically, let $H$ the set of all homomorphisms $h$ from $q_{\mathcal{S}}$ to $D$ with $h\left(\overrightarrow{t^{\prime}}\right)=\vec{c}$ (where $\overrightarrow{t^{\prime}}$ is the target list of $q_{\mathcal{S}}$ ), and let $\Gamma$ be the set of all facts in $D$ that partecipate in some homomorphism $h \in H$, i.e:

$$
\Gamma=\bigcup_{h \in H} h\left(q_{\mathcal{S}}\right)
$$

Consider now the $\mathcal{S}$-database $\Delta=D \backslash \Gamma$. Obviously, since $\Delta \subseteq D$, and since the left-hand side of mapping assertions are CQs, we have that $\mathcal{C}_{\mathcal{O}}^{\mathcal{M}}(\bar{\Delta}) \subseteq \mathcal{C}_{\mathcal{O}}^{\mathcal{M}(D)}$. In 
particular, let $\Lambda=\left\{\beta_{1}, \ldots, \beta_{k}\right\}$ be the set composed of all the facts in $\mathcal{C}_{\mathcal{O}}^{\mathcal{M}(D)}$ that are not in $\mathcal{C}_{\mathcal{O}}^{\mathcal{M}(\Delta)}$, i.e., $\Lambda=\mathcal{C}_{\mathcal{O}}^{\mathcal{M}(D)} \backslash \mathcal{C}_{\mathcal{O}}^{\mathcal{M}(\Delta)}$ (since $\mathcal{M}$ is a pure GAV mapping and $\mathcal{O}$ is a $D L$-Lite $e_{\text {RDFS }}$ ontology, clearly, both $\mathcal{C}_{\mathcal{O}}^{\mathcal{M}(D)}$ and $\mathcal{C}_{\mathcal{O}}^{\mathcal{M}(\Delta)}$ do not introduce variables). We now exhibit an $\mathcal{S}$-database $D^{\prime}$ for which $(i) \vec{c} \notin q_{\mathcal{S}}^{D^{\prime}}$, and $\mathcal{C}_{\mathcal{O}}^{\mathcal{M}(D)} \subseteq \mathcal{C}_{\mathcal{O}}^{\mathcal{M}\left(D^{\prime}\right)}$. To this aim, we exploit the following things:

- Let $q=\{\vec{t} \mid \exists \vec{y} . \phi(\vec{x}, \vec{y})\}=\left\{\vec{t} \mid \exists \vec{y} . \alpha_{1} \wedge \ldots \wedge \alpha_{\eta\left(q_{\mathcal{S}}\right)}\right\} \in$ HeadCompletion $\left(q_{\mathcal{S}}\right.$, con $)$ be the most specific query over $\mathcal{S}$ for which $\vec{c}=\left(c_{1}, \ldots, c_{n}\right) \in q^{D}$ still holds, i.e., the CQ whose target list $\vec{t}=\left(t_{1}, \ldots, t_{n}\right)$ is such that $(i)$ for each $i \in[1, n]$, if $c_{i}$ is a constant occurring either in $\operatorname{con}_{q_{\mathcal{S}}}$ or in $\operatorname{con}_{\mathcal{M}}$, then $t_{i}=c_{i}$ (otherwise term $t_{i}$ is a distinguished variable), and (ii) for each pair of numbers $i, j \in[1, n]$, $c_{i}=c_{j}$ if and only if $t_{i}=t_{j}$.

- Consider the target list $\vec{t}=\left(t_{1}, \ldots, t_{n}\right)$ of $q$ and the tuple of constants $\vec{c}=$ $\left(c_{1}, \ldots, c_{n}\right)$. Let $\Lambda^{\prime}=\left\{\beta_{1}^{\prime}, \ldots, \beta_{k}^{\prime}\right\}$ be the set of atoms over $\mathcal{O}$ obtained from the set of facts $\Lambda=\left\{\beta_{1}, \ldots, \beta_{k}\right\}$ by $(i)$ replacing everywhere the constant $c_{i} \in \vec{c}$ with the term $t_{i} \in \vec{t}$ (either a distinguished variable, or the constant $c_{i}$ itself), for each $i \in[1, n]$, and (ii) replacing everywhere each constant $c$ occurring neither in $q_{\mathcal{S}}$ nor in $\mathcal{M}$ with a fresh existential variable $y_{c}$.

In particular, there are two possible cases: either for each $i \in\left[1, \eta\left(q_{\mathcal{S}}\right)\right]$ there is an atom $\beta_{i}^{\prime} \in \Lambda^{\prime}$ such that $\beta_{i}^{\prime} \Sigma$-covers $\alpha_{i}$, or not.

In the former case, since for each atom $\alpha_{i}$ of $q$ there is an atom $\beta_{i}^{\prime} \in \Lambda^{\prime}$ such that $\beta_{i}^{\prime} \Sigma$-covers $\alpha_{i}$, by construction of the algorithm, it can be readily seen that the CQ $q^{\prime}=\left\{\vec{t} \mid \exists \overrightarrow{y^{\prime}} \cdot \beta_{1}^{\prime} \wedge \ldots \wedge \beta_{\eta\left(q_{\mathcal{S}}\right)}^{\prime}\right\}$ over $\mathcal{O}$ is a disjunct of $q_{\mathcal{O}}$. Furthermore, it is clear that $\vec{c} \in q^{\prime \Lambda}$. Two considerations are now in order: $(i)$ due to the facts that $\vec{c} \in q^{\prime \Lambda}$ and $\Lambda \subseteq \mathcal{C}_{\mathcal{O}}^{\mathcal{M}(D)}$, and since $q^{\prime}$ is a CQ, we derive that $\vec{c} \in q^{\mathcal{C}_{\mathcal{O}}^{\mathcal{M}(D)}}$ as well, and (ii) since $\vec{c} \in q^{\prime \mathcal{C}_{\mathcal{O}}^{\mathcal{M}(D)}}$ and since $q^{\prime}$ is a disjunct of $q_{\mathcal{O}}$, we have $\vec{c} \in q_{\mathcal{O}} \mathcal{C}^{\mathcal{M}(D)}$. Thus, as already observed, since in this setting for OBDM specifications $\operatorname{cert}_{q_{\mathcal{O}}, \Sigma}^{D}=q_{\mathcal{O}}^{\mathcal{C}_{\mathcal{O}}^{\mathcal{M}(D)}}$ for each UCQ $q_{\mathcal{O}}$ and $\mathcal{S}$-database $D$, we derive that $\vec{c} \in \operatorname{cert}_{q_{\mathcal{O}, \Sigma}}^{D}$, which is a contradiction on the initial assumption that $\vec{c} \notin \operatorname{cert}_{q_{\mathcal{O}}, \Sigma}^{D}$. It follows that the former case just considered is not possible because it leads to a contradiction. Therefore, we consider only the latter case.

Consider the latter case, that is, there exists at least an atom $\alpha_{i}$ of $q$ for which no atom $\beta^{\prime} \in \Lambda^{\prime}$ is such that $\beta^{\prime} \Sigma$-covers $\alpha_{i}$. It is not hard to ascertain that this implies that there is at least an atom $\alpha_{i}^{\prime}$ of $q_{\mathcal{S}}(\vec{c})$ for which no atom $\beta \in \Lambda$ is such that $\beta \Sigma$-covers $\alpha_{i}^{\prime}$, where we recall that $q_{\mathcal{S}}(\vec{c})=\left\{() \mid \exists \vec{y} \cdot \alpha_{1}^{\prime} \wedge \ldots \wedge \alpha_{\eta\left(q_{\mathcal{S}}\right)}^{\prime}\right\}$ is the boolean CQ obtained from $q_{\mathcal{S}}$ by replacing each occurrence of term $t_{i}^{\prime}$ in the body of $q_{\mathcal{S}}$ with constant $c_{i}$, for each $i \in[1, n]$ (where $\overrightarrow{t^{\prime}}=\left(t_{1}, \ldots, t_{n}\right)$ is the target list of $q_{\mathcal{S}}$ ). But then, consider the set of facts $\Omega$ obtained by unfolding each fact $\beta \in \Lambda$ with a disjunct of $\rho(\beta, \Sigma)$ such that there is no atom over $\mathcal{S}$ that instantiates $\alpha^{\prime}$ (clearly, since by assumption $\beta$ does not $\Sigma$-cover $\alpha^{\prime}$, following Definition 8.2 , at least one of such disjunct must exists). As a result, we trivially have that $\Omega \not \models q_{\mathcal{S}}(\vec{c})$, which implies that $\vec{c} \notin q_{\mathcal{S}}^{\Omega}$.

We now prove that the $\mathcal{S}$-database we are seeking is $D^{\prime}=\Delta \cup \Omega$. Observe that: $(i) q_{\mathcal{S}}$ is a CQJFE, and therefore it does not have existential variables in join 
occurring in its body, $(i i)$ from $(i)$ and by construction of $\Delta$, we know that there are no facts that may partecipate in a possible homomorphism from $q_{\mathcal{S}}$ to $D$ with $h\left(\overrightarrow{t^{\prime}}\right)=\vec{c}$ (where $\overrightarrow{t^{\prime}}$ is the target list of $q_{\mathcal{S}}$ ) in $\Delta$, (iii) $\vec{c} \notin q_{\mathcal{S}}^{\Omega}$. Putting together the above three observations, one can easily verify that they imply that $\vec{c} \notin q_{\mathcal{S}}^{D^{\prime}}$, where $D^{\prime}=\Delta \cup \Omega$. Furthermore, since $\mathcal{M}$ is a pure GAV mapping and $\mathcal{O}$ is a $D L$-Lite $e_{\text {RDFS }}$ ontology, and thus contains no assertions with $\exists R$ in the right-hand side for a basic role $R$, and since $\Omega$ is obtained by unfolding each atom $\beta \in \Lambda$ with a disjunct of $\rho(\beta, \Sigma)$, it is easy to verify that $\mathcal{C}_{\mathcal{O}}^{\mathcal{M}(\Omega)}$ is such that $\mathcal{C}_{\mathcal{O}}^{\mathcal{M}(D)} \subseteq \mathcal{C}_{\mathcal{O}}^{\mathcal{M}(\Omega)}$, which obviously implies that $\mathcal{C}_{\mathcal{O}}^{\mathcal{M}(D)} \subseteq \mathcal{C}_{\mathcal{O}}^{\mathcal{M}\left(D^{\prime}\right)}$ because $\Omega \subseteq D^{\prime}$ and the left-hand side of mapping assertions are CQs. Notice that $\vec{c} \in \operatorname{cert}_{q_{\mathcal{O}}^{\prime}, \Sigma}^{D}$ holds by assumption, and therefore $\vec{c} \in q_{\mathcal{O}}^{\prime}{ }^{\mathcal{C}_{\mathcal{O}}^{\mathcal{M}(D)}}$. Furthermore, since $\mathcal{C}_{\mathcal{O}}^{\mathcal{M}(D)} \subseteq \mathcal{C}_{\mathcal{O}}^{\mathcal{M}\left(D^{\prime}\right)}$ and $q_{\mathcal{O}}$ is a UCQ, we trivially derive that $\vec{c} \in q_{\mathcal{O}}^{\prime} \mathcal{C}^{\mathcal{M}\left(D^{\prime}\right)}$ as well, which, in turn, implies that $\vec{c} \in \operatorname{cert} q_{q_{\mathcal{O}}^{\prime}, \Sigma}^{D^{\prime}}$.

To complete the proof, consider the $\mathcal{S}$-database $D^{\prime}$. We have that, on the one hand, $\vec{c} \notin q_{\mathcal{S}}^{D^{\prime}}$, and, on the other hand, $\vec{c} \in \operatorname{cert}_{q_{\mathcal{O}}^{\prime}, \Sigma}^{D^{\prime}}$. It follows that $q_{\mathcal{O}}^{\prime}$ is not a sound $\mathcal{S}$-to- $\mathcal{O} \Sigma$-rewriting of $q_{\mathcal{S}}$, as required.

As a specialisation of Lemma 8.3 in the restricted scenario for CQJFEs, we have the following result which straightforward follows from the above theorem.

Corollary 8.2. Let $\Sigma=\langle\mathcal{O}, \mathcal{S}, \mathcal{M}\rangle$ be an $O B D M$ specification, and let $q_{\mathcal{S}}$ be a $C Q J F E$ over $\mathcal{S}$. If a $C Q q_{\mathcal{O}}$ over $\mathcal{O}$ is a sound $\mathcal{S}$-to- $\mathcal{O} \Sigma$-rewriting of $q_{\mathcal{S}}$, then there exists a $C Q q_{\mathcal{O}}^{\prime}$ over $\mathcal{O}$ with same target list of $q_{\mathcal{O}}$ such that (i) the body of $q_{\mathcal{O}}^{\prime}$ is the conjunction of at most $\eta\left(q_{\mathcal{S}}\right)$ atoms occurring in the body of $q_{\mathcal{O}}$ (and therefore, cert $_{q_{\mathcal{O}}, \Sigma} \sqsubseteq \operatorname{cert}_{q_{\mathcal{O}}^{\prime}, \Sigma}$ ), and (ii) $q_{\mathcal{O}}^{\prime}$ is a sound $\mathcal{S}$-to-O $\mathcal{O}$-rewriting of $q_{\mathcal{S}}$ as well.

Regarding the cost of the algorithm, we observe that the overall running time is exponential in the size of the input. Indeed, the computation of the head completion of $q_{\mathcal{S}}$ with respect to con $=\operatorname{con}_{\mathcal{M}} \cup \operatorname{con}_{q_{\mathcal{S}}}$ is, in general, exponential with respect to the size of the target list of $q_{\mathcal{S}}$, even when con $=\emptyset$. Furthermore, for each possible $q \in$ HeadCompletion $\left(q_{\mathcal{S}}\right.$, con $)$, the generated disjuncts added to the final UCQ $q_{\mathcal{O}}$ are, potentially, exponentially many with respect to $\eta\left(q_{\mathcal{S}}\right)$.

The next proposition shows that there exists OBDM specifications $\Sigma$ and CQJFEs $q_{\mathcal{S}}$ for which the unique (up to equivalence w.r.t. $\Sigma$ ) UCQ-maximally sound $\mathcal{S}$-to- $\mathcal{O}$ $\Sigma$-rewriting of $q_{\mathcal{S}}$ necessarily consists of an exponential number of disjuncts with respect to $\eta\left(q_{S}\right)$ for exponentially many source queries with respect to the size of the target list of $q_{\mathcal{S}}$.

Proposition 8.3. In the restricted scenario for CQJFEs, there are OBDM specifications $\Sigma$ and CQJFEs $q_{\mathcal{S}}$ for which the unique (up to equivalence w.r.t. $\Sigma$ )

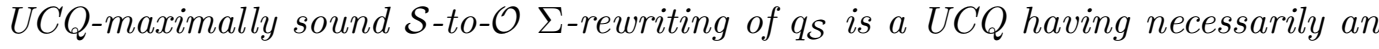
exponential number of disjuncts with respect to $\eta\left(q_{\mathcal{S}}\right)$, for exponentially many source queries with respect to the size of the target list of $q_{\mathcal{S}}$.

Proof. We provide here a small example showing the main reason of why the unique (up to equivalence w.r.t. the OBDM specification $\Sigma=\langle\mathcal{O}, \mathcal{S}, \mathcal{M}\rangle$ ) UCQ-maximally sound $\mathcal{S}$-to- $\mathcal{O} \Sigma$-rewriting of a CQJFE $q_{\mathcal{S}}$ may contain an exponential number of 
disjuncts with respect to $\eta\left(q_{\mathcal{S}}\right)$, for exponentially many source queries with respect to the size of the target list of $q_{\mathcal{S}}$.

Let $\Sigma=\langle\mathcal{O}, \mathcal{S}, \mathcal{M}\rangle$ be the following OBDM specification:

- $\mathcal{O}=\emptyset$

- $\mathcal{S}=\left\{s_{1}, s_{1,1}, s_{1,2}, s_{1,1}^{\prime}, s_{1,2}^{\prime}, s_{2}, s_{2,1}, s_{2,2}, s_{2,1}^{\prime}, s_{2,2}^{\prime}, s_{3}, s_{3,1}, s_{3,2}, s_{3,1}^{\prime}, s_{3,2}^{\prime}\right\}$

- $\mathcal{M}=\left\{m_{1}, m_{2}, m_{3}, m_{4}, m_{5}, m_{6}, m_{7}, m_{8}, m_{9}, m_{10}, m_{11}, m_{12}\right\}$, where:

$$
\begin{aligned}
& m_{1}: s_{1,1}\left(x_{1}, x_{2}\right) \wedge s_{1}\left(x_{1}, x_{2}\right) \quad \rightarrow \quad P_{1,1}\left(x_{1}, x_{2}\right), \\
& m_{2}: s_{1,2}\left(x_{1}, x_{2}\right) \wedge s_{1}\left(x_{1}, x_{2}\right) \quad \rightarrow \quad P_{1,2}\left(x_{1}, x_{2}\right), \\
& m_{3}: \quad s_{1,1}^{\prime}(x) \wedge s_{1}(x, x) \quad \rightarrow \quad A_{1,1}(x), \\
& m_{4}: \quad s_{1,2}^{\prime}(x) \wedge s_{1}(x, x) \quad \rightarrow \quad A_{1,2}(x), \\
& m_{5}: \quad s_{2,1}\left(x_{1}, x_{2}\right) \wedge s_{2}\left(x_{1}, x_{2}\right) \quad \rightarrow \quad P_{2,1}\left(x_{1}, x_{2}\right), \\
& m_{6}: s_{2,2}\left(x_{1}, x_{2}\right) \wedge s_{2}\left(x_{1}, x_{2}\right) \quad \rightarrow \quad P_{2,2}\left(x_{1}, x_{2}\right), \\
& m_{7}: \quad s_{2,1}^{\prime}(x) \wedge s_{2}(x, x) \rightarrow A_{2,1}(x), \\
& m_{8}: \quad s_{2,2}^{\prime}(x) \wedge s_{2}(x, x) \quad \rightarrow \quad A_{2,2}(x), \\
& m_{9}: \quad s_{3,1}\left(x_{1}, x_{2}\right) \wedge s_{3}\left(x_{1}, x_{2}\right) \quad \rightarrow \quad P_{3,1}\left(x_{1}, x_{2}\right), \\
& m_{10}: s_{3,2}\left(x_{1}, x_{2}\right) \wedge s_{3}\left(x_{1}, x_{2}\right) \quad \rightarrow \quad P_{3,2}\left(x_{1}, x_{2}\right) \text {, } \\
& m_{11}: \quad s_{3,1}^{\prime}(x) \wedge s_{3}(x, x) \quad \rightarrow \quad A_{3,1}(x), \\
& m_{12}: \quad s_{3,2}^{\prime}(x) \wedge s_{3}(x, x) \quad \rightarrow \quad A_{3,2}(x) .
\end{aligned}
$$

Let $q_{\mathcal{S}}$ be the following CQJFE over $\mathcal{S}: q_{\mathcal{S}}=\left\{\left(x_{1}, x_{2}, x_{3}, x_{4}, x_{5}, x_{6}\right)\right.$ $\left.s_{1}\left(x_{1}, x_{2}\right) \wedge s_{2}\left(x_{3}, x_{4}\right) \wedge s_{3}\left(x_{5}, x_{6}\right)\right\}$. Consider the following CQs occurring in HeadCompletion $\left(q_{\mathcal{S}},\{\}\right)$ :

$$
\begin{aligned}
& \text { 1. } q_{\mathcal{S}}^{1}=q_{\mathcal{S}}=\left\{\left(x_{1}, x_{2}, x_{3}, x_{4}, x_{5}, x_{6}\right) \mid s_{1}\left(x_{1}, x_{2}\right) \wedge s_{2}\left(x_{3}, x_{4}\right) \wedge s_{3}\left(x_{5}, x_{6}\right)\right\} ; \\
& \text { 2. } q_{\mathcal{S}}^{2}=\left\{\left(x_{1}, x_{1}, x_{3}, x_{4}, x_{5}, x_{6}\right) \mid s_{1}\left(x_{1}, x_{1}\right) \wedge s_{2}\left(x_{3}, x_{4}\right) \wedge s_{3}\left(x_{5}, x_{6}\right)\right\} ; \\
& \text { 3. } q_{\mathcal{S}}^{3}=\left\{\left(x_{1}, x_{2}, x_{3}, x_{3}, x_{5}, x_{6}\right) \mid s_{1}\left(x_{1}, x_{2}\right) \wedge s_{2}\left(x_{3}, x_{3}\right) \wedge s_{3}\left(x_{5}, x_{6}\right)\right\} ; \\
& \text { 4. } q_{\mathcal{S}}^{4}=\left\{\left(x_{1}, x_{2}, x_{3}, x_{4}, x_{5}, x_{5}\right) \mid s_{1}\left(x_{1}, x_{2}\right) \wedge s_{2}\left(x_{3}, x_{4}\right) \wedge s_{3}\left(x_{5}, x_{5}\right)\right\} ; \\
& \text { 5. } q_{\mathcal{S}}^{5}=\left\{\left(x_{1}, x_{1}, x_{3}, x_{3}, x_{5}, x_{6}\right) \mid s_{1}\left(x_{1}, x_{1}\right) \wedge s_{2}\left(x_{3}, x_{3}\right) \wedge s_{3}\left(x_{5}, x_{6}\right)\right\} ; \\
& \text { 6. } q_{\mathcal{S}}^{6}=\left\{\left(x_{1}, x_{1}, x_{3}, x_{4}, x_{5}, x_{5}\right) \mid s_{1}\left(x_{1}, x_{1}\right) \wedge s_{2}\left(x_{3}, x_{4}\right) \wedge s_{3}\left(x_{5}, x_{5}\right)\right\} ; \\
& \text { 7. } q_{\mathcal{S}}^{7}=\left\{\left(x_{1}, x_{2}, x_{3}, x_{3}, x_{5}, x_{5}\right) \mid s_{1}\left(x_{1}, x_{2}\right) \wedge s_{2}\left(x_{3}, x_{3}\right) \wedge s_{3}\left(x_{5}, x_{5}\right)\right\} ; \\
& \text { 8. } q_{\mathcal{S}}^{8}=\left\{\left(x_{1}, x_{1}, x_{3}, x_{3}, x_{5}, x_{5}\right) \mid s_{1}\left(x_{1}, x_{1}\right) \wedge s_{2}\left(x_{3}, x_{3}\right) \wedge s_{3}\left(x_{5}, x_{5}\right)\right\}
\end{aligned}
$$

One can verify that for each of the CQs illustrated above there are at least eight CQs over $\mathcal{O}$ that must necessarily appear in the unique (up to equivalence w.r.t. $\Sigma$ ) UCQ-maximally sound $\mathcal{S}$-to- $\mathcal{O} \Sigma$-rewriting of $q_{\mathcal{S}}$. For instance, consider the query $q_{\mathcal{S}}^{6}$ in case 6. We have that $B_{1}=\left\{P_{1,1}\left(x_{1}, x_{1}\right), P_{1,2}\left(x_{1}, x_{1}\right), A_{1,1}\left(x_{1}\right), A_{1,2}\left(x_{1}\right)\right\}, B_{2}=$ $\left\{P_{2,1}\left(x_{3}, x_{4}\right), P_{2,2}\left(x_{3}, x_{4}\right)\right\}$, and $B_{3}=\left\{P_{3,1}\left(x_{5}, x_{5}\right), P_{3,2}\left(x_{5}, x_{5}\right), A_{3,1}\left(x_{5}\right), A_{3,2}\left(x_{5}\right)\right\}$ are the set of all the atoms that $\Sigma$-cover $s_{1}\left(x_{1}, x_{1}\right), s_{2}\left(x_{1}, x_{2}\right)$, and $s_{3}\left(x_{5}, x_{5}\right)$, respectively. In particular, if we consider the subsets $B_{1}^{\prime}=\left\{A_{1,1}\left(x_{1}\right), A_{1,2}\left(x_{1}\right)\right\}$ and $B_{3}^{\prime}=\left\{A_{3,1}\left(x_{1}\right), A_{3,2}\left(x_{5}\right)\right\}$ of $B_{1}$ and $B_{3}$, respectively, then it is easy to verify that for each possible combination of atoms $\left(\beta_{1}, \beta_{2}, \beta_{3}\right)$ occurring in the Cartesian Product 
$B_{1}^{\prime} \times B_{2} \times B_{3}^{\prime}$, we have that the CQ $\left\{\left(x_{1}, x_{1}, x_{3}, x_{4}, x_{5}, x_{5}\right) \mid \exists \overrightarrow{y^{\prime}} \cdot \beta_{1} \wedge \beta_{2} \wedge \beta_{3}\right\}$ is necessarily a disjunct of the unique (up to equivalence w.r.t. $\Sigma$ ) UCQ-maximally sound $\mathcal{S}$-to- $\mathcal{O} \Sigma$-rewriting of $q_{\mathcal{S}}$, and, moreover, each of these CQs will not be produced when considering any other query in HeadCompletion $\left(q_{\mathcal{S}},\{\}\right)$.

By generalising the above construction, one can see that it is always possible to compose OBDM specifications $\Sigma=\langle\mathcal{O}, \mathcal{S}, \mathcal{M}\rangle$ and CQJFEs $q_{\mathcal{S}}$ for which the number of source queries to consider when computing the UCQ corresponding to the unique (up to equivalence w.r.t. $\Sigma$ ) UCQ-maximally sound $\mathcal{S}$-to- $\mathcal{O} \Sigma$-rewriting of $q_{\mathcal{S}}$ is equal to $2^{\frac{\eta\left(q_{\mathcal{S}}\right)}{2}}$ (and therefore, an exponential number of source queries with respect to the size of the target list of $q_{\mathcal{S}}$ ). Furthermore, for each of these source queries, the number of disjuncts occurring in the unique (up to equivalence w.r.t. $\Sigma$ ) UCQ-maximally sound $\mathcal{S}$-to- $\mathcal{O} \Sigma$-rewriting of $q_{\mathcal{S}}$ is at least $2^{\eta\left(q_{\mathcal{S}}\right)}$ (and therefore, an exponential number of disjuncts with respect to $\left.\eta\left(q_{\mathcal{S}}\right)\right)$.

\subsection{View-based Query Processing in the presence of Disjunctive Views}

In Subsection 3.3.3, we have figured out the relationship between the notion of sourceto-ontology rewriting and the view-based query processing approach. Specifically, for OBDM specifications $\Sigma=\langle\mathcal{O}, \mathcal{S}, \mathcal{M}\rangle$ with $\mathcal{M}$ being a pure GAV mapping and $\mathcal{O}=\emptyset$, the problem of computing a perfect (respectively, UCQ-maximally sound) $\mathcal{S}$-to- $\mathcal{O} \Sigma$-rewriting of a UCQ $q_{\mathcal{S}}$ is equivalent to the problem of computing an exact (respectively, UCQ-maximally sound) rewriting of $q_{\mathcal{S}}$ with respect to $\mathcal{V}_{\mathcal{M}}$, where $\mathcal{V}_{\mathcal{M}}$ denotes the set of UCQ view definitions associated to mapping $\mathcal{M}$ (cf. Theorem 3.2 and Corollary 3.1).

We are now ready to focus on the problem of computing UCQ-maximally sound rewritings of UCQs $q_{\mathcal{S}}$ with respect to sets of UCQ view definitions $\mathcal{V}$. Using results proven so far, in this section we delineate the precise dividing line between the existence and the non-existence cases along the dimension of join existential variables occurring in the bodies of the various disjuncts of $q_{\mathcal{S}}$.

As already sketched in Example 3.10 and formally proven in Duschka and Genesereth, 1998; Afrati and Chirkova, 2019, there are pairs of sets of UCQ view definitions $\mathcal{V}$ and CQs $q_{\mathcal{S}}$ for which no UCQ-maximally sound rewriting of $q_{\mathcal{S}}$ with respect to $\mathcal{V}$ exists. However, each $\mathrm{CQ} q_{\mathcal{S}}$ occurring in these pairs used to prove such a negative result has more than one join existential variable occurring in its body. We can strengthen this negative result. Indeed, by combining the proof of point 1 of Theorem 6.2 and Corollary 3.1 , we immediately obtain the following corollary.

Corollary 8.3. There exists a set of $U C Q$ view definitions $\mathcal{V}$ over a schema $\mathcal{S}$ and a boolean $C Q q_{\mathcal{S}}$ over $\mathcal{S}$ with only one variable occurring in its body for which no $U C Q$-maximally sound rewriting of $q_{\mathcal{S}}$ with respect to $\mathcal{V}$ exists. Furthermore, both $\mathcal{V}$ and $\mathcal{S}$ use only predicates with arity at most two, and all view definitions in $\mathcal{V}$ are single CQs except one view definition which is the union of only two CQs.

On the contrary, using results presented in this chapter, we are able to show that having no join existential variables occurring in the body of queries $q_{\mathcal{S}}$ is a sufficient condition that guarantees the existence of UCQ-maximally sound rewritings of 
UCQs $q_{\mathcal{S}}$ with respect to set of UCQ view definitions $\mathcal{V}$, i.e., UCQ-maximally sound rewritings of UCQJFEs $q_{\mathcal{S}}$ with respect to sets UCQ view definitions $\mathcal{V}$ always exist.

Towards this goal, we consider OBDM specifications $\Sigma=\langle\mathcal{O}, \mathcal{S}, \mathcal{M}\rangle$ where $\mathcal{M}$ is a pure GAV mapping and $\mathcal{O}=\emptyset$ is not a usual ontology, but it is simply a schema (without assertions) whose predicates have arity possibly greater than 2 . It is straightforward to argue that Algorithm MaximallySoundUCQJFEs (respectively, Algorithm MaximallySoundCQJFEs can be used for computing UCQ-maximally sound $\mathcal{S}$-to- $\mathcal{O} \Sigma$-rewritings of UCQJFEs (respectively, CQJFEs) $q_{\mathcal{S}}$ also for this kind of OBDM specifications $\Sigma=\langle\mathcal{O}, \mathcal{S}, \mathcal{M}\rangle$.

Let $\mathcal{V}=\left\{V_{1}, \ldots, V_{n}\right\}$ be a set of UCQ view definitions over a schema $\mathcal{S}$. We denote by $\Sigma_{\mathcal{V}}=\left\langle\mathcal{O}_{\mathcal{V}}, \mathcal{S}, \mathcal{M}_{\mathcal{V}}\right\rangle$ the OBDM specification where: $(i)$ for each $i \in[1, n]$ the schema $\mathcal{O}_{\mathcal{V}}$ comprises a predicate $V_{i}$ of the same arity of the UCQ associated to symbol $V_{i}$ in the view definitions $\mathcal{V} ;(i i) \mathcal{M}_{\mathcal{V}}$ is the pure GAV mapping relating schema $\mathcal{S}$ to schema $\mathcal{O}_{\mathcal{V}}$ obtained by including, for each $i \in[1, n]$ and for each disjunct $\{\vec{x} \mid \exists \vec{y} . \phi(\vec{x}, \vec{y})\}$ occurring in the UCQ associated to the symbol $V_{i}$, the mapping assertion $\exists \vec{y} \cdot \phi(\vec{x}, \vec{y}) \rightarrow V_{i}(\vec{x})$.

Example 8.9. Let $\mathcal{V}=\left\{V_{1}, V_{2}\right\}$ be the set of UCQ view definitions over schema $\mathcal{S}=\left\{s_{1}, s_{2}, s_{3}, s_{4}\right\}$, where:

- $V_{1}=\left\{\left(x_{1}, x_{2}, x_{3}\right) \mid s_{3}\left(x_{1}, x_{2}, x_{3}\right)\right\} \quad \cup\left\{\left(x_{1}, x_{2}, x_{3}\right) \quad \mid \exists y \cdot s_{1}\left(x_{1}, y\right) \wedge\right.$ $\left.s_{2}\left(y, x_{2}, x_{3}\right)\right\} \cup\left\{\left(x_{1}, x_{2}, x_{3}\right) \mid \exists y_{1}, y_{2} . s_{1}\left(x_{1}, y_{1}\right) \wedge s_{4}\left(x_{1}, x_{2}, x_{3}, y_{2}\right)\right\}$

- $V_{2}=\left\{\left(x_{1}, x_{2}, x_{3}, x_{4}\right) \mid s_{1}\left(x_{1}, x_{2}\right) \wedge s_{3}\left(x_{2}, x_{3}, x_{4}\right)\right\} \quad \cup\left\{\left(x_{1}, x_{2}, x_{3}, x_{4}\right) \mid\right.$ $\left.\exists y \cdot s_{2}\left(x_{1}, x_{2}, y\right) \wedge s_{4}\left(y, x_{3}, x_{3}, x_{4}\right)\right\}$

Then, the OBDM specification $\Sigma_{\mathcal{V}}=\left\langle\mathcal{O}_{\mathcal{V}}, \mathcal{S}, \mathcal{M}_{\mathcal{V}}\right\rangle$ is such that $\mathcal{O}_{\mathcal{V}}$ is a schema with a ternary predicate $V_{1}$ and a quaternary predicate $V_{2}$, and $\mathcal{M}_{\mathcal{V}}=$ $\left\{m_{1}, m_{2}, m_{3}, m_{4}, m_{5}\right\}$ is a pure GAV mapping relating $\mathcal{S}$ to $\mathcal{O}_{\mathcal{V}}$, where:

$$
\begin{array}{rrrl}
m_{1}: & s_{3}\left(x_{1}, x_{2}, x_{3}\right) & \rightarrow & V_{1}\left(x_{1}, x_{2}, x_{3}\right), \\
m_{2}: & \exists y \cdot s_{1}\left(x_{1}, y\right) \wedge s_{2}\left(y, x_{2}, x_{3}\right) & \rightarrow & V_{1}\left(x_{1}, x_{2}, x_{3}\right), \\
m_{3}: & \exists y_{1}, y_{2} . s_{1}\left(x_{1}, y_{1}\right) \wedge s_{4}\left(x_{1}, x_{2}, x_{3}, y_{2}\right) & \rightarrow & V_{1}\left(x_{1}, x_{2}, x_{3}\right), \\
m_{4}: & s_{1}\left(x_{1}, x_{2}\right) \wedge s_{3}\left(x_{2}, x_{3}, x_{4}\right) & \rightarrow & V_{2}\left(x_{1}, x_{2}, x_{3}, x_{4}\right), \\
m_{5}: & \exists y \cdot s_{2}\left(x_{1}, x_{2}, y\right) \wedge s_{4}\left(y, x_{3}, x_{3}, x_{4}\right) & \rightarrow & V_{2}\left(x_{1}, x_{2}, x_{3}, x_{4}\right) .
\end{array}
$$

We now provide the following theorem, which can be seen as the dual of Theorem 3.2 and can be shown using exactly the same arguments used in that proof.

Theorem 8.5. Let $\mathcal{V}$ be a set of $U C Q$ view definitions over a schema $\mathcal{S}$, and let $q_{\mathcal{S}}$ and $q_{\mathcal{V}}$ be two UCQs over $\mathcal{S}$ and over the view alphabet $\mathcal{V}$ (equivalently, $\mathcal{O}_{\mathcal{V}}$ ), respectively. We have that $q_{\mathcal{V}}$ is an exact (respectively, a sound) rewriting of $q_{\mathcal{S}}$ with respect to $\mathcal{V}$ if and only if $q_{\mathcal{V}}$ is a perfect (respectively, sound) $\mathcal{S}$-to- $\mathcal{O}_{\mathcal{V}} \Sigma_{\mathcal{V}}$-rewriting of $q_{\mathcal{S}}$.

Proof. As already observed in the proof of Theorem 3.2 .

1. By Levy et al., 1995, a UCQ $q \mathcal{V}$ is an exact (respectively, a sound) rewriting of a UCQ $q_{\mathcal{S}}$ with respect to a set of UCQ view definitions $\mathcal{V}$ if and only if 
$\exp _{\mathcal{V}}\left(q_{\mathcal{V}}\right) \equiv q_{\mathcal{S}}\left(\right.$ respectively, $\left.\exp _{\mathcal{V}}\left(q_{\mathcal{V}}\right) \sqsubseteq q_{\mathcal{S}}\right)$, where $\exp _{\mathcal{V}}(\cdot)$ is the function that, given a UCQ $q \mathcal{V}$ over the view alphabet, replace each atom occurring in $q \mathcal{V}$ by the definition of the views (being careful to use unique variables in place of those variables that appear in the bodies of the view but not in the heads of those), and then turning the resulting formula into an equivalent UCQ.

2. For OBDM specifications $\Sigma=\langle\mathcal{O}, \mathcal{S}, \mathcal{M}\rangle$ with $\mathcal{O}=\emptyset$ and $\mathcal{M}$ a pure GAV mapping, a UCQ $q$ is a perfect (respectively, sound) $\mathcal{S}$-to- $\mathcal{O} \Sigma$-rewriting of a $\mathrm{UCQ} q_{\mathcal{S}}$ if and only if $\operatorname{MapRef}(q, \mathcal{M}) \equiv q_{\mathcal{S}}$ (respectively, $\operatorname{MapRef}(q, \mathcal{M}) \sqsubseteq q_{\mathcal{S}}$ ), where $\operatorname{Map} \operatorname{Ref}(q, \mathcal{M})$ in this case is equivalent to unfolding the query $q$ with respect to $\mathcal{M}$ [Poggi et al., 2008, i.e., replacing each atom $\alpha$ occurring in $q$ by the logical disjunction of all the left-hand sides of mapping assertions in $\mathcal{M}$ having the predicate name $\alpha$ in the right-hand side (being careful to use unique variables in place of those variables that appear in the left-hand side of the mapping assertions but not in the right-hand side of those), and then turning the resulting formula into an equivalent UCQ. Technically speaking, in Poggi et al., 2008 the unfolding is specified for ontologies $\mathcal{O}$, and thus whose schema comprises only unary and binary predicates (i.e., atomic concepts and atomic roles, respectively). We point out that, however, it can be straightforwardly generalised in the data integration context when $\mathcal{O}$ is any schema whose predicates have also arity greater than 2, see, e.g., Lenzerini, 2002 .

3. By construction, $\exp _{\mathcal{V}}(q \mathcal{V})=\operatorname{MapRef}\left(q_{\mathcal{V}}, \mathcal{M}_{\mathcal{V}}\right)$ for any set of UCQ view definitions $\mathcal{V}$ and for any UCQ $q_{\mathcal{V}}$ over the view alphabet $\mathcal{V}$ (equivalently, $\mathcal{O}_{\mathcal{V}}$ ).

Thus, $q_{\mathcal{V}}$ is an exact (respectively, a sound) rewriting of $q_{\mathcal{S}}$ if and only if $\exp _{\mathcal{V}}\left(q_{\mathcal{V}}\right) \equiv q_{\mathcal{S}}$ (respectively, $\left.\exp _{\mathcal{V}}\left(q_{\mathcal{V}}\right) \sqsubseteq q_{\mathcal{S}}\right)$, which, since $\exp _{\mathcal{V}}\left(q_{\mathcal{V}}\right)=$ $\operatorname{MapRef}\left(q_{\mathcal{V}}, \mathcal{M}_{\mathcal{V}}\right)$, it is so if and only if $\operatorname{MapRef}\left(q_{\mathcal{V}}, \mathcal{M}_{\mathcal{V}}\right) \equiv q_{\mathcal{S}}$ (respectively, $\left.\operatorname{Map} \operatorname{Ref}\left(q_{\mathcal{V}}, \mathcal{M}_{\mathcal{V}}\right) \sqsubseteq q_{\mathcal{S}}\right)$, and therefore if and only if $q_{\mathcal{V}}$ is a perfect (respectively, sound) $\mathcal{S}$-to- $\mathcal{O}_{\mathcal{V}} \Sigma_{\mathcal{V}}$-rewriting of $q_{\mathcal{S}}$, as required.

From the sound part of the above theorem, we derive the following corollary, which can be seen as the dual of Corollary 3.1

Corollary 8.4. Let $\mathcal{V}$ be a set of $U C Q$ view definitions over a schema $\mathcal{S}$, and let $q_{\mathcal{S}}$ and $q_{\mathcal{V}}$ be two UCQs over $\mathcal{S}$ and over the view alphabet $\mathcal{V}$ (equivalently, $\mathcal{O}_{\mathcal{V}}$ ), respectively. We have that $q_{\mathcal{V}}$ is a UCQ-maximally sound rewriting of $q_{\mathcal{S}}$ with respect to $\mathcal{V}$ if and only if $q \mathcal{V}$ is the unique (up to equivalence w.r.t. $\Sigma_{\mathcal{V}}$ ) UCQ-maximally sound $\mathcal{S}$-to- $\mathcal{O}_{\mathcal{V}} \Sigma_{\mathcal{V}}$-rewriting of $q_{\mathcal{S}}$.

This, together with what illustrated in the previous sections, allow us to derive a technique to compute UCQ-maximally sound rewritings of UCQJFEs $q_{\mathcal{S}}$ with respect to sets of UCQ view definitions $\mathcal{V}$, thus proving that, for each pair composed by a set of UCQ view definitions $\mathcal{V}$ over a schema $\mathcal{S}$ and a UCQJFE $q_{\mathcal{S}}$ over $\mathcal{S}$, a UCQ-maximally sound rewriting of $q_{\mathcal{S}}$ with respect to $\mathcal{V}$ is guaranteed to exists.

In particular, given a set of UCQ view definitions $\mathcal{V}$ over a schema $\mathcal{S}$ and a UCQJFE (respectively, CQJFE) $q_{\mathcal{S}}$ over $\mathcal{S}$, we can first construct $\Sigma_{\mathcal{V}}$ and then run the MaximallySoundUCQJFEs (respectively, MaximallySoundCQJFEs) algorithm on $\Sigma_{\mathcal{V}}$ and $q_{\mathcal{S}}$. Due to Theorem 8.2 (respectively, Theorem 8.4), the UCQ returned 
by MaximallySoundUCQJFEs $\left(\Sigma_{\mathcal{V}}, q_{\mathcal{S}}\right)$ (respectively, MaximallySoundCQJFEs $\left(\Sigma_{\mathcal{V}}, q_{\mathcal{S}}\right)$ ) is the unique (up to equivalence w.r.t. $\Sigma_{\mathcal{V}}$ ) UCQ-maximally sound $\mathcal{S}$-to- $\mathcal{O}_{\mathcal{V}} \Sigma_{\mathcal{V}}$ rewriting of $q_{\mathcal{S}}$, and then, by Corollary 8.4 , it is also a UCQ-maximally sound rewriting of $q_{\mathcal{S}}$ with respect to $\mathcal{V}$.

By combining the above discussion with Theorem 3.3 , we can easily obtain the main result of this section.

Theorem 8.6. Let $\mathcal{V}$ be a set of $U C Q$ view definitions over a schema $\mathcal{S}$, and let $q_{\mathcal{S}}$ be a UCQJFE (respectively, CQJFE) over $\mathcal{S}$. Let denote by qv the UCQ over the schema $\mathcal{O}_{\mathcal{V}}$ (equivalently, view alphabet $\mathcal{V}$ ) returned by MaximallySoundUCQJFEs $\left(\Sigma_{\mathcal{V}}, q_{\mathcal{S}}\right)$ (respectively, MaximallySoundCQJFEs $\left(\Sigma_{\mathcal{V}}, q_{\mathcal{S}}\right)$ ). We have that:

- $q_{\mathcal{V}}$ is a UCQ-maximally sound rewriting of $q_{\mathcal{S}}$ with respect to $\mathcal{V}$;

- $q_{\mathcal{V}}$ is a perfect rewriting of $q_{\mathcal{S}}$ with respect to $\mathcal{V}$;

- $q_{\mathcal{V}}$ is a UCQ-exact rewriting of $q_{\mathcal{S}}$ wih respect to $\mathcal{V}$, if this latter exists.

As an interesting implication observe that, given any set of UCQ view definitions $\mathcal{V}$ over a schema $\mathcal{S}$ and any UCQJFE $q_{\mathcal{S}}$ over $\mathcal{S}$, a perfect rewriting of $q_{\mathcal{S}}$ with respect to $\mathcal{V}$ can be always expressed as a UCQ in which the body of each disjunct is the conjunction of at most bound $\left(\mathcal{M}_{\mathcal{V}}, q_{\mathcal{S}}\right)$ atoms (cf. Lemma 8.3). Furthermore, when $q_{\mathcal{S}}$ is a CQJFE, the body of each disjunct is the conjunction of at most $\eta\left(q_{\mathcal{S}}\right)$ atoms (cf. Corollary 8.2 , exactly as in the case of CQ view definitions $\mathcal{V}$ and UCQs $q_{\mathcal{S}}$ Levy et al., 1995.

We conclude with the following curious observations: the assumption that the target list of each disjunct of the various UCQ view definitions in $\mathcal{V}$ does not have repeated variables or constants is essential for the above theorem to hold. In fact, when removing this assumption, by the proof of point 5 of Theorem 6.2 we can easily construct a set of UCQ view definitions $\mathcal{V}$ and a CQJFE $q_{\mathcal{S}}$ for which no UCQ-maximally sound rewriting of $q_{\mathcal{S}}$ exists. Furthermore, when removing this assumption, there are cases where a UCQ-maximally sound rewriting of a CQJFE $q_{\mathcal{S}}$ with respect to a set of $\mathrm{CQ}$ view definitions $\mathcal{V}$ exists but it does not correspond to a perfect rewriting of $q_{\mathcal{S}}$ with respect to $\mathcal{V}$ (cf. Example 3.9). 



\section{Chapter 9}

\section{Non-Monotonic Source-to-Ontology Rewritings}

In this chapter, we investigate the notion of abstraction in the case where source-toontology rewritings can be expressed in a non-monotonic query language. One basic issue to address in this endeavour is selecting the non-monotonic query language.

Our choice in this chapter is to use EQL-Lite(UCQ) Calvanese et al., 2007a 1 . which is a language equipped with a single modal operator $\mathbf{K}$. The modal operator is used to formalise the epistemic state of the current OBDM system according to the minimal knowledge semantics (see later). Informally, the formula $\mathbf{K} \varrho$ is read as " $\varrho$ is known to hold in the OBDM system". Queries in EQL-Lite(UCQ) can use conjunction, negation, and existential quantification, and have atoms that are expressed exactly as $\mathbf{K} \varrho$, where $\varrho$ is a UCQ. With this combination of operators, it is possible to ask for those $x$ such that a given $\phi(x)$ is not known to hold, and this is crucial for characterising a set of tuples that are not certain answers to a given source query $q_{\mathcal{S}}$. The epistemic operator enables also other interesting features. For instance, we can distinguish between asking for those $x$ such that it is known that there is $y$ for which $P(x, y)$ holds (where $y$ can be unknown), and asking for those $x$ such that there is $y$ for which $P(x, y)$ is known to hold (and therefore $y$ is known).

$E Q L-L i t e(\mathrm{UCQ})$ is a particularly well-behaved fragment of $E Q L$, a variant of the well-known First-Order Modal Logic of knowledge/belief [Levesque, 1984, Reiter, 1992; Levesque and Lakemeyer, 2000. (see also Chellas, 1980). Before exploring $E Q L$-Lite(UCQ) source-to-ontology rewritings, we first recall the basis of EQL.

An epistemic interpretation for an ontology $\mathcal{O}$ is a pair $\langle E, \mathcal{I}\rangle$, where $E$ is a possibly infinite set of FOL interpretations for $\mathcal{O}$, and $\mathcal{I}=\left\langle\Delta^{\mathcal{I}},{ }^{\mathcal{I}}\right\rangle$ is an interpretation in $E$. We inductively define when an $E Q L$ sentence $\psi$ is true in an epistemic interpretation $\langle E, \mathcal{I}\rangle$, written $\langle E, \mathcal{I}\rangle \models \psi$, as follows:

$$
\begin{array}{lll}
\langle E, \mathcal{I}\rangle=A(c) & \text { iff } & \mathcal{I}=A(c), \\
\langle E, \mathcal{I}\rangle=P\left(c_{1}, c_{2}\right) & \text { iff } & \mathcal{I}=P\left(c_{1}, c_{2}\right), \\
\langle E, \mathcal{I}\rangle=\psi_{1} \wedge \psi_{2} & \text { iff } & \langle E, \mathcal{I}\rangle \models \psi_{1} \text { and }\langle E, \mathcal{I}\rangle \models \psi_{2}, \\
\langle E, \mathcal{I}\rangle=\neg \psi & \text { iff } & \langle E, \mathcal{I}\rangle \models \psi, \\
\langle E, \mathcal{I}\rangle=\exists x \cdot \psi & \text { iff } & \langle E, \mathcal{I}\rangle \models \psi_{c}^{x} \text { for some constant } c \in \Delta^{\mathcal{I}}, \\
\langle E, \mathcal{I}\rangle=\mathbf{K} \psi & \text { iff } & \left\langle E, \mathcal{I}^{\prime}\right\rangle \models \psi \text { for every } \mathcal{I}^{\prime} \in E,
\end{array}
$$

\footnotetext{
${ }^{1}$ In fact, we consider a restricted version of $E Q L-L i t e(\mathrm{UCQ})$, in which (in)equalities are disallowed.
} 
where $\psi, x$, and $\psi_{c}^{x}$ denote an arbitrary $E Q L$ sentence, a variable, and the $E Q L$ sentence obtained by replacing the variable $x$ with the constant $c$, respectively.

As in knowledge base scenarios, in OBDM, among the various epistemic interpretations, the interest is in the specific ones representing the minimal epistemic state of the OBDM system, i.e., the state in which the OBDM system has minimal knowledge. Namely: let $\Sigma=\langle\mathcal{O}, \mathcal{S}, \mathcal{M}\rangle$ be an OBDM specification and $D$ be an $\mathcal{S}$-database. Then, a $\langle\Sigma, D\rangle$-EQL-interpretation is an epistemic interpretation $\langle E, \mathcal{I}\rangle$ for which $E=\operatorname{Mod}_{D}(\Sigma)$. Finally, we say that an $E Q L$ sentence $\psi$ is EQL-logically implied by $\langle\Sigma, D\rangle$, denoted by $\langle\Sigma, D\rangle \models_{E Q L} \psi$, if for every $\langle\Sigma, D\rangle$-EQL-interpretation $\langle E, \mathcal{I}\rangle$ we have $\langle E, \mathcal{I}\rangle \models \psi_{\mathcal{I}}$, where $\psi_{\mathcal{I}}$ is obtained from $\psi$ by replacing each constant $c$ occurring in $\psi$ with the domain object $c^{\mathcal{I}} \in \Delta^{\mathcal{I}}$ (if defined, i.e., if $c \in \operatorname{dom}(D)$ ).

This led us to naturally define the certain answers of EQL queries in OBDM systems. First, we need to define $E Q L$ queries: an EQL query is a query of the form $q=\{\vec{t} \mid \psi(\vec{x})\}$, where the target list $\vec{t}$ is an $n$-tuple of terms, and the body $\psi(\vec{x})$ is an EQL formula in which the free variables are exactly the variables occurring in $\vec{t}$. For an EQL query $q=\left\{\left(t_{1}, \ldots, t_{n}\right) \mid \psi(\vec{x})\right\}$ of arity $n$ and an $n$-tuple of constants $\vec{c}=\left(c_{1}, \ldots, c_{n}\right)$, we denote by $q(\vec{c})=\{() \mid \psi(\vec{x} / \vec{c})\}$ the boolean EQL query in which the EQL sentence $\psi(\vec{x} / \vec{c})$ corresponds to $\perp$ in the case that there is some $i \in[1, n]$ for which $t_{i} \neq c_{i}$ and $t_{i}$ is a constant, otherwise $\psi(\vec{x} / \vec{c})$ is obtained from $\psi(\vec{x})$ by replacing all the occurrences of the term $t_{i}$ with the constant $c_{i}$, for each $i \in[1, n]$. Given an OBDM specification $\Sigma=\langle\mathcal{O}, \mathcal{S}, \mathcal{M}\rangle$, an $\mathcal{S}$-database $D$, and an $E Q L$ query $q_{\mathcal{O}}$ over $\mathcal{O}$ of arity $n$, the certain answers of $q_{\mathcal{O}}$ with respect to $\Sigma$ and $D$, denoted as always by $\operatorname{cert}_{q_{\mathcal{O}}, \Sigma}^{D}$, is the set $\operatorname{cert}_{q_{\mathcal{O}}, \Sigma}^{D}=\left\{\vec{c} \in \operatorname{dom}(D)^{n} \mid\langle\Sigma, D\rangle=_{E Q L} q_{\mathcal{O}}(\vec{c})\right\}$.

\subsection{Towards EQL-Lite(UCQ) Abstractions}

We start by recalling the EQL-Lite(UCQ) query language, and show how queries in such a language can be rewritten as FOL queries over the source schema to compute certain answers with respect to OBDM systems. We then show how the $E Q L-L i t e(\mathrm{UCQ})$ query language allows to obtain better abstractions of data services, compared to the usual language of UCQs.

\subsubsection{The EQL-Lite(UCQ) Query Language}

The EQL-Lite(UCQ) query language, introduced in Calvanese et al., 2007a is an epistemic query language whose epistemic atoms are formulas of the form $\mathbf{K} \varrho$, where $\varrho$ is a UCQ. Such a query language is a particularly well-behaved fragment of $E Q L$ queries. Formally, an EQL-Lite(UCQ) query over a DL ontology $\mathcal{O}$ is a query of the form $q_{\mathcal{O}}=\{\vec{t} \mid \psi(\vec{x})\}$, where the target list $\vec{t}$ is an $n$-tuple of terms, and the body $\psi(\vec{x})$ is a formula built according to the following syntax (we recall that the free variables occurring in formula $\psi(\vec{x})$ are exactly the variables occurring in $\vec{t}$ ):

$$
\psi::=\mathbf{K} \varrho|\exists x \cdot \psi| \psi_{1} \wedge \psi_{2}\left|\psi_{1} \vee \psi_{2}\right| \neg \psi
$$

where $\varrho$ is a disjunction of existentially quantified conjunction of atoms over $\mathcal{O}$ sharing the same free variables.

\footnotetext{
${ }^{2}$ In fact, we consider a restricted version of EQL-Lite(UCQ), in which (in)equalities are disallowed.
} 
Example 9.1. Let $\Sigma=\langle\mathcal{O}, \mathcal{S}, \mathcal{M}\rangle$ be the following OBDM specification:

- $\mathcal{O}=\{$ Professor $\sqsubseteq \exists$ Teaches, Teaches $\sqsubseteq$ Likes, $\exists$ WorksFor $\sqsubseteq \exists$ Likes $\}$

- $\mathcal{S}=\left\{s_{1}, s_{2}, s_{3}, s_{4}\right\}$

- $\mathcal{M}=\left\{m_{1}, m_{2}, m_{3}, m_{4}\right\}$, where:

$$
\begin{array}{rrrl}
m_{1}: & \exists y \cdot s_{1}\left(x_{1}, x_{2}, y\right) & \rightarrow \text { Teaches }\left(x_{1}, x_{2}\right), \\
m_{2}: & s_{2}(x) & \rightarrow & \text { Professor }(x), \\
m_{3}: & \exists y_{1}, y_{2} . s_{3}\left(x_{1}, x_{2}, y_{1}, y_{2}\right) & \rightarrow & \text { Likes }\left(x_{1}, x_{2}\right), \\
m_{4}: & \exists y_{1}, y_{2} \cdot s_{4}\left(x, y_{1}, y_{2}\right) & \rightarrow & \exists z \cdot \operatorname{WorksFor}(x, z) .
\end{array}
$$

Consider the following EQL-Lite(UCQ) queries over the ontology $\mathcal{O}$ :

- $q_{\mathcal{O}}^{1}=\{(x) \mid \exists y \cdot \mathbf{K}(\operatorname{Teaches}(x, y))\}$

- $q_{\mathcal{O}}^{2}=\{(x) \mid \exists y \cdot \mathbf{K}(\operatorname{Likes}(x, y))\}$

- $q_{\mathcal{O}}^{3}=\{(x) \mid \exists y \cdot \mathbf{K}(\operatorname{Likes}(x, y)) \wedge \neg \mathbf{K}(\operatorname{Teaches}(x, y))\}$.

Intuitively, $q_{\mathcal{O}}^{1}$ retrieves all the people for whom at least one subject is known they teach, $q_{\mathcal{O}}^{2}$ retrieves all the people for whom at least one subject is known they like, and finally $q_{\mathcal{O}}^{3}$ retrieves all the people for whom at least one subject is known they like and is not known they teach this subject.

Each EQL-Lite(UCQ) query $q_{\mathcal{O}}$ over $\mathcal{O}$ can be associated with a FOL query over a new schema. Formally, let $q_{\mathcal{O}}$ be an EQL-Lite(UCQ) query over $\mathcal{O}$ whose epistemic atoms are $\mathbf{K} \varrho_{1}, \ldots, \mathbf{K} \varrho_{m}$. For each $i \in[1, m]$, we denote by $q_{\mathcal{O}}^{\varrho_{i}}$ the UCQ over $\mathcal{O}$ associated to $\varrho_{i}$, i.e., the UCQ $q_{\mathcal{O}}^{\varrho_{i}}=\left\{\vec{z} \mid \exists \overrightarrow{y_{1}} \cdot \phi_{1}\left(\vec{z}, \overrightarrow{y_{1}}\right)\right\} \cup \ldots \cup\left\{\vec{z} \mid \exists \overrightarrow{y_{l}} \bullet \phi_{l}\left(\vec{z}, \overrightarrow{y_{l}}\right)\right\}$, where $\vec{z}=\left(z_{1}, \ldots, z_{a r\left(\varrho_{i}\right)}\right)$ is the tuple of all free variables occurring $\varrho_{i}$ (we recall that each free variable $z_{i} \in \vec{z}$ of $\varrho_{i}$ occurs also in the target list of $q_{\mathcal{O}}$ ), and $\varrho_{i}=\exists \overrightarrow{y_{1}} \cdot \phi_{1}\left(\vec{z}, \overrightarrow{y_{1}}\right) \vee \ldots \vee \exists \overrightarrow{y_{l}} \cdot \phi_{l}\left(\vec{z}, \overrightarrow{y_{l}}\right)$. Finally, we denote by $q_{\mathcal{O}}^{F O L}$ the FOL query over the schema $\mathcal{R}_{q_{\mathcal{O}}}=\left\{R_{\mathbf{K} \varrho_{1}}, \ldots, R_{\mathbf{K} \varrho_{m}}\right\}$ obtained from $q_{\mathcal{O}}$ in the following way: for each $i \in[1, m]$, the epistemic atom $\mathbf{K} \varrho_{i}$ is replaced with the atom $R_{\mathbf{K} \varrho_{i}}\left(z_{1}, \ldots, z_{a r\left(\varrho_{i}\right)}\right)$.

In order to reduce query answering of $E Q L-L i t e(\mathrm{UCQ})$ queries to the standard evaluation of Relational Algebra queries, and therefore of SQL queries (thus taking advantage of optimisation strategies provided by DBMSs), we have to introduce the notion of "domain independence", which is the semantical restriction on FOL queries needed to get equivalence with Relational Algebra queries Codd, 1972.

In the DL context, an FOL query $q_{\mathcal{O}}$ of arity $n$ over an ontology $\mathcal{O}$ is domain independent if $\left\{\vec{c} \in\right.$ Const $\left.^{n} \mid \mathcal{I}_{1} \models q_{\mathcal{O}}(\vec{c})\right\}=\left\{\vec{c} \in\right.$ Const $\left.^{n} \mid \mathcal{I}_{2} \models q_{\mathcal{O}}(\vec{c})\right\}$ for each pair of FOL interpretations $\mathcal{I}_{1}=\left\langle\Delta^{\mathcal{I}_{1}},{ }^{\mathcal{I}_{1}}\right\rangle$ and $\mathcal{I}_{2}=\left\langle\Delta^{\mathcal{I}_{2}},{ }^{\mathcal{I}_{2}}\right\rangle$ for $\mathcal{O}$ such that ${ }^{\mathcal{I}_{1}} \equiv \cdot \mathcal{I}_{2}$, where ${ }^{\mathcal{I}_{1}} \equiv \cdot^{\mathcal{I}_{2}}$ if $A^{\mathcal{I}_{1}}=A^{\mathcal{I}_{2}}$ (respectively, $P^{\mathcal{I}_{1}}=P^{\mathcal{I}_{2}}$ ) for each atomic concept $A$ (respectively, atomic role $P$ ) in the alphabet of $\mathcal{O}$. We say that an EQL-Lite(UCQ) query $q_{\mathcal{O}}$ over an ontology $\mathcal{O}$ is domain independent if its associated $q_{\mathcal{O}}^{F O L}$ is so.

Several syntactic sufficient conditions have been devised to guarantee domain independence of FOL queries, see, e.g., Abiteboul et al., 1995. Such syntactic conditions can be directly translated into syntactic conditions on EQL-Lite(UCQ) queries. As in relational database scenarios, where one allows only for FOL queries that 
are domain independent, in the sequel, whenever we speak about EQL-Lite(UCQ) queries, we will always mean domain independent EQL-Lite(UCQ) queries.

$E Q L-L i t e(\mathrm{UCQ})$ queries enjoy a very interesting computational property: one can decouple the reasoning needed for answering the epistemic atoms, which can be delegated to the underlying OBDM service for answering UCQs, from the reasoning needed for dealing with the other operators of the whole query. Formally, let $\Sigma=\langle\mathcal{O}, \mathcal{S}, \mathcal{M}\rangle$ be an OBDM specification, $D$ be an $\mathcal{S}$-database, and $q_{\mathcal{O}}$ be an EQL-Lite(UCQ) query over $\mathcal{O}$ whose epistemic atoms are $\mathbf{K} \varrho_{1}, \ldots, \mathbf{K} \varrho_{m}$. We denote by $\mathcal{I}_{q_{\mathcal{O}}, \Sigma}^{D}$ the set of facts over the schema $\mathcal{R}_{q_{\mathcal{O}}}=\left\{R_{\mathbf{K} \varrho_{1}}, \ldots, R_{\mathbf{K} \varrho_{m}}\right\}$ obtained by including, for each $i \in[1, m]$ and for each $\vec{c} \in \operatorname{cert}_{q_{\mathcal{O}}^{o_{i}, \Sigma}}^{D}$, the fact $R_{\mathbf{K}_{\varrho_{i}}}(\vec{c})$. By using [Calvanese et al., 2007a, Theorem 6], we easily get the following property.

Proposition 9.1. Let $\Sigma=\langle\mathcal{O}, \mathcal{S}, \mathcal{M}\rangle$ be an $O B D M$ specification and $q_{\mathcal{O}}$ be an EQL-Lite $(U C Q)$ query over $\mathcal{O}$ of arity $n$. Then, for each $\mathcal{S}$-database $D$ consistent with $\Sigma$, we have that $\operatorname{cert}_{q_{\mathcal{O}}, \Sigma}^{D}=\left\{\vec{c} \in \operatorname{dom}(D) \mid \mathcal{I}_{q_{\mathcal{O}}, \Sigma}^{D} \models q_{\mathcal{O}}^{F O L}(\vec{c})\right\}$.

The above proposition tells us that, in order to compute the certain answers of an EQL-Lite(UCQ) query $q_{\mathcal{O}}$, we can compute the certain answers of the UCQs $q_{\mathcal{O}}^{\varrho_{i}}$ associated to the epistemic atoms $\mathbf{K} \varrho_{i}$ of $q_{\mathcal{O}}$, and then consider $q_{\mathcal{O}}$ as an FOL query, where such certain answers are regarded as the extensions of the epistemic atoms.

In the setting for OBDM specifications $\Sigma=\langle\mathcal{O}, \mathcal{S}, \mathcal{M}\rangle$ considered in this thesis (i.e., $D L-$ Lite $_{\mathcal{R}}$ as ontology language and the GLAV approach for the mapping language), the certain answers of the UCQs $q_{\mathcal{O}}^{\varrho_{i}}$ over $\mathcal{O}$ associated to the epistemic atoms $\mathbf{K} \varrho_{i}$ of an $E Q L-L i t e(\mathrm{UCQ}) q_{\mathcal{O}}$ over $\mathcal{O}$ can be computed by means of a suitable query over the schema $\mathcal{S}$, which is $\operatorname{PerfRef}_{q_{\mathcal{O}}^{e_{i}}, \Sigma}$. Specifically, let $\Sigma=\langle\mathcal{O}, \mathcal{S}, \mathcal{M}\rangle$ be an OBDM specification and $q_{\mathcal{O}}$ be an EQL-Lite(UCQ) query over $\mathcal{O}$. We denote by $\mathrm{EQLPerfRef}_{q_{\mathcal{O}}, \Sigma}$ the FOL query over the schema $\mathcal{S}$ obtained from $q_{\mathcal{O}}$ by replacing

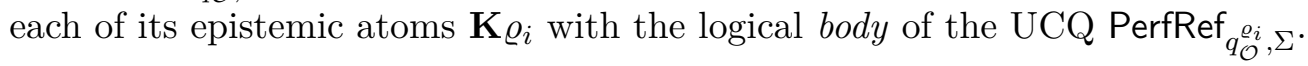

Example 9.2. Refer to Example 9.1 and let $\varrho_{1}=\operatorname{Teaches}(x, y)$ and $\varrho_{2}=\operatorname{Likes}(x, y)$. Then, $q_{\mathcal{O}}^{\underline{O}_{1}}=\{(x, y) \mid$ Teaches $(x, y)\}$ and $q_{\mathcal{O}}^{\varrho_{2}}=\{(x, y) \mid \operatorname{Likes}(x, y)\}$. Therefore:

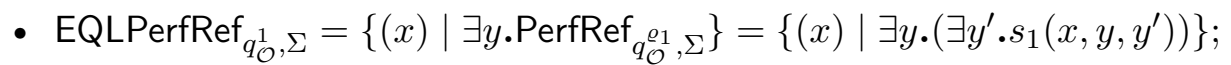

- EQLPerfRef $q_{q_{\mathcal{O}}^{2}, \Sigma}=\left\{(x) \mid \exists y \cdot \operatorname{PerfRef}_{q_{\mathcal{O}}^{\varrho_{2}, \Sigma}}\right\}=\left\{(x) \mid \exists y \cdot\left(\exists y_{1}^{\prime}, y_{2}^{\prime} \cdot s_{3}\left(x, y, y_{1}^{\prime}, y_{2}^{\prime}\right) \vee\right.\right.$ $\left.\left.\exists y^{\prime} . s_{1}\left(x, y, y^{\prime}\right)\right)\right\}$;

- $\mathrm{EQLPerfRef}_{q_{\mathcal{O}}^{3}, \Sigma}=\left\{(x) \mid \exists y \cdot\left(\operatorname{PerfRef}_{q_{\mathcal{O}}^{\varrho_{2}, \Sigma}} \wedge \neg \operatorname{PerfRef}_{q_{\mathcal{O}}^{\varrho_{1}, \Sigma}}\right)\right\}=\{(x) \mid$ $\left.\exists y \cdot\left(\left(\exists y_{1}^{\prime}, y_{2}^{\prime} \cdot s_{3}\left(x, y, y_{1}^{\prime}, y_{2}^{\prime}\right) \vee \exists y^{\prime} \cdot s_{1}\left(x, y, y^{\prime}\right)\right) \wedge \neg \exists y^{\prime} \cdot s_{1}\left(x, y, y^{\prime}\right)\right)\right\}$.

As a consequence of Proposition 9.1 and the above discussion, we can easily obtain the following theorem, which can be seen as the analogous of Calvanese et al., 2007a, Theorem 12] in the OBDM context.

Theorem 9.1. Let $\Sigma=\langle\mathcal{O}, \mathcal{S}, \mathcal{M}\rangle$ be an $O B D M$ specification and $q_{\mathcal{O}}$ be an $E Q L$-Lite $(U C Q)$ query over $\mathcal{O}$. Then, for each $\mathcal{S}$-database $D$ consistent with $\Sigma$, we have that $\operatorname{cert}_{q_{\mathcal{O}}, \Sigma}^{D}=E Q L P \operatorname{erfRef} f_{q_{\mathcal{O}}, \Sigma}^{D}$. 
From the above theorem, we can derive the following observation for OBDM specifications $\Sigma=\langle\mathcal{O}, \mathcal{S}, \mathcal{M}\rangle$ where $\mathcal{O}$ is a $D L$-Lite $\mathcal{R}_{\mathcal{R}}$ ontology and $\mathcal{M}$ is a GLAV mapping, which generalises the one given at the end of Chapter 2 for the sublanguage of UCQs: if $q_{\mathcal{O}}$ is an EQL-Lite(UCQ) query $q_{\mathcal{O}}$ over $\mathcal{O}$ of arity $n$, then the FOL query EQLPerfRef $q_{\mathcal{O}}, \Sigma \vee$ PerfRef $_{\mathcal{V}_{\mathcal{O}}^{n}, \Sigma}$ is the perfect $\mathcal{O}$-to- $\mathcal{S} \Sigma$-rewriting of $q_{\mathcal{O}}$, i.e. $\left(\text { EQLPerfRef }_{q_{\mathcal{O}}, \Sigma} \vee \operatorname{PerfRef}_{\mathcal{V}_{\mathcal{O}}^{n}, \Sigma}\right)^{D}=\operatorname{cert}_{q_{\mathcal{O}}, \Sigma}^{D}$ for every $\mathcal{S}$-database $D$.

This allows us to generalise also Lemmata 5.1 and 6.1 and Corollary 7.1 when the class $\mathcal{L}_{\mathcal{O}}$ of queries over ontologies $\mathcal{O}$ is the one of EQL-Lite(UCQ) queries, and thus is more general than the one of UCQs considered in the mentioned results.

Corollary 9.1. Let $n=\operatorname{ar}\left(q_{\mathcal{O}}\right)=\operatorname{ar}\left(q_{\mathcal{S}}\right)$. We have that:

- $q_{\mathcal{O}}$ is a complete $\mathcal{S}$-to-O $\mathcal{O}$-rewriting of $q_{\mathcal{S}}$ if and only if the following subsumption between queries hold: $q_{\mathcal{S}} \sqsubseteq\left(E Q L P e r f R f_{q_{\mathcal{O}}, \Sigma} \vee \operatorname{PerfRef}_{\mathcal{V}_{\mathcal{O}}^{n}, \Sigma}\right)$;

- $q_{\mathcal{O}}$ is a sound $\mathcal{S}$-to-O $\mathcal{O}$-rewriting of $q_{\mathcal{S}}$ if and only if the following subsumption between queries hold: EQLPerfRef $f_{q_{\mathcal{O}}, \Sigma} \sqsubseteq\left(q_{\mathcal{S}} \vee\right.$ PerfRef $\left._{\mathcal{V}_{\mathcal{O}}^{n}, \Sigma}\right)$;

- $q_{\mathcal{O}}$ is a perfect $\mathcal{S}$-to-O $\mathcal{O}$-rewriting of $q_{\mathcal{S}}$ if and only if both the following subsumptions between queries hold: $q_{\mathcal{S}} \sqsubseteq\left(E Q L P e r f R e f_{q_{\mathcal{O}}, \Sigma} \vee \operatorname{PerfRef}_{\mathcal{V}_{\mathcal{O}}^{n}, \Sigma}\right)$ and EQLPerfRef $_{q_{\mathcal{O}}, \Sigma} \sqsubseteq\left(q_{\mathcal{S}} \vee \operatorname{PerfRef}_{\mathcal{V}_{\mathcal{O}}^{n}, \Sigma}\right)$

\subsubsection{EQL-Lite(UCQ) Source-to-Ontology Rewritings}

As anticipated in the beginning of this section, we next show that considering EQL-Lite(UCQ) queries as target query language provides more expressivity in finding source-to-ontology rewritings, compared to UCQs. In particular, the next example shows that there are cases where no perfect $\mathcal{S}$-to- $\mathcal{O} \Sigma$-rewriting exists in the class of $\mathrm{UCQ}^{\neq} \mathrm{s}$, whereas it exists in the class of EQL-Lite(UCQ) queries.

Example 9.3. Consider the OBDM specification $\Sigma=\langle\mathcal{O}, \mathcal{S}, \mathcal{M}\rangle$ illustrated in Example 9.1, and let the data service be expressed as the CQJFE $q_{\mathcal{S}}=\{(x) \mid$ $\left.\exists y, y^{\prime} \cdot s_{1}\left(x, y, y^{\prime}\right)\right\}$ over $\mathcal{S}$. From results presented in Chapter 5, one can see that the query $q_{\mathcal{O}}^{c}=\{(x) \mid \exists y$. Teaches $(x, y)\}$ is the $\mathrm{UCQ}^{\neq}$-minimally complete $\mathcal{S}$-to- $\mathcal{O}$ $\Sigma$-rewriting of $q_{\mathcal{S}}$. Notice, however, that due to the presence of the ontology assertion Professor $\sqsubseteq \exists$ Teaches and of the mapping assertion $m_{2}$, the certain answers of $q_{\mathcal{O}}^{c}$ with respect to $\Sigma$ and a given $\mathcal{S}$-database $D$ include the values stored both in the first component of $s_{1}$ and in $s_{2}$. Thus, $q_{\mathcal{O}}^{c}$ is not a sound $\mathcal{S}$-to- $\mathcal{O} \Sigma$-rewriting of $q_{\mathcal{S}}$, which allows us to conclude that no $\mathrm{UCQ}^{\neq}$-perfect $\mathcal{S}$-to- $\mathcal{O} \Sigma$-rewriting of $q_{\mathcal{S}}$ exists.

On the other hand, consider the EQL-Lite(UCQ) query $q_{\mathcal{O}}^{1}$ illustrated in Example 9.1. One can verify that $q_{\mathcal{O}}^{1}$ is a perfect $\mathcal{S}$-to- $\mathcal{O} \Sigma$-rewriting of $q_{\mathcal{S}}$ (indeed, observe

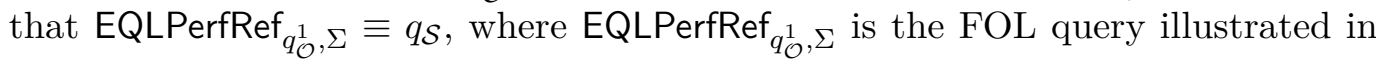
Example 9.2 corresponding to the perfect $\mathcal{O}$-to- $\mathcal{S} \Sigma$-rewriting of $q_{\mathcal{O}}^{1}$ ).

Unfortunately, exactly as in the case of $\mathrm{UCQ}^{\neq} \mathrm{S}$ as target query language, the next example shows that there are pairs of OBDM specifications $\Sigma=\langle\mathcal{O}, \mathcal{S}, \mathcal{M}\rangle$ and CQJFEs $q_{\mathcal{S}}$ over $\mathcal{S}$ for which no perfect $\mathcal{S}$-to- $\mathcal{O} \Sigma$-rewriting of $q_{\mathcal{S}}$ exists in the class of EQL-Lite(UCQ) queries. 
Example 9.4. Let $\Sigma=\langle\mathcal{O}, \mathcal{S}, \mathcal{M}\rangle$ be again the OBDM specification illustrated in Example 9.1, and let the data service be expressed as the CQJFE $q_{\mathcal{S}}=\{(x) \mid$ $\left.\exists y, y^{\prime}, y^{\prime \prime} . s_{3}\left(x, y, y^{\prime}, y^{\prime \prime}\right)\right\}$ over $\mathcal{S}$. Consider the EQL-Lite(UCQ) queries $q_{\mathcal{O}}^{2}$ and $q_{\mathcal{O}}^{3}$ illustrated in Example 9.1. One can see that, because of the presence of the ontology assertion Teaches $\sqsubseteq$ Likes and of the mapping assertion $m_{1}$, the query $q_{\mathcal{O}}^{2}$ is too general to be a perfect $\mathcal{S}$-to- $\mathcal{O} \Sigma$-rewriting. Indeed, its certain answers with respect to $\Sigma$ and a given $\mathcal{S}$-database $D$ include the values stored in the first component of both $s_{1}$ and $s_{3}$. On the other hand, the query $q_{\mathcal{O}}^{3}$ is too specific to be a perfect $\mathcal{S}$-to- $\mathcal{O}$ $\Sigma$-rewriting of $q_{\mathcal{S}}$. Indeed, there are $\mathcal{S}$-databases $D$ for which its certain answers with respect to $\Sigma$ and $D$ do not include the values stored in the first component of $s_{3}$. The above observations allow us to conclude that no perfect $\mathcal{S}$-to- $\mathcal{O} \Sigma$-rewriting of $q_{\mathcal{S}}$ exists in the class of EQL-Lite(UCQ) queries.

Clearly, as always, when perfect source-to-ontology rewritings in the class of EQL-Lite(UCQ) queries do not exist, the goal is to find queries in such a class that provide the best approximations of $q_{\mathcal{S}}$. By developing on the above example, we show that the class of EQL-Lite(UCQ) queries allows to find better approximations of source-to-ontology rewritings compared to the UCQ query language.

Example 9.5. Refer to Example 9.4, where $\Sigma=\langle\mathcal{O}, \mathcal{S}, \mathcal{M}\rangle$ is the OBDM specification illustrated in Example 9.1 and the data service is expressed as the CQJFE $q_{\mathcal{S}}=\left\{(x) \mid \exists y, y^{\prime}, y^{\prime \prime} . s_{3}\left(x, y, y^{\prime}, y^{\prime \prime}\right)\right\}$ over $\mathcal{S}$. From results of previous chapters, one can verify that the queries $q_{\mathcal{O}}^{c}=\{(x) \mid \exists y \cdot \operatorname{Likes}(x, y)\}$ and $q_{\mathcal{O}}^{\mathcal{S}}=\{(x) \mid \perp(x)\}$ are, respectively, the (unique up to equivalence w.r.t. $\Sigma$ ) $\mathrm{UCQ}^{\neq}$-minimally complete $\mathcal{S}$-to$\mathcal{O} \Sigma$-rewriting of $q_{\mathcal{S}}$ and the (unique up to equivalence w.r.t. $\Sigma$ ) $\mathrm{UCQ}^{\neq}$-maximally sound $\mathcal{S}$-to- $\mathcal{O} \Sigma$-rewriting of $q_{\mathcal{S}}$.

As for the best approximations of $q_{\mathcal{S}}$ (w.r.t. $\Sigma$ ) in the class of EQL-Lite(UCQ) queries, one can verify that the EQL-Lite(UCQ) queries $q_{\mathcal{O}}^{2}$ and $q_{\mathcal{O}}^{3}$ illustrated in Example 9.1 are, respectively, the (unique up to equivalence w.r.t. $\Sigma$ ) minimally complete $\mathcal{S}$-to- $\mathcal{O} \Sigma$-rewriting of $q_{\mathcal{S}}$ in the class of EQL-Lite(UCQ) queries and the unique (up to equivalence w.r.t. $\Sigma$ ) maximally sound $\mathcal{S}$-to- $\mathcal{O} \Sigma$-rewriting of $q_{\mathcal{S}}$ in the class of EQL-Lite(UCQ) queries. It is also clear that $q_{\mathcal{O}}^{2}$ and $q_{\mathcal{O}}^{3}$ are better approximations of $q_{\mathcal{S}}$ (w.r.t. $\Sigma$ ) compared to $q_{\mathcal{O}}^{c}$ and $q_{\mathcal{O}}^{s}$, respectively.

In the next sections, we carry out a study on the problem of computing the best abstractions of data services, expressed as queries over the source schema, in the class of EQL-Lite(UCQ) queries and also in a fragment of it.

Interestingly, for the class of EQL-Lite(UCQ) queries we have the following result, which is the analogous of Proposition 3.2 for the EQL-Lite(UCQ) query language, and can be easily proven by following exactly the same line of arguments used in the proof of that proposition.

Proposition 9.2. If $q_{1}$ and $q_{2}$ are minimally complete (respectively, maximally sound) $\mathcal{S}$-to- $\mathcal{O}$ E-rewritings of $q_{\mathcal{S}}$ in the class of EQL-Lite $(U C Q)$ queries, then they are equivalent w.r.t. $\Sigma$. 


\subsection{On the non-existence of $E Q L-L i t e(U C Q)$ Source-to- Ontology Rewritings}

Surprisingly, we now prove that neither minimally complete, nor maximally sound source-to-ontology rewritings in the class of EQL-Lite(UCQ) queries are guaranteed to exist, even in the case of OBDM specifications $\Sigma=\langle\mathcal{O}, \mathcal{S}, \mathcal{M}\rangle$ and data services $q_{\mathcal{S}}$ where $\mathcal{O}=\emptyset, \mathcal{M}$ is a pure GAV mapping, and $q_{\mathcal{S}}$ is a CQJFE over $\mathcal{S}$.

We start by looking at the minimally complete case.

Theorem 9.2. There exists an $O B D M$ specification $\Sigma=\langle\mathcal{O}, \mathcal{S}, \mathcal{M}\rangle$ with $\mathcal{O}=\emptyset$ and $\mathcal{M}$ being a pure GAV mapping and a CQJFE $q_{\mathcal{S}}$ for which no minimally complete $\mathcal{S}$-to-O $\mathcal{O}$-rewriting of $q_{\mathcal{S}}$ in the class of EQL-Lite $(U C Q)$ queries exists.

Proof. Let $\Sigma=\langle\mathcal{O}, \mathcal{S}, \mathcal{M}\rangle$ be the following OBDM specification:

- $\mathcal{O}=\emptyset$

- $\mathcal{S}=\left\{s, s_{1}, s_{2}, s_{3}, s_{4}, s_{5}\right\}$

- $\mathcal{M}=\left\{m_{1}, m_{2}, m_{3}, m_{4}\right\}$, where:

$$
\begin{array}{rrrl}
m_{1}: & s(x) & \rightarrow B(x), \\
m_{2}: & s_{1}(x) \wedge s_{2}(x) & \rightarrow B(x), \\
m_{3}: & s_{1}(x) & \rightarrow A_{1}(x), \\
m_{4}: & s_{2}\left(x_{1}\right) \wedge s_{3}\left(x_{1}, x_{2}\right) & \rightarrow & P\left(x_{1}, x_{2}\right), \\
m_{5}: & s_{1}\left(x_{2}\right) \wedge s_{5}\left(x_{1}, x_{2}\right) & \rightarrow P\left(x_{1}, x_{2}\right), \\
m_{6}: & s_{2}(x) \wedge s_{4}(x) & \rightarrow A_{2}(x), \\
m_{7}: & s\left(x_{1}\right) \wedge s_{1}\left(x_{2}\right) \wedge s_{2}\left(x_{2}\right) & \rightarrow & R\left(x_{1}, x_{2}\right) .
\end{array}
$$

Let $q_{\mathcal{S}}$ be the following boolean CQJFE over $\mathcal{S}: q_{\mathcal{S}}=\{() \mid \exists y . s(y)\}$.

From results of Chapter 5 , we know that the UCQ-minimally complete $\mathcal{S}$-to- $\mathcal{O}$ $\Sigma$-rewriting of $q_{\mathcal{S}}$ is the query $q_{\mathcal{O}}^{c}=\{() \mid \exists y \cdot B(y)\}$. Observe that, however, $q_{\mathcal{O}}^{c}$ is not a sound $\mathcal{S}$-to- $\mathcal{O} \Sigma$-rewriting of $q_{\mathcal{S}}$. This is because there are $\mathcal{S}$-databases $D$ for which $(i)$ there are no facts of the form $s(c)$ (i.e., the extension of source predicate $s$ in $D$ is empty) and (ii) $D$ contains both the facts $s_{1}(c)$ and $s_{2}(c)$ for a certain constant $c \in$ Const, thus resulting in $q_{\mathcal{S}}^{D}=\emptyset$ whereas $\operatorname{cert}_{q_{\mathcal{O}}, \Sigma}^{D}=\{\}$.

With the ability of the EQL-Lite(UCQ) query language of expressing epistemic forms of negations, we can detect some of those cases by adding $\neg \mathbf{K}(\varrho)$ in conjunction to the epistemic atom $\mathbf{K}(\exists y \cdot B(y))$, where $\varrho$ is the body of a CQ being a sound $\mathcal{S}$-to- $\mathcal{O} \Sigma$-rewriting of $\left\{() \mid \exists y \cdot s_{1}(y) \wedge s_{2}(y)\right\}$. At the same time, however, we need to ensure the fact that such new EQL-Lite(UCQ) query that we are building is still a complete $\mathcal{S}$-to- $\mathcal{O} \Sigma$-rewriting of $q_{\mathcal{S}}$. In other words, we want that whenever an $\mathcal{S}$-database $D$ contains a fact $s(c)$ for a constant $c \in$ Const, the certain answers of the new query are not empty, even if $\mathbf{K}(\varrho)$ is true in the current OBDM system. By looking at the mapping $\mathcal{M}$, we can ensure this by simply adding the epistemic atom $\mathbf{K}\left(\exists y, y^{\prime} . R\left(y, y^{\prime}\right)\right)$ in disjunction to the new EQL-Lite(UCQ) query.

With the above discussion at hand, we now introduce a pattern for an infinite number of EQL-Lite(UCQ) queries over $\mathcal{O}$ and related technical lemmata. Specifi- 
cally, for every $i \geq 0$, let $q_{\mathcal{O}}^{i}$ be the following $E Q L$-Lite(UCQ) query over $\mathcal{O}$ :

$$
q_{\mathcal{O}}^{i}=\left\{() \mid\left(\mathbf{K}(\exists y \cdot B(y)) \wedge\left(\bigwedge_{k=0}^{k=i} \neg \mathbf{K}\left(\varrho_{k}\right)\right)\right) \vee \mathbf{K}\left(\exists y, y^{\prime} \cdot R\left(y, y^{\prime}\right)\right)\right\}, \text { where }
$$

- $\varrho_{0}=\exists y_{0} \cdot A_{1}\left(y_{0}\right) \wedge A_{2}\left(y_{0}\right)$.

- $\varrho_{k}=\exists y_{0}, \ldots, y_{k} \cdot A_{1}\left(y_{0}\right) \wedge\left(\bigwedge_{j=0}^{j=k-1} P\left(y_{j}, y_{j+1}\right)\right) \wedge A_{2}\left(y_{k}\right)$, for each $k \in[1, i]$.

For instance, with $i=2$, we have $q_{\mathcal{O}}^{2}=\left\{() \mid\left(\mathbf{K}(\exists y \cdot B(y)) \wedge \neg \mathbf{K}\left(\exists y_{0} \cdot A_{1}\left(y_{0}\right) \wedge\right.\right.\right.$ $\left.A_{2}\left(y_{0}\right)\right) \wedge \neg \mathbf{K}\left(\exists y_{0}, y_{1} \cdot A_{1}\left(y_{0}\right) \wedge P\left(y_{0}, y_{1}\right) \wedge A_{2}\left(y_{1}\right)\right) \wedge \neg \mathbf{K}\left(\exists y_{0}, y_{1}, y_{2} \cdot A_{1}\left(y_{0}\right) \wedge P\left(y_{0}, y_{1}\right) \wedge\right.$ $\left.\left.\left.P\left(y_{1}, y_{2}\right) \wedge A_{2}\left(y_{2}\right)\right)\right) \vee \mathbf{K}\left(\exists y, y^{\prime} \cdot R\left(y, y^{\prime}\right)\right)\right\}$.

Observe that, for each $k \in[0, i]$, the body $\varrho_{k}$ in the epistemic atom $\mathbf{K}\left(\varrho_{k}\right)$ is exactly the body of the CQ $q_{\mathcal{O}}^{k}=\left\{() \mid \varrho_{k}\right\}$ illustrated in the proof of point 1 of Theorem 6.2, which we recall that is a sound $\mathcal{S}$-to- $\mathcal{O} \Sigma$-rewriting of the query $\left\{() \mid \exists y \cdot s_{1}(y) \wedge s_{2}(y)\right\}$ (note that the mapping assertions $m_{3}, m_{4}, m_{5}$, and $m_{6}$ are identical to the mapping assertions $m_{1}, m_{2}, m_{3}$, and $m_{4}$, respectively, of the mapping illustrated in the proof of point 1 of Theorem 6.2.

Lemma 9.1. For every natural number $i \geq 0$, we have that $q_{\mathcal{O}}^{i}$ is a complete $\mathcal{S}$-to-O $\Sigma$-rewriting of $q_{\mathcal{S}}$.

Proof. Consider any $i \geq 0$ and the associated EQL-Lite(UCQ) query $q_{\mathcal{O}}^{i}$. Let $D$ be any $\mathcal{S}$-database $D$ for which $q_{\mathcal{S}}^{D}=\{\}$, which is equivalent to say that $s(c) \in D$ for some constant $c \in$ Const. There are two possible cases: either for all $k \in[0, i]$ the epistemic atom $\mathbf{K}\left(\varrho_{k}\right)$ is false in the OBDM system $\langle\Sigma, D\rangle$, or not.

In the former case, due to the mapping assertion $m_{1}$ and the fact that $s(c) \in D$, we trivially have that the epistemic atom $\mathbf{K}(\exists y \cdot B(y))$ is true in $\langle\Sigma, D\rangle$. It follows that the formula $\left(\mathbf{K}(\exists y \cdot B(y)) \wedge \neg \mathbf{K}\left(\varrho_{0}\right) \wedge \ldots \wedge \neg \mathbf{K}\left(\varrho_{i}\right)\right)$ is as well true in $\langle\Sigma, D\rangle$, thus implying that $\operatorname{cert}_{q_{\mathcal{O}}^{i}, \Sigma}^{D}=\{\}$.

In the latter case, there is some $k \in[0, i]$ for which $\mathbf{K}\left(\varrho_{k}\right)$ is true in $\langle\Sigma, D\rangle$. Notice that, since $\varrho_{k}$ is the body of a CQ that is a sound $\mathcal{S}$-to- $\mathcal{O} \Sigma$-rewriting of $\left\{() \mid \exists y \cdot s_{1}(y) \wedge s_{2}(y)\right\}$ (cf. Lemma 6.2), we derive that $D$ contains both the facts $s_{1}\left(c^{\prime}\right)$ and $s_{2}\left(c^{\prime}\right)$ for some constant $c^{\prime} \in$ Const. This, together with the fact that $s(c) \in D$ for some constant $c \in$ Const, and by looking at the $m_{7}$ mapping assertion of $\mathcal{M}$, allows us to easily derive that the epistemic atom $\mathbf{K}\left(\exists y, y^{\prime} \cdot R\left(y, y^{\prime}\right)\right)$ is true in $\langle\Sigma, D\rangle$, thus implying that $\operatorname{cert}_{q_{\mathcal{O}}^{i}, \Sigma}^{D}=\{\}$ also in this case.

Thus, for any $\mathcal{S}$-database $D$, if $q_{\mathcal{S}}^{D}=\{\}$, then $\operatorname{cert}_{q_{\mathcal{O}}^{i}, \Sigma}^{D}=\{\}$. This clearly implies that $q_{\mathcal{O}}^{i}$ is a complete $\mathcal{S}$-to- $\mathcal{O} \Sigma$-rewriting of $q_{\mathcal{S}}$, as required.

Lemma 9.2. For every natural number $i \geq 0$, we have that cert $_{q_{\mathcal{O}}^{i+1, \Sigma}} \sqsubset \operatorname{cert}_{q_{\mathcal{O}}^{i}, \Sigma}$.

Proof. The proof immediately follows from the proof of Lemma 6.3, which implies that, for any pair of natural numbers $l, m \geq 0$ with $l \neq m$, the CQs $q_{\mathcal{O}}^{l}=\left\{() \mid \varrho_{l}\right\}$ and $q_{\mathcal{O}}^{m}=\left\{() \mid \varrho_{m}\right\}$ illustrated in the proof of point 1 of Theorem 6.2 are such that both $\operatorname{cert}_{q_{\mathcal{O}}^{l}, \Sigma} \nsubseteq \operatorname{cert}_{q_{\mathcal{O}}^{m}, \Sigma}$ and $\operatorname{cert}_{q_{\mathcal{O}}^{m}, \Sigma} \nsubseteq \operatorname{cert}_{q_{\mathcal{O}}^{l}, \Sigma}$ hold. Consider any natural number $i \geq 0$ and the EQL-Lite(UCQ) queries $q_{\mathcal{O}}^{i}$ and $q_{\mathcal{O}}^{i+1}$. Since the body of $q_{\mathcal{O}}^{i+1}$ contains also $\neg \mathbf{K}\left(\varrho_{i+1}\right)$ whereas $q_{\mathcal{O}}^{i}$ does not, from the above observation, we trivially 
derive that $\operatorname{cert}_{q_{\mathcal{O}}^{i+1, \Sigma}} \sqsubset \operatorname{cert}_{q_{\mathcal{O}}^{i}, \Sigma}$. Indeed, there is at least an $\mathcal{S}$-database $D$ (which contains both the facts $s_{1}(c)$ and $s_{2}(c)$ for some constant $c \in$ Const) in which the epistemic atom $\mathbf{K}\left(\varrho_{i+1}\right)$ is true in $\langle\Sigma, D\rangle$ whereas $\mathbf{K}\left(\varrho_{j}\right)$ is false in $\langle\Sigma, D\rangle$ for any $j \in[0, i]$, thus implying that $\operatorname{cert}_{q_{\mathcal{O}}^{i+1}, \Sigma}^{D}=\emptyset$ whereas $\operatorname{cert}_{q_{\mathcal{O}}^{i}, \Sigma}^{D}=\{\}$, as required.

Furthermore, due to Lemma 6.4, we know that each possible sound $\mathcal{S}$-to- $\mathcal{O} \Sigma$ rewriting of $\left\{() \mid \exists y \cdot s_{1}(y) \wedge s_{2}(y)\right\}$ is equivalent to the query $\left\{() \mid \varrho_{i}\right\}$, for some $i \geq 0$. Thus, using similar arguments as the ones given in the proof of Lemma 6.4 it is not difficult to see that each possible $E Q L$-Lite(UCQ) query $q_{\mathcal{O}}^{\prime}$ that is a complete $\mathcal{S}$-to$\mathcal{O} \Sigma$-rewriting of $q_{\mathcal{S}}$ is such that there exists an $i \geq 0$ for which $\operatorname{cert}_{q_{\mathcal{O}}^{i}, \Sigma} \sqsubset \operatorname{cert}_{q_{\mathcal{O}}^{\prime}, \Sigma}$ (including $q_{\mathcal{O}}^{c}=\{() \mid \exists y \cdot B(y)\}$, i.e., the UCQ-minimally complete $\mathcal{S}$-to- $\mathcal{O} \Sigma$-rewriting of $q_{\mathcal{S}}$, which is clearly such that $\operatorname{cert}_{q_{\mathcal{O}}^{i}, \Sigma} \sqsubset \operatorname{cert}_{q_{\mathcal{O}}^{\prime}, \Sigma}$ for any $\left.i \geq 0\right)$.

With this observation and the above lemmata at hand, we are now able to prove that no minimally complete $\mathcal{S}$-to- $\mathcal{O} \Sigma$-rewriting of $q_{\mathcal{S}}$ in the class of EQL-Lite(UCQ) queries exists. Indeed, since each possible $E Q L-L i t e(\mathrm{UCQ})$ query $q_{\mathcal{O}}^{\prime}$ that is a complete $\mathcal{S}$-to- $\mathcal{O} \Sigma$-rewriting of $q_{\mathcal{S}}$ is such that there exists an $i \geq 0$ for which cert $_{q_{\mathcal{O}, \Sigma}^{i} \Sigma} \sqsubset \operatorname{cert}_{q_{\mathcal{O}}^{\prime}, \Sigma}$, and since by Lemma $9.1 q_{\mathcal{O}}^{i}$ is a complete $\mathcal{S}$-to- $\mathcal{O} \Sigma$-rewriting of $q_{\mathcal{S}}$ for any $i \geq 0$, when seeking for a minimally complete $\mathcal{S}$-to- $\mathcal{O} \Sigma$-rewriting of $q_{\mathcal{S}}$ in the class of EQL-Lite(UCQ) queries one can limit the attention to only the illustrated queries $q_{\mathcal{O}}^{i}$ for $i \geq 0$. Notice that, however, by Lemma 9.2 , for any natural number $i \geq 0$, the query $q_{\mathcal{O}}^{i+1}$ is a better complete approximation of the $\mathcal{S}$-to- $\mathcal{O}$ $\Sigma$-rewriting of $q_{\mathcal{S}}$ compared to the query $q_{\mathcal{O}}^{i}$ (i.e., $\operatorname{cert}_{q_{\mathcal{O}}^{i+1}, \Sigma} \sqsubset \operatorname{cert}_{q_{\mathcal{O}}^{i}, \Sigma}$ ). Thus, since in any EQL-Lite(UCQ) query there are only a finite set of epistemic atoms, we can conclude that no minimally complete $\mathcal{S}$-to- $\mathcal{O} \Sigma$-rewriting of $q_{\mathcal{S}}$ in the class of EQL-Lite(UCQ) queries exists, as required.

We now turn to the maximally sound case.

Theorem 9.3. There exists an $O B D M$ specification $\Sigma=\langle\mathcal{O}, \mathcal{S}, \mathcal{M}\rangle$ with $\mathcal{O}=\emptyset$ and $\mathcal{M}$ being a pure GAV mapping and a CQJFE $q_{\mathcal{S}}$ for which no maximally sound $\mathcal{S}$-to-O $\mathcal{O}$-rewriting of $q_{\mathcal{S}}$ in the class of EQL-Lite $(U C Q)$ queries exists.

Proof. Let $\Sigma=\langle\mathcal{O}, \mathcal{S}, \mathcal{M}\rangle$ be the following OBDM specification:

- $\mathcal{O}=\emptyset$

- $\mathcal{S}=\left\{s, s^{\prime}, s_{1}, s_{2}, s_{3}, s_{4}, s_{5}\right\}$

- $\mathcal{M}=\left\{m_{1}, m_{2}, m_{3}, m_{4}\right\}$, where:

$$
\begin{array}{rrll}
m_{1}: & s(x) & \rightarrow B(x), \\
m_{2}: & s^{\prime}(x) & \rightarrow B(x), \\
m_{3}: & s_{1}(x) & \rightarrow A A_{1}(x), \\
m_{4}: & s_{2}\left(x_{1}\right) \wedge s_{3}\left(x_{1}, x_{2}\right) & \rightarrow P\left(x_{1}, x_{2}\right), \\
m_{5}: & s_{1}\left(x_{2}\right) \wedge s_{5}\left(x_{1}, x_{2}\right) & \rightarrow P\left(x_{1}, x_{2}\right), \\
m_{6}: & s_{2}(x) \wedge s_{4}(x) & \rightarrow A_{2}(x), \\
m_{7}: & s^{\prime}\left(x_{1}\right) \wedge s_{1}\left(x_{2}\right) \wedge s_{2}\left(x_{2}\right) & \rightarrow & R\left(x_{1}, x_{2}\right) .
\end{array}
$$

Let $q_{\mathcal{S}}$ be the following boolean CQJFE over $\mathcal{S}: q_{\mathcal{S}}=\{() \mid \exists y . s(y)\}$. 
From results of Chapter 8, we know that the UCQ-maximally sound $\mathcal{S}$-to- $\mathcal{O}$ $\Sigma$-rewriting of $q_{\mathcal{S}}$ is the CQ $q_{\mathcal{O}}^{s}=\{() \mid \perp\}$. Informally, this is because the CQ $q_{\mathcal{O}}^{c}=\{() \mid \exists y \cdot B(y)\}$ is not a sound $\mathcal{S}$-to- $\mathcal{O}$ $\Sigma$-rewriting of $q_{\mathcal{S}}$, since the query $q_{\mathcal{S}}^{\prime}=\left\{() \mid \exists y \cdot s^{\prime}(y)\right\}$ is a disjunct of $\operatorname{PerfRef}_{q_{\mathcal{O}}^{c}, \Sigma}$ such that $q_{\mathcal{S}}^{\prime} \nsubseteq q_{\mathcal{S}}$ (cf. Lemma 6.1).

With the ability of the EQL-Lite(UCQ) query language of expressing epistemic forms of negations, we can detect some of the cases where the epistemic atom $\mathbf{K}(\exists y \cdot B(y))$ is true in $\langle\Sigma, D\rangle$ for a certain $\mathcal{S}$-database $D$ and, nevertheless, it is sure that there are no facts of the form $s^{\prime}(c)$ in $D$ (i.e., the extension of source predicate $s^{\prime}$ in $D$ is empty). Observe that, by looking at the mapping assertions in $\mathcal{M}$ (in particular, to the $m_{7}$ mapping assertion), this latter requirement can be achieved by the following EQL-Lite(UCQ) formula: $\neg \mathbf{K}\left(\exists y, y^{\prime} \cdot R\left(y, y^{\prime}\right)\right) \wedge \mathbf{K}(\varrho)$, where $\varrho$ is the body of a UCQ being a sound $\mathcal{S}$-to- $\mathcal{O} \Sigma$-rewriting of the CQ $\left\{() \mid \exists y \cdot s_{1}(y) \wedge s_{2}(y)\right\}$.

With the above discussion at hand, we now introduce a pattern for an infinite number of $E Q L-L i t e(U C Q)$ queries over $\mathcal{O}$ and related technical lemmata. Specifically, for every $i \geq 0$, let $q_{\mathcal{O}}^{i}$ be the following EQL-Lite(UCQ) query over $\mathcal{O}$ :

$$
q_{\mathcal{O}}^{i}=\left\{() \mid \mathbf{K}(\exists y \cdot B(y)) \wedge \neg \mathbf{K}\left(\exists y, y^{\prime} \cdot R\left(y, y^{\prime}\right)\right) \wedge \mathbf{K}\left(\bigcup_{k=0}^{k=i} \varrho_{k}\right)\right\}, \text { where }
$$

- $\varrho_{0}=\exists y_{0} \cdot A_{1}\left(y_{0}\right) \wedge A_{2}\left(y_{0}\right)$.

- $\varrho_{k}=\exists y_{0}, \ldots, y_{k} \cdot A_{1}\left(y_{0}\right) \wedge\left(\bigwedge_{j=0}^{j=k-1} P\left(y_{j}, y_{j+1}\right)\right) \wedge A_{2}\left(y_{k}\right)$, for each $k \in[1, i]$.

For instance, with $i=2$, we have $q_{\mathcal{O}}^{2}=\left\{() \mid \mathbf{K}(\exists y \cdot B(y)) \wedge \neg \mathbf{K}\left(\exists y, y^{\prime} \cdot R\left(y, y^{\prime}\right)\right) \wedge\right.$ $\mathbf{K}\left(\left(\exists y_{0} \cdot A_{1}\left(y_{0}\right) \wedge A_{2}\left(y_{0}\right)\right) \cup\left(\exists y_{0}, y_{1} \cdot A_{1}\left(y_{0}\right) \wedge P\left(y_{0}, y_{1}\right) \wedge A_{2}\left(y_{1}\right)\right) \cup\left(\exists y_{0}, y_{1}, y_{2} \cdot A_{1}\left(y_{0}\right) \wedge\right.\right.$ $\left.\left.\left.P\left(y_{0}, y_{1}\right) \wedge P\left(y_{1}, y_{2}\right) \wedge A_{2}\left(y_{2}\right)\right)\right)\right\}$.

Observe that, for each $k \in[0, i]$, the disjunct $\varrho_{k}$ in the last epistemic atom is exactly the body of the CQ $q_{\mathcal{O}}^{k}=\left\{() \mid \varrho_{k}\right\}$ illustrated in the proof of point 1 of Theorem 6.2. which we recall that is a sound $\mathcal{S}$-to- $\mathcal{O} \Sigma$-rewriting of the query $\left\{() \mid \exists y \cdot s_{1}(y) \wedge s_{2}(y)\right\}$ (note that the mapping assertions $m_{3}, m_{4}, m_{5}$, and $m_{6}$ are identical to the mapping assertions $m_{1}, m_{2}, m_{3}$, and $m_{4}$, respectively, of the mapping illustrated in the proof of point 1 of Theorem 6.2.

Lemma 9.3. For every natural number $i \geq 0$, we have that $q_{\mathcal{O}}^{i}$ is a sound $\mathcal{S}$-to- $\mathcal{O}$ $\Sigma$-rewriting of $q_{\mathcal{S}}$.

Proof. Consider any $i \geq 0$ and the associated EQL-Lite(UCQ) query $q_{\mathcal{O}}^{i}$. Let $D$ be any $\mathcal{S}$-database for which $\operatorname{cert}_{q_{\mathcal{O}}^{i}, \Sigma}^{D}=\{\}$. Since $\operatorname{cert}_{q_{\mathcal{O}}^{i}, \Sigma}^{D}=\{\}$, we derive the following implications: $(i)$ the epistemic atom $\mathbf{K}\left(\bigcup_{k=0}^{k=i} \varrho_{k}\right)$ of $q_{\mathcal{O}}^{i}$ is true in $\langle\Sigma, D\rangle ;(i i)$ the epistemic atom $\mathbf{K}\left(\exists y, y^{\prime} \cdot R\left(y, y^{\prime}\right)\right)$ of $q_{\mathcal{O}}^{i}$ is false in $\langle\Sigma, D\rangle$; and (iii) the epistemic atom $\mathbf{K}(\exists y \cdot B(y))$ of $q_{\mathcal{O}}^{i}$ is true in $\langle\Sigma, D\rangle$.

From $(i)$, since $\bigcup_{k=0}^{k=i} \varrho_{k}$ is the body of a UCQ that is a sound $\mathcal{S}$-to- $\mathcal{O} \sum$-rewriting of $\left\{() \mid \exists y \cdot s_{1}(y) \wedge s_{2}(y)\right\}$ (indeed, by Lemma 6.2. for each $k \in[0, i], \varrho_{k}$ is the body of a CQ that is a $\mathcal{S}$-to- $\mathcal{O} \Sigma$-rewriting of $\left.\left\{() \mid \exists y \cdot s_{1}(y) \wedge s_{2}(y)\right\}\right)$, we derive that the $\mathcal{S}$-database $D$ contains both the facts $s_{1}(c)$ and $s_{2}(c)$ for some constant $c \in$ Const. This, together with $(i i)$, and by looking at the $m_{7}$ mapping assertion of $\mathcal{M}$, allows us to easily derive that there are no facts of the form $s^{\prime}\left(c^{\prime}\right)$ in $D$ (i.e., the extension of the source predicate $s^{\prime}$ in $D$ is empty). But then, since (iii) holds, by looking at 
the mapping $\mathcal{M}$, we derive that the $\mathcal{S}$-database $D$ necessarily contains a fact of the form $s\left(c^{\prime \prime}\right)$ for some constant $c^{\prime \prime} \in$ Const, thus implying that $q_{\mathcal{S}}^{D}=\{\}$.

Thus, for any $\mathcal{S}$-database $D$, if $\operatorname{cert}_{q_{\mathcal{O}}^{i}, \Sigma}^{D}=\{\}$, then $q_{\mathcal{S}}^{D}=\{\}$. This clearly implies that $q_{\mathcal{O}}^{i}$ is a sound $\mathcal{S}$-to- $\mathcal{O} \Sigma$-rewriting of $q_{\mathcal{S}}$, as required.

Lemma 9.4. For every natural number $i \geq 0$, we have that $\operatorname{cert}_{q_{\mathcal{O}}^{i}, \Sigma} \sqsubset \operatorname{cert}_{q_{\mathcal{O}}^{i+1}, \Sigma}$.

Proof. The proof immediately follows from the proof of Lemma 6.3, which implies that, for any pair of natural numbers $l, m \geq 0$ with $l \neq m$, the CQs $q_{\mathcal{O}}^{l}=\left\{() \mid \varrho_{l}\right\}$ and $q_{\mathcal{O}}^{m}=\left\{() \mid \varrho_{m}\right\}$ illustrated in the proof of point 1 of Theorem 6.2 are such that both $\operatorname{cert}_{q_{\mathcal{O}}^{l}, \Sigma} \nsubseteq \operatorname{cert}_{q_{\mathcal{O}, \Sigma}^{m},}$ and $\operatorname{cert}_{q_{\mathcal{O}}^{m}, \Sigma} \nsubseteq \operatorname{cert}_{q_{\mathcal{O}}^{l}, \Sigma}$ hold. Consider any natural number $i \geq 0$ and the EQL-Lite(UCQ) queries $q_{\mathcal{O}}^{i}$ and $q_{\mathcal{O}}^{i+1}$. Since the disjunct $\varrho_{i+1}$ occurs in the epistemic atom $\mathbf{K}\left(\bigcup_{k=0}^{k=i+1} \varrho_{k}\right)$ whereas it does not occur in the epistemic atom $\mathbf{K}\left(\bigcup_{k=0}^{k=i} \varrho_{k}\right)$, from the above observation, we trivially derive that $\operatorname{cert}_{q_{\mathcal{O}}^{i}, \Sigma} \sqsubset \operatorname{cert}_{q_{\mathcal{O}}^{i+1}, \Sigma}$. Indeed, there is at least an $\mathcal{S}$-database $D$ (which contains both the facts $s_{1}(c)$ and $s_{2}(c)$ for some constant $c \in$ Const) in which the formula $\varrho_{i+1}$ is true in $\langle\Sigma, D\rangle$ (and therefore, the epistemic atom $\mathbf{K}\left(\bigcup_{k=0}^{k=i+1} \varrho_{k}\right)$ is true in $\langle\Sigma, D\rangle$ ) whereas formula $\varrho_{j}$ is false in $\langle\Sigma, D\rangle$ for any $j \in[0, i]$ (and therefore, the epistemic atom $\mathbf{K}\left(\bigcup_{k=0}^{k=i} \varrho_{k}\right)$ is false in $\left.\langle\Sigma, D\rangle\right)$, thus implying that $\operatorname{cert}_{q_{\mathcal{O}}^{i+1}, \Sigma}^{D}=\{\}$ whereas $\operatorname{cert}_{q_{\mathcal{O}}^{i}, \Sigma}^{D}=\emptyset$, as required.

Furthermore, due to Lemma 6.4, we know that each possible sound $\mathcal{S}$-to- $\mathcal{O} \Sigma$ rewriting of $\left\{() \mid \exists y \cdot s_{1}(y) \wedge s_{2}(y)\right\}$ is equivalent to the query $\left\{() \mid \varrho_{i}\right\}$, for some $i \geq 0$. Thus, using similar arguments as the ones given in the proof of Lemma 6.4 it is not difficult to see that each possible EQL-Lite(UCQ) query $q_{\mathcal{O}}^{\prime}$ that is a sound $\mathcal{S}$-to- $\mathcal{O}$ $\Sigma$-rewriting of $q_{\mathcal{S}}$ is such that there exists an $i \geq 0$ for which $\operatorname{cert}_{q_{\mathcal{O}}^{\prime}, \Sigma} \sqsubset \operatorname{cert}_{q_{\mathcal{O}}^{i}, \Sigma}$.

With this observation and the above lemmata at hand, we are now able to prove that no maximally sound $\mathcal{S}$-to- $\mathcal{O} \Sigma$-rewriting of $q_{\mathcal{S}}$ in the class of EQL-Lite(UCQ) queries exists. Indeed, since each possible $E Q L-L i t e(\mathrm{UCQ})$ query $q_{\mathcal{O}}^{\prime}$ that is a sound $\mathcal{S}$ to- $\mathcal{O} \Sigma$-rewriting of $q_{\mathcal{S}}$ is such that there exists an $i \geq 0$ for which $\operatorname{cert}_{q_{\mathcal{O}}^{\prime}, \Sigma} \sqsubset \operatorname{cert}_{q_{\mathcal{O}}^{i}, \Sigma}$, and since by Lemma $9.3 q_{\mathcal{O}}^{i}$ is a sound $\mathcal{S}$-to- $\mathcal{O} \Sigma$-rewriting of $q_{\mathcal{S}}$ for any $i \geq 0$, when seeking for a maximally sound $\mathcal{S}$-to- $\mathcal{O} \Sigma$-rewriting of $q_{\mathcal{S}}$ in the class of EQL-Lite(UCQ) queries one can limit the attention to only the illustrated queries $q_{\mathcal{O}}^{i}$ for $i \geq 0$. Notice that, however, by Lemma 9.4 for any natural number $i \geq 0$, the query $q_{\mathcal{O}}^{i+1}$ is a better sound approximation of the $\mathcal{S}$-to- $\mathcal{O} \Sigma$-rewriting of $q_{\mathcal{S}}$ compared to the query $q_{\mathcal{O}}^{i}$ (i.e., $\operatorname{cert}_{q_{\mathcal{O}}^{i}, \Sigma} \sqsubset \operatorname{cert}_{q_{\mathcal{O}}^{i+1}, \Sigma}$ ). Thus, since in any EQL-Lite(UCQ) query there are only a finite set of epistemic atoms, and since each of its epistemic atoms $\mathbf{K}(\varrho)$ must be such that $\varrho$ is the body of a UCQ (and therefore, in $\varrho$ there are only a finite set of bodies of CQs), we can conclude that no maximally sound $\mathcal{S}$-to- $\mathcal{O}$ $\Sigma$-rewriting of $q_{\mathcal{S}}$ in the class of EQL-Lite(UCQ) queries exists, as required.

In the light of the above inexpressibility results, we explore two alternative special scenarios. In what follows, we will limit our attention to the DL ontology language $D L-L i t e_{\mathcal{R}}^{-}$, which is the fragment of $D L$-Lite $e_{\mathcal{R}}$ where disjointness assertions are disallowed. Observe that, for each OBDM specification $\Sigma=\langle\mathcal{O}, \mathcal{S}, \mathcal{M}\rangle$ where $\mathcal{O}$ is a $D L$-Lite $e_{\mathcal{R}}^{-}$ontology and $\mathcal{M}$ is a GLAV mapping, since inconsistencies can not 
arise, every $\mathcal{S}$-database $D$ is consistent with $\Sigma$. Thus, all the results we will present in the following hold even according to the semantics proposed in Lutz et al., 2018.

More specifically, in Section 9.3 we weaken the target query language by considering a fragment of the EQL-Lite(UCQ) query language that, notwithstanding, still enjoys a non-monotonic feature. In Section 9.4, instead, we weaken the mapping language by considering a special case of the GLAV approach that is incomparable with both the GAV, and the LAV approach.

\subsection{Source-to-Ontology Rewritings in a fragment of EQL-Lite(UCQ)}

In this section, we explore the possibility of expressing source-to-ontology rewritings where the target query language is a fragment, still non-monotonic, of the EQL-Lite(UCQ) query language considered so far.

In particular, while the proof of Theorem 9.3 shows that epistemic negation (even when not nested) already suffices to prevent the existence of maximally sound sourceto-ontology rewritings in the class of EQL-Lite(UCQ) queries (even with empty ontologies and pure GAV mappings), the proof of Theorem 9.2 suggests to remove the union (i.e., the rule $\psi::=\psi_{1} \vee \psi_{2}$ ) from the syntax of the EQL-Lite(UCQ) query language, in order to get a target query language $\mathcal{L}_{\mathcal{O}}$ ensuring the existence of minimally complete source-to-ontology rewritings in $\mathcal{L}_{\mathcal{O}}$. Thus, based on the observation that union can be expressed by means of conjunction and nested negation, we next consider the fragment of the EQL-Lite(UCQ) query language where both nested negation and union operator are disallowed.

Formally, an EQL-Lite ${ }^{-}$(UCQ) query over a DL ontology $\mathcal{O}$ is a query of the form $q_{\mathcal{O}}=\{\vec{t} \mid \psi(\vec{x})\}$, where the target list $\vec{t}$ is an $n$-tuple of terms, and the body $\psi(\vec{x})$ is a formula built according to the following syntax (we recall that the free variables occurring in formula $\psi(\vec{x})$ are exactly the variables occurring in $\vec{t}$ :

$$
\begin{array}{ll|l|l|l}
\psi & :=\mathbf{K} \varrho & \exists y \cdot \psi & \psi_{1} \wedge \psi_{2} & \mid \neg \delta \\
\delta & :=\mathbf{K} \varrho & \exists y . \delta & &
\end{array}
$$

The following example illustrates the $E Q L-L i t e^{-}(\mathrm{UCQ})$ query language.

Example 9.6. The queries $q_{\mathcal{O}}^{i}$, for $i \geq 0$, used in the proof of Theorem 9.3, as well as the queries $q_{\mathcal{O}}^{1}, q_{\mathcal{O}}^{2}$, and $q_{\mathcal{O}}^{3}$ illustrated in Example 9.1 are EQL-Lite ${ }^{-}(\mathrm{UCQ})$ queries. On the contrary, for any $i \geq 0$, the query $q_{\mathcal{O}}^{i}=\left\{() \mid \mathbf{K}(\varrho) \wedge \neg \mathbf{K}\left(\varrho_{0}\right) \wedge \neg \mathbf{K}\left(\varrho_{1}\right) \wedge\right.$ $\left.\ldots \wedge \neg \mathbf{K}\left(\varrho_{i}\right) \vee \mathbf{K}\left(\varrho^{\prime}\right)\right\}$ introduced in the proof of Theorem 9.2 is an EQL-Lite(UCQ) query but not an $E Q L-L_{i t e}-(\mathrm{UCQ})$ query.

Observe that, since all the queries over the ontology involved in the proof of Theorem 9.3 are EQL-Lite ${ }^{-}$(UCQ) queries, such proof in fact shows a stronger result than the one stated in the theorem: maximally sound source-to-ontology rewritings

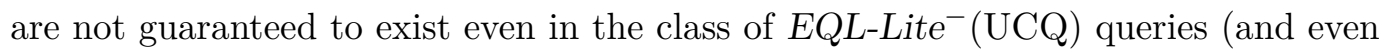
for OBDM specifications $\Sigma=\langle\mathcal{O}, \mathcal{S}, \mathcal{M}\rangle$ and data services $q_{\mathcal{S}}$ where $\mathcal{O}=\emptyset, \mathcal{M}$ is a pure GAV mapping, and $q_{\mathcal{S}}$ is a CQJFE over $\mathcal{S}$ ). 
It thus remains to study the minimally complete case. Specifically, we now provide the algorithm MinimallyCompleteEpistemic for computing minimally complete sourceto-ontology rewritings of CQs over the source schema when the target query language is the class of EQL-Lite ${ }^{-}(\mathrm{UCQ})$ queries. Thus proving that, for each pair composed by an OBDM specification $\Sigma=\langle\mathcal{O}, \mathcal{S}, \mathcal{M}\rangle$ where $\mathcal{O}$ is a $D L$-Lite $\mathcal{R}_{\mathcal{R}}^{-}$ontology and $\mathcal{M}$ is a GLAV mapping and a $\mathrm{CQ} q_{\mathcal{S}}$ over $\mathcal{S}$, the unique (up to equivalence w.r.t. $\Sigma$ ) minimally complete $\mathcal{S}$-to- $\mathcal{O} \Sigma$-rewriting of $q_{\mathcal{S}}$ in the class of $E Q L-L i t e^{-}$(UCQ) queries is guaranteed to exists.

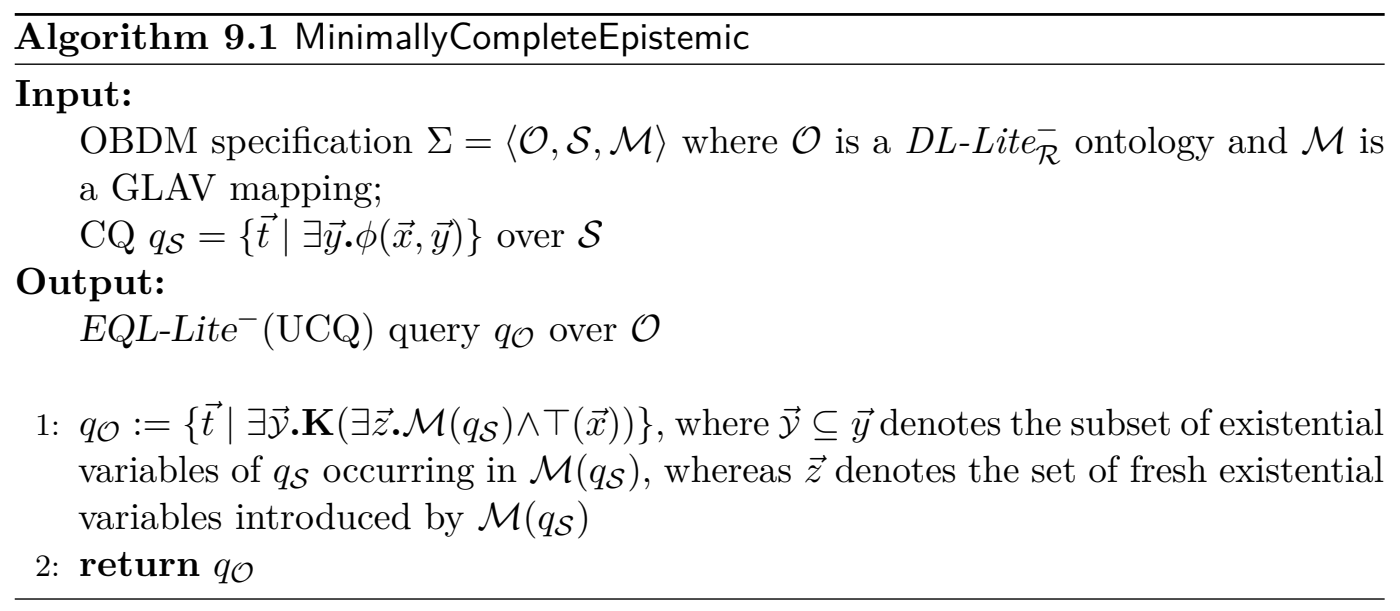

Roughly speaking, the algorithm computes an EQL-Lite ${ }^{-}$(UCQ) query $q_{\mathcal{O}}$ by first chasing (the incomplete $\mathcal{S}$-database associated to) $q_{\mathcal{S}}$ with respect to $\mathcal{M}$, using $\top$ to bind possible distinguished variables of $q_{\mathcal{S}}$ that are not involved in $\mathcal{M}\left(q_{\mathcal{S}}\right)$, and then using the epistemic operator to bind existential variables coming from $q_{\mathcal{S}}$. Note, in particular, that the latter is achieved by pushing the subset $\overrightarrow{\mathcal{Y}}$ of the existential variables $\vec{y}$ of $q_{\mathcal{S}}$ occurring in $\mathcal{M}\left(q_{\mathcal{S}}\right)$ inside the $\mathbf{K}$ operator. Finally, $\vec{z}$ denotes the set of fresh existential variables introduced by the chase.

Example 9.7. Let $\Sigma=\langle\mathcal{O}, \mathcal{S}, \mathcal{M}\rangle$ be the following OBDM specification:

- $\mathcal{O}=\emptyset$

- $\mathcal{S}=\left\{s_{1}, s_{2}, s_{3}\right\}$

- $\mathcal{M}=\left\{m_{1}, m_{2}, m_{3}\right\}$, where:

$$
\begin{array}{rrrl}
m_{1}: & \exists y \cdot s_{1}\left(x_{1}, x_{2}\right) \wedge s_{2}\left(x_{2}, y\right) & \rightarrow \exists z \cdot P\left(x_{1}, z\right) \wedge P\left(z, x_{2}\right), \\
m_{2}: & \exists y_{1}, y_{2}, y_{3} \cdot s_{2}\left(x, y_{1}\right) \wedge s_{3}\left(y_{1}, y_{2}, y_{3}\right) & \rightarrow \exists z \cdot P^{\prime}(x, z), \\
m_{3}: & \exists y \cdot s_{3}\left(y, x, c_{1}\right) & \rightarrow A(x) .
\end{array}
$$

Let the data service be the CQ $q_{\mathcal{S}}=\left\{\left(x_{1}, x_{2}\right) \mid \exists y_{1}, y_{2} \cdot s_{1}\left(x_{1}, y_{1}\right) \wedge s_{2}\left(y_{1}, y_{2}\right) \wedge\right.$ $\left.s_{3}\left(y_{2}, x_{2}, c_{2}\right)\right\}$ over $\mathcal{S}$. One can verify that MinimallyCompleteEpistemic $\left(\Sigma, q_{\mathcal{S}}\right)$ returns the EQL-Lite ${ }^{-}(\mathrm{UCQ})$ query $q_{\mathcal{O}}=\left\{\left(x_{1}, x_{2}\right) \mid \exists y_{1} \cdot \mathbf{K}\left(\exists z_{1}, z_{2} \cdot P\left(x_{1}, z_{1}\right) \wedge P\left(z_{1}, y_{1}\right) \wedge\right.\right.$ $\left.\left.P^{\prime}\left(y_{1}, z_{2}\right) \wedge \top\left(x_{2}\right)\right)\right\}$, which corresponds to the unique (up to equivalence w.r.t. $\Sigma$ ) minimally complete $\mathcal{S}$-to- $\mathcal{O} \Sigma$-rewriting in the class of EQL-Lite ${ }^{-}$(UCQ) queries. 
It is worth noting that, even though the " $\neg$ " operator is available in the EQL-Lite (UCQ) query language, the queries returned by the MinimallyCompleteEpistemic algorithm are free from this operator. The EQL-Lite(UCQ) queries without occurrences of the " $\neg$ " operator enjoy the following interesting and useful property:

Proposition 9.3. Let $\Sigma=\langle\mathcal{O}, \mathcal{S}, \mathcal{M}\rangle$ be an $O B D M$ specification, and let $q_{\mathcal{O}}$ be an EQL-Lite $(U C Q)$ query over $\mathcal{O}$ without occurrences of the " $\neg$ " operator. Then, the query $E Q L P e r f R e f_{q_{\mathcal{O}}, \Sigma}$ over $\mathcal{S}$ can be always expressed as an equivalent UCQ.

Proof. As illustrated in Subsection 9.1.1, the query EQLPerfRef $q_{\mathcal{O}}, \Sigma$ is obtained from $q_{\mathcal{O}}$ by replacing each of its epistemic atoms $\mathbf{K} \varrho_{i}$ with the logical body of the UCQ PerfRef $_{q_{\mathcal{O}}^{\varrho_{i}, \Sigma}}$ over $\mathcal{S}$, where $\varrho_{i}$ and $q_{\mathcal{O}}^{\varrho_{i}}$ are the body of a UCQ over $\mathcal{O}$ and the UCQ over $\mathcal{O}$ associated to $\varrho_{i}$, respectively. Thus, since by assumption in $q_{\mathcal{O}}$ there are no occurrences of the " $\neg$ " operator, and since it is so even in PerfRef $q_{q_{\mathcal{O}}^{e_{i}, \Sigma}}$ for each epistemic atom $\mathbf{K} \varrho_{i}$ occurring in $q_{\mathcal{O}}$, we have that $\mathrm{EQLPerfRe} \mathrm{f}_{q_{\mathcal{O}}, \Sigma}$ is a FOL query whose operators occurring in its body are only $\wedge, \vee$, and $\exists$. But then, it is trivial to see that a FOL query with only such operators occurring in its body can be always expressed as an equivalent UCQ, as required.

Moreover, the EQL-Lite ${ }^{-}$(UCQ) queries of the same shape as those returned by the MinimallyCompleteEpistemic algorithm further enjoy the following property:

Proposition 9.4. Let $\Sigma=\langle\mathcal{O}, \mathcal{S}, \mathcal{M}\rangle$ be an $O B D M$ specification, $D$ be an $\mathcal{S}$-database consistent with $\Sigma$, and $q_{\mathcal{O}}$ be an $E Q L-L_{i t e}{ }^{-}(U C Q)$ query $q_{\mathcal{O}}$ over $\mathcal{O}$ of the form $q_{\mathcal{O}}=\{\vec{t} \mid \exists \overrightarrow{\mathcal{Y}} \cdot \mathbf{K}(\phi)\}$, where $\phi$ is the body of a $C Q$. We have that $\vec{c} \in \operatorname{cert}_{q_{\mathcal{O}}, \Sigma}^{D}$ if and only if there is a function $h$ from the set of terms occurring in $\phi$ to the set of terms occurring in $\mathcal{C}_{\mathcal{O}}^{\mathcal{M}(D)}$ for which (i) $h(\mathcal{Y})$ is a constant, for each $\mathcal{Y} \in \overrightarrow{\mathcal{Y}}$; (ii) $h(c)=c$, for each constant $c$; (iii) $h(\phi) \subseteq \mathcal{C}_{\mathcal{O}}^{\mathcal{M}(D)}$, where $h(\phi)$ is the image of $\phi$ under $h$; and finally (vi) $h(\vec{t})=\vec{c}$ ( $h$ is also called a homomorphism from $q_{\mathcal{O}}$ to $\mathcal{C}_{\mathcal{O}}^{\mathcal{M}(D)}$ for which $h(\mathcal{Y})$ is a constant, for each $\mathcal{Y} \in \overrightarrow{\mathcal{Y}}$, and $h(\vec{t})=\vec{c})$.

Proof. We observe that $(i i),(i i i)$, and (vi) are the necessary and sufficient conditions that a CQ $q$ over $\mathcal{O}$ of the form $q=\{\vec{t} \mid \phi\}$ (where $\phi$ is the body of a CQ) must satisfy to be such that $\vec{c} \in \operatorname{cert}_{q, \Sigma}^{D}$ for a tuple of constants $\vec{c}$ (cf. Chapter 2).

We further observe that, by combining results of Fagin et al., 2005a, Proposition 4.2] with Calvanese et al., 2007b, Theorem 29], it is well-known that there is a homomorphism from $\mathcal{C}_{\mathcal{O}}^{\mathcal{M}(D)}$ to $\mathcal{I}$ (where this latter seen as a set of facts over $\mathcal{O}$ ), for each model $\mathcal{I}=\left\langle\Delta^{\mathcal{I}},{ }^{\mathcal{I}}\right\rangle$ of $\langle\Sigma, D\rangle$ (i.e., for each interpretation $\mathcal{I} \in \operatorname{Mod}_{D}(\Sigma)$ ).

From the above observations, and due to the semantic meaning of the existential variables occurring outside the epistemic operator $\mathbf{K}$ of an $E Q L$ formula, the claim of the proposition can be easily verified.

By exploiting the above results, we are now ready to establish termination and correctness of the MinimallyCompleteEpistemic algorithm.

Theorem 9.4. Let $\Sigma=\langle\mathcal{O}, \mathcal{S}, \mathcal{M}\rangle$ be an OBDM specification where $\mathcal{O}$ is a DL-Lite $\overline{\mathcal{R}}^{-}$ ontology and $\mathcal{M}$ is a GLAV mapping, and let $q_{\mathcal{S}}$ be a $C Q$ over $\mathcal{S}$. We have that MinimallyCompleteEpistemic $\left(\Sigma, q_{\mathcal{S}}\right)$ terminates and returns the unique (up to equivalence w.r.t. $\Sigma$ ) minimally complete $\mathcal{S}$-to- $\mathcal{O} \Sigma$-rewriting of $q_{\mathcal{S}}$ in the class of EQL-Lite $^{-}(U C Q)$ queries. 
Proof. Analogously to the MinimallyComplete algorithm illustrated in Chapter 5 , the termination of the MinimallyCompleteEpistemic algorithm easily follows from the termination of the chase of a source instance (possibly containing variables) with respect to a GLAV mapping, or, equivalently, with respect to a set of source-to-target tgds Fagin et al., 2005a.

As for the correctness, we first show that the query $q_{\mathcal{O}}=\left\{\vec{t} \mid \exists \overrightarrow{\mathcal{Y}} \cdot \mathbf{K}\left(\exists \vec{z} \cdot \mathcal{M}\left(q_{\mathcal{S}}\right) \wedge\right.\right.$ $\top(\vec{x}))\}$ returned by the algorithm is a complete $\mathcal{S}$-to- $\mathcal{O} \Sigma$-rewriting of $q_{\mathcal{S}}$. Observe that the possibly introduced fresh existential variables in $\vec{z}$ do not appear outside the epistemic operator $\mathbf{K}$, and therefore the CQ $q_{\mathcal{S}}$ corresponds to, or it is contained in, a disjunct of EQLPerfRef $q_{\mathcal{O}}, \Sigma$ (observe that, due to Proposition 9.3, the query EQLPerfRef $_{q_{\mathcal{O}}, \Sigma}$ can be expressed as an equivalent UCQ). Thus, due to Corollary 9.1 the fact that $q_{\mathcal{S}} \sqsubseteq$ EQLPerfRef $_{q_{\mathcal{O}}, \Sigma}$ implies that $q_{\mathcal{O}}$ is a complete $\mathcal{S}$-to- $\mathcal{O} \Sigma$-rewriting of $q_{\mathcal{S}}$. We now show that $q_{\mathcal{O}}$ is actually the unique (up to equivalence w.r.t. $\Sigma$ ) minimally complete $\mathcal{S}$-to- $\mathcal{O} \Sigma$-rewriting of $q_{\mathcal{S}}$ in the class of EQL-Lite ${ }^{-}$(UCQ) queries, that is, each $E Q L-L_{i t e}{ }^{-}(\mathrm{UCQ})$ query $q_{\mathcal{O}}^{\prime}$ that is a complete $\mathcal{S}$-to- $\mathcal{O} \Sigma$ rewriting of $q_{\mathcal{S}}$ is such that $\operatorname{cert}_{q_{\mathcal{O}}, \Sigma} \sqsubseteq \operatorname{cert}_{q_{\mathcal{O}}^{\prime}, \Sigma}$ (cf. Definition 3.7). We do this by way of contradiction.

Let $q_{\mathcal{O}}^{\prime}$ be an $E Q L-L_{i t e}{ }^{-}(\mathrm{UCQ})$ query such that $\operatorname{cert}_{q_{\mathcal{O}}, \Sigma} \nsubseteq \operatorname{cert}_{q_{\mathcal{O}}^{\prime}, \Sigma}$, that is, there exists an $\mathcal{S}$-database $D$ consistent with $\Sigma$ such that $\operatorname{cert}_{q_{\mathcal{O}}, \Sigma}^{D} \nsubseteq \operatorname{cert}_{q_{\mathcal{O}}^{\prime}, \Sigma}^{D}$. It follows that there is a tuple of constant $\vec{c}=\left(c_{1}, \ldots, c_{n}\right)$ such that $\vec{c} \notin \operatorname{cert}_{q_{\mathcal{O}}^{\prime}, \Sigma}^{D}$, but $\vec{c} \in \operatorname{cert}_{q_{\mathcal{O}}, \Sigma}^{D}$. Since $q_{\mathcal{O}}^{\prime}$ is an EQL-Lite $(\mathrm{UCQ})$, there are two possible cases: either $q_{\mathcal{O}}^{\prime}$ contains a part of the form $\neg \delta$ (with $\delta \not \equiv \perp$ ) in its body, or not.

In the former case, consider the $\mathcal{S}$-database $D^{\prime} \supseteq D$ in which each source predicate $s \in \mathcal{S}$ contains all possible tuples of constants occurring in $D$ whose arity is the one of $s$. Obviously, we have that $\vec{c} \in q_{\mathcal{S}}^{D^{\prime}}$, and, since $\mathcal{O}$ is a $D L$-Lite $e_{\mathcal{R}}^{-}$ontology, $D^{\prime}$ is consistent with $\Sigma$ (i.e., $\operatorname{Mod}_{D}(\Sigma) \neq \emptyset$ ). Furthermore, by construction of $D^{\prime}$ and the fact that in the EQL-Lite ${ }^{-}$UCQ) query language nested negation is disallowed (i.e., the $E Q L$ formula $\delta$ occurring in the body of $q_{\mathcal{O}}^{\prime}$ must be of the form $\exists \vec{y} \cdot \mathbf{K}(\varrho)$, where $\varrho$ is the body of a UCQ), we have that the formula $\delta$ occurring in the body of $q_{\mathcal{O}}^{\prime}$ is $E Q L$-logically implied by $\left\langle\Sigma, D^{\prime}\right\rangle$ (and thus, formula $\neg \delta$ is not EQL-logically implied by $\left.\left\langle\Sigma, D^{\prime}\right\rangle\right)$ when replacing the free variables of $q_{\mathcal{O}}^{\prime}$ with any tuple of constants. Since by construction of the EQL-Lite ${ }^{-}$(UCQ) query language formula $\neg \delta$ in $q_{\mathcal{O}}^{\prime}$ occurs either alone or in conjunction to a subformula of $q_{\mathcal{O}}^{\prime}$, this clearly implies that $q_{\mathcal{O}}^{\prime}$ is not EQL-logically implied by $\left\langle\Sigma, D^{\prime}\right\rangle$ when replacing the free variables of $q_{\mathcal{O}}^{\prime}$ with any tuple of constants, and therefore also for the tuple of constants $\vec{c}$. As a result, we have that $\vec{c} \notin \operatorname{cert}_{q_{\mathcal{O}}^{\prime}, \Sigma}^{D^{\prime}}$. But then, the facts that $\vec{c} \in q_{\mathcal{S}}^{D^{\prime}}$ and $\vec{c} \notin \operatorname{cert} t_{q_{\mathcal{O}}^{\prime}, \Sigma}^{D^{\prime}}$ imply that $q_{\mathcal{O}}^{\prime}$ is not a complete $\mathcal{S}$-to- $\mathcal{O} \Sigma$-rewriting of $q_{\mathcal{S}}$, as required.

Consider the latter case, that is, the EQL-Lite ${ }^{-}(\mathrm{UCQ})$ query $q_{\mathcal{O}}^{\prime}$ is of the form $q_{\mathcal{O}}^{\prime}=\left\{\overrightarrow{t^{\prime}} \mid \exists \overrightarrow{y^{\prime}} \cdot \mathbf{K}\left(\varrho_{1}\right) \wedge \ldots \wedge \mathbf{K}\left(\varrho_{m}\right)\right\}$ with $\varrho_{i}$ being the body of a UCQ, for each $i \in[1, m]$. In this case, it is possible to proceed in a similar way to the proof of Theorem 5.2. Consider the freezing of $q_{\mathcal{S}}=\{\vec{t} \mid \exists \vec{y} \cdot \phi(\vec{x}, \vec{y})\}$, i.e., the set $D^{\prime}$ of all facts over $\mathcal{S}$ obtained from $\phi(\vec{x}, \vec{y})$ by replacing each variable $v \in \vec{x} \cup \vec{y}$ with a different fresh constant denoted by $c_{v}$. Let, moreover, $\overrightarrow{c^{\prime}}=\left(c_{1}^{\prime}, \ldots, c_{n}^{\prime}\right)$ be the freezed tuple of constants where, for each $i \in[1, n], c_{i}^{\prime}=t_{i}$ if $t_{i}$ is a constant, and $c_{i}^{\prime}=c_{x}$ if $t_{i}=x$ (i.e., $t_{i} \in \vec{x}$ is a distinguished variable). Obviously, we have that $\overrightarrow{c^{\prime}} \in q_{\mathcal{S}}^{D^{\prime}}$ 
holds by construction, and, since $\mathcal{O}$ is a $D L$-Lite $e_{\mathcal{R}}^{-}$ontology, $D^{\prime}$ is consistent with $\Sigma$ (i.e., $\operatorname{Mod}_{D^{\prime}}(\Sigma) \neq \emptyset$ ). We now prove that $\overrightarrow{c^{\prime}} \notin \operatorname{cert} t_{q_{\mathcal{O}}^{\prime}, \Sigma}^{D^{\prime}}$.

Consider $\mathcal{C}_{\mathcal{O}}^{\mathcal{M}(D)}$, i.e., the canonical structure of $\mathcal{O}$ with respect to $\mathcal{M}$ and $D$. Since $\vec{c} \in \operatorname{cert}_{q_{\mathcal{O}}, \Sigma}^{D}$, due to Proposition 9.4, we derive that there exists a homomorphism $h$ from $q_{\mathcal{O}}$ to $\mathcal{C}_{\mathcal{O}}^{\mathcal{M}(D)}$ for which $(i) h(\mathcal{Y})$ is a constant, for each $\mathcal{Y} \in \overrightarrow{\mathcal{Y}}$, and $(i i) h(\vec{t})=\vec{c}$. Due to the facts that $\mathcal{M}$ is a GLAV mapping and $\mathcal{O}$ is a DL-Lite $e_{\mathcal{R}}^{-}$ontology, and due to the existence of such homomorphism $h$, by construction of $q_{\mathcal{O}}$ and $D^{\prime}$ it is easy to see the existence of a function $f$ from $\mathcal{C}_{\mathcal{O}}^{\mathcal{M}\left(D^{\prime}\right)}$ to $\mathcal{C}_{\mathcal{O}}^{\mathcal{M}(D)}$ for which $(i)$ $f(c \mathcal{Y})=h(\mathcal{Y})$, for each $\mathcal{Y} \in \overrightarrow{\mathcal{Y}}$ (recall that $\overrightarrow{\mathcal{Y}} \subseteq \vec{y}$ is the subset of existential variables of $q_{\mathcal{S}}$ occurring in $\mathcal{M}\left(q_{\mathcal{S}}\right)$ ), $(i i) f(c)=h(c)=c$, for each constant $c$ occurring in $q_{\mathcal{O}}$, (iii) $f\left(c_{x}\right)=h(x)$, for each distinguished variable $x \in \vec{x}$ of $q_{\mathcal{S}}$ occurring in $\mathcal{M}\left(q_{\mathcal{S}}\right)$, and $(v i) f\left(\mathcal{C}_{\mathcal{O}}^{\mathcal{M}\left(D^{\prime}\right)}\right) \subseteq \mathcal{C}_{\mathcal{O}}^{\mathcal{M}(D)}$, where $f\left(\mathcal{C}_{\mathcal{O}}^{\mathcal{M}\left(D^{\prime}\right)}\right)$ is the image of $\mathcal{C}_{\mathcal{O}}^{\mathcal{M}\left(D^{\prime}\right)}$ under $f$. Observe that $f\left(\overrightarrow{c^{\prime}}\right)=\vec{c}$ by construction.

Furthermore, by exploiting again Proposition 9.4, it is easy to verify that, since $\vec{c} \notin \operatorname{cert}_{q_{\mathcal{O}}^{\prime}, \Sigma}^{D}$ by assumption, then there is some epistemic atom $\mathbf{K}\left(\varrho_{i}\right)$ in the body of $q_{\mathcal{O}}^{\prime}$ such that for no disjunct $\phi$ in $\varrho_{i}$ there is a homomorphism $h$ from $\phi$ to $\mathcal{C}_{\mathcal{O}}^{\mathcal{M}(D)}$ for which $(i) h\left(y^{\prime}\right)$ is a constant, for each $y^{\prime} \in \overrightarrow{y^{\prime}}$ occurring in $\phi$, and $(i i)$ $h\left(\overrightarrow{t^{\prime}}\right)=\vec{c}$. But then, due to the existence of the function $f$, and due to the above consideration, we derive that there there is no disjunct $\phi$ in $\varrho_{i}$ such that that there is a homomorphism $h^{\prime}$ from $\phi$ to $\mathcal{C}_{\mathcal{O}}^{\mathcal{M}\left(D^{\prime}\right)}$ for which $(i) h^{\prime}\left(y^{\prime}\right)$ is a constant, for each $y^{\prime} \in \overrightarrow{y^{\prime}}$ occurring in $\phi$, and $(i i) h^{\prime}\left(\overrightarrow{t^{\prime}}\right)=\overrightarrow{c^{\prime}}$ (otherwise, the composition function $f \circ h^{\prime}$ would result in a homomorphism from $\phi$ to $\mathcal{C}_{\mathcal{O}}^{\mathcal{M}(D)}$ for which $(i) f\left(h\left(y^{\prime}\right)\right)$ is a constant, for each $y^{\prime} \in \overrightarrow{y^{\prime}}$ occurring in $\phi$, and $(i i) f\left(h^{\prime}\left(\overrightarrow{t^{\prime}}\right)\right)=\vec{c}$, and therefore this would contradict the assumption that for no disjunct $\phi$ in $\varrho_{i}$ there is such a homomorphism). Using Proposition 9.4, it can be easily proven that this implies that $\overrightarrow{c^{\prime}} \notin \operatorname{cert}_{q_{\mathcal{O}}^{\prime}, \Sigma}^{D^{\prime}}$. To conclude the proof, observe that $D^{\prime}$ is an $\mathcal{S}$-database consistent with $\Sigma$ for which $\overrightarrow{c^{\prime}} \in q_{\mathcal{S}}^{D^{\prime}}$ and $\overrightarrow{c^{\prime}} \notin \operatorname{cert} t_{q_{\mathcal{O}}^{\prime}, \Sigma}^{D^{\prime}}$, thus implying that $q_{\mathcal{O}}^{\prime}$ is not a complete $\mathcal{S}$-to- $\mathcal{O} \Sigma$-rewriting of $q_{\mathcal{S}}$, as required.

As for the running time of the MinimallyCompleteEpistemic algorithm, we observe that it is independent of both the size of $\mathcal{O}$ and $\mathcal{S}$, and, due to the application of the chase, it is polynomial in the size of $q_{\mathcal{S}}$ and exponential in the size of $\mathcal{M}$.

It remains to address the case of perfect source-to-ontology rewritings in the class of EQL-Lite ${ }^{-}$UCQ) queries. Consider any pair composed by an OBDM specification $\Sigma=\langle\mathcal{O}, \mathcal{S}, \mathcal{M}\rangle$ where $\mathcal{O}$ is a $D L-L i t e_{\mathcal{R}}^{-}$ontology and $\mathcal{M}$ is a GLAV mapping and a $\mathrm{CQ} q_{\mathcal{S}}$ over $\mathcal{S}$. Clearly, by definition, either the unique (up to equivalence w.r.t. $\Sigma$ ) minimally complete $\mathcal{S}$-to- $\mathcal{O} \Sigma$-rewriting of $q_{\mathcal{S}}$ in the class of $E Q L$-Lite ${ }^{-}(\mathrm{UCQ}$ ) queries is also a sound, and therefore a perfect, $\mathcal{S}$-to- $\mathcal{O} \Sigma$-rewriting of $q_{\mathcal{S}}$, or no perfect $\mathcal{S}$-to- $\mathcal{O} \Sigma$-rewriting of $q_{\mathcal{S}}$ in the class of $E Q L-L i t e^{-}(\mathrm{UCQ})$ queries exists.

With this observation at hand, we can easily derive the algorithm PerfectEpistemic together with its termination and correctness.

Essentially, the algorithm first computes the query $q_{\mathcal{O}}$ which is the unique (up to equivalence w.r.t. $\Sigma$ ) minimally complete $\mathcal{S}$-to- $\mathcal{O} \Sigma$-rewriting of $q_{\mathcal{S}}$ in the class of EQL-Lite ${ }^{-}$(UCQ) queries, and then, by exploiting Corollary 9.1, checks 


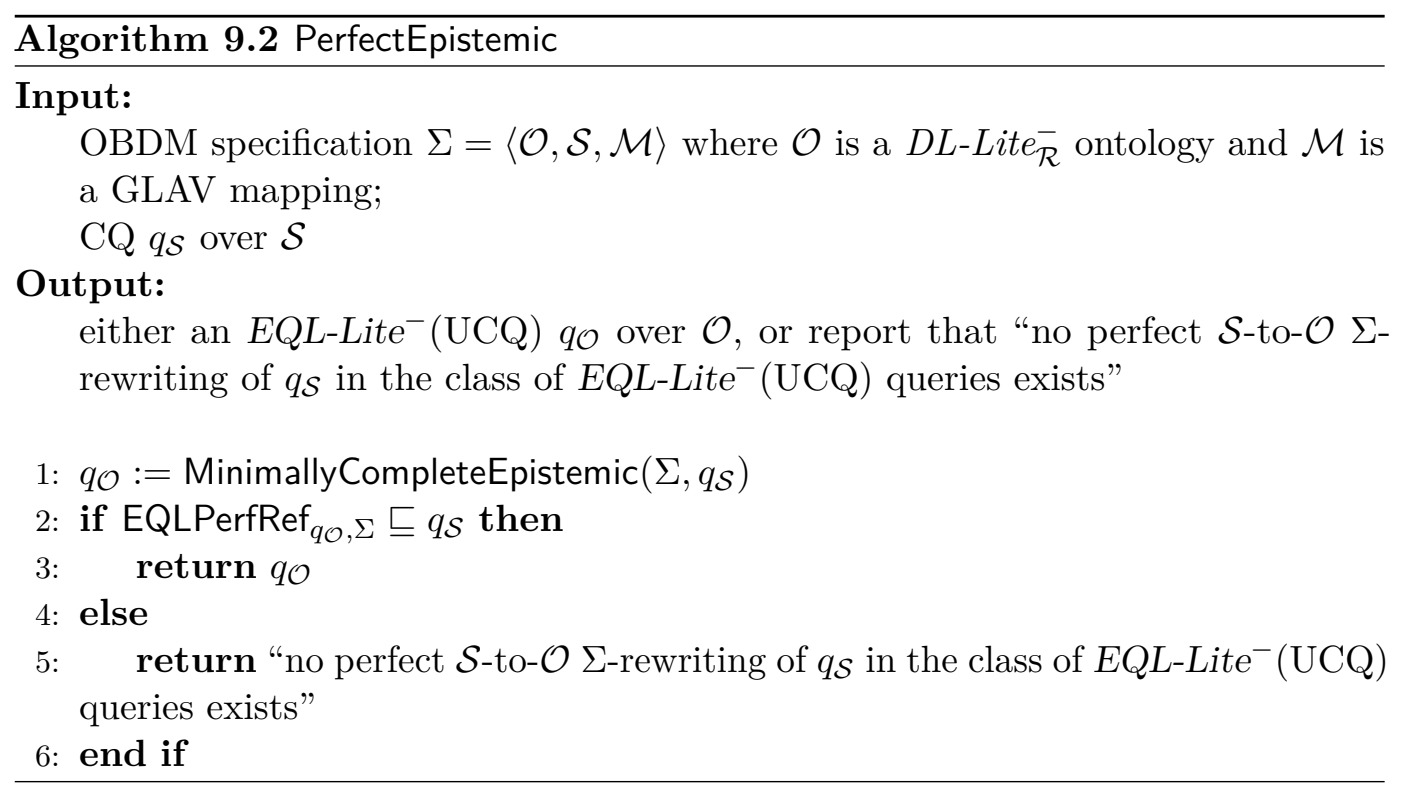

whether $q_{\mathcal{O}}$ is also a sound, and therefore a perfect, $\mathcal{S}$-to- $\mathcal{O} \Sigma$-rewriting of $q_{\mathcal{S}}$ via the following query containment check: EQLPerfRef $q_{\mathcal{O}}, \Sigma \sqsubseteq q_{\mathcal{S}}$ (note that $\mathcal{V}_{\mathcal{O}} \equiv \perp$ for each $D L$-Lite $e_{\mathcal{R}}^{-}$ontology $\left.\mathcal{O}\right)$. We point out that the above containment is actually a containment of UCQs. Indeed, on the one hand, the query $q_{\mathcal{S}}$ is a CQ, and, on the other hand, for each OBDM specification $\Sigma=\langle\mathcal{O}, \mathcal{S}, \mathcal{M}\rangle$ and EQL-Lite(UCQ) query $q_{\mathcal{O}}$ over $\mathcal{O}$ without any occurrence of the " $\neg$ " operator (as all those returned by the MinimallyCompleteEpistemic algorithm), the FOL query EQLPerfRef ${ }_{q_{\mathcal{O}}, \Sigma}$ can be in fact expressed as a UCQ. This allows us to conclude that the overall running time of the PerfectEpistemic algorithm is exponential in the size of the input.

Theorem 9.5. Let $\Sigma=\langle\mathcal{O}, \mathcal{S}, \mathcal{M}\rangle$ be an $O B D M$ specification where $\mathcal{O}$ is a DL-Lite $\overline{\mathcal{R}}^{-}$ ontology and $\mathcal{M}$ is a GLAV mapping, and let $q_{\mathcal{S}}$ be a $C Q$ over $\mathcal{S}$. We have that PerfectEpistemic $\left(\Sigma, q_{\mathcal{S}}\right)$ terminates and returns the perfect $\mathcal{S}$-to- $\mathcal{O} \Sigma$-rewriting of $q_{\mathcal{S}}$ if it exists and can be expressed as an EQL-Lite $(U C Q)$ query, otherwise it reports that no perfect $\mathcal{S}$-to- $\mathcal{O}$ $\Sigma$-rewriting of $q_{\mathcal{S}}$ in the class of $E Q L-L i t e^{-}(U C Q)$ queries exists.

\subsection{The case of One-To-One Mappings}

In this section, we study the problem of computing source-to-ontology rewritings in the class of EQL-Lite(UCQ) queries, for the case of OBDM specifications where the mapping assertions are a special case of GLAV mapping assertions considered so far.

Specifically, we consider OBDM specifications where the mapping language follows the "One-to-One approach". A One-To-One mapping assertion is a GLAV mapping assertion $\forall \vec{x} \cdot(\exists \vec{y} \cdot \phi(\vec{x}, \vec{y}) \rightarrow \exists \vec{z} \cdot \varphi(\vec{x}, \vec{z}))$ in which both $\phi(\vec{x}, \vec{y})$ and $\varphi(\vec{x}, \vec{z})$ are simply atoms without constants or repeated variables. We say that a mapping $\mathcal{M}$ is a One-To-One mapping if it consists of a finite set of One-To-One mapping assertions. Observe that GAV (respectively, pure GAV), LAV, and One-To-One are pairwise incomparable approaches for specifying mappings. 
Example 9.8. The mapping $\mathcal{M}$ defined in Example 9.1 is a One-To-One mapping. Observe that the mapping assertion $m_{4} \in \mathcal{M}$ is neither a GAV, nor a LAV mapping assertion. The pure GAV mapping assertion $s_{1}(x) \wedge s_{2}(x) \rightarrow A(x)$ is neither a OneTo-One, nor a LAV mapping assertion, whereas the LAV mapping assertion $s_{1}(x) \rightarrow$ $\exists z \cdot P(x, z) \wedge A(z)$ is neither a One-To-One, nor a GAV mapping assertion.

We now present a property for One-To-One mappings, which is crucial for the technical treatment of this section 3

Proposition 9.5. Let $\mathcal{M}$ be a One-To-One mapping relating a schema $\mathcal{S}$ to an ontology $\mathcal{O}$. For each $\mathcal{S}$-database $D$, we have that $\mathcal{M}(D)=\bigcup_{\alpha \in D} \mathcal{M}(\alpha)$.

Proof. The claim trivially follows by considering that the left-hand side of each One-to-One mapping assertion is constituted simply by a single atom. Thus, in order to compute $\mathcal{M}(D)$ for a One-To-One mapping $\mathcal{M}$ and an $\mathcal{S}$-database $D$, it is sufficient to consider separately each fact $\alpha \in D$ and take the union of the sets of atoms $\mathcal{M}(\alpha)$, as required.

In what follows in this section, we implicitly assume that each mapping $\mathcal{M}$ relating a schema $\mathcal{S}$ to an ontology $\mathcal{O}$ is a One-To-One mapping.

\subsubsection{Complete Source-to-Ontology Rewritings}

Interestingly, we now prove that, when the mapping language follows the One-To-One approach, one can use the MinimallyCompleteEpistemic algorithm to compute also minimally complete source-to-ontology rewritings of CQs over the source schema when the target query language is the class of EQL-Lite(UCQ) queries, rather than its fragment of EQL-Lite ${ }^{-}(\mathrm{UCQ})$ queries considered in the previous section. Thus proving that, for each pair composed by an OBDM specification $\Sigma=\langle\mathcal{O}, \mathcal{S}, \mathcal{M}\rangle$ where $\mathcal{O}$ is a $D L$-Lite $e_{\mathcal{R}}^{-}$ontology and $\mathcal{M}$ is a One-To-One mapping and a CQ $q_{\mathcal{S}}$ over $\mathcal{S}$, the unique (up to equivalence w.r.t. $\Sigma$ ) minimally complete $\mathcal{S}$-to- $\mathcal{O} \Sigma$-rewriting of $q_{\mathcal{S}}$ in the class of EQL-Lite(UCQ) queries is guaranteed to exists and, moreover, it can be expressed as an EQL-Lite ${ }^{-}$(UCQ) query. Before delving into the technical part, we now illustrate its application within a scenario with a One-To-One mapping.

Example 9.9. Let $\Sigma=\langle\mathcal{O}, \mathcal{S}, \mathcal{M}\rangle$ be the following OBDM specification:

- $\mathcal{O}=\emptyset$

- $\mathcal{S}=\left\{s_{1}, s_{2}, s_{3}\right\}$

- $\mathcal{M}=\left\{m_{1}, m_{2}, m_{3}, m_{4}, m_{5}, m_{6}\right\}$, where:

$$
\begin{aligned}
& m_{1}: \quad s_{1}\left(x_{1}, x_{2}\right) \rightarrow P_{1}\left(x_{1}, x_{2}\right), \\
& m_{2}: \quad \exists y \cdot s_{2}(x, y) \rightarrow \exists z \cdot P_{2}(x, z), \\
& m_{3}: \quad \exists y_{1}, y_{2} \cdot s_{3}\left(x, y_{1}, y_{2}\right) \quad \rightarrow \quad A(x), \\
& m_{4}: \quad \exists y_{1}, y_{2}, y_{3} \cdot s_{3}\left(y_{1}, y_{2}, y_{3}\right) \quad \rightarrow \quad \exists z_{1}, z_{2} \cdot P_{3}\left(z_{1}, z_{2}\right) .
\end{aligned}
$$

\footnotetext{
${ }^{3}$ Note that Proposition 9.5 is valid not only for One-To-One mapping, but also for LAV mapping.
} 
Let the data service be the CQ $q_{\mathcal{S}}=\left\{(x) \mid \exists y_{1}, y_{2} \cdot s_{1}\left(x, y_{1}\right) \wedge s_{2}\left(x, y_{2}\right) \wedge\right.$ $\left.s_{3}\left(y_{1}, y_{1}, y_{2}\right)\right\}$ over $\mathcal{S}$. One can verify that MinimallyCompleteEpistemic $\left(\Sigma, q_{\mathcal{S}}\right)$ returns the EQL-Lite ${ }^{-}(\mathrm{UCQ})$ query $q_{\mathcal{O}}=\left\{(x) \mid \exists y_{1} \cdot \mathbf{K}\left(\exists z_{1}, z_{2}, z_{3} \cdot P_{1}\left(x, y_{1}\right) \wedge P_{2}\left(x, z_{1}\right) \wedge\right.\right.$ $\left.\left.A\left(y_{1}\right) \wedge P_{3}\left(z_{2}, z_{3}\right)\right)\right\}$, which corresponds to the unique (up to equivalence w.r.t. $\Sigma$ ) minimally complete $\mathcal{S}$-to- $\mathcal{O} \Sigma$-rewriting in the class of EQL-Lite(UCQ) queries.

Theorem 9.6. Let $\Sigma=\langle\mathcal{O}, \mathcal{S}, \mathcal{M}\rangle$ be an $O B D M$ specification where $\mathcal{O}$ is a DL-Lite $\overline{\mathcal{R}}^{-}$ ontology and $\mathcal{M}$ is a One-To-One mapping, and let $q_{\mathcal{S}}$ be a $C Q$ over $\mathcal{S}$. We have that MinimallyCompleteEpistemic $\left(\Sigma, q_{\mathcal{S}}\right)$ terminates and returns the unique (up to equivalence w.r.t. $\Sigma$ ) minimally complete $\mathcal{S}$-to-O $\mathcal{O}$-rewriting of $q_{\mathcal{S}}$ in the class of EQL-Lite $(U C Q)$ queries.

Proof. Termination of the algorithm, as well as the fact that it returns a complete $\mathcal{S}$-to- $\mathcal{O} \Sigma$-rewriting of $q_{\mathcal{S}}$ (in fact, the unique (up to equivalence w.r.t. $\Sigma$ ) minimally complete $\mathcal{S}$-to- $\mathcal{O} \Sigma$-rewriting of $q_{\mathcal{S}}$ in the class of $E Q L-L i t e^{-}$(UCQ) queries), has already been discussed in the proof of Theorem 9.4 for the more general case of when $\mathcal{M}$ is a GLAV mapping rather than a One-To-One mapping. We now show that, when $\mathcal{M}$ is a One-To-One mapping, the computed EQL-Lite ${ }^{-}(\mathrm{UCQ})$ query $q_{\mathcal{O}}$ is actually the unique (up to equivalence w.r.t. $\Sigma$ ) minimally complete $\mathcal{S}$-to- $\mathcal{O} \Sigma$-rewriting of $q_{\mathcal{S}}$ even in the class of EQL-Lite(UCQ) queries, that is, each EQL-Lite(UCQ) query $q_{\mathcal{O}}^{\prime}$ that is a complete $\mathcal{S}$-to- $\mathcal{O}$ s-rewriting of $q_{\mathcal{S}}$ is such that $\operatorname{cert}_{q_{\mathcal{O}}, \Sigma} \sqsubseteq \operatorname{cert}_{q_{\mathcal{O}}^{\prime}, \Sigma}$ (cf. Definition 3.7). We do this by way of contradiction.

Let $q_{\mathcal{O}}^{\prime}$ be an EQL-Lite(UCQ) query such that $\operatorname{cert}_{q_{\mathcal{O}}, \Sigma} \nsubseteq \operatorname{cert}_{q_{\mathcal{O}}^{\prime}, \Sigma}$, that is, there exists an $\mathcal{S}$-database $D$ consistent with $\Sigma$ such that $\operatorname{cert}_{q_{\mathcal{O}, \Sigma}}^{D} \nsubseteq \operatorname{cert}_{q_{\mathcal{O}}^{\prime}, \Sigma}^{D}$. It follows that there is a tuple of constant $\vec{c}$ such that $\vec{c} \notin \operatorname{cert}_{q_{\mathcal{O}}^{\prime}, \Sigma}^{D}$, but $\vec{c} \in \operatorname{cert}_{q_{\mathcal{O}}, \Sigma}^{D}$. We now exhibit an $\mathcal{S}$-database $D^{\prime}$ consistent with $\Sigma$ for which $(i) \vec{c} \in q_{\mathcal{S}}^{D^{\prime}}$, and $(i i) \mathcal{C}_{\mathcal{O}}^{\mathcal{M}\left(D^{\prime}\right)}$ and $\mathcal{C}_{\mathcal{O}}^{\mathcal{M}(D)}$ are homomorphically equivalent, i.e., there is a homomorphism from $\mathcal{C}_{\mathcal{O}}^{\mathcal{M}\left(D^{\prime}\right)}$ to $\mathcal{C}_{\mathcal{O}}^{\mathcal{M}(D)}$ and vice versa.

Since $\vec{c} \in \operatorname{cert}_{q_{\mathcal{O}}, \Sigma}^{D}$, due to Proposition 9.4 we derive that there is a homomorphism $h$ from $q_{\mathcal{O}}$ to $\mathcal{C}_{\mathcal{O}}^{\mathcal{M}(D)}$ for which $(i) h(\mathcal{Y})$ is a constant, for each $\mathcal{Y} \in \overrightarrow{\mathcal{Y}}$, and $(i i) h(\vec{t})=\vec{c}$. Let $h^{\prime}$ be the function extending $h$ by assigning a different fresh constant $c_{y}$ to each existential variable $y \in \vec{y} \backslash \overrightarrow{\mathcal{Y}}$ (i.e., to each existential variable $y \in \vec{y}$ of $q_{\mathcal{S}}$ not occurring in $\mathcal{M}\left(q_{\mathcal{S}}\right)$ ). Consider now $h^{\prime}\left(q_{\mathcal{S}}\right)$ and $h\left(q_{\mathcal{O}}\right) \subseteq \mathcal{C}_{\mathcal{O}}^{\mathcal{M}}{ }^{(D)}$, i.e., the set of facts corresponding to the image of (the body of) $q_{\mathcal{S}}$ under $h^{\prime}$ and the set of atoms corresponding to the image of (the body of) $q_{\mathcal{O}}$ under $h$, respectively (this latter can be a set of atoms because, for some existential variable $z \in \vec{z}$ of $q_{\mathcal{O}}, h(z)$ is allowed to be a variable of $\left.\mathcal{C}_{\mathcal{O}}^{\mathcal{M}(D)}\right)$. Since the left-hand side of each mapping assertion in $\mathcal{M}$ is a single atom without constants or repeated variables, and since $h\left(q_{\mathcal{O}}\right) \subseteq \mathcal{C}_{\mathcal{O}}^{\mathcal{M}(D)}$ with $h(\mathcal{Y})$ being a constant for each $\mathcal{Y} \in \overrightarrow{\mathcal{Y}}$, by construction of $q_{\mathcal{S}}$ and $q_{\mathcal{O}}$, it is not hard to ascertain that, for each fact $\alpha \in h^{\prime}\left(q_{\mathcal{S}}\right)$, the chase of fact $\alpha$ with respect to $\mathcal{M}$, i.e., $\mathcal{M}(\alpha)$, is such that there exists a homomorphism from $\mathcal{M}(\alpha)$ to $\mathcal{C}_{\mathcal{O}}^{\mathcal{M}(D)}$.

But then, the $\mathcal{S}$-database we are seeking is $D^{\prime}=D \cup h^{\prime}\left(q_{\mathcal{S}}\right)$. Indeed, on the one hand, $\vec{c} \in q_{\mathcal{S}}^{D^{\prime}}$ trivially holds because $(i) \vec{c} \in q_{\mathcal{S}}^{D}$, (ii) $D \subseteq D^{\prime}$, and (iii) $q_{\mathcal{S}}$ is a CQ. On the other hand, using Proposition 9.5 and the fact that there exists a homomorphism from $\mathcal{M}(\alpha)$ to $\mathcal{C}_{\mathcal{O}}^{\mathcal{M}(D)}$ for each $\alpha \in h^{\prime}\left(q_{\mathcal{S}}\right)$, we easily derive that $\mathcal{C}_{\mathcal{O}}^{\mathcal{M}\left(D^{\prime}\right)}$ and $\mathcal{C}_{\mathcal{O}}^{\mathcal{M}(D)}$ are homomorphically equivalent. 
To conclude the proof observe that, since $\mathcal{C}_{\mathcal{O}}^{\mathcal{M}\left(D^{\prime}\right)}$ and $\mathcal{C}_{\mathcal{O}}^{\mathcal{M}(D)}$ are homomorphically equivalent, and since $\vec{c} \notin$ cert $t_{q_{\mathcal{O}}^{\prime}, \Sigma}^{D}$ by assumption, it is easy to see that $\vec{c} \notin \operatorname{cert} t_{q_{\mathcal{O}}^{\prime}, \Sigma}^{D^{\prime}}$ as well. It follows that $D^{\prime}$ is an $\mathcal{S}$-database consistent with $\Sigma$ (observe that $\mathcal{O}$ is a $D L$-Lite $e_{\mathcal{R}}^{-}$ontology, and therefore each $\mathcal{S}$-database is consistent with $\Sigma$ ) for which $\vec{c} \in q_{\mathcal{S}}^{D^{\prime}}$ and $\vec{c} \notin \operatorname{cert}_{q_{\mathcal{O}}^{\prime}, \Sigma}^{D^{\prime}}$, thus implying that $q_{\mathcal{O}}^{\prime}$ is not a complete $\mathcal{S}$-to- $\mathcal{O} \Sigma$-rewriting of $q_{\mathcal{S}}$, as required.

The following result is an immediate consequence of the above theorem.

Corollary 9.2. Let $\Sigma=\langle\mathcal{O}, \mathcal{S}, \mathcal{M}\rangle$ be an $O B D M$ specification where $\mathcal{O}$ is a DL-Lite $e_{\mathcal{R}}^{-}$ ontology and $\mathcal{M}$ is a One-To-One mapping, and let $q_{\mathcal{S}}$ be a $C Q$ over $\mathcal{S}$. Then, the unique (up to equivalence w.r.t. $\Sigma$ ) minimally complete $\mathcal{S}$-to- $\mathcal{O} \Sigma$-rewriting of $q_{\mathcal{S}}$ in the class of EQL-Lite $(U C Q)$ queries can be expressed as an $E Q L-L i t e^{-}(U C Q)$ query.

Observe that, when dealing with One-To-One mapping assertions, the application of the chase is feasible in polynomial time even in the size of the mapping $\mathcal{M}$, and therefore the overall running time of the MinimallyCompleteEpistemic algorithm becomes polynomial in the size of the input when $\mathcal{M}$ is a One-To-One mapping.

\subsubsection{Sound Source-to-Ontology Rewritings}

We now investigate the maximally sound case. Specifically, for OBDM specifications with One-To-One mapping assertions, we now provide the algorithm MaximallySoundEpistemic for computing maximally sound source-to-ontology rewritings of CQJFEs over the source schema when the target query language is the class of EQL-Lite(UCQ) queries. Thus proving that, for each pair composed by an OBDM specification $\Sigma=\langle\mathcal{O}, \mathcal{S}, \mathcal{M}\rangle$ where $\mathcal{O}$ is a $D L$-Lite $e_{\mathcal{R}}^{-}$ontology and $\mathcal{M}$ is a One-To-One mapping and a CQJFE $q_{\mathcal{S}}$ over $\mathcal{S}$, the unique (up to equivalence w.r.t. $\Sigma$ ) maximally sound $\mathcal{S}$-to- $\mathcal{O} \Sigma$-rewriting of $q_{\mathcal{S}}$ in the class of $E Q L$-Lite(UCQ) queries is guaranteed to exists and, moreover, as for the minimally complete case, it can be expressed as an EQL-Lite ${ }^{-}$(UCQ) query.

In the algorithm, since $\mathcal{M}$ is a One-To-One mapping, when computing the reformulation EQLPerfRef $q_{\mathcal{O}}^{c}, \Sigma$ over $\mathcal{S}$ of the $E Q L-L i t e^{-}(\mathrm{UCQ})$ query $q_{\mathcal{O}}^{c}$, we can assume that $\operatorname{PerfRef}_{q_{\mathcal{O}}^{\varrho}, \Sigma}$ of the epistemic atom $\mathbf{K} \varrho$ occurring in $q_{\mathcal{O}}^{c}$ is obtained by first reformulating $q_{\mathcal{O}}^{\varrho}$ with respect to $\mathcal{O}$, and then by unfolding the resulting UCQ with respect to $\mathcal{M}$ (with the proviso that, when unfolding an atom $\beta$ over $\mathcal{O}$, if a mapping assertion $m$ is such that the $k$-th argument of the atom in its right-hand side is an existential variable whilst the $k$-th argument of $\beta$ is either a distinguished variable or a constant, then $m$ have to be ignored). Furthermore, since the " $\neg$ " operator never occurs in the EQL-Lite ${ }^{-}(\mathrm{UCQ})$ query $q_{\mathcal{O}}^{c}$ of the algorithm, we can implicitly assume that its reformulation $\mathrm{EQLPerfRef} q_{q_{\mathcal{O}}^{c}, \Sigma}$ over $\mathcal{S}$ is first computed adhering to the above procedure, and then turned into an equivalent UCQ (cf. Proposition 9.3).

In a nutshell, the MaximallySoundEpistemic algorithm starts by checking whether there is some distinguished variable of $q_{\mathcal{S}}$ not appearing in $\mathcal{M}\left(q_{\mathcal{S}}\right)$, and if this is the case, then it returns the query $\{\vec{t} \mid \perp(\vec{x})\}$. Otherwise, the algorithm first computes the query $q_{\mathcal{O}}^{c}=\{\vec{t} \mid \psi(\vec{x})\}$, where $\psi(\vec{x})=\exists \overrightarrow{\mathcal{Y}} \cdot \mathbf{K}\left(\exists \vec{z} \cdot \mathcal{M}\left(q_{\mathcal{S}}\right)\right)$, which corresponds to the minimally complete $\mathcal{S}$-to- $\mathcal{O} \Sigma$-rewriting of $q_{\mathcal{S}}$ in the class of EQL-Lite(UCQ) queries, and then, for each disjunct $q_{\mathcal{S}}^{i}$ in its reformulation EQLPerfRef $q_{\mathcal{O}}^{c}, \Sigma$ over $\mathcal{S}$ such that 


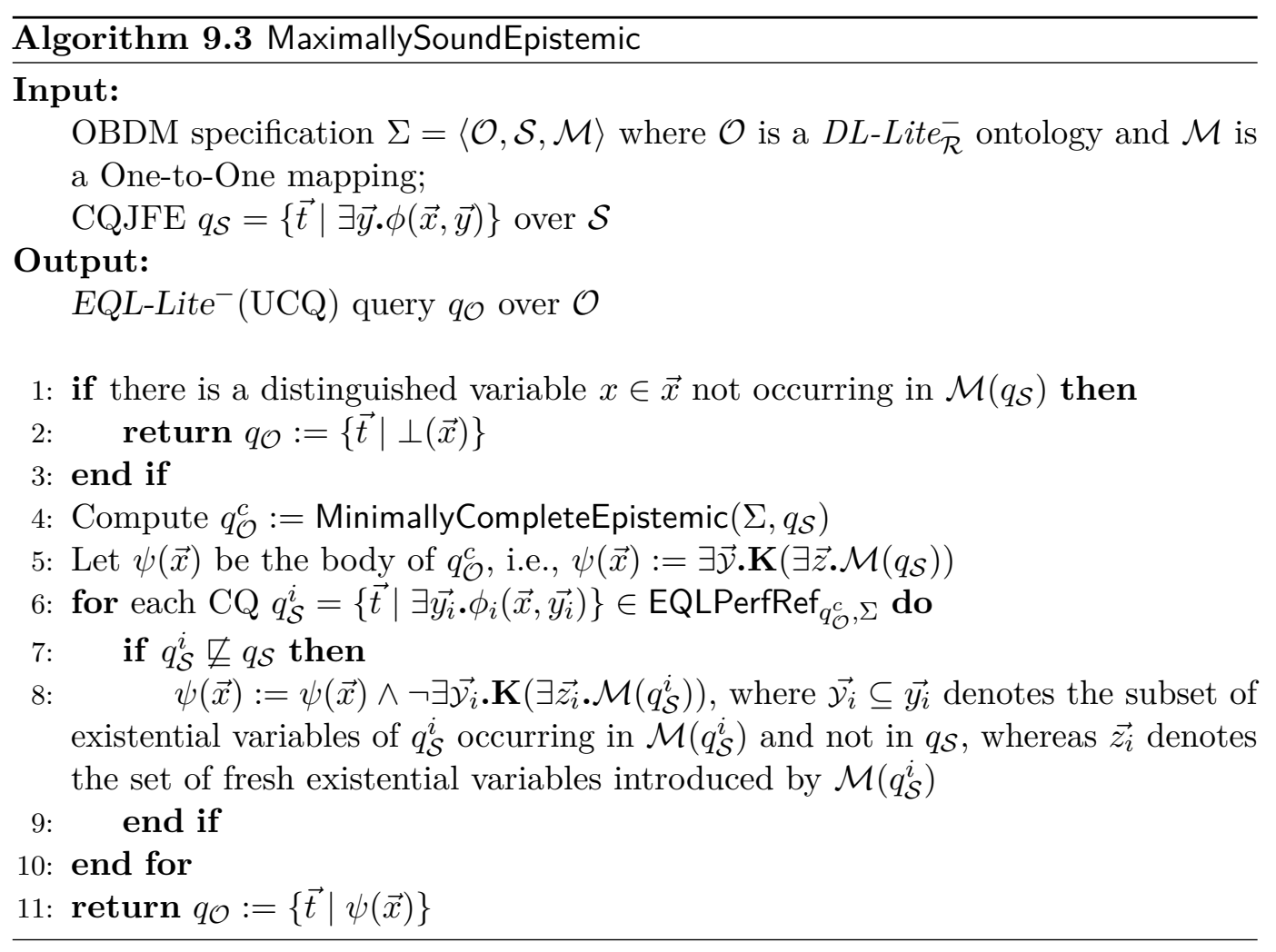

$q_{\mathcal{S}}^{i} \nsubseteq q_{\mathcal{S}}$, it adds in conjunction to $\psi(\vec{x})$ the negation of the minimally complete $\mathcal{S}$ to- $\mathcal{O} \Sigma$-rewriting of $q_{\mathcal{S}}^{i}$ in the class of EQL-Lite(UCQ) queries. Intuitively, by doing so, for each $\mathcal{S}$-database $D$, the algorithm prevents the returned EQL-Lite ${ }^{-}(\mathrm{UCQ})$ query $q_{\mathcal{O}}$ to have tuples of constants in its certain answers with respect to $\Sigma$ and $D$ that are not in the evaluation of $q_{\mathcal{S}}$ over $D$.

The next example illustrates the algorithm.

Example 9.10. Let $\Sigma=\langle\mathcal{O}, \mathcal{S}, \mathcal{M}\rangle$ be the following OBDM specification:

- $\mathcal{O}=\left\{A_{1} \sqsubseteq \exists P_{1}, A_{2} \sqsubseteq \exists P_{2}\right\} ;$

- $\mathcal{S}=\left\{s_{1}, s_{2}, s_{3}, s_{4}, s_{5}, s_{6}\right\}$

- $\mathcal{M}=\left\{m_{1}, m_{2}, m_{3}, m_{4}, m_{5}, m_{6}, m_{7}\right\}$, where:

$$
\begin{array}{rrrl}
m_{1}: & s_{1}\left(x_{1}, x_{2}\right) & \rightarrow & P_{1}\left(x_{1}, x_{2}\right), \\
m_{2}: & \exists y \cdot s_{2}(x, y) & \rightarrow & \exists \cdot P_{2}(x, z), \\
m_{3}: & \exists y \cdot s_{3}\left(x_{1}, x_{2}, y\right) & \rightarrow & P_{2}\left(x_{1}, x_{2}\right), \\
m_{4}: & \exists y_{1}, y_{2} \cdot s_{4}\left(x, y_{1}, y_{2}\right) & \rightarrow & A_{2}(x), \\
m_{5}: & \exists y_{1}, y_{2} \cdot s_{4}\left(y_{1}, y_{2}, x\right) & \rightarrow & \rightarrow z \cdot P_{3}(x, z), \\
m_{6}: & s_{5}(x) & \rightarrow & \exists \cdot P_{1}(x, z), \\
m_{7}: & s_{6}(x) & \rightarrow & A_{1}(x) .
\end{array}
$$

Let the data service be the CQJFE $q_{\mathcal{S}}=\left\{(x) \mid \exists y_{1}, y_{2} \cdot s_{1}\left(x, y_{1}\right) \wedge s_{2}\left(x, y_{2}\right)\right\}$ over $\mathcal{S}$. The algorithm first sets $q_{\mathcal{O}}^{c}$ to the unique (up to equivalence w.r.t. $\Sigma$ ) minimally complete $\mathcal{S}$-to- $\mathcal{O} \Sigma$-rewriting of $q_{\mathcal{S}}$, i.e., $q_{\mathcal{O}}^{c}=\left\{(x) \mid \exists y_{1} \cdot \mathbf{K}\left(\exists z_{1} \cdot P_{1}\left(x, y_{1}\right) \wedge P_{2}\left(x, z_{1}\right)\right)\right\}$. 
To compute EQLPerfRef $q_{\mathcal{O}}^{c}, \Sigma$, the query $q_{\mathcal{O}}^{\varrho}=\left\{\left(x, y_{1}\right) \mid \exists z_{1} \cdot P_{1}\left(x, y_{1}\right) \wedge P_{2}\left(x, z_{1}\right)\right\}$ associated to the epistemic atom $\mathbf{K} \varrho$ of $q_{\mathcal{O}}^{c}$ (with $\varrho=\exists z_{1} \cdot P_{1}\left(x, y_{1}\right) \wedge P_{2}\left(x, z_{1}\right)$ ) is first reformulated with respect to $\mathcal{O}$, thus obtaining the set $\left\{q_{\mathcal{O}}^{1}, q_{\mathcal{O}}^{2}\right\}$, where $q_{\mathcal{O}}^{1}=q_{\mathcal{O}}^{\varrho}$, while $q_{\mathcal{O}}^{2}=\left\{\left(x, y_{1}\right) \mid P_{1}\left(x, y_{1}\right) \wedge A_{2}(x)\right\}$ is obtained from $q_{\mathcal{O}}^{1}$ by applying the assertion $A_{2} \sqsubseteq \exists P_{2}$. Observe that the $A_{1} \sqsubseteq \exists P_{1}$ ontology assertion is applied neither in $q_{\mathcal{O}}^{1}$ nor in $q_{\mathcal{O}}^{2}$, since in both queries the variables $y_{1}$ is a distinguished variable. After that, each query in the set $\left\{q_{\mathcal{O}}^{1}, q_{\mathcal{O}}^{2}\right\}$ is unfolded with respect to $\mathcal{M}$, and therefore the computed query EQLPerfRef $q_{\mathcal{O}}^{c}, \Sigma$ over $\mathcal{S}$, which is then assumed to be turned into an equivalent UCQ, corresponds the union of the following CQs over $\mathcal{S}$ :

- $q_{\mathcal{S}}^{1}=\left\{(x) \mid \exists y_{1}, y_{2}^{1} \cdot s_{1}\left(x, y_{1}\right) \wedge s_{2}\left(x, y_{2}^{1}\right)\right\}$, obtained from $q_{\mathcal{O}}^{1}$ by unfolding the atom $P_{1}\left(x, y_{1}\right)$ through $m_{1}$, and the atom $P_{2}\left(y_{1}, z_{1}\right)$ through $m_{2}$;

- $q_{\mathcal{S}}^{2}=\left\{(x) \mid \exists y_{1}, y_{2}^{2}, y_{3}^{2} . s_{1}\left(x, y_{1}\right) \wedge s_{3}\left(x, y_{2}^{2}, y_{3}^{2}\right)\right\}$, obtained from $q_{\mathcal{O}}^{1}$ by unfolding the atom $P_{1}\left(x, y_{1}\right)$ through $m_{1}$, and the atom $P_{2}\left(y_{1}, z_{1}\right)$ through $m_{3}$ (after renaming variable $z_{1}$ with $\left.y_{2}^{2}\right)$;

- $q_{\mathcal{S}}^{3}=\left\{(x) \mid \exists y_{1}, y_{2}^{3}, y_{3}^{3} . s_{1}\left(x, y_{1}\right) \wedge s_{4}\left(x, y_{2}^{3}, y_{3}^{3}\right)\right\}$, obtained from $q_{\mathcal{O}}^{2}$ by unfolding the atom $P_{1}\left(x, y_{1}\right)$ through $m_{1}$, and the atom $A_{2}\left(y_{1}\right)$ through $m_{4}$.

Observe that the $m_{6}$ mapping assertion is applied neither in $q_{\mathcal{O}}^{1}$ nor in $q_{\mathcal{O}}^{2}$ for unfolding the atom $P_{1}\left(x, y_{1}\right)$, since in both queries the variable $y_{1}$ is a distinguished variable, whereas the corresponding argument (with same predicate name $P_{1}$ ) of the atom in the right-hand side of $m_{6}$ is only an existential variable.

While $q_{\mathcal{S}}^{1} \sqsubseteq q_{\mathcal{S}}$, it is easy to see that $q_{\mathcal{S}}^{i} \nsubseteq q_{\mathcal{S}}$ for both $i=2$ and $i=3$. Thus, the $E Q L-$ Lite $^{-}$(UCQ) query returned by the algorithm is:

$$
\begin{aligned}
q_{\mathcal{O}}=\left\{(x) \mid \exists y_{1} \cdot \mathbf{K}\left(\exists z_{1} \cdot P_{1}\left(x, y_{1}\right) \wedge P_{2}\left(x, z_{1}\right)\right) \wedge\right. & \\
& \neg \exists y_{2}^{2} \cdot \mathbf{K}\left(P_{1}\left(x, y_{1}\right) \wedge P_{2}\left(x, y_{2}^{2}\right)\right) \wedge \\
& \left.\neg \exists y_{3}^{3} \cdot \mathbf{K}\left(\exists z_{2} \cdot P_{1}\left(x, y_{1}\right) \wedge A_{2}(x) \wedge P_{3}\left(y_{3}^{3}, z_{2}\right)\right)\right\} .
\end{aligned}
$$

Note that for the $\mathcal{S}$-database $D=\left\{s_{1}\left(c, c_{1}\right), s_{2}\left(c_{1}, c_{2}\right)\right\}$ we have both $\left(c_{1}\right) \in q_{\mathcal{S}}^{D}$ and $\left(c_{1}\right) \in \operatorname{cert}_{q_{\mathcal{O}}, \Sigma}^{D}$. Conversely, consider the $\mathcal{S}$-databases $D_{1}=D \cup\left\{s_{3}\left(c_{1}, c_{3}, c_{4}\right)\right\}$ and $D_{2}=D \cup\left\{s_{4}\left(c_{1}, c_{5}, c_{6}\right)\right\}$. Then, we have $(c) \in q_{\mathcal{S}}^{D_{i}}$ but $(c) \notin \operatorname{cert}_{q_{\mathcal{O}}, \Sigma}^{D_{i}}$, for both $i=1$ and $i=2$. Thus, $q_{\mathcal{O}}$ is not a complete $\mathcal{S}$-to- $\mathcal{O} \Sigma$-rewriting of $q_{\mathcal{S}}$.

We are now ready to establish termination and correctness of the MaximallySoundEpistemic algorithm.

Theorem 9.7. Let $\Sigma=\langle\mathcal{O}, \mathcal{S}, \mathcal{M}\rangle$ be an $O B D M$ specification where $\mathcal{O}$ is a $D L$-Lite L $_{\mathcal{R}}^{-}$ ontology and $\mathcal{M}$ is a One-To-One mapping, and let $q_{\mathcal{S}}$ be a CQJFE over $\mathcal{S}$. We have that MaximallySoundEpistemic $\left(\Sigma, q_{\mathcal{S}}\right)$ terminates and returns the unique (up to equivalence w.r.t. $\Sigma$ ) maximally sound $\mathcal{S}$-to-O $\mathcal{O}$-rewriting of $q_{\mathcal{S}}$ in the class of EQL-Lite $(U C Q)$ queries.

Proof. Termination of the algorithm follows from the termination of the chase of a source instance (possibly containing variables) with respect to a One-To-One mapping, and the fact that it is always possible to compute EQLPerfRef $q_{q_{\mathcal{O}}^{c}, \Sigma}$ for an OBDM specification $\Sigma=\langle\mathcal{O}, \mathcal{S}, \mathcal{M}\rangle$ and an EQL-Lite(UCQ) query $q_{\mathcal{O}}^{c}$ over $\mathcal{O}$. 
Let $q_{\mathcal{O}}$ be the EQL-Lite ${ }^{-}$(UCQ) query returned by the algorithm. We now divide the proof into two parts: we first prove that $q_{\mathcal{O}}$ is a sound $\mathcal{S}$-to- $\mathcal{O} \Sigma$-rewriting of $q_{\mathcal{S}}$ and then we prove that each EQL-Lite(UCQ) $q_{\mathcal{O}}^{\prime}$ that is a sound $\mathcal{S}$-to- $\mathcal{O} \Sigma$-rewriting of $q_{\mathcal{S}}$ is such that $\operatorname{cert}_{q_{\mathcal{O}}^{\prime}, \Sigma} \sqsubseteq \operatorname{cert}_{q_{\mathcal{O}}, \Sigma}$.

Lemma 9.5. $q_{\mathcal{O}}$ is a sound $\mathcal{S}$-to-O $\mathcal{O}$-rewriting of $q_{\mathcal{S}}$.

Proof. If the algorithm returns the query $q_{\mathcal{O}}=\{\vec{t} \mid \perp(\vec{x})\}$, then the claim is trivial. Otherwise, let $D$ be any $\mathcal{S}$-database, and let $\vec{c}$ be any tuple of constants for which $\vec{c} \in \operatorname{cert}_{q_{\mathcal{O}}, \Sigma}^{D}$. We now prove that $\vec{c} \in q_{\mathcal{S}}^{D}$ as well. By construction of $q_{\mathcal{O}}$, it follows that $\vec{c} \in \operatorname{cert}_{q_{\mathcal{O}}^{c}, \Sigma}^{D}$, where $q_{\mathcal{O}}^{c}=\left\{\vec{t} \mid \exists \overrightarrow{\mathcal{Y}} \cdot \mathbf{K}\left(\exists \vec{z} \cdot \mathcal{M}\left(q_{\mathcal{S}}\right)\right)\right\}$ is the query returned by MinimallyCompleteEpistemic $\left(\Sigma, q_{\mathcal{S}}\right)$, i.e., the unique (up to equivalence w.r.t. $\Sigma$ ) minimally complete $\mathcal{S}$-to- $\mathcal{O} \Sigma$-rewriting of $q_{\mathcal{S}}$ in the class of EQL-Lite(UCQ) queries (cf. Theorem 9.6. Since $\vec{c} \in \operatorname{cert}_{q_{\mathcal{O}}^{c}, \Sigma}^{D}$, by combining Theorem 9.1 with Proposition 9.3 we derive that there is at least a disjunct $q_{\mathcal{S}}^{i}$ of the UCQ EQLPerfRef ${ }_{q_{\mathcal{O}}^{c}, \Sigma}$ witnessing that $\vec{c} \in \operatorname{cert}{q_{\mathcal{O}}^{c}, \Sigma}^{D}$, i.e., a disjunct $q_{\mathcal{S}}^{i}$ for which $\vec{c} \in q_{\mathcal{S}}^{i}{ }^{D}$. There are two possible cases for the disjunct $q_{\mathcal{S}}^{i}$ : either $q_{\mathcal{S}}^{i} \nsubseteq q_{\mathcal{S}}$, or $q_{\mathcal{S}}^{i} \sqsubseteq q_{\mathcal{S}}$.

In the former case, by construction of the algorithm, the query $q_{\mathcal{O}}$ contains in its body the formula $\neg \exists \overrightarrow{\mathcal{Y}_{i}} \cdot \mathbf{K}\left(\exists \overrightarrow{z_{i}} \cdot \mathcal{M}\left(q_{\mathcal{S}}^{i}\right)\right)$ in conjunction to the body of $q_{\mathcal{O}}^{c}$. Furthermore, observe that $q_{\mathcal{S}}^{i}$ is a disjunct of EQLPerfRef $q_{\mathcal{O}}^{c}, \Sigma$, and therefore each distinguished variable occurring in $q_{\mathcal{S}}^{i}$ occurs also in $\mathcal{M}\left(q_{\mathcal{S}}^{i}\right)$. So, by Theorem 9.6 we derive that $\exists \overrightarrow{\mathcal{y}_{i}} \cdot \mathbf{K}\left(\exists \overrightarrow{z_{i}} \cdot \mathcal{M}\left(q_{\mathcal{S}}^{i}\right)\right)$ is the body of a query being a complete $\mathcal{S}$-to$\mathcal{O} \Sigma$-rewriting of $q_{\mathcal{S}}^{i}$ (in fact, the unique (up to equivalence w.r.t. $\Sigma$ ) minimally complete $\mathcal{S}$-to- $\mathcal{O} \Sigma$-rewriting of $q_{\mathcal{S}}^{i}$ in the class of EQL-Lite(UCQ) queries). Thus, since $\vec{c} \in q_{\mathcal{S}}^{i}{ }^{D}$, from the above observation we have that formula $\exists \overrightarrow{\mathcal{Y}_{i}} \cdot \mathbf{K}\left(\exists \overrightarrow{z_{i}} \cdot \mathcal{M}\left(q_{\mathcal{S}}^{i}\right)\right)$ is $E Q L$-logically implied by $\langle\Sigma, D\rangle$ (and thus, formula $\neg \exists \overrightarrow{y_{i}} \cdot \mathbf{K}\left(\exists \overrightarrow{z_{i}} \cdot \mathcal{M}\left(q_{\mathcal{S}}^{i}\right)\right.$ ) is not $E Q L$-logically implied by $\langle\Sigma, D\rangle)$ when replacing its free variables with the tuple of constants $\vec{c}$. As a consequence, we have that $\vec{c} \notin \operatorname{cert}_{q_{\mathcal{O}}, \Sigma}^{D}$, which is a contradiction to the initial assumption that $\vec{c} \in \operatorname{cert}_{q_{\mathcal{O}}, \Sigma}^{D}$. It follows that the former case just considered is not possible because it leads to a contradiction. Therefore, we consider only the latter case. But then, as for the latter case, observe that $\vec{c} \in q_{\mathcal{S}}^{i}$ and $q_{\mathcal{S}}^{i} \sqsubseteq q_{\mathcal{S}}$ clearly imply that $\vec{c} \in q_{\mathcal{S}}^{D}$ as well, as required.

We now show that $q_{\mathcal{O}}$ is actually the unique (up to equivalence w.r.t. $\Sigma$ ) maximally sound $\mathcal{S}$-to- $\mathcal{O} \Sigma$-rewriting of $q_{\mathcal{S}}$ in the class of EQL-Lite(UCQ) queries. Since from the above lemma we know that $q_{\mathcal{O}}$ is a sound $\mathcal{S}$-to- $\mathcal{O} \Sigma$-rewriting of $q_{\mathcal{S}}$, it is enough to prove that each EQL-Lite(UCQ) $q_{\mathcal{O}}^{\prime}$ that is a sound $\mathcal{S}$-to- $\mathcal{O} \Sigma$-rewriting of $q_{\mathcal{S}}$ is such that $\operatorname{cert}_{q_{\mathcal{O}}^{\prime}, \Sigma} \sqsubseteq \operatorname{cert}_{q_{\mathcal{O}}, \Sigma}$ (cf. Definition 3.6). We do this by way of contradiction.

Let $q_{\mathcal{O}}^{\prime}$ be an $E Q L-L i t e(U C Q)$ query such that cert $_{q_{\mathcal{O}}^{\prime}, \Sigma} \nsubseteq \operatorname{cert}_{q_{\mathcal{O}}, \Sigma}$, that is, there exists an $\mathcal{S}$-database $D$ consistent with $\Sigma$ such that $\operatorname{cert}_{q_{\mathcal{O}}^{\prime}, \Sigma}^{D} \nsubseteq \operatorname{cert}_{q_{\mathcal{O}}, \Sigma}^{D}$. It follows that there is a tuple of constants $\vec{c}$ such that $\vec{c} \in \operatorname{cert}_{q_{\mathcal{O}}^{\prime}, \Sigma}^{D}$, but $\vec{c} \notin \operatorname{cert}_{q_{\mathcal{O}}, \Sigma}^{D}$. If $\vec{c} \notin q_{\mathcal{S}}^{D}$, then $q_{\mathcal{O}}^{\prime}$ is trivially not a sound $\mathcal{S}$-to- $\mathcal{O} \Sigma$-rewriting of $q_{\mathcal{S}}$, and we are done. Therefore, we assume that $\vec{c} \in q_{\mathcal{S}}^{D}$. We now exhibit an $\mathcal{S}$-database $D^{\prime}$ consistent with $\Sigma$ for which $(i) \vec{c} \notin q_{\mathcal{S}}^{D^{\prime}}$, and $(i i) \mathcal{C}_{\mathcal{O}}^{\mathcal{M}\left(D^{\prime}\right)}$ and $\mathcal{C}_{\mathcal{O}}^{\mathcal{M}(D)}$ are homomorphically equivalent, i.e., there is a homomorphism from $\mathcal{C}_{\mathcal{O}}^{\mathcal{M}\left(D^{\prime}\right)}$ to $\mathcal{C}_{\mathcal{O}}^{\mathcal{M}(D)}$ and vice versa. 
Consider first the case that the algorithm returns the query $q_{\mathcal{O}}=\{\vec{t} \mid \perp(\vec{x})\}$. In this case, in the body of $q_{\mathcal{S}}$ there is at least a distinguished variable $x \in \vec{x}$ occurring as $k$-th argument of some source predicate $s \in \mathcal{S}$ such that $x$ does not occur in $\mathcal{M}\left(q_{\mathcal{S}}\right)$. Let $h$ be any homomorphism from $q_{\mathcal{S}}=\{\vec{t} \mid \exists \vec{y} . \phi(\vec{x}, \vec{y})\}$ to $D$ with $h(\vec{t})=\vec{c}$, and consider the constant $c \in \vec{c}$ for which $h(x)=c$. The $\mathcal{S}$-database $D^{\prime}$ we are seeking is obtained from $D$ by replacing each fact of the form $s(\vec{a})$ with $s\left(\overrightarrow{a^{\prime}}\right)$, where $\vec{a}$ is any tuple of constants in which the $k$-th argument is $h(x)=c$, and $\overrightarrow{a^{\prime}}$ is obtained from $\vec{a}$ by replacing the $k$-th argument $h(x)=c$ with a fresh constant $c_{x}$. Two considerations follow for the $\mathcal{S}$-database $D^{\prime}$ : (i) Clearly, we have that $\vec{c} \notin q_{\mathcal{S}}^{D^{\prime}}$ because there can be no homomorphism $h$ from $q_{\mathcal{S}}$ to $D^{\prime}$ with $h(x)=c$ (and thus, with $h(\vec{t})=\vec{c}) ;(i i)$ Since $x$ does not occur in $\mathcal{M}\left(q_{\mathcal{S}}\right)$, and since the left-hand side of One-To-One mapping assertions are simply atoms without constants or repeated variables, it is easy to see that $D^{\prime}$ is such that $\mathcal{M}(D)=\mathcal{M}\left(D^{\prime}\right)$. It follows that $D^{\prime}$ is such that $(i) \vec{c} \notin q_{\mathcal{S}}^{D^{\prime}}$, and $(i i) \mathcal{C}_{\mathcal{O}}^{\mathcal{M}\left(D^{\prime}\right)}$ and $\mathcal{C}_{\mathcal{O}}^{\mathcal{M}(D)}$ are homomorphically equivalent.

We now consider the case that the algorithm does not return the query $q_{\mathcal{O}}=$ $\{\vec{t} \mid \perp(\vec{x})\}$, i.e., each distinguished variable of $q_{\mathcal{S}}$ occurs in $\mathcal{M}\left(q_{\mathcal{S}}\right)$. Consider any homomorphism $h$ from $q_{\mathcal{S}}=\{\vec{t} \mid \exists \vec{y} \cdot \phi(\vec{x}, \vec{y})\}$ to $D$ with $h(\vec{t})=\vec{c}$, and consider $h\left(q_{\mathcal{S}}\right)$, i.e., the set of facts corresponding to the image of $h$ under $q_{\mathcal{S}}$. By construction (see also Theorem 9.6), the formula $\exists \overrightarrow{\mathcal{Y}} \cdot \mathbf{K}\left(\exists \vec{z} \cdot \mathcal{M}\left(q_{\mathcal{S}}\right)\right)$ occurring in conjunction in the body of $q_{\mathcal{O}}$ is true in $\mathcal{C}_{\mathcal{O}}^{\mathcal{M}(D)}$ when replacing each free variable $x \in \vec{x}$ and each variable $\mathcal{Y} \in \overrightarrow{\mathcal{Y}}$ with $h(x)$ and $h(\mathcal{Y})$, respectively (observe that the existential variables $\overrightarrow{\mathcal{Y}}$ are those of $q_{\mathcal{S}}$ occurring also in $\left.\mathcal{M}\left(q_{\mathcal{S}}\right)\right)$. Since, however, $\vec{c} \notin \operatorname{cert}_{q_{\mathcal{O}}, \Sigma}^{D}$, by construction of $q_{\mathcal{O}}$ we derive that there is at least a formula $\neg \exists \overrightarrow{y_{i}} \cdot \mathbf{K}\left(\exists \overrightarrow{z_{i}} \cdot \mathcal{M}\left(q_{\mathcal{S}}^{i}\right)\right)$ occurring in conjunction in the body of $q_{\mathcal{O}}$ that is not EQL-logically implied by $\langle\Sigma, D\rangle$ when replacing each free variable $x \in \vec{x}$ and each variable $\mathcal{Y} \in \overrightarrow{\mathcal{Y}}$ with $h(x)$ and $h(\mathcal{Y})$, respectively, where $q_{\mathcal{S}}^{i}=\left\{\vec{t} \mid \exists \overrightarrow{y_{i}} \cdot \phi_{i}\left(\vec{x}, \overrightarrow{y_{i}}\right)\right\}$ is a disjunct of EQLPerfRef $_{q_{\mathcal{O}}^{c}, \Sigma}$ for which $q_{\mathcal{S}}^{i} \nsubseteq q_{\mathcal{S}}$ with $q_{\mathcal{O}}^{c}=\left\{\vec{t} \mid \exists \overrightarrow{\mathcal{Y}} \cdot \mathbf{K}\left(\exists \vec{z} \cdot \mathcal{M}\left(q_{\mathcal{S}}\right)\right)\right\}$. So, formula $\exists \overrightarrow{y_{i}} \cdot \mathbf{K}\left(\exists \overrightarrow{z_{i}} \cdot \mathcal{M}\left(q_{\mathcal{S}}^{i}\right)\right)$ must be true in $\mathcal{C}_{\mathcal{O}}^{\mathcal{M}(D)}$ when replacing each free variable $x \in \vec{x}$ and each variable $\mathcal{Y} \in \overrightarrow{\mathcal{Y}}$ with $h(x)$ and $h(\mathcal{Y})$, respectively. Therefore, using Proposition 9.4 , this implies that it is possible to extend $h$ with a new homomorphism $h_{i}$ from terms of $\mathcal{M}\left(q_{\mathcal{S}}^{i}\right)$ to $\mathcal{C}_{\mathcal{O}}^{\mathcal{M}(D)}$ for which $h_{i}\left(\mathcal{Y}_{i}\right)$ is a constant, for each $\mathcal{Y}_{i} \in \overrightarrow{\mathcal{Y}_{i}}$. Let now $h^{\prime}$ be the function extending $h_{i}$ by assigning a different fresh constant $c_{y_{i}}$ to each existential variable $y_{i} \notin \overrightarrow{\mathcal{Y}_{i}} \cup \overrightarrow{\mathcal{Y}}$ of $q_{\mathcal{S}}^{i}$, i.e., to each existential variable of $q_{\mathcal{S}}^{i}$ not occurring in $\mathcal{M}\left(q_{\mathcal{S}}^{i}\right)$.

Notice that $q_{\mathcal{S}}^{i}$ is a disjunct of EQLPerfRef $q_{\mathcal{O}}^{c}, \Sigma$ with $q_{\mathcal{O}}^{c}=\left\{\vec{t} \mid \exists \overrightarrow{\mathcal{Y}} \cdot \mathbf{K}\left(\exists \vec{z} \cdot \mathcal{M}\left(q_{\mathcal{S}}\right)\right)\right\}$. Thus, since the left-hand side of each mapping assertion in $\mathcal{M}$ is simply an atom without constants or repeated variables, it is easy to verify that all the possible logical consequences over $\mathcal{O}$ of the set of facts $h\left(q_{\mathcal{S}}\right)$ is a subset of the logical consequences over $\mathcal{O}$ of the set of facts $h^{\prime}\left(q_{\mathcal{S}}^{i}\right)$, i.e., there is a homomorphism from $\mathcal{C}_{\mathcal{O}}^{\mathcal{M}\left(h\left(q_{\mathcal{S}}\right)\right)}$ to $\mathcal{C}_{\mathcal{O}}^{\mathcal{M}\left(h^{\prime}\left(q_{\mathcal{S}}^{i}\right)\right)}$. Furthermore, similarly as already observed in the proof of Theorem 9.6 since formula $\exists \overrightarrow{\mathcal{Y}_{i}} \cdot \mathbf{K}\left(\exists \overrightarrow{z_{i}} \cdot \mathcal{M}\left(q_{\mathcal{S}}^{i}\right)\right)$ is true in $\mathcal{C}_{\mathcal{O}}^{\mathcal{M}(D)}$ when replacing each free variable $x \in \vec{x}$, each variable $\mathcal{Y} \in \overrightarrow{\mathcal{Y}}$, and each variable $\mathcal{Y}_{i} \in \overrightarrow{y_{i}}$ with $h(x)=h_{i}(x), h(\mathcal{Y})=h_{i}(\mathcal{Y})$, and $h_{i}\left(\mathcal{Y}_{i}\right)$, respectively, and since the left-hand side of each mapping assertion in $\mathcal{M}$ is a single atom without constants or repeated variables, we derive that each fact $\alpha \in h^{\prime}\left(q_{\mathcal{S}}^{i}\right)$ is such that there is a homomorphism from $\mathcal{M}(\alpha)$ to $\mathcal{C}_{\mathcal{O}}^{\mathcal{M}(D)}$. So, using Proposition 9.5. there is a homomorphism from $\mathcal{C}_{\mathcal{O}}^{\mathcal{M}\left(h^{\prime}\left(q_{\mathcal{S}}^{i}\right)\right)}$ to $\mathcal{C}_{\mathcal{O}}^{\mathcal{M}(D)}$. 
Due to the fact that there is a homomorphism from $\mathcal{C}_{\mathcal{O}}^{\mathcal{M}\left(h\left(q_{\mathcal{S}}\right)\right)}$ to $\mathcal{C}_{\mathcal{O}}^{\mathcal{M}\left(h^{\prime}\left(q_{\mathcal{S}}^{i}\right)\right)}$, and the fact that there is a homomorphism from $\mathcal{C}_{\mathcal{O}}^{\mathcal{M}\left(h^{\prime}\left(q_{\mathcal{S}}^{i}\right)\right)}$ to $\mathcal{C}_{\mathcal{O}}^{\mathcal{M}(D)}$, by making use of Proposition 9.5, it is possible to conclude that the $\mathcal{S}$-database $D_{h}$ obtained from $D$ by removing all the facts in $h\left(q_{\mathcal{S}}\right)$ and adding all the facts in $h^{\prime}\left(q_{\mathcal{S}}^{i}\right)$ is such that $\mathcal{C}_{\mathcal{O}}^{\mathcal{M}\left(D_{h}\right)}$ and $\mathcal{C}_{\mathcal{O}}^{\mathcal{M}(D)}$ are homomorphically equivalent, i.e., $D_{h}=\left(\left(D \backslash h\left(q_{\mathcal{S}}\right)\right) \cup h^{\prime}\left(q_{\mathcal{S}}^{i}\right)\right)$ is such that $\mathcal{C}_{\mathcal{O}}^{\mathcal{M}\left(D_{h}\right)}$ and $\mathcal{C}_{\mathcal{O}}^{\mathcal{M}(D)}$ are homomorphically equivalent.

Consider now the $\mathcal{S}$-database $D^{\prime}$ obtained by repeatedly iterating the above process for each possible homomorphism $h$ from $q_{\mathcal{S}}$ to $D$ with $h(\vec{t})=\vec{c}$, until one obtain a $D^{\prime}$ such that $\vec{c} \notin q_{\mathcal{S}}^{D^{\prime}}$. In other words, the $\mathcal{S}$-database $D^{\prime}$ can be obtained starting from $D$ and then repeatedly removing all the facts in $h\left(q_{\mathcal{S}}\right)$ and adding all the facts in $h^{\prime}\left(q_{\mathcal{S}}^{i}\right)$, for each homomorphism $h$ from $q_{\mathcal{S}}$ to $D$ with $h(\vec{t})=\vec{c}$, where $q_{\mathcal{S}}^{i}$ is a disjunct of EQLPerfRef $q_{\mathcal{O}}^{c}, \Sigma$ for which $(i) q_{\mathcal{S}}^{i} \nsubseteq q_{\mathcal{S}}$ and formula $\exists \overrightarrow{\mathcal{y}_{i}} \cdot \mathbf{K}\left(\exists \overrightarrow{z_{i}} \cdot \mathcal{M}\left(q_{\mathcal{S}}^{i}\right)\right)$ is true in $\mathcal{C}_{\mathcal{O}}^{\mathcal{M}(D)}$ when replacing each free variable $x \in \vec{x}$ and each variable $\mathcal{Y} \in \overrightarrow{\mathcal{Y}}$ with $h(x)$ and $h(\mathcal{Y})$, respectively (at least one must exists because $\vec{c} \notin \operatorname{cert} q_{\mathcal{O}^{,}, \Sigma}^{D}$ ), and (ii) $h^{\prime}$ is obtained from $h$ and $q_{\mathcal{S}}^{i}$ as illustrated above. Using again Proposition 9.5 from the previous observations, we derive that the obtained $\mathcal{S}$-database $D^{\prime}$ is such that $\mathcal{C}_{\mathcal{O}}^{\mathcal{M}\left(D^{\prime}\right)}$ and $\mathcal{C}_{\mathcal{O}}^{\mathcal{M}(D)}$ are homomorphically equivalent. Furthermore, since $q_{\mathcal{S}}$ is a CQJFE, and therefore it does not have existential variables in join occurring in its body, and since in $D^{\prime}$ we have removed all the facts $h\left(q_{\mathcal{S}}\right)$ for each homomorphism $h$ from $q_{\mathcal{S}}$ to $D$ with $h(\vec{t})=\vec{c}$, one can easily verify that $\vec{c} \notin q_{\mathcal{S}}^{D^{\prime}}$ by construction.

To conclude the proof note that, both in the case that the algorithm returns the query $\{\vec{t} \mid \perp(\vec{x})\}$ and in the case that it does not return $\{\vec{t} \mid \perp(\vec{x})\}$, it is possible to obtain an $\mathcal{S}$-database $D^{\prime}$ such that $(i) \vec{c} \notin q_{\mathcal{S}}^{D^{\prime}}$, and $(i i) \mathcal{C}_{\mathcal{O}}^{\mathcal{M}\left(D^{\prime}\right)}$ and $\mathcal{C}_{\mathcal{O}}^{\mathcal{M}(D)}$ are homomorphically equivalent. Since $\mathcal{C}_{\mathcal{O}}^{\mathcal{M}\left(D^{\prime}\right)}$ and $\mathcal{C}_{\mathcal{O}}^{\mathcal{M}(D)}$ are homomorphically equivalent, and since $\vec{c} \in \operatorname{cert}_{q_{\mathcal{O}}^{\prime}, \Sigma}^{D}$ by assumption, it is easy to see that $\vec{c} \in \operatorname{cert} t_{q_{\mathcal{O}}^{\prime}, \Sigma}^{D^{\prime}}$ as well. It follows that $D^{\prime}$ is an $\mathcal{S}$-database consistent with $\Sigma$ (observe that $\mathcal{O}$ is a $D L$-Lite $e_{\mathcal{R}}^{-}$ontology, and therefore each $\mathcal{S}$-database is consistent with $\Sigma$ ) for which $\vec{c} \notin q_{\mathcal{S}}^{D^{\prime}}$ and $\vec{c} \in \operatorname{cert} t_{q_{\mathcal{O}}^{\prime}, \Sigma}^{D^{\prime}}$, thus implying that $q_{\mathcal{O}}^{\prime}$ is not a sound $\mathcal{S}$-to- $\mathcal{O} \Sigma$-rewriting of $q_{\mathcal{S}}$, as required.

As for the running time of the MaximallySoundEpistemic algorithm, we observe that it is independent of the size of $\mathcal{S}$, polynomial in the size of both $\mathcal{O}$ and $\mathcal{M}$, and exponential in the size of $q_{\mathcal{S}}$. This latter is due to the fact that EQLPerfRef $q_{\mathcal{O}}^{c}, \Sigma$ is in general the union of an exponential number of CQs with respect to the number of atoms occurring in $q_{\mathcal{S}}$, and also due to the various containment check of CQs. Finally, note that the overall running time is exponential in the size of the input.

\subsubsection{Perfect Source-to-Ontology Rewritings}

We conclude this chapter with a consideration on perfect source-to-ontology rewritings in the class of EQL-Lite(UCQ) queries, for the case of OBDM specifications with One-To-One mapping assertions.

Consider any pair composed by an OBDM specification $\Sigma=\langle\mathcal{O}, \mathcal{S}, \mathcal{M}\rangle$ where $\mathcal{O}$ is a $D L$-Lite $e_{\mathcal{R}}^{-}$ontology and $\mathcal{M}$ is a One-to-One mapping and a CQ $q_{\mathcal{S}}$ over $\mathcal{S}$. Clearly, by definition, either the unique (up to equivalence w.r.t. $\Sigma$ ) minimally 
complete $\mathcal{S}$-to- $\mathcal{O} \Sigma$-rewriting of $q_{\mathcal{S}}$ in the class of EQL-Lite(UCQ) queries is also a sound, and therefore a perfect, $\mathcal{S}$-to- $\mathcal{O} \Sigma$-rewriting of $q_{\mathcal{S}}$, or no perfect $\mathcal{S}$-to- $\mathcal{O}$ $\Sigma$-rewriting of $q_{\mathcal{S}}$ in the class of EQL-Lite(UCQ) queries exists.

With this observation at hand, and making use of Theorem 9.6, we can specialise the PerfectEpistemic algorithm in an obvious way for the case of OBDM specifications with One-to-One mapping assertions. For the sake of completeness, we report here the algorithm PerfectEpistemicOneToOne together with its termination and correctness.

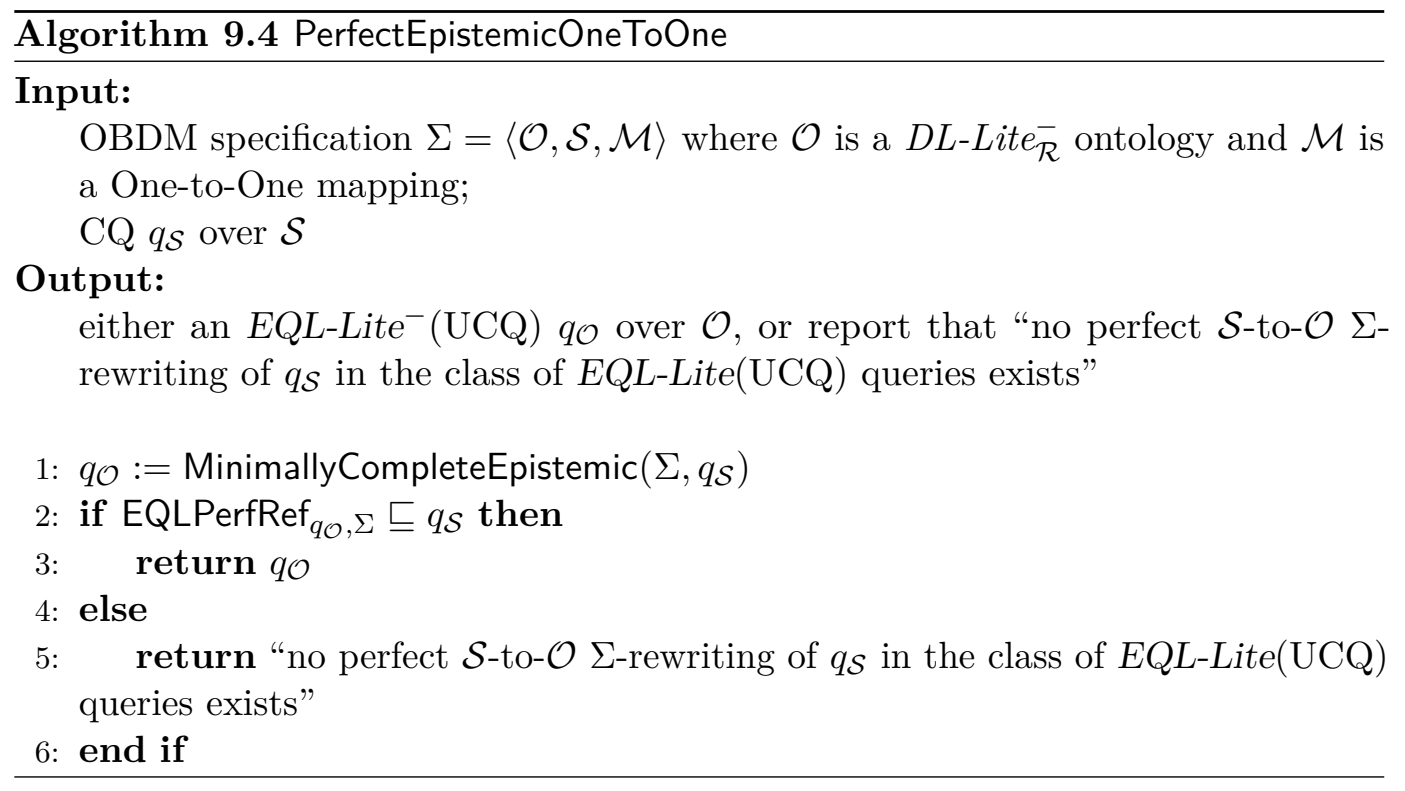

Theorem 9.8. Let $\Sigma=\langle\mathcal{O}, \mathcal{S}, \mathcal{M}\rangle$ be an $O B D M$ specification where $\mathcal{O}$ is a DL-Lite $\overline{\mathcal{R}}^{-}$ ontology and $\mathcal{M}$ is a One-To-One mapping, and let $q_{\mathcal{S}}$ be a $C Q$ over $\mathcal{S}$. We have that PerfectEpistemicOneToOne $\left(\Sigma, q_{\mathcal{S}}\right)$ terminates and returns the perfect $\mathcal{S}$ to-O $\mathcal{O}$-rewriting of $q_{\mathcal{S}}$ if it exists and can be expressed as an EQL-Lite $(U C Q)$ query, otherwise it reports that no perfect $\mathcal{S}$-to- $\mathcal{O} \Sigma$-rewriting of $q_{\mathcal{S}}$ in the class of EQL-Lite $(U C Q)$ queries exists.

Furthermore, as a straightforward consequence of Corollary 9.2 , we also get the following interesting result.

Corollary 9.3. Let $\Sigma=\langle\mathcal{O}, \mathcal{S}, \mathcal{M}\rangle$ be an OBDM specification where $\mathcal{O}$ is a DL-Lite $\overline{\mathcal{R}}^{-}$ ontology and $\mathcal{M}$ is a One-To-One mapping, and let $q_{\mathcal{S}}$ be a $C Q$ over $\mathcal{S}$. We have that the perfect $\mathcal{S}$-to-O $\mathcal{O}$-rewriting of $q_{\mathcal{S}}$ in the class of EQL-Lite $(U C Q)$ queries either does not exists, or it can be expressed as an EQL-Lite ${ }^{-}(U C Q)$ query. 


\section{Chapter 10}

\section{Conclusions}

This chapter concludes the thesis with a brief discussion and possible directions for future work.

\subsection{Discussion}

In this thesis we have introduced a novel reasoning task over the OBDM specification, called abstraction. The main purpose of this task is to automatically produce the semantic characterisation of data services through ontologies, and thus make data services automatically Findable, Accessible, Interoperable, and Reusable (FAIR). We have presented a formal framework for abstraction, via the semantically wellfounded notion(s) of source-to-ontology rewriting, which can be seen as the inverse of the well-known and well-studied notion(s) of ontology-to-source rewriting. We have carried out a comprehensive analysis of two important related computational problems within the most common languages used in OBDM, including two restricted scenarios and also the addition of non-monotonicity in the target query language.

We believe that the notions introduced and the technical results presented in this thesis are not only theoretically interesting in themselves, but also have many possible practical applications besides the semantic characterisations of data services, as for example in the fields mentioned in the introduction, namely open data, source profiling, updating, and explanation of classifiers. We point out that the thesis left some interesting and challenging open problems, which are detailed in the below list:

- When the UNA is not adopted, answering $\mathrm{CQ}^{\neq, b}$ s over DL-Lite $\mathcal{R}_{\mathcal{R}}$ knowledge bases has been shown to be FOL-rewritable. The FOL-rewritability question, however, is still open for unions thereof, although it has been proven that the problem remains in $\mathrm{AC}^{0}$ in data complexity.

- When the UNA is not adopted, answering $\mathrm{UCQ}^{2, \neq} \mathrm{s}$ over $D L$-Lite $e_{\mathrm{RDFS}}$ knowledge bases as well as answering general $\mathrm{CQ}^{\neq} \mathrm{S}$ over $D L$-Lite $\overrightarrow{\mathrm{RDFS}}_{\mathrm{S}}$ knowledge bases has been shown to be $\Pi_{2}^{p}$-complete in combined complexity. For the case of $\mathrm{CQ}^{2, F_{\mathrm{S}}}$ over $D L$-Lit $e_{\mathrm{RDFS}}$ knowledge bases, however, the $\Pi_{2}^{p}$-hardness part has only been conjectured.

- Strictly related to the above problem is the containment problem for $\mathrm{UCQ}^{\neq} \mathrm{s}$. Specifically, while checking whether $q^{\prime} \sqsubseteq q$ has been shown to be $\Pi_{2}^{p}$-hard 
(and therefore $\Pi_{2}^{p}$-complete) when both $q^{\prime}$ and $q$ are $\mathrm{CQ}^{\neq} \mathrm{S}$ as well as when $q^{\prime}$ is a $\mathrm{CQ}$ and $q$ is a $\mathrm{UCQ}^{2, \neq}$, the $\Pi_{2}^{p}$-hardness of the problem has only been conjectured for the case of $q^{\prime}$ being a $\mathrm{CQ}^{\neq}$and $q$ being a $\mathrm{CQ}^{2, \neq}$.

- For the case of mappings that are both pure GAV and LAV, the exact computational complexity of the verification problem for sound source-to-ontology rewritings is still open.

- For the case of LAV mappings, the exact computational complexity of the verification problem for perfect source-to-ontology rewritings is still open.

- Both in the restricted scenario for UCQJFEs and in the restricted scenario for CQJFEs, we point out that the verification problem has been studied for sound source-to-ontology rewritings only. While the proof of Theorem 5.1 already shows that the verification problem for complete source-to-ontology rewritings is NP-complete in both the restricted scenarios, the problem is still open for perfect source-to-ontology rewritings. In particular, it is trivial to establish membership in DP and in NP in the restricted scenario for UCQJFEs and in the restricted scenario for CQJFEs, respectively (for the former, just observe that the set of perfect source-to-ontology rewritings is the intersection between the set of sound source-to-ontology rewritings and the set of complete source-to-ontology rewritings, where, in the restricted scenario for UCQJFEs, soundness and completeness can be verified in coNP and in NP, respectively). However, we only conjecture matching lower bounds for both cases.

- The verification problem for all types of source-to-ontology rewritings when the target query language is the class of EQL-Lite(UCQ) queries has not been addressed at all, and thus it is still an open problem to determine the exact computational complexity of the verification problem for each type of source-to-ontology rewriting.

- The proofs of the non-existence cases of minimally complete, and maximally sound source-to-ontology rewritings in the class of EQL-Lite(UCQ) queries (Theorems 9.2 and 9.3 respectively) rely on pure GAV mapping assertions. For the case of LAV mappings, however, it is still an open problem to determine whether minimally complete (respectively, maximally sound) source-to-ontology rewritings are guaranteed to exist in the class of EQL-Lite(UCQ) queries.

- In addition to the previous cases, we point out that for the case of One-To-One mappings it is still an open problem to determine whether maximally sound source-to-ontology rewritings of CQs in the class of EQL-Lite(UCQ) queries are guaranteed to exist.

\section{$10.2 \quad$ Future Work}

In addition to tackling the open problems mentioned in the foregoing list, we see many other interesting avenues for future work, including the following: 
- Basically, the introduced notions of source-to-ontology rewriting are based on certain answers. In principle, there may be other meaningful properties that a query over the ontology have to satisfy to be considered a perfect (respectively, sound, complete) source-to-ontology rewriting. For instance, one may consider a model-based semantics where a query $q_{\mathcal{O}}$ is a perfect (respectively, sound, complete) $\mathcal{S}$-to- $\mathcal{O} \Sigma$-rewriting of a query $q_{\mathcal{S}}$ if and only if $q_{\mathcal{S}}^{D}=q_{\mathcal{O}}^{\mathcal{I}}$ (respectively, $\left.q_{\mathcal{O}}^{\mathcal{I}} \subseteq q_{\mathcal{S}}^{D}, q_{\mathcal{S}}^{D} \subseteq q_{\mathcal{O}}^{\mathcal{I}}\right)$ for each $\mathcal{S}$-database $D$ and model $\mathcal{I} \in \operatorname{Mod}_{\Sigma}(D)$.

- Throughout the thesis, we have implicitly assumed that both the evaluation of queries posed over source databases and the certain answers of queries posed over the ontology of OBDM systems are sets. This is in contrast with the standard semantics of DBMSs, which is based on bags (i.e., multisets) and duplicate tuples are retained by default. Considering as starting point the works Nikolaou et al., 2019, Cima et al., 2019e that propose a bag semantics for OBDM systems, it would be interesting to investigate the various notions of source-to-ontology rewriting under a bag-based semantics.

- Study the impact of integrity constraints over the source schemas $\mathcal{S}$.

- Extending the analysis to OBDM settings going beyond the one based on $D L$-Lite $e_{\mathcal{R}}$, e.g., by considering as ontology languages DLs equipped with role functionality assertions such as $D L$-Lite $\mathcal{A}$, or DLs of the $\mathcal{E} \mathcal{L}$ family.

- The class of queries for expressing data services in this thesis has been the one of UCQs (and its fragments). It would be interesting to examine cases where the source query language for expressing data services goes beyond UCQs.

- In Chapter 6, we have seen many cases where UCQ-maximally sound sourceto-ontology rewritings are not guaranteed to exists in the general scenario. It would be very useful singling out the minimal class of queries $\mathcal{L}_{\mathcal{O}}$ that guarantees the existence of $\mathcal{L}_{\mathcal{O}}$-maximally sound source-to-ontology rewritings. For instance, one may start to investigate whether such $\mathcal{L}_{\mathcal{O}}$ is the class of (unions of) conjunctive two-way regular path queries, a class of queries wellstudied in the context of lightweight DLs (see, e.g., Bienvenu et al., 2015]).

- Related to the above problem is to analyse another notable decision problem, namely the existence problem for a target query language $\mathcal{L}_{\mathcal{O}}$ : check whether an $\mathcal{L}_{\mathcal{O}}$ source-to-ontology rewriting (perfect, or approximated) exists for a given OBDM specification $\Sigma=\langle\mathcal{O}, \mathcal{S}, \mathcal{M}\rangle$ and a given source query $q_{\mathcal{S}}$ over $\mathcal{S}$.

- Singling out more interesting scenarios in which source-to-ontology rewritings expressed in the class of EQL-Lite(UCQ) queries (or its fragments still nonmonotonic) can be actually computed.

- Study the impact of our notions and technical results in other data interoperation architectures, such as peer-to-peer data integration Calvanese et al., 2004b.

We believe that each of the above issues is an interesting research problem that deserves to be investigated. 



\section{References}

[Abedjan et al., 2017] Ziawasch Abedjan, Lukasz Golab, and Felix Naumann. Data profiling: A tutorial. In Proceedings of the 2017 ACM International Conference on Management of Data (SIGMOD 2017), pages 1747-1751, 2017.

[Abedjan et al., 2018] Ziawasch Abedjan, Lukasz Golab, Felix Naumann, and Thorsten Papenbrock. Data Profiling. Synthesis Lectures on Data Management. Morgan \& Claypool Publishers, 2018.

[Abiteboul and Duschka, 1998] Serge Abiteboul and Oliver M. Duschka. Complexity of answering queries using materialized views. In Proceedings of the Seventeenth ACM SIGACT-SIGMOD-SIGART Symposium on Principles of Database Systems (PODS 1998), pages 254-265, 1998.

[Abiteboul et al., 1995] Serge Abiteboul, Richard Hull, and Victor Vianu. Foundations of Databases. Addison Wesley Publishing Company, 1995.

[Afrati and Chirkova, 2019] Foto N. Afrati and Rada Chirkova. Answering Queries Using Views, Second Edition. Synthesis Lectures on Data Management. Morgan \& Claypool Publishers, 2019.

[Afrati et al., 1999] Foto N. Afrati, Manolis Gergatsoulis, and Theodoros G. Kavalieros. Answering queries using materialized views with disjunctions. In Proceedings of the Seventh International Conference on Database Theory (ICDT 1999), volume 1540 of Lecture Notes in Computer Science, https://link.springer.com/ bookseries/558, pages 435-452, 1999.

[Aho et al., 1979] Alfred V. Aho, Yehoshua Sagiv, and Jeffrey D. Ullman. Equivalences among relational expressions. SIAM Journal on Computing, 8(2):218-246, 1979.

[Antonioli et al., 2014] Natalia Antonioli, Francesco Castanò, Spartaco Coletta, Stefano Grossi, Domenico Lembo, Maurizio Lenzerini, Antonella Poggi, Emanuela Virardi, and Patrizia Castracane. Ontology-based data management for the italian public debt. In Proceedings of the Eighth International Conference on Formal Ontology in Information Systems (FOIS 2014), volume 267 of Frontiers in Artificial Intelligence and Applications, http://ebooks.iospress.nl/bookseries/ frontiers-in-artificial-intelligence-and-applications, pages 372-385, 2014. 
[Arenas et al., 2009] Marcelo Arenas, Jorge Pérez, and Cristian Riveros. The recovery of a schema mapping: Bringing exchanged data back. ACM Transactions on Database Systems, 34(4):22:1-22:48, 2009.

[Arenas et al., 2010] Marcelo Arenas, Pablo Barceló, Leonid Libkin, and Filip Murlak. Relational and XML Data Exchange. Synthesis Lectures on Data Management. Morgan \& Claypool Publishers, 2010.

[Arenas et al., 2014] Marcelo Arenas, Pablo Barceló, Leonid Libkin, and Filip Murlak. Foundations of Data Exchange. Cambridge University Press, 2014.

[Arenas et al., 2018] Marcelo Arenas, Georg Gottlob, and Andreas Pieris. Expressive languages for querying the semantic web. ACM Transactions on Database Systems, 43(3):13:1-13:45, 2018.

[Arora and Barak, 2009] Sanjeev Arora and Boaz Barak. Computational Complexity - A Modern Approach. Cambridge University Press, 2009.

[Artale et al., 2009] Alessandro Artale, Diego Calvanese, Roman Kontchakov, and Michael Zakharyaschev. The DL-Lite family and relations. Journal of Artificial Intelligence Research, 36:1-69, 2009.

[Asratian et al., 1998] Armen S. Asratian, Tristan Denley, and Roland Haggkvist. Bipartite Graphs and their Applications. Cambridge University Press, 1998.

[Baader et al., 2003] Franz Baader, Diego Calvanese, Deborah McGuinness, Daniele Nardi, and Peter F. Patel-Schneider, editors. The Description Logic Handbook: Theory, Implementation and Applications. Cambridge University Press, 2003.

[Baader et al., 2005] Franz Baader, Sebastian Brandt, and Carsten Lutz. Pushing the $\mathcal{E} \mathcal{L}$ envelope. In Proceedings of the Nineteenth International Joint Conference on Artificial Intelligence (IJCAI 2005), pages 364-369, 2005.

[Baader et al., 2017] Franz Baader, Ian Horrocks, Carsten Lutz, and Ulrike Sattler. An Introduction to Description Logic. Cambridge University Press, 2017.

[Beame et al., 2014] Paul Beame, Paraschos Koutris, and Dan Suciu. Skew in parallel query processing. In Proceedings of the Thirty-Third ACM SIGMODSIGACT-SIGART Symposium on Principles of Database Systems (PODS 2014), pages 212-223, 2014.

[Beeri and Vardi, 1984] Catriel Beeri and Moshe Y. Vardi. A proof procedure for data dependencies. Journal of the ACM, 31(4):718-741, 1984.

[Berners-Lee et al., 2001] Tim Berners-Lee, James Hendler, and Ora Lassila. The semantic web. Scientific American, 284(5):34-43, 2001.

[Bernstein, 1976] Philip A. Bernstein. Synthesizing third normal form relations from functional dependencies. ACM Transactions on Database Systems, 1(4):277-298, 1976 . 
[Bienvenu et al., 2015] Meghyn Bienvenu, Magdalena Ortiz, and Mantas Simkus. Regular path queries in lightweight description logics: Complexity and algorithms. Journal of Artificial Intelligence Research, 53:315-374, 2015.

[Bienvenu, 2016] Meghyn Bienvenu. Ontology-mediated query answering: Harnessing knowledge to get more from data. In Proceedings of the Twenty-Fifth International Joint Conference on Artificial Intelligence (IJCAI 2016), pages 4058-4061, 2016.

[Bizer et al., 2009] Christian Bizer, Tom Heath, and Tim Berners-Lee. Linked data the story so far. International Journal on Semantic Web and Information Systems, $5(3): 1-22,2009$.

[Bondy and Murty, 2008] Adrian Bondy and M. Ram Murty. Graph Theory. Graduate Texts in Mathematics. Springer, 2008.

[Börger et al., 1997] Egon Börger, Erich Grädel, and Yuri Gurevich. The Classical Decision Problem. Perspectives in Mathematical Logic. Springer, 1997.

[Brickley and Guha, 2014] Dan Brickley and R. V. Guha. RDF Schema 1.1. W3C Recommendation, World Wide Web Consortium, 2014. Available at https: //www.w3.org/TR/2014/REC-rdf-schema-20140225/.

[Calì et al., 2002] Andrea Calì, Diego Calvanese, Giuseppe De Giacomo, and Maurizio Lenzerini. On the expressive power of data integration systems. In Proceedings of the Twenty-First International Conference on Conceptual Modeling (ER 2002), volume 2503 of Lecture Notes in Computer Science, https: //link.springer.com/bookseries/558, pages 338-350, 2002.

[Calì et al., 2013] Andrea Calì, Georg Gottlob, and Michael Kifer. Taming the infinite chase: Query answering under expressive relational constraints. Journal of Artificial Intelligence Research, 48:115-174, 2013.

[Calvanese and De Giacomo, 2005] Diego Calvanese and Giuseppe De Giacomo. Data integration: A logic-based perspective. AI Magazine, 26(1):59-70, 2005.

[Calvanese et al., 2000] Diego Calvanese, Giuseppe De Giacomo, Maurizio Lenzerini, and Moshe Y. Vardi. What is view-based query rewriting? In Proceedings of the Seventh International Workshop on Knowledge Representation meets Databases (KRDB 2000), volume 29 of CEUR Electronic Workshop Proceedings, http: //ceur-ws.org/, pages 17-27, 2000.

[Calvanese et al., 2004a] Diego Calvanese, Giuseppe De Giacomo, Maurizio Lenzerini, Riccardo Rosati, and Guido Vetere. DL-Lite: Practical reasoning for rich Dls. In Proceedings of the 2004 International Workshop on Description Logics (DL 2004), volume 104 of CEUR Electronic Workshop Proceedings, http://ceur-ws.org/, 2004.

[Calvanese et al., 2004b] Diego Calvanese, Giuseppe De Giacomo, Maurizio Lenzerini, and Riccardo Rosati. Logical foundations of peer-to-peer data integration. In Proceedings of the Twenty-Third ACM SIGACT-SIGMOD-SIGART Symposium on Principles of Database Systems (PODS 2004), pages 241-251, 2004. 
[Calvanese et al., 2005] Diego Calvanese, Giuseppe De Giacomo, Domenico Lembo, Maurizio Lenzerini, and Riccardo Rosati. Dl-lite: Tractable description logics for ontologies. In Proceedings of the Twentieth National Conference on Artificial Intelligence (AAAI 2005), pages 602-607, 2005.

[Calvanese et al., 2007a] Diego Calvanese, Giuseppe De Giacomo, Domenico Lembo, Maurizio Lenzerini, and Riccardo Rosati. EQL-lite: Effective first-order query processing in description logics. In Proceedings of the Twentieth International Joint Conference on Artificial Intelligence (IJCAI 2007), pages 274-279, 2007.

[Calvanese et al., 2007b] Diego Calvanese, Giuseppe De Giacomo, Domenico Lembo, Maurizio Lenzerini, and Riccardo Rosati. Tractable reasoning and efficient query answering in description logics: The DL-Lite family. Journal of Automated Reasoning, 39(3):385-429, 2007.

[Calvanese et al., 2007c] Diego Calvanese, Giuseppe De Giacomo, Maurizio Lenzerini, and Moshe Y. Vardi. View-based query processing: On the relationship between rewriting, answering and losslessness. Theoretical Computer Science, 371(3):169-182, 2007.

[Calvanese et al., 2009] Diego Calvanese, Giuseppe De Giacomo, Domenico Lembo, Maurizio Lenzerini, Antonella Poggi, Mariano Rodríguez-Muro, and Riccardo Rosati. Ontologies and databases: The DL-Lite approach. In Reasoning Web. Semantic Technologies for Information Systems, Fifth International Summer School, Tutorial Lectures (RW 2009), volume 5689 of Lecture Notes in Computer Science, https://link.springer.com/bookseries/558, pages 255-356. 2009.

[Calvanese et al., 2011] Diego Calvanese, Giuseppe De Giacomo, Domenico Lembo, Maurizio Lenzerini, Antonella Poggi, Mariano Rodriguez-Muro, Riccardo Rosati, Marco Ruzzi, and Domenico Fabio Savo. The Mastro system for ontology-based data access. Semantic Web Journal, 2(1):43-53, 2011.

[Calvanese et al., 2012] Diego Calvanese, Giuseppe De Giacomo, Maurizio Lenzerini, and Moshe Y. Vardi. Query processing under GLAV mappings for relational and graph databases. Proceedings of the VLDB Endowment, 6(2):61-72, 2012.

[Calvanese et al., 2017] Diego Calvanese, Benjamin Cogrel, Sarah Komla-Ebri, Roman Kontchakov, Davide Lanti, Martin Rezk, Mariano Rodriguez-Muro, and Guohui Xiao. Ontop: Answering SPARQL queries over relational databases. Semantic Web Journal, 8(3):471-487, 2017.

[Carey et al., 2012] Michael J. Carey, Nicola Onose, and Michalis Petropoulos. Data services. Communications of the ACM, 55(6):86-97, 2012.

[Chandra and Merlin, 1977] Ashok K. Chandra and Philip M. Merlin. Optimal implementation of conjunctive queries in relational data bases. In Proceedings of the Ninth ACM Symposium on Theory of Computing (STOC 1977), pages 77-90, 1977.

[Chellas, 1980] Brian F. Chellas. Modal Logic - An Introduction. Cambridge University Press, 1980. 
[Chen et al., 2014] Min Chen, Shiwen Mao, Yin Zhang, and Victor C. M. Leung. Big Data - Related Technologies, Challenges and Future Prospects. Springer Briefs in Computer Science. Springer, 2014.

[Cima et al., 2017a] Gianluca Cima, Giuseppe De Giacomo, Maurizio Lenzerini, and Antonella Poggi. On the SPARQL metamodeling semantics entailment regime for OWL 2 QL ontologies. In Proceedings of the Seventh International Conference on Web Intelligence, Mining and Semantics (WIMS 2017), pages 10:1-10:6, 2017.

[Cima et al., 2017b] Gianluca Cima, Giuseppe De Giacomo, Maurizio Lenzerini, and Antonella Poggi. Querying OWL 2 QL ontologies under the SPARQL metamodeling semantics entailment regime. In Proceedings of the Twenty-Fifth Italian Symposium on Advanced Database Systems (SEBD 2017), volume 2037 of CEUR Electronic Workshop Proceedings, http://ceur-ws.org/, page 165, 2017.

[Cima et al., 2017c] Gianluca Cima, Maurizio Lenzerini, and Antonella Poggi. Semantic technology for open data publishing. In Proceedings of the Seventh International Conference on Web Intelligence, Mining and Semantics (WIMS 2017), page 1:1, 2017.

[Cima et al., 2019a] Gianluca Cima, Federico Croce, Maurizio Lenzerini, Antonella Poggi, and Elian Toccacieli. On queries with inequalities in $D L$-LiteR $\neq$. In Proceedings of the Thirty-Second International Workshop on Description Logics (DL 2019), volume 2373 of CEUR Electronic Workshop Proceedings, http:// ceur-ws.org/, 2019.

[Cima et al., 2019b] Gianluca Cima, Maurizio Lenzerini, and Antonella Poggi. Exploiting ontologies for explaining data sources semantics. In Proceedings of Discussion and Doctoral Consortium Papers of the Eighteenth International Conference of the Italian Association for Artificial Intelligence (DDC@AI*IA 2019), volume 2495 of CEUR Electronic Workshop Proceedings, http://ceur-ws.org/, pages 33-35, 2019.

[Cima et al., 2019c] Gianluca Cima, Maurizio Lenzerini, and Antonella Poggi. Reverse engineering of data services. In Proceedings of the Twenty-Seventh Italian Symposium on Advanced Database Systems (SEBD 2019), volume 2400 of CEUR Electronic Workshop Proceedings, http://ceur-ws.org/, 2019.

[Cima et al., 2019d] Gianluca Cima, Maurizio Lenzerini, and Antonella Poggi. Semantic characterization of data services through ontologies. In Proceedings of the Twenty-Eighth International Joint Conference on Artificial Intelligence (IJCAI 2019), pages 1647-1653, 2019.

[Cima et al., 2019e] Gianluca Cima, Charalampos Nikolaou, Egor V. Kostylev, Mark Kaminski, Bernardo Cuenca Grau, and Ian Horrocks. Bag semantics of dl-lite with functionality axioms. In Proceedings of the Eighteenth International Semantic Web Conference (ISWC 2019), volume 11778 of Lecture Notes in Computer Science, https://link.springer.com/bookseries/558, pages 128-144, 2019. 
[Cima et al., 2019f] Gianluca Cima, Charalampos Nikolaou, Egor V. Kostylev, Mark Kaminski, Bernardo Cuenca Grau, and Ian Horrocks. Bagging the DL-Lite family further. In Proceedings of the Thirty-Second International Workshop on Description Logics (DL 2019), volume 2373 of CEUR Electronic Workshop Proceedings, http://ceur-ws.org/, 2019.

[Cima et al., 2020a] Gianluca Cima, Domenico Lembo, Lorenzo Marconi, Riccardo Rosati, and Domenico Fabio Savo. Controlled query evaluation in ontologybased data access. In Proceedings of the Nineteenth International Semantic Web Conference (ISWC 2020), volume 12506 of Lecture Notes in Computer Science, https://link.springer.com/bookseries/558, pages 128-146, 2020.

[Cima et al., 2020b] Gianluca Cima, Domenico Lembo, Riccardo Rosati, and Domenico Fabio Savo. Controlled query evaluation in description logics through instance indistinguishability. In Proceedings of the Twenty-Ninth International Joint Conference on Artificial Intelligence (IJCAI 2020), pages 1791-1797, 2020.

[Cima et al., 2020c] Gianluca Cima, Domenico Lembo, Riccardo Rosati, and Domenico Fabio Savo. Controlled query evaluation in description logics through instance indistinguishability (extended abstract). In Proceedings of the ThirtyThird International Workshop on Description Logics (DL 2020), volume 2663 of CEUR Electronic Workshop Proceedings, http://ceur-ws.org/, 2020.

[Cima et al., 2020d] Gianluca Cima, Maurizio Lenzerini, and Antonella Poggi. Answering conjunctive queries with inequalities in DL-Lite $\mathscr{R}$. In Proceedings of the Thirty-Fourth AAAI Conference on Artificial Intelligence (AAAI 2020), pages 2782-2789, 2020.

[Cima et al., 2020e] Gianluca Cima, Maurizio Lenzerini, and Antonella Poggi. Nonmonotonic ontology-based abstractions of data services. In Proceedings of the Seventeenth International Conference on Principles of Knowledge Representation and Reasoning (KR 2020), pages 243-252, 2020.

[Cima, 2017] Gianluca Cima. Preliminary results on ontology-based open data publishing. In Proceedings of the Thirtieth International Workshop on Description Logics (DL 2017), volume 1879 of CEUR Electronic Workshop Proceedings, http: //ceur-ws.org/, 2017.

[Civili et al., 2013] Cristina Civili, Marco Console, Giuseppe De Giacomo, Domenico Lembo, Maurizio Lenzerini, Lorenzo Lepore, Riccardo Mancini, Antonella Poggi, Riccardo Rosati, Marco Ruzzi, Valerio Santarelli, and Domenico Fabio Savo. MASTRO STUDIO: managing ontology-based data access applications. Proceedings of the VLDB Endowment, 6(12):1314-1317, 2013.

[Codd, 1970] Edgar F. Codd. A relational model of data for large shared data banks. Communications of the ACM, 13(6):377-387, 1970.

[Codd, 1972] Edgar F. Codd. Relational completeness of data base sublanguages. Research Report / RJ / IBM / San Jose, California, RJ987, 1972. 
[Cook, 1973] Stephen A. Cook. A hierarchy for nondeterministic time complexity. Journal of Computer and System Sciences, 7(4):343-353, 1973.

[Corona et al., 2009] Claudio Corona, Marco Ruzzi, and Domenico Fabio Savo. Filling the gap between OWL 2 QL and quonto: Rowlkit. In Proceedings of the Twenty-Second International Workshop on Description Logics (DL 2009), 2009.

[Croce et al., 2020] Federico Croce, Gianluca Cima, Maurizio Lenzerini, and Tiziana Catarci. Ontology-based explanation of classifiers. In Proceedings of the Workshops of the EDBT/ICDT 2020 Joint Conference, volume 2578 of CEUR Electronic Workshop Proceedings, http://ceur-ws.org/, 2020.

[Cuenca Grau et al., 2008] Bernardo Cuenca Grau, Ian Horrocks, Boris Motik, Bijan Parsia, Peter F. Patel-Schneider, and Ulrike Sattler. OWL 2: The next step for OWL. Journal of Web Semantics, 6(4):309-322, 2008.

[Cuenca Grau, 2004] Bernardo Cuenca Grau. A possible simplification of the semantic web architecture. In Proceedings of the Thirteenth International World Wide Web Conference (WWW 2004), pages 704-713, 2004.

[De Giacomo et al., 2018] Giuseppe De Giacomo, Domenico Lembo, Maurizio Lenzerini, Antonella Poggi, and Riccardo Rosati. Using ontologies for semantic data integration. In A Comprehensive Guide Through the Italian Database Research Over the Last 25 Years, pages 187-202. 2018.

[Doan et al., 2012] AnHai Doan, Alon Y. Halevy, and Zachary G. Ives. Principles of Data Integration. Morgan Kaufmann, 2012.

[Duschka and Genesereth, 1997] Oliver M. Duschka and Michael R. Genesereth. Answering recursive queries using views. In Proceedings of the Sixteenth ACM SIGACT-SIGMOD-SIGART Symposium on Principles of Database Systems (PODS 1997), pages 109-116, 1997.

[Duschka and Genesereth, 1998] Oliver M. Duschka and Michael R. Genesereth. Query planning with disjunctive sources. In Proceedings of the AAAI-98 Workshop on AI and Information Integration, 1998.

[Duval et al., 2002] Erik Duval, Wayne Hodgins, Stuart A. Sutton, and Stuart Weibel. Metadata principles and practicalities. D-Lib Magazine, 8(4), 2002.

[Eiter et al., 1997] Thomas Eiter, Georg Gottlob, and Heikki Mannila. Disjunctive datalog. ACM Transactions on Database Systems, 22(3):364-418, 1997.

[European Union, Parliament and Council, 2016] European Union, Parliament and Council. General data protection regulation. Official Journal of the European Union, L 119/1, 2016.

[Fagin et al., 2005a] Ronald Fagin, Phokion G. Kolaitis, Renée J. Miller, and Lucian Popa. Data exchange: Semantics and query answering. Theoretical Computer Science, 336(1):89-124, 2005. 
[Fagin et al., 2005b] Ronald Fagin, Phokion G. Kolaitis, and Lucian Popa. Data exchange: Getting to the core. Journal of the ACM, 30(1):174-210, 2005.

[Fagin et al., 2005c] Ronald Fagin, Phokion G. Kolaitis, Lucian Popa, and Wang Chiew Tan. Composing schema mappings: Second-order dependencies to the rescue. ACM Transactions on Database Systems, 30(4):994-1055, 2005.

[Fagin et al., 2008] Ronald Fagin, Phokion G. Kolaitis, Lucian Popa, and Wang Chiew Tan. Quasi-inverses of schema mappings. ACM Transactions on Database Systems, 33(2):11:1-11:52, 2008.

[Fagin, 1977] Ronald Fagin. Multivalued dependencies and a new normal form for relational databases. ACM Transactions on Database Systems, 2(3):262-278, 1977.

[Fagin, 2007] Ronald Fagin. Inverting schema mappings. ACM Transactions on Database Systems, 32(4):25, 2007.

[Friedman et al., 1999] Marc Friedman, Alon Levy, and Todd Millstein. Navigational plans for data integration. In Proceedings of the Sixteenth National Conference on Artificial Intelligence (AAAI 1999), pages 67-73, 1999.

[Garey and Johnson, 1979] Michael R. Garey and David S. Johnson. Computers and Intractability: A Guide to the Theory of NP-Completeness. W.H. Freeman and Company, 1979.

[Garey et al., 1976] Michael R. Garey, David S. Johnson, and Larry J. Stockmeyer. Some simplified NP-complete graph problems. Theoretical Computer Science, 1(3):237-267, 1976.

[Glimm and Ogbuji, 2013] Birte Glimm and Chimezie Ogbuji. SPARQL 1.1 entailment regimes. W3C Recommendation, World Wide Web Consortium, 2013. Available at https://www.w3.org/TR/sparql11-entailment/.

[Glimm, 2011] Birte Glimm. Using SPARQL with RDFS and OWL entailment. In Reasoning Web. Semantic Technologies for the Web of Data - Seventh International Summer School, Tutorial Lectures (RW 2011), volume 6848 of Lecture Notes in Computer Science, https://link.springer.com/bookseries/558, pages 137201, 2011.

[Gogacz and Marcinkowski, 2016] Tomasz Gogacz and Jerzy Marcinkowski. Red spider meets a rainworm: Conjunctive query finite determinacy is undecidable. In Proceedings of the Thirty-Fifth ACM SIGMOD-SIGACT-SIGAI Symposium on Principles of Database Systems (PODS 2016), pages 121-134, 2016.

[Goodman and Flaxman, 2017] Bryce Goodman and Seth R. Flaxman. European union regulations on algorithmic decision-making and a "right to explanation". AI Magazine, 38(3):50-57, 2017.

[Gottlob and Pieris, 2015] Georg Gottlob and Andreas Pieris. Beyond SPARQL under OWL 2 QL entailment regime: Rules to the rescue. In Proceedings of the Twenty-Fourth International Joint Conference on Artificial Intelligence (IJCAI 2015), pages 2999-3007, 2015. 
[Gottlob et al., 2014] Georg Gottlob, Stanislav Kikot, Roman Kontchakov, Vladimir V. Podolskii, Thomas Schwentick, and Michael Zakharyaschev. The price of query rewriting in ontology-based data access. Artificial Intelligence, 213:42-59, 2014.

[Gruber, 1993] Thomas R. Gruber. A translation approach to portable ontology specifications. Knowledge Acquisition, 5(2):199-220, 1993.

[Gruber, 2018] Thomas R. Gruber. Ontology. In Encyclopedia of Database Systems - Second Edition. 2018.

[Gutiérrez-Basulto et al., 2012] Víctor Gutiérrez-Basulto, Yazmin Angélica IbáñezGarcía, and Roman Kontchakov. An update on query answering with restricted forms of negation. volume 7497 of Lecture Notes in Computer Science, https: //link.springer.com/bookseries/558, pages 75-89, 2012.

[Gutiérrez-Basulto et al., 2015] Víctor Gutiérrez-Basulto, Yazmin Angélica IbáñezGarcía, Roman Kontchakov, and Egor V. Kostylev. Queries with negation and inequalities over lightweight ontologies. Journal of Web Semantics, 35:184-202, 2015.

[Harris and Seaborne, 2013] Steve Harris and Andy Seaborne. SPARQL 1.1 query language. W3C Recommendation, World Wide Web Consortium, 2013. Available at http://www .w3.org/TR/sparql11-query

[Hartmanis and Stearns, 1965] Juris Hartmanis and Richard E. Stearns. On the computational complexity of algorithms. Transactions of the American Mathematical Society, 117:285-306, 1965.

[Imielinski and Lipski Jr., 1984] Tomasz Imielinski and Witold Lipski Jr. Incomplete information in relational databases. Journal of the ACM, 31(4):761-791, 1984.

[Karp, 1972] Richard M. Karp. Reducibility among combinatorial problems. In Proceedings of a Symposium on the Complexity of Computer Computations, The IBM Research Symposia Series, https://link.springer.com/bookseries/10380, pages 85-103, 1972.

[Ketsman and Suciu, 2017] Bas Ketsman and Dan Suciu. A worst-case optimal multi-round algorithm for parallel computation of conjunctive queries. In Proceedings of the Thirty-Sixth ACM SIGMOD-SIGACT-SIGAI Symposium on Principles of Database Systems (PODS 2017), pages 417-428, 2017.

[Kharlamov et al., 2017] Evgeny Kharlamov, Dag Hovland, Martin G. Skjæveland, Dimitris Bilidas, Ernesto Jiménez-Ruiz, Guohui Xiao, Ahmet Soylu, Davide Lanti, Martin Rezk, Dmitriy Zheleznyakov, Martin Giese, Hallstein Lie, Yannis E. Ioannidis, Yannis Kotidis, Manolis Koubarakis, and Arild Waaler. Ontology based data access in statoil. Journal of Web Semantics, 44:3-36, 2017.

[Klug, 1988] Anthony C. Klug. On conjunctive queries containing inequalities. Journal of the ACM, 35(1):146-160, 1988. 
[Knopp, 1996] Konrad Knopp. Theory of Functions, Parts I and II. Dover Publications, 1996.

[Kolaitis and Vardi, 2000] Phokion G. Kolaitis and Moshe Y. Vardi. Conjunctivequery containment and constraint satisfaction. Journal of Computer and System Sciences, 61(2):302-332, 2000.

[Kolaitis and Vardi, 2008] Phokion G. Kolaitis and Moshe Y. Vardi. A logical approach to constraint satisfaction. In Complexity of Constraints - An Overview of Current Research Themes, volume 5250 of Lecture Notes in Computer Science, https://link.springer.com/bookseries/558, pages 125-155, 2008.

[Kolaitis et al., 1998] Phokion G. Kolaitis, David L. Martin, and Madhukar N. Thakur. On the complexity of the containment problem for conjunctive queries with built-in predicates. In Proceedings of the Seventeenth ACM SIGACT-SIGMODSIGART Symposium on Principles of Database Systems (PODS 1998), pages 197-204, 1998.

[Kontchakov et al., 2014] Roman Kontchakov, Martin Rezk, Mariano RodriguezMuro, Guohui Xiao, and Michael Zakharyaschev. Answering SPARQL queries over databases under OWL 2 QL entailment regime. In Proceedings of the Thirteenth International Semantic Web Conference (ISWC 2014), volume 8796 of Lecture Notes in Computer Science, https://link.springer.com/bookseries/ 558, pages 552-567, 2014.

[Koutris et al., 2016] Paraschos Koutris, Paul Beame, and Dan Suciu. Worst-case optimal algorithms for parallel query processing. In Proceedings of the Nineteenth International Conference on Database Theory (ICDT 2016), volume 48 of Leibniz International Proceedings in Informatics, https://www.dagstuhl.de/ en/publications/lipics, pages 8:1-8:18, 2016.

[Koutris et al., 2017] Paraschos Koutris, Tova Milo, Sudeepa Roy, and Dan Suciu. Answering conjunctive queries with inequalities. Theory of Computing Systems, 61(1):2-30, 2017.

[Lembo et al., 2015] Domenico Lembo, Maurizio Lenzerini, Riccardo Rosati, Marco Ruzzi, and Domenico Fabio Savo. Inconsistency-tolerant query answering in ontology-based data access. Journal of Web Semantics, 33:3-29, 2015.

[Lembo et al., 2017] Domenico Lembo, Riccardo Rosati, Valerio Santarelli, Domenico Fabio Savo, and Evgenij Thorstensen. Mapping repair in ontology-based data access evolving systems. In Proceedings of the Twenty-Sixth International Joint Conference on Artificial Intelligence (IJCAI 2017), pages 1160-1166, 2017.

[Lenzerini, 2002] Maurizio Lenzerini. Data integration: A theoretical perspective. In Proceedings of the Twenty-First ACM SIGACT-SIGMOD-SIGART Symposium on Principles of Database Systems (PODS 2002), pages 233-246, 2002.

[Lenzerini, 2011] Maurizio Lenzerini. Ontology-based data management. In Proceedings of the Twentieth International Conference on Information and Knowledge Management (CIKM 2011), pages 5-6, 2011. 
[Lenzerini, 2018] Maurizio Lenzerini. Managing data through the lens of an ontology. AI Magazine, 39(2):65-74, 2018.

[Levesque and Lakemeyer, 2000] Hector J. Levesque and Gerhard Lakemeyer. The Logic of Knowledge Bases. MIT Press, 2000.

[Levesque, 1984] Hector J. Levesque. Foundations of a functional approach to knowledge representation. Artificial Intelligence, 23(2):155-212, 1984.

[Levy et al., 1995] Alon Y. Levy, Alberto O. Mendelzon, Yehoshua Sagiv, and Divesh Srivastava. Answering queries using views. In Proceedings of the Fourteenth ACM SIGACT-SIGMOD-SIGART Symposium on Principles of Database Systems (PODS 1995), pages 95-104, 1995.

[Levy et al., 1996] Alon Y. Levy, Anand Rajaraman, and Joann J. Ordille. Querying heterogeneous information sources using source descriptions. In Proceedings of the Twenty-Second International Conference on Very Large Data Bases (VLDB 1996), pages 251-262, 1996.

[Lobo et al., 1992] Jorge Lobo, Jack Minker, and Arcot Rajasekar. Foundations of Disjunctive Logic Programming. Logic Programming. MIT Press, 1992.

[Lutz et al., 2018] Carsten Lutz, Johannes Marti, and Leif Sabellek. Query expressibility and verification in ontology-based data access. In Principles of Knowledge Representation and Reasoning: Proceedings of the Sixteenth International Conference (KR 2018), pages 389-398, 2018.

[Machan, 2009] Dyan Machan. DaaS: The new information goldmine. Wall Street Journal, pages 1-3, 2009.

[Madry, 2005] Aleksander Madry. Data exchange: On the complexity of answering queries with inequalities. Information Processing Letters, 94(6):253-257, 2005.

[Maier et al., 1979] David Maier, Alberto O. Mendelzon, and Yehoshua Sagiv. Testing implications of data dependencies. ACM Transactions on Database Systems, 4(4):455-469, 1979.

[Meseguer and Qian, 1993] José Meseguer and Xiaolei Qian. A logical semantics for object-oriented databases. In Proceedings of the 1993 ACM SIGMOD International Conference on Management of Data (SIGMOD 1993), pages 89-98, 1993.

[Miles and Bechhofer, 2009] Alistair Miles and Sean Bechhofer. SKOS Simple Knowledge Organization System. W3C Recommendation, World Wide Web Consortium, 2009. Available at http://www.w3.org/TR/skos-reference.

[Miles and Pérez-Agüera, 2007] Alistair Miles and José R. Pérez-Agüera. SKOS: Simple knowledge organisation for the web. Cataloging and Classification Quarterly, 43(3-4):69-83, 2007.

[Millstein et al., 2003] Todd D. Millstein, Alon Y. Halevy, and Marc Friedman. Query containment for data integration systems. Journal of Computer and System Sciences, 66(1):20-39, 2003. 
[Motik et al., 2012] Boris Motik, Bernardo Cuenca Grau, Ian Horrocks, Zhe Wu, Achille Fokoue, and Carsten Lutz. OWL 2 Web Ontology Language profiles (second edition). W3C Recommendation, World Wide Web Consortium, 2012. Available at http://www.w3.org/TR/ow12-profiles/.

[Nash et al., 2010] Alan Nash, Luc Segoufin, and Victor Vianu. Views and queries: Determinacy and rewriting. ACM Transactions on Database Systems, 35(3):21:121:41, 2010.

[Nikolaou et al., 2019] Charalampos Nikolaou, Egor V. Kostylev, George Konstantinidis, Mark Kaminski, Bernardo Cuenca Grau, and Ian Horrocks. Foundations of ontology-based data access under bag semantics. Artificial Intelligence, 274:91-132, 2019 .

[OECD, 2015] OECD. Making open science a reality. OECD Science, Technology and Industry Policy Papers, (25), 2015.

[Ortiz, 2018] Magdalena Ortiz. Improving data management using domain knowledge. In Proceedings of the Twenty-Seventh International Joint Conference on Artificial Intelligence (IJCAI 2018), pages 5709-5713, 2018.

[Papadimitriou, 1994] Christos H. Papadimitriou. Computational Complexity. Addison Wesley Publishing Company, 1994.

[Pentland, 2013] Alex Pentland. The data-driven society. Scientific American, 309(4):78-83, 2013.

[Poggi et al., 2008] Antonella Poggi, Domenico Lembo, Diego Calvanese, Giuseppe De Giacomo, Maurizio Lenzerini, and Riccardo Rosati. Linking data to ontologies. Journal on Data Semantics, X:133-173, 2008.

[Poggi, 2016] Antonella Poggi. On the SPARQL direct semantics entailment regime for OWL 2 QL. In Proceedings of the Twenty-Ninth International Workshop on Description Logics (DL 2016), volume 1577 of CEUR Electronic Workshop Proceedings, http://ceur-ws.org/, 2016.

[Rashid et al., 2020] Sabbir M. Rashid, James P. McCusker, Paulo Pinheiro, Marcello P. Bax, Henrique Santos, Jeanette A. Stingone, Amar K. Das, and Deborah L. McGuinness. The semantic data dictionary - an approach for describing and annotating data. Data Intelligence, pages 443-486, 2020.

[Reingold, 2008] Omer Reingold. Undirected connectivity in log-space. Journal of the ACM, 55(4):17:1-17:24, 2008.

[Reiter, 1992] Raymond Reiter. What should a database know? Journal of Logic Programming, 14(1\&2):127-153, 1992.

[Rosati and Almatelli, 2010] Riccardo Rosati and Alessandro Almatelli. Improving query answering over DL-Lite ontologies. In Principles of Knowledge Representation and Reasoning: Proceedings of the Twelfth International Conference (KR 2010), pages 290-300, 2010. 
[Rosati, 2007] Riccardo Rosati. The limits of querying ontologies. In Proceedings of the Eleventh International Conference on Database Theory (ICDT 2007), volume 4353 of Lecture Notes in Computer Science, https://link.springer.com/ bookseries/558, pages 164-178, 2007.

[Sagiv and Yannakakis, 1980] Yehoshua Sagiv and Mihalis Yannakakis. Equivalences among relational expressions with the union and difference operators. Journal of the ACM, 27(4):633-655, 1980.

[Stockmeyer, 1976] Larry J. Stockmeyer. The polynomial-time hierarchy. Theoretical Computer Science, 3(1):1-22, 1976.

[ten Cate et al., 2009] Balder ten Cate, Laura Chiticariu, Phokion G. Kolaitis, and Wang-Chiew Tan. Laconic schema mappings: Computing the core with SQL queries. Proceedings of the VLDB Endowment, 2(1):1006-1017, 2009.

[Turing, 1937] Alan M. Turing. On computable numbers, with an application to the Entscheidungsproblem. Proceedings of the London Mathematical Society, 42(1):230-265, 1937.

[van der Meyden, 1993] Ron van der Meyden. Recursively indefinite databases. Theoretical Computer Science, 116(1):151-194, 1993.

[van der Meyden, 1997] Ron van der Meyden. The complexity of querying indefinite data about linearly ordered domains. Journal of Computer and System Sciences, 54(1):113-135, 1997.

[Vardi, 1982] Moshe Y. Vardi. The complexity of relational query languages. In Proceedings of the Fourteenth ACM Symposium on Theory of Computing (STOC 1982), pages 137-146, 1982.

[Vollmer, 1999] Heribert Vollmer. Introduction to Circuit Complexity - A Uniform Approach. Texts in Theoretical Computer Science. An EATCS Series. Springer, 1999.

[Weibel et al., 1998] Stuart Weibel, John A. Kunze, Carl Lagoze, and Misha Wolf. Dublin core metadata for resource discovery. Request for Comments, 2413:1-8, 1998.

[Wilkinson et al., 2016] Mark D. Wilkinson, Michel Dumontier, IJsbrand Jan Aalbersberg, Gabrielle Appleton, Myles Axton, Arie Baak, Niklas Blomberg, JanWillem Boiten, Luiz Bonino da Silva Santos, Philip E. Bourne, Jildau Bouwman, Anthony J. Brookes, Tim Clark, Mercè Crosas, Ingrid Dillo, Olivier Dumon, Scott Edmunds, Chris T. Evelo, Richard Finkers, Alejandra Gonzalez-Beltran, Alasdair J. G. Gray, Paul Groth, Carole Goble, Jeffrey S. Grethe, Jaap Heringa, Peter A. C. 't Hoen, Rob Hooft, Tobias Kuhn, Ruben Kok, Joost Kok, Scott J. Lusher, Maryann E. Martone, Albert Mons, Abel L. Packer, Bengt Persson, Philippe Rocca-Serra, Marco Roos, Rene van Schaik, Susanna-Assunta Sansone, Erik Schultes, Thierry Sengstag, Ted Slater, George Strawn, Morris A. Swertz, Mark Thompson, Johan van der Lei, Erik van Mulligen, Jan Velterop, Andra Waagmeester, Peter Wittenburg, Katherine Wolstencroft, Jun Zhao, and Barend Mons. 
The FAIR guiding principles for scientific data management and stewardship. Scientific Data, 3, 2016.

[Xiao et al., 2018] Guohui Xiao, Diego Calvanese, Roman Kontchakov, Domenico Lembo, Antonella Poggi, Riccardo Rosati, and Michael Zakharyaschev. Ontologybased data access: A survey. In Proceedings of the Twenty-Seventh International Joint Conference on Artificial Intelligence (IJCAI 2018), pages 5511-5519, 2018.

[Zaniolo, 1982] Carlo Zaniolo. Database relations with null values. In Proceedings of the First ACM Symposium on Principles of Database Systems (PODS 1982), pages 27-33, 1982.

[Zheng et al., 2013] Zibin Zheng, Jieming Zhu, and Michael R. Lyu. Servicegenerated big data and big data-as-a-service: An overview. In Proceedings of the IEEE International Congress on Big Data (BigData Congress 2013), pages 403-410, 2013. 